Supporting Information

\title{
Enzymatic Pictet-Spengler Reaction: Computational Study of Mechanism and Enantioselectivity of Norcoclaurine Synthase
}

\author{
Xiang Sheng and Fahmi Himo* \\ Department of Organic Chemistry, Arrhenius Laboratory, Stockholm University, \\ SE-10691 Stockholm, Sweden \\ *Correspondence: fahmi.himo@su.se
}

\section{Table of Contents}

1. Results concerning the alternative "HPAA-first" mechanism. S2

2. The pathway with both Glu110 and Asp141 in the deprotonated states................................S6

3. Geometries and relative energies of enzyme-substrate complexes...................................S8

4. Optimized structures for the "dopamine-first" mechanism with full model...........................S11

5. Geometries and energy decomposition of the barrier-less transition states ...........................S14

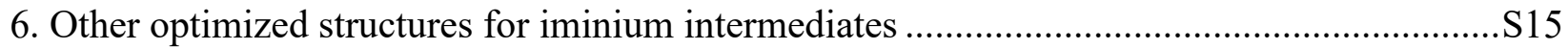

7. Other geometries and relative energies of the rate-limiting TS ......................................... 17

8. Absolute energies and energy corrections for stationary points ..........................................S19

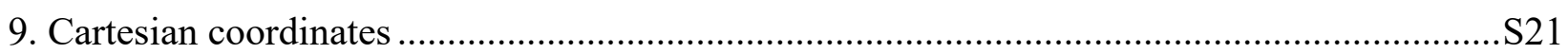




\section{Results concerning the alternative "HPAA-first" mechanism}

The alternative "HPAA-first" mechanism turned out to be associated with prohibitively high energies. The detailed "HPAA-first" mechanism is given in Scheme S1. The calculated energy profile is shown in Figure S1, and the optimized structures are shown in Figure S2.

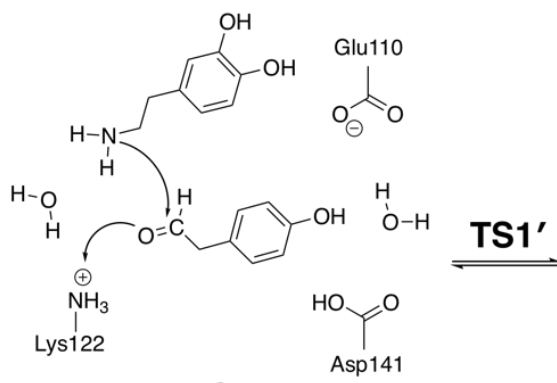

E:S'

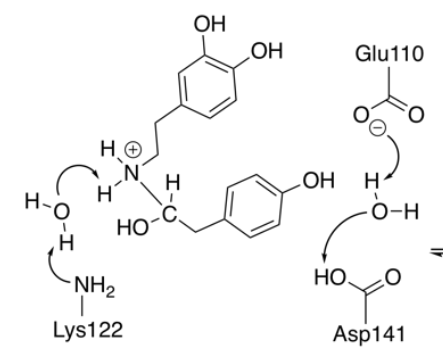

Int1'

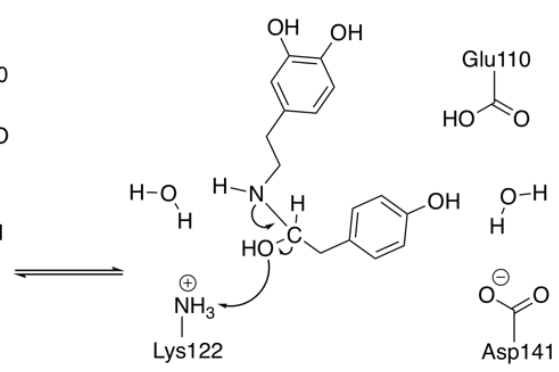

Int2'

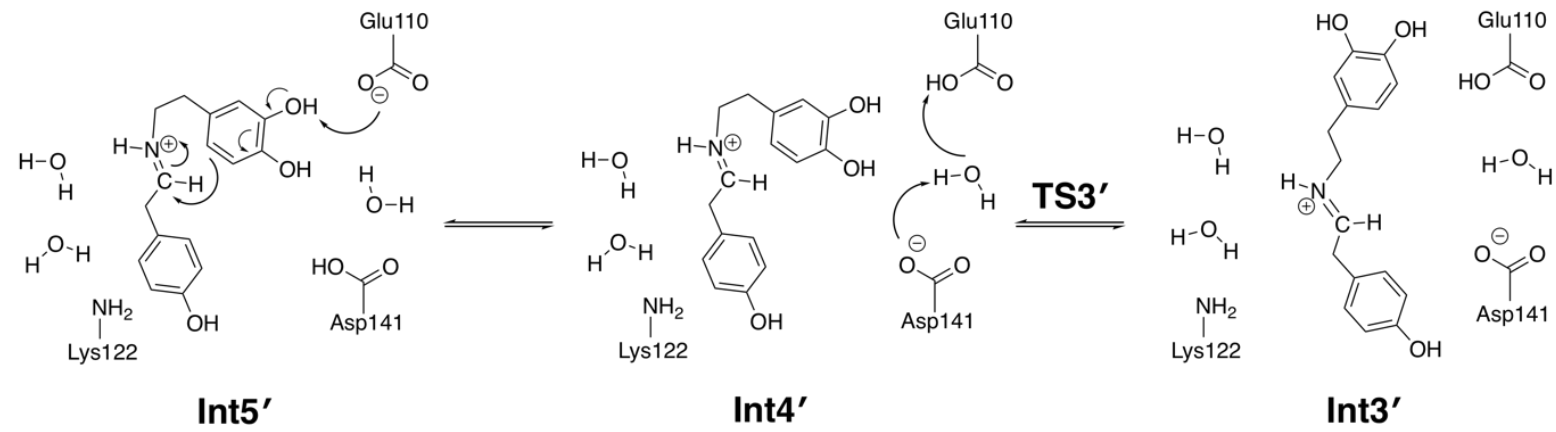

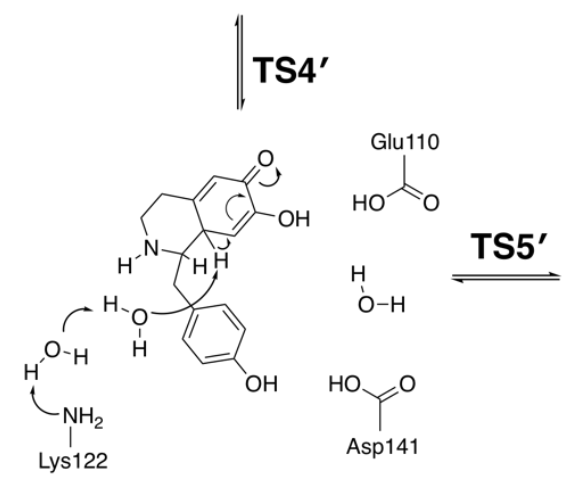

Int6'

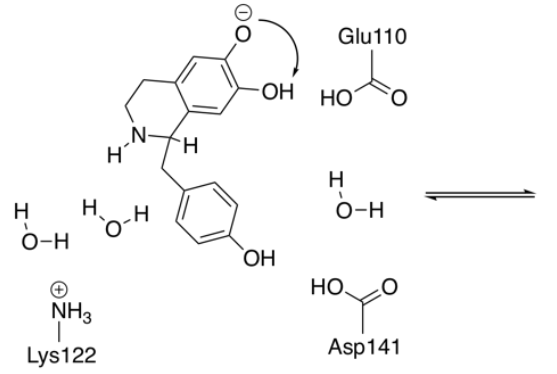

Int7'

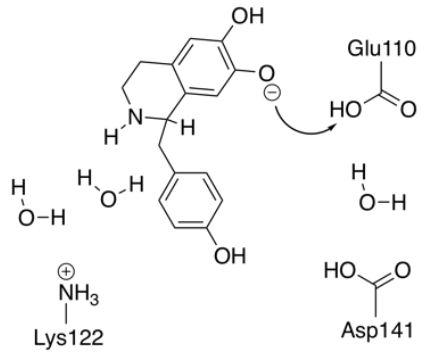

Int8'

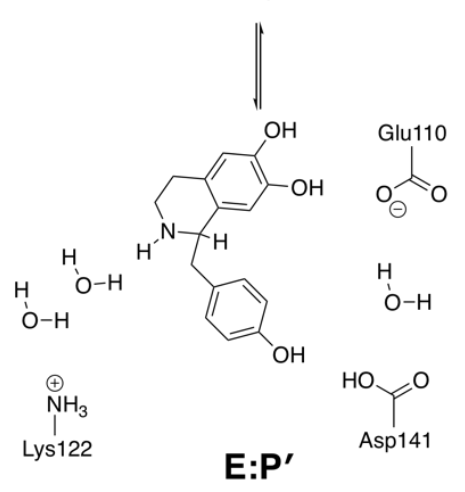

Scheme S1. The alternative "HPAA-first" mechanism considered in the present study. 
In the "HPAA-first" mechanism, the reaction starts with the formation of a $\mathrm{C}-\mathrm{N}$ bond between two substrates, concertedly with a proton transfer from Lys122 to the carbonyl oxygen of 4-HPAA. This step is calculated to have a barrier of $15.7 \mathrm{kcal} / \mathrm{mol}$ relative to $\mathbf{E}: \mathbf{S}$ (Figure $\mathrm{S} 1$ ), and the resulting Int1' is $11.4 \mathrm{kcal} / \mathrm{mol}$ higher than E:S. Next, a barrier-less proton transfer from the protonated amino group to the neutral Lys122 via a water molecule takes place to give Int2', which is $7.2 \mathrm{kcal} / \mathrm{mol}$ higher than E:S. The following dehydration is calculated to have a barrier of $18.8 \mathrm{kcal} / \mathrm{mol}$ relative to $\mathbf{E}: \mathbf{S}$, and the formed iminium intermediate (Int3') is $11.4 \mathrm{kcal} / \mathrm{mol}$ higher than $\mathbf{E}: \mathbf{S}$.

In Int3', the nucleophilic catechol moiety is not accessible to attack the iminium group unless Int3' undergoes a conformational change, which is a rotation around the $\mathrm{C} \alpha-\mathrm{C} \beta$ bond of the dopamine moiety. The energy barrier for this process is estimated to be ca $11 \mathrm{kcal} / \mathrm{mol}$ relative to Int3', i.e. ca $22 \mathrm{kcal} / \mathrm{mol}$ relative to $\mathbf{E}: \mathbf{S}$. This value is estimated by a constrained scan of the dihedral angle between the aromatic ring of the dopamine moiety and the iminium group $(\angle \mathrm{C} 4-\mathrm{C} \alpha-\mathrm{C} \beta-\mathrm{N})$. The result of this rotation is Int4', which is calculated to be $8.4 \mathrm{kcal} / \mathrm{mol}$ lower than Int3'.

Subsequently, after a proton transfer from Glu110 to Asp141, the cyclization step takes place, concertedly with the deprotonation of $\mathrm{C} 2-\mathrm{OH}$ by Glu110. Both the corresponding transition state (TS4') and formed intermediate (Int6') are calculated to be associated with high energies, +28.4 and $+21.7 \mathrm{kcal} / \mathrm{mol}$, respectively, relative to $\mathbf{E}: \mathbf{S}$.

Similarly to the dopamine-first" mechanism, the following deprotonation of $\mathrm{C} 5-\mathrm{H}$ is calculated to be rate-limiting. However, in the "HPAA-first" mechanism the only available general base is Lys122, and the proton transfer from $\mathrm{C} 5-\mathrm{H}$ to Lys122 takes place via two water molecules. The calculated energy of the transition state $\left(\mathbf{T S 5}^{\prime}\right)$ here is $+19.8 \mathrm{kcal} / \mathrm{mol}$ relative to Int6', which means that the overall barrier compared to $\mathbf{E}: \mathbf{S}$ is as high as $41.5 \mathrm{kcal} / \mathrm{mol}$.

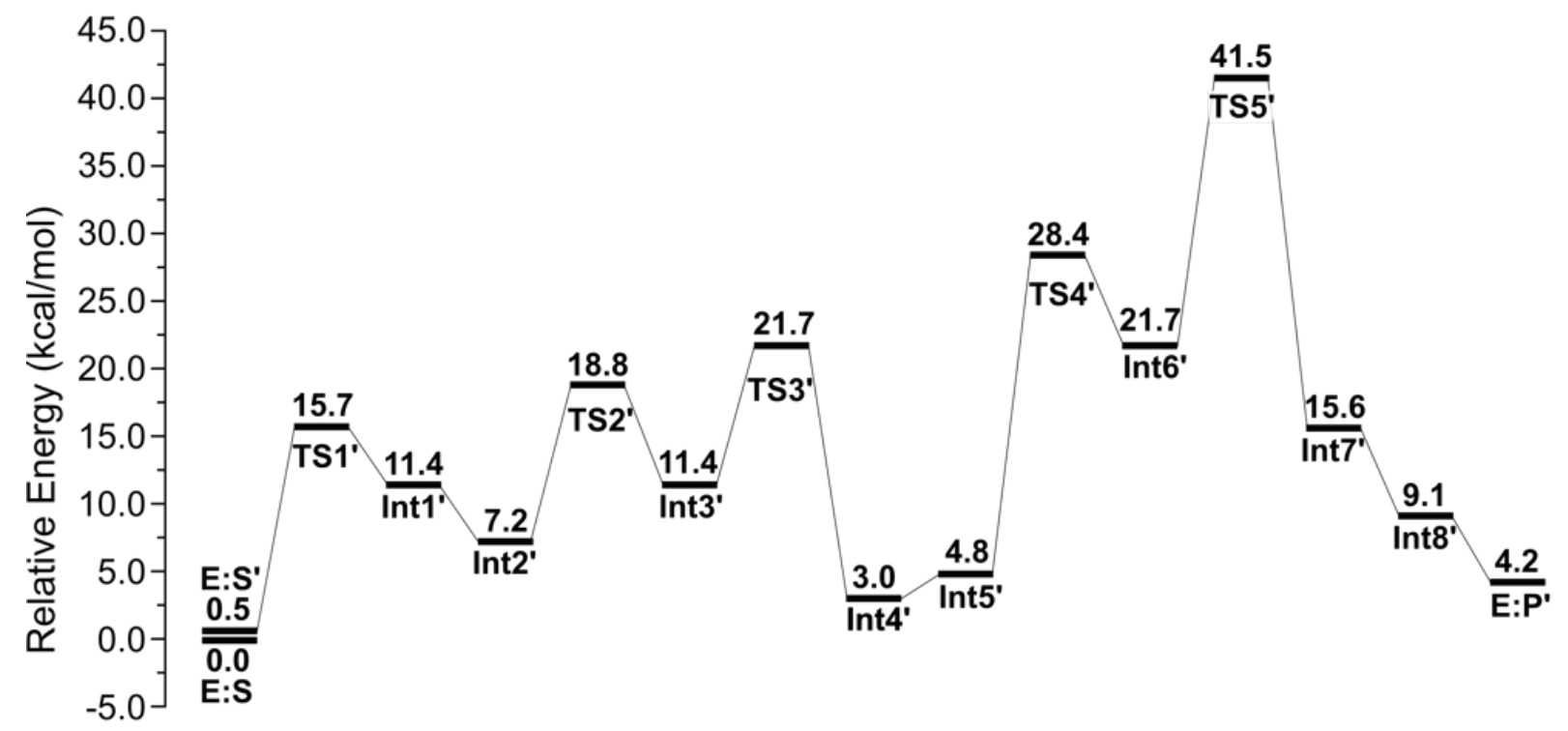

Figure S1. Calculated energy profile for the "HPAA-first" mechanism. 

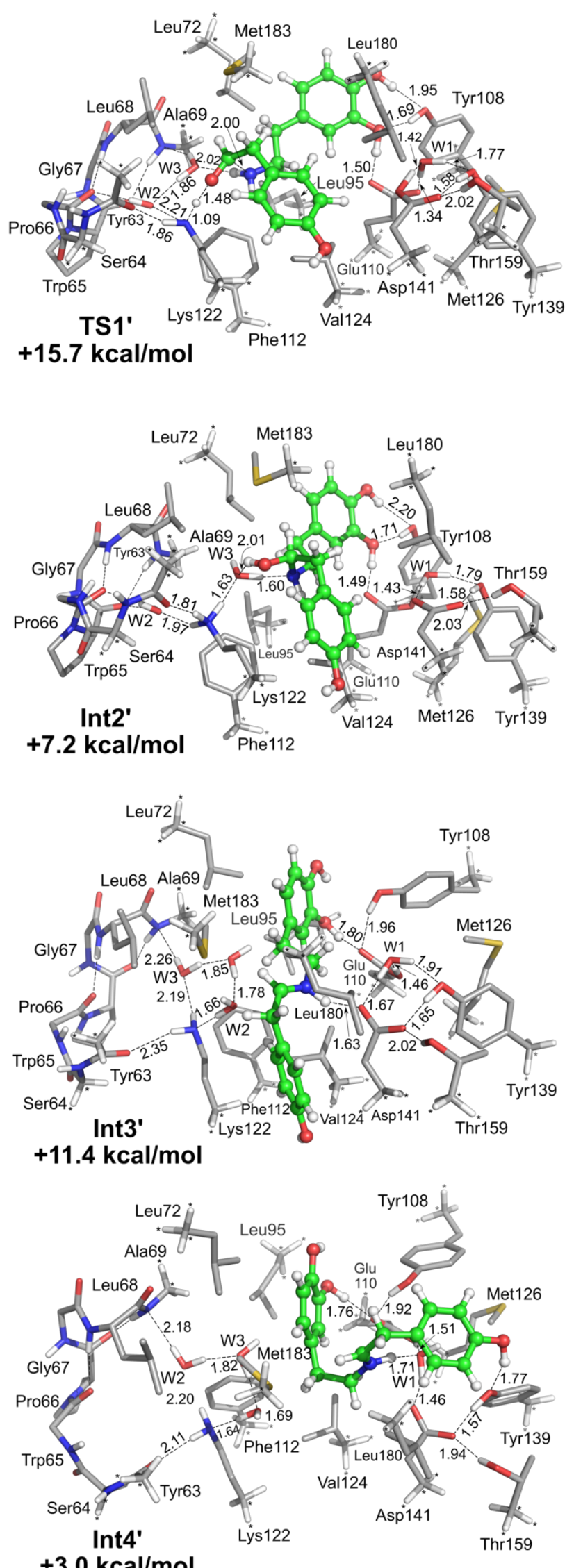
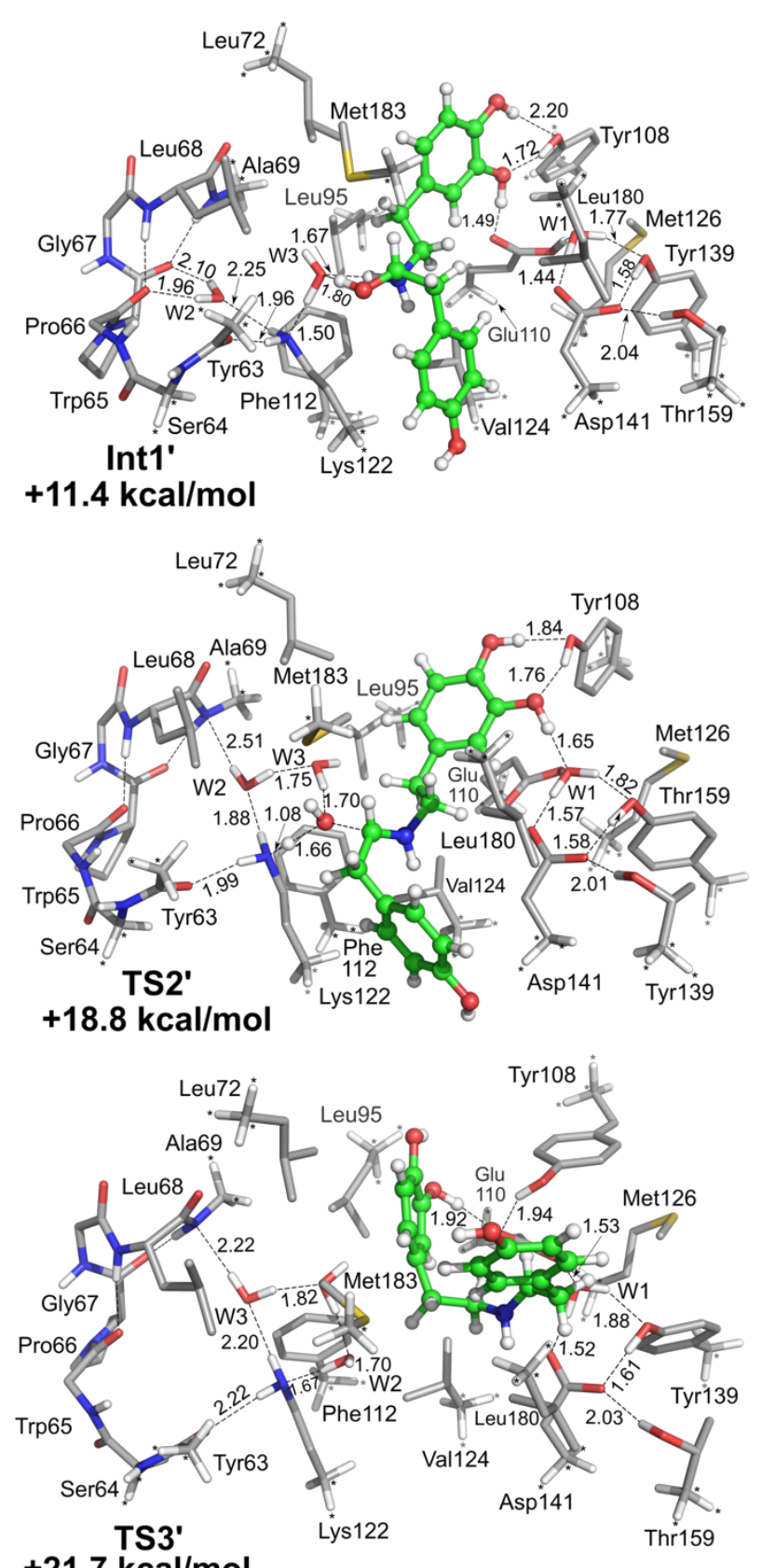

+21.7 kcal $/ \mathrm{mol}$

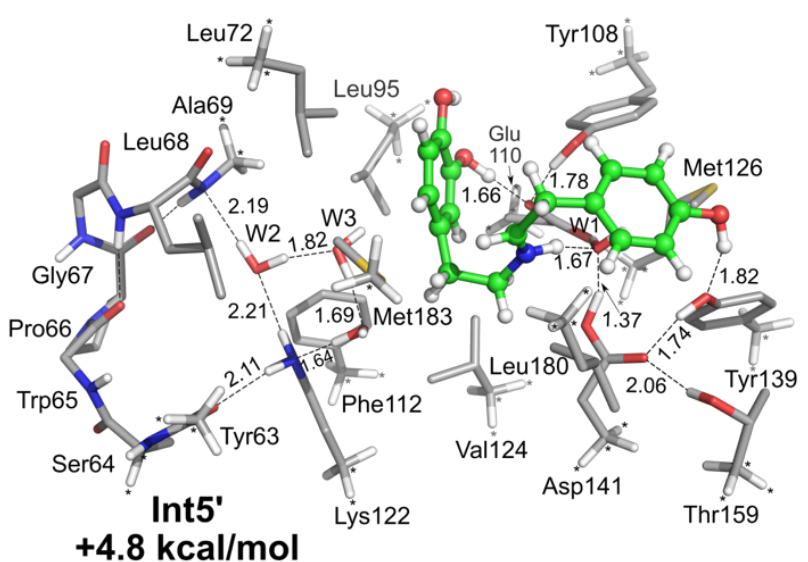

$+4.8 \mathrm{kcal} / \mathrm{mol}$ 


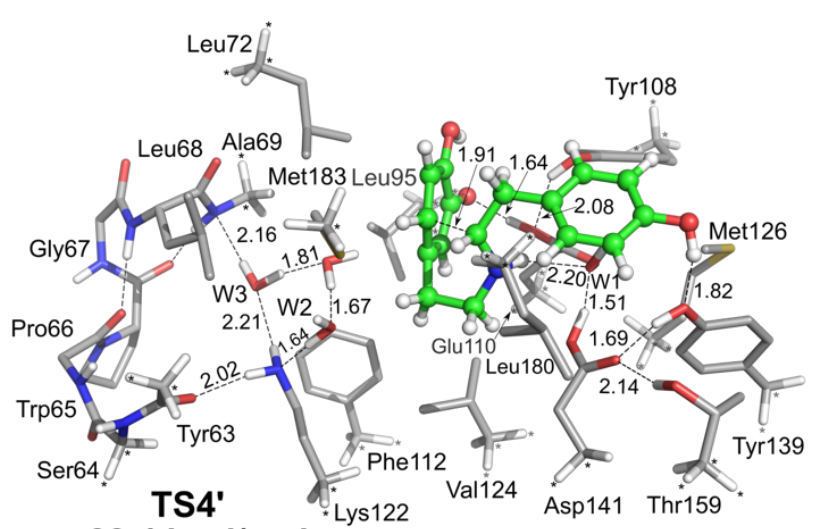

$+28.4 \mathrm{kcal} / \mathrm{mol}$

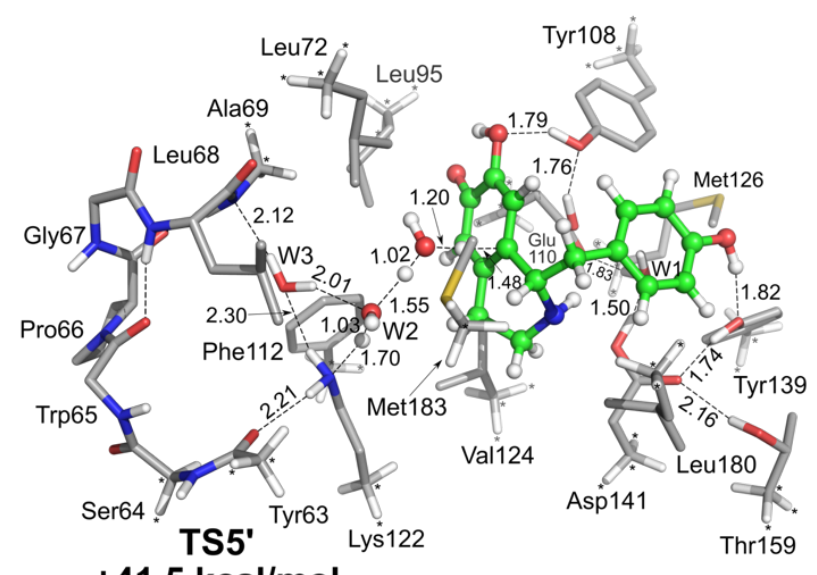

$+41.5 \mathrm{kcal} / \mathrm{mol}$

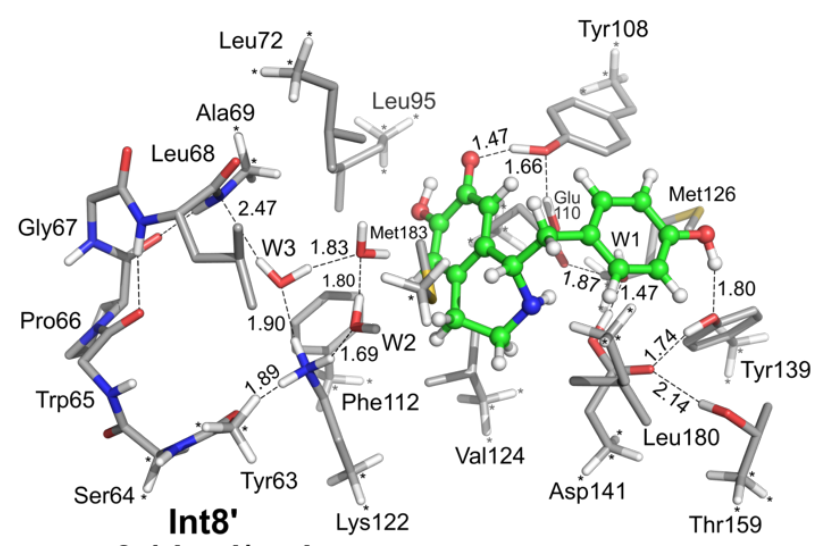

$+9.1 \mathrm{kcal} / \mathrm{mol}$

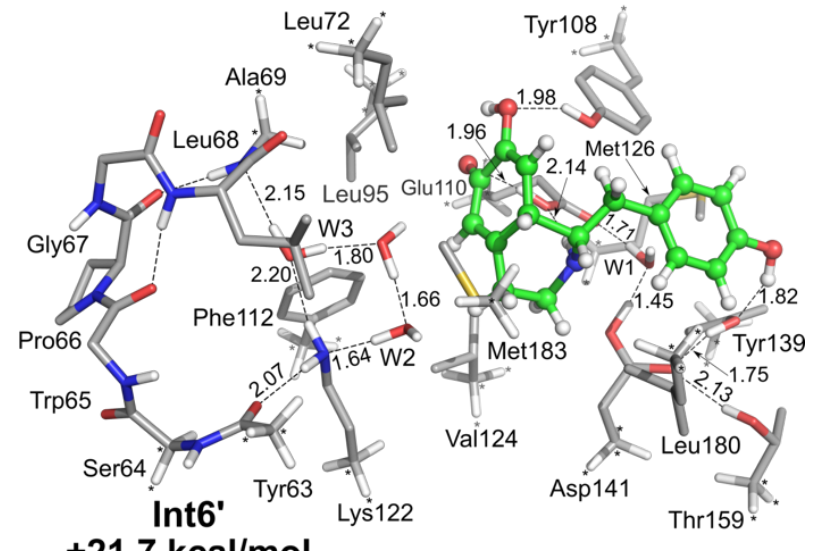

$+21.7 \mathrm{kcal} / \mathrm{mol}$

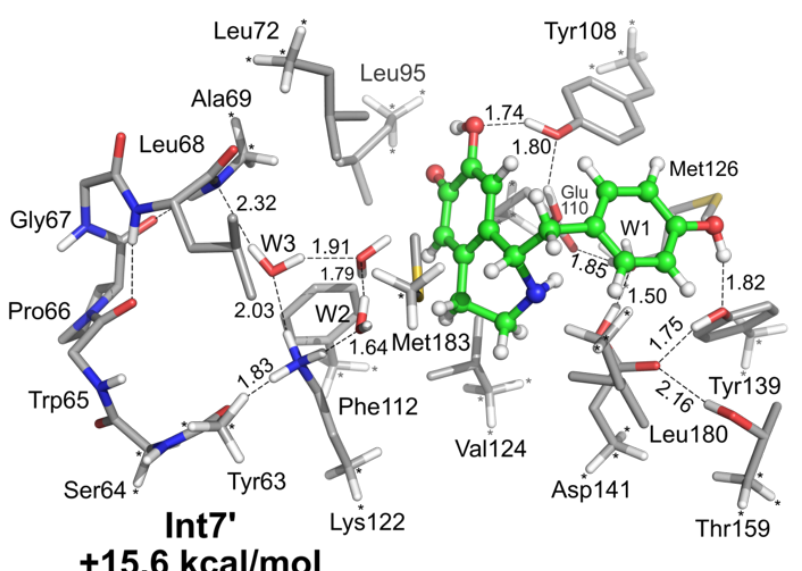

$+15.6 \mathrm{kcal} / \mathrm{mol}$

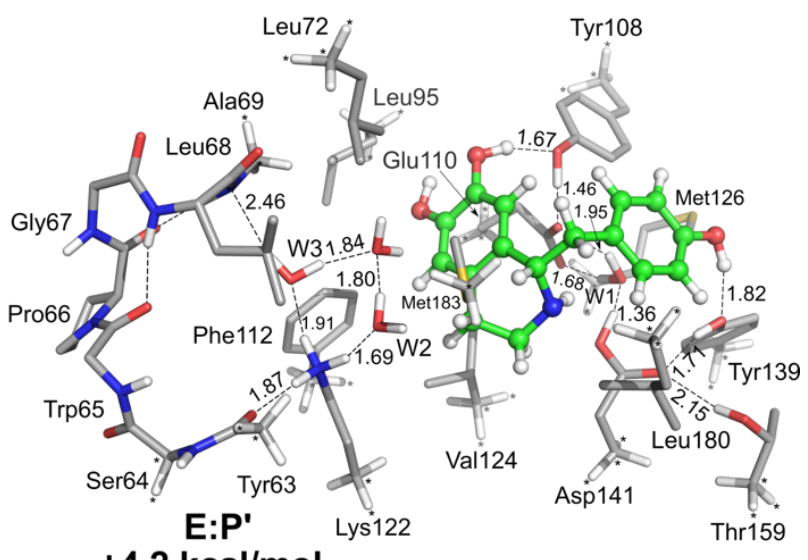

$+4.2 \mathrm{kcal} / \mathrm{mol}$

Figure S2. Optimized structures of the intermediates and transition states involved in the "HPAAfirst" mechanism. Energies relative to $\mathbf{E}: \boldsymbol{S}$ are given. 


\section{The pathway with both Glu110 and Asp141 in the deprotonated states}

For the Glu110 and Asp141 residues, that are bridged by a water molecule, the case with both two being in the deprotonated states was examined for the "dopamine-first" mechanism (Figure S3 for calculated energy profile and Figure S4 for optimized structures). During the geometry optimization of the enzyme-substrate complex, a proton transfer from the protonated amino group of dopamine to Glu110 took place spontaneously, resulting in both the amino group of dopamine and Glu110 being neutral $\left(\mathbf{E}: \mathbf{S}_{T}\right)$.

From there, the reaction pathway starts with the $\mathrm{C}-\mathrm{N}$ bond formation, resulting in the formation of Int1 $\mathbf{1}_{\mathbf{T}}$ with similar energy as the $\mathbf{E}: \mathbf{S}_{\mathbf{T}}$. The calculated energy of the corresponding transition state $\left(\mathbf{T S 1}_{\mathbf{T}}\right)$ is $+6.8 \mathrm{kcal} / \mathrm{mol}$ relative to $\mathbf{E}: \mathbf{S}_{\mathbf{T}}$. Next, a barrier-less proton transfer from Glu110 to the alkoxide takes place, and the formed intermediate (Int2 $\left.\mathbf{I}_{\mathrm{T}}\right)$ is very stable, with a calculated energy of $-12.4 \mathrm{kcal} / \mathrm{mol}$ relative to $\mathbf{E}: \mathbf{S}_{\mathbf{T}}$. From $\mathbf{I n t}_{\mathbf{T}}$, another proton transfer takes place, from the protonated amine group to Glu110, forming Int3 T. Subsequently, the dehydration step takes place with a reasonable barrier of $15.6 \mathrm{kcal} / \mathrm{mol}$ relative to Int2 $_{\mathbf{T}}$. The formed iminium intermediate $\left(\right.$ Int $\left._{\mathbf{T}}\right)$ is calculated to be $9.1 \mathrm{kcal} / \mathrm{mol}$ higher than $\mathbf{I n t} \mathbf{2}_{\mathbf{T}}$.

The reaction is then supposed to proceed with the cyclization by a $\mathrm{C}-\mathrm{C}$ formation. However, we could not locate the cyclized intermediate. All attempts to optimize this intermediate converged into the previous iminium intermediate (Int4 $\left.\mathbf{T}_{\mathrm{T}}\right)$. Forcing the formation of a cyclized intermediate (Int5T), by constraining the corresponding C-C bond distance to $1.6 \AA$, resulted in a very high energy, +21 $\mathrm{kcal} / \mathrm{mol}$ relative to Int $\mathbf{2}_{\mathrm{T}}$. This scenario is thus energetically unfavorable and can be ruled out.

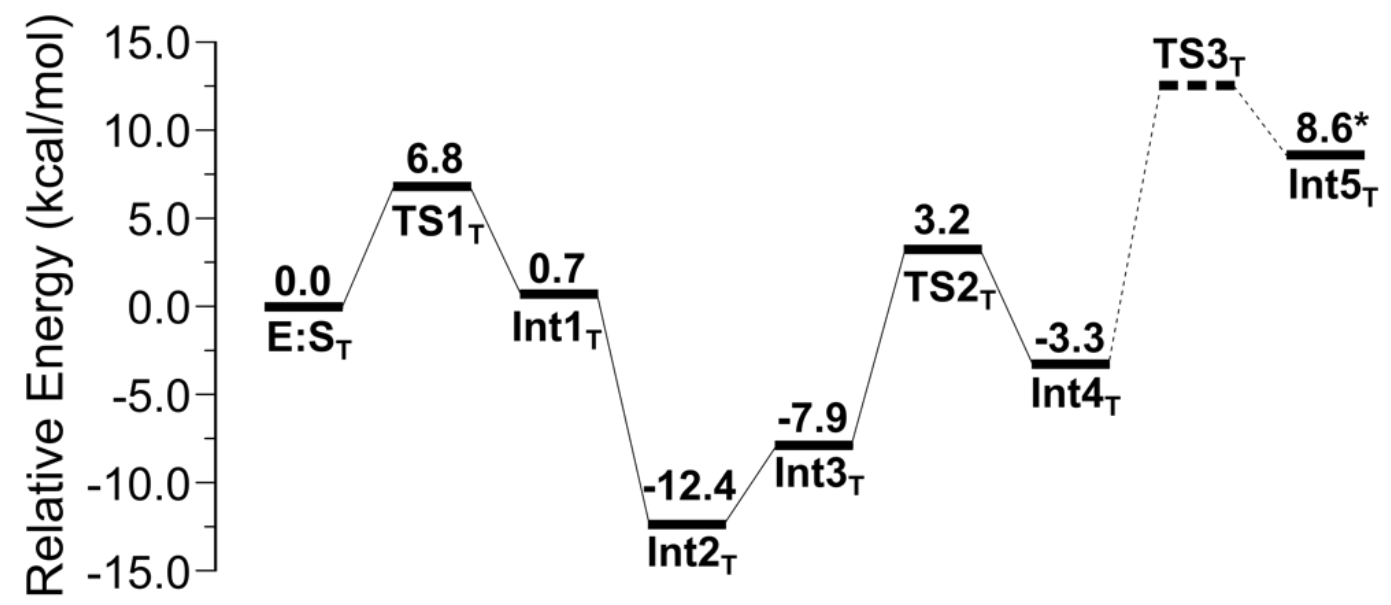

Figure S3. Energy profile for the "dopamine-first" mechanism with both Glu110 and Asp141 in the deprotonated states. * indicates that the cyclized intermediate (Int5T) was optimized with a constrained $C-C$ bond distance of $1.6 \AA$ 

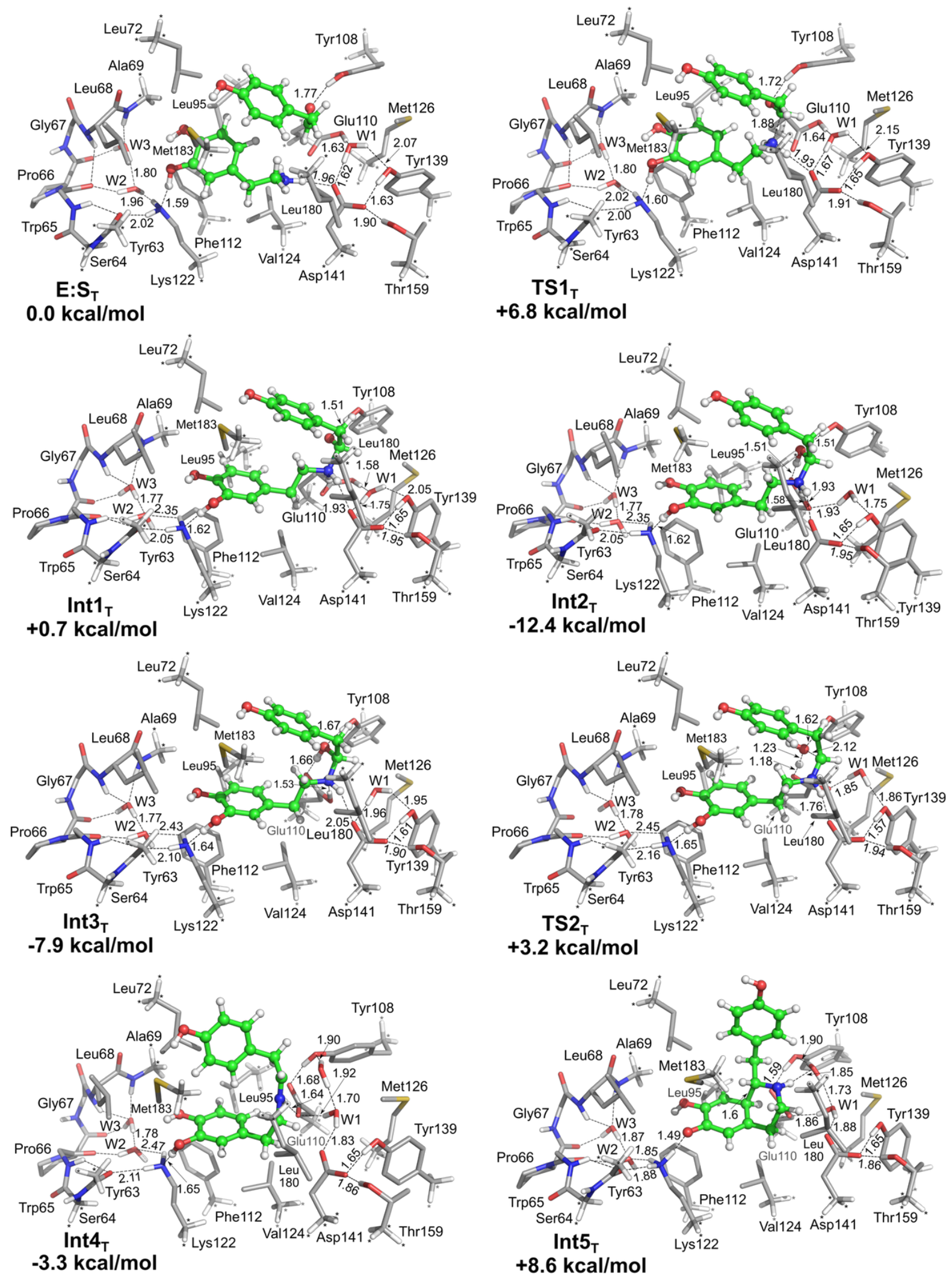

Figure S4. Optimized structures of the intermediates and transition states involved in the pathway with both Glu110 and Asp141 in the deprotonated states. The cyclized intermediate (Int5T) was optimized with a constrained $C-C$ bond distance of 1.6 A. Energies relative to $\boldsymbol{E}: \boldsymbol{S}_{\boldsymbol{T}}$ are given. 


\section{Geometries and relative energies of enzyme-substrate complexes}

The geometries of a large number of structures of the Michaelis complex have been optimized. Here, the structures and energies of the twenty lowest ones are given (Figure S5).

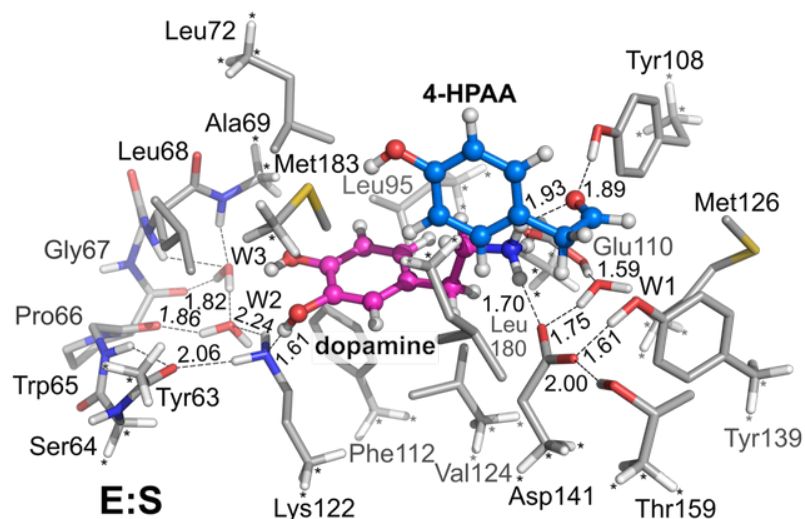

\section{$0.0 \mathrm{kcal} / \mathrm{mol}$}
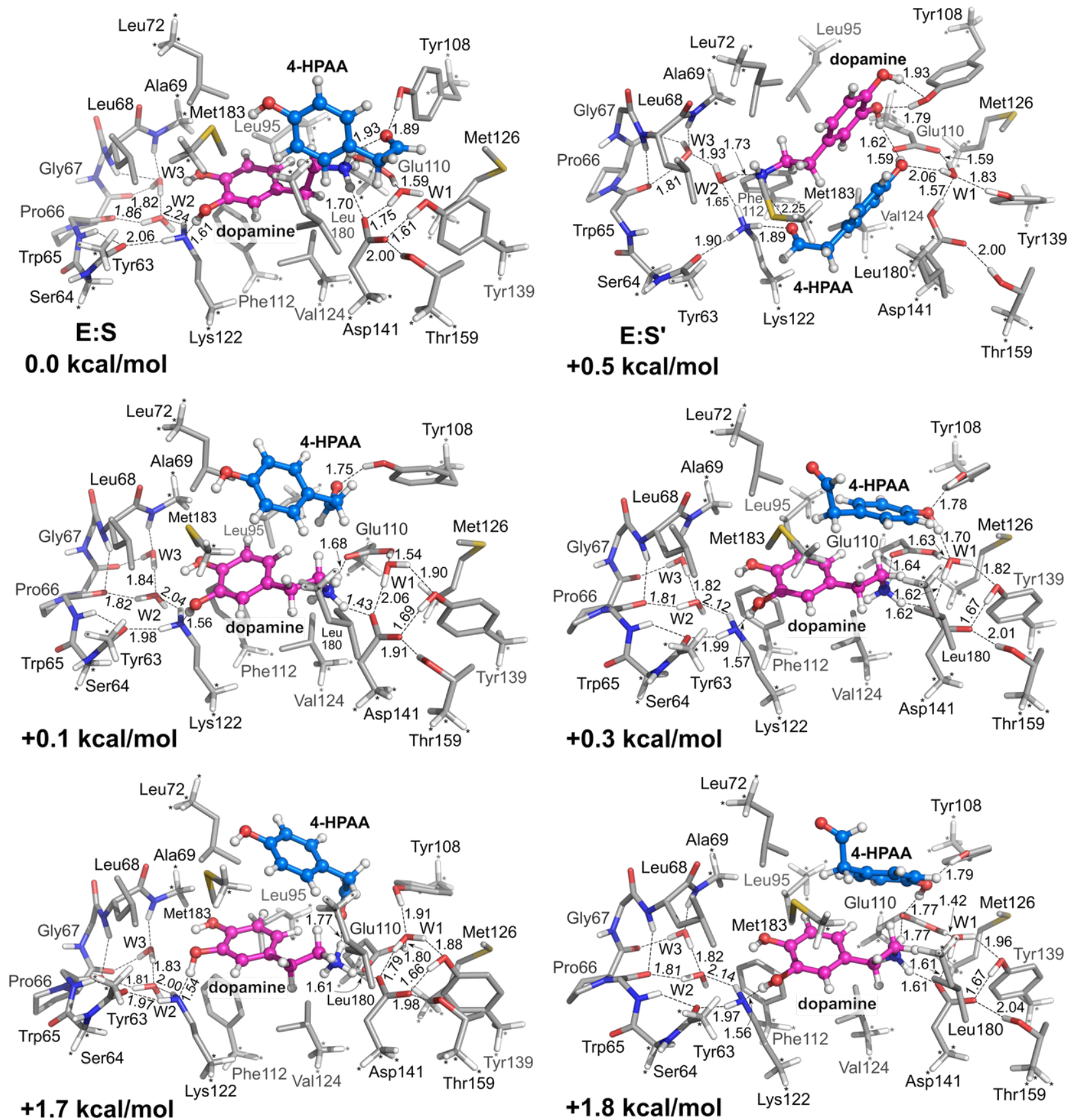


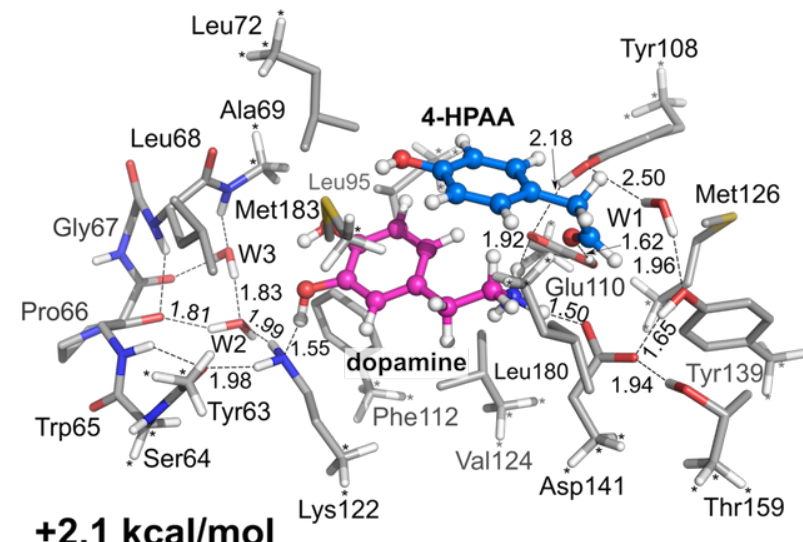

+2.1 kcal/mol Lysi22
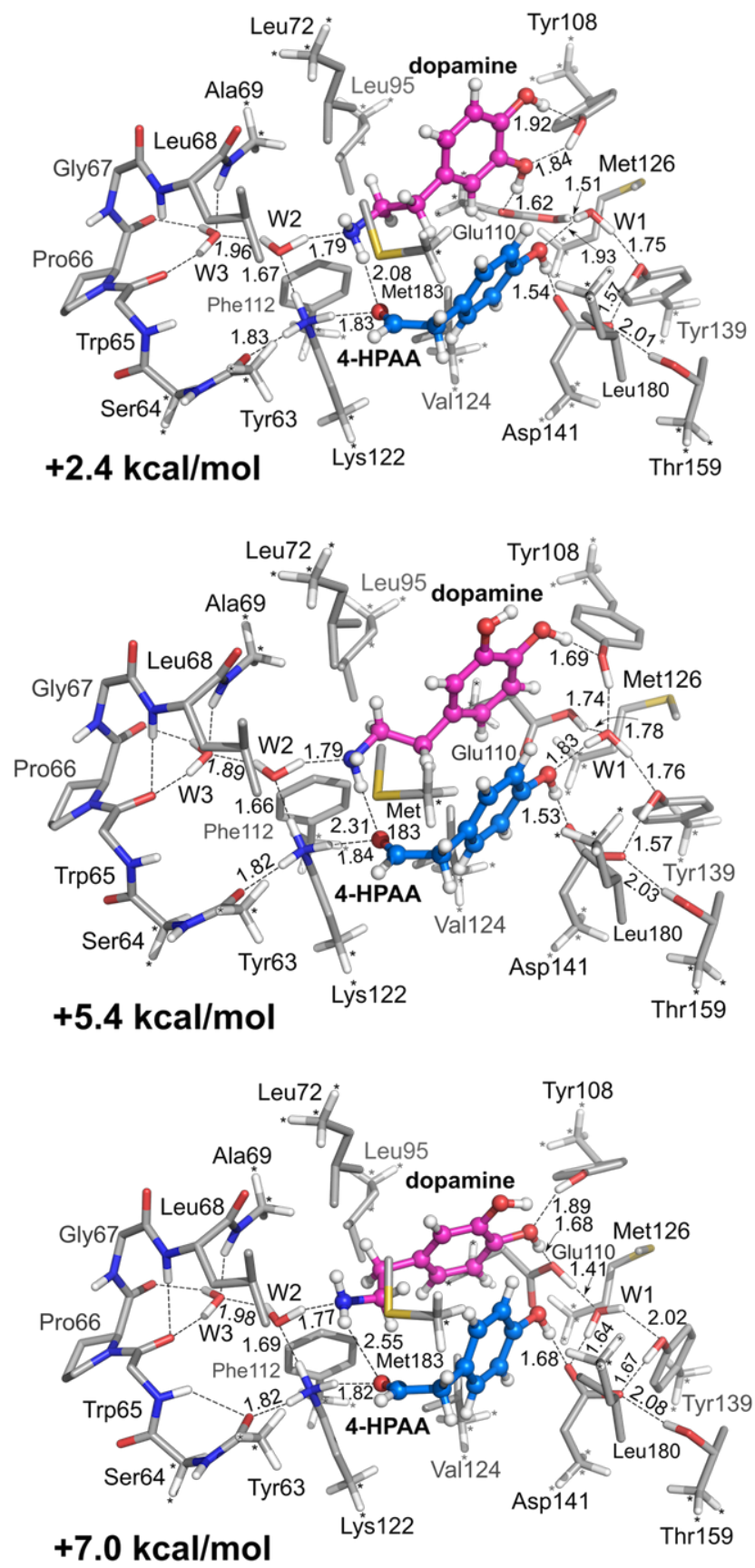
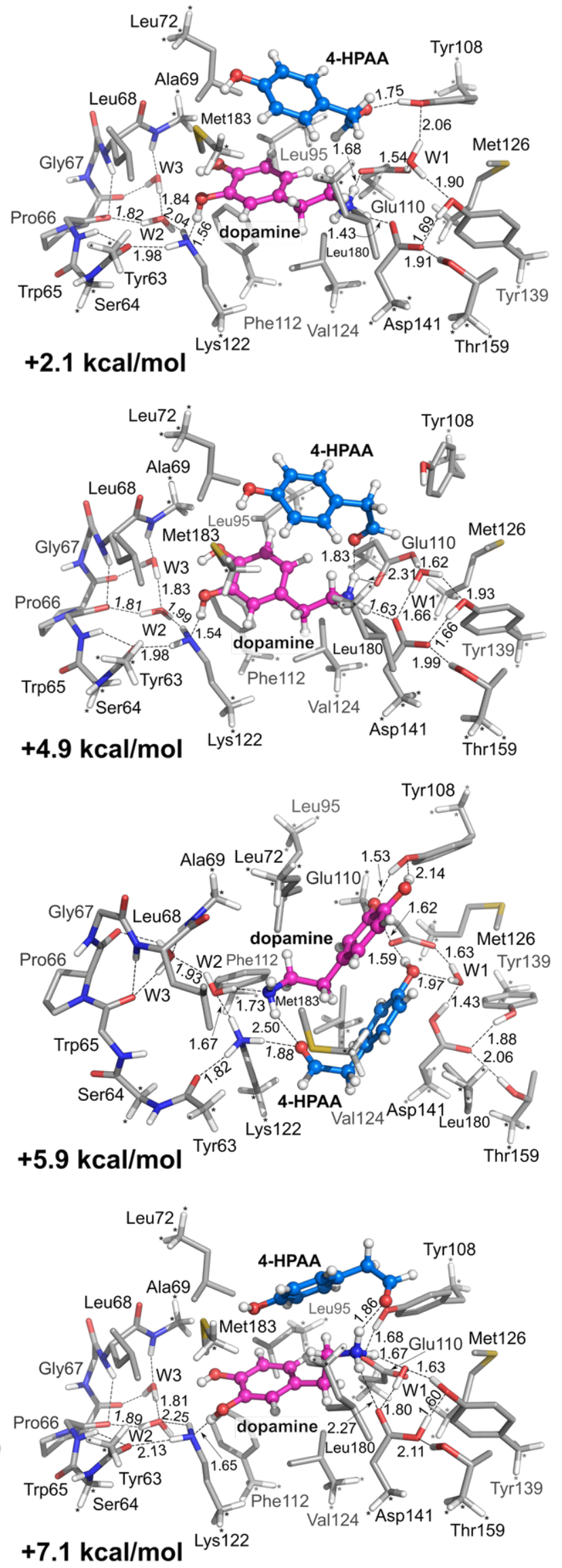

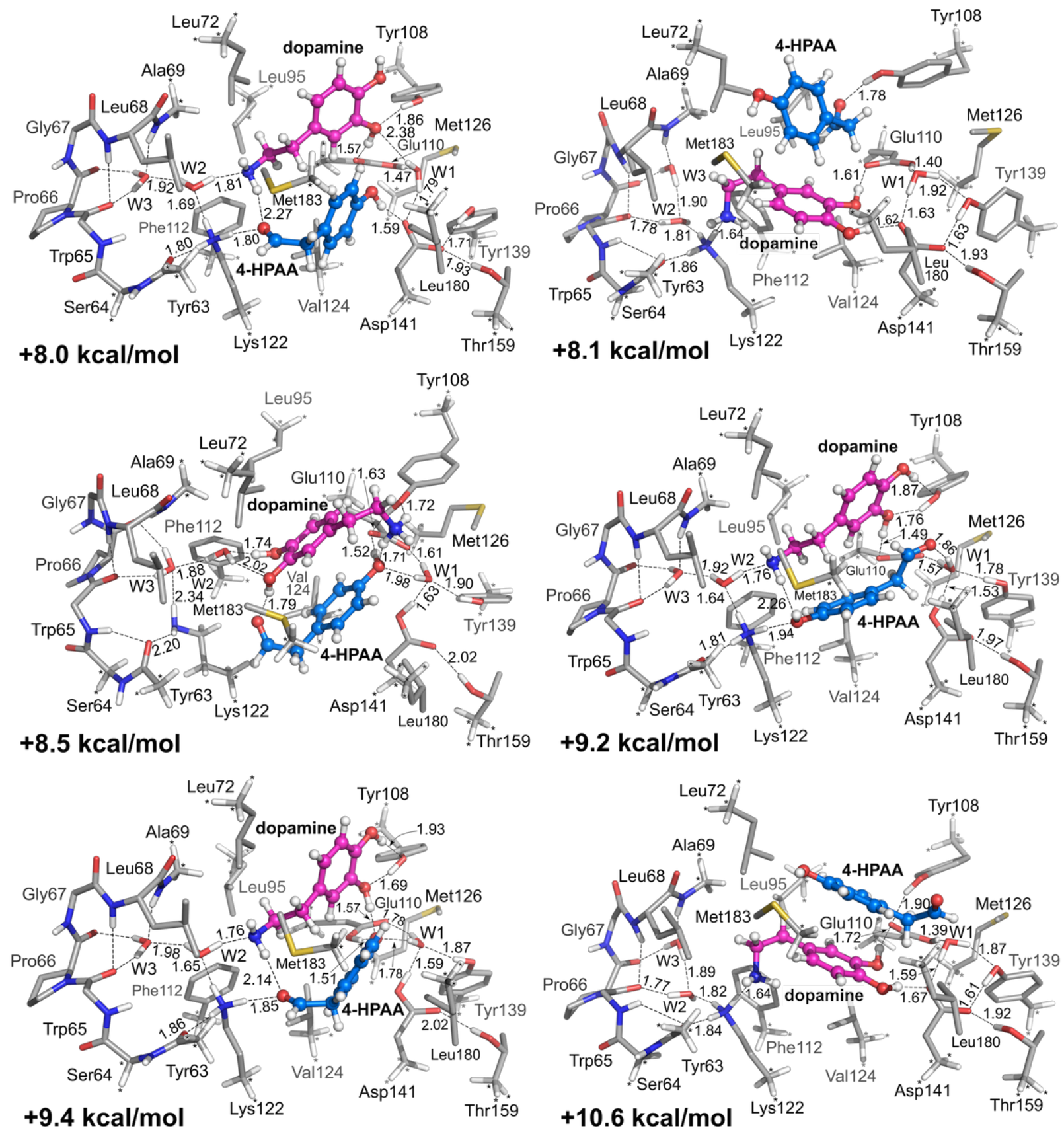

Figure S5. Optimized structures of the enzyme-substrate complexes with low energies. Energies relative to $\boldsymbol{E}: \boldsymbol{S}$ are given. 


\section{Optimized structures for the "dopamine-first" mechanism with full model}
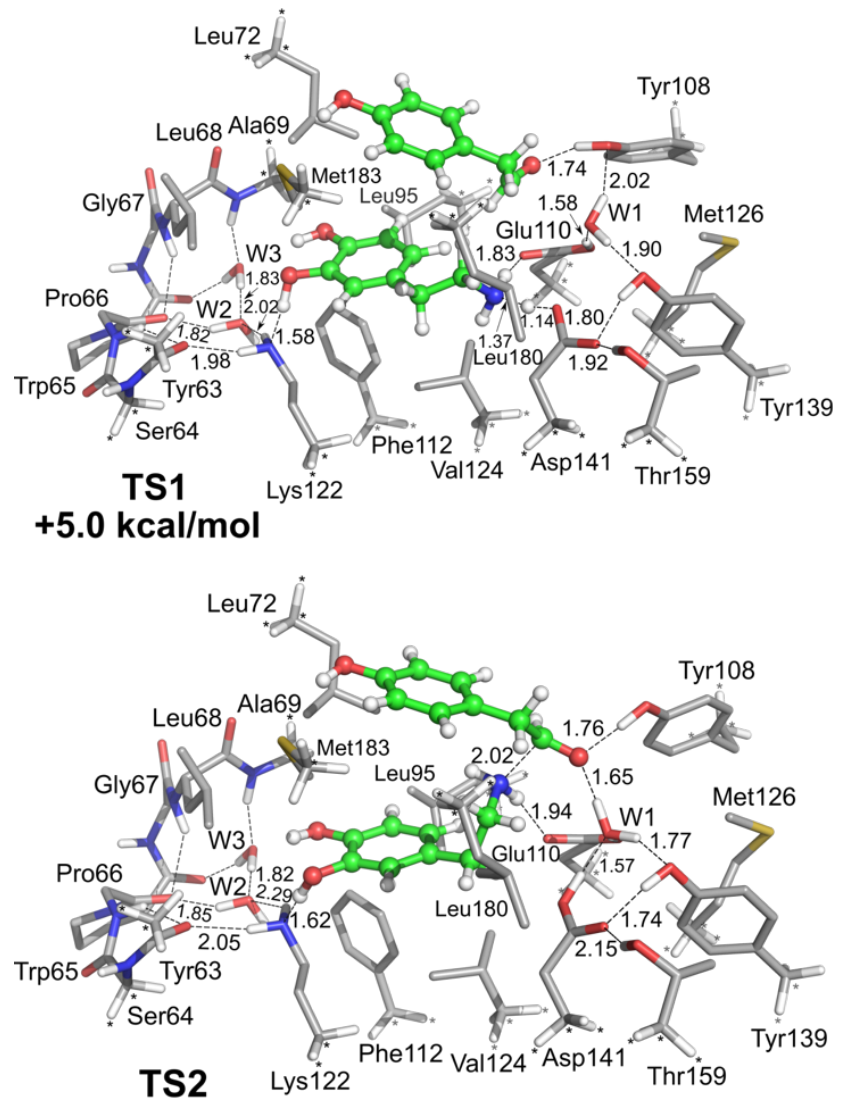

$+8.0 \mathrm{kcal} / \mathrm{mol}$

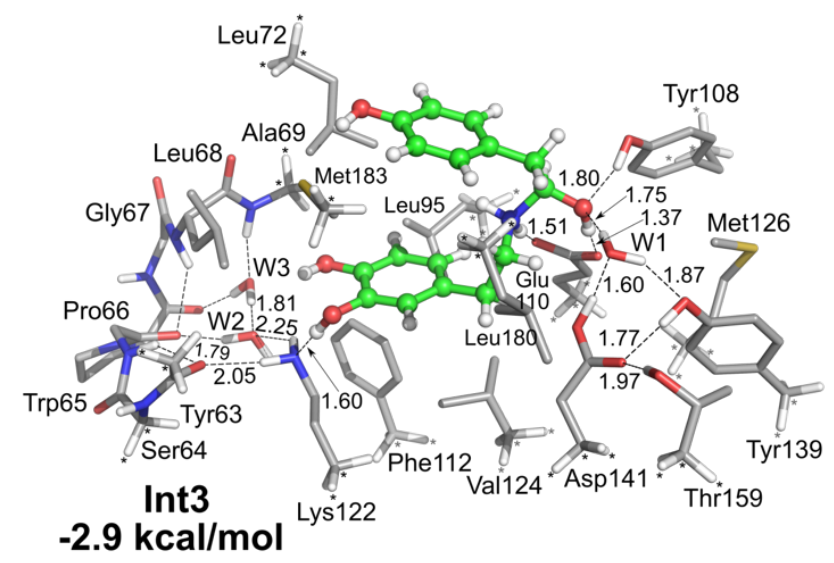

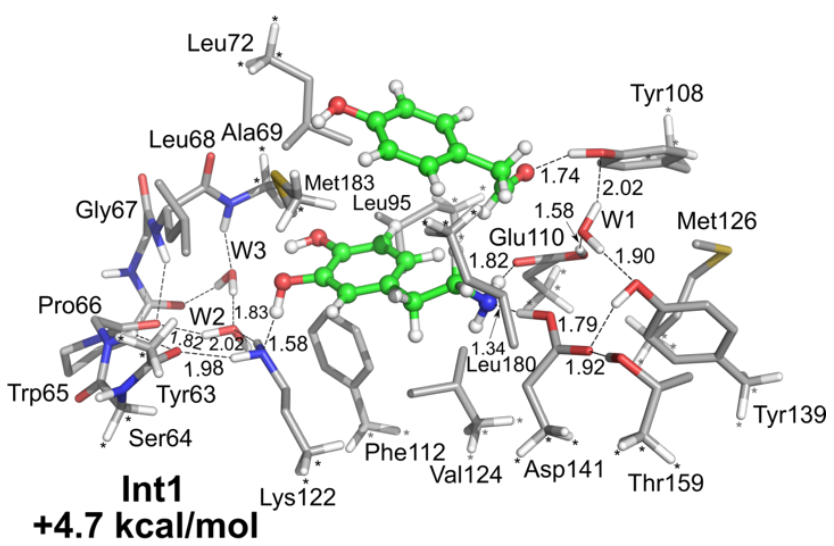
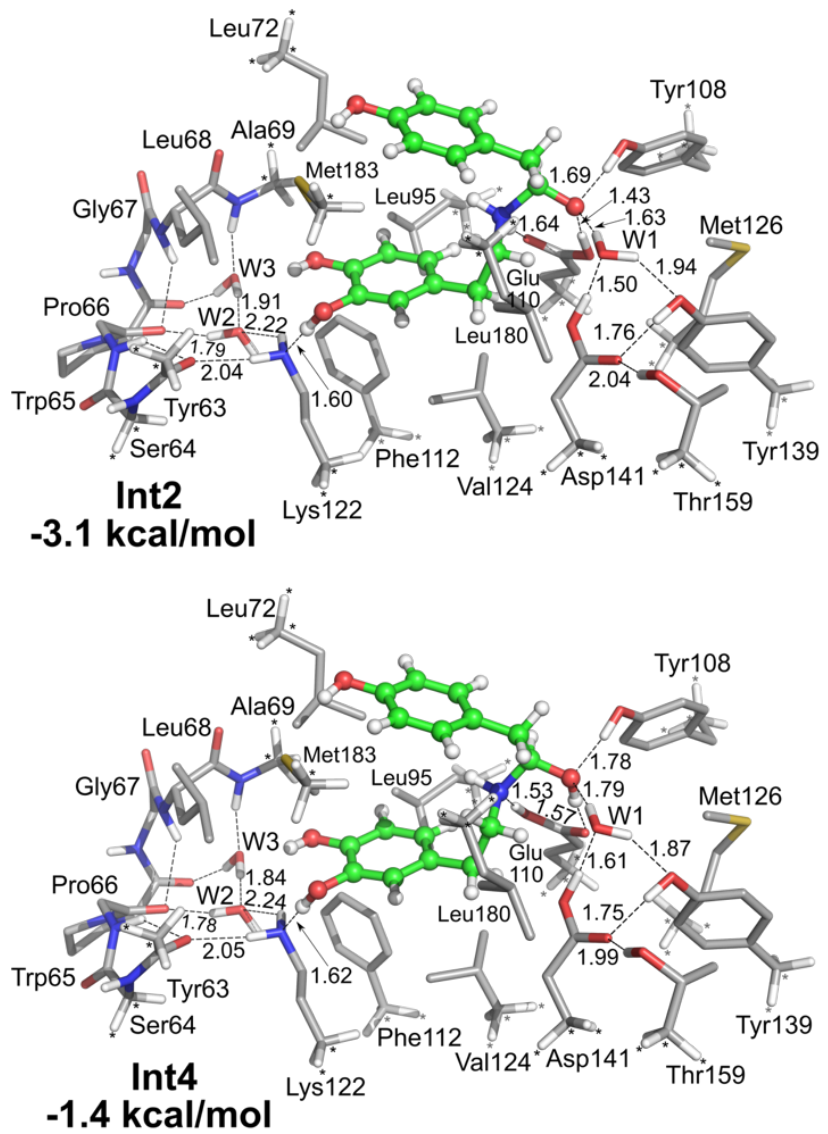


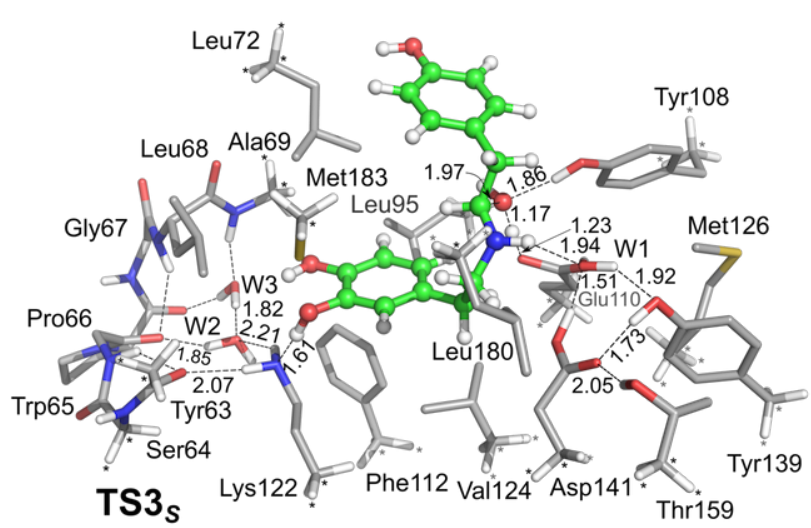

+13.6 kcal $/ \mathrm{mol}$

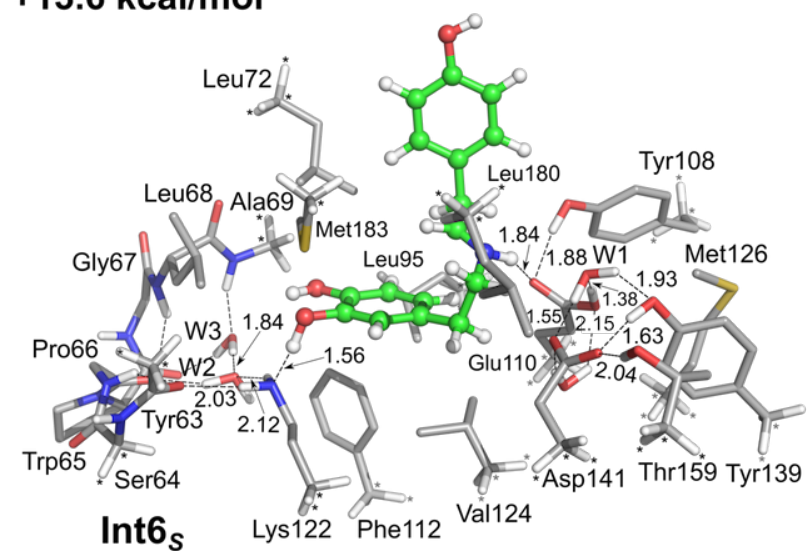

\section{$-0.8 \mathrm{kcal} / \mathrm{mol}$}

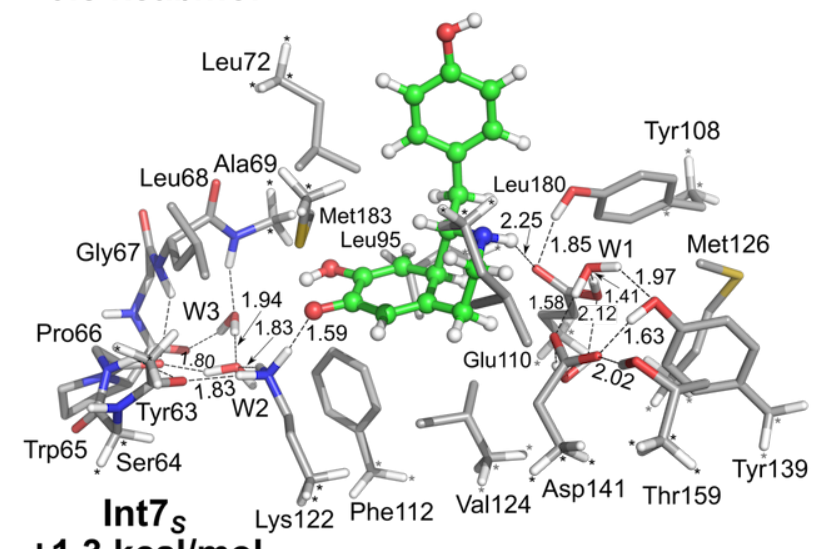

+1.3 kcal $/ \mathrm{mol}$

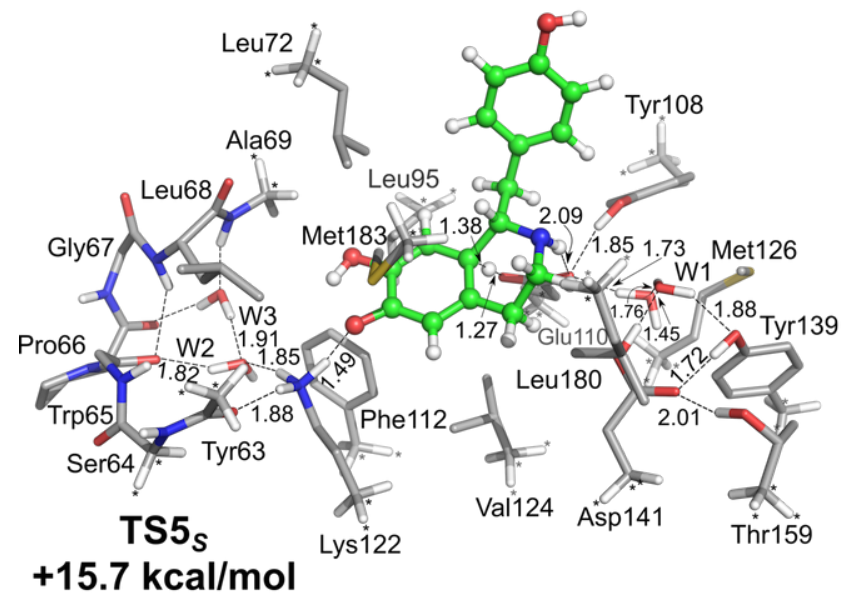

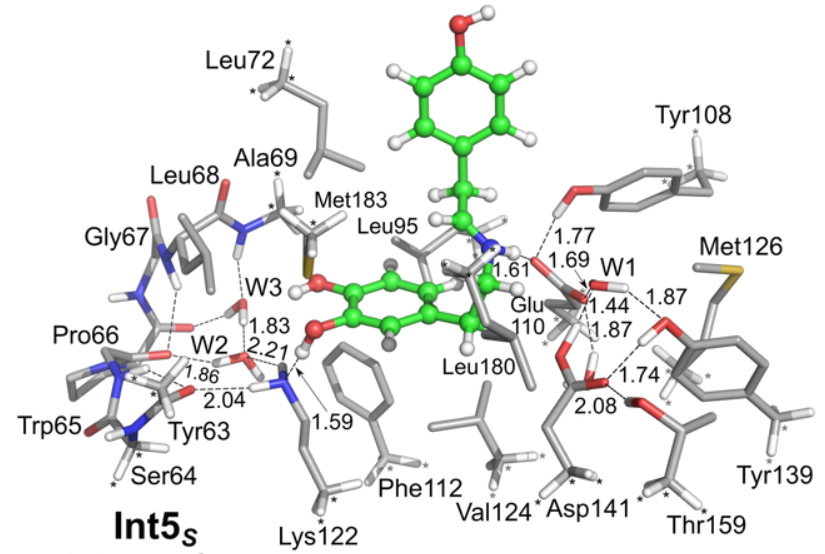

$-1.8 \mathrm{kcal} / \mathrm{mol}$

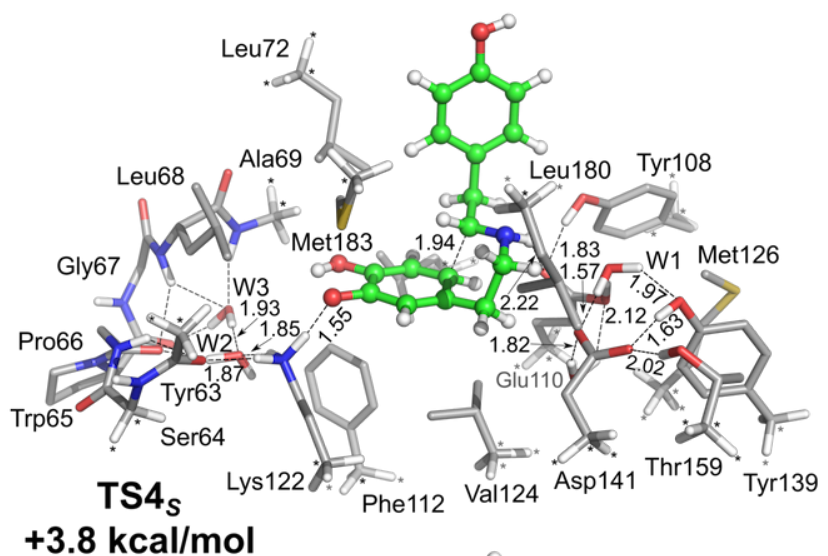

$+3.8 \mathrm{kcal} / \mathrm{mol}$

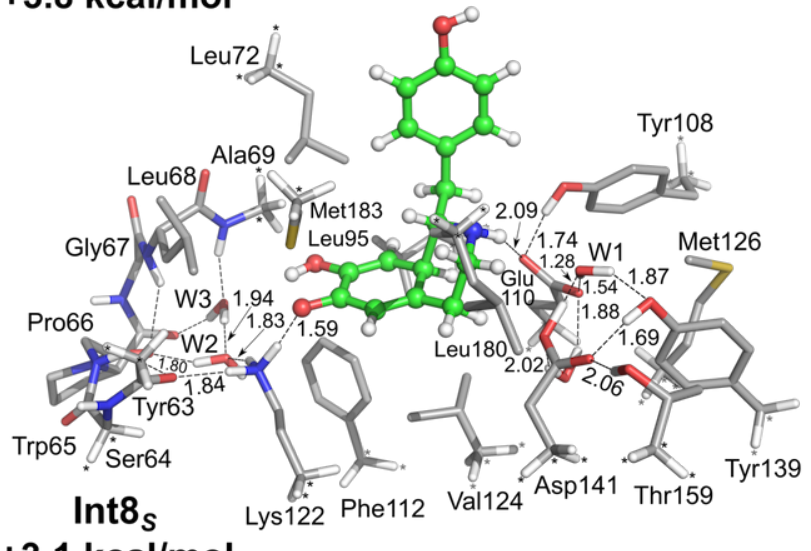

\section{$+3.1 \mathrm{kcal} / \mathrm{mol}$}

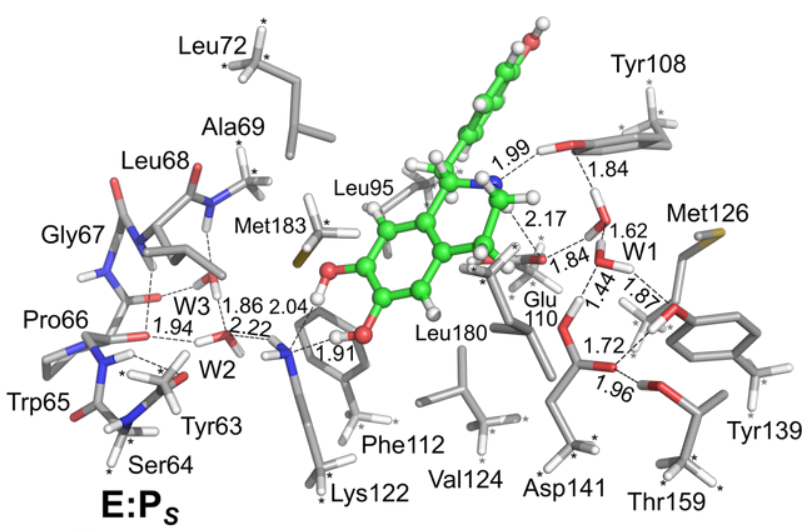

$-0.7 \mathrm{kcal} / \mathrm{mol}$ 


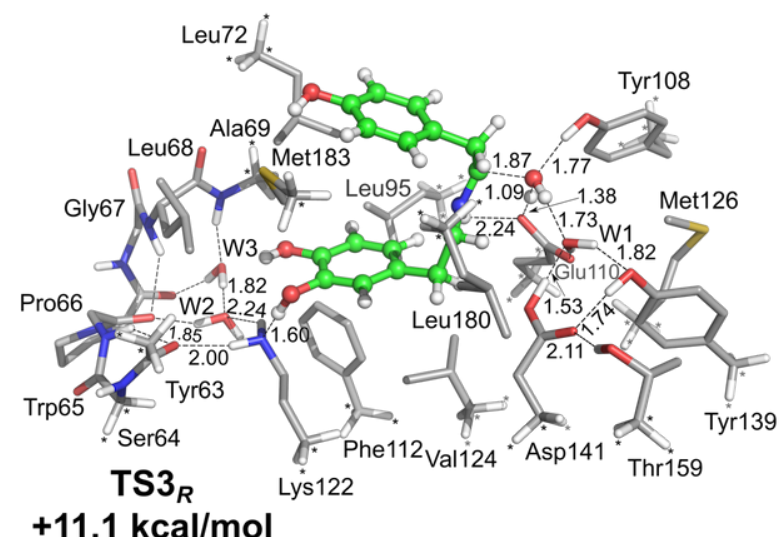

$+11.1 \mathrm{kcal} / \mathrm{mol}$

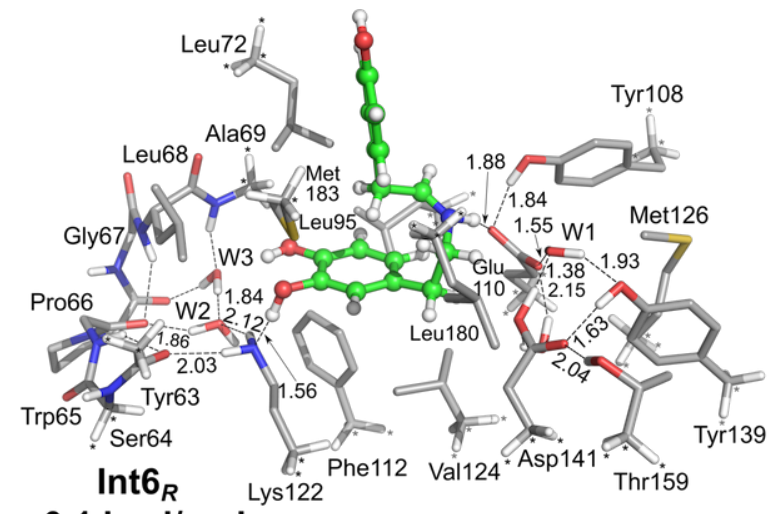

\section{$-0.1 \mathrm{kcal} / \mathrm{mol}$}
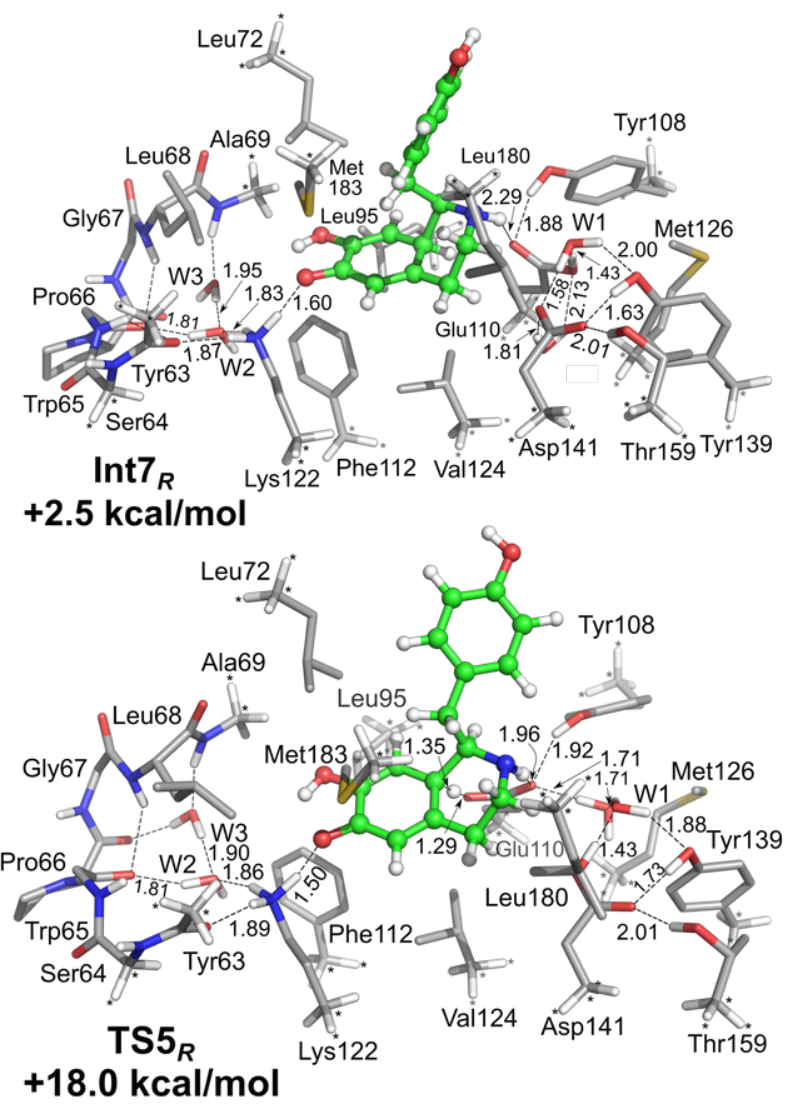

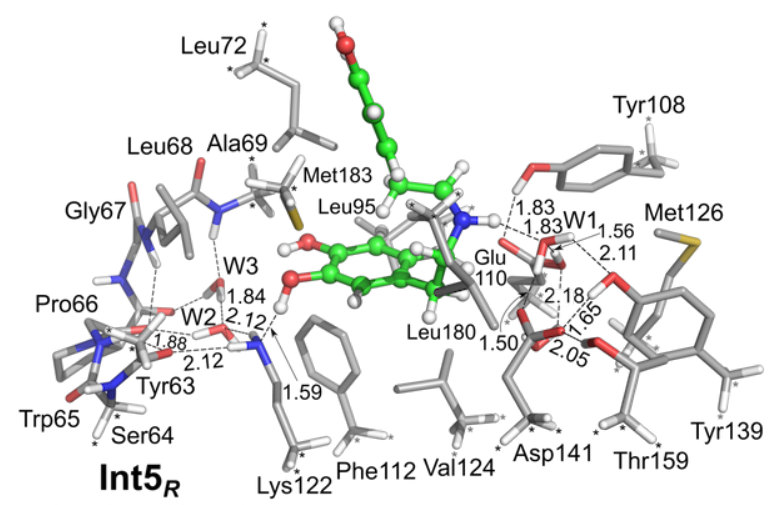

$-1.9 \mathrm{kcal} / \mathrm{mol}$

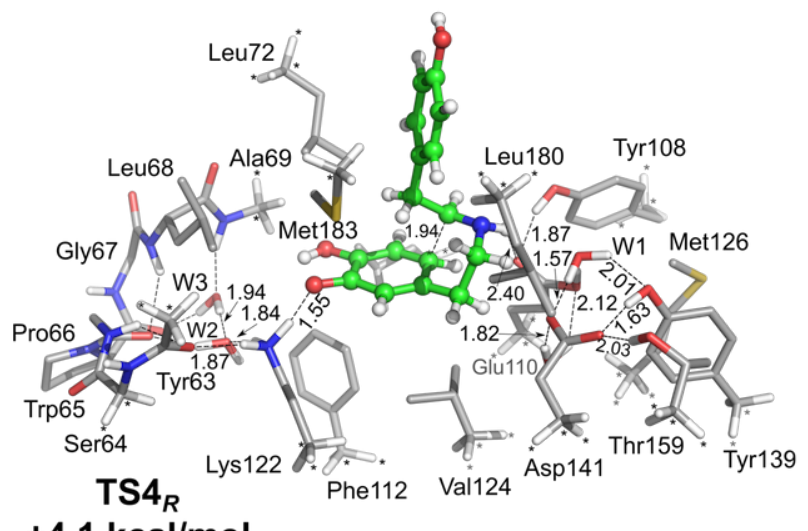

+4.1 $\mathrm{kcal} / \mathrm{mol}$
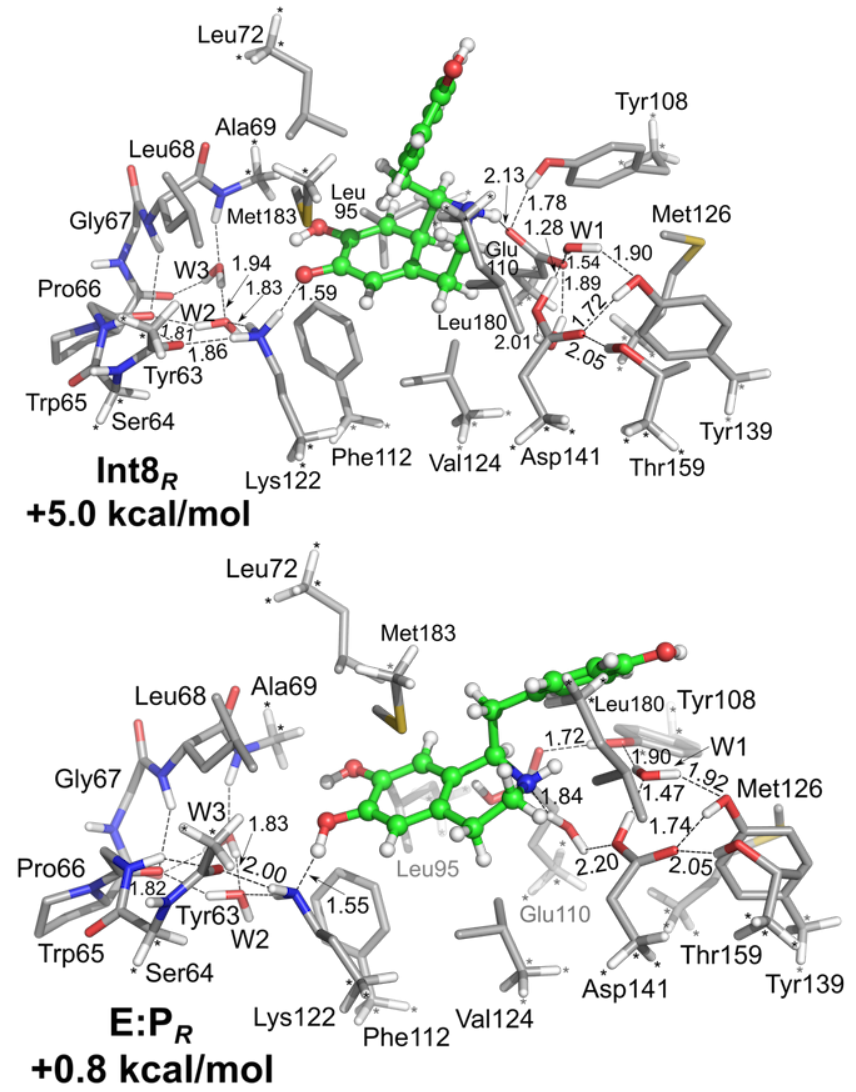

Figure S6. Optimized structures of the intermediates and transition states in the "dopamine-first" mechanism. Energies relative to $\boldsymbol{E}: \boldsymbol{S}$ are given. 
5. Geometries and energy decomposition of the barrier-less transition states
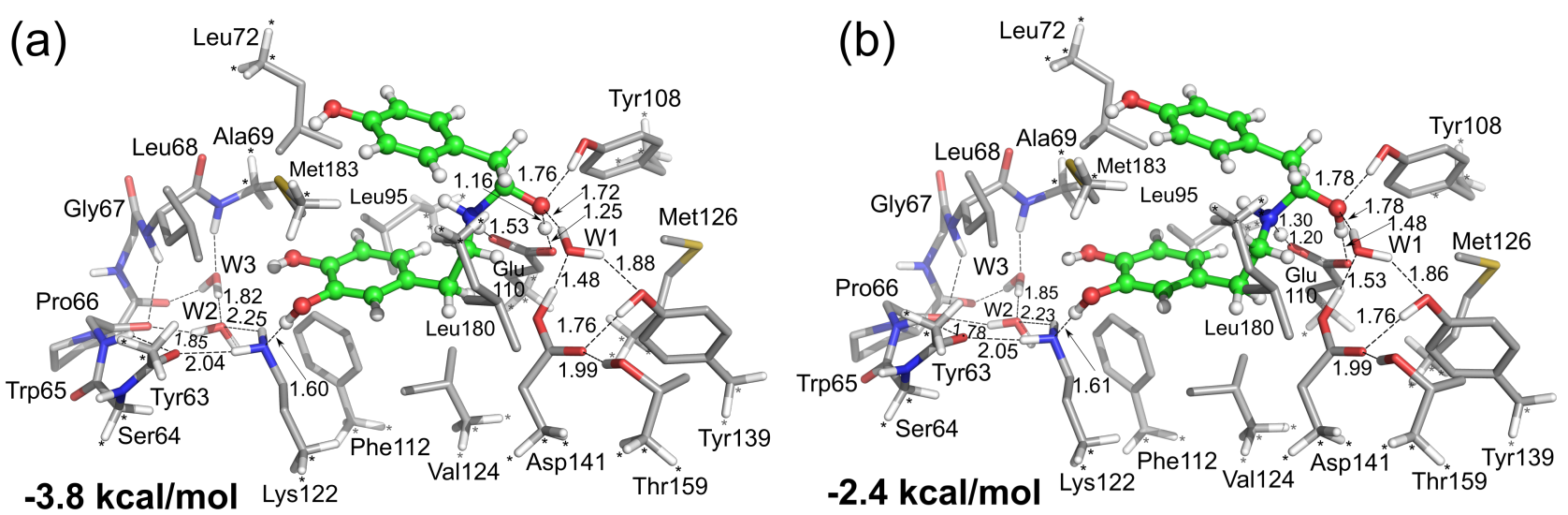

Figure S7. Optimized structures of the transition states for the (a) Int2-Int3 and (b) Int3-Int4 steps. Energies relative to $\mathbf{E}: \boldsymbol{S}$ are given. Note that both TSs could be optimized in the gas-phase, but after adding the corrections for solvation and ZPE the energies of these transition states were found to be slightly lower than one or both connecting intermediates, as shown in Tables S1 and S2 below.

Table S1. Energy decomposition of the Int2-Int3 step.

\begin{tabular}{lccc}
$B S 1=6-31 G(d, p), B S 2=6-311+G(2 d, 2 p)$ & & \\
\hline & Int2 & TS & Int3 \\
\hline BS1 & 0.0 & +3.2 & +2.8 \\
BS2 & 0.0 & +2.6 & +2.0 \\
BS2+solvation & 0.0 & +1.3 & +0.8 \\
BS2+solvation+ZPE & 0.0 & -0.7 & +0.2 \\
\hline
\end{tabular}

Table S2. Energy decomposition of the Int3-Int4 step. $B S 1=6-31 G(d, p), B S 2=6-311+G(2 d, 2 p)$

\begin{tabular}{lccc}
\hline & Int3 & TS & Int4 \\
\hline BS1 & 0.0 & +0.8 & -0.9 \\
BS2 & 0.0 & +1.8 & -0.4 \\
BS2+solvation & 0.0 & +4.0 & +1.9 \\
BS2+solvation+ZPE & 0.0 & +0.5 & +1.5 \\
\hline
\end{tabular}




\section{Other optimized structures for iminium intermediates}

The geometries of a large number of structures of the iminium intermediate have been optimized. The structures and the energies of the ten lowest ones are given (Figure S8). The first structure with a relative energy of $+3.5 \mathrm{kcal} / \mathrm{mol}$ corresponds to the unproductive conformation discussed in the main text.
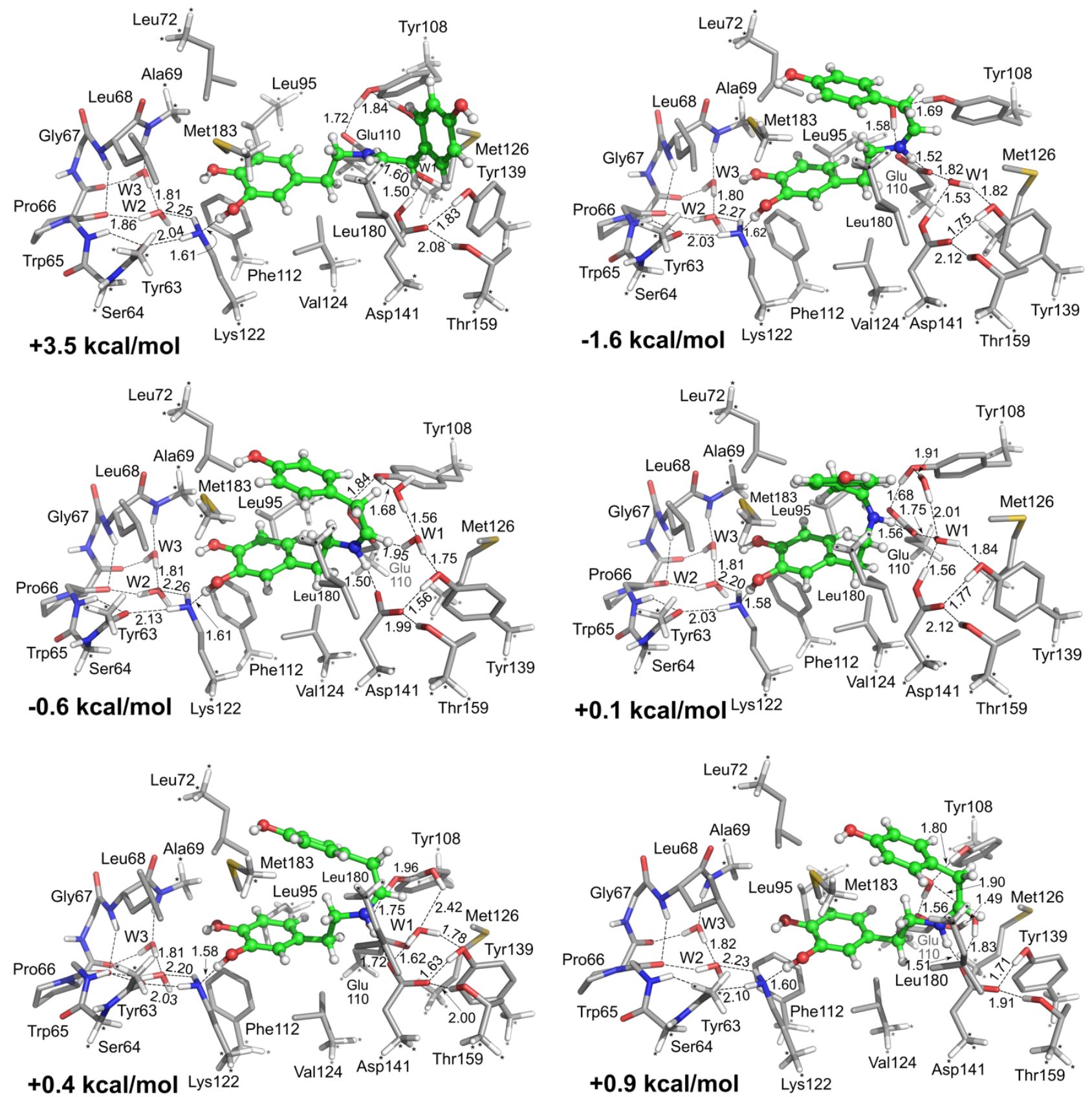

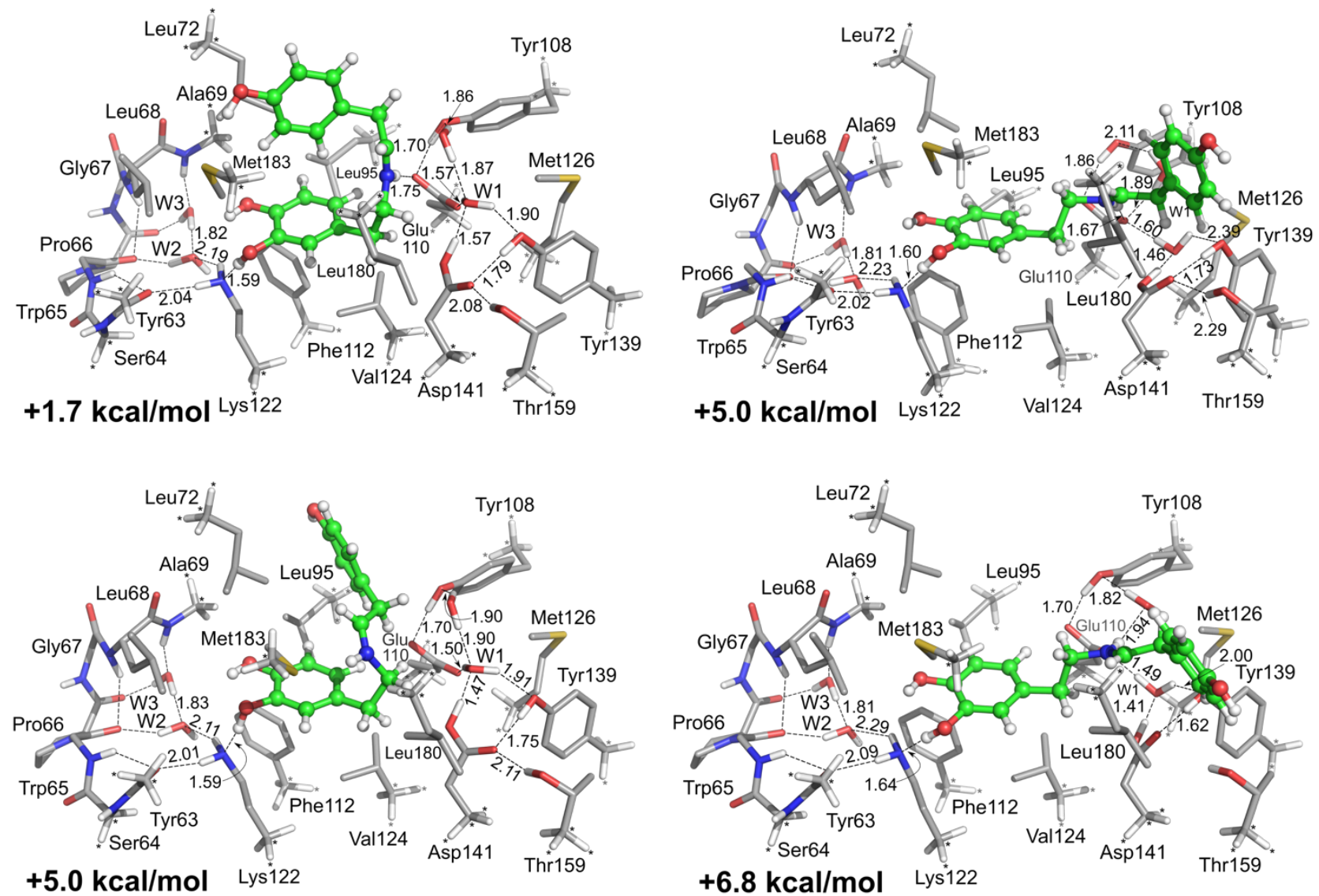

Figure S8. Optimized structures of iminium intermediates. Energies relative to $\mathbf{E}: \mathbf{S}$ are given. 


\section{Other geometries and relative energies of the rate-limiting TS}

The geometries of a large number of structures of the rate- and selectivity-determining transition state (TS5) have been optimized. In addition to $\mathbf{T S 5}_{S}$ and $\mathbf{T S 5}_{\boldsymbol{R}}$ discussed in the main text, the structures and the energies of the ten lowest ones are given in Figure S9.
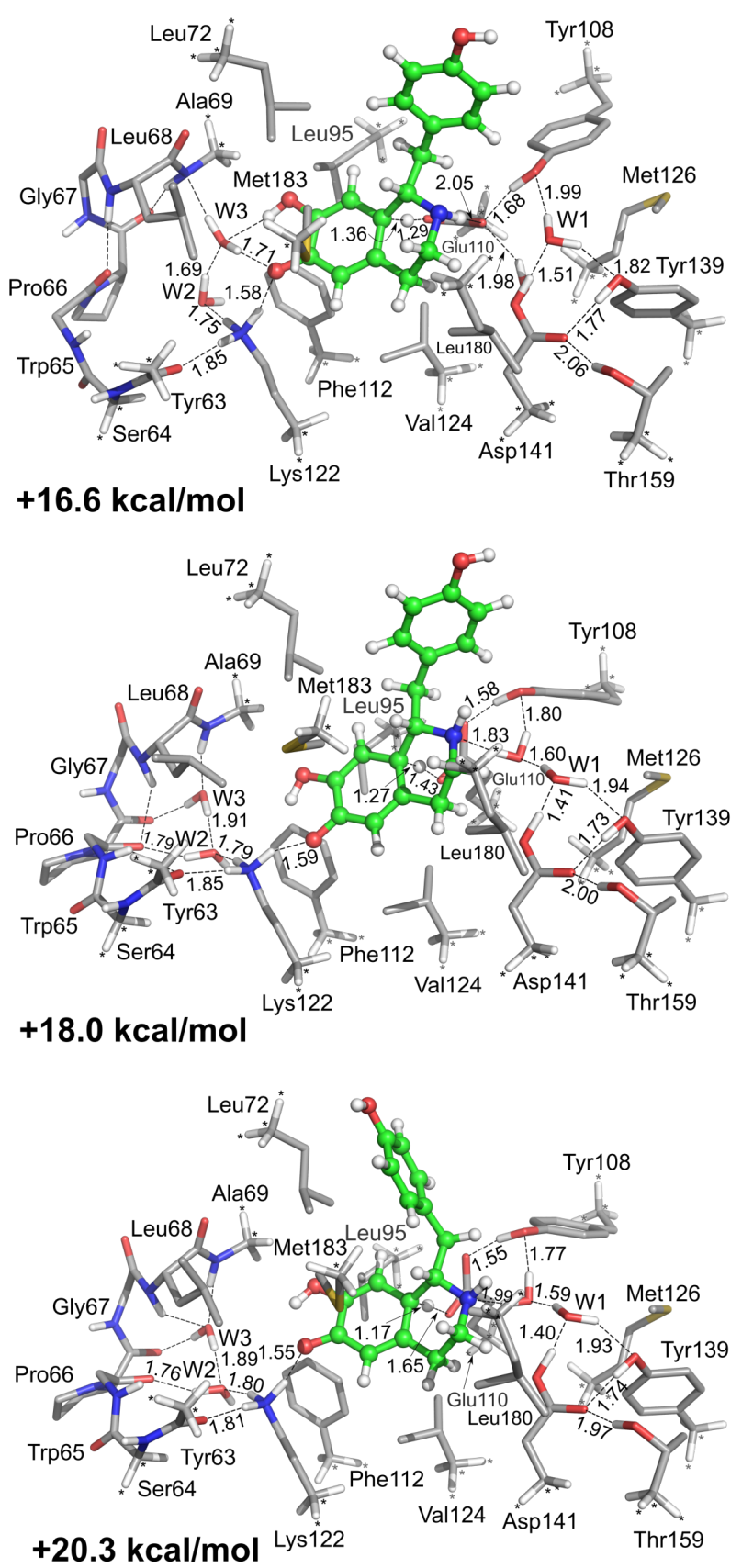
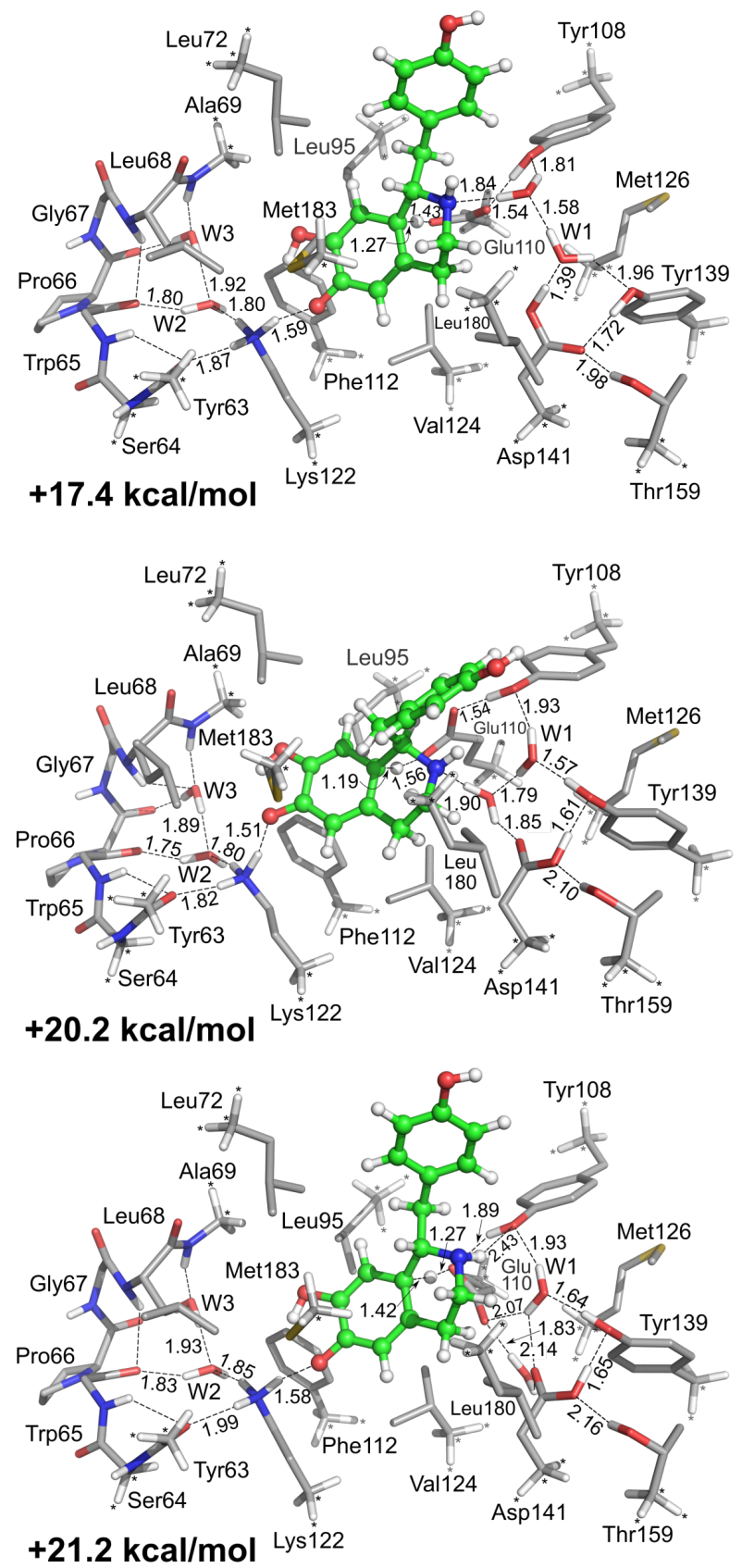

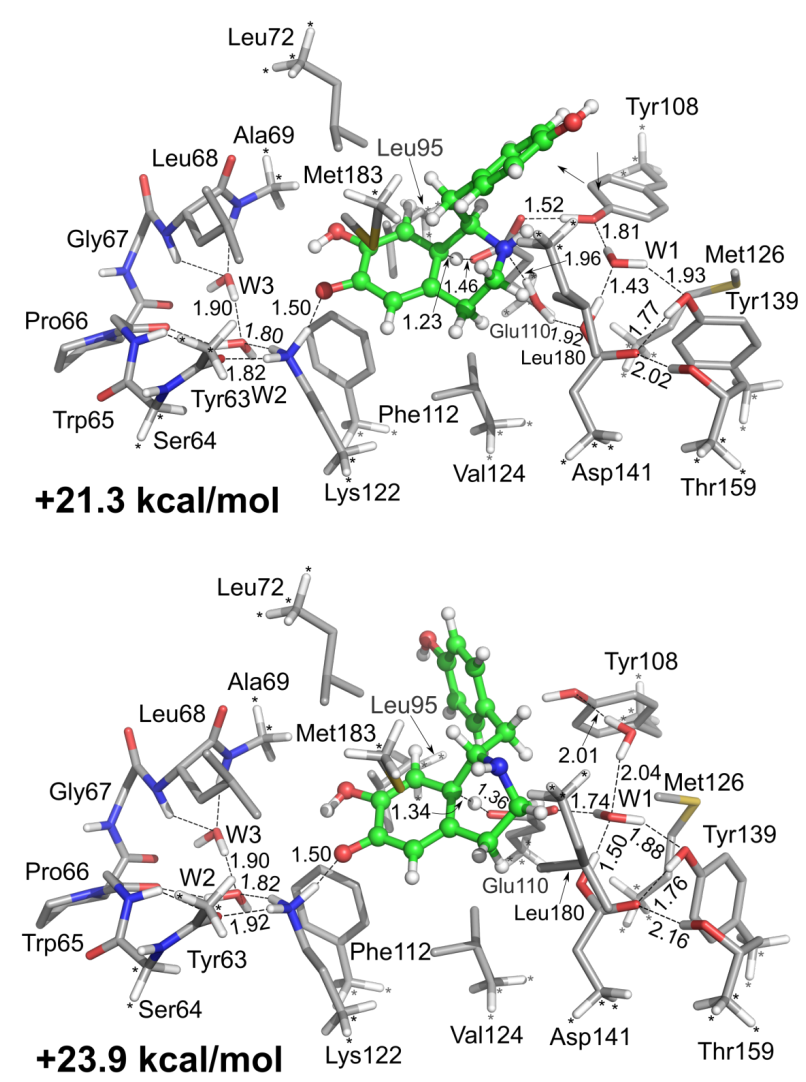
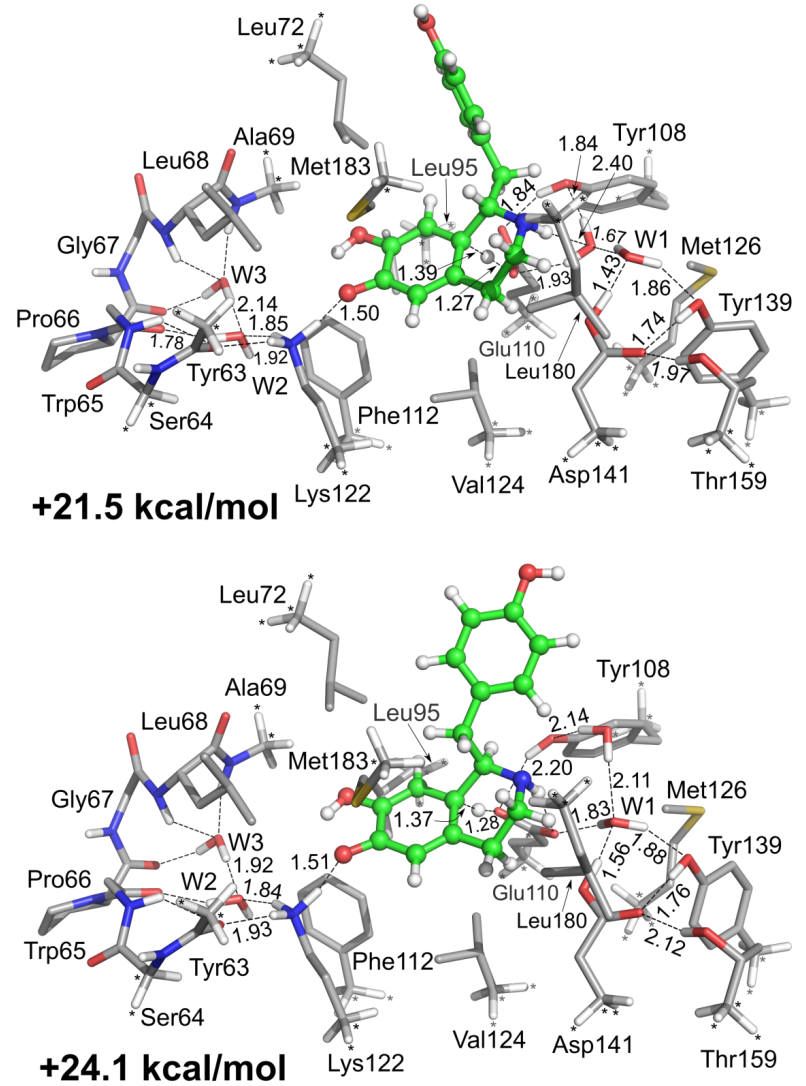

Figure S9. Optimized structures other conformations of the rate-determining transition state. Energies relative to $\mathbf{E}: \boldsymbol{S}$ are given. 


\section{Absolute energies and energy corrections for stationary points}

Table S3. Calculated absolute energies and energy corrections for the stationary points of the "dopamine-first" mechanism. $B S 1=6-31 G(d, p), B S 2=6-311+G(2 d, 2 p)$

\begin{tabular}{|c|c|c|c|c|c|}
\hline & $\begin{array}{l}\text { EBS1 }_{\text {B. }} \\
\text { (a.u.) }\end{array}$ & $\begin{array}{l}\text { EBS2 } \\
\text { (a.u.) }\end{array}$ & $\begin{array}{c}\text { Esolvation } \\
\text { (a.u.) }\end{array}$ & $\begin{array}{l}\text { E }_{Z P E} \\
\text { (a.u.) }\end{array}$ & $\begin{array}{c}\Delta \mathrm{E}_{\text {total }} \\
(\mathrm{kcal} / \mathrm{mol})\end{array}$ \\
\hline$E: S$ & -6505.509525 & -6507.193073 & -0.094967 & 2.673480 & 0.0 \\
\hline TS1 & -6505.496772 & -6507.176070 & -0.099991 & 2.669457 & 5.0 \\
\hline Int1 & -6505.496746 & -6507.175921 & -0.100175 & 2.668989 & 4.7 \\
\hline TS2 & -6505.501051 & -6507.179374 & -0.095495 & 2.673088 & 8.0 \\
\hline Int2 & -6505.518653 & -6507.196192 & -0.098088 & 2.674596 & -3.1 \\
\hline Int3 & -6505.514208 & -6507.193131 & -0.099830 & 2.673768 & -2.9 \\
\hline Int4 & -6505.515505 & -6507.194099 & -0.095834 & 2.673184 & -1.4 \\
\hline \multicolumn{6}{|c|}{$S$-pathway } \\
\hline $\mathbf{T S 3}_{S}$ & -6505.482331 & -6507.159633 & -0.101646 & 2.668488 & 13.6 \\
\hline $\operatorname{Int5} s$ & -6505.501256 & -6507.182977 & -0.105425 & 2.670904 & -1.8 \\
\hline Int6 $_{S}$ & -6505.498984 & -6507.179092 & -0.107486 & 2.670762 & -0.8 \\
\hline $\mathrm{TS}_{S}$ & -6505.489217 & -6507.168085 & -0.113405 & 2.672909 & 3.8 \\
\hline Int $7 s$ & -6505.490704 & -6507.170090 & -0.116112 & 2.673764 & 1.3 \\
\hline $\operatorname{Int8}_{S}$ & -6505.487938 & -6507.166627 & -0.115449 & 2.672499 & 3.1 \\
\hline $\mathbf{T S 5}_{S}$ & -6505.467542 & -6507.147266 & -0.111007 & 2.668751 & 15.7 \\
\hline $\mathbf{E}: \mathbf{P}_{S}$ & -6505.494518 & -6507.184649 & -0.105798 & 2.674822 & -0.7 \\
\hline \multicolumn{6}{|c|}{$R$-pathway } \\
\hline $\mathbf{T S 3}_{R}$ & -6505.489026 & -6507.167869 & -0.101140 & 2.672096 & 11.1 \\
\hline $\operatorname{Int5}_{R}$ & -6505.502974 & -6507.184430 & -0.104972 & 2.671739 & -1.9 \\
\hline Int6 $_{R}$ & -6505.499363 & -6507.182462 & -0.103853 & 2.671578 & -0.1 \\
\hline $\mathbf{T S 4}_{R}$ & -6505.486934 & -6507.167900 & -0.112664 & 2.672518 & 4.1 \\
\hline Int7 $7_{R}$ & -6505.488855 & -6507.169589 & -0.115370 & 2.674330 & 2.5 \\
\hline $\operatorname{Int8}_{R}$ & -6505.487246 & -6507.165266 & -0.113722 & 2.672409 & 5.0 \\
\hline $\mathbf{T S 5}_{R}$ & -6505.465689 & -6507.145821 & -0.108450 & 2.668455 & 18.0 \\
\hline$E: P_{R}$ & -6505.508967 & -6507.187347 & -0.100592 & 2.674731 & 0.8 \\
\hline
\end{tabular}


Table S4. Calculated absolute energies and energy corrections for the stationary points of the "HPAA-first" mechanism. $B S 1=6-31 G(d, p), B S 2=6-311+G(2 d, 2 p)$

\begin{tabular}{|c|c|c|c|c|c|}
\hline & $\begin{array}{l}\text { EBS1 }_{\text {B }} \\
\text { (a.u.) }\end{array}$ & $\begin{array}{l}\text { EBS2 }_{\text {B. }} \\
\text { (a.u.) }\end{array}$ & $\begin{array}{c}\text { E }_{\text {solvation }} \\
\text { (a.u.) }\end{array}$ & $\begin{array}{l}\text { E }_{\text {ZPE }} \\
\text { (a.u.) }\end{array}$ & $\begin{array}{c}\Delta \mathrm{E}_{\text {total }} \\
(\mathrm{kcal} / \mathrm{mol})\end{array}$ \\
\hline E:S' & -6505.495195 & -6507.171964 & -0.115169 & 2.673307 & 0.0 \\
\hline TS1' & -6505.464179 & -6507.140573 & -0.119728 & 2.670809 & 15.7 \\
\hline Int1' & -6505.467601 & -6507.150908 & -0.114758 & 2.669203 & 11.4 \\
\hline Int2' & -6505.469635 & -6507.153479 & -0.118974 & 2.669435 & 7.2 \\
\hline TS2' & -6505.462191 & -6507.145492 & -0.112149 & 2.673071 & 18.8 \\
\hline Int3' & -6505.476621 & -6507.165229 & -0.100572 & 2.669377 & 11.4 \\
\hline TS3' & -6505.461630 & -6507.149786 & -0.102191 & 2.671961 & 21.7 \\
\hline Int4' & -6505.498010 & -6507.181555 & -0.099499 & 2.671277 & 3.0 \\
\hline Int5' & -6505.493906 & -6507.177078 & -0.100019 & 2.670224 & 4.8 \\
\hline TS4' & -6505.460114 & -6507.145343 & -0.094801 & 2.670815 & 28.4 \\
\hline Int6' & -6505.473330 & -6507.155648 & -0.097746 & 2.673403 & 21.7 \\
\hline TS5' & -6505.441423 & -6507.117843 & -0.101028 & 2.670377 & 41.5 \\
\hline Int $7^{\prime}$ & -6505.477005 & -6507.154398 & -0.111547 & 2.676314 & 15.6 \\
\hline Int8' & -6505.491610 & -6507.167970 & -0.108429 & 2.676402 & 9.1 \\
\hline$E: P^{\prime}$ & -6505.494416 & -6507.170925 & -0.112554 & 2.675603 & 4.2 \\
\hline
\end{tabular}


The intermediates and transition states in the "dopamine-first" mechanism.

\section{E:S (0.0 kcal $/ \mathrm{mol})$}

\begin{tabular}{|c|c|c|c|}
\hline & -6.85030300 & -5.72322200 & -0.47365000 \\
\hline & -7.39424400 & -4.63311900 & -1.34271400 \\
\hline & -6.94698500 & -3.48081000 & -1.37971200 \\
\hline & -8.44487600 & -4.99553500 & -2.11267700 \\
\hline & -9.03781600 & -4.05806900 & -3.03043800 \\
\hline & -10.10995800 & -3.15016100 & -2.42953100 \\
\hline & -11.14403900 & -2.87825600 & -3.03180100 \\
\hline & -9.80847000 & -2.65452300 & -1.19576600 \\
\hline & -10.66956200 & -1.62296200 & -0.65915500 \\
\hline & 5157700 & -0.30573300 & 6300 \\
\hline & -9.30882000 & 0.16660500 & -1.48718200 \\
\hline & -11.52375000 & 0.37590600 & -1.84352900 \\
\hline & -11.31158800 & 1.72178300 & 8700 \\
\hline & -10.68072800 & 2.65915600 & -1.36867400 \\
\hline $\mathrm{O}$ & -9.86890900 & 3.52032200 & -1.7027 \\
\hline $\mathrm{C}$ & -12.722 & 9900 & 9900 \\
\hline & -13.38177800 & 0.79404100 & -3.21932100 \\
\hline & -12.8 & -0.16 & 3800 \\
\hline N & -11.11607000 & 2.46766700 & -0.10062000 \\
\hline 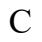 & -10.585 & 1700 & 1.0660 \\
\hline c & -9.95 & 2.19 & \\
\hline $\mathrm{O}$ & -10.14045900 & 2.412 & 3.3022 \\
\hline $\mathrm{N}$ & -9.2 & 1.12 & \\
\hline C & -8.364 & 0.43 & 2.5248 \\
\hline $\mathrm{C}$ & -7.277 & $1.36^{\circ}$ & 3.0863 \\
\hline $\mathrm{O}$ & -6.81 & 1.19 & 4.2 \\
\hline $\mathrm{C}$ & -7.71331500 & -0.752975 & 1.7858 \\
\hline C & -6.54698900 & -1.43167100 & 2.5272 \\
\hline C & -6.95 & -1.97 & 3.9034 \\
\hline & -5.963 & -2.54 & 1.6530 \\
\hline N & -6.83488200 & 2.30364600 & 2.2270 \\
\hline - & -5.72251400 & 3.15661400 & 2.5718 \\
\hline C & -4.29190400 & 1.44723500 & 7.39610200 \\
\hline c & -3.15671300 & 1.87590500 & 6.4586 \\
\hline $\mathrm{C}$ & -3.40437200 & 1.54574100 & 4.9786 \\
\hline $\mathrm{C}$ & -2.406 & 2.293 & 4.089 \\
\hline $\mathrm{C}$ & -3.34980100 & 0.03923600 & 4.68863900 \\
\hline & -0.59708600 & 7.88833900 & 0.19124800 \\
\hline $\mathrm{C}$ & -0.78058000 & 6.48472000 & 0.7743 \\
\hline $\mathrm{C}$ & -1.93510200 & 5.67468400 & 0.15259900 \\
\hline $\mathrm{C}$ & -1.59368300 & 5.18084800 & -1.26017600 \\
\hline $\mathrm{C}$ & -2.30162100 & 4.49153000 & 1.05497200 \\
\hline $\mathrm{C}$ & 7.55023900 & 8.26878900 & 1.91987900 \\
\hline $\mathrm{C}$ & 8.22524900 & 6.95193700 & 1.49877900 \\
\hline $\mathrm{C}$ & 7.72106700 & 5.67499700 & 2.14119000 \\
\hline $\mathrm{C}$ & 7.24857900 & 4.60171100 & 1.37687600 \\
\hline $\mathrm{C}$ & 7.84003900 & 5.47583600 & 3.52552100 \\
\hline 4 & 6.96339500 & 3.35864100 & 1.94915300 \\
\hline & 7.54090700 & 4.25460700 & 4.11632800 \\
\hline & 7.12790700 & 3.17271100 & 3.32564200 \\
\hline
\end{tabular}

$\begin{array}{lll}6.91185600 & 1.98575100 & 3.94807400\end{array}$ $\begin{array}{llll}3.21685100 & 5.44039900 & -2.26730800\end{array}$ $\begin{array}{llll}2.66665800 & 4.50472800 & -1.19022400\end{array}$ $\begin{array}{llll}3.64805500 & 4.30247500 & -0.00494300\end{array}$ $\begin{array}{llll}4.34390800 & 2.97788700 & -0.11123000\end{array}$ $\begin{array}{llll}3.96122600 & 2.00661900 & 0.54110500\end{array}$ $\begin{array}{llll}5.33270000 & 2.92946100 & -0.98651600\end{array}$ $\begin{array}{llll}-2.96480100 & 2.44785000 & -6.07431900\end{array}$ $\begin{array}{llll}-3.20884700 & 2.30417300 & -4.59122200\end{array}$ $\begin{array}{llll}-2.26909600 & 1.66625700 & -3.77627100\end{array}$ $\begin{array}{llll}-4.39109500 & 2.76684200 & -3.99657800\end{array}$ $\begin{array}{llll}-2.50505500 & 1.45722200 & -2.41768200\end{array}$ $\begin{array}{lll}-4.63261500 & 2.57480200 & -2.63327200\end{array}$ $\begin{array}{llll}-3.69681800 & 1.90044000 & -1.84079000\end{array}$ $\begin{array}{lll}-2.89715800 & -3.39275900 & -4.95378700\end{array}$ $\begin{array}{llll}-3.42138100 & -3.30708100 & -3.51579300\end{array}$ $\begin{array}{lll}-3.84797800 & -1.89695200 & -3.11182000\end{array}$ $-4.35009800 \quad-1.88235500 \quad-1.72521600$ $\begin{array}{llll}2.00126600 & -0.11534700 & -5.19338200\end{array}$ $\begin{array}{llll}1.47978800 & -0.27019200 & -3.76282600\end{array}$ $\begin{array}{llll}1.35011900 & 1.10897500 & -3.10037500\end{array}$ $\begin{array}{llll}0.16299000 & -1.05912700 & -3.74598100\end{array}$ $\begin{array}{llll}7.21989400 & 4.11420200 & -3.70378900\end{array}$ $\begin{array}{llll}8.37544600 & 3.59280300 & -2.84095600\end{array}$ $\begin{array}{llll}9.13527300 & 4.73798000 & -2.16656000\end{array}$ $\begin{array}{llll}10.73849900 & 4.28902900 & -1.39652800\end{array}$ $\begin{array}{llll}10.19676400 & 3.36341300 & 0.08067400\end{array}$ $10.98031300 \quad 1.15648200 \quad-4.39122600$ $10.28090800 \quad 0.39048400 \quad-3.30009000$ $\begin{array}{llll}9.28152600 & -0.53496300 & -3.61190500\end{array}$ $10.61484300 \quad 0.55486200 \quad-1.95042900$ $\begin{array}{llll}8.65409700 & -1.29624300 & -2.63124800\end{array}$ $9.99759400 \quad-0.19693400 \quad-0.95156600$ $\begin{array}{llll}9.02731400 & -1.15439000 & -1.28461600\end{array}$ $\begin{array}{llll}8.49030000 & -1.90827800 & -0.29539900\end{array}$ $\begin{array}{llll}5.50896000 & -3.80778200 & -4.16242500\end{array}$ $\begin{array}{llll}4.79945800 & -3.38268600 & -2.86097800\end{array}$ $\begin{array}{llll}5.66757700 & -2.82744500 & -1.73999300\end{array}$ $6.72033000 \quad-3.45454200 \quad-1.42923900$ $\begin{array}{llll}5.25994900 & -1.76599400 & -1.16564000\end{array}$ $9.25508300 \quad-6.21082200 \quad-3.56998600$ $\begin{array}{llll}9.71811500 & -5.71954800 & -2.19744000\end{array}$ $10.43039400 \quad-4.37001200-2.28096800$ $\begin{array}{llll}8.63109200 & -5.68022300 & -1.27816100\end{array}$ $\begin{array}{llll}4.12140600 & -6.81734100 & 3.23187100\end{array}$ $\begin{array}{llll}4.64356200 & -7.28075500 & 1.86247300\end{array}$ $\begin{array}{llll}4.81842900 & -6.17302400 & 0.80832700\end{array}$ $\begin{array}{llll}5.61030200 & -6.67854400 & -0.40225000\end{array}$ $\begin{array}{llll}3.46584100 & -5.60735400 & 0.35321800\end{array}$ $\begin{array}{llll}-1.25165300 & -5.26049200 & 4.15731200\end{array}$ $\begin{array}{llll}-0.70717600 & -3.58664600 & 4.64818800\end{array}$ $\begin{array}{lll}-0.78761000 & -2.75257300 & 3.02829400\end{array}$ $\begin{array}{llll}-7.43202300 & 2.69509600 & -0.50633200\end{array}$ $\begin{array}{llll}-6.56897600 & 0.27608600 & -1.61761500\end{array}$ $\begin{array}{llll}6.50847200 & 0.62030200 & -0.77600100\end{array}$ $\begin{array}{llll}4.26413200 & -0.57839300 & 1.05586200\end{array}$ $\begin{array}{llll}3.43876700 & -3.43748100 & 4.85982000\end{array}$ $\begin{array}{llll}3.30052300 & -3.44663100 & 3.46739100\end{array}$ $\begin{array}{llll}2.79167100 & -0.68896000 & 1.24553400\end{array}$ $\begin{array}{llll}2.07209800 & -0.67492100 & -0.10315800\end{array}$ $\begin{array}{llll}4.42106100 & -3.27220500 & 2.65271300\end{array}$ 


\begin{tabular}{|c|c|c|c|}
\hline $\mathrm{C}$ & 5.69381000 & -3.07678700 & \\
\hline $\mathrm{C}$ & .58099000 & & \\
\hline & & & \\
\hline & -0.28167500 & -1.63531600 & -0.09671600 \\
\hline $\mathrm{C}$ & -1.65076200 & -1.52662700 & \\
\hline $\mathrm{C}$ & 7.35887300 & -1.39273500 & \\
\hline $\mathrm{C}$ & -2.16065600 & -0.30784200 & 0.64456500 \\
\hline $\mathrm{C}$ & 5.81749900 & -3.07482300 & 4.58811100 \\
\hline $\mathrm{C}$ & 4.71627900 & -3.25517600 & 5.41321800 \\
\hline $\mathrm{C}$ & -1.31811800 & 579500 & 100 \\
\hline $\mathrm{C}$ & 0.04292500 & 1900 & 500 \\
\hline U & 2.40624800 & 752000 & \\
\hline C & 6.61042 & -0.4 & \\
\hline & 94500 & 7200 & 6800 \\
\hline O & 351800 & 67300 & 2700 \\
\hline & 2.32271500 & 754700 & \\
\hline H & 4.30013000 & 268100 & 7900 \\
\hline $\mathrm{H}$ & -3.85 & -1.0 & 700 \\
\hline & 6.69 & -3.1 & 3900 \\
\hline $\mathrm{H}$ & 0.69 & & \\
\hline & 2.48 & & \\
\hline & 7.75 & -3.4 & 300 \\
\hline $\mathrm{H}$ & 70200 & -1.5 & 300 \\
\hline & 8.41 & -1.2 & 3700 \\
\hline & 6.79 & -2.9 & 000 \\
\hline & 0.09 & -2.5 & 700 \\
\hline & 4.81 & -3.2 & 100 \\
\hline & & & 600 \\
\hline & 4.63 & -1.2 & \\
\hline & 1.55 & -3.6 & 300 \\
\hline & 2.44 & & 600 \\
\hline & 2.57 & -1.5 & 900 \\
\hline & 4.80 & -0.7 & 100 \\
\hline & -5.1 & -2. & 3000 \\
\hline & -3.22 & 38500 & 7300 \\
\hline $\mathrm{H}$ & & & \\
\hline & -4.60 & -1.5 & 5800 \\
\hline & -2.99890100 & 06800 & 0700 \\
\hline & -4.28 & 600 & 7500 \\
\hline & -2.65 & -3.6 & 2600 \\
\hline & -1.97154100 & 131300 & 300 \\
\hline & -3.62 & -2.9 & 300 \\
\hline & -5.12474900 & 3.29126400 & 1800 \\
\hline & & & \\
\hline & & & -0 . \\
\hline & -1.7603 & 0.9578 & 3600 \\
\hline & -1.33 & 2100 & 9800 \\
\hline & -0.57868200 & -0.60910700 & 5200 \\
\hline & 0.3268 & -2.08 & \\
\hline & -0.26901700 & -1.10 & 5800 \\
\hline & 2.21627700 & -0.84779700 & 8500 \\
\hline & 0.89 & 1.0 & \\
\hline & 2.33267000 & 1.58428400 & -3.002684 \\
\hline & 0.72577000 & & \\
\hline & 7.99772200 & 2.89352500 & -2.08734700 \\
\hline & 9.07406600 & 3.02665400 & -3.4606 \\
\hline & 8.51251600 & 5.244 & -1.42214500 \\
\hline & 9.40861300 & 5.48942800 & -2.91549100 \\
\hline & & 4.00897700 & \\
\hline & 9.56246100 & 2.51811700 & -0.19058600 \\
\hline & 11.10364500 & 2.97739700 & 0.55214300 \\
\hline & & -2.54221600 & -0.68266400 \\
\hline & 7.91384100 & -2.03661800 & -2.90309200 \\
\hline
\end{tabular}

H $\quad 10.28599800 \quad-0.08355100 \quad 0.08863400$

H $\quad 11.37512500 \quad 1.28042000 \quad-1.67977200$

$\mathrm{H} \quad 8.99761200 \quad-0.68178700 \quad-4.65100700$

$\mathrm{H} \quad 3.11338500 \quad 4.29712100 \quad 0.94597600$

$\begin{array}{llll}\mathrm{H} & 4.38884700 & 5.10660400 & 0.02027100\end{array}$

H $\quad 1.74217100 \quad 4.93994600 \quad-0.79721600$

$\mathrm{H} \quad 2.38197400 \quad 3.53505400 \quad-1.61386300$

$\mathrm{H} \quad 8.14994500 \quad 6.85527100 \quad 0.40934800$

$\mathrm{H} \quad 9.29991600 \quad 7.03330300 \quad 1.71010100$

H $\quad 8.20441400 \quad 6.28714200 \quad 4.15084900$

$\mathrm{H} \quad 7.13257400 \quad 4.71387200 \quad 0.30248300$

$\mathrm{H} \quad 6.65331800 \quad 2.52488100 \quad 1.32790900$

$\mathrm{H} \quad 7.65546000 \quad 4.10256700 \quad 5.18438800$

$\mathrm{H} \quad 6.79380600 \quad 1.27043900 \quad 3.29739600$

$\mathrm{H} \quad-3.07521500 \quad 3.86415700 \quad 0.60074500$

$\mathrm{H} \quad-1.42103100 \quad 3.86231700 \quad 1.23012900$

$\mathrm{H} \quad-2.66344500 \quad 4.82558700 \quad 2.03237300$

$\mathrm{H} \quad-2.44429000 \quad 4.66704100 \quad-1.71785700$

$\mathrm{H} \quad-1.30044200 \quad 6.00107400 \quad-1.92308000$

$\mathrm{H} \quad-0.76285200 \quad 4.46504600 \quad-1.22477800$

$\mathrm{H} \quad-2.81278500 \quad 6.33450400 \quad 0.08283200$

$\mathrm{H} \quad 0.14770000 \quad 5.90732500 \quad 0.65569700$

$\mathrm{H} \quad-0.94253100 \quad 6.56934800 \quad 1.85655400$

$\mathrm{H} \quad-7.22066000 \quad 2.40167900 \quad 1.28973400$

$\mathrm{H} \quad-8.93402500 \quad 0.08817600 \quad 3.38946100$

$\mathrm{H} \quad-8.49016700 \quad-1.50363900 \quad 1.57855800$

$\mathrm{H} \quad-7.34518600 \quad-0.39713300 \quad 0.81583600$

$\mathrm{H} \quad-6.67601300 \quad-3.37222000 \quad 1.54980900$

$\mathrm{H} \quad-5.74239900 \quad-2.19787800 \quad 0.64017300$

H $\quad-5.04464800 \quad-2.95330800 \quad 2.08936100$

$\mathrm{H} \quad-7.79233400 \quad-2.67091400 \quad 3.81807900$

$\mathrm{H} \quad-6.11530700 \quad-2.50926100 \quad 4.36199300$

$\mathrm{H} \quad-7.22839000 \quad-1.15399500 \quad 4.57216300$

H $\quad-5.76728200 \quad-0.67716000 \quad 2.68013500$

H $\quad-9.00570800 \quad 1.17874500 \quad 0.64330000$

$\mathrm{H} \quad-9.80551900 \quad 3.83019600 \quad 0.71929900$

$\mathrm{H} \quad-11.35767300 \quad 3.73162900 \quad 1.57186900$

H $\quad-11.73182600 \quad 1.68405400 \quad 0.05956300$

$\mathrm{H} \quad-13.50312800 \quad-0.12805200 \quad-1.24194700$

$\mathrm{H} \quad-14.47239100 \quad 0.84551800 \quad-3.24349300$

$\mathrm{H} \quad-13.25583600 \quad 2.61127900 \quad-2.02978600$

H $\quad-13.03536700 \quad 0.45467400 \quad-4.20068900$

$\mathrm{H} \quad-12.78313900 \quad-1.19595500 \quad-2.48099000$

$\mathrm{H} \quad-12.68773200 \quad 2.84326400 \quad-3.68796600$

$\mathrm{H} \quad-10.60786400 \quad 1.67770800 \quad-3.23852300$

$\mathrm{H} \quad-8.82968600 \quad-2.64981700 \quad-0.92365700$

$\mathrm{H} \quad-8.85620300 \quad-5.91007200 \quad-2.00552900$

$\mathrm{H} \quad 4.29441500 \quad-4.26496200 \quad-2.44567800$

$\mathrm{H} \quad 4.02250700 \quad-2.64487100 \quad-3.07948700$

$\mathrm{H} \quad 9.80213900 \quad-3.62658700 \quad-2.78057500$

$\mathrm{H} \quad 10.65392000 \quad-3.99760600 \quad-1.27775200$

H $\quad 11.36578700 \quad-4.44791700 \quad-2.84572200$

H $\quad 10.41203700 \quad-6.45747700 \quad-1.77484600$

H $\quad 3.24847400 \quad-6.16755700 \quad 3.12595300$

H $\quad 5.60591700 \quad-7.78952400 \quad 2.00086900$

H $\quad 3.96164300 \quad-8.03882800 \quad 1.45018700$

H $\quad 5.10599700 \quad-7.53734900 \quad-0.86379300$

$\mathrm{H} \quad 5.71216400 \quad-5.89573500 \quad-1.15846800$

$\mathrm{H} \quad 6.62471300 \quad-6.98054200 \quad-0.13039400$

$\mathrm{H} \quad 2.84446500 \quad-5.27929000 \quad 1.19140100$

H $\quad 3.60158000 \quad-4.74950500 \quad-0.31511400$

$\mathrm{H} \quad 2.90073900 \quad-6.36828200 \quad-0.19902600$

$\mathrm{H} \quad 5.39568300 \quad-5.36129100 \quad 1.26900900$ 


\begin{tabular}{|c|c|c|c|}
\hline H & 805300 & & \\
\hline & -2.26879000 & -5.22234400 & \\
\hline & & & \\
\hline & -0.04619300 & -3.15384300 & .33437800 \\
\hline & -1.78026700 & -2.86210500 & \\
\hline & -4.13080400 & -0.50632000 & \\
\hline & -3.48813800 & -0.14919200 & 75800 \\
\hline & -2.37985100 & -0.38362500 & \\
\hline & -2.45111500 & 3.37530700 & \\
\hline & -1.37807800 & & 300 \\
\hline & -2.60861700 & 2.107208 & 900 \\
\hline & & & \\
\hline & -3.00 & & \\
\hline & -2.214 & 1.40600 & 300 \\
\hline & -7.136 & & 300 \\
\hline & -8.1628 & & 800 \\
\hline & -7.4816 & -0.04441 & 400 \\
\hline & -6.25 & & \\
\hline & 5.752 & & \\
\hline & 4.458 & & \\
\hline & -8.23 & & \\
\hline & -6.140 & & 300 \\
\hline & & & \\
\hline & $8.466^{\prime}$ & -5.5 & \\
\hline & 8.008 & -4.9 & 200 \\
\hline & -4.504 & & \\
\hline & -11.703 & & 2300 \\
\hline & -4.824 & & \\
\hline & & & \\
\hline & -3.4 & & 300 \\
\hline & & & 300 \\
\hline & & & \\
\hline & 6.421 & & \\
\hline & & & \\
\hline & 4.098 & & \\
\hline & & & \\
\hline & & & \\
\hline & & -4.2549 & \\
\hline & & -2.9 & 700 \\
\hline & & & \\
\hline & & & \\
\hline & & & \\
\hline & & & \\
\hline & & & \\
\hline & & & \\
\hline & & & \\
\hline & -1.90 & 2.510343 & 80600 \\
\hline & $-2.696^{\prime}$ & -4.43293700 & \\
\hline & -7.65 & & \\
\hline & -6.33 & -6.47349000 & 4400 \\
\hline & -9.4655 & -4.59971200 & \\
\hline & & & \\
\hline & -5.94472400 & 3.77058200 & \\
\hline & & & \\
\hline & -5.22059500 & 1.96510100 & 80500 \\
\hline & -3.9514 & & \\
\hline & & 8.33275500 & \\
\hline & -0.56150300 & 7.87481600 & -0.90380000 \\
\hline & & & \\
\hline & & 8.35302500 & 1.49435700 \\
\hline & 8.86521000 & -7.23328300 & -3.52480300 \\
\hline & 10.04961400 & -6.25242800 & -4.32234700 \\
\hline & 3.79003400 & -7.68718800 & 3.80875300 \\
\hline
\end{tabular}

$\begin{array}{rrrr}\mathrm{H} & 4.87368700 & -6.24583700 & 3.78617800 \\ \mathrm{H} & -0.57947100 & -5.69109900 & 3.41108100 \\ \mathrm{H} & 6.14358700 & -0.25612900 & -1.04749700 \\ \mathrm{H} & 7.45955000 & 0.57045000 & -0.95120700\end{array}$

\section{TS1 (+5.0 kcal $/ \mathrm{mol})$}

\begin{tabular}{|c|c|c|c|}
\hline & 6.64497500 & 5.74161100 & \\
\hline & 7.17600900 & 4.76239800 & -0.87951600 \\
\hline & 6.74523600 & 3.61304300 & -1.02456700 \\
\hline & 8.19854600 & 5.21771900 & -1.63735900 \\
\hline & 8.75105200 & 4.39833300 & -2.68434600 \\
\hline & 9.82488600 & 3.40076000 & -2.24855400 \\
\hline & 10.83028600 & 3.20174100 & -2.92472600 \\
\hline & 9.56021200 & 2.74177900 & -1.08608100 \\
\hline $\mathrm{C}$ & 10.42291500 & 1.63640200 & -0.72781200 \\
\hline & 10.12589900 & 0.42419400 & -1.60013200 \\
\hline & 8.96642200 & -0.01270900 & -1.66658700 \\
\hline $\mathrm{N}$ & 11.14447300 & -0.20819100 & -2.21559600 \\
\hline $\mathrm{C}$ & 10.85745800 & -1.45150400 & -2.95147000 \\
\hline $\mathrm{C}$ & 10.23554200 & -2.51734100 & -2.04502600 \\
\hline $\mathrm{O}$ & 9.39904500 & -3.30472600 & -2.48500200 \\
\hline $\mathrm{C}$ & 12.22990800 & -1.83457700 & -3.53320400 \\
\hline $\mathrm{C}$ & 12.91147400 & -0.474 & 03000 \\
\hline $\mathrm{C}$ & 12.48751800 & 0.33862800 & -2.51374300 \\
\hline $\mathrm{N}$ & 10.70843000 & -2.527 & 12400 \\
\hline $\mathrm{C}$ & 10.19481600 & -3.3680 & 14200 \\
\hline $\mathrm{C}$ & 9.64228400 & -2.58151000 & 22700 \\
\hline $\mathrm{O}$ & 9.79403400 & -3.005 & 600 \\
\hline $\mathrm{N}$ & 8.97493000 & -1.421 & 24700 \\
\hline $\mathrm{C}$ & 8.13200900 & -0.824 & 100 \\
\hline $\mathrm{C}$ & 6.98095200 & -1.7542 & 2600 \\
\hline $\mathrm{O}$ & 6.52211200 & -1.69521500 & 0500 \\
\hline $\mathrm{C}$ & 7.59336800 & 0.5336 & 500 \\
\hline $\mathrm{C}$ & 6.62193200 & 1.245 & 000 \\
\hline $\mathrm{C}$ & 7.21287700 & 1.42281800 & 3000 \\
\hline $\mathrm{C}$ & 6.20603100 & 2.595 & 3300 \\
\hline $\mathrm{N}$ & 6.49036300 & -2.5538 & 5700 \\
\hline $\mathrm{C}$ & 5.38292000 & -3.4470 & 6300 \\
\hline $\mathrm{C}$ & 4.07432500 & -2.3818 & 200 \\
\hline $\mathrm{C}$ & 2.87850100 & -2.65827300 & 7000 \\
\hline $\mathrm{C}$ & 3.08329600 & -2.28066300 & 8300 \\
\hline $\mathrm{C}$ & 1.86714700 & -2.70061200 & 3600 \\
\hline $\mathrm{C}$ & 3.37152800 & -0.78466700 & 4.42463300 \\
\hline $\mathrm{C}$ & 0.11714300 & -7.71472700 & -0.93825700 \\
\hline $\mathrm{C}$ & 0.32800000 & -6.39285900 & -0.19622200 \\
\hline $\mathrm{C}$ & 1.56180800 & -5.59103300 & 3200 \\
\hline $\mathrm{C}$ & 1.42290900 & -5.08196300 & 39500 \\
\hline $\mathrm{C}$ & 1.81491200 & -4.42011900 & 71000 \\
\hline $\mathrm{C}$ & -8.00474400 & -8.17487600 & 0.88936700 \\
\hline $\mathrm{C}$ & -8.76762400 & -6.86554100 & 0.66024400 \\
\hline $\mathrm{C}$ & -8.27232200 & -5.65228700 & 1.40921800 \\
\hline $\mathrm{C}$ & -6.91627000 & -5.31559800 & 1.45109600 \\
\hline & -9.17681700 & -4.79262800 & 2.04358000 \\
\hline $\mathrm{C}$ & -6.47516900 & -4.14706300 & 2.06656200 \\
\hline $\mathrm{C}$ & -8.75576300 & -3.62067500 & 2.66574600 \\
\hline $\mathrm{C}$ & -7.40317100 & -3.28151300 & 2.65611300 \\
\hline $\mathrm{O}$ & -7.02924400 & -2.08697600 & 3.22483000 \\
\hline & -3.68759400 & -4.88579900 & -2.96521500 \\
\hline 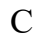 & -3.41474600 & -3.83820600 & -1.89321100 \\
\hline & -4.71301500 & -3.53019800 & -1.13394200 \\
\hline
\end{tabular}




\begin{tabular}{|c|c|c|c|}
\hline & -4.81361900 & -2.14568700 & -0.54185500 \\
\hline & -6.06441800 & -1.81095500 & -0.29062700 \\
\hline & -3.83683100 & -1.42524900 & -0.33548400 \\
\hline & 2.48851600 & -1.51995200 & -6.45641900 \\
\hline & 2.77218700 & -1.66687100 & -4.98258900 \\
\hline & 1.89001500 & -1.14988700 & -4.02829800 \\
\hline & 3.94298700 & -2.29288600 & -4.53286900 \\
\hline & 2.17386200 & -1.22032600 & 414100 \\
\hline & 4.22797800 & -2.38399800 & -3.16825700 \\
\hline & 3.35141000 & -1.83119600 & -2.22977700 \\
\hline & 2.56465400 & 4.11492300 & -4.55447100 \\
\hline & 3.23367900 & 3.78114400 & -3.21721900 \\
\hline & 3.67365100 & 2.31828000 & -3.14136300 \\
\hline $\mathrm{N}$ & 4.39465900 & 2.03133600 & -1.85 \\
\hline $\mathrm{C}$ & -2.40513900 & 0.99191200 & -5.13504 \\
\hline & -1.87381000 & 1.02313900 & -3.6996 \\
\hline $\mathrm{C}$ & -1.84213100 & -0.37871300 & -3.08088 \\
\hline $\mathrm{C}$ & -0.50794400 & 1887900 & -3.62107000 \\
\hline $\mathrm{C}$ & -7.68574600 & -3.3 & -4.12584200 \\
\hline $\mathrm{C}$ & -8.72952400 & -2.89976300 & -3.08863000 \\
\hline$c$ & -9.14975600 & -4.06628100 & -2.19429600 \\
\hline S & -10.54759700 & -3.67557000 & -1.07853800 \\
\hline $\mathrm{C}$ & -9.83059200 & -2.24492000 & -0.19955100 \\
\hline $\mathrm{C}$ & -11.3940 & -0.20912400 & -4.32995000 \\
\hline $\mathrm{C}$ & -10.66351100 & 0.33644900 & -3.12687300 \\
\hline $\mathrm{C}$ & -9.26767500 & 0.46348 & -3.13122700 \\
\hline $\mathrm{C}$ & -11.3482 & 0.71475500 & -1.96718300 \\
\hline $\mathrm{C}$ & -8.57782500 & 0.94258600 & -2.02149500 \\
\hline $\mathrm{C}$ & -10.672 & 1.21 & -0.85406 \\
\hline $\mathrm{C}$ & -9.28476100 & 1.33593600 & -0.87961600 \\
\hline $\mathrm{O}$ & -8.6301 & 1.834638 & 0.22948 \\
\hline $\mathrm{C}$ & -5.815 & 4.5 & -3.54313 \\
\hline $\mathrm{C}$ & -5.18286200 & 3.955508 & -2.28964800 \\
\hline $\mathrm{C}$ & -6.17127200 & 3.335648 & \\
\hline $\mathrm{O}$ & -7.308 & 3.8 & -1.17406 \\
\hline $\mathrm{O}$ & -5.78721600 & 533000 & -0.60834400 \\
\hline $\mathrm{C}$ & -9.4985 & 6.9 & -2.55420100 \\
\hline $\mathrm{C}$ & -9.98001700 & 6.250942 & -1.27933 \\
\hline $\mathrm{C}$ & -10.65714100 & 4.91272700 & -1.58480200 \\
\hline $\mathrm{O}$ & -8.913 & 6.0 & -0.35211 \\
\hline $\mathrm{C}$ & -4.23392600 & 6.52728300 & 4.16094600 \\
\hline $\mathrm{C}$ & -4.77253900 & 18300 & 2.88305600 \\
\hline $\mathrm{C}$ & -5.49539800 & 6.235469 & 1.91 \\
\hline $\mathrm{C}$ & -5.86917900 & 6.97821800 & 0.62295900 \\
\hline $\mathrm{C}$ & -4.66533800 & 4.99020500 & 1.59047600 \\
\hline $\mathrm{C}$ & 1.12052000 & 4.75917800 & 4.75729100 \\
\hline $\mathrm{S}$ & 2.29433800 & 3.38991000 & 5.04606600 \\
\hline $\mathrm{C}$ & 2.26310200 & 2.59845200 & 3.40194200 \\
\hline $\mathrm{O}$ & 7.01242500 & -2.82856300 & -1.06224700 \\
\hline $\mathrm{O}$ & 6.27820600 & -0.31514200 & -2.07285900 \\
\hline $\mathrm{O}$ & -6.62487600 & 0.09278600 & 1.34774100 \\
\hline $\mathrm{N}$ & -3.48418500 & 1.37430500 & -0.50811700 \\
\hline $\mathrm{C}$ & -0.44649400 & 0.75504100 & 5.72804400 \\
\hline $\mathrm{C}$ & -1.07558600 & 1.86032500 & 5.13953800 \\
\hline $\mathrm{C}$ & -2.77188100 & 2.16016100 & 0.53199400 \\
\hline $\mathrm{C}$ & -1.36215500 & 2.59015500 & 0.13315200 \\
\hline $\mathrm{C}$ & -2.28879200 & 1.70742400 & 4.47345300 \\
\hline $\mathrm{C}$ & -2.92022700 & 0.46378100 & 4.41252000 \\
\hline$C$ & -0.26177800 & 1.55526600 & 0.27772200 \\
\hline $\mathrm{C}$ & -4.21781500 & 0.26321400 & 3.65176100 \\
\hline $\mathrm{C}$ & 1.07089400 & 1.99722300 & 0.22126200 \\
\hline $\mathrm{C}$ & 2.12828100 & 1.12269600 & 0.44175100 \\
\hline & -3.93582600 & -0.81581100 & \\
\hline
\end{tabular}

C

\section{C}

C

8$$
\text { 每 }
$$

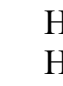

\section{H}

\section{$\mathrm{H}$}

\section{H}

$\mathrm{H}$

$\mathrm{H}$
$\mathrm{H}$

$\mathrm{H}$

\section{$\mathrm{H}$}

$\mathrm{H}$

$\mathrm{H}$

$\begin{array}{rrrr}\mathrm{H} & -8.70902100 & 0.16283500 & -4.01405200 \\ \mathrm{H} & -4.88317700 & -4.23793100 & -0.31500200\end{array}$ $\begin{array}{llll}\mathrm{H} & -4.88317700 & -4.23793100 & -0.31500200 \\ \mathrm{H} & -5.58117300 & -3.64963600 & -1.78973700\end{array}$

H $\quad-2.62760900 \quad-4.15521900 \quad-1.20118900$

$\begin{array}{llll}1.86278500 & -0.23313000 & 0.71719200\end{array}$ $\begin{array}{lll}-2.29307700 & -0.63205500 & 5.02758800\end{array}$ $\begin{array}{lll}-1.07223200 & -0.49738800 & 5.67506200\end{array}$ $\begin{array}{lll}0.55338500 & -0.69223100 & 0.75456600\end{array}$ $\begin{array}{lll}-0.50174300 & 0.20110300 & 0.52857400\end{array}$ $\begin{array}{llll}0.74326100 & 0.84350000 & 6.36869600\end{array}$ $\begin{array}{lll}-4.40484200 & -1.94119600 & 2.69951700\end{array}$ $\begin{array}{llll}2.92568300 & -1.06228400 & 0.92920700\end{array}$ $\begin{array}{lll}3.43725500 & 1.49587400 & 0.46222800\end{array}$ $\begin{array}{lll}-0.61903300 & 2.83702700 & 5.22416700\end{array}$ $\begin{array}{lll}-2.75997800 & 2.57394600 & 4.01589600\end{array}$ $\begin{array}{llll}3.70980200 & -0.48327500 & 0.86985600\end{array}$ $\begin{array}{lll}-4.51053200 & 1.17742200 & 3.12612400\end{array}$ $\begin{array}{lll}-1.51169600 & -0.18999800 & 0.54959000\end{array}$ $\begin{array}{lll}-2.73654500 & 1.59079300 & 1.46314800\end{array}$ $\begin{array}{lll}-5.04511200 & -0.04311900 & 4.29938900\end{array}$ $\begin{array}{lll}-1.09712100 & 3.45427300 & 0.75507600\end{array}$ $\begin{array}{lll}-3.18641400 & -0.58160600 & 1.87798300\end{array}$ $\begin{array}{llll}-2.76542100 & -1.60936200 & 4.98582100\end{array}$ $\begin{array}{llll}1.29477000 & 3.04664300 & 0.04708700\end{array}$ $\begin{array}{llll}-0.58205400 & -1.34730800 & 6.13636300\end{array}$ $\begin{array}{llll}0.35899900 & -1.73685600 & 0.96347700\end{array}$ $\begin{array}{llll}-4.72735200 & 1.91613800 & -0.68285400\end{array}$ $\begin{array}{llll}1.17170500 & 1.69789400 & 6.15181100\end{array}$ $\begin{array}{llll}-1.39318900 & 2.97968900 & -0.89597200\end{array}$ $\begin{array}{lll}-3.36692400 & 3.05209000 & 0.72869900\end{array}$ $\begin{array}{llll}-3.64096200 & 0.38893400 & -0.25540100\end{array}$ $\begin{array}{llll}5.13924600 & 2.71277500 & -1.74356700\end{array}$ $\begin{array}{llll}3.72652800 & 1.82425000 & -0.47674700\end{array}$ $\begin{array}{llll}4.87446200 & 1.13032500 & -1.95389300\end{array}$ $\begin{array}{llll}4.29802900 & 2.09505300 & -4.02202400\end{array}$ $2.80563300 \quad 1.65365000 \quad-3.20119800$ $\begin{array}{llll}4.11771200 & 4.41904300 & -3.07598000\end{array}$ $\begin{array}{llll}2.55764500 & 3.99869700 & -2.38127900\end{array}$ $\begin{array}{llll}1.60731400 & 3.59437900 & -4.65594600\end{array}$ $\begin{array}{llll}3.19806600 & 3.79673300 & -5.39093100\end{array}$ $\begin{array}{lll}4.62691200 & -2.72737700 & -5.25809100\end{array}$ $\begin{array}{llll}5.11974700 & -2.89898400 & -2.82129800\end{array}$ $\begin{array}{llll}3.56786600 & -1.89260700 & -1.17033900\end{array}$ $\begin{array}{llll}1.47407700 & -0.81533800 & -1.94117500\end{array}$ $\begin{array}{llll}0.96511700 & -0.69270500 & -4.36186700\end{array}$ $\begin{array}{llll}0.21511000 & 1.24455600 & -4.29189900\end{array}$ $\begin{array}{llll}-0.58758300 & 2.77016300 & -3.91879000\end{array}$ $\begin{array}{llll}-0.09361600 & 1.67844300 & -2.60838700\end{array}$ $\begin{array}{llll}-2.58053600 & 1.64396600 & -3.12741600\end{array}$ $\begin{array}{llll}-1.44099100 & -0.37095600 & -2.06333600\end{array}$ $-2.84473400 \quad-0.81374400 \quad-3.04026700$ $-1.21232300 \quad-1.04899600 \quad-3.67615800$ $\begin{array}{lll}-8.32087000 & -2.10276200 & -2.46224400\end{array}$ $\begin{array}{lll}-9.61668400 & -2.48687000 & -3.57722900\end{array}$ $\begin{array}{lll}-8.30384600 & -4.40711600 & -1.58467700\end{array}$ $-9.49206800 \quad-4.91655200 \quad-2.79440300$ $\begin{array}{lll}-8.82764600 & -2.48521000 & 0.15734900\end{array}$ $-9.80833400 \quad-1.36412100 \quad-0.83708500$ $\begin{array}{lll}-10.47028300 & -2.04452200 & 0.66130000\end{array}$ $\begin{array}{llll}-8.07849900 & 2.58786700 & -0.09285400\end{array}$ $\begin{array}{lll}-7.49565100 & 1.00058400 & -2.01945600\end{array}$ $\begin{array}{llll}-11.20821600 & 1.52571500 & 0.03758800\end{array}$ $\begin{array}{llll}-12.42986600 & 0.61899300 & -1.93342100\end{array}$ $-8.70902100 \quad 0.16283500 \quad-4.01405200$ $\begin{array}{lll}-2.62760900 & -4.15521900 & -1.20118900\end{array}$ 


\begin{tabular}{|c|c|c|c|}
\hline $\mathrm{H}$ & 20000 & -2.92250200 & \\
\hline & & & \\
\hline & -9.81949500 & -7.02388500 & \\
\hline & -10.23501300 & -5.03404000 & 02775600 \\
\hline & & -5.97377100 & \\
\hline & -5.42232000 & -3.89477200 & 2.07739000 \\
\hline & -9.46374300 & -2.95184300 & \\
\hline & -6.04769600 & -2.06471100 & \\
\hline & & -3.82 & \\
\hline & 0.95 & -3.743 & \\
\hline & 1.97 & -4.761 & \\
\hline & & -4.529 & 7500 \\
\hline & 1.26 & -5.897 & -2.8047 \\
\hline & 0.56 & -4.397 & \\
\hline & 2.43 & -6.26 & \\
\hline & -0.5 & -5.7 & \\
\hline & & & \\
\hline & 6.9 & -2.6 & \\
\hline & 8.7 & -0.6 & \\
\hline & & & \\
\hline & 7.1 & & \\
\hline & 7.0 & & \\
\hline & & & \\
\hline & 5.4 & & \\
\hline & & & \\
\hline & & & \\
\hline & 7.3 & & \\
\hline & & & \\
\hline & 8.7 & -1.26 & \\
\hline & & & \\
\hline & & & \\
\hline & 11.3 & -1.7 & -0. \\
\hline & & & \\
\hline & & & \\
\hline & 12.7 & -2.4 & \\
\hline & & & \\
\hline & 12.4 & 1.40 & \\
\hline & & & \\
\hline & & -1.26 & \\
\hline & & 2.71 & -0.776 \\
\hline & & & \\
\hline & -4.6 & & \\
\hline & & & \\
\hline $\mathrm{H}$ & -9.9 & 4.23 & -2.1 \\
\hline & -10.9 & & \\
\hline & & & \\
\hline $\mathrm{H}$ & -10.6 & & -0.7 \\
\hline & -3.3 & & \\
\hline $\mathrm{H}$ & -5.4 & 7.99 & \\
\hline $\mathrm{H}$ & -3.93 & & \\
\hline $\mathrm{H}$ & & & \\
\hline $\mathrm{H}$ & -6.4 & 6.344 & -0.06150300 \\
\hline $\mathrm{H}$ & -6.4 & & \\
\hline $\mathrm{H}$ & -4.40 & 4.423 & 2.49095100 \\
\hline $\mathrm{H}$ & -5.21 & 4.31492600 & 0.93432300 \\
\hline $\mathrm{H}$ & -3.72 & 5.270 & \\
\hline $\mathrm{H}$ & -6.42 & 5.90022000 & 2.39195900 \\
\hline $\mathrm{H}$ & 0.90 & 5.217 & \\
\hline $\mathrm{H}$ & & 5.51056000 & 4.09951000 \\
\hline & 2.94211800 & 1.74746400 & 3.43977100 \\
\hline $\mathrm{H}$ & 1.260 & 2.23988300 & \\
\hline $\mathrm{H}$ & 2.61244100 & 3.28585700 & 2.63031200 \\
\hline & 4.33093200 & -0.50660700 & 4.86621900 \\
\hline
\end{tabular}

$\begin{array}{llll}3.41383100 & -0.53838400 & 3.35946700\end{array}$ $\begin{array}{llll}2.57816100 & -0.18665800 & 4.88781700\end{array}$ $\begin{array}{lll}1.63520300 & -3.76319700 & 3.90931600\end{array}$ $\begin{array}{llll}0.97933900 & -2.12502400 & 4.06235200\end{array}$ $\begin{array}{llll}2.04293900 & -2.52402400 & 2.70787500\end{array}$ $\begin{array}{llll}3.96378900 & -2.82610400 & 4.24827300\end{array}$ $\begin{array}{llll}2.61961900 & -3.72489800 & 6.13177300\end{array}$ $\begin{array}{llll}2.00575900 & -2.11033500 & 6.46102600\end{array}$ $\begin{array}{llll}6.72210800 & -1.95113800 & -1.38008800\end{array}$ $\begin{array}{llll}7.73979400 & -3.10191900 & -1.64907400\end{array}$ $\begin{array}{llll}7.20547600 & -0.01879600 & -2.13847900\end{array}$ $\begin{array}{llll}6.00598100 & -0.64661600 & -2.94065100\end{array}$ $\begin{array}{lll}-6.17178600 & -0.97005200 & 0.26861200\end{array}$ $\begin{array}{llll}-2.94071700 & 1.35192500 & -1.36587700\end{array}$ $\begin{array}{llll}7.93195300 & 3.82170300 & -3.12765400\end{array}$ $\begin{array}{llll}5.93967500 & 5.24280800 & 0.78113000\end{array}$ $\begin{array}{llll}-6.58337700 & 5.30031200 & -3.26909200\end{array}$ $\begin{array}{llll}-8.72903400 & 6.33736900 & -3.04513800\end{array}$ $\begin{array}{llll}-8.36950000 & 5.33831200 & -0.67266800\end{array}$ $\begin{array}{llll}4.30320700 & -1.30931300 & 6.98602600\end{array}$ $\begin{array}{llll}11.46144000 & 1.95683600 & -0.79220800\end{array}$ $\begin{array}{lll}4.48802400 & -2.89438200 & 2.24686200\end{array}$ $\begin{array}{llll}0.92549600 & -8.42576500 & -0.74015600\end{array}$ $\begin{array}{llll}2.96031400 & -2.32137700 & -7.03295900\end{array}$ $\begin{array}{llll}2.88983300 & -0.57224400 & -6.83824400\end{array}$ $\begin{array}{llll}-1.82023900 & 0.32038500 & -5.77183600\end{array}$ $\begin{array}{lll}-6.85133800 & -3.82696500 & -3.64422800\end{array}$ $\begin{array}{lll}-8.56104000 & -8.97760500 & 0.38592300\end{array}$ $\begin{array}{llll}-4.56559900 & -4.57807600 & -3.54835700\end{array}$ $\begin{array}{llll}-12.47193100 & -0.24660900 & -4.15181000\end{array}$ $\begin{array}{llll}-11.06654800 & -1.22692500 & -4.56851700\end{array}$ $\begin{array}{lll}-7.06722100 & -0.48308400 & 2.00047400\end{array}$ $\begin{array}{llll}-5.01838900 & 5.08499400 & -4.09393600\end{array}$ $\begin{array}{llll}-6.26937300 & 3.80854700 & -4.17919600\end{array}$ $-2.90702400 \quad-5.05318500 \quad-3.71522100$ $\begin{array}{lll}-3.92150100 & -5.89082000 & -2.59753500\end{array}$ $\begin{array}{llll}-7.24962300 & -2.43986200 & -4.64042900\end{array}$ $\begin{array}{lll}-8.07468300 & -3.98051400 & -4.89312600\end{array}$ $\begin{array}{llll}-11.22598200 & 0.40045800 & -5.22315900\end{array}$ $\begin{array}{llll}-2.38161900 & 1.99807000 & -5.56651100\end{array}$ $\begin{array}{llll}-3.46105200 & 0.70677000 & -5.18620400\end{array}$ $\begin{array}{llll}1.41930500 & -1.52771400 & -6.69213800\end{array}$ $\begin{array}{llll}2.38145500 & 5.18660200 & -4.68436100\end{array}$ $\begin{array}{llll}7.46874600 & 6.15186100 & 0.71156600\end{array}$ $\begin{array}{llll}6.14219900 & 6.57343200 & -0.38552100\end{array}$ $\begin{array}{llll}9.17510900 & 5.04229300 & -3.46162400\end{array}$ $\begin{array}{llll}10.20866900 & 1.33496900 & 0.30085900\end{array}$ $\begin{array}{llll}5.60752000 & -4.17848700 & 2.73935800\end{array}$ $\begin{array}{llll}5.16968600 & -3.98315300 & 1.02927900\end{array}$ $\begin{array}{llll}4.98714000 & -2.87680000 & 6.64883000\end{array}$ $\begin{array}{llll}3.75067500 & -2.59901900 & 8.01892900\end{array}$ $\begin{array}{llll}-0.83504700 & -8.18101800 & -0.66507800\end{array}$ $\begin{array}{llll}0.06214200 & -7.55296300 & -2.01990700\end{array}$ $\begin{array}{lll}-7.96400700 & -8.47301300 & 1.94212500\end{array}$ $\begin{array}{llll}-7.00930200 & -8.21945000 & 0.43564800\end{array}$ $\begin{array}{llll}-9.08652900 & 7.94454700 & -2.37922400\end{array}$ $\begin{array}{lll}-10.30534600 & 7.10334500 & -3.27789600\end{array}$ $\begin{array}{lll}-3.87385700 & 7.30403600 & 4.84339500\end{array}$ $\begin{array}{lll}-4.98804800 & 5.89937200 & 4.64788500\end{array}$ $\begin{array}{llll}0.19201000 & 4.39716600 & 4.31012300\end{array}$ $\begin{array}{llll}-7.29797700 & 0.71502100 & 0.99292700\end{array}$ 
Int1 (+4.7 kcal/mol)

\begin{tabular}{|c|c|c|c|}
\hline & 6.64631500 & 5.74377400 & \\
\hline & 7.17695400 & 4.76639900 & -0.87334900 \\
\hline & 6.74477200 & 3.61800100 & -1.02229900 \\
\hline & 8.20060300 & 5.22224200 & -1.62932500 \\
\hline & 8.75307300 & 4.40409200 & -2.67738600 \\
\hline & 9.82651400 & 3.40585700 & -2.24201000 \\
\hline & 10.83292000 & 3.20781900 & -2.91691500 \\
\hline & 9.56035500 & 2.74527500 & -1.08072900 \\
\hline & 10.42326100 & 1.64023100 & -0.722 \\
\hline & 10.13032900 & 0.42897200 & -1.597 \\
\hline & 8.97168900 & -0.00933700 & -1.66 \\
\hline N & 11.15154200 & -0.20210400 & -2.20931500 \\
\hline & 10.86786400 & -1.44570000 & -2.94606100 \\
\hline 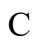 & 10.24278100 & -2.51098000 & -2.04100300 \\
\hline D & 9.40564500 & -3.29687300 & -2.48237200 \\
\hline & 12.24273 & -1.82804400 & -3.5 \\
\hline $\mathrm{C}$ & 12.92405300 & -0.46733 & -3.7 \\
\hline C & 12.49507000 & 0.34583100 & -2.50295100 \\
\hline $\mathrm{N}$ & 10.71369300 & -2.52232600 & -0.77229 \\
\hline $\mathrm{C}$ & 10.19794900 & -3.36199200 & 0.29253 \\
\hline $\mathrm{C}$ & 9.64405700 & -2.57515600 & 1.48651000 \\
\hline $\mathrm{O}$ & 9.7952 & -2.998203 & \\
\hline $\mathrm{N}$ & 8.97580600 & -1.41615300 & 1.193 \\
\hline & 8.130 & -0.8210 & 2.23 \\
\hline C & 6.981 & -1.753 & \\
\hline O & 6.52337600 & -1.69849000 & 3.81000 \\
\hline C & 7.58721900 & 0.53536600 & 1.74969 \\
\hline $\mathrm{C}$ & 6.61097500 & 1.24261600 & 2.70915600 \\
\hline C & 7.19852800 & 1.42260000 & 4.11355000 \\
\hline C & 6.19004800 & 2.59078600 & 2.116 \\
\hline N & 6.49215800 & -2.55253200 & 1.701 \\
\hline C & 5.38559200 & -3.4472 & 1.95 \\
\hline $\mathrm{C}$ & 4.07604100 & -2.3881 & 6.9 \\
\hline C & 2.87932800 & -2.66501000 & 6.075 \\
\hline C & 3.08088900 & -2.28711000 & 4.60 \\
\hline $\mathrm{C}$ & 1.86177600 & -2.70554 & 3.77385 \\
\hline $\mathrm{C}$ & 3.37054900 & -0.79136300 & 4.42406 \\
\hline $\mathrm{C}$ & 0.12120000 & -7.71246300 & -0.94691 \\
\hline C & 0.33282400 & -6.38986700 & -0.20662600 \\
\hline $\mathrm{C}$ & 1.58059600 & -5.60151800 & -0.64 \\
\hline $\mathrm{C}$ & 1.48063100 & -5.11957400 & -2.09948100 \\
\hline $\mathrm{C}$ & 1.81658600 & -4.41345600 & 0.29188600 \\
\hline $\mathrm{C}$ & -8.00055800 & -8.17564300 & 0.87910600 \\
\hline $\mathrm{C}$ & -8.76416700 & -6.86635400 & 0.65178500 \\
\hline $\mathrm{C}$ & -8.27195200 & -5.65383200 & 1.40401600 \\
\hline $\mathrm{C}$ & -6.91640400 & -5.31575400 & 1.45000500 \\
\hline $\mathrm{C}$ & -9.17914600 & -4.79590000 & 2.03691800 \\
\hline $\mathrm{C}$ & -6.47836200 & -4.14721700 & 2.06769700 \\
\hline $\mathrm{C}$ & -8.76114100 & -3.62395500 & 2.66110400 \\
\hline $\mathrm{C}$ & -7.40896100 & -3.28294000 & 2.65496200 \\
\hline $\mathrm{O}$ & -7.03860700 & -2.08755800 & 3.22434100 \\
\hline $\mathrm{C}$ & -3.68375300 & -4.88192900 & -2.97123800 \\
\hline $\mathrm{C}$ & -3.40637200 & -3.83933000 & -1.89550200 \\
\hline $\mathrm{C}$ & -4.69706300 & -3.54296600 & -1.11741200 \\
\hline $\mathrm{C}$ & -4.80681400 & -2.15366500 & -0.53819600 \\
\hline $\mathrm{O}$ & -6.05892100 & -1.82272100 & -0.29093000 \\
\hline $\mathrm{O}$ & -3.83355100 & -1.42676700 & -0.33679400 \\
\hline $\mathrm{C}$ & 2.49209900 & -1.51063500 & -6.45706600 \\
\hline $\mathrm{C}$ & 2.77491500 & -1.65980100 & -4.98324800 \\
\hline $\mathrm{C}$ & 1.88789200 & -1.15247100 & -4.02829100 \\
\hline
\end{tabular}

Con

C 


\begin{tabular}{|c|c|c|c|}
\hline & .41515200 & -1.93242200 & \\
\hline & & & \\
\hline & & & \\
\hline & -0.62622500 & 2.83324000 & 5.22491600 \\
\hline & -2.77046600 & 2.57715900 & 4.02120200 \\
\hline & 3.69487600 & -0.49768300 & 0.87205300 \\
\hline & -4.52661900 & 1.18610100 & 13100 \\
\hline & -1.52623200 & -0.19241200 & 25700 \\
\hline & -2.74990200 & 1.60195500 & 42000 \\
\hline & -5.05838600 & -0.04023700 & 6300 \\
\hline & -1.10248200 & 3.45076300 & 3100 \\
\hline & -3.20654500 & -0.56472700 & 7500 \\
\hline & -2.78 & -1.6 & 200 \\
\hline & 1.28 & 3.0 & \\
\hline & -0.59 & 600 & 100 \\
\hline & 0.339 & -1.7 & 600 \\
\hline & -4.711 & & 700 \\
\hline & & & \\
\hline & -1.39 & & 900 \\
\hline & -3.3 & & \\
\hline & -3.6 & & \\
\hline & 5.12 & 2.7 & -1.7 \\
\hline & & & \\
\hline & 4.88 & & \\
\hline & 4.30 & 2.1 & -4. \\
\hline & 2.81 & & \\
\hline & 4.11 & & 400 \\
\hline & 2.55 & & \\
\hline & 1.6 & & \\
\hline & 3.2 & 3.8 & -5 . \\
\hline & & & 600 \\
\hline & & -2.8 & \\
\hline & 3.56 & -1.8 & \\
\hline & 1.46 & -0.8 & 300 \\
\hline & 0.95 & -0.7 & 900 \\
\hline & & & \\
\hline & & & \\
\hline & -0.0 & & 600 \\
\hline & -2.5 & & 600 \\
\hline & -1.43 & -0.3 & 300 \\
\hline & -2.844 & -0.8 & \\
\hline & -1.21 & -1.0 & 900 \\
\hline & -8.317 & -2.0 & 3300 \\
\hline & -9.6 & -2.4 & \\
\hline & -8.30 & -4.4 & 600 \\
\hline & -9.49 & -4.9 & 3300 \\
\hline & -8.82 & -2.4 & 900 \\
\hline & -9.802 & -1.36250500 & 8000 \\
\hline & -10.468 & 42600 & \\
\hline & -8.07 & & -0.0 \\
\hline & -7.4920 & 1.00 & -2. \\
\hline & & & \\
\hline & -12.42568100 & 0.61775900 & 26200 \\
\hline & & & \\
\hline & -4.83913200 & -4.24337400 & 686600 \\
\hline & -5.576 & -3.68242200 & 4800 \\
\hline & -2.609 & -4.15664800 & 6500 \\
\hline & -3.05130600 & -2.91850200 & 4700 \\
\hline & & -6.64275200 & \\
\hline & -9.81620400 & -7.02676200 & 0.91068400 \\
\hline & -10.23702100 & -5.03847800 & 2.01799200 \\
\hline & & -5.97251000 & 0.98832400 \\
\hline & -5.42591200 & -3.89357500 & 2.08151500 \\
\hline
\end{tabular}

\begin{tabular}{|c|c|c|c|}
\hline & -9.47119700 & -2.95615400 & \\
\hline $\mathrm{H}$ & -6.05726000 & -2.06219600 & \\
\hline & 2.70739300 & -3.84377300 & \\
\hline & 0.96694400 & -3.72217500 & \\
\hline & 1.93008800 & -4.73379100 & \\
\hline & 2.37600700 & -4.56480100 & -2.39315400 \\
\hline & 1.35605600 & -5.94873400 & \\
\hline & 0.62466500 & -4.44406800 & 03200 \\
\hline & 2.44732600 & -6.27581100 & 7200 \\
\hline & -0.54 & & 3000 \\
\hline & 0.39777800 & -6.58429100 & 6000 \\
\hline & 6.91025800 & -2.5 & \\
\hline & 8.74 & -0.6 & \\
\hline & 709700 & 1.1 & \\
\hline & 7.09 & & \\
\hline & 7.05872200 & 3.257 & 600 \\
\hline & 5.74 & 2.480 & \\
\hline & & & \\
\hline & 8.15 & 1.96 & \\
\hline & 6.51 & & \\
\hline $\mathrm{H}$ & 7.35 & 0.45 & \\
\hline & 5.71 & 0.6 & \\
\hline & 8.70 & -1.2 & 600 \\
\hline & 9.39 & -3.9 & 1700 \\
\hline & 10.9 & -4.0 & 800 \\
\hline & & & 7400 \\
\hline & 13.1 & 0.1 & 1900 \\
\hline & 14.0 & 1000 & \\
\hline & 12.7 & -2.42 & 7600 \\
\hline & 12.5 & & -4. \\
\hline & & & 600 \\
\hline & 12.1 & -2.41 & 8500 \\
\hline & 10.1 & 4100 & 3500 \\
\hline & & & -0.7 \\
\hline & 8.60 & 6.12 & -1.4 \\
\hline & -4.6 & & 100 \\
\hline & & 3.2 & 700 \\
\hline & -9.96 & 4.2 & \\
\hline & 00 & & 400 \\
\hline & 400 & 6500 & 3900 \\
\hline & & & \\
\hline & & & \\
\hline & -5.45 & & \\
\hline & -3.93 & 7.6 & 100 \\
\hline & -4.95 & 7.31 & \\
\hline & -6.45 & & \\
\hline & -6.46 & & \\
\hline $\mathrm{H}$ & -4.44 & & \\
\hline & & & \\
\hline & -3.74 & 5.25 & \\
\hline & -6.43 & & \\
\hline & & 5.213 & 5600 \\
\hline & 1.56078500 & 5.502 & \\
\hline & & & \\
\hline & 1.22241300 & 2.24009700 & \\
\hline & 2.57 & 3.277 & \\
\hline & 4.33125200 & -0.51467700 & 4.86359000 \\
\hline & 3.41073900 & -0.54468900 & \\
\hline & 2.57880400 & -0.19282700 & \\
\hline $\mathrm{H}$ & 1.62864600 & -3.76769000 & 3.91375100 \\
\hline & & -2.12850500 & \\
\hline $\mathrm{H}$ & 2.03498700 & -2.52987800 & 2.71025900 \\
\hline & 3.95963200 & -2.83358700 & 4.24477900 \\
\hline
\end{tabular}




\begin{tabular}{|c|c|c|c|}
\hline & 2.62107100 & -3.73177400 & \\
\hline & 2.00687500 & -2.11765300 & \\
\hline & & & \\
\hline & & & \\
\hline & & & \\
\hline & 6.01500600 & -0.63151400 & \\
\hline & & & \\
\hline & & & \\
\hline & & & \\
\hline & & & \\
\hline & & & \\
\hline & & & -3.0 \\
\hline & -8.3 & & \\
\hline & & & \\
\hline & & & \\
\hline & & & \\
\hline & 0.9 & & \\
\hline & & & \\
\hline & & & \\
\hline & & & \\
\hline & & & \\
\hline & & & \\
\hline & & & \\
\hline & & & \\
\hline & & 900 & \\
\hline & & & \\
\hline & & & \\
\hline & & & \\
\hline & & & \\
\hline & & & \\
\hline & & & \\
\hline & & & \\
\hline & & & \\
\hline & & & \\
\hline & & & \\
\hline & & & \\
\hline & & & \\
\hline & & & \\
\hline & & & \\
\hline & & & \\
\hline & & & \\
\hline & & & \\
\hline & & & \\
\hline & & & \\
\hline & & & \\
\hline & & & \\
\hline & & & \\
\hline & & & \\
\hline & & & \\
\hline & & & \\
\hline & & & \\
\hline & & & \\
\hline & & & \\
\hline & & & \\
\hline & & & \\
\hline
\end{tabular}

\section{TS2 (+8.0 kcal $/ \mathrm{mol})$}

$\begin{array}{lrrr}\mathrm{C} & 6.70633500 & 5.65128100 & 0.11635400 \\ \mathrm{C} & 7.22095800 & 4.67585000 & -0.89478100 \\ \mathrm{O} & 6.77819700 & 3.53165400 & -1.04993000\end{array}$
$\begin{array}{llll}\mathrm{N} & 8.24591000 & 5.13364000 & -1.64785900\end{array}$

$\begin{array}{llll}\text { C } & 8.81845800 & 4.31769800 & -2.68611300\end{array}$

$\begin{array}{llll}\text { C } & 9.88569600 & 3.32354300 & -2.22834400\end{array}$

$\begin{array}{llll}\mathrm{O} & 10.90305700 & 3.12074800 & -2.88466500\end{array}$

$\mathrm{N} \quad 9.59974500 \quad 2.67018100 \quad-1.06703600$

$\begin{array}{llll}\text { C } & 10.44921500 & 1.55904200 & -0.69551400\end{array}$

$\begin{array}{llll}\text { C } & 10.17742900 & 0.35944500 & -1.59304900\end{array}$

$\begin{array}{llll}\mathrm{O} & 9.01977800 & -0.06844000 & -1.71489800\end{array}$

$\mathrm{N} \quad 11.21637100 \quad-0.27497800 \quad-2.17318200$

C $\quad 10.94565100 \quad-1.50877300 \quad-2.93111000$

$\begin{array}{llll}\text { C } & 10.29491900 & -2.57790300 & -2.04897400\end{array}$

$\begin{array}{llll}\text { O } & 9.45435600 & -3.34959100 & -2.50762500\end{array}$

$\begin{array}{lrrr}\text { C } & 12.33271600 & -1.89053700 & -3.47732900\end{array}$

$\begin{array}{llll}\text { C } & 13.02279800 & -0.53004400 & -3.65666500\end{array}$

$\begin{array}{llll}\text { C } & 12.56708500 & 0.27284600 & -2.43087700\end{array}$

$\begin{array}{llll}\mathrm{N} & 10.74762400 & -2.60638000 & -0.77322500\end{array}$

$\begin{array}{llll}\text { C } & 10.20162700 & -3.44685200 & 0.27549400\end{array}$

$\begin{array}{llll}\text { C } & 9.64246600 & -2.65759700 & 1.46523500\end{array}$

$\begin{array}{llll}\text { O } & 9.78941400 & -3.07281400 & 2.60440200\end{array}$

$\begin{array}{llll}\mathrm{N} & 8.97098300 & -1.50252600 & 1.16028900\end{array}$

$\begin{array}{llll}\text { C } & 8.09025400 & -0.92487200 & 2.18214800\end{array}$

$\begin{array}{llll}\text { C } & 6.97124300 & -1.89556300 & 2.60949800\end{array}$

$\begin{array}{llll}\text { O } & 6.52654800 & -1.88375300 & 3.75739900\end{array}$

$\begin{array}{llll}\text { C } & 7.49178800 & 0.39284000 & 1.65720800\end{array}$

$\begin{array}{llll}\text { C } & 6.41207700 & 1.03467800 & 2.54839800\end{array}$

$\begin{array}{llll}\text { C } & 6.89475000 & 1.28338900 & 3.98147200\end{array}$

$\begin{array}{llll}\text { C } & 5.92487600 & 2.33834300 & 1.90767800\end{array}$

$\begin{array}{llll}\mathrm{N} & 6.48461800 & -2.67390600 & 1.62533100\end{array}$

$\begin{array}{llll}\text { C } & 5.35813300 & -3.54648100 & 1.85825000\end{array}$

$\begin{array}{llll}\text { C } & 4.02746500 & -2.51355300 & 6.89947800\end{array}$

$\begin{array}{llll}\text { C } & 2.83635100 & -2.78787700 & 5.97092000\end{array}$

$\begin{array}{llll}\text { C } & 3.06019100 & -2.44247900 & 4.49382400\end{array}$

$\begin{array}{llll}\text { C } & 1.83403400 & -2.83059300 & 3.65902400\end{array}$

$\begin{array}{llll}\text { C } & 3.39639000 & -0.96120600 & 4.28730900\end{array}$

$\begin{array}{llll}\text { C } & 0.07521200 & -7.74096200 & -1.10821300\end{array}$

$\begin{array}{llll}\text { C } & 0.27444600 & -6.42718400 & -0.34506500\end{array}$

$\begin{array}{llll}\text { C } & 1.45831700 & -5.56433700 & -0.82102900\end{array}$

$\begin{array}{llll}\text { C } & 1.22644000 & -4.98498900 & -2.22331400\end{array}$

$\begin{array}{llll}\text { C } & 1.72086100 & -4.43362200 & 0.18100100\end{array}$

$\begin{array}{llll}\text { C } & -8.06114500 & -8.15100700 & 0.66520800\end{array}$

$\begin{array}{llll}\text { C } & -8.77182900 & -6.80384500 & 0.44771200\end{array}$

$\begin{array}{llll}\text { C } & -8.33707800 & -5.66015800 & 1.33389200\end{array}$

$\begin{array}{llll}\text { C } & -7.14230600 & -4.96914800 & 1.11155500\end{array}$

$\begin{array}{llll}\text { C } & -9.14783500 & -5.22473500 & 2.39004700\end{array}$

$\begin{array}{llll}\text { C } & -6.77266100 & -3.87285100 & 1.89050500\end{array}$

$\begin{array}{llll}\text { C } & -8.80069100 & -4.13058600 & 3.17493900\end{array}$

$\begin{array}{llll}\text { C } & -7.61561800 & -3.42986000 & 2.91843000\end{array}$

$\begin{array}{llll}\text { O } & -7.33899800 & -2.34473500 & 3.68348800\end{array}$

$\begin{array}{llll}\text { C } & -3.69354200 & -4.86099100 & -3.13142400\end{array}$

$\begin{array}{llll}\text { C } & -3.41797400 & -3.81120800 & -2.06260600\end{array}$

$\begin{array}{llll}\text { C } & -4.72087100 & -3.49345200 & -1.31561200\end{array}$

$\begin{array}{llll}\text { C } & -4.79605600 & -2.12460000 & -0.68543000\end{array}$

$\begin{array}{llll}\mathrm{O} & -6.05390600 & -1.73759300 & -0.51050300\end{array}$

$\begin{array}{llll}\mathrm{O} & -3.81738800 & -1.45802000 & -0.36895800\end{array}$

$\begin{array}{llll}\text { C } & 2.53088200 & -1.51269600 & -6.55240400\end{array}$

$\begin{array}{llll}\text { C } & 2.78735600 & -1.58059500 & -5.06600900\end{array}$

$\begin{array}{llll}\text { C } & 1.87202200 & -1.03534400 & -4.16015000\end{array}$

$\begin{array}{llll}\text { C } & 3.95903800 & -2.15526800 & -4.55458100\end{array}$

$\begin{array}{llll}\text { C } & 2.12458100 & -1.02422000 & -2.78831200\end{array}$

$\begin{array}{llll}\text { C } & 4.21678700 & -2.16175200 & -3.18092500\end{array}$

$\begin{array}{llll}\text { C } & 3.30718100 & -1.57744800 & -2.29228000\end{array}$

$\begin{array}{llll}\text { C } & 2.64097300 & 4.10329000 & -4.59670600\end{array}$

$\begin{array}{llll}\text { C } & 3.22541100 & 3.80241800 & -3.21216300\end{array}$ 


\begin{tabular}{|c|c|c|c|}
\hline $\mathrm{C}$ & & & \\
\hline $\mathrm{N}$ & & & -1.67034600 \\
\hline $\mathrm{C}$ & -2.34979200 & 02706200 & -5.23701200 \\
\hline $\mathrm{C}$ & & & -3.77778300 \\
\hline $\mathrm{C}$ & -1.81396700 & -0.38854000 & -3.21055400 \\
\hline $\mathrm{C}$ & -0.52916800 & 1.76812300 & -3.63044900 \\
\hline $\mathrm{C}$ & -7.67153800 & -3.23401700 & 10000 \\
\hline $\mathrm{C}$ & -8.70256500 & -2.82220300 & 92400 \\
\hline $\mathrm{C}$ & -9.13065700 & -3.99030300 & 6500 \\
\hline S & -10.44184000 & -3.58957200 & 7800 \\
\hline $\mathrm{C}$ & -9.56089200 & -2.36393800 & 300 \\
\hline $\mathrm{C}$ & -11.35341800 & -0.10838900 & 5700 \\
\hline $\mathrm{C}$ & -10.66073200 & 26400 & 5200 \\
\hline $\mathrm{C}$ & -9.27598200 & 7700 & 7800 \\
\hline $\mathrm{C}$ & -11.37 & 100 & 3400 \\
\hline $\mathrm{C}$ & -8.6 & & 9700 \\
\hline $\mathrm{C}$ & -10.7 & & \\
\hline $\mathrm{C}$ & -9.36111800 & & 0800 \\
\hline $\mathrm{O}$ & -8.748 & & 2800 \\
\hline $\mathrm{C}$ & -5.7 & & 700 \\
\hline $\mathrm{C}$ & -5.0 & & 2800 \\
\hline $\mathrm{C}$ & & & \\
\hline $\mathrm{O}$ & -6.9 & & 600 \\
\hline $\mathrm{O}$ & -5.2 & & 300 \\
\hline $\mathrm{C}$ & -9.4 & & 600 \\
\hline $\mathrm{C}$ & -9.8 & & 800 \\
\hline $\mathrm{C}$ & -10.4 & & 200 \\
\hline $\mathrm{O}$ & -8.7 & & 700 \\
\hline $\mathrm{C}$ & -4.1 & & 100 \\
\hline $\mathrm{C}$ & -4.6 & & \\
\hline $\mathrm{C}$ & -5.0 & & \\
\hline $\mathrm{C}$ & -5.6 & & 00 \\
\hline $\mathrm{C}$ & -3.8 & & 000 \\
\hline $\mathrm{C}$ & & & \\
\hline S & 2.4 & & \\
\hline $\mathrm{C}$ & & & \\
\hline $\mathrm{O}$ & & & 500 \\
\hline $\mathrm{O}$ & & & \\
\hline $\mathrm{O}$ & & & \\
\hline $\mathrm{N}$ & -2.9 & & 700 \\
\hline $\mathrm{C}$ & -0.1 & & \\
\hline $\mathrm{C}$ & -0.8 & & \\
\hline $\mathrm{C}$ & -2.9 & & \\
\hline $\mathrm{C}$ & -2.2 & & 500 \\
\hline $\mathrm{C}$ & -2.17258700 & & \\
\hline $\mathrm{C}$ & -2.8 & & 300 \\
\hline $\mathrm{C}$ & $-0.7^{\prime}$ & & \\
\hline $\mathrm{C}$ & -4.1 & & \\
\hline $\mathrm{C}$ & & & \\
\hline $\mathrm{C}$ & & & \\
\hline $\mathrm{C}$ & & & \\
\hline $\mathrm{C}$ & & & \\
\hline $\mathrm{C}$ & -2.0905 & & \\
\hline $\mathrm{C}$ & -0.78830100 & -0.46783400 & 5.80993600 \\
\hline $\mathrm{C}$ & 1.00926600 & -0.56 & 01100 \\
\hline $\mathrm{C}$ & -0.3 & -0.25 & 6500 \\
\hline $\mathrm{O}$ & & & 74000 \\
\hline $\mathrm{O}$ & -5.53 & -0.52 & 5200 \\
\hline $\mathrm{O}$ & & 0.14637900 & 0.70502200 \\
\hline $\mathrm{O}$ & 2.50803000 & 2.77518600 & 0.30116200 \\
\hline $\mathrm{H}$ & -0.42844700 & & 5.95189300 \\
\hline $\mathrm{H}$ & -2.70664500 & 2.79203700 & 4.99754500 \\
\hline $\mathrm{H}$ & 3.74988900 & 0.98393600 & 0.85322500 \\
\hline
\end{tabular}

$\begin{array}{llll}\mathrm{H} & -4.46109000 & 1.55688300 & 3.96166500\end{array}$

$\mathrm{H} \quad-1.06987200 \quad-1.05883000 \quad 0.30403000$

$\mathrm{H} \quad-2.50731600 \quad 1.93443300 \quad 2.31176600$

$\mathrm{H} \quad-4.94419200 \quad 0.43521500 \quad 5.21532500$

$\mathrm{H} \quad-2.37041800 \quad 2.45907800 \quad-0.13373000$

$\mathrm{H} \quad-3.97105000 \quad-1.46154700 \quad 3.63032700$

$\mathrm{H} \quad-2.55359800 \quad-1.49596900 \quad 5.20242700$

$\mathrm{H} \quad-0.08720500 \quad 3.13080300 \quad 0.14197100$

$\mathrm{H} \quad-0.23905800 \quad-1.37340300 \quad 6.04163000$

$\mathrm{H} \quad 1.34451800 \quad-1.58746700 \quad 0.60931300$

$\mathrm{H} \quad-3.25948200 \quad-0.72507200 \quad 1.34377100$

$\mathrm{H} \quad 1.49766200 \quad 1.66490300 \quad 6.32605300$

$\mathrm{H} \quad-2.76370500 \quad 0.78864000 \quad-0.51618500$

$\begin{array}{llll}\mathrm{H} & -4.00891900 & 1.60347600 & 1.43838500\end{array}$

$\mathrm{H} \quad-2.06295000 \quad-0.34732400 \quad 2.43587700$

$\mathrm{H} \quad 5.02691200 \quad 2.61888500 \quad-1.59148100$

$\mathrm{H} \quad 3.16195500 \quad 2.56605500 \quad-0.46737200$

$\mathrm{H} \quad 4.39152800 \quad 1.12872600 \quad-1.54368700$

$\mathrm{H} \quad 4.21410000 \quad 1.97292400 \quad-3.80660800$

$\mathrm{H} \quad 2.63809000 \quad 1.72353900 \quad-3.06585000$

$\mathrm{H} \quad 4.14558000 \quad 4.38659000 \quad-3.06718200$

$\mathrm{H} \quad 2.53151200 \quad 4.12136000 \quad-2.42534700$

$\mathrm{H} \quad \begin{array}{llll}1.68862900 & 3.58503900 & -4.74634500\end{array}$

$\mathrm{H} \quad 3.32349100 \quad 3.78404000 \quad-5.39244400$

$\mathrm{H} \quad 4.67202200 \quad-2.61090400 \quad-5.23754300$

$\mathrm{H} \quad 5.11422800 \quad-2.63510100 \quad-2.79246800$

$\mathrm{H} \quad 3.51901700 \quad-1.55442300 \quad-1.22844200$

$\mathrm{H} \quad 1.39963900 \quad-0.59322700 \quad-2.10693600$

$\mathrm{H} \quad 0.94766900 \quad-0.61335900 \quad-4.53767600$

$\mathrm{H} \quad 0.23777800 \quad 1.32885400 \quad-4.27677000$

$\mathrm{H} \quad-0.62876000 \quad 2.82199200 \quad-3.91424800$

$\mathrm{H} \quad-0.16424100 \quad 1.72914600 \quad-2.59924600$

$\mathrm{H} \quad-2.60962100 \quad 1.58991700 \quad-3.18452400$

$\mathrm{H} \quad-1.46134900 \quad-0.39678800 \quad-2.17659600$

$\mathrm{H} \quad-2.80736600 \quad-0.84660100 \quad-3.22560500$

$\mathrm{H} \quad-1.14335100 \quad-1.02541000 \quad-3.79896000$

$\mathrm{H} \quad-8.27336600 \quad-2.03786400 \quad-2.61904900$

$\mathrm{H} \quad-9.58814700 \quad-2.38939200 \quad-3.72401800$

$\mathrm{H} \quad-8.26795700 \quad-4.38843300 \quad-1.81394300$

$\mathrm{H} \quad-9.54215700 \quad-4.80692200 \quad-2.96539100$

$\mathrm{H} \quad-8.54670700 \quad-2.70949000 \quad 0.08679900$

$\mathrm{H} \quad-9.54064000 \quad-1.38258300 \quad-0.59460300$

$\mathrm{H} \quad-10.09866800 \quad-2.29892200 \quad 0.82914000$

H $\quad-8.12379400 \quad 2.70000500 \quad-0.18853100$

$\mathrm{H} \quad-7.54529200 \quad 1.26317300 \quad-2.15527000$

$\mathrm{H} \quad-11.29032000 \quad 1.50849500 \quad-0.08800500$

$\mathrm{H} \quad-12.44491400 \quad 0.57004400 \quad-2.08538900$

$\mathrm{H} \quad-8.69654000 \quad 0.39462700 \quad-4.15905500$

$\mathrm{H} \quad-4.90105500 \quad-4.22439500 \quad-0.51926300$

$\mathrm{H} \quad-5.58061500 \quad-3.57832300 \quad-1.98622000$

$\mathrm{H} \quad-2.63718300 \quad-4.12780000 \quad-1.36337900$

$\mathrm{H} \quad-3.04604400 \quad-2.90117000 \quad-2.54107900$

$\mathrm{H} \quad-8.65090600 \quad-6.51286100 \quad-0.60275500$

$\mathrm{H} \quad-9.84816600 \quad-6.94907000 \quad 0.58717500$

$\mathrm{H} \quad-10.08644100 \quad-5.73815500 \quad 2.58177600$

$\mathrm{H} \quad-6.49639700 \quad-5.29016800 \quad 0.29883600$

$\mathrm{H} \quad-5.85439400 \quad-3.33039800 \quad 1.69182100$

$\mathrm{H} \quad-9.44619900 \quad-3.78265000 \quad 3.97454900$

$\mathrm{H} \quad-6.64132900 \quad-1.79771000 \quad 3.26271000$

$\mathrm{H} \quad 2.54237900 \quad-3.78870000 \quad-0.14757700$

H $\quad 0.82527800 \quad-3.80849400 \quad 0.28587100$

$\mathrm{H} \quad 1.96874500 \quad-4.82185700 \quad 1.17369500$

H $\quad 2.07841800 \quad-4.37900500 \quad-2.54618300$ 


\begin{tabular}{|c|c|c|c|}
\hline $\mathrm{H}$ & 1.06912100 & -5.76719600 & \\
\hline $\mathrm{H}$ & 0.34238100 & -4.33491500 & -2.22744100 \\
\hline & .35320000 & -6.20372500 & -0.85669000 \\
\hline & -0.64002000 & -5.82034200 & 3500 \\
\hline & 0.40213100 & -6.64974900 & \\
\hline & 6.86751600 & -2.65534000 & 0.68211600 \\
\hline & & -0.75308900 & \\
\hline & 8.31269400 & 1.11208800 & 1.52100200 \\
\hline & 7.05911400 & 0.210 & \\
\hline & 6.7241 & & \\
\hline & 5.63023500 & 2.206 & \\
\hline & 5.0762 & 2.755 & 394000 \\
\hline & 7.80292000 & 1.90 & 3800 \\
\hline & 6.12947000 & 1.81 & \\
\hline & 7.099 & & \\
\hline & 5.565 & 0.3 & \\
\hline & & -1.37 & \\
\hline & 9.3974 & -4.03 & 0400 \\
\hline & 10.956 & -4.12 & \\
\hline & & & \\
\hline & $13.224 \mathrm{C}$ & & \\
\hline & & -0.6 & \\
\hline & & -2.4 & \\
\hline & 12.661 & -0.0 & -4. \\
\hline & 12.498 & & \\
\hline & 12.2578 & -2.4 & 66000 \\
\hline & 10.232 & -1.3 & -3.7 \\
\hline & & & \\
\hline & & & \\
\hline & & & \\
\hline & -4.242 & & -2.7 \\
\hline & -9.749 & 4.28 & -2.2 \\
\hline $\mathrm{H}$ & 300 & & 000 \\
\hline & -11.36330 & & \\
\hline & -10.56727200 & & \\
\hline & & & \\
\hline & -5.539 & & \\
\hline & & & \\
\hline & -4.877 & & \\
\hline & -5.86625300 & & \\
\hline $\mathrm{H}$ & -6.53 & & \\
\hline & -3.51639500 & & \\
\hline & -4.209 & & \\
\hline & -3.064 & & \\
\hline & -5.86833400 & & \\
\hline & & & \\
\hline & & & \\
\hline & 2.958 & & \\
\hline & 1.240 & 1.99 & 0800 \\
\hline & 2.47232500 & & \\
\hline & & -0.7 & \\
\hline & 3.42587800 & -0.72 & 7700 \\
\hline & & -0.3 & \\
\hline & 1.57615700 & -3.88858600 & 3.78372700 \\
\hline & 0.96000800 & -2.23506400 & 14900 \\
\hline & & -2.64916100 & 2.59408800 \\
\hline & 3.91987300 & -3.02740400 & 4.14933200 \\
\hline & 2.55954900 & -3.848 & \\
\hline & 1.97134100 & & \\
\hline & 6.78015100 & -1.83661900 & -1.42173600 \\
\hline & & -3.01856200 & -1.73346900 \\
\hline & 7.19630500 & 0.17287200 & -1.93327300 \\
\hline & 5.92743000 & -0.30223400 & -2.71911300 \\
\hline
\end{tabular}

\begin{tabular}{|c|c|c|c|}
\hline $\mathrm{H}$ & & & \\
\hline & & & \\
\hline & & & \\
\hline & & & \\
\hline & & & \\
\hline & & & \\
\hline & & & \\
\hline & & 8900 & \\
\hline & 11.49016 & 718000 & 800 \\
\hline & & -2.97 & \\
\hline & & -8.4 & \\
\hline & & & \\
\hline & 2.958 & -0.5 & -6. \\
\hline & & & \\
\hline & & & \\
\hline & -8.6 & -8.9 & \\
\hline & & -4.5 & \\
\hline & -12.4 & 8000 & -4.3 \\
\hline & & & -4. \\
\hline & & & \\
\hline & -4.9 & & \\
\hline & & & \\
\hline & -2.9 & & \\
\hline & -3.9 & & -2.7 \\
\hline & -7.2 & & \\
\hline & -8.0 & & \\
\hline & -11.1 & & \\
\hline & & & \\
\hline & -3.4 & & \\
\hline & & & \\
\hline & & & \\
\hline & & & \\
\hline & 6.2 & & -0.37 \\
\hline & & & -3.45 \\
\hline & 10.2 & & \\
\hline $\mathrm{H}$ & & -4.2 & \\
\hline & & & \\
\hline & & & \\
\hline & & -2.7 & \\
\hline & -0.8 & -8.2 & \\
\hline $\mathrm{H}$ & & -7.56 & -2.188 \\
\hline & & -8.4 & \\
\hline $\mathrm{H}$ & & -8.1 & \\
\hline $\mathrm{H}$ & & & -2.45 \\
\hline & & & \\
\hline $\mathrm{H}$ & & & \\
\hline & -4.95295400 & 5.86181600 & 4.57639300 \\
\hline & & 4.20115600 & 4.39946800 \\
\hline & -5.79344100 & 1.67433800 & -0.26055100 \\
\hline
\end{tabular}

\section{Int2 (-3.1 kcal/mol)}

$\begin{array}{cccc}\text { C } & 6.65621300 & 5.71074500 & 0.26570200 \\ \mathrm{C} & 7.17912600 & 4.76679400 & -0.77091600 \\ \mathrm{O} & 6.72639100 & 3.63447400 & -0.97760000 \\ \mathrm{~N} & 8.21866900 & 5.24099500 & -1.49327100 \\ \mathrm{C} & 8.79131600 & 4.45661000 & -2.55581700 \\ \mathrm{C} & 9.85996200 & 3.45357800 & -2.12381300 \\ \mathrm{O} & 10.87882600 & 3.26766200 & -2.78233900 \\ \mathrm{~N} & 9.57355200 & 2.77096400 & -0.97909900 \\ \mathrm{C} & 10.42577000 & 1.65244300 & -0.63873800\end{array}$


C $\quad 10.18144100 \quad 0.48579800 \quad-1.58615400$

$\begin{array}{lllll}\text { O } & 9.02921800 & 0.06383500 & -1.76235100\end{array}$

N $\quad 11.24075500 \quad-0.13417100 \quad-2.14586200$

C $\quad 10.99978500 \quad-1.36115800 \quad-2.92413900$

C $\quad 10.35044600 \quad-2.44549700 \quad-2.05826800$

$\begin{array}{llll}\text { O } & 9.51297900 & -3.21584800 & -2.52520800\end{array}$

C $\quad 12.40139200 \quad-1.71812600 \quad-3.45098400$

C $\quad 13.08174200 \quad-0.34782200 \quad-3.59072000$

$\begin{array}{llll}\text { C } & 12.59248400 & 0.42784000 & -2.36047500\end{array}$

N $\quad 10.79901900 \quad-2.48392100 \quad-0.78122000$

C $\quad 10.25470300 \quad-3.33636700 \quad 0.25877700$

C $\quad 9.69722900 \quad-2.55591000 \quad 1.45553300$

$\begin{array}{llll}\text { O } & 9.87703500 & -2.95788200 & 2.59439900\end{array}$

$\begin{array}{llll}\mathrm{N} & 8.99120800 & -1.41876200 & 1.15374500\end{array}$

$\begin{array}{llll}\text { C } & 8.09846500 & -0.87407100 & 2.18417000\end{array}$

$\begin{array}{llll}\text { C } & 7.00674400 & -1.87892800 & 2.60374000\end{array}$

$\begin{array}{llll}\text { O } & 6.57100400 & -1.90125800 & 3.75450000\end{array}$

$\begin{array}{llll}\text { C } & 7.45928000 & 0.43090500 & 1.67414700\end{array}$

$\begin{array}{lllll}\text { C } & 6.37881942 & 1.03808296 & 2.58798043\end{array}$

$\begin{array}{lllll}\text { C } & 6.89059850 & 1.32559404 & 4.00338488\end{array}$

$\begin{array}{llll}\text { C } & 5.81328248 & 2.30929148 & 1.94768797\end{array}$

$\begin{array}{llll}\mathrm{N} & 6.53015500 & -2.64896100 & 1.60673200\end{array}$

$\begin{array}{llll}\text { C } & 5.41066900 & -3.53409700 & 1.82481600\end{array}$

$\begin{array}{llll}\text { C } & 4.05440000 & -2.61478000 & 6.88118400\end{array}$

$\begin{array}{llll}\text { C } & 2.87052700 & -2.87986100 & 5.94116600\end{array}$

$\begin{array}{llll}\text { C } & 3.10774704 & -2.50237410 & 4.47436198\end{array}$

$\begin{array}{llll}\text { C } & 1.90617403 & -2.90624810 & 3.61252899\end{array}$

$\begin{array}{llll}\text { C } & 3.41001211 & -1.00917513 & 4.30116389\end{array}$

C $\quad 0.18501100 \quad-7.73117500 \quad-1.23806600$

$\begin{array}{lllll}\text { C } & 0.37256900 & -6.42823800 & -0.45180200\end{array}$

$\begin{array}{lllll}\text { C } & 1.49237167 & -5.50844318 & -0.97598864\end{array}$

$\begin{array}{llll}\text { C } & 1.12968879 & -4.86836127 & -2.32327535\end{array}$

$\begin{array}{llll}\text { C } & 1.81402205 & -4.41976417 & 0.05505869\end{array}$

$\begin{array}{llll}\text { C } & -7.95064800 & -8.27054800 & 0.50311700\end{array}$

$\begin{array}{llll}\text { C } & -8.63389800 & -6.90347700 & 0.32605100\end{array}$

$\begin{array}{llll}\text { C } & -8.10412300 & -5.76284700 & 1.16787200\end{array}$

$\begin{array}{llll}\text { C } & -6.80964532 & -5.25003115 & 1.01398388\end{array}$

$\begin{array}{llll}\text { C } & -8.93321864 & -5.14440472 & 2.11184813\end{array}$

$\begin{array}{llll}\text { C } & -6.36905199 & -4.14543584 & 1.74730647\end{array}$

$\begin{array}{llll}\text { C } & -8.51341364 & -4.03876704 & 2.84393286\end{array}$

$\begin{array}{llll}\text { C } & -7.23314845 & -3.50939656 & 2.65059252\end{array}$

O $\quad-6.87871070 \quad-2.40579696 \quad 3.36670833$

C $\quad-3.61229200 \quad-4.85533000 \quad-3.21596000$

C $\quad-3.29301900 \quad-3.86049400 \quad-2.10464000$

C $\quad-4.58533500 \quad-3.22573200 \quad-1.59168600$

C $\quad-4.41557800 \quad-2.25807400 \quad-0.45461500$

O $\quad-5.48514700 \quad-1.55910100 \quad-0.18659900$

$\begin{array}{llll}\mathrm{O} & -3.34952300 & -2.17481000 & 0.17890900\end{array}$

$\begin{array}{llll}\text { C } & 2.58264000 & -1.37017600 & -6.55301100\end{array}$

C $\quad 2.82833600 \quad-1.45452100 \quad-5.06514300$

C $\quad 1.86286683 \quad-1.00378716 \quad-4.15967105$

$\begin{array}{llll}\text { C } & 4.03339380 & -1.95269321 & -4.55011870\end{array}$

$\begin{array}{llll}\text { C } & 2.09563142 & -1.00788579 & -2.78401375\end{array}$

C $\quad 4.27142704 \quad-1.97611260 \quad-3.17318896$

$\begin{array}{llll}\text { C } & 3.30937492 & -1.48375476 & -2.28423428\end{array}$

$\begin{array}{llll}\text { C } & 2.62203600 & 4.20767300 & -4.48844700\end{array}$

C $\quad 3.17190100 \quad 3.88504100 \quad-3.09485600$

C $\quad 3.57216000 \quad 2.42078400 \quad-2.92437600$

$\mathrm{N} \quad 4.10650000 \quad 2.18555300 \quad-1.56983000$

$\begin{array}{llll}\mathrm{C} & -2.33094300 & 1.08671200 & -5.20291900\end{array}$

$\begin{array}{llll}\text { C } & -1.84315100 & 1.04615800 & -3.74871600\end{array}$

C $\quad-1.78693100 \quad-0.39991600 \quad-3.23987400$

C $\quad-0.50307300 \quad 1.77632000 \quad-3.58736500$ C $\quad-8.99242900 \quad-4.01815800 \quad-2.37455600$

$\mathrm{S} \quad-10.24132600 \quad-3.61053300 \quad-1.10133700$

C $\quad-9.33488400 \quad-2.31636400 \quad-0.18075500$

C $\quad-11.32191100 \quad-0.16792100 \quad-4.51285900$

$\begin{array}{llll}\text { C } & -10.68992300 & 0.39636200 & -3.26586400\end{array}$

C $\quad-9.31081300 \quad 0.63064100 \quad-3.19943200$

C $\quad-11.45846300 \quad 0.70724200 \quad-2.13942200$

C $\quad-8.72255300 \quad 1.19006300 \quad-2.06819300$

C $\quad-10.88895400 \quad 1.28585900 \quad-1.00615900$

$\begin{array}{llll}\text { C } & -9.52209600 & 1.55723600 & -0.97925700\end{array}$

$\begin{array}{llll}\text { O } & -8.99829100 & 2.19802600 & 0.12130800\end{array}$

$\begin{array}{llll}\text { C } & -5.76699500 & 4.60937100 & -3.53784200\end{array}$

C $\quad-5.10207000 \quad 3.94622600 \quad-2.32639200$

C $\quad-6.03357900 \quad 3.35871200 \quad-1.29484300$

O $\quad-7.15812600 \quad 3.81874600 \quad-1.06914800$

$\begin{array}{lllll}\text { O } & -5.51226600 & 2.34635200 & -0.64144900\end{array}$

C $\quad-9.46695400 \quad 6.93762400 \quad-2.51387700$

$\begin{array}{llll}\text { C } & -9.93211700 & 6.22661800 & -1.24499100\end{array}$

C $\quad-10.51581284 \quad 4.84602273 \quad-1.54513681$

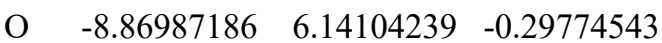

C $\quad-4.26027500 \quad 6.34258500 \quad 4.23327200$

$\begin{array}{llll}\text { C } & -4.75368800 & 6.93918600 & 2.91495600\end{array}$

$\begin{array}{llll}\text { C } & -5.14353100 & 5.89813200 & 1.84693400\end{array}$

$\begin{array}{llll}\text { C } & -5.68866900 & 6.60401800 & 0.60012300\end{array}$

C $\quad-3.97551200 \quad 4.97243100 \quad 1.47542000$

C $\quad 1.09459600 \quad 4.57602000 \quad 4.82519000$

S $\quad 2.22038800 \quad 3.15676500 \quad 5.04920900$

C $\quad 1.96815795 \quad 2.33577405 \quad 3.43930320$

O $\quad 7.13430545 \quad-2.59327806 \quad-1.20887179$

$\begin{array}{lllll}\text { O } & 6.29215309 & 0.08013702 & -1.85143958\end{array}$

$\begin{array}{llll}\text { O } & -6.64618632 & 1.69061578 & 1.50360673\end{array}$

$\mathrm{N} \quad-3.10324500 \quad-0.20623400 \quad 1.99060400$

C $\quad-0.42930600 \quad 0.50052300 \quad 5.87317500$

C $\quad-1.13122800 \quad 1.66106300 \quad 5.52403000$

$\begin{array}{llll}\text { C } & -3.12764400 & 1.17038500 & 1.40939400\end{array}$

C $\quad-2.36309800 \quad 1.22192400 \quad 0.08209100$

C $\quad-2.41127400 \quad 1.56914500 \quad 4.98074800$

C $\quad-3.04044500 \quad 0.33273200 \quad 4.80284700$

$\begin{array}{llll}\text { C } & -0.88324400 & 0.93171500 & 0.21063300\end{array}$

C $\quad-4.38409500 \quad 0.23491500 \quad 4.12150000$

C $\quad 0.04564000 \quad 1.98055500 \quad 0.17486600$

$\begin{array}{lllll}\text { C } & 1.41447800 & 1.74765300 & 0.30913800\end{array}$

$\begin{array}{llll}\text { C } & -4.32553200 & -0.61646000 & 2.84767900\end{array}$

$\begin{array}{llll}\text { C } & 1.86194900 & 0.43435900 & 0.55868800\end{array}$

C $\quad-2.33916600 \quad-0.81981100 \quad 5.19905300$

C $\quad-1.05010900 \quad-0.74624800 \quad 5.71640800$

$\begin{array}{llll}\text { C } & 0.95597800 & -0.62063300 & 0.56695700\end{array}$

C $\quad-0.40556300 \quad-0.37772000 \quad 0.37464000$

$\begin{array}{llll}\text { O } & 0.82907700 & 0.52727200 & 6.37605800\end{array}$

$\begin{array}{llll}\mathrm{O} & -5.44625700 & -0.55038800 & 2.08475500\end{array}$

$\begin{array}{llll}\text { O } & 3.19085200 & 0.18605200 & 0.77843100\end{array}$

O $\quad 2.31448100 \quad 2.76918500 \quad 0.26681200$

$\mathrm{H} \quad-0.67530700 \quad 2.62912300 \quad 5.69176100$

$\mathrm{H} \quad-2.93776500 \quad 2.47961200 \quad 4.70441200$

$\mathrm{H} \quad 3.62515300 \quad 1.04534600 \quad 0.90196700$

$\mathrm{H} \quad-4.76457200 \quad 1.22736100 \quad 3.85987700$

$\mathrm{H} \quad-1.08731700 \quad-1.22112600 \quad 0.32682900$

$\mathrm{H} \quad-2.69875900 \quad 1.85071600 \quad 2.14714300$

H $\quad-5.13921000 \quad-0.23598100 \quad 4.76063600$

$\mathrm{H} \quad-2.51864300 \quad 2.22092500 \quad-0.33448800$

H $\quad-4.06579400 \quad-1.65345700 \quad 3.11609600$ 


\begin{tabular}{|c|c|c|c|}
\hline & 80881200 & .79488700 & \\
\hline & & 3.00474200 & \\
\hline & & & \\
\hline & 1.32557900 & -1.62737300 & .71778600 \\
\hline & -3.11406700 & -0.92850800 & 1.22389500 \\
\hline & 1.21776100 & 1.41585100 & \\
\hline & -2.83823800 & 0.52193600 & 92100 \\
\hline & -4.17029800 & & \\
\hline & -2.24873600 & -0.34096700 & 92400 \\
\hline & 4.94583200 & 2.749 & -1.424 \\
\hline & 3.03326200 & 2.560 & -0.449 \\
\hline & 4.4207 & & \\
\hline & 4.3037 & & -3.7 \\
\hline & 2.70 & 1.76 & -3.0 \\
\hline & 4.0520 & & -2.893 \\
\hline & 2.4330 & 4.13 & -2.324 \\
\hline & 1.6829 & & -4.678 \\
\hline & 3.33 & & -5.2 \\
\hline & 4.7873 & -2.3 & -5.23 \\
\hline & 5.19 & & \\
\hline & 3.50 & & \\
\hline & 1.33 & -0.6 & 476 \\
\hline & & & \\
\hline & 0.256 & & -4.261 \\
\hline & -0.611 & & -3.824 \\
\hline & -0.12 & & 900 \\
\hline & -2.5801 & & 2000 \\
\hline & & & \\
\hline & -2.7791 & & -3.2 \\
\hline & -1.1170 & -1.0 & 900 \\
\hline & -8.25 & & 200 \\
\hline & -9.5590 & & 300 \\
\hline & -8.0909 & -4.3 & \\
\hline & -9.41 & & -2.9 \\
\hline & -8.3028 & -2.6 & 300 \\
\hline & & & \\
\hline & -9.82 & -2.2 & \\
\hline & -8.35 & & -0.2 \\
\hline & -7.6 & & 6700 \\
\hline & -11.4 & & 6200 \\
\hline & -12.527 & & 7700 \\
\hline & -8.683 & & -4.0 \\
\hline & -5.2897 & & \\
\hline H & -5.1155 & & \\
\hline & -2.7562 & & -1.27 \\
\hline & -2.6341 & -3.0 & \\
\hline & -8.5796 & -6.6 & -0.73 \\
\hline & -9.7016 & -7.0 & 0000 \\
\hline & -9.9439 & & \\
\hline & -6.1272 & -5.7 & 7458 \\
\hline & -5.35420855 & & \\
\hline & & & \\
\hline & -6.36085 & -1.773 & 78376 \\
\hline & 2.5889 & & -0.310 \\
\hline & 0.91857 & -3.822 & 0.26780765 \\
\hline & 2.15605 & -4.848 & 27509 \\
\hline & $1.9451 \mathrm{C}$ & -4.240 & -2.69542413 \\
\hline & 0.90735964 & -5.61783596 & -3.08895779 \\
\hline & & -4.22786447 & -2.21577212 \\
\hline & 2.39607388 & -6.12004564 & -1.11813584 \\
\hline & -0.56743299 & -5.85768927 & -0.45687337 \\
\hline & & -6.67071936 & 0.59998887 \\
\hline & 6.89021400 & -2.58090400 & 0.65701600 \\
\hline
\end{tabular}

$\begin{array}{llll}8.69221500 & -0.69435500 & 3.08070800\end{array}$

$\begin{array}{llll}8.25918379 & 1.17108035 & 1.52520866\end{array}$

$\begin{array}{llll}7.01327897 & 0.24053485 & 0.69006123\end{array}$

$\begin{array}{llll}6.57463956 & 3.09779438 & 1.91631079\end{array}$

$\begin{array}{lll}5.49533061 & 2.14549513 & 0.91384730\end{array}$

$\begin{array}{lll}4.96368325 & 2.69422670 & 2.52154260\end{array}$

$\begin{array}{lll}7.76036889 & 1.99535764 & 3.97561812\end{array}$

$\begin{array}{llll}6.11275208 & 1.81733701 & 4.59718752\end{array}$

$\begin{array}{lll}7.16629067 & 0.40050892 & 4.51212843\end{array}$

$\begin{array}{llll}5.56561121 & 0.30857974 & 2.66878062\end{array}$

$\begin{array}{llll}8.67381000 & -1.33233600 & 0.19225400\end{array}$

$\begin{array}{llll}9.45165500 & -3.92635800 & -0.19135900\end{array}$

$\begin{array}{lll}11.01158900 & -4.02061600 & 0.64942000\end{array}$

$\begin{array}{llll}11.43166500 & -1.75089300 & -0.49582700\end{array}$

$\begin{array}{llll}13.23275800 & 0.23716400 & -1.49025800\end{array}$

$14.17112700 \quad-0.41207200 \quad-3.63310800$

$\begin{array}{llll}12.92929500 & -2.33345600 & -2.71432500\end{array}$

$12.73468400 \quad 0.15356500 \quad-4.49971300$

$\begin{array}{llll}12.51992200 & 1.50280300 & -2.53127400\end{array}$

$12.35053100 \quad-2.28071000 \quad-4.38426000$

$10.29582200 \quad-1.16340000 \quad-3.73677200$

$\begin{array}{llll}8.59973200 & 2.73385600 & -0.69200100\end{array}$

$\begin{array}{llll}8.63533900 & 6.12736200 & -1.25281800\end{array}$

$\begin{array}{llll}-4.50626700 & 4.68972200 & -1.77888700\end{array}$

$\begin{array}{llll}-4.40032500 & 3.16541600 & -2.63424200\end{array}$

$\begin{array}{llll}-9.78546534 & 4.22526721 & -2.07599000\end{array}$

$\begin{array}{llll}-10.77509680 & 4.33425979 & -0.61550483\end{array}$

$\begin{array}{llll}-11.41118244 & 4.91743227 & -2.17149329\end{array}$

$\begin{array}{llll}-10.69840502 & 6.83941102 & -0.75376206\end{array}$

$\begin{array}{llll}-3.38908700 & 5.69971300 & 4.05381900\end{array}$

$\begin{array}{lll}-5.62036500 & 7.58508300 & 3.10332400\end{array}$

$\begin{array}{lll}-3.97353600 & 7.59134200 & 2.49612500\end{array}$

$\begin{array}{lll}-4.93892100 & 7.29071500 & 0.18633100\end{array}$

$\begin{array}{llll}-5.95384600 & 5.88519200 & -0.17768000\end{array}$

$\begin{array}{lll}-6.59492300 & 7.17471600 & 0.81881400\end{array}$

$\begin{array}{lll}-3.59685000 & 4.41824800 & 2.33974900\end{array}$

$\begin{array}{llll}-4.28797500 & 4.23281800 & 0.73295400\end{array}$

$\begin{array}{lll}-3.13942800 & 5.54604900 & 1.05456500\end{array}$

$\begin{array}{lll}-5.94558000 & 5.26851200 & 2.25841600\end{array}$

$\begin{array}{lll}1.09650900 & 5.15339900 & 5.75155600\end{array}$

$\begin{array}{lll}1.44389200 & 5.20948600 & 4.00658200\end{array}$

$\begin{array}{lll}2.58610617 & 1.43855874 & 3.43359325\end{array}$

$\begin{array}{lll}0.92326106 & 2.04138939 & 3.31669658\end{array}$

$\begin{array}{llll}2.27217895 & 2.98792727 & 2.61992145\end{array}$

$\begin{array}{lll}4.37446412 & -0.74454315 & 4.73885489\end{array}$

$\begin{array}{lll}3.44636314 & -0.74825319 & 3.23757688\end{array}$

$\begin{array}{llll}2.62544314 & -0.41166206 & 4.77957784\end{array}$

$\begin{array}{llll}1.66390498 & -3.96894808 & 3.72623005\end{array}$

$\begin{array}{lll}1.01585506 & -2.32745204 & 3.88960194\end{array}$

$\begin{array}{lll}2.10039805 & -2.71833917 & 2.55222598\end{array}$

$\begin{array}{llll}3.98894502 & -3.05723717 & 4.13443903\end{array}$

$\begin{array}{llll}2.60323895 & -3.94468698 & 5.99029106\end{array}$

$\begin{array}{lll}1.99681402 & -2.32445693 & 6.31154295\end{array}$

$\begin{array}{llll}6.76375789 & -1.69908790 & -1.35283226\end{array}$

$\begin{array}{llll}7.88365266 & -2.69994403 & -1.82098595\end{array}$

$\begin{array}{llll}7.25312452 & 0.22829960 & -1.93337571\end{array}$

$\begin{array}{lll}5.93902340 & -0.01855262 & -2.74666371\end{array}$

$\begin{array}{lll}-6.31873010 & 0.76932668 & 1.70432470\end{array}$

$\begin{array}{llll}-5.41860700 & -1.10407800 & 0.76486100\end{array}$

$\begin{array}{llll}7.98079900 & 3.88806700 & -3.02431200\end{array}$

$\begin{array}{llll}5.94380000 & 5.19338200 & 0.90689700\end{array}$

$\begin{array}{llll}-6.53364300 & 5.32330900 & -3.23186600\end{array}$

$\begin{array}{llll}-8.69139900 & 6.34759500 & -3.01705600\end{array}$ 


$\begin{array}{cccc}\mathrm{H} & -8.22349554 & 5.50415684 & -0.64146406 \\ \mathrm{H} & 4.27679300 & -1.54132000 & 6.90042100 \\ \mathrm{H} & 11.46454600 & 1.97904800 & -0.63397300 \\ \mathrm{H} & 4.52097000 & -2.97961500 & 2.13139300 \\ \mathrm{H} & 0.99705700 & -8.44088000 & -1.05324700 \\ \mathrm{H} & 3.05354700 & -2.20336100 & -7.08352500 \\ \mathrm{H} & 3.00411400 & -0.44550600 & -6.96708400 \\ \mathrm{H} & -1.73448900 & 0.43823200 & -5.85287300 \\ \mathrm{H} & -6.77169700 & -3.78737000 & -3.89201300 \\ \mathrm{H} & -8.53124300 & -9.02691100 & -0.04011800 \\ \mathrm{H} & -4.48321900 & -4.51595000 & -3.79036700 \\ \mathrm{H} & -12.40288300 & -0.27656200 & -4.39132400 \\ \mathrm{H} & -10.91959500 & -1.15766100 & -4.75593900 \\ \mathrm{H} & -7.61309657 & 1.66414253 & 1.36856558 \\ \mathrm{H} & -4.96810900 & 5.13897400 & -4.06670700 \\ \mathrm{H} & -6.21368300 & 3.86110100 & -4.20032900 \\ \mathrm{H} & -2.82350300 & -4.99992400 & -3.96308300 \\ \mathrm{H} & -3.84511400 & -5.87261700 & -2.87970100 \\ \mathrm{H} & -7.16740100 & -2.37462300 & -4.85090800 \\ \mathrm{H} & -7.98485500 & -3.91004400 & -5.15542300 \\ \mathrm{H} & -11.14893800 & 0.46868700 & -5.38599400 \\ \mathrm{H} & -2.30764500 & 2.10428400 & -5.60494100 \\ \mathrm{H} & -3.38531900 & 0.79842800 & -5.27191000 \\ \mathrm{H} & 1.51583700 & -1.37507900 & -6.79888100 \\ \mathrm{H} & 2.43667600 & 5.28121700 & -4.58857500 \\ \mathrm{H} & 7.47289700 & 6.10659400 & 0.87797900 \\ \mathrm{H} & 6.15113300 & 6.55800700 & -0.20982900 \\ \mathrm{H} & 9.21958100 & 5.12395900 & -3.31052400 \\ \mathrm{H} & 10.16444900 & 1.29383800 & 0.36058100 \\ \mathrm{H} & 5.63085900 & -4.28268000 & 2.58961300 \\ \mathrm{H} & 5.19532600 & -4.04634100 & 0.88482700 \\ \mathrm{H} & 4.97120800 & -3.09991400 & 6.52898200 \\ \mathrm{H} & 3.72202700 & -2.86618800 & 7.89509000 \\ \mathrm{H} & -0.76794400 & -8.20702100 & -0.98739000 \\ \mathrm{H} & 0.13965800 & -7.53757700 & -2.31518900 \\ \mathrm{H} & -7.91816900 & -8.59840400 & 1.54735100 \\ \mathrm{H} & -6.95106600 & -8.29808400 & 0.05747500 \\ \mathrm{H} & -9.06117400 & 7.93376000 & -2.30589500 \\ \mathrm{H} & -10.26909900 & 7.11529500 & -3.23905400 \\ \mathrm{H} & -3.90949600 & 7.10110800 & 4.94079600 \\ \mathrm{H} & -5.01688600 & 5.69924300 & 4.69478300 \\ \mathrm{H} & 0.07747400 & 4.23998100 & 4.61021200 \\ \mathrm{H} & -6.05029915 & 2.07730725 & 0.18642809\end{array}$

\section{Int3 (-2.9 kcal/mol)}

$\begin{array}{cccc}\mathrm{C} & 6.64961000 & 5.71529300 & 0.38987500 \\ \mathrm{C} & 7.17317600 & 4.79288000 & -0.66574400 \\ \mathrm{O} & 6.71826200 & 3.66657300 & -0.89832400 \\ \mathrm{~N} & 8.21532400 & 5.28119900 & -1.37514900 \\ \mathrm{C} & 8.78919600 & 4.51981300 & -2.45362300 \\ \mathrm{C} & 9.85696700 & 3.50752300 & -2.04170900 \\ \mathrm{O} & 10.87686000 & 3.33546000 & -2.70242200 \\ \mathrm{~N} & 9.56856800 & 2.80078200 & -0.91227300 \\ \mathrm{C} & 10.41962200 & 1.67432900 & -0.59576300 \\ \mathrm{C} & 10.17694300 & 0.52992800 & -1.57049600 \\ \mathrm{O} & 9.02495100 & 0.11374300 & -1.76050100 \\ \mathrm{~N} & 11.23760100 & -0.07888300 & -2.14013600 \\ \mathrm{C} & 10.99756200 & -1.28824700 & -2.94569000 \\ \mathrm{C} & 10.34666100 & -2.39075200 & -2.10410600 \\ \mathrm{O} & 9.50956800 & -3.15076900 & -2.58824100\end{array}$

$\begin{array}{llll}\text { C } & 12.39992100 & -1.63408800 & -3.47816900\end{array}$

$\begin{array}{llll}\text { C } & 13.08199600 & -0.26153300 & -3.58468900\end{array}$

$\begin{array}{llll}\text { C } & 12.59016100 & 0.48646700 & -2.33848200\end{array}$

$\begin{array}{llll}\mathrm{N} & 10.79349900 & -2.45599200 & -0.82748700\end{array}$

$\begin{array}{llll}\text { C } & 10.24717800 & -3.32889700 & 0.19436100\end{array}$

$\begin{array}{llll}\text { C } & 9.69114800 & -2.57173800 & 1.40666300\end{array}$

$\begin{array}{llll}\text { O } & 9.87242100 & -2.99539800 & 2.53740900\end{array}$

$\begin{array}{llll}\mathrm{N} & 8.98472300 & -1.42894500 & 1.12749400\end{array}$

$\begin{array}{llll}\text { C } & 8.09182200 & -0.90607400 & 2.16914000\end{array}$

$\begin{array}{llll}\text { C } & 7.00241700 & -1.92122700 & 2.56956500\end{array}$

$\begin{array}{llll}\text { O } & 6.56821600 & -1.96790500 & 3.72017900\end{array}$

$\begin{array}{llll}\text { C } & 7.44923500 & 0.40756800 & 1.68587600\end{array}$

$\begin{array}{llll}\text { C } & 6.33709000 & 0.96970500 & 2.59032700\end{array}$

$\begin{array}{llll}\mathrm{C} & 6.80703700 & 1.21447800 & 4.02814100\end{array}$

$\begin{array}{llll}\text { C } & 5.77206000 & 2.25527700 & 1.97873400\end{array}$

$\begin{array}{llll}\mathrm{N} & 6.52556400 & -2.67134400 & 1.55754900\end{array}$

$\begin{array}{llll}\text { C } & 5.40544100 & -3.55992800 & 1.75807800\end{array}$

$\begin{array}{llll}\text { C } & 4.04164100 & -2.74455200 & 6.83020300\end{array}$

$\begin{array}{llll}\text { C } & 2.86090000 & -2.99290400 & 5.88173300\end{array}$

$\begin{array}{llll}\text { C } & 3.10614400 & -2.59312600 & 4.42210000\end{array}$

$\begin{array}{llll}\text { C } & 1.91125100 & -2.98578700 & 3.54598700\end{array}$

$\begin{array}{llll}\text { C } & 3.40872200 & -1.09734300 & 4.27232900\end{array}$

$\begin{array}{llll}\text { C } & 0.18576900 & -7.69543700 & -1.39733000\end{array}$

$\begin{array}{llll}\text { C } & 0.37235400 & -6.40886600 & -0.58441700\end{array}$

$\begin{array}{llll}\text { C } & 1.43447048 & -5.43960662 & -1.13861595\end{array}$

$\begin{array}{llll}\text { C } & 0.97855261 & -4.76914207 & -2.44208500\end{array}$

$\begin{array}{llll}\text { C } & 1.78179570 & -4.37465197 & -0.09142331\end{array}$

$\begin{array}{llll}\text { C } & -7.95214100 & -8.27320100 & 0.32087200\end{array}$

$\begin{array}{llll}\text { C } & -8.62681400 & -6.89765800 & 0.18511200\end{array}$

$\begin{array}{llll}\text { C } & -8.08610900 & -5.80328600 & 1.07792000\end{array}$

$\begin{array}{llll}\text { C } & -6.81037900 & -5.25454900 & 0.89675900\end{array}$

$\begin{array}{llll}\text { C } & -8.87698900 & -5.27838700 & 2.10634100\end{array}$

$\begin{array}{llll}\text { C } & -6.35110800 & -4.20032700 & 1.68824800\end{array}$

$\begin{array}{llll}\text { C } & -8.43722900 & -4.22290300 & 2.90002500\end{array}$

$\begin{array}{llll}\text { C } & -7.17793100 & -3.66037100 & 2.68109800\end{array}$

$\begin{array}{llll}\text { O } & -6.79289200 & -2.60181300 & 3.46413700\end{array}$

$\begin{array}{llll}\text { C } & -3.60984600 & -4.78116300 & -3.32149600\end{array}$

$\begin{array}{llll}\text { C } & -3.28657800 & -3.81238400 & -2.18697500\end{array}$

$\begin{array}{llll}\text { C } & -4.57606300 & -3.21061000 & -1.62867700\end{array}$

$\begin{array}{llll}\text { C } & -4.41639900 & -2.24220300 & -0.47906300\end{array}$

$\begin{array}{llll}\text { O } & -5.45040600 & -1.56953600 & -0.16225700\end{array}$

$\begin{array}{llll}\text { O } & -3.30129900 & -2.17335800 & 0.11848300\end{array}$

$\begin{array}{llll}\text { C } & 2.58845900 & -1.22635100 & -6.57782700\end{array}$

$\begin{array}{llll}\text { C } & 2.83460700 & -1.34130400 & -5.09206600\end{array}$

$\begin{array}{llll}\text { C } & 1.88853600 & -0.87021500 & -4.17664600\end{array}$

$\begin{array}{llll}\text { C } & 4.02279900 & -1.88945000 & -4.58870700\end{array}$

$\begin{array}{llll}\text { C } & 2.12495700 & -0.90330300 & -2.80199600\end{array}$

$\begin{array}{llll}\text { C } & 4.26381900 & -1.94208500 & -3.21312200\end{array}$

$\begin{array}{llll}\text { C } & 3.32237800 & -1.42950000 & -2.31355400\end{array}$

$\begin{array}{llll}\text { C } & 2.62275900 & 4.30818100 & -4.39972400\end{array}$

$\begin{array}{llll}\text { C } & 3.16623900 & 3.95732400 & -3.01036000\end{array}$

$\begin{array}{llll}\text { C } & 3.56760300 & 2.49039500 & -2.86814600\end{array}$

$\mathrm{N} \quad 4.08995900 \quad 2.22625400 \quad-1.51414500$

$\begin{array}{llll}\text { C } & -2.32798900 & 1.20068400 & -5.18484600\end{array}$

$\begin{array}{llll}\text { C } & -1.83200700 & 1.12390300 & -3.73566100\end{array}$

$\begin{array}{llll}\text { C } & -1.76728900 & -0.33545500 & -3.26766700\end{array}$

$\begin{array}{llll}\text { C } & -0.49323400 & 1.85481000 & -3.56625200\end{array}$

$\begin{array}{llll}\text { C } & -7.60174600 & -3.15727400 & -4.44383900\end{array}$

$\begin{array}{llll}\text { C } & -8.63014300 & -2.79061300 & -3.37238800\end{array}$

$\begin{array}{llll}\text { C } & -8.94654400 & -3.95974200 & -2.44035400\end{array}$

S $\quad-10.14396700 \quad-3.57193200 \quad-1.11228500$

$\begin{array}{llll}\text { C } & -9.19106900 & -2.31120400 & -0.19034900\end{array}$

$\begin{array}{llll}\text { C } & -11.31943800 & -0.07101100 & -4.53337300\end{array}$ 


\begin{tabular}{|c|c|c|c|}
\hline $\boldsymbol{r}$ & -10.69945600 & 0.47463900 & -3.27235400 \\
\hline & -9.31938200 & 0.69714600 & -3.18692900 \\
\hline & -11.48012100 & 0.78006000 & -2.15295400 \\
\hline & -8.74146900 & 1.24209100 & -2.04350400 \\
\hline & -10.92062200 & 1.34405100 & -1.00732200 \\
\hline & -9.55287400 & 1.60515000 & -0.96276300 \\
\hline & -9.03700200 & 2.23829600 & 0.14901700 \\
\hline & -5.76776500 & 4.68736500 & -3.45307100 \\
\hline & -5.11184800 & 4.00227900 & -2.2504 \\
\hline & -6.05559900 & 3.41590600 & -1.231 \\
\hline $\mathrm{O}$ & -7.18479000 & 3.86832600 & -1.020 \\
\hline $\mathrm{O}$ & -5.53679600 & 2.40658700 & -0.567 \\
\hline & -9.47007600 & 6.99288900 & -2.38704300 \\
\hline $\mathrm{C}$ & -9.94716300 & 6.26460000 & -1.13 \\
\hline C & -10.58152800 & 4.91396400 & -1.46397500 \\
\hline $\mathrm{O}$ & -8.88080700 & 6.11773400 & -0.19735000 \\
\hline $\mathrm{C}$ & -4.27273400 & 6.26204300 & 4.353 \\
\hline 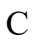 & -4.76641100 & 6.88772100 & 3.0492 \\
\hline $\mathrm{C}$ & -5.14671900 & 5.87083400 & 1.95500600 \\
\hline $\mathrm{C}$ & -5.70475900 & 6.60381600 & 0.72957300 \\
\hline $\mathrm{C}$ & -3.96794000 & 4.97079300 & 1.555 \\
\hline $\mathrm{C}$ & 1.08197400 & 4.48572200 & 4.91731100 \\
\hline 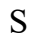 & 2.20592200 & 3.05923700 & 5.10425500 \\
\hline $\mathrm{C}$ & 1.77107298 & 2.147 & 3.58418434 \\
\hline $\mathrm{O}$ & 7.11919200 & -2.49056600 & -1.21012200 \\
\hline $\mathrm{O}$ & 6.28507000 & 0.09582500 & -1.84 \\
\hline $\mathrm{O}$ & -6.79628456 & 1.70 & 1.6 \\
\hline $\mathrm{N}$ & -3.14769500 & -0.31845200 & 1.90371400 \\
\hline $\mathrm{C}$ & -0.41072600 & 0.34671800 & 5.8396 \\
\hline $\mathrm{C}$ & -1.12458300 & 1.50567100 & 5.50965800 \\
\hline $\mathrm{C}$ & -3.17774200 & 1.07177000 & 1.35776000 \\
\hline $\mathrm{C}$ & -2.38427900 & 1.160486 & 0.04953400 \\
\hline $\mathrm{C}$ & -2.39941400 & 1.40898700 & 4.95649800 \\
\hline $\mathrm{C}$ & -3.00943300 & 0.16812700 & 4.74 \\
\hline $\mathrm{C}$ & -0.90309200 & 0.8 & 215200 \\
\hline $\mathrm{C}$ & -4.36139000 & 39700 & 297700 \\
\hline $\mathrm{C}$ & 0.01302300 & $1.94 \mathrm{C}$ & 0.19109300 \\
\hline $\mathrm{C}$ & 1.38324100 & 1.73168600 & 0.33620200 \\
\hline $\mathrm{C}$ & -4.30775100 & -0.73292600 & 2.78681400 \\
\hline $\mathrm{C}$ & 1.84413400 & 0.42059000 & 0.57160100 \\
\hline $\mathrm{C}$ & -2.29533400 & -0.98454700 & 5.11792000 \\
\hline $\mathrm{C}$ & -1.01178900 & -0.90506200 & 24300 \\
\hline $\mathrm{C}$ & 0.95092700 & -0.645151 & 0.55749200 \\
\hline $\mathrm{C}$ & -0.41157600 & -0.41716300 & 0.35360700 \\
\hline $\mathrm{O}$ & 0.84083000 & 0.37985000 & 6.35671100 \\
\hline $\mathrm{O}$ & -5.49277800 & -0.61495800 & 2.07724600 \\
\hline $\mathrm{O}$ & 3.17449200 & 0.18476200 & 0.79817200 \\
\hline $\mathrm{O}$ & 2.27186300 & 2.76466700 & 0.31522800 \\
\hline $\mathrm{H}$ & -0.68179100 & 2.47550600 & 5.70066100 \\
\hline $\mathrm{H}$ & -2.93714700 & 2.31817100 & 4.69846800 \\
\hline $\mathrm{H}$ & 3.59822800 & 1.04732500 & 0.93434600 \\
\hline $\mathrm{H}$ & -4.75253500 & 1.06970100 & 3.85437300 \\
\hline $\mathrm{H}$ & -1.08384900 & -1.26648200 & 0.28211200 \\
\hline $\mathrm{H}$ & -2.77654300 & 1.74538200 & 2.11782000 \\
\hline $\mathrm{H}$ & -5.10148600 & -0.42119900 & 4.72182200 \\
\hline $\mathrm{H}$ & -2.54510200 & 2.16639600 & -0.34820600 \\
\hline $\mathrm{H}$ & -4.08740300 & -1.78590300 & 3.00831000 \\
\hline $\mathrm{H}$ & -2.75087500 & -1.96349400 & 4.99263400 \\
\hline $\mathrm{H}$ & -0.32375700 & 2.97178000 & 0.04384100 \\
\hline $\mathrm{H}$ & -0.46020200 & -1.79777000 & 5.91992600 \\
\hline $\mathrm{H}$ & 1.33188600 & -1.64916000 & 0.69748400 \\
\hline $\mathrm{H}$ & -3.16976047 & -1.04289195 & 1.11692080 \\
\hline 4 & 1.21802500 & 1.27737100 & 6.24528100 \\
\hline
\end{tabular}

$\mathrm{H} \quad-2.83428800 \quad 0.46615900 \quad-0.66657300$

$\mathrm{H} \quad-4.21823000 \quad 1.32409700 \quad 1.17646100$

$\mathrm{H} \quad-2.27888000 \quad-0.46288100 \quad 2.42518000$

$\mathrm{H} \quad 4.92818200 \quad 2.78656200 \quad-1.35048800$

$\mathrm{H} \quad 2.99896200 \quad 2.57377400 \quad-0.39657700$

$\mathrm{H} \quad 4.40383700 \quad 1.25870900 \quad-1.44192700$

$\mathrm{H} \quad 4.30650200 \quad 2.23176600 \quad-3.64333000$

$\mathrm{H} \quad 2.70277800 \quad 1.84059900 \quad-3.03095600$

$\mathrm{H} \quad 4.04446600 \quad 4.58137600 \quad-2.79103600$

$\mathrm{H} \quad 2.42299200 \quad 4.18965600 \quad-2.23853600$

$\mathrm{H} \quad 1.68501100 \quad 3.78243900 \quad-4.60467900$

$\mathrm{H} \quad 3.33710200 \quad 4.03589400 \quad-5.18496800$

$\mathrm{H} \quad 4.76067100 \quad-2.28999300 \quad-5.27973500$

H $\quad 5.17302400 \quad-2.39749700 \quad-2.83106900$

$\mathrm{H} \quad 3.51990000 \quad-1.44297600 \quad-1.24672900$

$\mathrm{H} \quad 1.37538700 \quad-0.53011100 \quad-2.11345300$

$\mathrm{H} \quad 0.95222300 \quad-0.47249600 \quad-4.54973700$

$\mathrm{H} \quad 0.26070600 \quad 1.46958200 \quad-4.25996700$

$\mathrm{H} \quad-0.60771700 \quad 2.92499000 \quad-3.77209000$

$\mathrm{H} \quad-0.10265300 \quad 1.74655300 \quad-2.55021300$

$\mathrm{H} \quad-2.56610300 \quad 1.64103700 \quad-3.10011600$

$\mathrm{H} \quad-1.38935600 \quad-0.42072600 \quad-2.24605100$

$\mathrm{H} \quad-2.75979500 \quad-0.79720300 \quad-3.30242200$

$\mathrm{H} \quad-1.10998700 \quad-0.92905300 \quad-3.91269500$

$\mathrm{H} \quad-8.23732600 \quad-1.96221600 \quad-2.77486300$

$\mathrm{H} \quad-9.55846400 \quad-2.42845500 \quad-3.82393600$

$\mathrm{H} \quad-8.02659500 \quad-4.32988600 \quad-1.97106700$

$\mathrm{H} \quad-9.38978700 \quad-4.79382500 \quad-2.99538800$

$\mathrm{H} \quad-8.14588300 \quad-2.61617600 \quad-0.09864600$

$\mathrm{H} \quad-9.25586300 \quad-1.33277700 \quad-0.66932300$

$\mathrm{H} \quad-9.62246300 \quad-2.25705800 \quad 0.81028800$

$\mathrm{H} \quad-8.45492000 \quad 2.96702900 \quad-0.16991600$

$\mathrm{H} \quad-7.66897100 \quad 1.38201800 \quad-1.98283000$

$\mathrm{H} \quad-11.53472700 \quad 1.61704400 \quad-0.15620400$

$\mathrm{H} \quad-12.55020700 \quad 0.59557400 \quad-2.18466900$

$\mathrm{H} \quad-8.68351200 \quad 0.43864200 \quad-4.02974800$

$\mathrm{H} \quad-5.25373000 \quad-3.99800700 \quad-1.27261800$

$\mathrm{H} \quad-5.13577500 \quad-2.69040600 \quad-2.41447100$

$\mathrm{H} \quad-2.72454100 \quad-4.30184600 \quad-1.38584200$

$\mathrm{H} \quad-2.64645300 \quad-3.00306100 \quad-2.55242300$

$\mathrm{H} \quad-8.56076900 \quad-6.57564400 \quad-0.86147800$

$\mathrm{H} \quad-9.69676300 \quad-7.00438000 \quad 0.39103400$

$\mathrm{H} \quad-9.87215700 \quad-5.68402500 \quad 2.26580000$

H $\quad-6.16036000 \quad-5.65908700 \quad 0.12431900$

$\mathrm{H} \quad-5.35410800 \quad-3.79852800 \quad 1.54083700$

$\mathrm{H} \quad-9.06657800 \quad-3.80066400 \quad 3.67639800$

$\mathrm{H} \quad-6.48727000 \quad-1.86876900 \quad 2.89118500$

$\mathrm{H} \quad 2.51233686 \quad-3.65631872 \quad-0.47788362$

H $\quad 0.88203858 \quad-3.81504215 \quad 0.19245614$

$\mathrm{H} \quad 2.19185758 \quad-4.82224364 \quad 0.81942198$

H $\quad \begin{array}{llll}1.75426464 & -4.10670502 & -2.83847254\end{array}$

$\mathrm{H} \quad 0.73449415 \quad-5.50123316 \quad-3.21792150$

H $\quad 0.08379960 \quad-4.15918152 \quad-2.26530379$

H $\quad 2.34606100 \quad-6.01864215 \quad-1.35027833$

$\mathrm{H} \quad-0.58381074 \quad-5.86933372 \quad-0.52567924$

H $\quad 0.63267978 \quad-6.67323128 \quad 0.44857208$

H $\quad 6.88418200 \quad-2.58265700 \quad 0.60903600$

$\mathrm{H} \quad 8.68585000 \quad-0.74277500 \quad 3.06858200$

$\mathrm{H} \quad 8.24303800 \quad 1.16210200 \quad 1.58368500$

H $\quad 7.03197400 \quad 0.24546800 \quad 0.68432500$

$\mathrm{H} \quad \begin{array}{llll}6.52381400 & 3.05342600 & 1.99352900\end{array}$

$\mathrm{H} \quad 5.48255300 \quad 2.12260500 \quad 0.93214600$

H $\quad 4.90334600 \quad 2.61040700 \quad 2.54326300$ 


\begin{tabular}{|c|c|c|c|}
\hline & & & \\
\hline & & & \\
\hline & & & \\
\hline & 53149000 & 0.22830000 & \\
\hline & & -1.32598400 & 6838600 \\
\hline & 9.44313300 & -3.90798600 & -0.26799200 \\
\hline & 11.00269900 & -4.02247000 & 0.57097900 \\
\hline & 11.42537400 & -1.72873400 & -0.52611400 \\
\hline & 13.22820200 & 0.27581300 & \\
\hline & 14.17143200 & -0.325 & -3 . \\
\hline & 12.92596600 & 01900 & 520500 \\
\hline & & & \\
\hline & & & \\
\hline & & -2.1 & \\
\hline & & -1.0 & 300 \\
\hline & & 2.7 & \\
\hline & & 6.16 & \\
\hline & & & \\
\hline & -4.41884100 & 3.2 & 0600 \\
\hline & & & \\
\hline & & & \\
\hline & & & \\
\hline & & & \\
\hline & & & \\
\hline & 752700 & & \\
\hline & & & \\
\hline & -4.96695400 & & \\
\hline & & & \\
\hline & & & \\
\hline & -3.5 & & \\
\hline & & & \\
\hline & & & \\
\hline & -5.9 & & \\
\hline & & & \\
\hline & 1.4259 & & \\
\hline & & & \\
\hline & & & \\
\hline & & & \\
\hline & $4.3^{\circ}$ & -0.8 & \\
\hline & & & \\
\hline & & & \\
\hline & & & \\
\hline & & -2.4 & \\
\hline & & & \\
\hline & & & \\
\hline & & & \\
\hline & 1.98681600 & -2.4 & 40900 \\
\hline & & & \\
\hline & & -2.7 & \\
\hline & & & 5400 \\
\hline & & -0.0 & \\
\hline & & & \\
\hline $\mathrm{H}$ & -5.43839045 & -1.036 & 70242 \\
\hline & & & 67300 \\
\hline $\mathrm{H}$ & 5.93652300 & 5.1848 & 57800 \\
\hline $\mathrm{H}$ & -6.53544200 & & 63200 \\
\hline $\mathrm{H}$ & & & 72700 \\
\hline & -8.36409200 & 5.34201200 & 624900 \\
\hline & & $-1.671<$ & \\
\hline $\mathrm{H}$ & 11.45848100 & 2.00048000 & -0.58107000 \\
\hline & 4.51652300 & -3.01085100 & 2.07598500 \\
\hline $\mathrm{H}$ & & -8.40869800 & -1.22636500 \\
\hline & 3.06008200 & -2.04821000 & -7.12515000 \\
\hline
\end{tabular}

$\begin{array}{cccc}\text { H } & 3.00997400 & -0.29328500 & -6.97266900 \\ \mathrm{H} & -1.73371900 & 0.56747900 & -5.85187300 \\ \mathrm{H} & -6.76431000 & -3.69902900 & -3.98602700 \\ \mathrm{H} & -8.53582200 & -9.01352100 & -0.24050200 \\ \mathrm{H} & -4.48092500 & -4.42658000 & -3.88633000 \\ \mathrm{H} & -12.40034200 & -0.18994300 & -4.42126300 \\ \mathrm{H} & -10.90693300 & -1.05251200 & -4.79182300 \\ \mathrm{H} & -7.69852300 & 1.63830500 & 1.30371200 \\ \mathrm{H} & -4.96833400 & 5.22794400 & -3.96987000 \\ \mathrm{H} & -6.21322100 & 3.95262200 & -4.13134000 \\ \mathrm{H} & -2.81993300 & -4.91018300 & -4.07028000 \\ \mathrm{H} & -3.84275100 & -5.80518500 & -3.00640700 \\ \mathrm{H} & -7.16359400 & -2.26885600 & -4.91049500 \\ \mathrm{H} & -7.98001900 & -3.79803100 & -5.24747100 \\ \mathrm{H} & -11.14547200 & 0.58335700 & -5.39307300 \\ \mathrm{H} & -2.30451400 & 2.22626300 & -5.56596900 \\ \mathrm{H} & -3.38215300 & 0.91349300 & -5.26121500 \\ \mathrm{H} & 1.52200900 & -1.22661600 & -6.82527200 \\ \mathrm{H} & 2.43712900 & 5.38348000 & -4.47817100 \\ \mathrm{H} & 7.46527300 & 6.09884600 & 1.01127000 \\ \mathrm{H} & 6.14473800 & 6.57193100 & -0.06880200 \\ \mathrm{H} & 9.21827700 & 5.20259000 & -3.19393300 \\ \mathrm{H} & 10.15622600 & 1.29274400 & 0.39443100 \\ \mathrm{H} & 5.62486000 & -4.32388200 & 2.50773800 \\ \mathrm{H} & 5.18928000 & -4.05303600 & 0.80812000 \\ \mathrm{H} & 4.95916500 & -3.22207500 & 6.46951700 \\ \mathrm{H} & 3.70794900 & -3.01671900 & 7.83831400 \\ \mathrm{H} & -0.76735500 & -8.17664500 & -1.15778500 \\ \mathrm{H} & 0.14186800 & -7.47989600 & -2.47033700 \\ \mathrm{H} & -7.92101700 & -8.62228000 & 1.35824400 \\ \mathrm{H} & -6.95191700 & -8.29124400 & -0.12381000 \\ \mathrm{H} & -9.06497600 & 7.98471600 & -2.15818100 \\ \mathrm{H} & -10.27125900 & 7.18504800 & -3.10958500 \\ \mathrm{H} & -3.92325300 & 7.00608200 & 5.07732600 \\ \mathrm{H} & -5.02975000 & 5.60913800 & 4.80115700 \\ \mathrm{H} & 0.06157400 & 4.15754400 & 4.70531600 \\ \mathrm{H} & -6.09818100 & 2.13028600 & 0.25102400\end{array}$

\section{Int4 (-1.4 kcal/mol)}

$\begin{array}{cccc}\mathrm{C} & 6.65329700 & 5.70855900 & 0.36081100 \\ \mathrm{C} & 7.17720200 & 4.78122600 & -0.69026900 \\ \mathrm{O} & 6.72568900 & 3.65201300 & -0.91507400 \\ \mathrm{~N} & 8.21690500 & 5.26786700 & -1.40448700 \\ \mathrm{C} & 8.79126000 & 4.50096200 & -2.47878700 \\ \mathrm{C} & 9.85944800 & 3.49111300 & -2.06162100 \\ \mathrm{O} & 10.87859800 & 3.31488200 & -2.72251700 \\ \mathrm{~N} & 9.57239000 & 2.79117300 & -0.92770200 \\ \mathrm{C} & 10.42382600 & 1.66676000 & -0.60519100 \\ \mathrm{C} & 10.18227700 & 0.51773800 & -1.57473200 \\ \mathrm{O} & 9.03060700 & 0.10064000 & -1.76420000 \\ \mathrm{~N} & 11.24345900 & -0.09376800 & -2.14068200 \\ \mathrm{C} & 11.00336600 & -1.30534700 & -2.94287300 \\ \mathrm{C} & 10.34984900 & -2.40486200 & -2.09945700 \\ \mathrm{O} & 9.51341000 & -3.16580100 & -2.58333500 \\ \mathrm{C} & 12.40618400 & -1.65407200 & -3.47203500 \\ \mathrm{C} & 13.08880700 & -0.28220800 & -3.58351100 \\ \mathrm{C} & 12.59592400 & 0.47115000 & -2.34088900 \\ \mathrm{~N} & 10.79400000 & -2.46685900 & -0.82180000 \\ \mathrm{C} & 10.24498200 & -3.33679700 & 0.20113800 \\ \mathrm{C} & 9.68826600 & -2.57628200 & 1.41101000\end{array}$




\begin{tabular}{|c|c|c|c|}
\hline & 9.86840700 & -2.99746000 & 2.54291300 \\
\hline & 8.98293400 & -1.43382100 & 1.12843200 \\
\hline & 8.08984700 & -0.90706300 & 2.16808300 \\
\hline & 6.99909000 & -1.92006400 & 2.57019300 \\
\hline ) & 6.56471600 & -1.96419000 & 3.72073200 \\
\hline & 7.45024000 & 0.40665700 & 1.68144700 \\
\hline & 6.34049300 & 0.97519100 & 2.58500900 \\
\hline & 6.81059000 & 1.21804400 & 500 \\
\hline & 5.78372700 & 2.26408100 & 1.97263000 \\
\hline & 6.52240000 & -2.67292900 & 1.55994900 \\
\hline 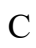 & 5.40214000 & -3.56070300 & 1.76326600 \\
\hline & 4.04009900 & -2.72557600 & 6.83268900 \\
\hline & 2.85773100 & -2.97818800 & 5.88742400 \\
\hline $\mathrm{C}$ & 3.09953100 & -2.58677300 & 4.425 \\
\hline & 1.90162000 & -2.98414400 & 3.55510300 \\
\hline C & 3.40205200 & -1.09183400 & 4.2663 \\
\hline C & 0.17841600 & -7.70351200 & -1.3757 \\
\hline C & 0.36740000 & -6.41328900 & -0.5690 \\
\hline C & 1.51600900 & -5.50727300 & -1.05 \\
\hline 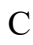 & 1.19875800 & -4.84400100 & -2.40105000 \\
\hline $\mathrm{C}$ & 1.83172000 & -4.43750500 & -0.00126600 \\
\hline $\mathrm{C}$ & -7.95955200 & -8.26853900 & 0.346 \\
\hline$C$ & -8.63653200 & -6.89452900 & 0.1979 \\
\hline C & -8.11609000 & -5.79141500 & 1.09 \\
\hline $\mathrm{C}$ & -6.85047800 & -5.21823200 & 0.9187 \\
\hline $\mathrm{C}$ & -8.91952700 & -5.28176600 & $2.11 \mathrm{C}$ \\
\hline $\mathrm{C}$ & -6.41181100 & -4.15798100 & 1.7156 \\
\hline $\mathrm{C}$ & -8.50066900 & -4.22404600 & 2.9204 \\
\hline $\mathrm{C}$ & -7.24903400 & -3.63823000 & 2.711 \\
\hline $\mathrm{O}$ & -6.88852700 & -2.59007700 & 3.51265400 \\
\hline $\mathrm{C}$ & -3.61534800 & -4.79329900 & -3.30968800 \\
\hline $\mathrm{C}$ & -3.2938 & -3.81770200 & -2.18327200 \\
\hline & -4.59353900 & -3.25373900 & -1.60383900 \\
\hline $\mathrm{C}$ & -4.45682400 & -2.22862600 & -0.51287300 \\
\hline 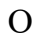 & -5.39899400 & -1.47330800 & -0.233 \\
\hline & -3.31100700 & -2.22684000 & 0.124 \\
\hline C & 2.58500200 & -1.25535900 & -6.58046100 \\
\hline $\mathrm{C}$ & 2.82994600 & -1.36312800 & -5.09367600 \\
\hline $\mathrm{C}$ & 1.88328200 & -0.88734200 & -4.18 \\
\hline $\mathrm{C}$ & 4.01787300 & -1.908544 & $-4.586^{\prime}$ \\
\hline $\mathrm{C}$ & 2.11895600 & -0.91318000 & -2.80597900 \\
\hline $\mathrm{C}$ & 4.25842500 & -1.95356600 & -3.210 \\
\hline $\mathrm{C}$ & 3.31648100 & -1.43645300 & -2.3144 \\
\hline $\mathrm{C}$ & 2.62420300 & 4.28709200 & -4.42266000 \\
\hline $\mathrm{C}$ & 3.17177300 & 3.94198700 & -3.03369000 \\
\hline $\mathrm{C}$ & 3.57019400 & 2.47489500 & -2.88537000 \\
\hline $\mathrm{N}$ & 4.09507500 & 2.21705400 & $-1.531^{\prime}$ \\
\hline $\mathrm{C}$ & -2.32918900 & 1.18066500 & -5.19523100 \\
\hline $\mathrm{C}$ & -1.83788100 & 1.11563200 & -3.74308200 \\
\hline $\mathrm{C}$ & -1.77527600 & -0.33889400 & -3.25996100 \\
\hline $\mathrm{C}$ & -0.49923900 & 1.84628800 & -3.57266300 \\
\hline $\mathrm{C}$ & -7.60621900 & -3.17036400 & -4.43703600 \\
\hline $\mathrm{C}$ & -8.63845900 & -2.79997000 & -3.37036200 \\
\hline & -8.96517000 & -3.96905800 & -2.44174100 \\
\hline S & -10.18619900 & -3.58715900 & -1.13375400 \\
\hline & -9.25757300 & -2.32043300 & -0.19687600 \\
\hline $\mathrm{C}$ & -11.32149300 & -0.08150200 & -4.53702000 \\
\hline $\mathrm{C}$ & -10.69802800 & 0.46574000 & -3.27840300 \\
\hline & -9.31814900 & 0.69107300 & -3.19727400 \\
\hline C & -11.47578800 & 0.77107600 & -2.15685900 \\
\hline & -8.73779900 & 1.23930800 & -2.05652500 \\
\hline & -10.91409100 & 1.33853300 & -1.01406300 \\
\hline & -9.54674000 & 1.60356100 & -0.97405500 \\
\hline
\end{tabular}

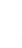

c

(c)

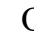

.

(

(c)

C

8

$$
\mathrm{H}
$$$$
\text { H }
$$ \\ $\mathrm{H}$}

$\mathrm{H}$

(1)

$\mathrm{H}$

\section{H}

$\begin{array}{llll}-9.03005400 & 2.24188700 & 0.13331200\end{array}$ $\begin{array}{llll}-5.76579300 & 4.67639400 & -3.47544800\end{array}$ $\begin{array}{llll}-5.10919700 & 3.99499900 & -2.27069300\end{array}$ $\begin{array}{llll}-6.05087400 & 3.41107800 & -1.24833600\end{array}$ $\begin{array}{llll}-7.17941400 & 3.86468400 & -1.03527500\end{array}$ $\begin{array}{llll}-5.53067700 & 2.40199700 & -0.58485300\end{array}$ $\begin{array}{llll}-9.46602600 & 6.98874900 & -2.41702000\end{array}$ $\begin{array}{llll}-9.94317500 & 6.26411600 & -1.16045400\end{array}$

$\begin{array}{llll}-10.57667500 & 4.91208200 & -1.48828100\end{array}$ $\begin{array}{llll}-8.87779700 & 6.12058700 & -0.22397200\end{array}$ $\begin{array}{llll}-4.26767700 & 6.27848600 & 4.32540800\end{array}$

$-4.76164700$

$-5.13974800$

$-5.70038700$

$-3.95883900$

1.08576300

2.23088200

1.88475154

7.11874200 6.29128317 $-6.79848479$

$-3.17674800$

$-0.36793800$

$-1.07827000$

$-3.16334300$

$-2.37947600$

$-2.35166200$

$-2.96569700$

$-0.89525400$

$-4.31767600$

0.01688700

1.39019900

$-4.26195200$

1.86291300

$-2.25797400$

$-0.97429500$

0.97579000

$6.90015400 \quad 3.01889400$

$5.88008000 \quad 1.92674000$ $\begin{array}{ll}5.88008000 & 1.92674000 \\ 6.60999700 & 0.70059000\end{array}$ $4.98270600 \quad 1.52798600$ $4.50017400 \quad 4.89409800$ $3.09074800 \quad 5.08489200$ $2.22509926 \quad 3.51581846$ $-2.50300500-1.21003700$ $0.11242641-1.85027824$ $\begin{array}{ll}1.74439277 & 1.63124697\end{array}$ $\begin{array}{rr}1.74439277 & 1.63124697 \\ -0.35911700 & 1.90869100\end{array}$ $0.38577600 \quad 5.88731200$ $1.54829800 \quad 5.56451700$ $1.03530500 \quad 1.41001400$ $\begin{array}{ll}1.15308700 & 0.09545000\end{array}$ $1.45854200 \quad 5.00685000$ $0.22171000 \quad 4.79139400$ $\begin{array}{ll}0.88533600 & 0.22562000\end{array}$ $\begin{array}{ll}0.13581900 & 4.12473900\end{array}$ $1.94963600 \quad 0.19411600$ $\begin{array}{ll}1.74170500 & 0.32629100\end{array}$ $-0.38999100$ $-0.70485500 \quad 2.84670400$ $\begin{array}{ll}0.43623900 & 0.56590500\end{array}$ $-0.93377000 \quad 5.16149400$ $-0.86219300 \quad 5.69219900$ $\begin{array}{lll}-5.51552900 & -0.60311300 & 2.18888400\end{array}$ $\begin{array}{llll}3.19742500 & 0.20823600 & 0.78247600\end{array}$ $\begin{array}{lll}2.27051300 & 2.78265300 & 0.29154700\end{array}$ $\begin{array}{lll}-0.63264100 & 2.51548000 & 5.76193900\end{array}$ $-2.88371100$

3.61634100 $-4.68362500$

$-1.05591800$ $2.37051800 \quad 4.74734200$ $\begin{array}{ll}1.07284100 & 0.91912000\end{array}$ $-1.26883400 \quad 0.33961800$ $-2.74291500-1.70872800$ $\begin{array}{lll}-5.06871900 & -0.32134100 & 4.77973800\end{array}$ $\begin{array}{llll}-2.53907200 & 2.16663000 & -0.28470800\end{array}$ $\begin{array}{lll}-4.08195100 & -1.75477400 & 3.11492700\end{array}$ $\begin{array}{lll}-2.71618700 & -1.91094200 & 5.03252600\end{array}$ $\begin{array}{lll}-0.32772200 & 2.96887100 & 0.04560400\end{array}$ $\begin{array}{lll}-0.42662300 & -1.75845600 & 5.96133100\end{array}$ $\begin{array}{lll}1.36364500 & -1.63451000 & 0.71742800\end{array}$ $\begin{array}{llll}-3.26704800 & -1.43681700 & 0.82144800\end{array}$ $\begin{array}{lll}1.26862900 & 1.30717900 & 6.27473100\end{array}$ $\begin{array}{llll}-2.83515900 & 0.47543000 & -0.63364300\end{array}$ $\begin{array}{llll}-4.19362300 & 1.33397600 & 1.22983100\end{array}$ $\begin{array}{lll}-2.29150000 & -0.54965900 & 2.37955200\end{array}$ $\begin{array}{llll}4.93881900 & 2.77049300 & -1.37455600\end{array}$

$\mathrm{H} \quad 2.99226000 \quad 2.58671500 \quad-0.40555500$

$\mathrm{H} \quad 4.39763100 \quad 1.24687600 \quad-1.45031600$ 


\begin{tabular}{|c|c|c|c|}
\hline $\mathrm{H}$ & .30539500 & & \\
\hline & & & \\
\hline & 4.05206800 & 4.56528100 & \\
\hline & 2.43173600 & & \\
\hline & & & \\
\hline & & & \\
\hline & & -2.31211200 & \\
\hline & .16813900 & -2.40563600 & -2.82604600 \\
\hline & & -1.442 & -1.247 \\
\hline & 1.370146 & -0.535 & -2.11 \\
\hline & & -0.490 & -4.55 \\
\hline & 0.259 & & -4.25 \\
\hline & 9700 & $2.91<$ & -3.79 \\
\hline & -0.1 & 1.748 & -2.552 \\
\hline $\mathrm{H}$ & -2.57 & & \\
\hline & -1.40 & -0.4 & -2.23 \\
\hline & -2.76 & -0.8 & -3.30 \\
\hline I & -1.10 & -0.937 & -3.890 \\
\hline & -8.24 & -1.972 & -2.770 \\
\hline $\mathrm{H}$ & -9.5 & -2.4 & -3.8 \\
\hline 1 & -8.0 & -4.3 & -1.95 \\
\hline $\mathrm{H}$ & -9.3 & -4.8 & -3.0 \\
\hline $\mathrm{H}$ & -8.2 & -2.6 & -0.0 \\
\hline$F$ & -9.2 & -1.3 & -0.69 \\
\hline $\mathrm{H}$ & -9.7 & -2.2 & \\
\hline H & -8.3 & 2.92 & $-0.1 \xi$ \\
\hline & -7.6 & 1.38 & -1.9 \\
\hline $\mathrm{H}$ & -11.5 & 300 & -0.1 \\
\hline $\mathrm{H}$ & -12.5 & 800 & -2.1 \\
\hline $\mathrm{H}$ & & & \\
\hline $\mathrm{H}$ & -5.2 & & \\
\hline $\mathrm{F}$ & -5.2( & -2.7 & -2.3 \\
\hline $\mathrm{H}$ & -2.7 & -4.2 & -1.3 \\
\hline $\mathrm{H}$ & -2.6 & -2.9 & -2.5 \\
\hline $\mathrm{H}$ & -8.5 & -6.5 & -0.8 \\
\hline $\mathrm{H}$ & -9.7 & -7.0 & \\
\hline $\mathrm{H}$ & -9.90 & -5.7 & \\
\hline & & & \\
\hline $\mathrm{H}$ & -5.4 & -3.7 & \\
\hline & -9.13 & -3.8 & \\
\hline F & -6.4 & -1.8 & 300 \\
\hline $\mathrm{H}$ & 2.62 & -3.76 & -0.33 \\
\hline & 0.9 & -3.8 & \\
\hline H & 2.14 & -4.88 & \\
\hline $\mathrm{H}$ & & $-4.22 t$ & -2.74 \\
\hline $\mathrm{H}$ & & & \\
\hline $\mathrm{H}$ & 0.32 & $-4.1 \xi$ & -2.30 \\
\hline & 2.41 & -6.13 & \\
\hline $\mathrm{H}$ & -0.5 & -5.8 & -0.59 \\
\hline $\mathrm{H}$ & & -6.67 & \\
\hline $\mathrm{H}$ & & & \\
\hline $\mathrm{H}$ & & -0.74 & \\
\hline $\mathrm{H}$ & 8.24626600 & & \\
\hline $\mathrm{H}$ & 600 & 34600 & 0.680 \\
\hline $\mathrm{H}$ & 5600 & & \\
\hline $\mathrm{H}$ & & & 0.9252 \\
\hline $\mathrm{H}$ & 4.91592200 & 2.62376100 & 2.53560800 \\
\hline & & & \\
\hline $\mathrm{H}$ & & & 48100 \\
\hline & 7.07768500 & 0.27809600 & 4.50874400 \\
\hline & & & \\
\hline $\mathrm{H}$ & 8.66440600 & -1.33241400 & 0.16870100 \\
\hline & 9.44082800 & -3.91576800 & -0.26114600 \\
\hline
\end{tabular}

$\begin{array}{llll}\mathrm{H} & 10.99904100 & -4.03051000 & 0.58043300\end{array}$

H $\quad 11.42511400 \quad-1.73871200 \quad-0.52094200$

$\mathrm{H} \quad 13.23351900 \quad 0.26469900 \quad-1.47230000$

$\mathrm{H} \quad 14.17827200 \quad-0.34711800 \quad-3.62312100$

$\mathrm{H} \quad 12.93099100 \quad-2.28406600 \quad-2.74560700$

$\begin{array}{lllll}\mathrm{H} & 12.74576200 & 0.23657400 & -4.48424200\end{array}$

H $\quad 12.52417500 \quad 1.54902100 \quad-2.49263100$

$\mathrm{H} \quad 12.35773800 \quad-2.19855500 \quad-4.41612200$

$\mathrm{H} \quad 10.30206800 \quad-1.09125900 \quad-3.75368200$

$\mathrm{H} \quad 8.59826600 \quad 2.74974800 \quad-0.64209100$

$\mathrm{H} \quad 8.63290600 \quad 6.15037600 \quad-1.14922700$

$\mathrm{H} \quad-4.50602800 \quad 4.72546300 \quad-1.71389200$

$\mathrm{H} \quad-4.41526700 \quad 3.20862600 \quad-2.58204600$

$\mathrm{H} \quad-9.87347200 \quad 4.28060700 \quad-2.04250900$

$\mathrm{H} \quad-10.84465600 \quad 4.38665500 \quad-0.56885900$

$\mathrm{H} \quad-11.47502000 \quad 5.03003500 \quad-2.10330200$

$\mathrm{H} \quad-10.68425500 \quad 6.89060500 \quad-0.64838100$

$\mathrm{H} \quad-3.39711900 \quad 5.63862500 \quad 4.13396200$

$\mathrm{H} \quad-5.63363200 \quad 7.53540700 \quad 3.21911700$

$\mathrm{H} \quad-3.98523100 \quad 7.56717700 \quad 2.61688200$

H $\quad-4.96433300 \quad 7.32194200 \quad 0.30541900$

$\mathrm{H} \quad-5.95187400 \quad 5.90912800 \quad-0.09806700$

$\mathrm{H} \quad-6.61697500 \quad 7.15740200 \quad 0.93555900$

$\mathrm{H} \quad-3.56276000 \quad 4.41721800 \quad 2.37674000$

H $\quad-4.26309400 \quad 4.25148200 \quad 0.77394500$

$\mathrm{H} \quad-3.13661100 \quad 5.57921700 \quad 1.11183600$

$\mathrm{H} \quad-5.93069100 \quad 5.22809700 \quad 2.32521200$

$\mathrm{H} \quad 1.08933700 \quad 5.06231800 \quad 5.82981900$

H $\quad \begin{array}{llll}1.41954100 & 5.15106300 & 4.08269800\end{array}$

H $\quad 2.50474273 \quad 1.32927139 \quad 3.49696922$

H $\quad \begin{array}{llll}0.83568910 & 1.92504561 & 3.46480399\end{array}$

H $\quad 2.13598546 \quad 2.85549424 \quad 2.66211707$

$\mathrm{H} \quad 4.36591400 \quad-0.83203900 \quad 4.70840600$

H $\quad 3.44061800 \quad-0.81983200 \quad 3.20569600$

$\mathrm{H} \quad 2.61729100 \quad-0.49838100 \quad 4.74906300$

H $\quad 1.65664800 \quad-4.04693900 \quad 3.66319500$

$\mathrm{H} \quad 1.01110900 \quad-2.40415800 \quad 3.82814700$

H $\quad 2.10263000 \quad-2.79286800 \quad 2.49677600$

$\mathrm{H} \quad 3.98203500 \quad-3.13879800 \quad 4.08362500$

H $\quad 2.58809500 \quad-4.04296900 \quad 5.92507000$

$\mathrm{H} \quad 1.98514400 \quad-2.42441700 \quad 6.26209300$

$\mathrm{H} \quad 6.83179600 \quad-1.60652000 \quad-1.47862400$

$\mathrm{H} \quad 7.82617600 \quad-2.77723500 \quad-1.81976400$

H $\quad \begin{array}{lllll}7.25829088 & 0.22841513 & -1.90842203\end{array}$

H $\quad \begin{array}{lllll}5.96005795 & 0.00255247 & -2.75245473\end{array}$

$\mathrm{H} \quad-6.42707500 \quad 0.91094900 \quad 1.88904000$

$\mathrm{H} \quad-5.44334300 \quad-0.93696700 \quad 1.23785700$

H $\quad 7.98154000 \quad 3.94002900 \quad-2.95775700$

$\mathrm{H} \quad 5.93964800 \quad 5.18138800 \quad 0.99255300$

$\mathrm{H} \quad-6.53276700 \quad 5.38694400 \quad-3.16218200$

$\mathrm{H} \quad-8.69078900 \quad 6.40286200 \quad-2.92509700$

$\mathrm{H} \quad-8.24486200 \quad 5.48781100 \quad-0.59822300$

$\mathrm{H} \quad 4.26125900 \quad-1.65233000 \quad 6.86816800$

$\mathrm{H} \quad 11.46261800 \quad 1.99322100 \quad-0.59123800$

$\mathrm{H} \quad 4.51310700 \quad-3.01045300 \quad 2.07906800$

H $\quad 0.98979600 \quad-8.41676900 \quad-1.20217600$

$\mathrm{H} \quad 3.05590600 \quad-2.08051300 \quad-7.12343200$

$\mathrm{H} \quad 3.00753500 \quad-0.32467600 \quad-6.97970400$

$\mathrm{H} \quad-1.73328000 \quad 0.54362700 \quad-5.85701800$

$\mathrm{H} \quad-6.77070800 \quad-3.71330600 \quad-3.97664400$

$\mathrm{H} \quad-8.54194400 \quad-9.01238100 \quad-0.21151500$

H $\quad-4.48582300 \quad-4.44185200 \quad-3.87738400$

$\mathrm{H} \quad-12.40253300 \quad-0.19694300 \quad-4.42275800$ 


$\begin{array}{cccc}\mathrm{H} & -10.91287600 & -1.06516400 & -4.79378000 \\ \mathrm{H} & -7.69810400 & 1.66765500 & 1.30729800 \\ \mathrm{H} & -4.96605300 & 5.21443800 & -3.99441000 \\ \mathrm{H} & -6.21199300 & 3.93952600 & -4.15091900 \\ \mathrm{H} & -2.82570500 & -4.92568600 & -4.05816700 \\ \mathrm{H} & -3.84898600 & -5.81597500 & -2.99079400 \\ \mathrm{H} & -7.16747400 & -2.28401000 & -4.90704500 \\ \mathrm{H} & -7.98518900 & -3.81376100 & -5.23822700 \\ \mathrm{H} & -11.14720100 & 0.56957100 & -5.39915000 \\ \mathrm{H} & -2.30498500 & 2.20482200 & -5.58012200 \\ \mathrm{H} & -3.38359800 & 0.89403400 & -5.27031300 \\ \mathrm{H} & 1.51849700 & -1.25567800 & -6.82765600 \\ \mathrm{H} & 2.43940900 & 5.36224500 & -4.50500900 \\ \mathrm{H} & 7.46941300 & 6.09373600 & 0.98060500 \\ \mathrm{H} & 6.14948300 & 6.56375000 & -0.10166500 \\ \mathrm{H} & 9.22071200 & 5.18068300 & -3.22169000 \\ \mathrm{H} & 10.15964200 & 1.28992200 & 0.38662000 \\ \mathrm{H} & 5.62116500 & -4.32205200 & 2.51568600 \\ \mathrm{H} & 5.18639400 & -4.05714700 & 0.81489400 \\ \mathrm{H} & 4.95722600 & -3.20506700 & 6.47356300 \\ \mathrm{H} & 3.70645300 & -2.99376100 & 7.84186200 \\ \mathrm{H} & -0.77503100 & -8.18307900 & -1.13419800 \\ \mathrm{H} & 0.13443000 & -7.49186500 & -2.44951000 \\ \mathrm{H} & -7.92845100 & -8.61381700 & 1.38510900 \\ \mathrm{H} & -6.95943800 & -8.28898100 & -0.09836800 \\ \mathrm{H} & -9.06008200 & 7.98108300 & -2.19189000 \\ \mathrm{H} & -10.26722500 & 7.17889000 & -3.14007300 \\ \mathrm{H} & -3.91743000 & 7.02489700 & 5.04595800 \\ \mathrm{H} & -5.02510200 & 5.62782900 & 4.77517100 \\ \mathrm{H} & 0.07117300 & 4.15441700 & 4.68285400 \\ \mathrm{H} & -6.08281300 & 2.13655300 & 0.23754300\end{array}$

\section{$\mathrm{TS3}_{S}(+13.6 \mathrm{kcal} / \mathrm{mol})$}

$\begin{array}{cccc}\mathrm{C} & 6.64102900 & 5.62098900 & 1.54812500 \\ \mathrm{C} & 7.14634800 & 4.93900500 & 0.31626100 \\ \mathrm{O} & 6.68180100 & 3.88985700 & -0.14556400 \\ \mathrm{~N} & 8.18683200 & 5.55927100 & -0.28576300 \\ \mathrm{C} & 8.75551100 & 5.02882700 & -1.49739600 \\ \mathrm{C} & 9.81839300 & 3.94972000 & -1.29359400 \\ \mathrm{O} & 10.84170000 & 3.90597400 & -1.96978800 \\ \mathrm{~N} & 9.52152500 & 3.03800500 & -0.32407300 \\ \mathrm{C} & 10.37384000 & 1.87504700 & -0.21169800 \\ \mathrm{C} & 10.13206000 & 0.91604600 & -1.36911800 \\ \mathrm{O} & 8.98233500 & 0.52076000 & -1.61505800 \\ \mathrm{~N} & 11.19128600 & 0.43446100 & -2.05049300 \\ \mathrm{C} & 10.95717500 & -0.61201300 & -3.05967700 \\ \mathrm{C} & 10.31015700 & -1.85270100 & -2.43670400 \\ \mathrm{O} & 9.49292500 & -2.52405000 & -3.06387900 \\ \mathrm{C} & 12.36164000 & -0.85263600 & -3.64179000 \\ \mathrm{C} & 13.04023700 & 0.51834200 & -3.49915500 \\ \mathrm{C} & 12.54212700 & 1.03045100 & -2.14081500 \\ \mathrm{~N} & 10.73924700 & -2.14133300 & -1.18499100 \\ \mathrm{C} & 10.19651900 & -3.19584300 & -0.35054000 \\ \mathrm{C} & 9.60341600 & -2.69278100 & 0.97105400 \\ \mathrm{O} & 9.73247000 & -3.34912900 & 1.99285200 \\ \mathrm{~N} & 8.92171200 & -1.50408200 & 0.91593400 \\ \mathrm{C} & 7.99895100 & -1.19227000 & 2.01468400 \\ \mathrm{C} & 6.89233800 & -2.25516600 & 2.16655400 \\ \mathrm{O} & 6.40591100 & -2.51397200 & 3.26859300 \\ \mathrm{C} & 7.38755800 & 0.20323900 & 1.79219600\end{array}$

\begin{tabular}{|c|c|c|c|}
\hline & 6.26142900 & 0.59915500 & \\
\hline & 6.69399000 & 0.51868700 & \\
\hline & & & \\
\hline & 6.46952100 & -2.80322600 & \\
\hline & 5.36508400 & & \\
\hline & 4.03930600 & -3.97724100 & 6.13046100 \\
\hline & 2.85795500 & -4.05559300 & \\
\hline & & -3.33484800 & \\
\hline & 1.88690700 & -3.64151600 & 36950300 \\
\hline & & & \\
\hline & & -7.11688600 & \\
\hline & 0.31885200 & & \\
\hline & & -5.07383300 & \\
\hline & & & \\
\hline & & & \\
\hline & -8.02231000 & & \\
\hline & -8.5 & -6.5 & \\
\hline & & & \\
\hline & & & \\
\hline & & & \\
\hline & -5.7 & & \\
\hline$C$ & & & \\
\hline & -6.3 & & \\
\hline & -5.7 & & \\
\hline & -3.6 & & \\
\hline & -3.4 & & \\
\hline & & & \\
\hline & & & \\
\hline & & & \\
\hline 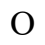 & & -1.2 & \\
\hline & & & \\
\hline & & & \\
\hline & & & \\
\hline & & & 7300 \\
\hline & & & \\
\hline & & & \\
\hline 0 & & & \\
\hline & & & \\
\hline & & & \\
\hline & & & \\
\hline N & & & \\
\hline & & & \\
\hline & & & \\
\hline $\mathrm{C}$ & -1.8 & & \\
\hline C & & & \\
\hline & & & \\
\hline$C$ & & & \\
\hline $\mathrm{C}$ & -9.0 & & -3 \\
\hline$S$ & & & \\
\hline$C$ & & & \\
\hline $\mathrm{C}$ & & & \\
\hline $\mathrm{C}$ & & & \\
\hline & & & \\
\hline & & & \\
\hline $\mathrm{C}$ & & & \\
\hline $\mathrm{C}$ & & 1.40208500 & \\
\hline & & & \\
\hline & & & \\
\hline C & & 5.44231400 & -2.32767200 \\
\hline $\mathrm{C}$ & & & \\
\hline 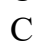 & & & \\
\hline 0 & & & -0.0632000 \\
\hline & -5.37481600 & 2.35920800 & -0.5194180 \\
\hline
\end{tabular}




\begin{tabular}{|c|c|c|c|}
\hline & .49221500 & 1800 & \\
\hline & -9.91267600 & 6.42776100 & \\
\hline & -10.60693400 & & \\
\hline & -8.79508500 & 5.97823500 & 0.99815300 \\
\hline & -4.25117500 & 5.37410800 & \\
\hline & -4.77564900 & 6.36647200 & 4.57756600 \\
\hline & -5.59493200 & & \\
\hline & -5.91405500 & & 2.39678100 \\
\hline & & & \\
\hline & 1.10007900 & 3.50302400 & 5.76881900 \\
\hline & & & \\
\hline & & 1.51373500 & \\
\hline & & & \\
\hline & 6.24641600 & & -1.78856500 \\
\hline & & & \\
\hline & 1000 & & \\
\hline & & & \\
\hline & & & \\
\hline & & & \\
\hline & & & \\
\hline & & & \\
\hline & -2.9 & & \\
\hline & & & \\
\hline & & & \\
\hline & & & \\
\hline & & & \\
\hline & & & \\
\hline & & & \\
\hline & -1.6 & -1.7 & \\
\hline & & & \\
\hline & & -0.8 & \\
\hline & & & \\
\hline & & & \\
\hline & & & \\
\hline & & & \\
\hline 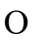 & & & \\
\hline & & & \\
\hline & & & \\
\hline & & & \\
\hline $\mathrm{H}$ & & & \\
\hline & & & \\
\hline & & & \\
\hline & & & \\
\hline $\mathrm{H}$ & & & \\
\hline & & & \\
\hline & & & \\
\hline $\mathrm{H}$ & & & 1300 \\
\hline & & & \\
\hline & & & \\
\hline & & & \\
\hline $\mathrm{H}$ & & -4.81 & \\
\hline & & & \\
\hline & & & \\
\hline H & & & \\
\hline $\mathrm{H}$ & & & -0.77580900 \\
\hline & & & \\
\hline & & & \\
\hline $\mathrm{H}$ & & 5800 & -3.14757700 \\
\hline & & & \\
\hline & & & \\
\hline & & & \\
\hline $\mathrm{H}$ & 1.63329500 & 4.77099200 & -3.69722300 \\
\hline & 3.27936800 & 5.13475400 & 122112200 \\
\hline
\end{tabular}

$\begin{array}{llll}5.09581300 & -1.67314300 & -3.26355600\end{array}$

$\mathrm{H} \quad 3.44465800 \quad-1.08451300 \quad-1.50703900$

$\mathrm{H} \quad 1.29772500 \quad-0.01020300 \quad-2.15192400$

$\mathrm{H} \quad 0.86764100 \quad 0.56872100 \quad-4.51849600$

$\mathrm{H} \quad 0.19048100 \quad 2.40870200 \quad-3.75676300$

$\mathrm{H} \quad-0.67430000 \quad 3.75445600 \quad-3.01287500$

$\mathrm{H} \quad-0.21073900 \quad 2.35017400 \quad-2.03585700$

$\mathrm{H} \quad-2.66290100 \quad 2.37926000 \quad-2.64549800$

H $\quad-1.53746800 \quad 0.18233800 \quad-2.19510400$

$\mathrm{H} \quad-2.86347800 \quad 0.03761800 \quad-3.35370000$

$\mathrm{H} \quad-1.19117300 \quad 0.01770000 \quad-3.92013800$

$\mathrm{H} \quad-8.25183800 \quad-1.24424000 \quad-2.98501100$

$\mathrm{H} \quad-9.60895100 \quad-1.41809900 \quad-4.07711800$

$\mathrm{H} \quad-8.09386600 \quad-3.74063700 \quad-2.76475000$

$\mathrm{H} \quad-9.49858000 \quad-3.91610200 \quad-3.82343400$

H $\quad-8.12588300 \quad-2.47195300 \quad-0.52009200$

$\mathrm{H} \quad-9.18347700 \quad-1.06627100 \quad-0.84646400$

H $\quad-9.59236600 \quad-2.22095400 \quad 0.44237700$

$\mathrm{H} \quad-8.08523000 \quad 2.60524500 \quad 0.52109400$

$\mathrm{H} \quad-7.53494900 \quad 1.74359200 \quad-1.76567900$

H $\quad-11.23662100 \quad 1.39387700 \quad 0.36244900$

$\mathrm{H} \quad-12.42964900 \quad 1.02209500 \quad-1.78957100$

$\mathrm{H} \quad-8.72667000 \quad 1.45515000 \quad-3.90555700$

$\mathrm{H} \quad-5.51190600 \quad-3.19523600 \quad-2.26872000$

$\mathrm{H} \quad-5.07301500 \quad-1.72605000 \quad-3.09958500$

$\mathrm{H} \quad-3.01439400 \quad-3.77421100 \quad-2.08897800$

$\mathrm{H} \quad-2.65797000 \quad-2.33451800 \quad-3.00806900$

$\mathrm{H} \quad-8.67887500 \quad-6.10365800 \quad-2.04960800$

$\mathrm{H} \quad-9.61357000 \quad-6.66012200 \quad-0.68727200$

$\mathrm{H} \quad-9.38996100 \quad-5.43479900 \quad 1.26034600$

$\mathrm{H} \quad-5.97542200 \quad-5.71432400 \quad-1.31769000$

$\mathrm{H} \quad-4.73506300 \quad-4.17349200 \quad 0.15451900$

$\mathrm{H} \quad-8.14768400 \quad-3.86694200 \quad 2.72627600$

H $\quad-4.90237800 \quad-2.68820500 \quad 2.01255200$

$\mathrm{H} \quad 2.65001400 \quad-3.56572100 \quad-1.05843100$

$\mathrm{H} \quad 0.99439800 \quad-3.77590900 \quad-0.47122100$

$\mathrm{H} \quad 2.22339800 \quad-4.98125200 \quad-0.07710100$

$\mathrm{H} \quad 2.00353900 \quad-3.44737600 \quad-3.49758400$

$\mathrm{H} \quad 0.86395900 \quad-4.61215900 \quad-4.18661200$

$\mathrm{H} \quad 0.31675300 \quad-3.44376000 \quad-2.97535800$

$\mathrm{H} \quad 2.35407200 \quad-5.67534400 \quad-2.44467900$

$\mathrm{H} \quad-0.59807700 \quad-5.44047700 \quad-1.73197900$

$\mathrm{H} \quad 0.48451600 \quad-6.52095600 \quad-0.87281700$

$\mathrm{H} \quad 6.86430900 \quad-2.54026800 \quad 0.11193800$

$\mathrm{H} \quad 8.56459600 \quad-1.22485900 \quad 2.94632900$

$\mathrm{H} \quad 8.19549100 \quad 0.94611800 \quad 1.86480400$

$\mathrm{H} \quad \begin{array}{llll}6.99785000 & 0.25644500 & 0.76776200\end{array}$

$\begin{array}{llll}\mathrm{H} & 6.54816200 & 2.74435500 & 2.61843400\end{array}$

$\mathrm{H} \quad 5.48187700 \quad 2.10701000 \quad 1.37432500$

$\mathrm{H} \quad 4.89215600 \quad 2.28019200 \quad 3.03455700$

$\mathrm{H} \quad 7.57715100 \quad 1.14559500 \quad 4.41465400$

H $\quad 5.89258500 \quad 0.88270800 \quad 4.88555100$

$\mathrm{H} \quad 6.91926000 \quad-0.51008700 \quad 4.51855400$

H $\quad 5.43515900 \quad-0.10724500 \quad 2.62317100$

$\mathrm{H} \quad 8.65757000 \quad-1.17324100 \quad-0.00720300$

$\mathrm{H} \quad 9.41153200 \quad-3.69504900 \quad-0.92560800$

H $\quad 10.95990800 \quad-3.93469900 \quad-0.09508000$

$\mathrm{H} \quad 11.36601700 \quad-1.48032400 \quad-0.75022900$

H $\quad 13.17843600 \quad 0.66890400 \quad-1.32349900$

$\mathrm{H} \quad 14.12997500 \quad 0.46418600 \quad-3.54620100$

$\mathrm{H} \quad 12.88798100 \quad-1.60315300 \quad-3.04200500$

H $\quad 12.69883300 \quad 1.19243000 \quad-4.29116200$ 


\begin{tabular}{|c|c|c|c|}
\hline $\mathrm{H}$ & 12.46750300 & & \\
\hline & & -1.21564000 & \\
\hline & 0.25596300 & -0.25613400 & -3.81915700 \\
\hline & .54565400 & & \\
\hline & & & \\
\hline & & & \\
\hline & & & \\
\hline & -9.94690900 & 4.78322100 & 739200 \\
\hline & -10.85442500 & & 762000 \\
\hline & -11.52575300 & & -0 \\
\hline & -10.59592500 & & 8600 \\
\hline & -3.40241500 & & \\
\hline & 702600 & & \\
\hline & & & \\
\hline & -4.9 & & \\
\hline & -6.6 & 6.5 & \\
\hline & -6.3 & & \\
\hline & -4.69 & $3.7^{\prime}$ & \\
\hline & -5.52 & & \\
\hline & -3.9 & & \\
\hline & -6.5 & & \\
\hline & 0.80 & & \\
\hline & 0.22 & & \\
\hline & 2.58 & 1.2 & \\
\hline & & & \\
\hline & 1.20 & & \\
\hline & 4.12 & -1.5 & \\
\hline & & & \\
\hline & 2.3 & & \\
\hline & & & \\
\hline & 0.9 & & \\
\hline & 2.0 & -3.1 & \\
\hline & $3.9^{\circ}$ & -3.7 & \\
\hline & & & \\
\hline & & -3.6 & \\
\hline 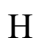 & & & \\
\hline & & -2.3 & \\
\hline & & & \\
\hline f & & & \\
\hline & -2.57 & -2.3 & \\
\hline I & -6.0 & -0.2 & \\
\hline & & & \\
\hline & 5.92 & & \\
\hline I & -6.57 & & \\
\hline & -8.71 & & \\
\hline & & & \\
\hline $\mathrm{H}$ & & -2.93 & \\
\hline & 11.4 & & \\
\hline $\mathrm{H}$ & 4.45 & -3.2 & 8300 \\
\hline & & -7.85 & \\
\hline F & & -0.4 & \\
\hline $\mathrm{H}$ & 2.92208600 & & -6.8 \\
\hline & -1.80 & & \\
\hline $\mathrm{H}$ & -6.83892000 & 220000 & 36700 \\
\hline $\mathrm{H}$ & -8.65455700 & -8.5 & 732800 \\
\hline $\mathrm{H}$ & -4.560 & -3.4 & -4.6 \\
\hline $\mathrm{H}$ & -12.46703300 & 0.93774100 & 938600 \\
\hline $\mathrm{H}$ & -11.05 & 0.10089300 & \\
\hline $\mathrm{H}$ & & & \\
\hline $\mathrm{H}$ & -5.00825100 & 6.07367000 & -2.72906700 \\
\hline $\mathrm{H}$ & -6.25949000 & & -3.13949800 \\
\hline $\mathrm{H}$ & -2.90714800 & -3.83278500 & -4.92847900 \\
\hline & -3.92539200 & -4.92467300 & -4.06312400 \\
\hline
\end{tabular}

$\begin{array}{cccc}\mathrm{H} & -7.24371400 & -1.06268000 & -5.17332100 \\ \mathrm{H} & -8.06929100 & -2.48728100 & -5.81117100 \\ \mathrm{H} & -11.21565300 & 1.83933600 & -5.02897200 \\ \mathrm{H} & -2.36864500 & 3.45708400 & -4.92853500 \\ \mathrm{H} & -3.45080500 & 2.11332900 & -4.89144900 \\ \mathrm{H} & 1.43293800 & 0.32542700 & -6.90000000 \\ \mathrm{H} & 2.39349200 & 6.30952000 & -3.25148700 \\ \mathrm{H} & 7.46349900 & 5.86590000 & 2.22834300 \\ \mathrm{H} & 6.13612100 & 6.55606200 & 1.28345000 \\ \mathrm{H} & 9.18315800 & 5.84850200 & -2.08433700 \\ \mathrm{H} & 10.11380600 & 1.32692600 & 0.69813000 \\ \mathrm{H} & 5.58687300 & -4.63768800 & 1.56387400 \\ \mathrm{H} & 5.18093600 & -4.01068600 & -0.04816500 \\ \mathrm{H} & 4.95469300 & -4.36911300 & 5.67405200 \\ \mathrm{H} & 3.71348100 & -4.44620800 & 7.06572100 \\ \mathrm{H} & -0.84753900 & -7.63511000 & -2.76478300 \\ \mathrm{H} & 0.05481600 & -6.68514700 & -3.91306400 \\ \mathrm{H} & -7.98579700 & -8.56660700 & -0.33861900 \\ \mathrm{H} & -7.02566900 & -7.94133200 & -1.72868400 \\ \mathrm{H} & -9.07966200 & 8.41159900 & -0.35755700 \\ \mathrm{H} & -10.29611100 & 7.82791600 & -1.44406600 \\ \mathrm{H} & -3.89191900 & 5.95095900 & 6.48239000 \\ \mathrm{H} & -5.00695400 & 4.64391700 & 5.93364500 \\ \mathrm{H} & 1.88055600 & 3.26992600 & 6.49890300 \\ \mathrm{H} & -5.82955900 & 1.69144200 & 0.12680300 \\ & & & \end{array}$

\section{Int5 $S(-1.8 \mathrm{kcal} / \mathrm{mol})$}

$\begin{array}{lccc}\mathrm{C} & 6.62349100 & 5.52818200 & 1.82570700 \\ \mathrm{C} & 7.12158100 & 4.91061600 & 0.55778300 \\ \mathrm{O} & 6.65711600 & 3.88343300 & 0.04801100 \\ \mathrm{~N} & 8.15524600 & 5.56204700 & -0.02099100 \\ \mathrm{C} & 8.71721400 & 5.08999100 & -1.25989600 \\ \mathrm{C} & 9.78168700 & 4.00317000 & -1.11153400 \\ \mathrm{O} & 10.80585800 & 3.99594000 & -1.78767700 \\ \mathrm{~N} & 9.48468400 & 3.04305900 & -0.19010300 \\ \mathrm{C} & 10.33914800 & 1.87802500 & -0.12994800 \\ \mathrm{C} & 10.09753400 & 0.97110200 & -1.32870800 \\ \mathrm{O} & 8.94875400 & 0.57879700 & -1.58590600 \\ \mathrm{~N} & 11.15583200 & 0.53248700 & -2.03885500 \\ \mathrm{C} & 10.92582100 & -0.46279000 & -3.09976000 \\ \mathrm{C} & 10.28510900 & -1.73649100 & -2.54005000 \\ \mathrm{O} & 9.46725000 & -2.37658600 & -3.19824600 \\ \mathrm{C} & 12.33072600 & -0.66614600 & -3.69515600 \\ \mathrm{C} & 13.00379800 & 0.69858000 & -3.48133400 \\ \mathrm{C} & 12.50489400 & 1.13669700 & -2.09820500 \\ \mathrm{~N} & 10.71911800 & -2.08895400 & -1.30637900 \\ \mathrm{C} & 10.17836400 & -3.18544800 & -0.52648900 \\ \mathrm{C} & 9.58183100 & -2.75276600 & 0.81799900 \\ \mathrm{O} & 9.70562700 & -3.46425200 & 1.80293000 \\ \mathrm{~N} & 8.90255100 & -1.56160500 & 0.82629300 \\ \mathrm{C} & 7.98436600 & -1.30452000 & 1.94250600 \\ \mathrm{C} & 6.86906600 & -2.36373000 & 2.03962700 \\ \mathrm{O} & 6.37416700 & -2.66222900 & 3.12801200 \\ \mathrm{C} & 7.38425100 & 0.10618300 & 1.80275900 \\ \mathrm{C} & 6.26805000 & 0.45962300 & 2.80405700 \\ \mathrm{C} & 6.70547900 & 0.29162200 & 4.26320700 \\ \mathrm{C} & 5.78414600 & 1.89005200 & 2.54648400 \\ \mathrm{~N} & 6.44634700 & -2.86082500 & 0.86409000 \\ \mathrm{C} & 5.33777600 & -3.78362100 & 0.80544400 \\ \mathrm{C} & 4.04754600 & -4.28789700 & 5.93574300\end{array}$




\begin{tabular}{|c|c|c|c|}
\hline & & -4.33696900 & \\
\hline $\mathrm{C}$ & & & \\
\hline & & & \\
\hline & 3.07512600 & -1.99068600 & 95340400 \\
\hline & & -6.96635800 & \\
\hline & & -5.94620600 & \\
\hline & 1.39762600 & -4.93136900 & 7200 \\
\hline & & & \\
\hline & 84703300 & -4.27626700 & 800 \\
\hline C & 300 & -7.94011600 & -1.5 \\
\hline & 500 & -6.54325 & 900 \\
\hline & & & \\
\hline & & 3100 & \\
\hline & 200 & -5.1 & 700 \\
\hline & & 600 & \\
\hline & -7.26340 & -4.3 & 200 \\
\hline & -5.9778 & -4.0 & \\
\hline & & -3.2 & \\
\hline & -3.75 & -3.6 & 700 \\
\hline & 00 & -3.0 & \\
\hline $\mathrm{C}$ & -4.61 & & \\
\hline & -4.54 & -1.6 & -0.9 \\
\hline & & & \\
\hline & -5.42 & -0.7 & -0.7 \\
\hline & & 0.60 & \\
\hline $\mathrm{C}$ & & & \\
\hline$C$ & 1.775 & & \\
\hline $\mathrm{C}$ & & -0.5 & \\
\hline & & & \\
\hline & & -0.9 & -3.6 \\
\hline $\mathrm{C}$ & & & \\
\hline & & & \\
\hline & & & \\
\hline & & & \\
\hline & & & \\
\hline & & & \\
\hline & & & \\
\hline & & & \\
\hline & & & -2.8 \\
\hline & -7.74 & -1.7 & \\
\hline & -8.73 & & \\
\hline & & -3.0 & \\
\hline & 600 & -2.9 & \\
\hline & & -1.9 & \\
\hline & -11 . & & \\
\hline & & 5900 & \\
\hline & & & -2.780 \\
\hline & -11.428 & 100 & \\
\hline & & & \\
\hline & -10.77 & 300 & 00 \\
\hline & & & -0.3 \\
\hline 0 & & & \\
\hline & -5.85102900 & 5.54922400 & -1.970 \\
\hline & & & \\
\hline $\mathrm{C}$ & -5.9583 & 3.6601 & -0.21006100 \\
\hline $\mathrm{O}$ & -7.070 & 3.94214200 & \\
\hline $\mathrm{O}$ & & 2.50603400 & -0.03067500 \\
\hline C & -9.52387300 & 7.51698400 & -0.29883100 \\
\hline $\mathrm{C}$ & & & \\
\hline $\mathrm{C}$ & -10.54956900 & 5.22145100 & 0.01957600 \\
\hline & -8.82054700 & 6.06052600 & 1.51224500 \\
\hline & & 5.07884500 & 5.95643800 \\
\hline $\mathrm{C}$ & -4.73987000 & 6.02259300 & 4.86101800 \\
\hline
\end{tabular}

\begin{tabular}{|c|c|c|c|}
\hline & -5.15128500 & 415700 & \\
\hline & & & \\
\hline & -4.00018500 & 4.51673200 & \\
\hline & 1.10937600 & 3.20143600 & 97107700 \\
\hline & 1.72233500 & & \\
\hline & 2.16157700 & 1.32216600 & \\
\hline & 7.02037100 & -2.11696700 & 092400 \\
\hline & 6.20853500 & 0.56625300 & -1.73 \\
\hline & -5.50251500 & 1.15889700 & \\
\hline & -2.91657800 & 280800 & \\
\hline & -2.33194900 & 15300 & 300 \\
\hline & -1.09334700 & -3.325 & \\
\hline & -3.01922800 & 77300 & 700 \\
\hline & -2.28344100 & 57300 & \\
\hline & -0.91164 & 4500 & \\
\hline & 692200 & -3.0 & \\
\hline & 875500 & & \\
\hline & -1.75059100 & -2.521 & \\
\hline & 0.16819000 & 1.867 & \\
\hline & & & \\
\hline & -2.01645000 & -1.0 & \\
\hline & 1.85360700 & & \\
\hline & -3.1 & & \\
\hline & -3.3 & -3.9 & \\
\hline & 2500 & & \\
\hline & -0.4 & -0.3 & \\
\hline & -2.4 & -4.2 & \\
\hline & -6.3 & & \\
\hline & 83500 & -0.2 & \\
\hline & & & \\
\hline $\mathrm{H}$ & -0.2 & -3.2 & \\
\hline & 0.0 & -2.5 & \\
\hline & 3.6 & & \\
\hline & & & \\
\hline & -1.19 & -1.0 & 300 \\
\hline & -2.56 & & 300 \\
\hline & -0.70901800 & -2.6 & 600 \\
\hline & & & \\
\hline & & -0.4 & \\
\hline & -4.00164500 & -3.5 & \\
\hline & -0.0 & 000 & \\
\hline & -4.33994600 & & \\
\hline & 1.17474600 & -1.8 & \\
\hline & -3.393968 & -1.0 & \\
\hline & -3.35298200 & -4.58 & \\
\hline & & & \\
\hline & -4.07428000 & & \\
\hline & -7.36313200 & & \\
\hline & 4.88158100 & 4800 & -0.6 \\
\hline & 3.09743300 & & \\
\hline & & & \\
\hline & & & \\
\hline & 2.57009200 & & \\
\hline & 4.00357700 & 2900 & 5500 \\
\hline & 2.38881600 & 01600 & \\
\hline & & & \\
\hline & 3.21643300 & 5.33354500 & -3.95318600 \\
\hline & 4.62773300 & -0.75500900 & 45700 \\
\hline & & & \\
\hline & 3.48522500 & -1.03003400 & -1.57172800 \\
\hline & & & \\
\hline $\mathrm{H}$ & 0.82487900 & 0.74905000 & -4.43562800 \\
\hline $\mathrm{H}$ & 0.14980700 & 2.55951400 & -3.67683700 \\
\hline
\end{tabular}




\begin{tabular}{|c|c|c|c|}
\hline & -0.66464600 & & \\
\hline & -0.17912000 & & \\
\hline & & & -2.45617700 \\
\hline & -1.49847000 & .25135300 & -2.21926700 \\
\hline & -2.96094200 & & -3.23003500 \\
\hline & -1.35418400 & 0.18729700 & -3.97578100 \\
\hline & -8.31658400 & -1.02037200 & -2.98463800 \\
\hline & -9.68402500 & -1.24780100 & \\
\hline & -8.06215000 & -3.53354 & 8400 \\
\hline & -9.51707500 & -3.7276 & 7800 \\
\hline & -7.99730100 & -2.3765 & -0. \\
\hline & -9.00269100 & -0.9283 & \\
\hline & -9.43502000 & -2.0497 & \\
\hline & -8.17608200 & 2.699 & \\
\hline & -7.60172800 & & \\
\hline & -11.31953400 & & 400 \\
\hline & -12.50101700 & 1.1692 & 100 \\
\hline & -8.7729 & & \\
\hline & -5.469 & -2.9630 & 000 \\
\hline & -4.919819 & -1.4757 & \\
\hline & -3.028 & & \\
\hline & -2.5773 & -2.2805 & -3. \\
\hline & & & \\
\hline & -9.616 & -6.625 & \\
\hline & -9.120 & -5.370 & \\
\hline & -6.0 & -5.8 & \\
\hline & -4.535 & -4.455 & \\
\hline & -7.588 & & \\
\hline & -4.5 & -2.86 & \\
\hline & 2.5 & -3.488 & \\
\hline & 0.9 & -3.8 & \\
\hline & & -5.008 & \\
\hline & 1.790 & -3.180 & \\
\hline & 0.62 & -4.3 & \\
\hline & 0.14 & -3.26 & \\
\hline & & & \\
\hline & -0.6 & & \\
\hline & & -6.4 & \\
\hline & 000 & -2.5 & -0 . \\
\hline & & -1.3 & \\
\hline & & & \\
\hline & & & \\
\hline & & & \\
\hline & & & \\
\hline & & & \\
\hline & & & \\
\hline & 5.91 & & \\
\hline & & -0.7551 & \\
\hline & & -0.2289 & \\
\hline & & -1.1730 & -0.0 \\
\hline & & -3.6568 & \\
\hline & & & \\
\hline & 11.34601000 & -1.45052800 & -0.8 \\
\hline & & & \\
\hline & 14.09369200 & 0.65139000 & -3.5318 \\
\hline & 12.86085700 & -1.44506200 & 7900 \\
\hline & 12.65905100 & 1.41166800 & -4.2368 \\
\hline & 12.42707100 & 2.21952100 & -1.99196900 \\
\hline & & -0.97485200 & \\
\hline & 10.22168700 & -0.07226300 & -3.83923100 \\
\hline & 8.50953400 & 2.93723700 & 0.07518000 \\
\hline & & 6.34295500 & 0.45079200 \\
\hline & -4.59689200 & 5.23806700 & -0.24657400 \\
\hline
\end{tabular}$$
\mathrm{H}
$$

\section{$\mathrm{H}$}

$\mathrm{H}$

$\mathrm{H}$

$\mathrm{H}$

H

$\mathrm{H}$

\section{$\mathrm{H}$}

$\mathrm{H}$
$\mathrm{H}$

\section{$\mathrm{H}$}

H

$$
\text { H }
$$$$
\mathrm{H}
$$

\section{$\mathrm{H}$}

$\mathrm{H}$

\section{$\mathrm{H}$
$\mathrm{H}$}

$\mathrm{H}$

$$
\mathrm{H}
$$

\section{$\mathrm{H}$}

\section{$\mathrm{H}$
$\mathrm{H}$}

$-4.33917400$

$-9.84602900$

$-10.78564400$

$-11.46477100$

$-10.66222200$

$-3.37715400$

$-5.59643800$

$-3.95377500$

$-4.91753400$

$-5.98556600$

$-6.56954700$

$-3.63840200$

$-4.31925600$

$-3.14941400$

$-5.96612700$

0.79130800

0.25221800

2.62659200

2.87115000

1.27556600

3.88034000

3.19715500

2.12219300

2.18475800

1.05022800

2.16592600

4.10343100

2.71793700

1.95391700

6.74767100

7.75676600

7.12542000

5.89232300

$-5.08261600$

$-6.12516300$

7.90075300

5.91574000

$-6.60999100$

$-8.75501900$

$-8.24087900$

4.25625200

11.37686000

4.43815900

0.85679000

2.88734300

2.84004200

$-1.87488000$

$-6.90063600$

$-8.70051000$

$-4.62657400$

$-12.52548300$

$-11.09900600$

$-5.69205800$

$-5.05382900$

$-6.30858500$

$-2.97353200$

$-3.98662000$

$-7.31008100$

$-8.14077700$

$-11.27913500$

$-2.43063300$

$-3.51329600$

H 1.35579900
$4.05906900-1.51713300$ $4.78743400-0.70069100$ $\begin{array}{lll}4.45003200 & 0.75693400\end{array}$

$5.48153300-0.52223400$ $6.86393800 \quad 1.40941300$

$4.50774700 \quad 5.59574200$

$6.60065000 \quad 5.22985600$

$6.75611900 \quad 4.63096500$

$7.10207400 \quad 2.31675200$

$5.87401000 \quad 1.61233000$

$6.87049600 \quad 2.92831000$

$3.73074400 \quad 3.60008000$

$4.02923300 \quad 2.00435300$

$5.16815600 \quad 2.69318600$

$4.62279600 \quad 3.78055500$

$4.23267400 \quad 6.13894300$

$2.54083800 \quad 6.13041100$

$1.12363900 \quad 3.27847300$

$1.08563300 \quad 5.03828200$

$0.69018400 \quad 4.34706400$

$\begin{array}{ll}-1.69550100 & 4.63080800\end{array}$

$-1.42472400 \quad 3.02578600$

$\begin{array}{ll}1.68802100 & 4.40798100\end{array}$

$\begin{array}{ll}-4.90792500 & 2.27531800\end{array}$

$\begin{array}{ll}-3.76127600 & 2.98240800\end{array}$

$-3.22862400 \quad 1.71442100$

$-3.72340400 \quad 3.29260400$

$\begin{array}{ll}-5.38237700 & 4.64218800\end{array}$

$-4.01667500 \quad 5.42764600$

$-1.17792500-1.80294800$

$-2.21226700-2.41993200$

$0.89833700-1.71612400$

$0.63614600-2.64651800$

$1.73502200-2.03337500$

$\begin{array}{ll}-0.07132200 & 0.64326200\end{array}$

$4.67189400-1.85817900$

$\begin{array}{ll}4.85684900 & 2.31020900\end{array}$

$6.15063800-1.46696600$

$7.11340000-0.97074600$

$\begin{array}{ll}5.49819500 & 0.97343100\end{array}$

$\begin{array}{ll}-3.25208700 & 6.22785900\end{array}$

$2.19773200-0.04891300$

$\begin{array}{ll}-3.34579500 & 1.24059500\end{array}$

$-7.70506000-3.26007800$

$-0.03384500-7.38235100$

$\begin{array}{ll}1.60980600 & -6.75148000\end{array}$

$2.16628400-5.40114100$

$-2.41863500-4.61620500$

$-8.47834000-2.30550200$

$-3.15533000-4.72318200$

$1.13475700-4.00231000$

$0.34399100-4.67632500$

$0.37370300-2.13551500$

$6.19970900-2.34516400$

$\begin{array}{ll}5.01132700 & -2.80769400\end{array}$

$-3.58390200-5.05551200$

$-4.71754300-4.23970600$

$-0.80336200-5.13156100$

$-2.19377600-5.83495200$

$2.08862700-4.81420100$

$3.69675800-4.69142400$

$2.35314400-4.71501200$

$0.66751900-6.84390000$ 


$\begin{array}{cccc}\mathrm{H} & 2.34437300 & 6.45943500 & -2.90450500 \\ \mathrm{H} & 7.45054700 & 5.73828900 & 2.51186900 \\ \mathrm{H} & 6.11668200 & 6.47537300 & 1.61280100 \\ \mathrm{H} & 9.14140500 & 5.93813200 & -1.80760200 \\ \mathrm{H} & 10.08112300 & 1.29034600 & 0.75528900 \\ \mathrm{H} & 5.56297000 & -4.71778200 & 1.33052100 \\ \mathrm{H} & 5.14328400 & -4.00971700 & -0.24467500 \\ \mathrm{H} & 4.95771900 & -4.65728000 & 5.45280500 \\ \mathrm{H} & 3.72604900 & -4.80398000 & 6.84714000 \\ \mathrm{H} & -0.90192500 & -7.49103000 & -3.10070100 \\ \mathrm{H} & -0.00664300 & -6.48458300 & -4.20560400 \\ \mathrm{H} & -8.02444300 & -8.54165900 & -0.67701500 \\ \mathrm{H} & -7.07323600 & -7.84714700 & -2.03987000 \\ \mathrm{H} & -9.10776200 & 8.41606500 & 0.16861800 \\ \mathrm{H} & -10.33189200 & 7.88834100 & -0.93772200 \\ \mathrm{H} & -3.87632800 & 5.61188200 & 6.84073500 \\ \mathrm{H} & -4.99559800 & 4.33438900 & 6.23411000 \\ \mathrm{H} & 1.89547200 & 2.95308400 & 6.68985100 \\ \mathrm{H} & -5.87838000 & 1.79135900 & 0.54388800\end{array}$

\section{Int6s $(-0.8 \mathrm{kcal} / \mathrm{mol})$}

$\begin{array}{cccc}\mathrm{C} & 6.64215500 & 5.63931900 & 1.41360900 \\ \mathrm{C} & 7.14331400 & 4.92287200 & 0.20022000 \\ \mathrm{O} & 6.67032100 & 3.86543700 & -0.23442100 \\ \mathrm{~N} & 8.18717400 & 5.51840400 & -0.41968200 \\ \mathrm{C} & 8.73687700 & 4.95889000 & -1.62708600 \\ \mathrm{C} & 9.79576100 & 3.87796600 & -1.41696600 \\ \mathrm{O} & 10.81170800 & 3.81929100 & -2.10301900 \\ \mathrm{~N} & 9.50311700 & 2.98221700 & -0.43153700 \\ \mathrm{C} & 10.34973300 & 1.81616600 & -0.30942400 \\ \mathrm{C} & 10.07097200 & 0.82696800 & -1.43277700 \\ \mathrm{O} & 8.91418300 & 0.42149300 & -1.62731800 \\ \mathrm{~N} & 11.10753600 & 0.33286100 & -2.13888300 \\ \mathrm{C} & 10.84555000 & -0.74121900 & -3.11141800 \\ \mathrm{C} & 10.22274200 & -1.96630700 & -2.43681100 \\ \mathrm{O} & 9.39826500 & -2.66162100 & -3.02773300 \\ \mathrm{C} & 12.23149200 & -0.99465900 & -3.73164100 \\ \mathrm{C} & 12.91109300 & 0.38031300 & -3.64481300 \\ \mathrm{C} & 12.45618800 & 0.92401000 & -2.28395900 \\ \mathrm{~N} & 10.67878300 & -2.21519000 & -1.18616500 \\ \mathrm{C} & 10.15750700 & -3.24583600 & -0.30921200 \\ \mathrm{C} & 9.58645000 & -2.70681100 & 1.00795900 \\ \mathrm{O} & 9.72331500 & -3.34178300 & 2.04210800 \\ \mathrm{~N} & 8.91376700 & -1.51389900 & 0.93711900 \\ \mathrm{C} & 8.01957800 & -1.16343900 & 2.04692900 \\ \mathrm{C} & 6.89526600 & -2.19968500 & 2.24295000 \\ \mathrm{O} & 6.40001400 & -2.39317700 & 3.35476100 \\ \mathrm{C} & 7.43215600 & 0.24074200 & 1.81574700 \\ \mathrm{C} & 6.35909400 & 0.68850800 & 2.82635300 \\ \mathrm{C} & 6.85376800 & 0.62628900 & 4.27564600 \\ \mathrm{C} & 5.87970800 & 2.10033300 & 2.47673200 \\ \mathrm{~N} & 6.46729900 & -2.79703800 & 1.11742900 \\ \mathrm{C} & 5.36237800 & -3.72489400 & 1.13548600 \\ \mathrm{C} & 4.07124400 & -3.82115500 & 6.28938200 \\ \mathrm{C} & 2.90277600 & -3.93151100 & 5.29986300 \\ \mathrm{C} & 3.11264100 & -3.16423300 & 3.98823100 \\ \mathrm{C} & 2.04178400 & -3.54425200 & 2.95891300 \\ \mathrm{C} & 3.14547800 & -1.64777100 & 4.20397600 \\ \mathrm{C} & 0.07756600 & -7.22070700 & -2.62901600 \\ \mathrm{C} & 0.31709500 & -6.13347200 & -1.57945200\end{array}$

\begin{tabular}{|c|c|c|c|}
\hline & 1.46652000 & -5.16328900 & \\
\hline & 1.03793400 & -4.12452500 & \\
\hline & 7279000 & -4.47663400 & \\
\hline & & & \\
\hline & -8.62084100 & -6.65331800 & \\
\hline & -7.72024100 & -5.72094000 & \\
\hline & -6.41584200 & -5.45798600 & \\
\hline & & & \\
\hline & -5.57886400 & -4.58752800 & \\
\hline & & & \\
\hline & & & \\
\hline & -5.27392500 & & \\
\hline$U$ & -3.72469600 & & \\
\hline & & & \\
\hline & & & \\
\hline & -4.88 & & -0.9646030 \\
\hline & -3.9 & & \\
\hline & & & \\
\hline & & & \\
\hline & & & \\
\hline & & & \\
\hline & & & \\
\hline & & & \\
\hline & & & \\
\hline & & & \\
\hline & & & \\
\hline & & & \\
\hline & & & \\
\hline & & & \\
\hline 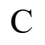 & -2.43 & & \\
\hline & & & \\
\hline & & & \\
\hline$C$ & & & \\
\hline & & & \\
\hline & & & \\
\hline$C$ & & & \\
\hline $\mathrm{S}$ & 600 & -3.2 & -1.5 \\
\hline & & & \\
\hline & & & \\
\hline C & & & -2.92 \\
\hline$C$ & & & -2.948097 \\
\hline & & & \\
\hline & & & \\
\hline C & -10.5 & 200 & -0.4 \\
\hline & & & \\
\hline & & & \\
\hline & & & \\
\hline C & & & \\
\hline & & & \\
\hline & & & \\
\hline $\mathrm{O}$ & -5.37139300 & & -0.23 \\
\hline $\mathrm{C}$ & & & \\
\hline & & & \\
\hline & & & \\
\hline $\mathrm{O}$ & -8.77 & 6.10709100 & \\
\hline & & & \\
\hline & & & \\
\hline & & & \\
\hline $\mathrm{C}$ & -5.69377500 & 6.54578700 & 2.08052800 \\
\hline & & & \\
\hline & & & \\
\hline & & & 4.02138800 \\
\hline & 2.25711300 & 1.64656400 & 4.18249900 \\
\hline
\end{tabular}




\begin{tabular}{|c|c|c|c|}
\hline & 6.98410100 & -2.35164400 & \\
\hline & 6.16442600 & 0.33512400 & \\
\hline & & 97751100 & \\
\hline & -3.02164600 & 0.00690700 & \\
\hline & -2.33126300 & -2.60530300 & \\
\hline & -1.09537200 & -2.25652500 & \\
\hline & -2.95240300 & & \\
\hline & -2.18505900 & & \\
\hline & & & \\
\hline & & & \\
\hline & 2300 & & \\
\hline & & -2.0 & \\
\hline & & & \\
\hline & & & \\
\hline & -2.072 & & \\
\hline & & & \\
\hline & & & \\
\hline & & & \\
\hline & & & \\
\hline & & & \\
\hline & & & \\
\hline & -6.2 & & \\
\hline & & & \\
\hline & & & \\
\hline & & & \\
\hline & & & \\
\hline & & & \\
\hline & & & \\
\hline & & & \\
\hline & & & \\
\hline & & & \\
\hline & & & \\
\hline & & & \\
\hline & & & \\
\hline & & & \\
\hline & & & \\
\hline & & & \\
\hline & & & \\
\hline & & & \\
\hline & & & \\
\hline & & & \\
\hline & & & \\
\hline & & & \\
\hline & & & \\
\hline & & & \\
\hline & & & \\
\hline & & & \\
\hline & & & \\
\hline & & & \\
\hline & & & \\
\hline & & & -4.3 \\
\hline & & & \\
\hline & & & \\
\hline & & & \\
\hline & & & \\
\hline & & & \\
\hline & & & \\
\hline & & & \\
\hline & & 2.22300600 & -2.17910600 \\
\hline & & & \\
\hline & & & \\
\hline & & -0.09908900 & \\
\hline & & & \\
\hline
\end{tabular}

$\mathrm{H}$ 


\begin{tabular}{|c|c|c|c|}
\hline $\mathrm{H}$ & -5.57217400 & 6.99174200 & \\
\hline $\mathrm{H}$ & -3.93799200 & 7.08089700 & 4.11240000 \\
\hline & -4.90867100 & 7.25150900 & 1.77903400 \\
\hline & -6.02047000 & 5.99554500 & 1.19610600 \\
\hline & -6.55698800 & 7.11814800 & 2.42968700 \\
\hline & -3.69648400 & 3.96986300 & 3.31345300 \\
\hline & -4.38912800 & 4.17377300 & 1.70394400 \\
\hline & -3.18154600 & 5.32601700 & 2.29984100 \\
\hline & -5.99714900 & 4.92600900 & 73600 \\
\hline & 0.75719000 & 8500 & 5200 \\
\hline & 0.31288700 & 2.96 & 800 \\
\hline & & 1.37 & \\
\hline & 2.99 & & \\
\hline & 1.41 & 0.98 & 4.38 \\
\hline & 4.00 & 1200 & 6900 \\
\hline & 3.21 & -1.12 & 7400 \\
\hline & 2.23 & -1.30 & $4.70^{\prime}$ \\
\hline & 2.08 & -4.6 & 2.71 \\
\hline & 1.03 & -3.34 & 9600 \\
\hline & & -2.9 & 5000 \\
\hline & 4.09 & -3.4 & \\
\hline & 2.73 & -4.95 & 000 \\
\hline & & & \\
\hline & 6.70 & -1.4 & -1.68 \\
\hline & 7.71 & -2.4 & -2.23 \\
\hline $\mathrm{H}$ & & & -1.80 \\
\hline & 5.83 & 0.27 & -2.68 \\
\hline H & -5.0 & & 300 \\
\hline & -6.1 & -0.5 & 100 \\
\hline & 7.91 & 4.5 & -2.18 \\
\hline $\mathrm{H}$ & & & 500 \\
\hline & -6.59 & & 7800 \\
\hline & -8.73 & 6.95 & 300 \\
\hline $\mathrm{H}$ & -8.20 & & 400 \\
\hline & 4.28 & -2.76 & 400 \\
\hline ( & 11.35 & & 5100 \\
\hline & & -3.2 & \\
\hline & & -7.96 & 600 \\
\hline $\mathrm{H}$ & 2.9 & -0.6 & 1900 \\
\hline & & & 7400 \\
\hline H & -1.86 & & 500 \\
\hline $\mathrm{H}$ & -6.8 & -2.8 & 8600 \\
\hline & -8.67 & -8.66 & 2700 \\
\hline $\mathrm{H}$ & & -3.5 & 600 \\
\hline & -12.4 & & 1400 \\
\hline & -11.12060900 & -0.06 & 84600 \\
\hline & & & \\
\hline & -5.03458500 & 5.97 & 8900 \\
\hline & -6.288 & 4.7 & -3.16 \\
\hline & -2.94766500 & -3.99 & -4.72 \\
\hline & -3.96025500 & -5.06220400 & 35100 \\
\hline & & & \\
\hline & -8.11557700 & -2.67563900 & -5.61267500 \\
\hline & -11.25679300 & 1.67230400 & 66400 \\
\hline & -2.40930700 & 3.29066600 & -4.93927500 \\
\hline & -3.49113800 & 1.94873600 & 33000 \\
\hline & & 0.10250200 & -6.84379000 \\
\hline & 2.36359800 & 6.18946600 & -3.37640100 \\
\hline & 7.46892600 & 5.903 & 2.08107900 \\
\hline & & & 1.12441700 \\
\hline & 9.16068100 & 5.76114700 & -2.24030400 \\
\hline & 10.11168400 & 1.29551500 & 0.62216700 \\
\hline & 5.58802000 & -4.61428700 & 1.73310000 \\
\hline
\end{tabular}

$\begin{array}{cccc}\mathrm{H} & 5.16982800 & -4.03490200 & 0.10680900 \\ \mathrm{H} & 4.98176000 & -4.22713500 & 5.83746400 \\ \mathrm{H} & 3.74985000 & -4.26346300 & 7.23881200 \\ \mathrm{H} & -0.87413600 & -7.73458500 & -2.46520900 \\ \mathrm{H} & 0.02080500 & -6.81846800 & -3.64634800 \\ \mathrm{H} & -7.99657900 & -8.59398700 & 0.03289700 \\ \mathrm{H} & -7.04547600 & -8.00925200 & -1.38061000 \\ \mathrm{H} & -9.09046300 & 8.37666900 & -0.47042800 \\ \mathrm{H} & -10.31401100 & 7.76202100 & -1.53161500 \\ \mathrm{H} & -3.85889400 & 6.11424600 & 6.40420600 \\ \mathrm{H} & -4.97723800 & 4.79192700 & 5.90069100 \\ \mathrm{H} & 1.94155900 & 3.51805200 & 6.44962400 \\ \mathrm{H} & -5.96917400 & 1.25899100 & 0.58318500\end{array}$

\section{$\mathrm{TS}_{S}(+3.8 \mathrm{kcal} / \mathrm{mol})$}

$\begin{array}{lccc}\mathrm{C} & 6.62443700 & 5.69332300 & 1.27973700 \\ \mathrm{C} & 7.09439900 & 4.97672900 & 0.05657900 \\ \mathrm{O} & 6.51644700 & 3.99924200 & -0.44278500 \\ \mathrm{~N} & 8.21272900 & 5.47737000 & -0.50979900 \\ \mathrm{C} & 8.73789700 & 4.90845300 & -1.72260300 \\ \mathrm{C} & 9.77066200 & 3.80472800 & -1.52035400 \\ \mathrm{O} & 10.75936500 & 3.69993600 & -2.23795800 \\ \mathrm{~N} & 9.48954800 & 2.93539300 & -0.50655700 \\ \mathrm{C} & 10.31083600 & 1.74829800 & -0.40749500 \\ \mathrm{C} & 10.00686100 & 0.77939200 & -1.54194900 \\ \mathrm{O} & 8.83743200 & 0.41507100 & -1.74972600 \\ \mathrm{~N} & 11.03313300 & 0.24927600 & -2.23379200 \\ \mathrm{C} & 10.75585100 & -0.84819900 & -3.17592400 \\ \mathrm{C} & 10.12206000 & -2.03916000 & -2.44989200 \\ \mathrm{O} & 9.24867900 & -2.71935200 & -2.98713200 \\ \mathrm{C} & 12.13718100 & -1.13740500 & -3.79279600 \\ \mathrm{C} & 12.84684600 & 0.22356800 & -3.72442000 \\ \mathrm{C} & 12.40038000 & 0.79684600 & -2.37351300 \\ \mathrm{~N} & 10.61728300 & -2.26780400 & -1.21103000 \\ \mathrm{C} & 10.10859400 & -3.25888100 & -0.28131500 \\ \mathrm{C} & 9.60868100 & -2.65802100 & 1.03922800 \\ \mathrm{O} & 9.80833000 & -3.23552500 & 2.09508500 \\ \mathrm{~N} & 8.92457000 & -1.47044700 & 0.94190500 \\ \mathrm{C} & 8.06100400 & -1.08543100 & 2.06614700 \\ \mathrm{C} & 6.96297600 & -2.13104500 & 2.34990500 \\ \mathrm{C} & -7.67921100 & -5.75803900 & 0.22305600 \\ \mathrm{O} & 6.52125600 & -2.29286400 & 3.48677000 \\ \mathrm{C} & 7.42952300 & 0.28978300 & 1.78044200 \\ \mathrm{C} & 6.35331900 & 0.74895900 & 2.78180500 \\ \mathrm{C} & 6.88300300 & 0.81455700 & 4.21807700 \\ \mathrm{C} & 5.78478900 & 2.10127500 & 2.34352400 \\ \mathrm{~N} & 6.49283000 & -2.76591200 & 1.26014700 \\ \mathrm{C} & 5.37754400 & -3.67924400 & 1.34112900 \\ \mathrm{C} & 4.05875300 & -3.58962300 & 6.48805700 \\ \mathrm{C} & 2.89722500 & -3.73578300 & 5.49703500 \\ \mathrm{C} & 3.13975200 & -3.06197100 & 4.14108800 \\ \mathrm{C} & 2.05001400 & -3.46278600 & 3.13935000 \\ \mathrm{C} & 3.24437400 & -1.53691100 & 4.26196400 \\ \mathrm{C} & 0.12487000 & -7.33038400 & -2.31950200 \\ \mathrm{C} & 0.34875600 & -6.19607800 & -1.31632200 \\ \mathrm{C} & 1.49065600 & -5.22964500 & -1.68841600 \\ \mathrm{C} & 1.96234100 & -4.46024000 & -0.45021900 \\ \mathrm{C} & -8.57957700 & -6.72200800 & -0.51748200 \\ \mathrm{C} & & & \\ \mathrm{C} & & & \\ \mathrm{C} & & & \end{array}$




\begin{tabular}{|c|c|c|c|}
\hline & -6.37933200 & -5.49966900 & -0.23078400 \\
\hline & -8.10887600 & -5.08521200 & 1.37240400 \\
\hline & -5.54425800 & -4.59999400 & 0.42633100 \\
\hline & -7.29606600 & -4.16088100 & 2.02711100 \\
\hline & -6.01249000 & -3.89943600 & 1.54381800 \\
\hline & -5.24398100 & -2.96058800 & 2.17831000 \\
\hline C & -3.68092300 & -4.17323000 & -3.78069400 \\
\hline C & -3.43195000 & -3.32713800 & -2.52063300 \\
\hline t & -4.73362200 & -2.59569900 & -2.17823900 \\
\hline C & -4.83244400 & -1.82297700 & -0.88566300 \\
\hline $\mathrm{O}$ & -3.95081400 & -1.80382100 & -0.01160300 \\
\hline $\mathrm{O}$ & -5.98465000 & -1.21100300 & -0.77 \\
\hline C & 2.49364800 & -0.19799400 & -6.559 \\
\hline $\mathrm{C}$ & 2.76666100 & -0.54483700 & -5.11 \\
\hline & 1.85808600 & -0.19453700 & -4.113 \\
\hline C & 3.94536000 & -1.20444300 & -4.74 \\
\hline C & 2.12267500 & -0.46106100 & -2.76 \\
\hline C & 4.21025400 & -1.49 & -3.40 \\
\hline C & 3.30649100 & -1.10582700 & -2.40428900 \\
\hline f & 2.55593900 & 4.97424100 & -3.62491200 \\
\hline & 3.10656200 & 78200 & -2.301 \\
\hline $\mathrm{C}$ & 3.61485300 & 2.99082200 & -2.437 \\
\hline $\mathrm{N}$ & 4.20402400 & 2.49586100 & 57300 \\
\hline$C$ & -2.40744800 & 2.00954700 & -4.797 \\
\hline $\mathrm{C}$ & -1.85685200 & 1.72767700 & -3.39920100 \\
\hline C & -1.77269400 & 0.21718800 & -3.15317100 \\
\hline C & -0.51333200 & 2.44404000 & -3.194 \\
\hline C & -7.68067400 & -2.40699600 & -4.629 \\
\hline C & -8.69183800 & -2.19894000 & -3.50 \\
\hline $\mathrm{C}$ & -9.01759300 & -3.49858400 & -2.764 \\
\hline 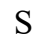 & -10.21058400 & -3.31267500 & -1.38 \\
\hline $\mathrm{C}$ & -9.25945200 & -2.19304100 & -0.301 \\
\hline $\mathrm{C}$ & -11.39474600 & 0.66312700 & 82900 \\
\hline $\mathrm{C}$ & -10.63971200 & 618800 & -2.98 \\
\hline $\mathrm{C}$ & -9.27461800 & 1.278 & -3.00 \\
\hline C & -11.27379000 & 813100 & 09800 \\
\hline $\mathrm{C}$ & -8.56752200 & 1.57 & -1.84 \\
\hline $\mathrm{C}$ & -10.58679300 & 1.24723200 & -0.561 \\
\hline $\mathrm{C}$ & -9.23213900 & 89900 & -0.608 \\
\hline $\mathrm{O}$ & -8.59498100 & 1.90 & 0400 \\
\hline $\mathrm{C}$ & -5.82736200 & 5.22118600 & -2.5617 \\
\hline $\mathrm{C}$ & -5.10135000 & 4.42 & $-1.4^{\top}$ \\
\hline $\mathrm{C}$ & -5.93553200 & 3.519 & -0.58 \\
\hline $\mathrm{O}$ & -7.06144700 & 3.903 & 8400 \\
\hline $\mathrm{O}$ & -5.39006000 & 2.40279400 & -0.28623100 \\
\hline $\mathrm{C}$ & -9.51693100 & 7.35443200 & 66100 \\
\hline $\mathrm{C}$ & -9.91613200 & 6.38 & -0.03 \\
\hline $\mathrm{C}$ & -10.50501800 & 5.08815000 & -0.60306400 \\
\hline $\mathrm{O}$ & -8.80530100 & 6.10612900 & 0.80708800 \\
\hline $\mathrm{C}$ & -4.26288900 & 5.68140500 & 5.37523800 \\
\hline $\mathrm{C}$ & -4.77383500 & 6.50778800 & 4.19189700 \\
\hline $\mathrm{C}$ & -5.14981400 & 5.68696200 & 2.94382600 \\
\hline $\mathrm{C}$ & -5.76146500 & 6.59103600 & 1.86854400 \\
\hline $\mathrm{C}$ & -3.95110400 & 4.91801500 & 2.37122600 \\
\hline $\mathrm{C}$ & 1.09413100 & 3.84081100 & 5.63713000 \\
\hline $\mathrm{S}$ & 1.72941500 & 3.52269000 & 3.95098500 \\
\hline $\mathrm{C}$ & 2.03223400 & 1.72986400 & 4.08043100 \\
\hline $\mathrm{O}$ & 6.95490700 & -2.23776600 & -1.47013400 \\
\hline $\mathrm{O}$ & 6.10740800 & 0.50375500 & -1.91236100 \\
\hline $\mathrm{O}$ & -5.04914500 & 0.89440300 & -2.58727200 \\
\hline $\mathrm{N}$ & -2.95224400 & 0.12944500 & 2.13687000 \\
\hline $\mathrm{C}$ & -2.17164900 & -2.05751600 & 6.95674200 \\
\hline & -0.95003600 & -1.67629100 & 6.39222700 \\
\hline
\end{tabular}

\begin{tabular}{|c|c|c|c|}
\hline & -2.99408900 & .50624500 & \\
\hline $\mathrm{C}$ & -2.35689600 & & \\
\hline & -0.81808100 & & .00877000 \\
\hline C & -1.88226900 & -1.99583800 & 16557800 \\
\hline & -0.99895500 & & \\
\hline & -1.70832700 & -1.92015900 & 2.66409900 \\
\hline & 0.14770200 & 1.81199400 & \\
\hline & 1.41814400 & & \\
\hline & -1.72471600 & -0.468 & \\
\hline & 1.48242300 & -0.2 & \\
\hline & -3.09898 & -2.3 & \\
\hline $\mathrm{C}$ & -3.2434 & -2.402 & \\
\hline & 0.3694 & -1.0 & \\
\hline $\mathrm{C}$ & -0.9322 & -0.39 & \\
\hline C & -2.2579 & -2.0738 & \\
\hline $\mathrm{O}$ & -6.2853 & & 00 \\
\hline $\mathrm{C}$ & 2.73619400 & -0.7 & \\
\hline $\mathrm{O}$ & 2.5360 & & \\
\hline $\mathrm{H}$ & -0.124 & -1. & \\
\hline $\mathrm{H}$ & 0.13 & & \\
\hline $\mathrm{H}$ & 3.2903 & & \\
\hline $\mathrm{H}$ & -2.4941 & $-2.4^{\prime}$ & \\
\hline $\mathrm{H}$ & -1.718 & -0.9 & \\
\hline $\mathrm{H}$ & -2.429 & & \\
\hline $\mathrm{H}$ & -0.750 & -2.3 & \\
\hline $\mathrm{H}$ & -2.32 & & \\
\hline $\mathrm{H}$ & -0.98 & & \\
\hline $\mathrm{H}$ & -3.93 & -2.6 & \\
\hline $\mathrm{H}$ & 0.096 & & \\
\hline $\mathrm{H}$ & -4.194 & -2.6 & \\
\hline $\mathrm{H}$ & & -2.0 & \\
\hline $\mathrm{H}$ & -3.683 & -0.4 & \\
\hline $\mathrm{H}$ & -3.148 & -2.3 & \\
\hline $\mathrm{H}$ & & & \\
\hline & -4.02 & & \\
\hline & -7.23 & & \\
\hline $\mathrm{H}$ & 4.86 & & \\
\hline & 3.45 & & \\
\hline & 4.77 & & \\
\hline & 4.39 & & \\
\hline & & & \\
\hline & & & \\
\hline & 2.335 & & \\
\hline & 1.62 & 4.4 & -3.5 \\
\hline $\mathrm{H}$ & 3.2728 & & \\
\hline & 4.6502 & -1.5 & \\
\hline $\mathrm{H}$ & & -2.0 & \\
\hline & 3.50466700 & -1.3 & \\
\hline & 1.391 & -0.1 & \\
\hline $\mathrm{H}$ & 0.923 & & -4.3 \\
\hline $\mathrm{H}$ & & & \\
\hline $\mathrm{H}$ & -0.64 & & -3.21 \\
\hline $\mathrm{H}$ & -0.0592 & & \\
\hline $\mathrm{H}$ & & & \\
\hline $\mathrm{H}$ & -1.27680000 & -0.01200000 & 1700 \\
\hline & -2.78321100 & -0.19 & \\
\hline $\mathrm{H}$ & -1.21526600 & -0.28779500 & -3.95132600 \\
\hline $\mathrm{H}$ & -8.27728800 & -1.48115000 & 54100 \\
\hline $\mathrm{H}$ & -9.61679300 & -1.75307500 & -3.87869500 \\
\hline $\mathrm{H}$ & -8.10282100 & -3.95141600 & -2.36100800 \\
\hline & & -4.22962400 & \\
\hline $\mathrm{H}$ & -8.25242500 & -2.58191900 & -0.14173500 \\
\hline & -9.21997900 & -1.18334500 & -0.70872300 \\
\hline
\end{tabular}




\begin{tabular}{|c|c|c|c|}
\hline & & & \\
\hline & & & \\
\hline & & & \\
\hline & -11.08930300 & 1.24985000 & \\
\hline & -12.32907000 & 0.68434100 & \\
\hline & -8.74677900 & 1.28893000 & \\
\hline & -5.56594900 & -3.30887700 & 54300 \\
\hline & -4.98192300 & -1.89511800 & \\
\hline & -3.10224700 & -3.94691900 & 76800 \\
\hline & -2.63743400 & -2.59483100 & \\
\hline & -8.76607100 & -6.33031200 & -1.5 \\
\hline & & -6.751 & \\
\hline & & -5.263 & \\
\hline & -6.0 & -6.01 & 6000 \\
\hline & -4.5 & -4.4 & \\
\hline & -7.6 & -3.61 & \\
\hline & -4.6 & -2.53 & \\
\hline & & -3.68 & \\
\hline & 1.1 & -3.974 & \\
\hline & & -5.12 & \\
\hline & & -3.6 & \\
\hline & 0.6 & -4.7 & \\
\hline & & & \\
\hline & & -5.82 & \\
\hline & -0.57 & -5.6 & \\
\hline & & -6.6 & \\
\hline & & -2.5 & \\
\hline & & -1.0 & \\
\hline & & & \\
\hline & & 0.2 & \\
\hline & & & \\
\hline & & & \\
\hline & & & \\
\hline & & & \\
\hline & & & \\
\hline & & & \\
\hline & & & \\
\hline & & -1.2 & \\
\hline & & -3.7 & -0.7 \\
\hline & & & 2000 \\
\hline & & -1.60 & \\
\hline & & & \\
\hline & & & \\
\hline & & & \\
\hline & & & \\
\hline & & & \\
\hline & 12.0 & 600 & 3400 \\
\hline & & -0.525 & 29500 \\
\hline & & & \\
\hline & & 6.21 & -0.0 \\
\hline & -4.603 & & \\
\hline & & & \\
\hline & & 4.58967600 & -1.2 \\
\hline & & & \\
\hline & & 5.276 & 37600 \\
\hline & & & \\
\hline & & & \\
\hline & & 7.091 & \\
\hline & & & \\
\hline & & 7.37388500 & \\
\hline & -6.02602600 & 6.01549400 & \\
\hline & & & \\
\hline & -3.50890900 & 4.23374800 & 3.10088700 \\
\hline
\end{tabular}

\begin{tabular}{|c|c|c|c|}
\hline $\mathrm{H}$ & -4.25282400 & .32079100 & \\
\hline $\mathrm{H}$ & & & \\
\hline & -5.91168200 & & \\
\hline & 0.86224400 & 4.90572500 & 70200100 \\
\hline & & & \\
\hline & 2.46002900 & 1.42205600 & .12705300 \\
\hline & 2.73945500 & 1.50520700 & \\
\hline & 1.10105000 & & \\
\hline & 4.12940000 & -1.23899900 & \\
\hline & & & \\
\hline & 900 & 000 & 800 \\
\hline & 58800 & -4.5 & \\
\hline & 1.0 & & \\
\hline & 1500 & -2.5 & \\
\hline & & & \\
\hline & 2.7 & -4.8 & 100 \\
\hline & & & \\
\hline & & & \\
\hline & 7.6 & -2.4 & -2 . \\
\hline & & & \\
\hline $\mathrm{H}$ & 5.8 & & \\
\hline & 5100 & & -1. \\
\hline $\mathrm{H}$ & 2600 & -0.5 & \\
\hline 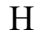 & 4800 & & -2.2 \\
\hline & & & \\
\hline $\mathrm{H}$ & 900 & & \\
\hline & 500 & & -1. \\
\hline $\mathrm{H}$ & & & \\
\hline I & 100 & -2.5 & \\
\hline $\mathrm{H}$ & 7300 & & \\
\hline $\mathrm{H}$ & & & \\
\hline $\mathrm{H}$ & & & \\
\hline $\mathrm{H}$ & & & \\
\hline $\mathrm{H}$ & & & \\
\hline $\mathrm{H}$ & -1.8 & & \\
\hline $\mathrm{H}$ & -6.8 & & \\
\hline $\mathrm{H}$ & 73900 & & \\
\hline $\mathrm{H}$ & 73800 & -3.7 & \\
\hline $\mathrm{H}$ & 700 & 900 & 300 \\
\hline $\mathrm{H}$ & -11. & 9900 & 400 \\
\hline $\mathrm{H}$ & & & \\
\hline $\mathrm{H}$ & & & \\
\hline $\mathrm{H}$ & 15400 & & \\
\hline $\mathrm{H}$ & -2.8 & -4.1 & \\
\hline $\mathrm{H}$ & -3.9 & -5.2 & \\
\hline $\mathrm{H}$ & -7.2 & -1.4 & \\
\hline $\mathrm{H}$ & -8.0 & -2.9 & \\
\hline $\mathrm{H}$ & -11.22642700 & & 7800 \\
\hline $\mathrm{H}$ & & & \\
\hline $\mathrm{H}$ & & & \\
\hline $\mathrm{H}$ & & -0.1 & \\
\hline $\mathrm{H}$ & 015200 & 6.0509 & -3.5 \\
\hline $\mathrm{H}$ & 7.44668900 & & \\
\hline $\mathrm{H}$ & & & \\
\hline $\mathrm{H}$ & 35100 & 5.68900400 & -2.36279800 \\
\hline $\mathrm{H}$ & & & \\
\hline $\mathrm{H}$ & 5.60287200 & -4.54514100 & 1.97241200 \\
\hline $\mathrm{H}$ & 5.15865200 & -4.03299600 & \\
\hline $\mathrm{H}$ & & -4.00879600 & \\
\hline $\mathrm{H}$ & 3.73369000 & -3.99762600 & 7.45158400 \\
\hline & -0.82582900 & -7.84134400 & \\
\hline $\mathrm{H}$ & 0.07236700 & -6.96627100 & -3.35139600 \\
\hline & -7.95890500 & -8.63305400 & 0.34846400 \\
\hline
\end{tabular}


H $\quad-7.00209500 \quad-8.09757100 \quad-1.08064400$

$\mathrm{H} \quad-9.10680500 \quad 8.30345300 \quad-0.78759000$

$\mathrm{H} \quad-10.32250400 \quad 7.64567400 \quad-1.83180200$

$\mathrm{H} \quad-3.90512000 \quad 6.31511200 \quad 6.19363000$

$\mathrm{H} \quad-5.01625600 \quad 4.97114300 \quad 5.73340400$

$\begin{array}{llll}\mathrm{H} & 1.84393700 & 3.59210400 & 6.39290800\end{array}$

$\mathrm{H} \quad-5.95949600 \quad 1.24125400 \quad 0.60121400$

\section{$\operatorname{Int}_{S}(+1.3 \mathrm{kcal} / \mathrm{mol})$}

$\begin{array}{llll}\text { C } & 6.63151200 & 5.70949900 & 1.23335800\end{array}$

$\begin{array}{llll}\text { C } & 7.10382500 & 4.98277800 & 0.01743000\end{array}$

$\begin{array}{lllll}\mathrm{O} & 6.52424000 & 4.00286500 & -0.47641900\end{array}$

$\begin{array}{llll}\mathrm{N} & 8.22502200 & 5.47501400 & -0.54924400\end{array}$

C $\quad 8.75215200 \quad 4.89510600 \quad-1.75599900$

C $\quad 9.78483200 \quad 3.79334600 \quad-1.54218100$

$\begin{array}{llll}\text { O } & 10.77349000 & 3.68149400 & -2.25853200\end{array}$

$\begin{array}{llll}\mathrm{N} & 9.50373600 & 2.93385400 & -0.51996500\end{array}$

C $\quad 10.32582600 \quad 1.74822600 \quad-0.40921400$

C $\quad 10.02568400 \quad 0.76984700 \quad-1.53646600$

O $\quad 8.85662000 \quad 0.40320400 \quad-1.74416600$

$\begin{array}{lllll}\mathrm{N} & 11.05360600 & 0.23413400 & -2.22059200\end{array}$

C $\quad 10.78023400 \quad-0.87336600 \quad-3.15231100$

$\begin{array}{llll}\text { C } & 10.14286500 & -2.05576400 & -2.41526900\end{array}$

$\begin{array}{lllll}\text { O } & 9.26797900 & -2.73875900 & -2.94662800\end{array}$

C $\quad 12.16413500 \quad-1.16899800 \quad-3.76048100$

C $\quad 12.87420000 \quad 0.19231500 \quad-3.70228300$

$\begin{array}{lllll}\text { C } & 12.42207300 & 0.77906700 & -2.35915800\end{array}$

$\mathrm{N} \quad 10.63598000 \quad-2.27292300 \quad-1.17359700$

C $\quad 10.12444300 \quad-3.25289300 \quad-0.23358600$

$\begin{array}{llll}\text { C } & 9.62131000 & -2.63614300 & 1.07848100\end{array}$

$\begin{array}{llll}\mathrm{O} & 9.81988300 & -3.19969700 & 2.14187600\end{array}$

N $\quad 8.93505400 \quad-1.45091100 \quad 0.96512900$

$\begin{array}{llll}\text { C } & 8.06810700 & -1.05475600 & 2.08290500\end{array}$

$\begin{array}{llll}\text { C } & 6.97502400 & -2.10161800 & 2.38078800\end{array}$

O $\quad 6.53411000 \quad-2.25030300 \quad 3.51943800$

$\begin{array}{llll}\text { C } & 7.42552500 & 0.31083300 & 1.77641700\end{array}$

$\begin{array}{llll}\text { C } & 6.34651600 & 0.77459800 & 2.77282400\end{array}$

$\begin{array}{llll}\text { C } & 6.88284700 & 0.87964300 & 4.20429000\end{array}$

$\begin{array}{llll}\text { C } & 5.75179000 & 2.10629500 & 2.30728700\end{array}$

$\begin{array}{llll}\mathrm{N} & 6.50479900 & -2.74898600 & 1.29805500\end{array}$

$\begin{array}{llll}\text { C } & 5.39065000 & -3.66284500 & 1.38877200\end{array}$

$\begin{array}{llll}\text { C } & 4.06039300 & -3.52101600 & 6.53177300\end{array}$

$\begin{array}{llll}\text { C } & 2.89666700 & -3.68845400 & 5.54641400\end{array}$

C $\quad 3.13293100 \quad-3.04562500 \quad 4.17517200$

$\begin{array}{llll}\text { C } & 2.04152400 & -3.47244300 & 3.18653500\end{array}$

$\begin{array}{llll}\text { C } & 3.22966000 & -1.51792300 & 4.26096800\end{array}$

C $\quad 0.14825100 \quad-7.35509200 \quad-2.24563700$

C $\quad 0.38100900 \quad-6.19804600 \quad-1.27116000$

$\begin{array}{llll}\text { C } & 1.55257200 & -5.27524600 & -1.66271500\end{array}$

C $\quad 1.18777000 \quad-4.36446900 \quad-2.84340900$

$\begin{array}{llll}\text { C } & 2.00287300 & -4.43693600 & -0.46260400\end{array}$

$\begin{array}{llll}\text { C } & -7.97801800 & -8.15478100 & -0.56512300\end{array}$

$\begin{array}{llll}\text { C } & -8.56665100 & -6.73668600 & -0.47328900\end{array}$

$\begin{array}{llll}\text { C } & -7.67864200 & -5.75587100 & 0.26005000\end{array}$

$\begin{array}{llll}\text { C } & -6.37703400 & -5.49338200 & -0.18624200\end{array}$

$\begin{array}{llll}\text { C } & -8.12451300 & -5.06739200 & 1.39392900\end{array}$

$\begin{array}{llll}\text { C } & -5.55631200 & -4.57395700 & 0.46200700\end{array}$

$\begin{array}{llll}\text { C } & -7.32646400 & -4.12412300 & 2.03919200\end{array}$

$\begin{array}{llll}\text { C } & -6.04115800 & -3.85726700 & 1.56242100\end{array}$

$\begin{array}{llll}\text { O } & -5.29083100 & -2.90042300 & 2.18857900\end{array}$
C $\quad-3.65597000 \quad-4.21585300 \quad-3.74759500$

C $\quad-3.42005500 \quad-3.35091800 \quad-2.49855100$

C $\quad-4.72672000 \quad-2.61816400 \quad-2.17851200$

C $\quad-4.83530000 \quad-1.82817100 \quad-0.89722200$

$\begin{array}{llll}\text { O } & -3.95969900 & -1.79961100 & -0.01894500\end{array}$

$\begin{array}{lllll}\mathrm{O} & -5.98837800 & -1.21412400 & -0.80737800\end{array}$

C $\quad 2.52200000 \quad-0.26536100 \quad-6.55421400$

$\begin{array}{llll}\text { C } & 2.78882500 & -0.60076200 & -5.10756600\end{array}$

$\begin{array}{lll}1.86759500 & -0.26147500 & -4.11062900\end{array}$

$3.97294300-1.24502000 \quad-4.72139700$

$\begin{array}{lll}2.12381300 & -0.52670400 & -2.76404100\end{array}$

$\begin{array}{llll}4.22956800 & -1.53206500 & -3.37799300\end{array}$

$\begin{array}{llll}3.31188400 & -1.15772900 & -2.38980600\end{array}$

$\begin{array}{llll}2.57431500 & 4.93696700 & -3.67258400\end{array}$

$\begin{array}{llll}3.13692200 & 4.39686600 & -2.35127200\end{array}$

$\begin{array}{lll}3.67005800 & 2.97383200 & -2.49360700\end{array}$

$\begin{array}{llll}4.25357500 & 2.47971900 & -1.20831100\end{array}$

$-2.38441200 \quad 1.95706400 \quad-4.82512400$

$\begin{array}{lll}-1.84520100 & 1.71020500 & -3.41411400\end{array}$

$\begin{array}{lll}-1.77707300 & 0.20977800 & -3.10932000\end{array}$

$\begin{array}{lll}-0.49486900 & 2.41831700 & -3.22474400\end{array}$

$-7.65513000-2.46126700 \quad-4.62388100$

$\begin{array}{lll}-8.66957400 & -2.24150900 & -3.50119800\end{array}$

$-9.00073100 \quad-3.53382100-2.75331300$

$-10.20601300 \quad-3.33382800-1.38890500$

$-9.27133000 \quad-2.19021800 \quad-0.31359400$

$\begin{array}{llll}-11.37206400 & 0.61004500 & -4.28572700\end{array}$

$\begin{array}{llll}-10.62494500 & 0.93135100 & -3.01434300\end{array}$

$\begin{array}{lll}-9.25902000 & 1.24054600 & -3.03777300\end{array}$

$\begin{array}{llll}-11.26764200 & 0.92607400 & -1.77223400\end{array}$

$\begin{array}{llll}-8.55928800 & 1.55455500 & -1.87508300\end{array}$

$\begin{array}{lll}-10.58788500 & 1.25350600 & -0.60016900\end{array}$

$\begin{array}{llll}-9.23225600 & 1.58772200 & -0.64457400\end{array}$

$\begin{array}{lll}-8.60234200 & 1.92543000 & 0.52290900\end{array}$

$\begin{array}{llll}-5.81147500 & 5.18911700 & -2.63042900\end{array}$

$\begin{array}{llll}-5.08740300 & 4.40481700 & -1.53238300\end{array}$

$\begin{array}{llll}-5.92314700 & 3.50904600 & -0.63579400\end{array}$

$\begin{array}{llll}-7.04945800 & 3.90045200 & -0.23351100\end{array}$

$\begin{array}{lll}-5.37992000 & 2.39546500 & -0.32457800\end{array}$

$\begin{array}{llll}-9.50557700 & 7.33432700 & -1.24873900\end{array}$

$\begin{array}{llll}-9.90782500 & 6.37495200 & -0.12872200\end{array}$

$\begin{array}{llll}-10.49331300 & 5.07339200 & -0.68011600\end{array}$

$\begin{array}{lll}-8.79999700 & 6.10931700 & 0.72460400\end{array}$

$\begin{array}{lll}-4.26491900 & 5.73242000 & 5.30472600\end{array}$

$\begin{array}{llll}-4.77637900 & 6.54661300 & 4.11293000\end{array}$

$\begin{array}{lll}-5.12444800 & 5.71456600 & 2.86412500\end{array}$

$\begin{array}{lll}-5.76897200 & 6.59712400 & 1.79031700\end{array}$

$\begin{array}{lll}-3.89910600 & 4.99065200 & 2.28927200\end{array}$

$\begin{array}{lll}1.09276500 & 3.89822900 & 5.59741100\end{array}$

$\begin{array}{lll}1.78047200 & 3.55232600 & 3.93667800\end{array}$

$\begin{array}{lll}1.95697100 & 1.74128200 & 4.05532400\end{array}$

$\begin{array}{llll}6.97247700 & -2.25426500 & -1.44081600\end{array}$

$\begin{array}{llll}6.13646700 & 0.48871500 & -1.94175400\end{array}$

$\begin{array}{llll}-5.05035200 & 0.88213900 & -2.62532900\end{array}$

$\begin{array}{llll}-2.99165400 & 0.15403000 & 2.20424000\end{array}$

$\begin{array}{lll}-2.31069000 & -1.86390300 & 7.02504900\end{array}$

$\begin{array}{lll}-1.06199200 & -1.57454900 & 6.46644000\end{array}$

$\begin{array}{lll}-3.01161600 & 1.52443800 & 1.76626400\end{array}$

$\begin{array}{lll}-2.36481000 & 1.68411200 & 0.33923700\end{array}$

$\begin{array}{lll}-0.91096300 & -1.60199900 & 5.08440500\end{array}$

$\begin{array}{lll}-1.98191700 & -1.91625200 & 4.23613000\end{array}$

$\begin{array}{lll}-1.02810400 & 1.04114200 & 0.38125200\end{array}$

$\begin{array}{lll}-1.78090400 & -1.90387900 & 2.73951300\end{array}$ 


\begin{tabular}{|c|c|c|c|}
\hline & 167200 & 1.76037000 & \\
\hline & 40136100 & 1.11812500 & \\
\hline & -1.72287200 & -0.46719900 & \\
\hline & 1.42534200 & -0.33599300 & 1200250 \\
\hline & -3.22425100 & -2.19916400 & \\
\hline & -3.38907500 & -2.17645900 & 6.19317500 \\
\hline & 0.28824400 & -1.06502900 & \\
\hline & -1.02774000 & -0.41033600 & 0.6618630 \\
\hline & -2.41694000 & & \\
\hline & -6.30562900 & 0.40826600 & \\
\hline & 2.67150900 & & \\
\hline & 2.52841800 & & \\
\hline & -0.231 & & \\
\hline & & & \\
\hline & 3.25732000 & & \\
\hline & -2.58 & & \\
\hline & & & \\
\hline & -2.4 & & \\
\hline & -0.84413800 & & \\
\hline & -2.3 & & \\
\hline & & & \\
\hline & -4.0 & & \\
\hline & & & \\
\hline & -4.3 & & \\
\hline & & & \\
\hline & -3.6 & & \\
\hline & & & \\
\hline & -3.0 & & \\
\hline & -4.0 & & \\
\hline & -7.2 & & \\
\hline & & & -0.8 \\
\hline & & & \\
\hline & & & \\
\hline & & & \\
\hline & & & 900 \\
\hline & & & -2.0 \\
\hline & & & \\
\hline & & & \\
\hline & & & \\
\hline & & & -5.4 \\
\hline & & & \\
\hline & & & \\
\hline & & & \\
\hline & & & \\
\hline & & & \\
\hline & & & \\
\hline 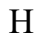 & & & -2.2 \\
\hline & & & \\
\hline & & & \\
\hline & & & \\
\hline $\mathrm{H}$ & & & 27800 \\
\hline & & & \\
\hline & & & \\
\hline & & & \\
\hline $\mathrm{H}$ & & & -3.42680100 \\
\hline & & & \\
\hline & & & \\
\hline $\mathrm{H}$ & -9.80277400 & -2.14893400 & 10600 \\
\hline & & & \\
\hline & & & \\
\hline & & & \\
\hline $\mathrm{H}$ & -12.32387600 & 0.67554700 & -1.72108600 \\
\hline & -8.72459600 & 1.23324300 & $500107<0$ \\
\hline
\end{tabular}

$\mathrm{H} \quad-5.55685000 \quad-3.33364600 \quad-2.13998400$

$\mathrm{H} \quad-4.97124000 \quad-1.93084100 \quad-2.99511100$

$\mathrm{H} \quad-3.09653000 \quad-3.95668300 \quad-1.64620600$

$\mathrm{H} \quad-2.62591800 \quad-2.61890600 \quad-2.67572300$

$\mathrm{H} \quad-8.75057600 \quad-6.35900700 \quad-1.48714700$

H $\quad-9.54762300 \quad-6.76653100 \quad 0.01120700$

$\mathrm{H} \quad-9.13135300 \quad-5.24882100 \quad 1.75890200$

$\mathrm{H} \quad-5.98982500 \quad-6.01959600 \quad-1.05533900$

$\mathrm{H} \quad-4.54211800 \quad-4.40190500 \quad 0.12013800$

$\mathrm{H} \quad-7.69375400 \quad-3.56573800 \quad 2.89355300$

$\mathrm{H} \quad-4.70958200 \quad-2.48506300 \quad 1.52171800$

$\mathrm{H} \quad 2.79202000 \quad-3.72874800 \quad-0.73250900$

$\mathrm{H} \quad 1.16170600 \quad-3.85428300 \quad-0.07299100$

$\mathrm{H} \quad 2.37064800 \quad-5.06469500 \quad 0.35511900$

$\mathrm{H} \quad 2.04348000 \quad-3.76010100 \quad-3.15924500$

$\mathrm{H} \quad 0.84192500 \quad-4.93580700 \quad-3.71038400$

$\mathrm{H} \quad 0.38467200 \quad-3.67327900 \quad-2.55875400$

$\mathrm{H} \quad 2.39598200 \quad-5.91255100 \quad-1.96891200$

$\mathrm{H} \quad-0.52924000 \quad-5.58717100 \quad-1.19043300$

$\mathrm{H} \quad 0.56124900 \quad-6.60560600 \quad-0.26829500$

$\mathrm{H} \quad 6.86580400 \quad-2.56093700 \quad 0.36715900$

H $\quad 8.68108400 \quad-1.00235500 \quad 2.98284200$

$\begin{array}{llll}\mathrm{H} & 8.22294800 & 1.06794500 & 1.74517600\end{array}$

$\mathrm{H} \quad 6.98700400 \quad 0.27200700 \quad 0.76986200$

$\mathrm{H} \quad \begin{array}{llll}6.52488200 & 2.88387300 & 2.28053800\end{array}$

H $\quad 5.32450400 \quad 2.02653600 \quad 1.30420000$

$\mathrm{H} \quad 4.95633000 \quad 2.44416300 \quad 2.97838900$

$\mathrm{H} \quad 7.75095900 \quad 1.55040300 \quad 4.25176200$

$\mathrm{H} \quad 6.11384200 \quad 1.28500100 \quad 4.86950300$

H $\quad 7.16745600 \quad-0.10316600 \quad 4.58325000$

H $\quad 5.54523500 \quad 0.02720600 \quad 2.77676600$

$\mathrm{H} \quad 8.60183700 \quad-1.21535100 \quad 0.03527200$

H $\quad 9.29975300 \quad-3.77756200 \quad-0.72368500$

H $\quad 10.89025400 \quad-3.98526900 \quad 0.03189400$

H $\quad 11.31481100 \quad-1.61211800 \quad-0.82528500$

$\mathrm{H} \quad 13.06212600 \quad 0.43048900 \quad-1.53905800$

$\mathrm{H} \quad 13.96081700 \quad 0.11136500 \quad-3.77306300$

H $\quad 12.69337700 \quad-1.90150300 \quad-3.14156100$

$\mathrm{H} \quad 12.52691200 \quad 0.83534300 \quad-4.51709000$

$\mathrm{H} \quad 12.38404600 \quad 1.86884000 \quad-2.35455500$

$\mathrm{H} \quad 12.08255700 \quad-1.57847800 \quad-4.76821000$

H $\quad 10.05649200 \quad-0.56270400 \quad-3.90991200$

H $\quad 8.54343800 \quad 2.87487200 \quad-0.20127400$

$\mathrm{H} \quad 8.73117100 \quad 6.21894800 \quad-0.09301900$

$\mathrm{H} \quad-4.59150200 \quad 5.12119000 \quad-0.86298000$

$\mathrm{H} \quad-4.29399100 \quad 3.79214200 \quad-1.97002900$

$\mathrm{H} \quad-9.76360000 \quad 4.56913800 \quad-1.32387200$

$\mathrm{H} \quad-10.73734000 \quad 4.39001900 \quad 0.13734300$

$\mathrm{H} \quad-11.39813700 \quad 5.25401300 \quad-1.27038600$

$\mathrm{H} \quad-10.65499500 \quad 6.86239300 \quad 0.51159300$

$\mathrm{H} \quad-3.39863600 \quad 5.12795700 \quad 5.01103400$

$\mathrm{H} \quad-5.66526000 \quad 7.11586500 \quad 4.41326300$

$\mathrm{H} \quad-4.01903800 \quad 7.29294200 \quad 3.83130100$

$\mathrm{H} \quad-5.08758000 \quad 7.40700300 \quad 1.49725700$

$\mathrm{H} \quad-6.01056200 \quad 6.01386000 \quad 0.89954400$

$\mathrm{H} \quad-6.70584400 \quad 7.04228600 \quad 2.13422000$

$\mathrm{H} \quad-3.42488800 \quad 4.32894500 \quad 3.01944700$

$\mathrm{H} \quad-4.18145600 \quad 4.37609900 \quad 1.42970600$

$\mathrm{H} \quad-3.14437500 \quad 5.71488300 \quad 1.95558200$

$\mathrm{H} \quad-5.85891900 \quad 4.95062300 \quad 3.15767700$

$\mathrm{H} \quad 0.92872800 \quad 4.97557300 \quad 5.66245200$

$\mathrm{H} \quad 0.13787100 \quad 3.38529200 \quad 5.73693500$

$\mathrm{H} \quad 2.41559500 \quad 1.41121500 \quad 3.12359100$ 


\begin{tabular}{|c|c|c|c|}
\hline $\mathrm{H}$ & .60466800 & 1.46276700 & \\
\hline & 0.98404900 & & \\
\hline & & & 83966300 \\
\hline & 31708900 & -1.08358600 & 3.26146000 \\
\hline & .33292400 & -1.09519300 & 4.73096300 \\
\hline & .03864200 & -4.55857300 & 3.04236100 \\
\hline & 1.05045500 & -3.19275900 & 3.56166600 \\
\hline & 2.18117600 & -2.99781800 & \\
\hline & 3000 & -3.40371400 & 3.80993400 \\
\hline & .70458100 & -4.76064600 & 5200 \\
\hline & .97523500 & -3.27478100 & 4500 \\
\hline & & -1.3 & -1.57 \\
\hline & & -2.4 & 0300 \\
\hline & 5800 & 0.6 & 3600 \\
\hline & & & \\
\hline & 22600 & & 5600 \\
\hline & 1600 & 262400 & 3200 \\
\hline & & & 600 \\
\hline & 00 & & \\
\hline & 00 & & \\
\hline & & & 700 \\
\hline & -8.21 & & 1500 \\
\hline & & & \\
\hline & 11.3 & & 7800 \\
\hline & 4.49 & -3.1 & 500 \\
\hline & 0.9 & -8.0 & -2.18 \\
\hline & 300 & -0.9 & 6900 \\
\hline & 2.9 & & \\
\hline & -1.7 & & 0000 \\
\hline & -6.8 & -3. & 6400 \\
\hline F & -8.6 & -8.7 & 500 \\
\hline & -4.52 & -3.8 & 4900 \\
\hline & -12.4 & & 4500 \\
\hline & 300 & & 48700 \\
\hline & -5.6 & & 5400 \\
\hline & & & \\
\hline & -6.2 & & \\
\hline & -2.8 & -4.2 & 7500 \\
\hline & -3.8 & -5.2 & 4300 \\
\hline & -7.21 & -1.5 & 5800 \\
\hline & -8.03 & -2.9 & \\
\hline & -11.2 & & 9100 \\
\hline & & & 8800 \\
\hline & -3.4 & & \\
\hline & & -0.2 & 4500 \\
\hline & 2.38 & & -3.60 \\
\hline & 7.45 & & 200 \\
\hline & & & \\
\hline & & 5.6 & -2.4 \\
\hline & 10.0 & 53600 & 2300 \\
\hline & & -4.52 & 7100 \\
\hline & & & \\
\hline & & -3.94385400 & 31200 \\
\hline & & -3.9 & \\
\hline & -0.80215800 & -7.86494100 & -2.06460700 \\
\hline & 9300 & -7.00 & 10300 \\
\hline & -7.94026700 & -8.63 & 94700 \\
\hline & -6.98065000 & -8.11440800 & -1.01449000 \\
\hline & & & \\
\hline & -10.30979800 & 7.61796300 & -1.93560300 \\
\hline & -3.90930600 & 6.37474300 & 6.11728200 \\
\hline & & 5.02533200 & 5.66853600 \\
\hline & 1.79163000 & 3.59448500 & 6.38114000 \\
\hline
\end{tabular}

\section{$\operatorname{Int8}_{S}(+3.1 \mathrm{kcal} / \mathrm{mol})$}

C $\quad \begin{array}{llll}6.61151800 & 5.67975700 & 1.42690100\end{array}$

C $\quad 7.08522200 \quad 4.99780600 \quad 0.18575300$

O $\quad 6.50714800 \quad 4.03621300 \quad-0.34411200$

$\mathrm{N} \quad 8.20620200 \quad 5.51004800 \quad-0.36325100$

C $\quad 8.73054000 \quad 4.97331200 \quad-1.59103500$

C $\quad 9.76501200 \quad 3.86579400 \quad-1.41893700$

O $\quad 10.75295600 \quad 3.78185600 \quad-2.14012400$

N $\quad 9.48764300 \quad 2.96987300 \quad-0.42767500$

C $\quad 10.31529200 \quad 1.78512600 \quad-0.35649000$

C $\quad 10.01969900 \quad 0.84233000 \quad-1.51491900$

O $\quad 8.85245900 \quad 0.47638000 \quad-1.73380100$

$\mathrm{N} \quad 11.04984700 \quad 0.33476500 \quad-2.21670500$

C $\quad 10.78208900 \quad-0.74268500 \quad-3.18476400$

C $\quad 10.14476400 \quad-1.95051500 \quad-2.48979800$

O $\quad 9.27146300 \quad-2.61563100 \quad-3.04562800$

C $\quad 12.16899100 \quad-1.01568900 \quad-3.79621200$

C $\quad 12.87497300 \quad 0.34502300 \quad-3.69404100$

C $\quad 12.41627600 \quad 0.88957600 \quad-2.33515400$

$\begin{array}{llll}\mathrm{N} & 10.63729700 & -2.21048700 & -1.25622100\end{array}$

C $\quad 10.12744300 \quad-3.22484900 \quad-0.35254900$

C $\quad 9.62094100 \quad-2.65685100 \quad 0.97995000$

$\begin{array}{llll}\text { O } & 9.82023700 & -3.25720200 & 2.02290900\end{array}$

N $\quad 8.93218800 \quad-1.46999500 \quad 0.90801200$

C $\quad 8.06480500 \quad-1.11474600 \quad 2.03887700$

C $\quad 6.96847700 \quad-2.16863000 \quad 2.29631900$

O $\quad 6.52575700 \quad-2.35507500 \quad 3.42892600$

C $\quad 7.43034900 \quad 0.26532200 \quad 1.78557100$

$\begin{array}{llll}\text { C } & 6.34660300 & 0.69364000 & 2.79255300\end{array}$

$\begin{array}{llll}\text { C } & 6.86908600 & 0.72552400 & 4.23257800\end{array}$

$\begin{array}{llll}\text { C } & 5.77441300 & 2.05462500 & 2.38685800\end{array}$

$\begin{array}{llll}\mathrm{N} & 6.49953200 & -2.77961400 & 1.19253600\end{array}$

C $\quad 5.38306500 \quad-3.69404000 \quad 1.25501900$

C $\quad 4.05716500 \quad-3.73401600 \quad 6.40116000$

$\begin{array}{llll}\text { C } & 2.90403000 & -3.86449900 & 5.39896100\end{array}$

C $\quad 3.16326800 \quad-3.16475100 \quad 4.05913700$

C $\quad 2.11431100 \quad-3.58414400 \quad 3.02307200$

C $\quad 3.21840000 \quad-1.63906800 \quad 4.20162300$

C $\quad 0.14221900 \quad-7.26351600 \quad-2.50147400$

C $\quad 0.35858100 \quad-6.14794700 \quad-1.47653000$

C $\quad \begin{array}{llll}\text { C } & 1.50258000 & -5.17609400 & -1.82639100\end{array}$

C $\quad 1.09391500 \quad-4.19892400 \quad-2.93707600$

C $\quad \begin{array}{llll}1.95167000 & -4.41365000 & -0.57610500\end{array}$

C $\quad-7.98125900 \quad-8.13275700 \quad-0.84351300$

C $\quad-8.57889400 \quad-6.72344000 \quad-0.70226200$

C $\quad-7.68867600 \quad-5.76270200 \quad 0.05240600$

C $\quad-6.39146000 \quad-5.48834800 \quad-0.39834700$

C $\quad-8.12514300 \quad-5.10481100 \quad 1.20744500$

C $\quad-5.56564200 \quad-4.58637200 \quad 0.26480200$

C $\quad-7.32133700 \quad-4.17843400 \quad 1.86967800$

C $\quad-6.04045200 \quad-3.89560200 \quad 1.38729600$

$\begin{array}{llll}\text { O } & -5.28703800 & -2.95189400 & 2.02022200\end{array}$

C $\quad-3.66721300 \quad-4.07896600 \quad-3.88987300$

C $\quad-3.38188400 \quad-3.27281500 \quad-2.60928200$

C $\quad-4.63841600-2.48200400 \quad-2.23254400$

C $\quad-4.69405900 \quad-1.72956000 \quad-0.90832500$

O $\quad-3.81553900 \quad-1.92457900 \quad-0.01643300$

$\begin{array}{llll}\mathrm{O} & -3.81553900 & -1.92457900 & -0.01643300 \\ \mathrm{O} & -5.70655100 & -0.97057000 & -0.78324600\end{array}$ 


\begin{tabular}{|c|c|c|c|}
\hline & 2.50304000 & -0.02430600 & -6.56171800 \\
\hline & 2.76969400 & -0.40426200 & -5.12638800 \\
\hline & 1.84953900 & -0.09211800 & -4.11964200 \\
\hline & 3.95237000 & -1.06222900 & -4.75979200 \\
\hline & 2.10813800 & -0.39218400 & -2.78093300 \\
\hline & 4.20999800 & -1.38669500 & -3.42516000 \\
\hline & 3.29606200 & -1.03472600 & -2.42534800 \\
\hline & 2.55093800 & 5.07396500 & 946700 \\
\hline c & 3.12555400 & 4.48769800 & -2.20261500 \\
\hline C & 3.65471300 & 3.06924100 & -2.39916300 \\
\hline $\mathrm{N}$ & 4.25096800 & 89300 & 773700 \\
\hline $\mathrm{C}$ & -2.40475300 & 2.12946500 & 176100 \\
\hline $\mathrm{C}$ & -1.90120900 & 1.83732700 & 519300 \\
\hline $\mathrm{C}$ & -1.86789500 & 0.32790500 & 36400 \\
\hline $\mathrm{C}$ & -0.54882700 & 2.51914200 & 206000 \\
\hline $\mathrm{C}$ & -7.66947100 & -2.30026100 & 078900 \\
\hline $\mathrm{C}$ & -8.66940000 & -2.11739600 & -3.56027500 \\
\hline $\mathrm{C}$ & -8.98239400 & -3.42537700 & 304400 \\
\hline $\mathrm{S}$ & -10.14563200 & -3.24 & 0200 \\
\hline $\mathrm{C}$ & -9.17969800 & -2.10140000 & -0.37911700 \\
\hline $\mathrm{C}$ & -11.39014400 & 0.75231300 & \\
\hline $\mathrm{C}$ & -10.67888100 & 1.04789100 & 596300 \\
\hline $\mathrm{C}$ & -9.29812300 & 1.28134700 & -2.93176100 \\
\hline $\mathrm{C}$ & -11.36944400 & 1.08 & 98200 \\
\hline $\mathrm{C}$ & -8.62733100 & 1.56443400 & 31700 \\
\hline $\mathrm{C}$ & -10.71739800 & 1.38527600 & -0.5 \\
\hline $\mathrm{C}$ & -9.347318 & 1.640 & -0.54 \\
\hline $\mathrm{O}$ & -8.73321000 & 1.94274300 & 94400 \\
\hline $\mathrm{C}$ & -5.83 & 12000 & 36700 \\
\hline $\mathrm{C}$ & -5.10692900 & $\$ 20300$ & 10700 \\
\hline $\mathrm{C}$ & -5.94858500 & 3.56588 & -0.4 \\
\hline $\mathrm{O}$ & -7.07403700 & 3.90 & -0.0 \\
\hline $\mathrm{O}$ & -5.348191 & 2.45041900 & 93700 \\
\hline $\mathrm{C}$ & -9.52980400 & 5869900 & 34200 \\
\hline $\mathrm{C}$ & -9.94 & $6.38^{\prime}$ & 1900 \\
\hline $\mathrm{C}$ & -10.54626300 & 5.10 & 19000 \\
\hline $\mathrm{O}$ & -8.84 & 6.08 & 08100 \\
\hline $\mathrm{C}$ & -4.28114200 & 5.545352 & 65500 \\
\hline $\mathrm{C}$ & -4.78807100 & 6.39463500 & 74400 \\
\hline $\mathrm{C}$ & -5.154262 & 5.59660100 & 65600 \\
\hline $\mathrm{C}$ & -5.75988800 & 6.52418800 & 46800 \\
\hline $\mathrm{C}$ & -3.95017200 & 4.83936400 & 22100 \\
\hline $\mathrm{C}$ & 1.07912800 & 3.709401 & 38900 \\
\hline S & 1.74989100 & 3.43164400 & 4.04 \\
\hline $\mathrm{C}$ & 2.01550100 & 1.62944000 & 4.12805100 \\
\hline $\mathrm{O}$ & 6.97062400 & -2.18753800 & 748900 \\
\hline $\mathrm{O}$ & 6.13630800 & 0.57006300 & 18900 \\
\hline $\mathrm{O}$ & -5.33906400 & 0.99705000 & -2.72257800 \\
\hline $\mathrm{N}$ & -2.92318600 & -0.09474500 & 2.19560500 \\
\hline $\mathrm{C}$ & -2.28827900 & -2.51723400 & 371600 \\
\hline $\mathrm{C}$ & -1.04293100 & -2.10330100 & 213500 \\
\hline $\mathrm{C}$ & -3.00027000 & 1.30613800 & 98200 \\
\hline $\mathrm{C}$ & -2.35800800 & 1.61332200 & 0.47971000 \\
\hline $\mathrm{C}$ & -0.84638700 & -1.99089500 & 4.96985200 \\
\hline $\mathrm{C}$ & -1.86674200 & -2.28670800 & 525800 \\
\hline $\mathrm{C}$ & -0.99264900 & 1.02851200 & 0.47346900 \\
\hline $\mathrm{C}$ & -1.61920300 & -2.12640700 & 2.57354900 \\
\hline $\mathrm{C}$ & 0.13876400 & 1.80293200 & 0.56366500 \\
\hline & 1.43154800 & 1.21780600 & 0.68251600 \\
\hline $\mathrm{C}$ & -1.62513300 & -0.64610300 & 2.17717000 \\
\hline $\mathrm{C}$ & 1.52787300 & -0.23646000 & 0.72374200 \\
\hline $\mathrm{C}$ & -3.10824300 & -2.69185100 & 4.55255500 \\
\hline & -3.31806700 & -2.80992200 & 5.92576200 \\
\hline
\end{tabular}

8

8

H

\section{$\mathrm{H}$ \\ .}

\section{$\mathrm{H}$}

H

\section{$\mathrm{H}$}

$\mathrm{H}$
$\mathrm{H}$

\section{$\mathrm{H}$}

$\mathrm{H}$

$\mathrm{H}$

\section{$\mathrm{H}$}

\section{H}

\section{$\mathrm{H}$
$\mathrm{H}$}

$\mathrm{H}$
$\mathrm{H}$$$
\mathrm{H}
$$

$\begin{array}{llll}\mathrm{H} & -4.87022300 & -1.76386900 & -3.02634500\end{array}$

$\mathrm{H} \quad-3.07868400 \quad-3.92515300 \quad-1.78440400$

$\begin{array}{llll}\mathrm{H} & -2.54949000 & -2.57862200 & -2.76899400\end{array}$

$\begin{array}{llll}\mathrm{H} & -8.77438600 & -6.31699300 & -1.70297800\end{array}$

$\begin{array}{llll}\mathrm{H} & -9.55515900 & -6.77626800 & -0.20969800\end{array}$

$\begin{array}{llll}0.42947800 & -1.01882100 & 0.61757700\end{array}$

$\begin{array}{lll}-0.91757500 & -0.43622700 & 0.63786000\end{array}$

$\begin{array}{lll}-2.44134200 & -2.61680200 & 8.18076400\end{array}$

$\begin{array}{lll}-6.34758000 & 0.65123200 & 1.05822100\end{array}$

$\begin{array}{llll}2.79854300 & -0.72618400 & 0.80727000\end{array}$

$\begin{array}{rrr}2.52489400 & 1.87597300 & 0.75859500 \\ -0.24979000 & -1.88058500 & 7.04782400\end{array}$

$\begin{array}{llll}0.12417300 & -1.67030000 & 4.60216400\end{array}$

$\begin{array}{llll}3.34814200 & 0.07719300 & 0.90813700\end{array}$

$\begin{array}{lll}-2.37300500 & -2.65551500 & 1.98517700\end{array}$

$\begin{array}{lll}-1.63118600 & -0.98442500 & 0.01611800\end{array}$

$\begin{array}{lll}-2.46125100 & 1.88217300 & 2.64502800\end{array}$

$\begin{array}{lll}-0.64797600 & -2.55693100 & 2.32145600\end{array}$

$\begin{array}{rrr}-0.96036900 & -0.07546500 & 2.83516000\end{array}$

$\begin{array}{lll}-3.92011200 & -2.91049700 & 3.86504300\end{array}$

$\begin{array}{lll}0.07713700 & 2.88640600 & 0.54485600\end{array}$

$\begin{array}{lll}-4.28934800 & -3.12769500 & 6.29935900\end{array}$

$\begin{array}{llll}0.54283600 & -2.09369100 & 0.57672800\end{array}$

$\begin{array}{lll}-3.56474800 & -0.64456600 & 1.62223700\end{array}$

$\begin{array}{lll}-3.34357600 & -2.90728600 & 8.36746000\end{array}$

$\begin{array}{rrr}-4.04285800 & 1.62096700 & 1.88118100\end{array}$

$\begin{array}{llll}-7.31794100 & 0.78730700 & 1.06828600\end{array}$

$\begin{array}{llll}4.90581000 & 3.20936800 & -0.71737300\end{array}$

$\begin{array}{llll}3.50914500 & 2.29015300 & -0.41887500\end{array}$

$\begin{array}{llll}4.82637300 & 1.69266300 & -1.33897400\end{array}$

$\begin{array}{llll}4.43978600 & 3.05183300 & -3.16108100\end{array}$

$\begin{array}{llll}2.87078700 & 2.37422300 & -2.71058900\end{array}$

$\begin{array}{lll}2.36395900 & 4.48153600 & -1.41419000\end{array}$

$\begin{array}{llll}1.61385600 & 4.58246000 & -3.77467500\end{array}$

$\begin{array}{llll}3.25396700 & 4.94690400 & -4.33066000\end{array}$

$\begin{array}{llll}4.66480300 & -1.34694300 & -5.53031900\end{array}$

$\begin{array}{llll}5.10330500 & -1.94365700 & -3.15578600\end{array}$

$3.48919400-1.28195100-1.38740600$

$1.36779500 \quad-0.15619800 \quad-2.02425500$

$\begin{array}{llll}0.18697500 & 2.23366000 & -3.84229000\end{array}$

$\begin{array}{llll}-0.65212600 & 3.60995700 & -3.12404900\end{array}$

$\begin{array}{llll}-0.14313500 & 2.25440800 & -2.10091400\end{array}$

$\begin{array}{llll}-2.62301100 & 2.27032000 & -2.62922500\end{array}$

$\begin{array}{llll}-1.45964400 & 0.09818300 & -2.08514800\end{array}$

$\begin{array}{lll}-2.88384500 & -0.07160300 & -3.12463100 \\ -1.25314700 & -0.19461100 & -3.81634100\end{array}$

$\begin{array}{lll}-1.25314700 & -0.19461100 & -3.81634100 \\ -8.24340300 & -1.41105900 & -2.84222800\end{array}$

$\begin{array}{llll}-9.60143200 & -1.66867200 & -3.91664900\end{array}$

$\begin{array}{llll}-8.06136400 & -3.88305400 & -2.45080000\end{array}$

$\begin{array}{lll}-9.45406600 & -4.14878300 & -3.50742600\end{array}$

$\begin{array}{lll}-9.22159200 & -1.08204500 & -0.76167800\end{array}$

$\begin{array}{lll}-9.62860100 & -2.12942800 & 0.61538200\end{array}$

$\begin{array}{lll}-8.12150100 & 2.70691800 & 0.48671100\end{array}$

$\begin{array}{lll}-7.55258900 & 1.69615300 & -1.76502600\end{array}$

$\begin{array}{llll}-11.25778400 & 1.43558100 & 0.39418400\end{array}$

$\begin{array}{lll}-12.43859700 & 0.89430800 & -1.72938700\end{array}$

$\begin{array}{llll}-8.72529500 & 1.22917500 & -3.85403200\end{array}$ 


\begin{tabular}{|c|c|c|c|}
\hline $\mathrm{H}$ & -9.12862300 & -5.29719600 & \\
\hline & & -5.98915700 & -1.28621800 \\
\hline & -4.55879000 & -4.39518900 & -0.08611400 \\
\hline & -7.68149600 & -3.64308300 & 600 \\
\hline & -4.66446300 & -2.56847400 & \\
\hline & 2.68395300 & -3.63449900 & -0.81006800 \\
\hline & & -3.92883400 & -0.10 \\
\hline & 2.39110900 & -5.08376000 & \\
\hline & 1.93198500 & 93300 & 400 \\
\hline & 0.73476400 & -4.722 & -3.8285 \\
\hline & 0.28608300 & -3.54 & -2.5917 \\
\hline & 537200 & $-5.76 c$ & 300 \\
\hline & -0.56488000 & -5.56 & 400 \\
\hline & 0.5 & -6.6 & -0.4 \\
\hline & & -2.5 & \\
\hline & 8.6 & -1.10 & 500 \\
\hline & & & \\
\hline & 6.95 & $0.2^{\prime}$ & \\
\hline & 6.5 & 2.82 & \\
\hline & & & \\
\hline & 4.967 & & \\
\hline & & & \\
\hline & & & \\
\hline & 7.1 & -0.2 & 700 \\
\hline & & -0.0 & \\
\hline & & -1.2 & 800 \\
\hline & 9.30 & -3.7 & -0 . \\
\hline & 10.8 & & 100 \\
\hline & 11.3 & & \\
\hline & & & \\
\hline & & & \\
\hline & 12.6 & -1.7 & 900 \\
\hline & 12.5 & & \\
\hline & $12.3^{\prime}$ & & \\
\hline & 12.0 & -1.3 & \\
\hline & 10. & & \\
\hline & & & \\
\hline & & & \\
\hline & & & \\
\hline & -4.3 & & \\
\hline & -9.8 & & -1 . \\
\hline & -10.7 & & \\
\hline & & & \\
\hline & 900 & & 000 \\
\hline & -3.41354700 & & \\
\hline & & & \\
\hline & -4.02 & & \\
\hline & -5.0 & & \\
\hline $\mathrm{H}$ & -6.00 & 5.97 & \\
\hline & -6.68641800 & 6.989 & \\
\hline & & & \\
\hline & -4.241 & 4.25 & 240800 \\
\hline & -3.160 & & \\
\hline & -5.91732600 & 4.85416700 & 3.34670300 \\
\hline & 0.86382200 & 4.77582400 & 5.82078900 \\
\hline & & 3.14730800 & \\
\hline & 2.48723700 & 1.34489400 & 3.18844700 \\
\hline & & $1.36 ?$ & \\
\hline & & 1.09305900 & 4.23245300 \\
\hline & 4.05779000 & -1.32383400 & 4.82658800 \\
\hline & & & 3.22190000 \\
\hline & 2.29462700 & -1.25267500 & 4.64944500 \\
\hline & 2.17202600 & -4.65757800 & 2.81214800 \\
\hline
\end{tabular}

H $\quad 1.10317500 \quad-3.38125000 \quad 3.39141200$

$\begin{array}{llll}\mathrm{H} & 2.24634500 & -3.04480900 & 2.08118700\end{array}$

$\mathrm{H} \quad 4.15219200 \quad-3.48247000 \quad 3.71139400$

$\mathrm{H} \quad 2.71805700 \quad-4.93052500 \quad 5.21040200$

$\mathrm{H} \quad 1.97490200 \quad-3.47209800 \quad 5.83662000$

$\mathrm{H} \quad 6.69103500 \quad-1.26278300 \quad-1.63133900$

$\mathrm{H} \quad 7.66743000 \quad-2.35690600 \quad-2.18885700$

$\mathrm{H} \quad 7.09279300 \quad 0.77806800 \quad-1.96444300$

$\mathrm{H} \quad 5.85717600 \quad 0.28128100 \quad-2.81995300$

$\mathrm{H} \quad-5.05656200 \quad 1.54798100 \quad-1.97740800$

$\mathrm{H} \quad-6.12194800 \quad-0.13070700 \quad 0.44144900$

H $\quad 7.88635900 \quad 4.54397200 \quad-2.14018900$

$\mathrm{H} \quad 5.92073300 \quad 5.02950400 \quad 1.96325300$

$\mathrm{H} \quad-6.60239800 \quad 5.92049800 \quad-2.00771700$

$\mathrm{H} \quad-8.75335700 \quad 6.90797100 \quad-1.60777900$

$\mathrm{H} \quad-8.25532900 \quad 5.48046800 \quad 0.49231000$

$\mathrm{H} \quad 4.26369000 \quad-2.67682200 \quad 6.60652200$

H $\quad 11.36157200 \quad 2.08403500 \quad-0.31859300$

$\mathrm{H} \quad 4.49612900 \quad-3.20665300 \quad 1.66155100$

$\mathrm{H} \quad 0.95086900 \quad-8.00084400 \quad-2.46740900$

$\mathrm{H} \quad 2.97326300 \quad-0.72972500 \quad-7.25313700$

H $\quad 2.91453300 \quad 0.96808400 \quad-6.78637700$

$\mathrm{H} \quad-1.80723300 \quad 1.61359600 \quad-5.51150500$

$\mathrm{H} \quad-6.82332000 \quad-2.90571800 \quad-4.35312700$

$\mathrm{H} \quad-8.60124500 \quad-8.74464800 \quad-1.50922500$

$\mathrm{H} \quad-4.54067500 \quad-3.65665600 \quad-4.40098900$

$\mathrm{H} \quad-12.46887500 \quad 0.66026200 \quad-4.09960000$

$\mathrm{H} \quad-11.03998400 \quad-0.18624100 \quad-4.69702300$

$\mathrm{H} \quad-5.66328200 \quad 0.20641900 \quad-2.24657200$

$\mathrm{H} \quad-5.03770100 \quad 5.89634600 \quad-2.86919000$

$\mathrm{H} \quad-6.28263700 \quad 4.66310100 \quad-3.22956900$

$\mathrm{H} \quad-2.88497200 \quad-4.08175100 \quad-4.65700800$

$\mathrm{H} \quad-3.89812700 \quad-5.14192300 \quad-3.74782000$

$\mathrm{H} \quad-7.23561800 \quad-1.34590200 \quad-5.01774200$

$\mathrm{H} \quad-8.05359700 \quad-2.79986300 \quad-5.59652800$

$\mathrm{H} \quad-11.22232000 \quad 1.53914600 \quad-4.99436500$

$\mathrm{H} \quad-2.38362200 \quad 3.20469900 \quad-4.95786500$

$\mathrm{H} \quad-3.45897800 \quad 1.85818100 \quad-4.86575700$

H $\quad 1.43488500 \quad 0.01382300 \quad-6.79727200$

$\mathrm{H} \quad 2.36316200 \quad 6.14814800 \quad-3.39870900$

$\mathrm{H} \quad 7.43232300 \quad 5.95682300 \quad 2.09650300$

$\mathrm{H} \quad 6.07539500 \quad 6.59718000 \quad 1.15929500$

$\mathrm{H} \quad 9.15438300 \quad 5.77030400 \quad-2.21113600$

H $\quad 10.07520600 \quad 1.23120600 \quad 0.55501900$

H $\quad 5.60956900 \quad-4.57454800 \quad 1.86541500$

$\mathrm{H} \quad 5.16070300 \quad-4.02384100 \quad 0.23844600$

$\mathrm{H} \quad 4.97316500 \quad-4.14025400 \quad 5.96030300$

$\mathrm{H} \quad 3.73187300 \quad-4.16622400 \quad 7.35367100$

$\mathrm{H} \quad-0.80705300 \quad-7.78096200 \quad-2.33801500$

$\mathrm{H} \quad 0.09134100 \quad-6.87464700 \quad-3.52448500$

$\mathrm{H} \quad-7.94196800 \quad-8.64778500 \quad 0.12210600$

$\mathrm{H} \quad-6.98432400 \quad-8.07534300 \quad-1.29201800$

$\mathrm{H} \quad-9.12208100 \quad 8.30929500 \quad-0.59736700$

$\mathrm{H} \quad-10.33507300 \quad 7.67512600 \quad-1.65917500$

$\mathrm{H} \quad-3.92587100 \quad 6.15954700 \quad 6.33986600$

$\mathrm{H} \quad-5.03369300 \quad 4.82516900 \quad 5.84512500$

H $\quad \begin{array}{llll}1.80705400 & 3.42507700 & 6.49381000\end{array}$

H $\quad-5.88586600 \quad 1.66891500 \quad 0.43260000$ 
$\mathrm{TS5}_{S}(+15.7 \mathrm{kcal} / \mathrm{mol})$

$\begin{array}{llll}\text { C } & 6.71445000 & 5.60200400 & 1.68883200\end{array}$

$\begin{array}{llll}\mathrm{C} & 7.15854700 & 5.01446300 & 0.39175800\end{array}$

$\begin{array}{lllll}\mathrm{O} & 6.43653500 & 4.29431500 & -0.30510600\end{array}$

$\mathrm{N} \quad 8.41504300 \quad 5.34050100 \quad-0.00406700$

C $\quad 8.91086300 \quad 4.89056200 \quad-1.27251500$

C $\quad 9.91336800 \quad 3.74551800 \quad-1.22047400$

O $\quad 10.71183500 \quad 3.56016600 \quad-2.13504000$

$\begin{array}{llll}\mathrm{N} & 9.83726600 & 2.91766300 & -0.14550200\end{array}$

C $\quad 10.60124200 \quad 1.69057600 \quad-0.19631100$

C $\quad 10.19898300 \quad 0.83806600 \quad-1.39822100$

$\begin{array}{lllll}\mathrm{O} & 9.00838100 & 0.56236200 & -1.59707200\end{array}$

$\mathrm{N} \quad 11.18239500 \quad 0.28800300 \quad-2.14283100$

C $\quad 10.82331800 \quad-0.73191100 \quad-3.14202700$

C $\quad 10.17348400 \quad-1.94939000 \quad-2.47569100$

$\begin{array}{llll}\text { O } & 9.28175300 & -2.58049500 & -3.04363300\end{array}$

C $\quad 12.16583000 \quad-1.03105700 \quad-3.83387100$

$\begin{array}{llll}\text { C } & 12.91777500 & 0.30361100 & -3.72921100\end{array}$

$\begin{array}{llll}\text { C } & 12.55146300 & 0.81726600 & -2.33052200\end{array}$

N $\quad 10.68244900 \quad-2.25670000 \quad-1.26044300$

C $\quad 10.14548600 \quad-3.25000600 \quad-0.34714600$

C $\quad 9.61495800 \quad-2.63322900 \quad 0.95725700$

$\begin{array}{llll}\mathrm{O} & 9.84111800 & -3.15648700 & 2.03622200\end{array}$

N $\quad 8.88727000 \quad-1.48205500 \quad 0.80489000$

C $\quad 8.01419200 \quad-1.04188700 \quad 1.89382900$

$\begin{array}{llll}\text { C } & 6.94750300 & -2.08733100 & 2.28381500\end{array}$

$\begin{array}{llll}\text { O } & 6.45294400 & -2.08199800 & 3.40974700\end{array}$

C $\quad 7.28141200 \quad 0.25259800 \quad 1.48735200$

$\begin{array}{llll}\text { C } & 6.69654600 & 1.02779200 & 2.68207400\end{array}$

$\begin{array}{llll}\text { C } & 7.78617200 & 1.84822100 & 3.38200800\end{array}$

$\begin{array}{llll}\text { C } & 5.53660000 & 1.92441900 & 2.24140800\end{array}$

$\begin{array}{llll}\mathrm{N} & 6.54847100 & -2.89685700 & 1.28102000\end{array}$

C $\quad 5.39303500 \quad-3.75632800 \quad 1.42432400$

$\begin{array}{llll}\text { C } & 3.92051800 & -3.81250300 & 6.52995800\end{array}$

$\begin{array}{llll}\text { C } & 2.79011200 & -3.97718100 & 5.50510900\end{array}$

C $\quad 3.02093600 \quad-3.22471800 \quad 4.18840700$

$\begin{array}{llll}\text { C } & 2.17868500 & -3.83852800 & 3.06585100\end{array}$

$\begin{array}{llll}\text { C } & 2.74149700 & -1.72260200 & 4.32746700\end{array}$

$\begin{array}{llll}\text { C } & 0.22354700 & -7.24777600 & -2.50202800\end{array}$

C $\quad 0.28050200 \quad-6.08365000 \quad-1.50259500$

C $\quad \begin{array}{llll}\text { C } & 1.38531400 & -5.03439500 & -1.71619700\end{array}$

$\begin{array}{llll}\text { C } & 1.31743300 & -4.37846700 & -3.10199100\end{array}$

C $\quad 1.28243300 \quad-3.96436700 \quad-0.62271800$

C $\quad-7.95251800 \quad-8.03956200 \quad-1.08073200$

C $\quad-8.59828000 \quad-6.64718500 \quad-1.00824500$

C $\quad-7.82942900 \quad-5.59711500 \quad-0.23477100$

C $\quad-6.48890300 \quad-5.30667000 \quad-0.51906600$

$\begin{array}{llll}\text { C } & -8.45948500 & -4.83360200 & 0.75536400\end{array}$

$\begin{array}{llll}\text { C } & -5.80863500 & -4.28578700 & 0.14375900\end{array}$

$\begin{array}{llll}\text { C } & -7.80070000 & -3.79692300 & 1.41176600\end{array}$

C $\quad-6.47463600 \quad-3.49876500 \quad 1.09045800$

$\begin{array}{llll}\text { O } & -5.87512000 & -2.45197500 & 1.72958700\end{array}$

C $\quad-3.51075600 \quad-4.01474800 \quad-3.97798800$

C $\quad-2.98986200 \quad-3.38422300 \quad-2.67030200$

$\begin{array}{llll}\text { C } & -3.91572300 & -2.31609900 & -2.09480000\end{array}$

C $\quad-3.39444400 \quad-1.65712000 \quad-0.82520300$

O $\quad-2.13406200 \quad-1.72478900 \quad-0.61831700$

O $\quad-4.21375300 \quad-1.07283400 \quad-0.06671200$

$\begin{array}{llll}\text { C } & 2.77521200 & -0.01059800 & -6.44807500\end{array}$

$\begin{array}{llll}\text { C } & 3.00866000 & -0.44496700 & -5.02242400\end{array}$

$\begin{array}{llll}\text { C } & 2.15284700 & -0.02776300 & -3.99766800\end{array}$ $\begin{array}{llll}4.09888100 & -1.25716800 & -4.68297000\end{array}$

$\begin{array}{llll}2.37414000 & -0.39417200 & -2.66916900\end{array}$

$\begin{array}{llll}4.32448600 & -1.63779000 & -3.35707500\end{array}$

$\begin{array}{llll}3.46818400 & -1.19769500 & -2.34308400\end{array}$

$\begin{array}{llll}2.79008600 & 5.06885600 & -3.35515000\end{array}$

$\begin{array}{llll}3.35295800 & 4.43748000 & -2.08080500\end{array}$

$3.94115000 \quad 3.05900300 \quad-2.36862600$

$\begin{array}{llll}4.44597000 & 2.42253800 & -1.12090100\end{array}$

$\begin{array}{llll}-2.15900600 & 2.18482700 & -4.76545600\end{array}$

$\begin{array}{lll}-1.66524500 & 1.87782000 & -3.34604000\end{array}$

$\begin{array}{lll}-1.51654000 & 0.37071200 & -3.12498000\end{array}$

$\begin{array}{lll}-0.35758000 & 2.62623000 & -3.05105400\end{array}$

$-7.46953000 \quad-2.18818200 \quad-4.89156700$

$\begin{array}{lll}-8.55333200 & -2.00344100 & -3.82658600\end{array}$

$\begin{array}{lll}-8.95237000 & -3.32151900 & -3.16068700\end{array}$

$\begin{array}{lll}-10.43003600 & -3.21161800 & -2.08304100\end{array}$

$\begin{array}{llll}-9.88164400 & -1.94457200 & -0.88545600\end{array}$

$\begin{array}{lll}-11.16901100 & 0.90145300 & -4.53020100\end{array}$

$\begin{array}{llll}-10.58398400 & 1.24391800 & -3.18545200\end{array}$

$\begin{array}{llll}-9.21803700 & 1.51609200 & -3.04739300\end{array}$

$\begin{array}{lll}-11.38341500 & 1.32748200 & -2.04035000\end{array}$

$\begin{array}{lll}-8.67317800 & 1.89375100 & -1.82249600\end{array}$

$\begin{array}{llll}-10.85663200 & 1.71362800 & -0.80940600\end{array}$

$\begin{array}{llll}-9.50237400 & 2.02683300 & -0.70016200\end{array}$

$\begin{array}{lll}-9.03107800 & 2.47190800 & 0.50907900\end{array}$

$\begin{array}{lll}-5.61968700 & 5.35718600 & -2.53493900\end{array}$

$\begin{array}{llll}-4.97210100 & 4.49523300 & -1.44139600\end{array}$

$\begin{array}{llll}-5.91469100 & 3.68572200 & -0.58341700\end{array}$

$\begin{array}{llll}-7.02653500 & 4.11208000 & -0.24215000\end{array}$

$\begin{array}{llll}-5.42706900 & 2.52393900 & -0.22937400\end{array}$

$\begin{array}{llll}-9.33315500 & 7.47841200 & -1.16882600\end{array}$

$\begin{array}{llll}-9.84546900 & 6.54902600 & -0.07142900\end{array}$

$\begin{array}{lll}-10.50935400 & 5.30075200 & -0.65167600\end{array}$

$\begin{array}{lll}-8.79942800 & 6.20735100 & 0.83323400\end{array}$

$\begin{array}{lll}-4.29114700 & 5.56099800 & 5.45535000\end{array}$

$\begin{array}{lll}-4.76847100 & 6.43753100 & 4.29387900\end{array}$

$\begin{array}{lll}-5.08164100 & 5.67540200 & 2.99218500\end{array}$

$\begin{array}{lll}-5.70776500 & 6.61973600 & 1.95932700\end{array}$

$\begin{array}{lll}-3.83613400 & 4.99541700 & 2.40717500\end{array}$

$\begin{array}{llll}1.04125700 & 3.66590200 & 5.82001000\end{array}$

$\begin{array}{lll}2.08566200 & 3.62718000 & 4.31835100\end{array}$

$\begin{array}{lll}1.87354700 & 1.88295600 & 3.82342700\end{array}$

$\begin{array}{llll}\text { O } & 7.00904400 & -2.31737800 & -1.48030700\end{array}$

$\begin{array}{lllll}\mathrm{O} & 6.29801200 & 0.41518000 & -2.01743200\end{array}$

O $\quad-6.79894700 \quad-0.75868700 \quad-0.74555200$

$\begin{array}{llll}\mathrm{N} & -3.14178100 & 0.00490500 & 2.56675900\end{array}$

C $\quad-3.23606700 \quad-3.30350000 \quad 6.77063300$

$\begin{array}{llll}\text { C } & -1.90376300 & -3.27742500 & 6.34993200\end{array}$

C $\quad-2.93702000 \quad 1.42588300 \quad 2.34829000$

C $\quad-2.20082400 \quad 1.71621900 \quad 1.01965300$

C $\quad-1.61179900 \quad-2.95767400 \quad 5.02778300$

$\begin{array}{llll}\text { C } & -2.62280700 & -2.66007200 & 4.10432500\end{array}$

C $\quad-0.91611700 \quad 0.93264900 \quad 0.95868400$

$\begin{array}{llll}\text { C } & -2.26612700 & -2.27295400 & 2.69046400\end{array}$

$\begin{array}{llll}\text { C } & 0.26896400 & 1.53403400 & 0.55734400\end{array}$

$\begin{array}{llll}\text { C } & 1.49803400 & 0.83321000 & 0.59049800\end{array}$

C $\quad-1.92182500 \quad-0.77470100 \quad 2.56770500$

$\begin{array}{llll}\text { C } & 1.46880500 & -0.55416100 & 0.97004900\end{array}$

$\begin{array}{llll}\text { C } & -3.94964600 & -2.68870000 & 4.54197600\end{array}$

C $\quad-4.25706200 \quad-3.01019900 \quad 5.86338400$

C $\quad 0.31614900 \quad-1.15093100 \quad 1.38217600$

C $\quad-0.94664600 \quad-0.44941000 \quad 1.36761900$

O $\quad-3.48287100 \quad-3.62663900 \quad 8.08052000$ 


\begin{tabular}{|c|c|c|c|}
\hline & .79304700 & & \\
\hline $\mathrm{O}$ & & & \\
\hline & & & \\
\hline & .11957200 & & \\
\hline & -0.57290000 & -2.93918600 & \\
\hline & 3.29388300 & -0.44511600 & 0.720 \\
\hline & -3.09401500 & -2.50221500 & 200 \\
\hline & -1.62846500 & -1.06772400 & \\
\hline & & & \\
\hline & & -2.876 & \\
\hline & 58600 & 2.7871 & \\
\hline & -1.3 & -0.4931 & \\
\hline & -4.7 & -2.4 & \\
\hline & 0.28 & 2.5 & \\
\hline & -5.2 & -3.0 & \\
\hline & 0.3 & -2.17 & 1.7 \\
\hline & -3.7 & -0.3 & \\
\hline & -4.4 & -3.6 & \\
\hline & -2.8 & & \\
\hline & -3.9 & & \\
\hline & -7.7 & & \\
\hline & & & \\
\hline & & & \\
\hline & & & \\
\hline & & & \\
\hline & & & \\
\hline & & & \\
\hline & & & \\
\hline & & & \\
\hline & & & \\
\hline & & & \\
\hline & & & \\
\hline & & -1.4 & \\
\hline & & -0. & \\
\hline & & & \\
\hline & & & \\
\hline & & & \\
\hline & & & \\
\hline & & & \\
\hline & -1.2 & & \\
\hline & & & \\
\hline & & & \\
\hline & $-8.1 \xi$ & & \\
\hline & -9.4 & & 300 \\
\hline & & & -2.5 \\
\hline & -9.2 & & \\
\hline & -8.8 & & -0 . \\
\hline & -9.8 & -0.9 & \\
\hline & & & \\
\hline & -8.3 & & \\
\hline & -7.6 & & \\
\hline $\mathrm{H}$ & -11. & & 300 \\
\hline & -12.4 & & 2900 \\
\hline & & & \\
\hline & -4.91 & -2.70 & 200 \\
\hline & & -1.5 & \\
\hline $\mathrm{H}$ & -2.82967400 & -4.15895000 & -1.912 \\
\hline & -2.00 & -2.92936800 & -2.82 \\
\hline & & -6.28197600 & -2.03 \\
\hline & -9.60330500 & -6.73279900 & -0.58214900 \\
\hline & & -5.03441000 & \\
\hline $\mathrm{H}$ & & -5.88789200 & -1.26885300 \\
\hline & -4.75920500 & -4.10512400 & -0.06310000 \\
\hline
\end{tabular}
$-5.18457300-2.07097200$ $2.09883400 \quad-3.24004500$ $0.34279900-3.40679000$ $1.30360700-4.41514600$ $2.07366500-3.59392700$ $\begin{array}{lll}1.47653000 & -5.09695000 & -3.91178400\end{array}$ $\begin{array}{llll}0.33663400 & -3.91046000 & -3.25500700\end{array}$ $\begin{array}{llll}2.36089500 & -5.53621200 & -1.62278100\end{array}$ $-0.68356100 \quad-5.55561700 \quad-1.53471400$ $\begin{array}{llll}0.36730000 & -6.48153700 & -0.48334300\end{array}$ $\begin{array}{llll}6.90222300 & -2.76149400 & 0.33860700\end{array}$ $\begin{array}{lll}8.62636000 & -0.88647000 & 2.78421400\end{array}$ $\begin{array}{llll}7.96704500 & 0.89501200 & 0.91898800\end{array}$ $\begin{array}{llll}6.48306200 & -0.01704500 & 0.78519300\end{array}$ $\begin{array}{lll}5.87346400 & 2.65107800 & 1.49282600\end{array}$ $\begin{array}{lll}4.71866200 & 1.34783200 & 1.79965700\end{array}$ $\begin{array}{lll}5.12099300 & 2.48188700 & 3.08786600\end{array}$ $\begin{array}{llll}8.17573100 & 2.63170700 & 2.71303000\end{array}$ $\begin{array}{llll}7.39556300 & 2.34732900 & 4.27397500\end{array}$ $\begin{array}{lll}8.63207100 & 1.22740000 & 3.69418100\end{array}$ $\begin{array}{llll}6.31136700 & 0.28964700 & 3.39369300\end{array}$ $\begin{array}{llll}8.56091500 & -1.27702700 & -0.13304800\end{array}$ $\begin{array}{llll}9.33331500 & -3.76903900 & -0.86278300\end{array}$ $\begin{array}{lll}10.90718300 & -3.98200400 & -0.07044500\end{array}$ $\begin{array}{llll}11.36150200 & -1.61203700 & -0.88280400\end{array}$ $\begin{array}{llll}13.23112500 & 0.41546100 & -1.56900200\end{array}$ $\begin{array}{llll}13.99667300 & 0.19933000 & -3.86219300\end{array}$ $12.69955500 \quad-1.81450000 \quad-3.28511800$ $\begin{array}{llll}12.54744100 & 1.00523100 & -4.48321500\end{array}$ $\begin{array}{llll}12.53246000 & 1.90553100 & -2.26686400\end{array}$ $12.02327400 \quad-1.37629800 \quad-4.85875700$ $\begin{array}{llll}10.08316800 & -0.33733400 & -3.84306700\end{array}$ $\begin{array}{llll}9.04409100 & 2.97504800 & 0.47614700\end{array}$ $\begin{array}{llll}8.98457000 & 5.93259400 & 0.58118700\end{array}$ $\begin{array}{llll}-4.43306300 & 5.14836100 & -0.74223400\end{array}$ $\begin{array}{llll}-4.22599000 & 3.81529500 & -1.86140100\end{array}$ $\begin{array}{llll}-9.81795500 & 4.76880300 & -1.31463800\end{array}$ $\begin{array}{llll}-10.79576900 & 4.61717600 & 0.15027100\end{array}$ $\begin{array}{llll}-11.39976900 & 5.55666800 & -1.23575100\end{array}$ $\begin{array}{lll}-10.57865600 & 7.08994500 & 0.53992500\end{array}$ $\begin{array}{llll}-3.42224900 & 4.96370100 & 5.15573900\end{array}$ $\begin{array}{lll}-5.66536700 & 6.99119900 & 4.59959300\end{array}$ $\begin{array}{lll}-4.00416500 & 7.19691100 & 4.07323200\end{array}$ $\begin{array}{lll}-5.03423000 & 7.46082900 & 1.74994800\end{array}$ $\begin{array}{lll}-5.90178000 & 6.10182200 & 1.01687200\end{array}$ $\begin{array}{lll}-6.66582400 & 7.02210100 & 2.29829200\end{array}$ $\begin{array}{lll}-3.36005500 & 4.31406800 & 3.11662100\end{array}$ $\begin{array}{lll}-4.09096400 & 4.40434900 & 1.52217600\end{array}$ $\begin{array}{lll}-3.09038800 & 5.74444500 & 2.11093400\end{array}$ $\begin{array}{lll}-5.81642800 & 4.89107900 & 3.22567700\end{array}$ $\begin{array}{llll}1.08293600 & 4.68112300 & 6.21892600\end{array}$ $\begin{array}{lll}0.00391700 & 3.42061400 & 5.57767600\end{array}$ $\begin{array}{llll}2.46058600 & 1.74391100 & 2.91464500\end{array}$ $\begin{array}{llll}2.24013700 & 1.20257200 & 4.59536900\end{array}$ $\begin{array}{llll}0.82816400 & 1.66369100 & 3.59639200\end{array}$ $\begin{array}{llll}3.33005400 & -1.27975200 & 5.13759900\end{array}$ $\begin{array}{llll}2.99143100 & -1.19844300 & 3.40229900\end{array}$ $\begin{array}{llll}1.68020800 & -1.54150400 & 4.54173600\end{array}$ $\begin{array}{llll}2.43952800 & -4.89120100 & 2.90680000\end{array}$ $\begin{array}{llll}1.11089100 & -3.80469700 & 3.31767100\end{array}$ $\begin{array}{lll}2.32060900 & -3.30429500 & 2.12581700\end{array}$ $\begin{array}{llll}4.08146300 & -3.32971400 & 3.92808700\end{array}$ 


\begin{tabular}{crrc}
$\mathrm{H}$ & 2.67129200 & -5.04801900 & 5.29207000 \\
$\mathrm{H}$ & 1.83459300 & -3.65187800 & 5.94177200 \\
$\mathrm{H}$ & 6.69254800 & -1.40756500 & -1.62303900 \\
$\mathrm{H}$ & 7.68800700 & -2.48850100 & -2.16014000 \\
$\mathrm{H}$ & 7.24840100 & 0.64337300 & -2.03879800 \\
$\mathrm{H}$ & 6.01695300 & 0.16525600 & -2.91067700 \\
$\mathrm{H}$ & -6.97097400 & -0.11337300 & -1.44294600 \\
$\mathrm{H}$ & -6.86193900 & 0.23107700 & 0.70620000 \\
$\mathrm{H}$ & 8.04580800 & 4.51919600 & -1.83096700 \\
$\mathrm{H}$ & 6.04304100 & 4.90124300 & 2.18781000 \\
$\mathrm{H}$ & -6.39278700 & 6.00804300 & -2.12276600 \\
$\mathrm{H}$ & -8.54976800 & 6.98127000 & -1.75337900 \\
$\mathrm{H}$ & -8.15890200 & 5.65668200 & 0.35535600 \\
$\mathrm{H}$ & 4.11342000 & -2.75128800 & 6.72691200 \\
$\mathrm{H}$ & 11.66835900 & 1.91580000 & -0.19465300 \\
$\mathrm{H}$ & 4.56318700 & -3.20953700 & 1.87271000 \\
$\mathrm{H}$ & 1.04996300 & -7.95539200 & -2.39781500 \\
$\mathrm{H}$ & 3.26186500 & -0.69076400 & -7.15284400 \\
$\mathrm{H}$ & 3.19189700 & 0.98919500 & -6.62621000 \\
$\mathrm{H}$ & -1.55724700 & 1.67156500 & -5.52334100 \\
$\mathrm{H}$ & -6.65440800 & -2.80844800 & -4.49816500 \\
$\mathrm{H}$ & -8.53513900 & -8.66937000 & -1.76423700 \\
$\mathrm{H}$ & -4.37514800 & -3.49306500 & -4.39644900 \\
$\mathrm{H}$ & -12.25181800 & 0.76635400 & -4.46742300 \\
$\mathrm{H}$ & -10.74653600 & -0.02531900 & -4.93318800 \\
$\mathrm{H}$ & -5.81930600 & -0.85532500 & -0.67177200 \\
$\mathrm{H}$ & -4.80467600 & 5.96866900 & -2.93542300 \\
$\mathrm{H}$ & -6.05145500 & 4.75091300 & -3.33865000 \\
$\mathrm{H}$ & -2.70745200 & -4.02080100 & -4.72295000 \\
$\mathrm{H}$ & -3.75716200 & -5.07554000 & -3.84939000 \\
$\mathrm{H}$ & -7.01682500 & -1.23666500 & -5.19018000 \\
$\mathrm{H}$ & -7.83327100 & -2.67834200 & -5.80095500 \\
$\mathrm{H}$ & -10.97195400 & 1.69076400 & -5.26227100 \\
$\mathrm{H}$ & -2.12078900 & 3.26131200 & -4.96438900 \\
$\mathrm{H}$ & -3.21248900 & 1.92593800 & -4.91114500 \\
$\mathrm{H}$ & 1.71475400 & 0.04046200 & -6.71437000 \\
$\mathrm{H}$ & 2.61023100 & 6.14435600 & -3.25241000 \\
$\mathrm{H}$ & 7.51879000 & 5.86656800 & 2.38303900 \\
$\mathrm{H}$ & 6.13471900 & 6.50896600 & 1.48263200 \\
$\mathrm{H}$ & -6.03463500 & 1.96513500 & 0.42940500 \\
$\mathrm{H}$ & 9.36085400 & 5.68678400 & -1.87464000 \\
$\mathrm{H}$ & 10.36975400 & 1.09275700 & 0.68962800 \\
$\mathrm{H}$ & 5.59199100 & -4.64357800 & 2.03501200 \\
$\mathrm{H}$ & 5.09285700 & -4.08820800 & 0.42800300 \\
$\mathrm{H}$ & 4.84399400 & -4.22649900 & 6.11306800 \\
$\mathrm{H}$ & 3.56334400 & -4.24701000 & 7.47027300 \\
$\mathrm{H}$ & -0.73546800 & -7.75599700 & -2.36860800 \\
$\mathrm{H}$ & 0.20582300 & -6.85234500 & -3.52334200 \\
$\mathrm{H}$ & -7.94638100 & -8.56063600 & -0.11770800 \\
$\mathrm{H}$ & -6.94280400 & -7.99017000 & -1.50047900 \\
$\mathrm{H}$ & -10.115219000 & 8.41196800 & -0.76562400 \\
& -3.95270800 & 6.16588100 & -1.86547900 \\
\hline
\end{tabular}

\section{$\mathrm{E}: \mathrm{P}_{S}(\mathbf{- 0 . 7 \mathrm { kcal } / \mathrm { mol } )}$}

$\begin{array}{llll}\text { C } & 6.92047800 & 5.65603000 & 1.68641300 \\ \text { C } & 7.41339800 & 4.93314900 & 0.47206500 \\ \text { O } & 6.89690800 & 3.90762700 & 0.01460600\end{array}$
$\begin{array}{llll}\mathrm{N} & 8.49685200 & 5.50130300 & -0.11261400\end{array}$

$\begin{array}{llll}\text { C } & 9.05912200 & 4.95682000 & -1.31928100\end{array}$

$\begin{array}{llll}\text { C } & 10.08410000 & 3.84638400 & -1.12320700\end{array}$

$\begin{array}{llll}\mathrm{O} & 11.08049400 & 3.74812400 & -1.83346200\end{array}$

$\begin{array}{llll}\mathrm{N} & 9.77907500 & 2.95819900 & -0.13512800\end{array}$

$\begin{array}{llll}\text { C } & 10.56117300 & 1.74340400 & -0.08146300\end{array}$

$\begin{array}{llll}\text { C } & 10.23604300 & 0.84354500 & -1.26738000\end{array}$

$\begin{array}{lllll}\mathrm{O} & 9.05933900 & 0.53786000 & -1.51222900\end{array}$

$\mathrm{N} \quad 11.25734500 \quad 0.30801500 \quad-1.96992200$

$\begin{array}{llll}\text { C } & 10.95508300 & -0.73093900 & -2.96753600\end{array}$

$\begin{array}{llll}\text { C } & 10.29332000 & -1.94383600 & -2.30526000\end{array}$

$\begin{array}{llll}\text { O } & 9.42859600 & -2.59676500 & -2.88767900\end{array}$

C $\quad 12.33031800 \quad-1.02330700 \quad-3.59656000$

$\begin{array}{llll}\text { C } & 13.07324800 & 0.31418600 & -3.45788200\end{array}$

$\begin{array}{llll}\text { C } & 12.63685200 & 0.82896400 & -2.08011800\end{array}$

$\mathrm{N} \quad 10.76121600 \quad-2.22411600 \quad-1.06555300$

C $\quad 10.19547300 \quad-3.21221400 \quad-0.16700800$

$\begin{array}{llll}\text { C } & 9.64088500 & -2.60454100 & 1.12898900\end{array}$

$\begin{array}{llll}\text { O } & 9.79284300 & -3.17543300 & 2.19731200\end{array}$

$\begin{array}{llll}\mathrm{N} & 8.96483600 & -1.41930900 & 0.99104600\end{array}$

$\begin{array}{llll}\text { C } & 8.04522400 & -1.01578900 & 2.06029600\end{array}$

$\begin{array}{llll}\text { C } & 6.97765800 & -2.09043900 & 2.34554400\end{array}$

$\begin{array}{llll}\text { O } & 6.47196300 & -2.21092300 & 3.46044100\end{array}$

$\begin{array}{llll}\text { C } & 7.32102300 & 0.28571500 & 1.66936900\end{array}$

$\begin{array}{llll}\text { C } & 6.58303500 & 0.95837300 & 2.84472800\end{array}$

$\begin{array}{llll}\text { C } & 7.54603400 & 1.83285800 & 3.65565100\end{array}$

$\begin{array}{llll}\text { C } & 5.38802000 & 1.77610200 & 2.34262800\end{array}$

$\begin{array}{llll}\mathrm{N} & 6.59114200 & -2.79047700 & 1.25835000\end{array}$

$\begin{array}{llll}\text { C } & 5.45488800 & -3.67701700 & 1.30558800\end{array}$

$\begin{array}{llll}\text { C } & 4.06262300 & -3.78749900 & 6.43255100\end{array}$

$\begin{array}{llll}\text { C } & 2.91834600 & -3.87920500 & 5.41670600\end{array}$

$\begin{array}{llll}\text { C } & 3.19355400 & -3.16037000 & 4.08943600\end{array}$

$\begin{array}{llll}\text { C } & 2.20517600 & -3.63776200 & 3.02343000\end{array}$

$\begin{array}{llll}\text { C } & 3.13888400 & -1.63448600 & 4.21947600\end{array}$

$\begin{array}{llll}\text { C } & 0.17289900 & -7.03289400 & -2.58881700\end{array}$

$\begin{array}{llll}\text { C } & 0.33749700 & -5.89489000 & -1.57591200\end{array}$

$\begin{array}{llll}\text { C } & 1.54827600 & -4.97181900 & -1.80616200\end{array}$

$\begin{array}{llll}\text { C } & 1.43395300 & -4.17508200 & -3.11267400\end{array}$

$\begin{array}{llll}\text { C } & 1.71654400 & -4.01834300 & -0.61793100\end{array}$

$\begin{array}{llll}\text { C } & -7.99089400 & -7.72529500 & -1.04892800\end{array}$

$\begin{array}{llll}\text { C } & -8.52270900 & -6.29073900 & -0.87858800\end{array}$

$\begin{array}{llll}\text { C } & -7.74034900 & -5.41266900 & 0.08210000\end{array}$

$\begin{array}{llll}\text { C } & -6.34487900 & -5.28992300 & 0.04029900\end{array}$

$\begin{array}{llll}\text { C } & -8.42105900 & -4.65128600 & 1.04235900\end{array}$

$\begin{array}{llll}\text { C } & -5.65145300 & -4.43726200 & 0.90705400\end{array}$

$\begin{array}{llll}\text { C } & -7.74962600 & -3.80284800 & 1.91585800\end{array}$

$\begin{array}{llll}\text { C } & -6.35852500 & -3.68747100 & 1.84894700\end{array}$

$\begin{array}{llll}\text { O } & -5.77631600 & -2.78951500 & 2.71159900\end{array}$

$\begin{array}{llll}\text { C } & -3.53567000 & -3.72332400 & -3.95705900\end{array}$

$\begin{array}{llll}\text { C } & -3.05721200 & -3.00384700 & -2.70146700\end{array}$

$\begin{array}{llll}\text { C } & -4.19941900 & -2.35471400 & -1.91871700\end{array}$

$\begin{array}{llll}\text { C } & -3.76084500 & -1.32130100 & -0.91890100\end{array}$

$\begin{array}{llll}\mathrm{O} & -2.43649400 & -1.26201600 & -0.75626900\end{array}$

$\begin{array}{llll}\text { O } & -4.52555400 & -0.60392900 & -0.28854700\end{array}$

$\begin{array}{llll}\text { C } & 2.76981300 & 0.22439000 & -6.46826400\end{array}$

$\begin{array}{llll}\text { C } & 2.97878800 & -0.20395800 & -5.03469500\end{array}$

$\begin{array}{llll}\text { C } & 2.02198100 & 0.09552100 & -4.05904600\end{array}$

$\begin{array}{llll}\text { C } & 4.13508400 & -0.88838400 & -4.63572800\end{array}$

$\begin{array}{llll}\text { C } & 2.21497100 & -0.24796600 & -2.72037000\end{array}$

$\begin{array}{llll}\text { C } & 4.33204800 & -1.24852100 & -3.30010200\end{array}$

$\begin{array}{llll}\text { C } & 3.37773600 & -0.91481400 & -2.33359100\end{array}$

$\begin{array}{llll}\text { C } & 2.90885300 & 5.25591800 & -3.30068500\end{array}$

$\begin{array}{llll}\text { C } & 3.12551200 & 4.56262700 & -1.94490700\end{array}$ 


\begin{tabular}{|c|c|c|c|}
\hline $\mathrm{C}$ & 3.60923300 & 3.11770800 & \\
\hline $\mathrm{N}$ & & 2.44999800 & -0.77100300 \\
\hline $\mathrm{C}$ & -2.10394400 & 2.46696800 & -4.67499600 \\
\hline $\mathrm{C}$ & -1.58831100 & 2.19554900 & -3.25810300 \\
\hline & -1.51156000 & & -2.96489000 \\
\hline & -0.24085800 & 2.89455600 & -3.03255200 \\
\hline $\mathrm{C}$ & -7.48060000 & -1.82537900 & 26600 \\
\hline & -8.53846000 & -1.63329800 & 777500 \\
\hline C & & -2.92585500 & 76300 \\
\hline $\mathrm{S}$ & -10.24194800 & -2.79882200 & 55200 \\
\hline $\mathrm{C}$ & -9.60591500 & -1.52875400 & 97300 \\
\hline $\mathrm{C}$ & -11.12715300 & 1.31245300 & 420900 \\
\hline $\mathrm{C}$ & -10.54237300 & 1700 & 1300 \\
\hline & -9.16237500 & & -2.7 \\
\hline 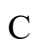 & -11.35 & & 95600 \\
\hline & -8.615 & 2.17 & -1.5 \\
\hline C & -10.8 & & 189400 \\
\hline & -9.45 & & -0.4 \\
\hline & -8.97 & 2.9 & 0.7 \\
\hline $\mathrm{C}$ & $-5.4 \varepsilon$ & & \\
\hline 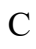 & -4.86 & & -1.2 \\
\hline & -5.84 & & -0.3 \\
\hline & -6.9 & & -0 . \\
\hline & -5.37 & & \\
\hline C & -9.12 & & $-0 . \varepsilon$ \\
\hline & -9.6 & & \\
\hline$C$ & -10.3 & 300 & 400 \\
\hline & -8.6 & & \\
\hline$C$ & -4.02 & & \\
\hline & -4.49 & & \\
\hline & -4.8 & & \\
\hline & -5.4 & & \\
\hline & -3.6 & & \\
\hline & & & \\
\hline & 2.29 & & \\
\hline $\mathrm{C}$ & 1.8 & & \\
\hline & 7.06 & & -1 \\
\hline $\mathrm{O}$ & 6.25 & & -1. \\
\hline & -6.8 & & 200 \\
\hline & -3.25 & & \\
\hline & & & \\
\hline & -2.88 & & \\
\hline & -3.60 & & \\
\hline & -3.0 & 200 & 3800 \\
\hline & -2.20 & & \\
\hline & -2.20 & & \\
\hline C & -1.6 & & \\
\hline & -1.42366800 & & \\
\hline $\mathrm{C}$ & -0.8 & & \\
\hline & & & \\
\hline & -1.79 & & \\
\hline $\mathrm{C}$ & 1.060 & 0.392 & 5400 \\
\hline & -2.91230700 & -4.39 & 67900 \\
\hline $\mathrm{C}$ & & & \\
\hline & 0.33 & -0.654 & \\
\hline C & -1.00 & & \\
\hline $\mathrm{O}$ & -4.28603400 & -7.79274700 & 2.96502700 \\
\hline $\mathrm{O}$ & -6.73923600 & 1.87876700 & 1.95343000 \\
\hline $\mathrm{O}$ & & & 0.62060100 \\
\hline C & 1.07390400 & 2.71483900 & 0.17556300 \\
\hline & -2.88172600 & -7.35547500 & 0.85701100 \\
\hline П & & & 0.33385300 \\
\hline & 2.76972800 & 0.93116500 & 0.14404800 \\
\hline
\end{tabular}

$\begin{array}{llll}\mathrm{H} & -1.50201000 & -2.87446900 & 0.68427800\end{array}$

$\mathrm{H} \quad-2.19639000 \quad-0.56996600 \quad-0.10156900$

$\mathrm{H} \quad-3.21577400 \quad-0.12938500 \quad 4.01292200$

$\mathrm{H} \quad-0.36437900 \quad-3.20736800 \quad 1.95521600$

H $\quad-3.08769100 \quad 1.93157700 \quad 2.73895000$

$\mathrm{H} \quad-1.53367400 \quad-1.78315100 \quad 3.52641700$

$\mathrm{H} \quad-2.94729400 \quad-3.57211500 \quad 4.05622500$

$\mathrm{H} \quad-1.35536700 \quad 2.76178900 \quad 0.97406400$

$\mathrm{H} \quad-4.17881700 \quad-5.63592100 \quad 4.56750800$

$\mathrm{H} \quad 0.85966300 \quad-1.59012400 \quad 1.61269200$

$\mathrm{H} \quad-3.54854800 \quad-1.45017100 \quad 1.45013000$

$\mathrm{H} \quad-4.79474100 \quad-7.70623900 \quad 3.78170000$

$\mathrm{H} \quad-3.69079600 \quad 1.16454000 \quad 1.28762000$

H $\quad-4.69503700 \quad-0.08259800 \quad 3.05283300$

$\mathrm{H} \quad-7.67021500 \quad 2.13526300 \quad 1.80174200$

$\mathrm{H} \quad 4.32466500 \quad 3.06825700 \quad-0.16547800$

$\mathrm{H} \quad 2.01710900 \quad 2.53819400 \quad-0.05511700$

$\mathrm{H} \quad 4.41298000 \quad 1.65614500 \quad-0.91255100$

$\mathrm{H} \quad 4.55792600 \quad 3.11283600 \quad-2.64541700$

$\mathrm{H} \quad 2.89303200 \quad 2.52940300 \quad-2.66949000$

$\mathrm{H} \quad 3.87275900 \quad 5.11924900 \quad-1.36211900$

$\mathrm{H} \quad 2.19693400 \quad 4.57903700 \quad-1.36333300$

$\mathrm{H} \quad 2.04500600 \quad 4.83562600 \quad-3.82428100$

$\mathrm{H} \quad 3.78455600 \quad 5.13030100 \quad-3.94838400$

$\mathrm{H} \quad 4.88117800 \quad-1.15566000 \quad-5.38029700$

$\mathrm{H} \quad 5.21900500 \quad-1.79954300 \quad-3.00282900$

$\mathrm{H} \quad 3.52860600 \quad-1.17918000 \quad-1.29279700$

$\mathrm{H} \quad 1.45686000 \quad-0.00425700 \quad-1.98377900$

$\mathrm{H} \quad 1.11243000 \quad 0.60375300 \quad-4.35689100$

$\mathrm{H} \quad 0.50584300 \quad 2.55241400 \quad-3.75761500$

$\mathrm{H} \quad-0.34194900 \quad 3.97831200 \quad-3.15792900$

$\mathrm{H} \quad 0.14946200 \quad 2.71152400 \quad-2.02821900$

$\mathrm{H} \quad-2.30487800 \quad 2.63449400 \quad-2.54850900$

$\mathrm{H} \quad-1.11760800 \quad 0.50845200 \quad-1.96381400$

$\mathrm{H} \quad-2.50110000 \quad 0.23644600 \quad-3.03241900$

$\mathrm{H} \quad-0.86288100 \quad 0.18235000 \quad-3.68097200$

$\mathrm{H} \quad-8.19177700 \quad-0.87884300 \quad-2.97507600$

$\mathrm{H} \quad-9.46563400 \quad-1.24173600 \quad-4.11665400$

$\mathrm{H} \quad-7.96052600 \quad-3.28101500 \quad-2.38941900$

$\mathrm{H} \quad-9.14507300 \quad-3.71933300 \quad-3.62350500$

$\mathrm{H} \quad-8.64968900 \quad-1.82341700 \quad-0.16030200$

H $\quad-9.52101600 \quad-0.55541300-1.08094200$

$\mathrm{H} \quad-10.34048300 \quad-1.44827200 \quad 0.20692700$

H $\quad-8.26588500 \quad 3.55408100 \quad 0.49824300$

$\mathrm{H} \quad-7.54481600 \quad 2.29302200 \quad-1.46148400$

$\mathrm{H} \quad-11.46696500 \quad 2.34839100 \quad 0.25422600$

$\mathrm{H} \quad-12.42684500 \quad 1.63829600 \quad-1.92661800$

$\mathrm{H} \quad-8.50216400 \quad 1.66268100 \quad-3.65120800$

$\mathrm{H} \quad-4.80757300 \quad-3.08925500 \quad-1.37505200$

$\mathrm{H} \quad-4.89649000 \quad-1.84155200 \quad-2.59225200$

$\mathrm{H} \quad-2.49306900 \quad-3.67874600 \quad-2.04918300$

$\mathrm{H} \quad-2.35565300 \quad-2.21829100 \quad-2.99395400$

$\mathrm{H} \quad-8.54654900 \quad-5.80602900 \quad-1.86404500$

$\mathrm{H} \quad-9.56522900 \quad-6.31845900 \quad-0.54600100$

$\mathrm{H} \quad-9.50359500 \quad-4.71377500 \quad 1.09351700$

$\mathrm{H} \quad-5.77046700 \quad-5.87220300 \quad-0.67518100$

$\mathrm{H} \quad-4.56972900 \quad-4.37976500 \quad 0.86811000$

$\mathrm{H} \quad-8.29047900 \quad-3.21141500 \quad 2.64686300$

$\mathrm{H} \quad-4.79676600 \quad-2.71105600 \quad 2.62354100$

$\mathrm{H} \quad 2.60737600 \quad-3.39272600 \quad-0.72898200$

$\mathrm{H} \quad 0.85400900 \quad-3.34591600 \quad-0.53312800$

H $\quad 1.80390900 \quad-4.56928100 \quad 0.32266700$

H $\quad 2.29913100 \quad-3.52038200 \quad-3.25161400$ 


\begin{tabular}{|c|c|c|c|}
\hline 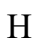 & 36279400 & -4.82507400 & \\
\hline t & .54152300 & -3.53668400 & -3.09289300 \\
\hline & & -5.60423300 & -1.86581300 \\
\hline & -0.56827900 & -5.27041900 & -1.59790200 \\
\hline & 0.39498200 & -6.31253900 & -0.56264900 \\
\hline & 6.94246800 & -2.55379400 & 0.33282000 \\
\hline & 8.61490700 & -0.89153500 & 14100 \\
\hline & 8.05007200 & & \\
\hline & 6.61636500 & 0.05687100 & \\
\hline & 5.71833000 & 2.534 & 400 \\
\hline & 4.65402500 & & 600 \\
\hline & & & \\
\hline & 7.9206 & & 400 \\
\hline & 7.0493 & 2.262 & 700 \\
\hline & 7100 & & 900 \\
\hline & 6.20558600 & & 600 \\
\hline & 8.69058000 & -1.1 & 200 \\
\hline & & -3.7 & 000 \\
\hline & 10.938 & -3.9 & 2700 \\
\hline & 11.418 & & 7600 \\
\hline & & & \\
\hline & 14.15769 & & \\
\hline & & & \\
\hline & 12.739 & & \\
\hline & 12.6238 & & -2.0 \\
\hline & 12.237 & & \\
\hline & 10.23760800 & & 300 \\
\hline & & & \\
\hline & 8.9508 & & \\
\hline & -4.2372 & & -0.5 \\
\hline & -4.2000 & & 100 \\
\hline & -9.74331300 & & -1.0 \\
\hline F & -10.69750000 & & 000 \\
\hline $\mathrm{H}$ & 30600 & & 800 \\
\hline & -10.38552 & & \\
\hline $\mathrm{H}$ & & & \\
\hline & & & \\
\hline & -3.70970900 & & \\
\hline $\mathrm{H}$ & -4.730 & & 000 \\
\hline & -5.68830100 & & \\
\hline $\mathrm{H}$ & -6.37914600 & & \\
\hline $\mathrm{H}$ & -3.2495 & & 000 \\
\hline & -3.99119500 & 4.47924600 & \\
\hline $\mathrm{H}$ & -2.88923600 & & \\
\hline & -5.655 & & \\
\hline & & 4.749 & \\
\hline & 0.21903600 & 3.6 & 600 \\
\hline & & 1.46380000 & \\
\hline & & & \\
\hline & & 1.724 & 3.71 \\
\hline & & -1.26425500 & \\
\hline & & & \\
\hline & & -1.30844200 & 4.53 \\
\hline & & -4.71265400 & \\
\hline & 1.17507400 & -3.45378100 & 3.35211700 \\
\hline & 2.35375100 & -3.11493 & 00800 \\
\hline & 4.21169700 & -3.41779500 & 3.77849000 \\
\hline & 2.72018800 & -4.93973600 & 5.20927600 \\
\hline & & -3.48144300 & \\
\hline & 6.80350600 & -1.15400600 & -1.53916700 \\
\hline & 7.77037300 & -2.26350700 & -2.09659800 \\
\hline & & 0.95771600 & -1.55217900 \\
\hline & 5.99903000 & 0.62621100 & -2.54051900 \\
\hline
\end{tabular}

\begin{tabular}{|c|c|c|c|}
\hline & 16593200 & & \\
\hline & & & \\
\hline & 8.23530100 & & \\
\hline & & 5.03684700 & \\
\hline & -6.23679200 & & \\
\hline & -8.37726500 & 7.29288700 & 4000 \\
\hline & -8.00549900 & & \\
\hline & 4.29155100 & -2.7 & \\
\hline & 11.61849200 & & \\
\hline & & & \\
\hline & 0.977 & -7.7 & \\
\hline & & -0.4 & \\
\hline & 3.21 & & \\
\hline & -1.513 & & \\
\hline & -6.66 & & \\
\hline & -8.62 & -8.2 & -1. \\
\hline & -4.3 & & \\
\hline & & & \\
\hline & -10.7 & & \\
\hline & & -1.2 & \\
\hline & -4.6 & & \\
\hline & -5.9 & & \\
\hline & -2.7 & & \\
\hline & -3.79 & & \\
\hline & & & \\
\hline & -7.8 & & \\
\hline & -10. & & -5 . \\
\hline & -2.0 & & \\
\hline & -3.1 & & \\
\hline & 1.7 & & \\
\hline & & & \\
\hline & 7.73 & & \\
\hline & 6.43 & & \\
\hline & & & \\
\hline & 10.2 & & \\
\hline & 5.65 & -4.5 & \\
\hline & & -3.9 & \\
\hline $\mathrm{H}$ & & & \\
\hline & & -4.2 & \\
\hline & -0.79 & -7.5 & \\
\hline & 0.144 & -6.62 & -3.6038 \\
\hline & -7.977 & 5092600 & -0.09378700 \\
\hline & & -7.6 & \\
\hline $\mathrm{H}$ & -8.71360300 & & -0.47576500 \\
\hline & -9.928 & & \\
\hline $\mathrm{H}$ & -3.66345200 & & \\
\hline & -4.79852500 & 5.01271500 & 5.94278900 \\
\hline & & & \\
\hline & -5.99118900 & 2.51752500 & 0.90491000 \\
\hline
\end{tabular}

\section{$\mathrm{TS3}_{R}(+11.1 \mathrm{kcal} / \mathrm{mol})$}

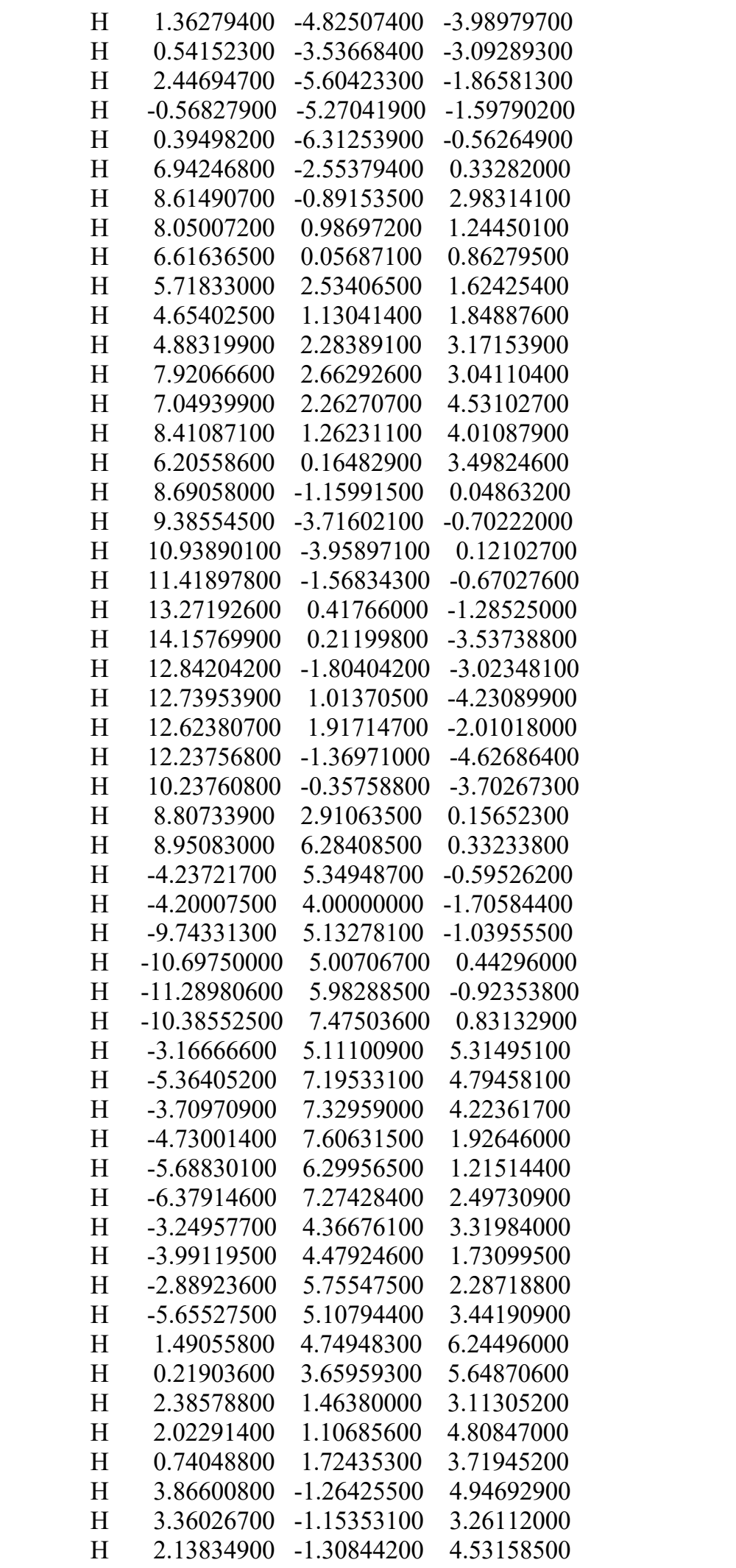

$\begin{array}{llll}\text { C } & 6.68317400 & 5.65250200 & 0.25338400\end{array}$

C $\quad 7.19937000 \quad 4.70612500 \quad-0.78395100$

O $\quad 6.75413800 \quad 3.56836500 \quad-0.97735900$

$\mathrm{N} \quad 8.22784000 \quad 5.17867500 \quad-1.52188800$

C $\quad 8.78209900 \quad 4.39036400 \quad-2.59158700$

C $\quad 9.84530800 \quad 3.37316300 \quad-2.17621700$

O $\quad 10.85447900 \quad 3.18250200 \quad-2.84847000$

$\mathrm{N} \quad 9.56546600 \quad 2.68642500 \quad-1.03288700$

C $\quad 10.41131400 \quad 1.55966200 \quad-0.70338500$ 
C $\quad 10.12991200 \quad 0.38947800 \quad-1.63615400$

$\begin{array}{lllll}\mathrm{O} & 8.96943000 & -0.02588200 & -1.77245700\end{array}$

$\begin{array}{llll}\mathrm{N} & 11.16525500 & -0.23797600 & -2.23040400\end{array}$

C $\quad 10.88744200 \quad-1.45334500 \quad-3.01493600$

C $\quad 10.23639000 \quad-2.53903800 \quad-2.15315000$

O $\quad 9.39017100 \quad-3.29750900 \quad-2.62356800$

C $\quad 12.27122900 \quad-1.82791900 \quad-3.57481800$

$\begin{array}{llll}\text { C } & 12.96745700 & -0.46669700 & -3.72274500\end{array}$

$\begin{array}{lllll}\text { C } & 12.51840200 & 0.30859600 & -2.47695800\end{array}$

N $\quad 10.69392700 \quad-2.59638700 \quad-0.88013600$

C $\quad 10.14914900 \quad-3.45835800 \quad 0.15177700$

$\begin{array}{llll}\text { C } & 9.60435600 & -2.69345000 & 1.36381700\end{array}$

$\begin{array}{llll}\text { O } & 9.76442000 & -3.13095600 & 2.49271700\end{array}$

$\begin{array}{llll}\mathrm{N} & 8.93131200 & -1.53089300 & 1.08956300\end{array}$

$\begin{array}{llll}\text { C } & 8.06073000 & -0.97680600 & 2.13286200\end{array}$

$\begin{array}{llll}\text { C } & 6.93411400 & -1.94897000 & 2.53664400\end{array}$

O $\quad 6.48465300 \quad-1.95602500 \quad 3.68295600$

$\begin{array}{llll}\text { C } & 7.47748700 & 0.36532500 & 1.65553900\end{array}$

$\begin{array}{llll}\text { C } & 6.42259300 & 0.99948600 & 2.58127000\end{array}$

$\begin{array}{lllll}\text { C } & 6.92718100 & 1.18874000 & 4.01595700\end{array}$

$\begin{array}{llll}\mathrm{C} & 5.96120400 & 2.33647200 & 1.99153400\end{array}$

$\begin{array}{llll}\mathrm{N} & 6.45151000 & -2.71183000 & 1.53865300\end{array}$

$\begin{array}{llll}\text { C } & 5.32256300 & -3.58578100 & 1.75388500\end{array}$

$\begin{array}{llll}\text { C } & 4.01278800 & -2.68536100 & 6.82589000\end{array}$

$\begin{array}{llll}\text { C } & 2.82550700 & -2.94803300 & 5.89126300\end{array}$

$\begin{array}{llll}\text { C } & 3.04237600 & -2.54324800 & 4.42877400\end{array}$

$\begin{array}{llll}\text { C } & 1.83006400 & -2.93627800 & 3.57652200\end{array}$

$\begin{array}{llll}\text { C } & 3.34025100 & -1.04693200 & 4.27814900\end{array}$

$\begin{array}{llll}\text { C } & 0.01993000 & -7.68936500 & -1.30379500\end{array}$

C $\quad 0.23326100 \quad-6.39414300 \quad-0.51137000$

C $\quad 1.40529200 \quad-5.52050100 \quad-0.99991600$

C $\quad 1.11745200 \quad-4.87686200 \quad-2.36345800$

$\begin{array}{llll}\text { C } & 1.73287500 & -4.43746600 & 0.03536500\end{array}$

$\begin{array}{llll}\text { C } & -8.11014900 & -8.13023900 & 0.48858800\end{array}$

$\begin{array}{llll}\text { C } & -8.81667500 & -6.77805500 & 0.31131400\end{array}$

$\begin{array}{llll}\text { C } & -8.32629200 & -5.67826800 & 1.21830700\end{array}$

$\begin{array}{llll}\text { C } & -7.06073700 & -5.10578400 & 1.06012700\end{array}$

$\begin{array}{llll}\text { C } & -9.13044400 & -5.20074100 & 2.25978000\end{array}$

$\begin{array}{llll}\text { C } & -6.61060000 & -4.08879400 & 1.90041800\end{array}$

C $\quad-8.70386700 \quad-4.18010200 \quad 3.10282700$

$\begin{array}{llll}\text { C } & -7.44138700 & -3.60649700 & 2.92206400\end{array}$

O $\quad-7.06947000 \quad-2.60016700 \quad 3.76040400$

C $\quad-3.75082900 \quad-4.74821100 \quad-3.23417200$

C $\quad-3.57114100 \quad-3.71586200 \quad-2.11087900$

C $\quad-4.95044900 \quad-3.09837000 \quad-1.82778900$

C $\quad-5.13363200 \quad-2.08476500 \quad-0.71409400$

O $\quad-6.07603400 \quad-1.26431700 \quad-0.85664200$

O $\quad-4.38313200 \quad-2.16568200 \quad 0.32446000$

C $\quad 2.46809900-1.32235200 \quad-6.58878600$

$\begin{array}{lllll}\text { C } & 2.73535500 & -1.42620700 & -5.10686400\end{array}$

C $\quad \begin{array}{llll}\text { C } & 1.83853500 & -0.87996200 & -4.18373700\end{array}$

$\begin{array}{llll}\text { C } & 3.89914500 & -2.03439400 & -4.61640700\end{array}$

$\begin{array}{llll}\text { C } & 2.10105700 & -0.90127800 & -2.81413100\end{array}$

C $\quad 4.16656400 \quad-2.07327300 \quad-3.24512500$

$\begin{array}{llll}\text { C } & 3.27516800 & -1.48886500 & -2.33865800\end{array}$

$\begin{array}{llll}\text { C } & 2.59712800 & 4.23923300 & -4.48395500\end{array}$

C $\quad 3.20102400 \quad 3.91050400 \quad-3.11416100$

C $\quad 3.55311800 \quad 2.43431800 \quad-2.94234000$

N $\quad 4.15895400 \quad 2.19862300 \quad-1.61988900$

C $\quad-2.40240200 \quad 1.19094400 \quad-5.18726200$

$\begin{array}{llll}\text { C } & -1.90277600 & 1.13658700 & -3.73693100\end{array}$

C $\quad-1.82192500 \quad-0.31471600 \quad-3.24712400$

$\begin{array}{llll}\text { C } & -0.56977600 & 1.88001000 & -3.57583800\end{array}$
S $\quad-10.37220400 \quad-3.48484500 \quad-1.09205200$

$\begin{array}{llll}\text { C } & -9.41294400 & -2.30295000 & -0.07986000\end{array}$

C $\quad-11.40515800 \quad 0.05344700 \quad-4.44519400$

C $\quad-10.70902900 \quad 0.56198000 \quad-3.21027400$

$\begin{array}{llll}\text { C } & -9.32105800 & 0.74389500 & -3.19324700\end{array}$

C $\quad-11.42241500 \quad 0.84805900 \quad-2.04212900$

C $\quad-8.66559900 \quad 1.21358600 \quad-2.05930100$

C $\quad-10.78478400 \quad 1.33774900 \quad-0.90326100$

C $\quad-9.40720500 \quad 1.53895900 \quad-0.91880200$

$\begin{array}{llll}\text { O } & -8.79952800 & 2.04622700 & 0.21635900\end{array}$

C $\quad-5.77954000 \quad 4.74767200 \quad-3.47315600$

C $\quad-5.04973000 \quad 4.09786900 \quad-2.28990200$

C $\quad-5.89764000 \quad 3.35200000 \quad-1.29467600$

O $\quad-7.01557900 \quad 3.73421000 \quad-0.93739000$

O $\quad-5.29271600 \quad 2.27931200 \quad-0.82766500$

C $\quad-9.44047300 \quad 7.11830700 \quad-2.40731400$

C $\quad-9.85772400 \quad 6.34504900 \quad-1.15813800$

C $\quad-10.49817300 \quad 5.00005000 \quad-1.50284100$

O $\quad-8.74123200 \quad 6.16348500 \quad-0.28721600$

C $\quad-4.19716900 \quad 6.40314700 \quad 4.29964600$

$\begin{array}{llll}\text { C } & -4.68288600 & 7.00848900 & 2.98236000\end{array}$

$\begin{array}{llll}\text { C } & -5.10311000 & 5.97119600 & 1.92164000\end{array}$

$\begin{array}{llll}\text { C } & -5.62284700 & 6.67682600 & 0.66431700\end{array}$

C $\quad-3.96542300 \quad 5.00282900 \quad 1.56314900$

C $\quad 1.13726500 \quad 4.55970500 \quad 4.84234700$

S $\quad 2.43053300 \quad 3.31330600 \quad 5.17174200$

C $\quad 2.30305200 \quad 2.34667100 \quad 3.62908000$

O $\quad 6.99604000 \quad-2.67242500 \quad-1.23822800$

O $\quad 6.22924000 \quad-0.06236900 \quad-1.88054400$

$\begin{array}{llll}\mathrm{O} & -6.51050000 & 0.67258200 & 0.72906100\end{array}$

N $\quad-2.88433900 \quad-0.19759700 \quad 1.87591700$

C $\quad-0.03250000 \quad 0.60883100 \quad 6.16011300$

$\begin{array}{llll}\text { C } & -0.78163300 & 1.72852900 & 5.77884500\end{array}$

$\begin{array}{llll}\text { C } & -2.88179000 & 1.20919400 & 1.46913300\end{array}$

C $\quad-2.14711200 \quad 1.34944700 \quad 0.12899300$

C $\quad-2.00598500 \quad 1.56653000 \quad 5.13434800$

$\begin{array}{llll}\text { C } & -2.53125900 & 0.29487800 & 4.90053700\end{array}$

$\begin{array}{llll}\text { C } & -0.67113400 & 1.02702000 & 0.21615200\end{array}$

C $\quad-3.82995800 \quad 0.11154700 \quad 4.14353700$

$\begin{array}{llll}\text { C } & 0.27299600 & 2.06263400 & 0.24402200\end{array}$

$\begin{array}{lllll}\text { C } & 1.63621700 & 1.80280800 & 0.37831600\end{array}$

$\begin{array}{llll}\text { C } & -3.56903900 & -0.69775300 & 2.90074800\end{array}$

$\begin{array}{llll}\text { C } & 2.06618800 & 0.47372600 & 0.55747800\end{array}$

C $\quad-1.80291600 \quad-0.81718600 \quad 5.34812000$

C $\quad-0.56728200 \quad-0.67115100 \quad 5.96382100$

C $\quad 1.14762800 \quad-0.56846400 \quad 0.50000100$

C $\quad-0.20584500 \quad-0.29253300 \quad 0.30786200$

$\begin{array}{llll}\text { O } & 1.19406900 & 0.71106000 & 6.72922100\end{array}$

O $\quad-5.31005500 \quad-1.06731800 \quad 2.32505200$

$\begin{array}{llll}\text { O } & 3.39133800 & 0.19888600 & 0.76291600\end{array}$

O $\quad 2.56113200 \quad 2.80648600 \quad 0.38006300$

$\mathrm{H} \quad-0.40479300 \quad 2.71886100 \quad 5.99850200$

$\mathrm{H} \quad-2.56740900 \quad 2.44592200 \quad 4.82810900$

$\mathrm{H} \quad 3.83291800 \quad 1.04928600 \quad 0.92055400$

$\mathrm{H} \quad-4.25996000 \quad 1.08092500 \quad 3.87765200$

$\mathrm{H} \quad-0.89280300 \quad-1.12857000 \quad 0.22535400$

$\mathrm{H} \quad-2.37875600 \quad 1.79804700 \quad 2.24212800$

H $\quad-4.57525300 \quad-0.42472100 \quad 4.73737000$

$\mathrm{H} \quad-2.28949800 \quad 2.37607300 \quad-0.21877600$

H $\quad-3.39775100 \quad-1.75820100 \quad 3.05638400$ 


\begin{tabular}{|c|c|c|c|}
\hline & 20092000 & .81829500 & \\
\hline & & 3.09851400 & \\
\hline & & & \\
\hline & 1.49974000 & -1.58795300 & \\
\hline & -4.98529800 & -1.56091400 & 9800 \\
\hline & 1.56130300 & & \\
\hline & -2.65288800 & 0.70833300 & 6600 \\
\hline & -3.90945300 & 1.57456700 & \\
\hline & -2.74123700 & -0.86079200 & 7000 \\
\hline & 5.02796600 & 900 & \\
\hline & 3.21088200 & 2.625 & \\
\hline & 4.4320 & & \\
\hline & & & \\
\hline & 2.6 & & \\
\hline & & & \\
\hline & 2.50 & & \\
\hline & 000 & 3.7 & \\
\hline & & & \\
\hline & 4.5 & -2.4 & \\
\hline & & & \\
\hline & & -1.4 & \\
\hline & 1.3 & -0.4 & \\
\hline & & -0.4 & \\
\hline & 0.1 & & \\
\hline & -0.6 & & \\
\hline & & & \\
\hline & -2.64 & & \\
\hline & & & \\
\hline & -2.81 & -0.7 & \\
\hline & -1.16 & -0.9 & \\
\hline & -8.26 & & \\
\hline & -9.62 & & \\
\hline & -8.25 & -4.3 & \\
\hline & -9.5 & -4.6 & \\
\hline & -8.40 & -2.6 & \\
\hline & -9.35 & & \\
\hline & -9.92 & -2.2 & \\
\hline & -8.1( & 2.7 & \\
\hline & -7.5 & & \\
\hline & 112 & & \\
\hline & -12.497 & & \\
\hline & -8.73 & & \\
\hline $\mathrm{H}$ & -5.65 & -3.9 & \\
\hline & & & \\
\hline & -3.15 & -4.1 & \\
\hline & -2.87 & -2.9 & \\
\hline & -8.72 & -6.4 & 100 \\
\hline & -9.8895 & -6.9 & \\
\hline & -10.12 & -5.6 & \\
\hline & -6.40 & -5.4 & \\
\hline & -5.62 & -3.6 & \\
\hline & & & \\
\hline & -6.36 & -2.0 & \\
\hline & & -3.79 & \\
\hline & 0.85 & -3.7990 & \\
\hline & & -4.87 & \\
\hline $\mathrm{H}$ & & $-4.2^{\prime}$ & \\
\hline & 0.90245700 & -5.62 & -3.1 \\
\hline & & -4.20 & \\
\hline 11 & 2.28965700 & -6.16688500 & -1.10521000 \\
\hline & -0.68321400 & -5.78759900 & -0.54691500 \\
\hline & & -6.64296300 & \\
\hline & 6.82460600 & -2.66265200 & 0.59266500 \\
\hline
\end{tabular}

$\begin{array}{llll}8.66320100 & -0.83990200 & 3.03129000\end{array}$ $\begin{array}{llll}8.30987400 & 1.07339500 & 1.53139500\end{array}$ $\begin{array}{llll}7.02967300 & 0.22140900 & 0.66409000\end{array}$ $\begin{array}{llll}6.78060300 & 3.06590100 & 2.01206900\end{array}$ $\begin{array}{llll}5.65023400 & 2.24988900 & 0.94613200\end{array}$ $\begin{array}{lll}5.13138500 & 2.75706400 & 2.56817500\end{array}$ $\begin{array}{llll}7.84913000 & 1.78507700 & 4.03086300\end{array}$ $\begin{array}{llll}6.18069000 & 1.72037500 & 4.61572900\end{array}$ $\begin{array}{llll}7.11562800 & 0.22466200 & 4.49066100\end{array}$ $\begin{array}{llll}5.56163700 & 0.32223600 & 2.61684000\end{array}$ $\begin{array}{llll}8.63766400 & -1.39042400 & 0.12762800\end{array}$ $\begin{array}{llll}9.33796800 & -4.03493300 & -0.30088100\end{array}$ $\begin{array}{lll}10.90228200 & -4.15518600 & 0.52722200\end{array}$ $\begin{array}{llll}11.33816400 & -1.87499900 & -0.59104700\end{array}$ $\begin{array}{llll}13.17706400 & 0.10511900 & -1.62366800\end{array}$ $14.05454700 \quad-0.54572300 \quad-3.79098500$ $12.80625500 \quad-2.45471300 \quad-2.85301600$ $\begin{array}{llll}12.60649500 & 0.04476500 & -4.62065200\end{array}$ $\begin{array}{llll}12.45543800 & 1.38524000 & -2.64094100\end{array}$ $12.19080000 \quad-2.38493100 \quad-4.50932900$ $\begin{array}{llll}10.17137400 & -1.23399100 & -3.81145500\end{array}$ $\begin{array}{llll}8.59424400 & 2.65866500 & -0.73488400\end{array}$ $\begin{array}{llll}8.63991800 & 6.07187800 & -1.29959000\end{array}$ $\begin{array}{llll}-4.53905600 & 4.88070600 & -1.71300500\end{array}$ $\begin{array}{llll}-4.27040500 & 3.41790200 & -2.64378900\end{array}$ $\begin{array}{llll}-9.80845300 & 4.38378400 & -2.09196700\end{array}$ $\begin{array}{llll}-10.73720900 & 4.44886700 & -0.58993900\end{array}$ $\begin{array}{llll}-11.41504300 & 5.12749000 & -2.08733200\end{array}$ $\begin{array}{llll}-10.57156200 & 6.94584300 & -0.58110700\end{array}$ $\begin{array}{lll}-3.33590300 & 5.74853400 & 4.11487600\end{array}$ $\begin{array}{lll}-5.53286000 & 7.67607000 & 3.17089200\end{array}$ $\begin{array}{lll}-3.88858800 & 7.63886400 & 2.55729100\end{array}$ $\begin{array}{lll}-4.84511000 & 7.32026400 & 0.23302200\end{array}$ $\begin{array}{llll}-5.92576700 & 5.95307000 & -0.09526000\end{array}$ $\begin{array}{lll}-6.50106700 & 7.29180200 & 0.87577700\end{array}$ $\begin{array}{lll}-3.61956600 & 4.42871900 & 2.42815600\end{array}$ $\begin{array}{llll}-4.28916900 & 4.28114300 & 0.80682800\end{array}$ $\begin{array}{llll}-3.10210300 & 5.54722200 & 1.15939100\end{array}$ $\begin{array}{lll}-5.92896200 & 5.37705600 & 2.33951800\end{array}$ $\begin{array}{llll}0.97142300 & 5.12246300 & 5.76317100\end{array}$ $\begin{array}{llll}1.46221700 & 5.24624000 & 4.05706700\end{array}$ $\begin{array}{llll}3.04295700 & 1.54917800 & 3.68915900\end{array}$ $\begin{array}{llll}1.31221300 & 1.89590600 & 3.53895200\end{array}$ $\begin{array}{lll}2.51358800 & 2.97014100 & 2.75914400\end{array}$ $\begin{array}{lll}4.31333000 & -0.79315800 & 4.70410900\end{array}$ $\begin{array}{llll}3.36011400 & -0.76575000 & 3.21974200\end{array}$ $\begin{array}{llll}2.56928900 & -0.45248900 & 4.78133000\end{array}$ $\begin{array}{llll}1.59614300 & -4.00273300 & 3.67260600\end{array}$ $\begin{array}{llll}0.94121300 & -2.36791800 & 3.87742500\end{array}$ $\begin{array}{llll}2.00671500 & -2.72569800 & 2.51754400\end{array}$ $\begin{array}{llll}3.92087000 & -3.08915700 & 4.06845500\end{array}$ $\begin{array}{llll}2.56812900 & -4.01583700 & 5.92634700\end{array}$ $\begin{array}{llll}1.95107500 & -2.40957800 & 6.28107700\end{array}$ $6.72848900-1.76681400-1.49499900$ $\begin{array}{llll}7.71073400 & -2.94256200 & -1.84122200\end{array}$ $\begin{array}{llll}7.15076800 & 0.24797000 & -1.95587400\end{array}$ $\begin{array}{llll}5.89997000 & -0.21424200 & -2.77752100\end{array}$ $\begin{array}{llll}-6.41977800 & -0.13401000 & 0.09663800\end{array}$ $\begin{array}{lll}-5.78954000 & -0.26348700 & 1.99217800\end{array}$ $\begin{array}{llll}7.96227400 & 3.83379300 & -3.05823800\end{array}$ $\begin{array}{llll}5.96656500 & 5.14105100 & 0.89429400\end{array}$ $\begin{array}{lll}-6.53414200 & 5.46441400 & -3.14476500\end{array}$ $\begin{array}{llll}-8.67307300 & 6.54756400 & -2.94699600\end{array}$ 


$\begin{array}{cccc}\mathrm{H} & -8.17422800 & 5.47484000 & -0.67007400 \\ \mathrm{H} & 4.23978700 & -1.61343300 & 6.84557500 \\ \mathrm{H} & 11.45369300 & 1.87342600 & -0.72581400 \\ \mathrm{H} & 4.44099300 & -3.02260800 & 2.06773300 \\ \mathrm{H} & 0.82304400 & -8.41195400 & -1.12971900 \\ \mathrm{H} & 2.92283900 & -2.15449600 & -7.13476500 \\ \mathrm{H} & 2.89608900 & -0.39819800 & -6.99773400 \\ \mathrm{H} & -1.81848300 & 0.54035200 & -5.84629300 \\ \mathrm{H} & -6.89724700 & -3.63775700 & -3.89879600 \\ \mathrm{H} & -8.68545200 & -8.89550300 & -0.05073900 \\ \mathrm{H} & -4.61391400 & -4.44789000 & -3.84441900 \\ \mathrm{H} & -12.48594800 & -0.00556400 & -4.29174800 \\ \mathrm{H} & -11.05900500 & -0.95123100 & -4.71318300 \\ \mathrm{H} & -7.45532000 & 0.94711400 & 0.75182900 \\ \mathrm{H} & -4.97651700 & 5.26992800 & -4.00384000 \\ \mathrm{H} & -6.24022000 & 4.01029400 & -4.13833700 \\ \mathrm{H} & -2.96979000 & -4.89690600 & -3.98881500 \\ \mathrm{H} & -3.99569600 & -5.76406300 & -2.90455200 \\ \mathrm{H} & -7.28317900 & -2.20694800 & -4.82750900 \\ \mathrm{H} & -8.12340100 & -3.72893400 & -5.13750600 \\ \mathrm{H} & -11.22898600 & 0.69370200 & -5.31495700 \\ \mathrm{H} & -2.36823000 & 2.21111300 & -5.58208700 \\ \mathrm{H} & -3.46119900 & 0.91751200 & -5.25130600 \\ \mathrm{H} & 1.39956600 & -1.31087300 & -6.82716400 \\ \mathrm{H} & 2.42565400 & 5.31587500 & -4.57505500 \\ \mathrm{H} & 7.50919500 & 6.03275500 & 0.86297000 \\ \mathrm{H} & 6.19002700 & 6.50831700 & -0.21893700 \\ \mathrm{H} & 9.21422600 & 5.05731700 & -3.34436300 \\ \mathrm{H} & 10.16881400 & 1.21315600 & 0.30484000 \\ \mathrm{H} & 5.53754300 & -4.34295300 & 2.51166400 \\ \mathrm{H} & 5.09915400 & -4.08734300 & 0.81009300 \\ \mathrm{H} & 4.92055100 & -3.18026400 & 6.46392500 \\ \mathrm{H} & 3.68384900 & -2.93970600 & 7.84013800 \\ \mathrm{H} & -0.93790300 & -8.15366000 & -1.05008400 \\ \mathrm{H} & -0.03019600 & -7.48690200 & -2.37906900 \\ \mathrm{H} & -8.07581100 & -8.46705200 & 1.53055900 \\ \mathrm{H} & -7.11458600 & -8.16905300 & 0.03625900 \\ \mathrm{H} & -9.01933400 & 8.10708900 & -2.19495300 \\ \mathrm{H} & -10.24449800 & 7.31189900 & -3.12593900 \\ \mathrm{H} & -3.83090500 & 7.15146700 & 5.01022500 \\ \mathrm{H} & -4.95902100 & 5.76659800 & 4.76167900 \\ \mathrm{H} & 0.20630300 & 4.07789900 & 4.53737700 \\ \mathrm{H} & -5.84024100 & 1.73269000 & -0.15310600 \\ & & & \end{array}$

\section{$\operatorname{Int5}_{R}(-1.9 \mathrm{kcal} / \mathrm{mol})$}

$\begin{array}{cccc}\mathrm{C} & 6.52379500 & 5.75095000 & -0.52597800 \\ \mathrm{C} & 7.00937200 & 4.69504300 & -1.46795500 \\ \mathrm{O} & 6.51461800 & 3.56600900 & -1.56517000 \\ \mathrm{~N} & 8.05778400 & 5.06559200 & -2.23917400 \\ \mathrm{C} & 8.60473400 & 4.16107500 & -3.21646100 \\ \mathrm{C} & 9.67296100 & 3.20921200 & -2.68384500 \\ \mathrm{O} & 10.69422900 & 2.95279200 & -3.31405600 \\ \mathrm{~N} & 9.38075500 & 2.65861000 & -1.47089700 \\ \mathrm{C} & 10.23477600 & 1.59604000 & -0.98816700 \\ \mathrm{C} & 9.96511200 & 0.30098600 & -1.74215000 \\ \mathrm{O} & 8.81395500 & -0.16070400 & -1.78754900 \\ \mathrm{~N} & 11.00664900 & -0.37339400 & -2.26901600 \\ \mathrm{C} & 10.76265800 & -1.70595800 & -2.84631600 \\ \mathrm{C} & 10.17551500 & -2.66348100 & -1.80527200 \\ \mathrm{O} & 9.35794000 & -3.52412700 & -2.12616400\end{array}$

$\begin{array}{llll}\text { C } & 12.14979700 & -2.11635100 & -3.37406500\end{array}$

$\begin{array}{llll}\text { C } & 12.80383700 & -0.77202000 & -3.72791000\end{array}$

$\begin{array}{llll}\text { C } & 12.34615100 & 0.16096200 & -2.59971000\end{array}$

$\begin{array}{llll}\mathrm{N} & 10.65383300 & -2.49193500 & -0.54969600\end{array}$

$\begin{array}{llll}\text { C } & 10.16695200 & -3.19476200 & 0.62177000\end{array}$

$\begin{array}{llll}\text { C } & 9.60794500 & -2.26865400 & 1.70890700\end{array}$

$\begin{array}{llll}\text { O } & 9.77460000 & -2.53473100 & 2.88901100\end{array}$

$\begin{array}{llll}\mathrm{N} & 8.91080000 & -1.17148500 & 1.27196600\end{array}$

$\begin{array}{llll}\text { C } & 8.02110400 & -0.49515000 & 2.22419700\end{array}$

$\begin{array}{llll}\text { C } & 6.92838200 & -1.43522900 & 2.77095900\end{array}$

$\begin{array}{llll}\text { O } & 6.47342300 & -1.29139500 & 3.90660300\end{array}$

$\begin{array}{llll}\text { C } & 7.38112600 & 0.72988200 & 1.54621800\end{array}$

$\begin{array}{llll}\text { C } & 6.26713900 & 1.42861900 & 2.34793200\end{array}$

$\begin{array}{llll}\text { C } & 6.73971800 & 1.89648600 & 3.72856800\end{array}$

$\begin{array}{llll}\text { C } & 5.70436700 & 2.59733200 & 1.53498700\end{array}$

$\begin{array}{llll}\mathrm{N} & 6.47200700 & -2.33964800 & 1.88662900\end{array}$

$\begin{array}{llll}\text { C } & 5.38203200 & -3.22758800 & 2.21026100\end{array}$

$\begin{array}{llll}\text { C } & 4.13249100 & -1.69073400 & 7.14143000\end{array}$

$\begin{array}{llll}\text { C } & 2.94408700 & -2.11747200 & 6.26937300\end{array}$

$\begin{array}{llll}\text { C } & 3.11852500 & -1.85314800 & 4.77014400\end{array}$

$\begin{array}{llll}\text { C } & 1.96392600 & -2.46956000 & 3.97262300\end{array}$

$\begin{array}{llll}\text { C } & 3.25307100 & -0.35869800 & 4.46115300\end{array}$

$\begin{array}{llll}\text { C } & 0.12096700 & -7.80267000 & -0.17939200\end{array}$

$\begin{array}{llll}\text { C } & 0.38411300 & -6.44315400 & 0.47012600\end{array}$

$\begin{array}{llll}\text { C } & 1.49850600 & -5.62826200 & -0.21771500\end{array}$

$\begin{array}{llll}\text { C } & 0.96873600 & -4.91254200 & -1.46787600\end{array}$

$\begin{array}{llll}\text { C } & 2.12570300 & -4.63086300 & 0.76204500\end{array}$

$\begin{array}{llll}\text { C } & -7.96914400 & -8.15650500 & 1.80291700\end{array}$

$\begin{array}{llll}\text { C } & -8.52916300 & -6.74452100 & 1.53741400\end{array}$

$\begin{array}{llll}\text { C } & -7.59767300 & -5.62242600 & 1.95356200\end{array}$

$\begin{array}{llll}\text { C } & -6.31345300 & -5.50842200 & 1.40453800\end{array}$

$\begin{array}{llll}\text { C } & -7.98710500 & -4.64374400 & 2.87658400\end{array}$

$\begin{array}{llll}\text { C } & -5.46163600 & -4.46006800 & 1.74710000\end{array}$

$\begin{array}{llll}\text { C } & -7.15766300 & -3.57241900 & 3.21111500\end{array}$

$\begin{array}{llll}\text { C } & -5.89183000 & -3.46195500 & 2.63035400\end{array}$

$\begin{array}{llll}\text { O } & -5.10547500 & -2.38076300 & 2.93714600\end{array}$

$\begin{array}{llll}\text { C } & -3.73908600 & -5.21544000 & -2.41369400\end{array}$

$\begin{array}{llll}\text { C } & -3.47742000 & -4.04380900 & -1.45876900\end{array}$

$\begin{array}{llll}\text { C } & -4.77816800 & -3.24908900 & -1.32423800\end{array}$

$\begin{array}{llll}\text { C } & -4.83939700 & -2.14978000 & -0.30221900\end{array}$

$\begin{array}{llll}\text { O } & -3.93344700 & -1.85811900 & 0.48460000\end{array}$

$\begin{array}{llll}\text { O } & -6.01126500 & -1.53960400 & -0.31552500\end{array}$

$\begin{array}{llll}\text { C } & 2.35231000 & -2.15000900 & -6.30575200\end{array}$

$\begin{array}{llll}\text { C } & 2.65746700 & -2.11744900 & -4.82936800\end{array}$

$\begin{array}{llll}\text { C } & 1.76380500 & -1.52729200 & -3.93024400\end{array}$

$\begin{array}{llll}\text { C } & 3.85607100 & -2.64063300 & -4.32511900\end{array}$

$\begin{array}{llll}\text { C } & 2.06278600 & -1.42711100 & -2.57219900\end{array}$

$\begin{array}{llll}\text { C } & 4.15736400 & -2.56064400 & -2.96273300\end{array}$

$\begin{array}{llll}\text { C } & 3.26692400 & -1.93707900 & -2.08212500\end{array}$

$\begin{array}{llll}\text { C } & 2.39713700 & 3.64261500 & -4.95887900\end{array}$

$\begin{array}{llll}\text { C } & 2.89951200 & 3.47090600 & -3.51947000\end{array}$

$\begin{array}{llll}C & 3.36430000 & 2.04665500 & -3.21799200\end{array}$

$\begin{array}{llll}\mathrm{N} & 3.89906000 & 1.93176700 & -1.84553900\end{array}$

$\begin{array}{llll}\text { C } & -2.54850300 & 0.43356400 & -5.16185100\end{array}$

$\begin{array}{llll}\text { C } & -1.98797100 & 0.51802800 & -3.74466900\end{array}$

$\begin{array}{llll}\text { C } & -1.91126400 & -0.88874400 & -3.13955400\end{array}$

$\begin{array}{llll}\text { C } & -0.64064800 & 1.25331800 & -3.72449600\end{array}$

$\begin{array}{llll}\text { C } & -7.76965800 & -3.78985900 & -3.66390000\end{array}$

$\begin{array}{llll}\text { C } & -8.76734800 & -3.27802500 & -2.62458100\end{array}$

$\begin{array}{llll}\text { C } & -9.07787100 & -4.32199700 & -1.55083100\end{array}$

S $\quad-10.23443500 \quad-3.75523800 \quad-0.24757400$

$\begin{array}{llll}\text { C } & -9.24362400 & -2.40445200 & 0.47768500\end{array}$

C $\quad-11.51205600 \quad-0.76387100 \quad-4.11151400$ 


\begin{tabular}{|c|c|c|c|}
\hline & -10.71805200 & -0.12629200 & -2.99404300 \\
\hline & -9.37047000 & 0.21442200 & -3.16636100 \\
\hline & -11.29362200 & 0.13144300 & -1.74475100 \\
\hline & -8.62642700 & 0.80408400 & -2.14601200 \\
\hline & -10.56943500 & 0.73733100 & -0.71812800 \\
\hline & -9.23204300 & 1.09162900 & -0.91378700 \\
\hline & -8.55325900 & 1.70431300 & 0.10842700 \\
\hline & -5.97319700 & 4.12407100 & -3.87343900 \\
\hline & -5.20697500 & 3.65810000 & -2.63098600 \\
\hline & -6.00380900 & 3.02932900 & -1.50617500 \\
\hline 0 & -7.11049600 & 3.51056400 & -1.16382000 \\
\hline O & -5.43966200 & 2.02222200 & -0.94426800 \\
\hline & -9.66665100 & 6.54511300 & -3.06453000 \\
\hline $\mathrm{C}$ & -10.03362700 & 5.91908800 & -1.71946800 \\
\hline C & -10.61719400 & 4.51454600 & -1.88684700 \\
\hline $\mathrm{O}$ & -8.90353400 & 5.89608500 & -0.85215700 \\
\hline $\mathrm{C}$ & -4.30597200 & 6.82727600 & 3.58087800 \\
\hline $\mathrm{C}$ & -4.85461600 & 7.29026200 & 2.22735500 \\
\hline $\mathrm{C}$ & -5.109 & 6.1 & \\
\hline $\mathrm{C}$ & -5.86811300 & 6.68799700 & -0.00860200 \\
\hline $\mathrm{C}$ & -3.803 & 313600 & 0.77308200 \\
\hline $\mathrm{C}$ & 1.07430700 & 5.17291600 & 4.26693600 \\
\hline , & 1.10151300 & 4.12046900 & 2.77477100 \\
\hline $\mathrm{C}$ & 2.00942100 & 2.70020300 & 3.46 \\
\hline $\mathrm{O}$ & 6.96079900 & -2.75782100 & -0.84406900 \\
\hline $\mathrm{O}$ & 6.05 & 907300 & -1.85506000 \\
\hline $\mathrm{O}$ & -5.23175100 & -0.09656500 & -2.75590800 \\
\hline $\mathrm{N}$ & -3.44305800 & 0.92305700 & 1.97582400 \\
\hline $\mathrm{C}$ & -0.92816600 & 2.37872000 & 7.19140200 \\
\hline $\mathrm{C}$ & -1.53988000 & 820400 & 6.17099900 \\
\hline $\mathrm{C}$ & -3.00992400 & 1.94068300 & 1.00595600 \\
\hline $\mathrm{C}$ & -2.3761 & 1.30516700 & -0.25143200 \\
\hline $\mathrm{C}$ & -1.67607500 & 2.56194600 & 4.90496700 \\
\hline $\mathrm{C}$ & -1.21193700 & 1.26771100 & 4.63096 \\
\hline $\mathrm{C}$ & -1.016 & 0.709680 & $0.00886^{\circ}$ \\
\hline $\mathrm{C}$ & -1.31242000 & 0.70632600 & 3.22576300 \\
\hline $\mathrm{C}$ & 0.12661700 & 1.52852400 & -0.00063400 \\
\hline $\mathrm{C}$ & 1.37849300 & 1.00794300 & 0.31451500 \\
\hline $\mathrm{C}$ & -2.72662600 & 0.36840200 & 978600 \\
\hline $\mathrm{C}$ & 1.49201700 & -0.35120800 & 0.67410500 \\
\hline $\mathrm{C}$ & -0.61275800 & 0.53762700 & 5.66047800 \\
\hline $\mathrm{C}$ & -0.46443500 & 502300 & 6.93281100 \\
\hline $\mathrm{C}$ & 0.37135800 & -1.174734 & 0.67 \\
\hline $\mathrm{C}$ & -0.87669500 & -0.64463400 & 0.33520300 \\
\hline $\mathrm{O}$ & -0.81009800 & 2.97507700 & 8.41004400 \\
\hline $\mathrm{O}$ & -6.08118900 & 0.63271700 & 1.05542200 \\
\hline $\mathrm{O}$ & 2.72999100 & -0.83038900 & 0.98730600 \\
\hline $\mathrm{O}$ & 2.52492800 & 1.74542100 & 0.36778800 \\
\hline $\mathrm{H}$ & -1.89596100 & 4.11896900 & 6.38818200 \\
\hline $\mathrm{H}$ & -2.12831200 & 3.15258100 & 4.11382400 \\
\hline $\mathrm{H}$ & 3.31740700 & -0.05208100 & 0.92904600 \\
\hline $\mathrm{H}$ & -0.73388300 & -0.22017000 & 3.14805200 \\
\hline $\mathrm{H}$ & -1.74953700 & -1.28927300 & 0.32571900 \\
\hline $\mathrm{H}$ & -2.31389000 & 2.62652400 & 1.49077000 \\
\hline $\mathrm{H}$ & -0.88218300 & 1.39735700 & 2.50083400 \\
\hline $\mathrm{H}$ & -2.31261000 & 2.11042400 & -0.98892100 \\
\hline $\mathrm{H}$ & -3.23313000 & -0.39044700 & 3.48560500 \\
\hline $\mathrm{H}$ & -0.23780900 & -0.46254400 & 5.46672700 \\
\hline $\mathrm{H}$ & 0.05127300 & 2.58460600 & -0.24392000 \\
\hline $\mathrm{H}$ & 0.01847800 & 0.50922100 & 7.71832100 \\
\hline $\mathrm{H}$ & 0.48244000 & -2.22362600 & 0.91762800 \\
\hline $\mathrm{H}$ & -4.42829900 & 0.61819000 & 1.84431400 \\
\hline $\mathrm{H}$ & -0.37553800 & 2.36677900 & 9.02241900 \\
\hline
\end{tabular}

\begin{tabular}{|c|c|c|c|}
\hline & 3.06699100 & 0.56333400 & \\
\hline $\mathrm{H}$ & & & \\
\hline & -7.01872000 & 0.81533700 & \\
\hline & 4.60837300 & 2.65319200 & -1.69939400 \\
\hline & 2.95420400 & & -0.56700700 \\
\hline & 4.41775900 & 1.05565500 & -1.75562700 \\
\hline & 4.12415500 & 1.75590400 & -3.9606 \\
\hline & 2.53524300 & 1.34057700 & -3.3244 \\
\hline & 3.74142100 & & \\
\hline & & & -2.8 \\
\hline & 1.47540 & 3.07715300 & -5.1 \\
\hline & & 3.28 & -5.6 \\
\hline & 4.55 & -3.12 & \\
\hline & 5.07 & -2.99 & -2.5 \\
\hline & & -1.86 & \\
\hline & 1.3493 & -0.96 & -1.8 \\
\hline & 0.819 & -1.14 & -4.2 \\
\hline $\mathrm{H}$ & & & \\
\hline & -0.76 & 2.3 & -4. \\
\hline & & & \\
\hline $\mathrm{H}$ & -2.65 & 1.0 & -3.1 \\
\hline & -1.43 & -0.8 & -2.1 \\
\hline & -2.9 & -1.2 & \\
\hline & -1.33 & -1.5 & -3 . \\
\hline & -8.3 & -2.3 & \\
\hline $\mathrm{H}$ & -9.65 & -2.9 & \\
\hline $\mathrm{H}$ & -8.15 & -4.6 & $-1 .($ \\
\hline & -9.5 & -5.2 & -1.9 \\
\hline & -8.24 & -2.7 & \\
\hline & -9.18 & -1.5 & -0.1 \\
\hline & -9.7 & -2.0 & \\
\hline & -7.98 & 2.42 & -0.2 \\
\hline & -7.58 & 1.03 & \\
\hline $\mathrm{H}$ & -11.0 & 100 & 0.2 \\
\hline $\mathrm{H}$ & -12.3 & -0.1 & -1 . \\
\hline & -8.8 & 0.0 & -4.1 \\
\hline & -5.61 & -3.9 & \\
\hline & -5.03 & -2.7 & -2.2 \\
\hline $\mathrm{H}$ & -3.1 & -4.3 & -0. \\
\hline $\mathrm{H}$ & -2.69 & -3.3 & \\
\hline & & & \\
\hline & -9.45 & -6.6 & \\
\hline & -8.97 & -4.7 & \\
\hline & -5.96 & -6.2 & 0.6 \\
\hline & -4.46 & -4.4 & \\
\hline & -7.48 & -2.8 & \\
\hline & -4.50 & -2.2 & \\
\hline & 2.81 & -3.94 & \\
\hline & & & \\
\hline & 2.669 & $-5.1<$ & \\
\hline & 1.773 & -4.42 & -2.0 \\
\hline & 0.46 & -5.60 & -2.14 \\
\hline & 0.24419300 & -4.13 & -1.1 \\
\hline & & & \\
\hline & -0.53431900 & -5.83 & 0.47464600 \\
\hline & & -6.60 & \\
\hline & 6.86427700 & -2.42724300 & 0.95123100 \\
\hline & 8.615 & -0.19769000 & \\
\hline & 8.17444600 & 1.46158200 & \\
\hline $\mathrm{H}$ & 6.96982600 & 0.41747100 & 0.57803300 \\
\hline & & 3.37530300 & \\
\hline $\mathrm{H}$ & & 2.28402600 & 0.53905700 \\
\hline & 4.84118100 & 3.04673900 & 2.03477100 \\
\hline
\end{tabular}




\begin{tabular}{|c|c|c|c|}
\hline $\mathrm{H}$ & 7.60475200 & 2.56685200 & \\
\hline $\mathrm{H}$ & & & \\
\hline & .00769400 & 1.04682100 & 4.35859100 \\
\hline & .46036900 & 0.70258800 & 2300 \\
\hline & 62223000 & -1.16970000 & 0.29834500 \\
\hline & 37482500 & -3.87187400 & 0700 \\
\hline & 10.95654300 & -3.78989600 & 1300 \\
\hline & 11.27822400 & -1.71359600 & -0.3 \\
\hline & 01427700 & 0.09017500 & -1.7 \\
\hline & 93800 & -0.83081700 & \\
\hline & 35800 & -2.61751400 & -2.5 \\
\hline & 35300 & -0.40451700 & -4.6 \\
\hline & 12.25939400 & 1.20313300 & -2.9 \\
\hline & 12.07327900 & -2.80391800 & -4.2 \\
\hline & 100 & -1.6420 & \\
\hline & 00 & 2.66 & -1.1 \\
\hline & & & -2.0 \\
\hline & -4.6971 & 4.526 & -2.19 \\
\hline & -4.4218 & 2.9536 & -2.9 \\
\hline & & & -2.3 \\
\hline & -10.83528300 & 4.07449500 & -0.9 \\
\hline & -11.53769100 & 4.52690600 & -2. \\
\hline & & 200 & \\
\hline & -3.43 & 6.17 & \\
\hline & & & \\
\hline & -4.15 & 8.00 & \\
\hline & -5.29 & & -0.5 \\
\hline & -6.04 & & -0.7 \\
\hline & -6.84 & & \\
\hline & & & \\
\hline & -4.00 & & \\
\hline & -3.14 & 6.2 & 0.2 \\
\hline & -5.7 & & \\
\hline & 0.53 & & \\
\hline & 0.55 & & \\
\hline & 2.2 & & \\
\hline & 2.93 & & \\
\hline & & & \\
\hline & & & \\
\hline & 3.26 & -0.1 & \\
\hline & 2.4 & & \\
\hline & & & \\
\hline & & -2.0 & \\
\hline & & -2.3 & \\
\hline & & -2.32999700 & \\
\hline & & & \\
\hline & & & \\
\hline & & & -1.2 \\
\hline & 7400 & -3.0 & -1.4 \\
\hline & & & -2.0 \\
\hline & 5.71 & -0.6 & -2.6 \\
\hline & 395300 & & -2.1 \\
\hline & & 2300 & \\
\hline & 28600 & 3.54739400 & -3.59 \\
\hline & 5.83520500 & 5.30980900 & \\
\hline & -6.73881500 & 4.86225900 & -3.62 \\
\hline & -8.89334600 & 5.92989500 & -3.54370400 \\
\hline & -8.32399300 & 5.16010500 & 1900 \\
\hline & & & \\
\hline & 11.27371600 & 1.91410600 & -1.05929300 \\
\hline & & -2.67162900 & 2.44281300 \\
\hline & 0.92705200 & -8.50751900 & 0.05113100 \\
\hline & 2.82359400 & -3.01071700 & -6.79027100 \\
\hline
\end{tabular}

$\begin{array}{cccc}\mathrm{H} & 2.74140400 & -1.25151100 & -6.80191800 \\ \mathrm{H} & -1.97692400 & -0.27888500 & -5.76912000 \\ \mathrm{H} & -6.91651500 & -4.25928900 & -3.15901700 \\ \mathrm{H} & -8.61406100 & -8.92025200 & 1.35687400 \\ \mathrm{H} & -4.62138700 & -4.99247300 & -3.02691500 \\ \mathrm{H} & -12.58251400 & -0.75609000 & -3.88842700 \\ \mathrm{H} & -11.21957800 & -1.80937700 & -4.26855200 \\ \mathrm{H} & -5.89715900 & -0.62059700 & -2.28916900 \\ \mathrm{H} & -5.18898600 & 4.58480700 & -4.48291100 \\ \mathrm{H} & -6.42732100 & 3.29488300 & -4.42599300 \\ \mathrm{H} & -2.96839500 & -5.45016300 & -3.15628000 \\ \mathrm{H} & -3.95784300 & -6.18434800 & -1.94833100 \\ \mathrm{H} & -7.34955900 & -2.97709400 & -4.26592700 \\ \mathrm{H} & -8.16214500 & -4.54223000 & -4.35628000 \\ \mathrm{H} & -11.36269600 & -0.24237000 & -5.06217500 \\ \mathrm{H} & -2.54065700 & 1.39370100 & -5.68875300 \\ \mathrm{H} & -3.60162800 & 0.13568700 & -5.16935200 \\ \mathrm{H} & 1.28045100 & -2.19077900 & -6.52373300 \\ \mathrm{H} & 2.20037600 & 4.69510400 & -5.18925500 \\ \mathrm{H} & 7.35171200 & 6.22401600 & 0.01233700 \\ \mathrm{H} & 5.99386800 & 6.53392400 & -1.07904600 \\ \mathrm{H} & 9.01176000 & 4.73086700 & -4.05846500 \\ \mathrm{H} & 9.99929800 & 1.39341400 & 0.05999600 \\ \mathrm{H} & 5.62562200 & -3.87723000 & 3.05742900 \\ \mathrm{H} & 5.18902900 & -3.85157200 & 1.33606900 \\ \mathrm{H} & 5.04563300 & -2.20871500 & 6.83137400 \\ \mathrm{H} & 3.82530100 & -1.81086300 & 8.18614300 \\ \mathrm{H} & -0.82162900 & -8.24860500 & 0.15121400 \\ \mathrm{H} & 0.05029600 & -7.74649000 & -1.27108700 \\ \mathrm{H} & -7.91083200 & -8.34993400 & 2.87918500 \\ \mathrm{H} & -6.97956400 & -8.23530200 & 1.34157400 \\ \mathrm{H} & -9.26215900 & 7.56060600 & -2.99306100 \\ \mathrm{H} & -10.48458900 & 6.62542400 & -3.78809700 \\ \mathrm{H} & -3.94403800 & 7.66943400 & 4.18011200 \\ \mathrm{H} & -5.04637200 & 6.24260800 & 4.13788300 \\ \mathrm{H} & 2.09148800 & 5.42918800 & 4.57518800 \\ \mathrm{H} & -5.90991500 & 1.25171700 & 0.25293600\end{array}$

\section{Int6 $_{R}(-0.1 \mathrm{kcal} / \mathrm{mol})$}

$\begin{array}{cccc}\mathrm{C} & 6.44351000 & 5.79796000 & -0.07745100 \\ \mathrm{C} & 6.95234000 & 4.81585700 & -1.08448600 \\ \mathrm{O} & 6.49388000 & 3.67841200 & -1.24627200 \\ \mathrm{~N} & 7.98528800 & 5.25568700 & -1.83731000 \\ \mathrm{C} & 8.53374400 & 4.42526300 & -2.87803000 \\ \mathrm{C} & 9.61030300 & 3.44133000 & -2.42287200 \\ \mathrm{O} & 10.62827600 & 3.24065100 & -3.07842900 \\ \mathrm{~N} & 9.33126000 & 2.79730000 & -1.25418800 \\ \mathrm{C} & 10.19714300 & 1.70842100 & -0.85930000 \\ \mathrm{C} & 9.93029200 & 0.47226400 & -1.70684600 \\ \mathrm{O} & 8.78014200 & 0.01268800 & -1.78492400 \\ \mathrm{~N} & 10.97154000 & -0.15964500 & -2.28419900 \\ \mathrm{C} & 10.72345400 & -1.43960100 & -2.96933100 \\ \mathrm{C} & 10.12851100 & -2.47865100 & -2.01484400 \\ \mathrm{O} & 9.30924000 & -3.30522600 & -2.41187600 \\ \mathrm{C} & 12.10953800 & -1.80954100 & -3.52777000 \\ \mathrm{C} & 12.76198500 & -0.44182000 & -3.77971800 \\ \mathrm{C} & 12.30811800 & 0.40225000 & -2.58181800 \\ \mathrm{~N} & 10.60299600 & -2.41794000 & -0.74768500 \\ \mathrm{C} & 10.10959300 & -3.21887000 & 0.35610500 \\ \mathrm{C} & 9.53802300 & -2.39167100 & 1.51390400\end{array}$




\begin{tabular}{|c|c|c|c|}
\hline & 9.69427200 & -2.75917600 & 2.66804800 \\
\hline & 8.84333900 & -1.26173100 & 1.16775000 \\
\hline & 7.95666600 & -0.66071000 & 2.17085000 \\
\hline & 6.84814800 & -1.62827700 & 2.63048500 \\
\hline & 6.37822700 & -1.55928300 & 3.76775800 \\
\hline & 7.34494300 & 0.63231500 & 1.60307500 \\
\hline & 6.25412900 & 1.29474000 & 2.46568100 \\
\hline & 6.72972000 & 1.59874100 & 3.89042100 \\
\hline & 5.76126700 & 2.56833900 & 1.77290900 \\
\hline & 6.40034300 & -2.47028900 & 1.68394100 \\
\hline & 5.30068700 & -3.36820400 & 1.942 \\
\hline & 4.03520700 & -2.22364400 & 6.975 \\
\hline & 2.86178200 & -2.57902300 & 6.05208600 \\
\hline $\mathrm{C}$ & 3.07172500 & -2.18285300 & 000 \\
\hline & 1.98869700 & -2.79460600 & 3.69 \\
\hline C & 3.12503800 & -0.66381800 & 4.39 \\
\hline C & 0.05042800 & -7.74466400 & -0.81 \\
\hline C & 0.28967500 & -6.43825300 & -0.05 \\
\hline $\mathrm{C}$ & 1.42888800 & -5.57505600 & -0.6 \\
\hline $\mathrm{C}$ & 0.96068000 & -4.78082200 & -1.86 \\
\hline $\mathrm{C}$ & 1.98554300 & -4.63832300 & \\
\hline $\mathrm{C}$ & -8.04534900 & -8.25743000 & 600 \\
\hline $\mathrm{C}$ & -8.59882000 & -6.82922600 & 0.95 \\
\hline $\mathrm{C}$ & -7.63394800 & -5.78882000 & 1.47 \\
\hline $\mathrm{C}$ & -6.35754400 & -5.66947500 & 0.91 \\
\hline $\mathrm{C}$ & -7.95403400 & -4.938317 & 2.53 \\
\hline $\mathrm{C}$ & -5.43439900 & -4.745792 & \\
\hline $\mathrm{C}$ & -7.05117300 & -3.98711800 & 3.01 \\
\hline $\mathrm{C}$ & -5.78479900 & -3.8783 & 2.43 \\
\hline $\mathrm{O}$ & -4.91493000 & -2.93149200 & 2.88 \\
\hline $\mathrm{C}$ & -3.80510500 & -4.99216100 & -2.8 \\
\hline $\mathrm{C}$ & -3.41691500 & -3.95810000 & $-1.7^{\prime}$ \\
\hline $\mathrm{C}$ & -4.62443500 & -3.08851800 & -1.40 \\
\hline $\mathrm{C}$ & -4.49492300 & -2.06828200 & 300 \\
\hline $\mathrm{O}$ & -3.58481300 & -2.20295 & 0.6 \\
\hline $\mathrm{O}$ & -5.34768100 & -1.13963000 & -0.27 \\
\hline $\mathrm{C}$ & 2.29554900 & -1.62749000 & -6.47 \\
\hline $\mathrm{C}$ & 2.60420900 & -1.70204600 & -4.99 \\
\hline $\mathrm{C}$ & 1.73415700 & -1.13285000 & -4.06 \\
\hline $\mathrm{C}$ & 3.78454400 & -2.29482400 & -4.53 \\
\hline $\mathrm{C}$ & 2.04169000 & -1.11454200 & -2.70 \\
\hline $\mathrm{C}$ & 4.09307800 & -2.298 & -3.16 \\
\hline $\mathrm{C}$ & 3.22888800 & -1.69135900 & -2.25 \\
\hline $\mathrm{C}$ & 2.33177400 & 4.04164000 & -4.67 \\
\hline $\mathrm{C}$ & 2.91362400 & 3.77206200 & -3.28151800 \\
\hline $\mathrm{C}$ & 3.35920600 & 2.32274600 & -3.09 \\
\hline $\mathrm{N}$ & 3.96317900 & 2.11877200 & -1.76 \\
\hline $\mathrm{C}$ & -2.61069500 & 0.85563800 & -5.14 \\
\hline $\mathrm{C}$ & -2.05467100 & 0.83039200 & -3.72 \\
\hline $\mathrm{C}$ & -1.97497300 & -0.62094300 & -3.23 \\
\hline $\mathrm{C}$ & -0.71312700 & 1.57162100 & -3.64 \\
\hline $\mathrm{C}$ & -7.83293000 & -3.47517600 & -3.99764300 \\
\hline $\mathrm{C}$ & -8.80864800 & -3.03069100 & -2.90691600 \\
\hline $\mathrm{C}$ & -9.02145900 & -4.08876000 & -1.822 \\
\hline S & -10.00134500 & -3.50643700 & 85700 \\
\hline $\mathrm{C}$ & -8.83031900 & -2.28583600 & 0.30357500 \\
\hline $\mathrm{C}$ & -11.57643700 & -0.42547600 & -4.21786100 \\
\hline $\mathrm{C}$ & -10.84365200 & 0.15224400 & -3.03365300 \\
\hline $\mathrm{C}$ & -9.46377600 & 0.38654000 & -3.08551400 \\
\hline $\mathrm{C}$ & -11.51292200 & 0.44755900 & -1.84219100 \\
\hline $\mathrm{C}$ & -8.77196000 & 0.91095700 & -1.99679800 \\
\hline & -10.84081100 & 0.98927800 & -0.74757000 \\
\hline & -9.47262200 & 1.23727900 & -0.83019700 \\
\hline
\end{tabular}

O

c

(

\section{O}

c

c

c

S

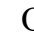

\section{.}

\section{,}

\section{C}

C

\section{C}

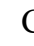

C

\section{O}

$\mathrm{H}$

\section{$\mathrm{H}$}

$\mathrm{H}$

$\mathrm{H}$

$\mathrm{H}$

\section{$\mathrm{H}$}

$\begin{array}{lll}-8.83772800 & 1.79200200 & 0.26601400\end{array}$

$\begin{array}{llll}-6.04216400 & 4.43177100 & -3.58015500\end{array}$

$\begin{array}{llll}-5.27739200 & 3.87879900 & -2.37579100\end{array}$

$\begin{array}{llll}-6.08198700 & 3.21004900 & -1.29481700\end{array}$

$\begin{array}{llll}-7.21298500 & 3.57611200 & -0.96436100\end{array}$

$\begin{array}{llll}-5.41930900 & 2.22949700 & -0.72083700\end{array}$

$\begin{array}{llll}-9.73993300 & 6.77984300 & -2.59508800\end{array}$

$\begin{array}{llll}-10.12429800 & 6.07005100 & -1.29835400\end{array}$

$\begin{array}{llll}-10.71853800 & 4.68476800 & -1.55681100\end{array}$

$\begin{array}{llll}-8.99872800 & 5.98199000 & -0.42523500\end{array}$

$\begin{array}{llll}-4.39934900 & 6.54274900 & 4.06825600\end{array}$

$\begin{array}{lll}-4.91560400 & 7.07531200 & 2.73110800\end{array}$

$\begin{array}{lll}-5.28238300 & 5.98388300 & 1.70634000\end{array}$

$\begin{array}{lll}-5.87005100 & 6.62155500 & 0.44244600\end{array}$

$\begin{array}{lll}-4.08539000 & 5.08931100 & 1.35000300\end{array}$

$\begin{array}{lll}0.98017000 & 4.84267300 & 4.63876300\end{array}$

$\begin{array}{lll}1.16007400 & 3.96095700 & 3.05155100\end{array}$

$\begin{array}{lll}2.21559000 & 2.59527600 & 3.63273400\end{array}$

$\begin{array}{llll}6.89909400 & -2.70202600 & -1.07101400\end{array}$

$\begin{array}{llll}6.03319000 & -0.14796100 & -1.88346800\end{array}$

$\begin{array}{llll}-5.49624500 & 0.09172000 & -2.76292100\end{array}$

$\begin{array}{lll}-2.83124300 & -0.04706700 & 1.97980200\end{array}$

$\begin{array}{lll}-1.18301300 & 1.33626900 & 7.50004800\end{array}$

$\begin{array}{lll}-1.54942500 & 2.29003500 & 6.54315500\end{array}$

$\begin{array}{lll}-2.94360300 & 1.22715900 & 1.27590900\end{array}$

$-2.26912800 \quad 1.09978500 \quad-0.11343200$

$\begin{array}{lll}-1.42038100 & 1.99400300 & 5.19107300\end{array}$

$\begin{array}{lll}-0.91885100 & 0.75657700 & 4.76737400\end{array}$

$\begin{array}{lll}-0.82312600 & 0.69075100 & 0.01157900\end{array}$

$\begin{array}{lll}-0.76906100 & 0.47126600 & 3.28454600\end{array}$

$\begin{array}{llll}0.19381600 & 1.65505300 & 0.07936200\end{array}$

$\begin{array}{lll}1.51459100 & 1.28725300 & 0.32857600\end{array}$

$\begin{array}{lll}-1.88088300 & -0.38653700 & 2.77632200\end{array}$

$\begin{array}{llll}1.82291300 & -0.06871900 & 0.55659500\end{array}$

$\begin{array}{lll}-0.56113800 & -0.18825700 & 5.73321400\end{array}$

$\begin{array}{lll}-0.68895600 & 0.09356600 & 7.09095400\end{array}$

$\begin{array}{llll}0.83189800 & -1.03939200 & 0.46427600\end{array}$

$\begin{array}{lll}-0.48185600 & -0.66094600 & 0.18090900\end{array}$

$\begin{array}{lll}-1.32529100 & 1.67859700 & 8.81117600\end{array}$

$\begin{array}{lll}-6.39987500 & 0.84560700 & 1.08787500\end{array}$

$\begin{array}{llll}3.11403400 & -0.41518400 & 0.83495800\end{array}$

$\begin{array}{llll}2.53561900 & 2.18614500 & 0.41356700\end{array}$

$\begin{array}{lll}-1.93157800 & 3.24851200 & 6.87619600\end{array}$

$\begin{array}{lll}-1.68803800 & 2.74301600 & 4.45194400\end{array}$

$\begin{array}{llll}3.60561500 & 0.42524900 & 0.87480800\end{array}$

$\begin{array}{lll}0.15383800 & -0.08895900 & 3.09288200\end{array}$

$\begin{array}{llll}-1.25154000 & -1.42308300 & 0.10046200\end{array}$

$\begin{array}{lll}-2.47793500 & 2.01604600 & 1.86630900\end{array}$

$\begin{array}{lll}-0.68711800 & 1.40319000 & 2.72753200\end{array}$

$\begin{array}{lll}-2.37048100 & 2.07058000 & -0.60556300\end{array}$

$\begin{array}{lll}-1.91538000 & -1.42127300 & 3.11482000\end{array}$

$-0.16205900 \quad-1.15085600 \quad 5.42937900$

$\begin{array}{llll}-0.02884000 & 2.71020700 & -0.04791900\end{array}$

$\begin{array}{lll}-0.39589100 & -0.64853500 & 7.82936100\end{array}$

$\begin{array}{lll}1.09678800 & -2.08035800 & 0.58567900\end{array}$

$\begin{array}{lll}-3.39048700 & -0.84589900 & 1.55239500\end{array}$

$\begin{array}{lll}-1.05121300 & 0.93654600 & 9.36611700\end{array}$

$\begin{array}{llll}-2.84185300 & 0.37856200 & -0.70032100\end{array}$

$\begin{array}{lll}-4.00510600 & 1.44500200 & 1.17418300\end{array}$

$\begin{array}{lll}-7.37541300 & 0.89362700 & 0.99853900\end{array}$

$\begin{array}{llll}4.72613600 & 2.78399100 & -1.62441400\end{array}$

H $\quad 3.05189200 \quad 2.22242000 \quad-0.49449400$

H $\quad 4.41458500 \quad 1.20325500 \quad-1.71947700$ 


\begin{tabular}{|c|c|c|c|}
\hline & 06710400 & .05707400 & \\
\hline $\mathrm{H}$ & & & -3.19217600 \\
\hline & & & \\
\hline & 2.18068100 & 4.02254900 & -2.50517800 \\
\hline & & 3.49682700 & -4.82492400 \\
\hline & 3.02970400 & 3.72534600 & \\
\hline & 4.46243900 & -2.76581100 & -5.23946000 \\
\hline & 4.99473000 & -2.78608200 & -2.80689100 \\
\hline & 3.47736600 & -1.67038700 & 9700 \\
\hline & 1.35013300 & 346300 & \\
\hline & 0.80275900 & 927000 & 600 \\
\hline & -0.00714000 & 233500 & -4 \\
\hline $\mathrm{H}$ & & 50500 & \\
\hline $\mathrm{H}$ & -0.24 & 374300 & 400 \\
\hline & -2.76 & 54100 & \\
\hline $\mathrm{H}$ & $-1.49^{\prime}$ & 800 & 3500 \\
\hline r & -2.98 & 2900 & 0700 \\
\hline $\mathrm{H}$ & -1.39 & -1.2 & 400 \\
\hline $\mathrm{H}$ & -8.40 & -2.1 & 900 \\
\hline $\mathrm{F}$ & -9.77 & -2.7 & \\
\hline $\mathrm{H}$ & -8.05 & -4.4 & \\
\hline $\mathrm{r}$ & -9.56 & -4.9 & -2. \\
\hline $\mathrm{H}$ & -7.84 & & \\
\hline $\mathrm{H}$ & -8.74 & -1.4 & -0 . \\
\hline $\mathrm{F}$ & -9.22 & -1.9 & \\
\hline $\mathrm{H}$ & -8.26 & 100 & \\
\hline $\mathrm{H}$ & -7.69 & 1.0 & 200 \\
\hline $\mathrm{H}$ & & & \\
\hline $\mathrm{H}$ & -12.58 & 700 & 300 \\
\hline $\mathrm{H}$ & -8.90 & 500 & \\
\hline $\mathrm{H}$ & -5.46 & -3.7 & \\
\hline $\mathrm{H}$ & -4.96 & -2.5 & 500 \\
\hline $\mathrm{H}$ & -3.01 & -4.4 & \\
\hline $\mathrm{H}$ & -2.6 & -3.3 & \\
\hline $\mathrm{H}$ & -8.80 & 800 & 900 \\
\hline $\mathrm{H}$ & & & \\
\hline $\mathrm{H}$ & -8.94 & & \\
\hline $\mathrm{H}$ & -6.07 & -6.3 & \\
\hline $\mathrm{H}$ & -4.4 & -4. & \\
\hline $\mathrm{H}$ & -7.31 & -3.3 & \\
\hline $\mathrm{H}$ & -4.29 & -2.7 & \\
\hline $\mathrm{H}$ & & -3.9 & \\
\hline $\mathrm{H}$ & 1.171 & -4.06 & \\
\hline $\mathrm{H}$ & & & \\
\hline $\mathrm{H}$ & 1.78 & -4.2 & -2.3 \\
\hline $\mathrm{H}$ & 0.510 & -5.43 & \\
\hline $\mathrm{H}$ & 0.20 & 86900 & -1.5 \\
\hline $\mathrm{H}$ & 2.24033200 & -6.24731400 & -0.9 \\
\hline $\mathrm{H}$ & & & \\
\hline $\mathrm{H}$ & & -6.67750500 & \\
\hline $\mathrm{H}$ & 6.79774600 & -2.49519700 & \\
\hline $\mathrm{H}$ & 855 & -0.4 & \\
\hline $\mathrm{H}$ & & & \\
\hline $\mathrm{H}$ & 6.922 & 0.41 & \\
\hline $\mathrm{H}$ & 6.56436000 & 3.31525700 & \\
\hline $\mathrm{H}$ & 5.449 & 2.38 & \\
\hline $\mathrm{H}$ & & & \\
\hline $\mathrm{H}$ & 7.62795400 & 2.23010700 & \\
\hline & & & \\
\hline $\mathrm{H}$ & 6.94606100 & 0.67744100 & 4.43319600 \\
\hline & 5.41498700 & 0.59224100 & 2.54140300 \\
\hline $\mathrm{H}$ & & & \\
\hline$H$ & 9.32291300 & -3.86803800 & -0.03795000 \\
\hline
\end{tabular}

H $\quad 10.89815200 \quad-3.84849000 \quad 0.77515800$

$\begin{array}{llll}\mathrm{H} & 11.22897400 & -1.65749000 & -0.52690100\end{array}$

$\mathrm{H} \quad 12.98078100 \quad 0.26870100 \quad-1.72546900$

$\mathrm{H} \quad 13.84983200 \quad-0.49404500 \quad-3.86007200$

$\mathrm{H} \quad 12.67511400 \quad-2.36941700 \quad-2.77498400$

H $\quad 12.37331300 \quad-0.00299600 \quad-4.70413400$

H $\quad 12.21754300 \quad 1.46458000 \quad-2.81239600$

$\mathrm{H} \quad 12.03132200 \quad-2.43138700 \quad-4.42058900$

$\mathrm{H} \quad 9.98577400 \quad-1.30644600 \quad-3.76509200$

$\mathrm{H} \quad 8.36037900 \quad 2.77169600 \quad-0.95556400$

$\mathrm{H} \quad 8.40641500 \quad 6.15127700 \quad-1.64346900$

$\mathrm{H} \quad-4.75245000 \quad 4.70431400 \quad-1.87560500$

$\mathrm{H} \quad-4.49990600 \quad 3.18302000 \quad-2.70353200$

H $\quad-10.00948800 \quad 4.05755800 \quad-2.10997200$

$\mathrm{H} \quad-10.93637200 \quad 4.18210400 \quad-0.61098500$

$\mathrm{H} \quad-11.64108800 \quad 4.74403000 \quad-2.14349200$

$\begin{array}{lllll}\mathrm{H} & -10.85683600 & 6.68021800 & -0.75514200\end{array}$

$\mathrm{H} \quad-3.52633000 \quad 5.89851000 \quad 3.90492500$

$\mathrm{H} \quad-5.79766400 \quad 7.70577200 \quad 2.89920300$

$\mathrm{H} \quad-4.15437000 \quad 7.73006600 \quad 2.28277000$

$\mathrm{H} \quad-5.14774000 \quad 7.31110000 \quad-0.01319800$

$\mathrm{H} \quad-6.12813500 \quad 5.86261200 \quad-0.29917800$

$\mathrm{H} \quad-6.78908100 \quad 7.17402800 \quad 0.65339700$

$\mathrm{H} \quad-3.67354200 \quad 4.57895600 \quad 2.22596300$

$\mathrm{H} \quad-4.37622600 \quad 4.31482600 \quad 0.63420800$

$\mathrm{H} \quad-3.27782500 \quad 5.68035900 \quad 0.89930900$

$\mathrm{H} \quad-6.05720100 \quad 5.34475300 \quad 2.15407600$

$\mathrm{H} \quad 0.34640800 \quad 5.71490900 \quad 4.46370400$

$\mathrm{H} \quad 0.50708900 \quad 4.20492400 \quad 5.39046300$

$\mathrm{H} \quad 2.51551500 \quad 2.03343000 \quad 2.75064400$

$\mathrm{H} \quad 3.11105700 \quad 2.98598700 \quad 4.12416600$

$\mathrm{H} \quad 1.67175000 \quad 1.95229200 \quad 4.32705100$

H $\quad 3.98533800 \quad-0.22293300 \quad 4.90574900$

H $\quad 3.20666700 \quad-0.41607200 \quad 3.33562200$

H $\quad 2.21470400 \quad-0.19360000 \quad 4.78886000$

$\mathrm{H} \quad 1.97659100 \quad-3.88774700 \quad 3.75653800$

$\mathrm{H} \quad 0.98971100 \quad-2.43986600 \quad 3.97927900$

$\mathrm{H} \quad 2.14188000 \quad-2.51896500 \quad 2.64474300$

$\mathrm{H} \quad 4.04611000 \quad-2.57562000 \quad 4.27680000$

$\mathrm{H} \quad 2.68033700 \quad-3.66111700 \quad 6.10710400$

H $\quad 1.94420900 \quad-2.09909100 \quad 6.42576600$

$\mathrm{H} \quad 6.60818200 \quad-1.81965800 \quad-1.37348600$

$\mathrm{H} \quad 7.62934700 \quad-2.97843600 \quad-1.65224500$

H $\quad 6.94190100 \quad 0.18012100 \quad-2.01663200$

H $\quad 5.69407000 \quad-0.43883400 \quad-2.74304700$

$\mathrm{H} \quad-5.10886200 \quad 0.86132600 \quad-2.32411700$

H $\quad-6.09080300 \quad-0.03188400 \quad 0.74267200$

$\mathrm{H} \quad 7.71189200 \quad 3.83864900 \quad-3.30216900$

$\mathrm{H} \quad 5.74917900 \quad 5.30333500 \quad 0.60023200$

$\mathrm{H} \quad-6.80729700 \quad 5.15135300 \quad-3.28356000$

$\mathrm{H} \quad-8.96655100 \quad 6.19607100 \quad-3.11163100$

$\mathrm{H} \quad-8.41524500 \quad 5.28168100 \quad-0.75968700$

$\mathrm{H} \quad 4.23664200 \quad-1.14720100 \quad 6.92902300$

H $\quad 11.23334800 \quad 2.03826600 \quad-0.91378400$

$\mathrm{H} \quad 4.40092500 \quad-2.81781500 \quad 2.22092700$

$\mathrm{H} \quad 0.85994300 \quad-8.45934800 \quad-0.63216700$

$\mathrm{H} \quad 2.76613600 \quad-2.45000100 \quad-7.01953700$

$\mathrm{H} \quad 2.68521200 \quad-0.69545600 \quad-6.90157200$

$\mathrm{H} \quad-2.03725900 \quad 0.19174400 \quad-5.80109300$

$\mathrm{H} \quad-6.97270200 \quad-3.97771700 \quad-3.53602500$

H $\quad-8.68747000 \quad-8.98751900 \quad 0.60444600$

$\mathrm{H} \quad-4.69157600 \quad-4.66078800 \quad-3.40254200$

$\mathrm{H} \quad-12.65230200 \quad-0.47766900 \quad-4.03001400$ 


$\begin{array}{cccc} & & & \\ \mathrm{H} & -11.23297500 & -1.44208800 & -4.44252200 \\ \mathrm{H} & -5.65446800 & -0.48887100 & -1.99127100 \\ \mathrm{H} & -5.25649600 & 4.93938100 & -4.14926400 \\ \mathrm{H} & -6.49396000 & 3.64827700 & -4.19750600 \\ \mathrm{H} & -3.03187200 & -5.16754700 & -3.60721800 \\ \mathrm{H} & -4.02437600 & -5.99475400 & -2.46366500 \\ \mathrm{H} & -7.41172000 & -2.61744700 & -4.53278400 \\ \mathrm{H} & -8.22279900 & -4.17110400 & -4.74811200 \\ \mathrm{H} & -11.42459400 & 0.16909300 & -5.12420600 \\ \mathrm{H} & -2.60205300 & 1.85418200 & -5.59367000 \\ \mathrm{H} & -3.66357600 & 0.55872200 & -5.17779000 \\ \mathrm{H} & 1.22436900 & -1.65160600 & -6.69568600 \\ \mathrm{H} & 2.13483900 & 5.10885200 & -4.82229400 \\ \mathrm{H} & 7.26940800 & 6.22782500 & 0.49882800 \\ \mathrm{H} & 5.92010600 & 6.61934500 & -0.57816500 \\ \mathrm{H} & 8.94277800 & 5.05956000 & -3.67151500 \\ \mathrm{H} & 9.97069100 & 1.42594600 & 0.17233900 \\ \mathrm{H} & 5.54224100 & -4.08215900 & 2.73679500 \\ \mathrm{H} & 5.09845200 & -3.92182600 & 1.02400200 \\ \mathrm{H} & 4.94966300 & -2.71521700 & 6.62821800 \\ \mathrm{H} & 3.72498400 & -2.42555500 & 8.00631000 \\ \mathrm{H} & -0.89283900 & -8.21561400 & -0.52302800 \\ \mathrm{H} & -0.01707600 & -7.60296600 & -1.89887000 \\ \mathrm{H} & -7.99004100 & -8.53458900 & 2.16704900 \\ \mathrm{H} & -7.05429300 & -8.29915900 & 0.64602600 \\ \mathrm{H} & -9.33644900 & 7.78684600 & -2.44291500 \\ \mathrm{H} & -10.55576200 & 6.91625600 & -3.31258100 \\ \mathrm{H} & -4.03986100 & 7.33550900 & 4.73280600 \\ \mathrm{H} & -5.14093400 & 5.91578300 & 4.57541500 \\ \mathrm{H} & 1.95348700 & 5.18067700 & 5.00498100 \\ \mathrm{H} & -5.92196600 & 1.71435500 & 0.05801000\end{array}$

\section{$\mathrm{TS}_{R}(+4.1 \mathrm{kcal} / \mathrm{mol})$}

$\begin{array}{cccc}\mathrm{C} & 6.51407300 & 5.79672700 & -0.30499400 \\ \mathrm{C} & 6.98369700 & 4.78947200 & -1.30270300 \\ \mathrm{O} & 6.39365800 & 3.72622200 & -1.54464800 \\ \mathrm{~N} & 8.11213000 & 5.11903500 & -1.96709900 \\ \mathrm{C} & 8.63491100 & 4.25828600 & -2.99420900 \\ \mathrm{C} & 9.67876500 & 3.25262100 & -2.52110900 \\ \mathrm{O} & 10.66692100 & 2.97746300 & -3.19282600 \\ \mathrm{~N} & 9.40921600 & 2.66861800 & -1.31733600 \\ \mathrm{C} & 10.24350500 & 1.55605700 & -0.91733000 \\ \mathrm{C} & 9.93303300 & 0.31504300 & -1.74311700 \\ \mathrm{O} & 8.76607800 & -0.10595400 & -1.81271400 \\ \mathrm{~N} & 10.95431300 & -0.36598500 & -2.29542200 \\ \mathrm{C} & 10.68239500 & -1.67912900 & -2.90470300 \\ \mathrm{C} & 10.07729000 & -2.64150700 & -1.87724600 \\ \mathrm{O} & 9.20996400 & -3.45198000 & -2.20100300 \\ \mathrm{C} & 12.06033100 & -2.10545600 & -3.44520900 \\ \mathrm{C} & 12.74725800 & -0.76696200 & -3.75714900 \\ \mathrm{C} & 12.31062600 & 0.14126400 & -2.60108900 \\ \mathrm{~N} & 10.59108300 & -2.52557000 & -0.63026500 \\ \mathrm{C} & 10.11047100 & -3.23855900 & 0.53885100 \\ \mathrm{C} & 9.60710600 & -2.31403100 & 1.65574700 \\ \mathrm{O} & 9.82020300 & -2.58596900 & 2.82588200 \\ \mathrm{~N} & 8.90489800 & -1.20606600 & 1.25029200 \\ \mathrm{C} & 8.05022900 & -0.53071200 & 2.23473400 \\ \mathrm{C} & 6.95546300 & -1.45518900 & 2.80493600 \\ \mathrm{O} & 6.51664500 & -1.28984100 & 3.94257000 \\ \mathrm{C} & 7.41019800 & 0.70907700 & 1.58550400\end{array}$

\begin{tabular}{|c|c|c|c|}
\hline & 6.35103500 & 1.43748400 & \\
\hline & 6.90859500 & 1.91080300 & 3.77941800 \\
\hline & 5.77581400 & 2.60679200 & 1.63043000 \\
\hline & 6.48084700 & -2.36659000 & 1.93607700 \\
\hline & 5.37281800 & -3.22903400 & 2.27138600 \\
\hline & 4.05529600 & -1.77673300 & 7.21051500 \\
\hline & 2.87976600 & -2.20315000 & 6.32127800 \\
\hline & 3.06666200 & -1.92475200 & 4.82580600 \\
\hline & 1.96103000 & -2.60428400 & 4.01013800 \\
\hline & 3.12363300 & -0.42336700 & 4.51989100 \\
\hline & 0.16010500 & -7.78424100 & -0.25811100 \\
\hline & 0.41146200 & -6.41225100 & 0.36924300 \\
\hline & 1.62066000 & -5.67622800 & -0.24352200 \\
\hline & 1.31238200 & -5.14648100 & -1.65116000 \\
\hline & 2.08495800 & -4.53189700 & 0.66069200 \\
\hline & -7.95296300 & -8.19987900 & 1.61562900 \\
\hline & -8.53920500 & -6.80324800 & 1.34365500 \\
\hline & -7.63703600 & -5.67388100 & 1.79135000 \\
\hline & -6.34175100 & -5.54240500 & 1.27503100 \\
\hline 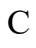 & -8.05808300 & -4.72024700 & 2.72557500 \\
\hline & -5.50170500 & -4.50333700 & 1.66790900 \\
\hline & -7.24068700 & -3.65886000 & 3.11042400 \\
\hline $\mathcal{C}$ & -5.95725900 & -3.53370400 & 2.57031900 \\
\hline & -5.18530200 & -2.47409400 & 2.94750400 \\
\hline$C$ & -3.68163900 & -5.17534200 & 914600 \\
\hline & -3.42790100 & -4.02389500 & -1.51171200 \\
\hline & -4.73004400 & -3.23403800 & -1.35241400 \\
\hline 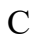 & -4.80784300 & -2.14638300 & -0.30794200 \\
\hline & -3.91265800 & -1.89074600 & 0.51034800 \\
\hline & -5.96306800 & -1.52771600 & 55700 \\
\hline & 2.44643900 & -2.02500300 & -6.26380600 \\
\hline & 2.72538900 & -2.00523100 & 204400 \\
\hline C & 1.81088400 & -1.43607400 & 949300 \\
\hline & 3.91362000 & -2.53801000 & -4.26263800 \\
\hline & & & 2070500 \\
\hline & 4.18016000 & -2.49511600 & -2.89122900 \\
\hline & 3.26675200 & -1.89780000 & -2.01533700 \\
\hline & 2.45179900 & 21100 & 23200 \\
\hline & 3.02006500 & 3.56196200 & -3.41004500 \\
\hline & & & 82800 \\
\hline & 4.14658800 & 1.99346400 & -1.82455300 \\
\hline & -2.47838700 & 0.52165600 & -5.14054300 \\
\hline & -1.92257200 & 0.62877100 & -3.72054300 \\
\hline & -1.83690300 & -0.75637600 & -3.06994700 \\
\hline & -0.57652800 & 1.36973000 & -3.72683000 \\
\hline & -7.70152800 & -3.74461900 & -3.77705100 \\
\hline & -8.71086000 & -3.25050100 & -2.74017600 \\
\hline r & -9.01991200 & -4.30534800 & -1.67671400 \\
\hline & -10.20050000 & -3.76244200 & -0.38573700 \\
\hline & -9.24621800 & -2.38963900 & 0.34904000 \\
\hline & -11.44978500 & -0.72558300 & -4.22315400 \\
\hline & -10.68776800 & -0.08906300 & -3.08486600 \\
\hline & -9.33112700 & 0.23463300 & -3.21473200 \\
\hline & -11.30483300 & 0.18925400 & -1.86067700 \\
\hline & -8.61625700 & 0.82923800 & -2.17716700 \\
\hline & -10.61063100 & 0.80074900 & -0.81769200 \\
\hline & -9.26418900 & 1.14065100 & -0.97223300 \\
\hline & -8.61970200 & 1.75798900 & 0.06569100 \\
\hline & -5.93337400 & 4.17820300 & -3.83613400 \\
\hline & -5.18547900 & 3.70523800 & -2.58592700 \\
\hline & -5.99312100 & 3.06941300 & -1.46988600 \\
\hline & -7.12671400 & 3.52754200 & -1.17460600 \\
\hline & -5.41609100 & 2.09701800 & -0.87356900 \\
\hline
\end{tabular}




\begin{tabular}{|c|c|c|c|}
\hline$\tau^{2}$ & -9.64610100 & 6.57223800 & -3.03516300 \\
\hline & -10.02601200 & 5.92516500 & -1.70348900 \\
\hline & -10.60163000 & 4.52091100 & -1.89773800 \\
\hline & -8.90574800 & 5.89567900 & -0.82539200 \\
\hline & -4.37069500 & 6.76661400 & 3.68127400 \\
\hline & -4.89452000 & 7.24174300 & 2.32299100 \\
\hline C & -5.19909500 & 6.11503000 & 1.31818000 \\
\hline $\mathrm{C}$ & -5.87637500 & 6.67817500 & 0.06450800 \\
\hline $\mathrm{C}$ & -3.93860100 & 5.32981700 & 0.93065200 \\
\hline $\mathrm{C}$ & 1.00690600 & 5.12121200 & 4.40861700 \\
\hline S & 1.27941300 & 4.27375300 & 2.81051800 \\
\hline $\mathrm{C}$ & 2.22687100 & 2.83134700 & 78700 \\
\hline $\mathrm{O}$ & 6.91803900 & -2.63727500 & -0.83390400 \\
\hline $\mathrm{O}$ & 6.03687900 & -0.13622000 & -2.029 \\
\hline J & -5.11775300 & 0.01939300 & -2.68958400 \\
\hline $\mathrm{N}$ & -3.00656700 & 0.72497500 & 2.08380000 \\
\hline $\mathrm{C}$ & -1.74065000 & 2.03002100 & 7.15135600 \\
\hline $\mathrm{C}$ & -2.01159100 & 2.90877800 & 6.09663600 \\
\hline $\mathrm{C}$ & -2.9710 & 1.87892 & 47500 \\
\hline C & -2.31624100 & 1.50995100 & -0.16395200 \\
\hline & -1.69429700 & 2.53915700 & 420900 \\
\hline $\mathrm{C}$ & -1.10084800 & 1.30125900 & 4.51182100 \\
\hline $\mathrm{C}$ & -1.00064700 & 0.85102500 & 0.07516800 \\
\hline $\mathrm{C}$ & -0.73881600 & 0.92167600 & 686600 \\
\hline $\mathrm{C}$ & 0.20452900 & 1.51481000 & -0.08581900 \\
\hline $\mathrm{C}$ & 1.43112500 & 0.90273400 & 97600 \\
\hline $\mathrm{C}$ & -1.82144600 & 0.1078 & 74300 \\
\hline $\mathrm{C}$ & 1.39678900 & -0.44798500 & 0.80569900 \\
\hline $\mathrm{C}$ & -0.83747600 & 0.43626600 & 331400 \\
\hline $\mathrm{C}$ & -1.15164400 & 0.79005100 & 6.88919300 \\
\hline $\mathrm{C}$ & 0.22188600 & -1.11386100 & 677300 \\
\hline $\mathrm{C}$ & -1.03148900 & -0.44733800 & 369500 \\
\hline $\mathrm{O}$ & -2.06498500 & 2.44157000 & 301800 \\
\hline $\mathrm{O}$ & -6.25150600 & 0.5379 & 6000 \\
\hline $\mathrm{O}$ & 2.62144300 & -0.97597000 & 331400 \\
\hline $\mathrm{O}$ & 2.59718800 & 1.44554700 & 829800 \\
\hline $\mathrm{H}$ & -2.47169300 & 3.86629000 & 489800 \\
\hline $\mathrm{H}$ & -1.89808700 & 3.22942400 & 159000 \\
\hline $\mathrm{H}$ & 3.22804300 & -0.22754700 & 139100 \\
\hline $\mathrm{H}$ & 0.17074900 & 0.31947000 & 29300 \\
\hline $\mathrm{H}$ & -1.86725700 & -1.09351400 & 568200 \\
\hline $\mathrm{H}$ & -2.39715700 & 2.68345200 & 1.68130700 \\
\hline $\mathrm{H}$ & -0.51131300 & 1.82397900 & 2.52757600 \\
\hline $\mathrm{H}$ & -2.21243600 & 2.42572400 & -0.75117900 \\
\hline $\mathrm{H}$ & -1.98195000 & -0.86958100 & 2.85339500 \\
\hline $\mathrm{H}$ & -0.37165900 & -0.52650800 & 5.38667000 \\
\hline $\mathrm{H}$ & 0.23135600 & 2.52897400 & -0.47294800 \\
\hline $\mathrm{H}$ & -0.93336500 & 0.10622900 & 7.70623200 \\
\hline $\mathrm{H}$ & 0.21659300 & -2.12523400 & 1.36495100 \\
\hline $\mathrm{H}$ & -3.76910000 & 0.08961400 & 1.86310800 \\
\hline $\mathrm{H}$ & -1.85700300 & 1.73387700 & 9.03688900 \\
\hline $\mathrm{H}$ & -3.00917200 & 0.84778500 & -0.68668200 \\
\hline $\mathrm{H}$ & -3.98662500 & 2.22736300 & 1.04260200 \\
\hline $\mathrm{H}$ & -7.20825200 & 0.72986200 & 1.05692700 \\
\hline $\mathrm{H}$ & 4.77756500 & 2.78072500 & -1.61400400 \\
\hline $\mathrm{H}$ & 3.41320200 & 1.89613000 & -1.04836400 \\
\hline $\mathrm{H}$ & 4.74602800 & 1.14929400 & -1.79252900 \\
\hline $\mathrm{H}$ & 4.30486800 & 1.89226100 & -3.91581600 \\
\hline $\mathrm{H}$ & 2.73943500 & 1.39784600 & -3.26186500 \\
\hline $\mathrm{H}$ & 3.85132200 & 4.26062900 & -3.24719000 \\
\hline $\mathrm{H}$ & 2.25594000 & 3.79821700 & -2.66015000 \\
\hline & 1.52057600 & 3.18648600 & -4.95014000 \\
\hline & 3.16224600 & 3.38957300 & -5.57824500 \\
\hline
\end{tabular}

H

$\mathrm{H}$

H

$\mathrm{H}$

$\mathrm{H}$

H$$
\mathrm{H}
$$$$
\mathrm{H}
$$

$\mathrm{H}$

$\mathrm{H}$

H$$
\text { (1) }
$$

\section{$\mathrm{H}$}$$
\begin{aligned}
& \mathrm{H} \\
& \mathrm{H}
\end{aligned}
$$

\section{H}

\section{H}

$\mathrm{H}$

$\begin{array}{lrrr}\mathrm{H} & 10.89474600 & -3.86332100 & 0.97240100 \\ \mathrm{H} & 11.25922600 & -1.78508000 & -0.47523800\end{array}$

$\begin{array}{rrrr}\mathrm{H} & 12.97168600 & 0.02355400 & -1.73335300\end{array}$

H $\quad 13.83358700 \quad-0.85021700 \quad-3.82972800$

H $\quad 12.61276200 \quad-2.64551300 \quad-2.66876000$

H $\quad 12.37305000 \quad-0.36098700 \quad-4.70225200$

$\begin{array}{llll}4.62330400 & -3.01400600 & -4.93504800\end{array}$

$\begin{array}{llll}5.07914200 & -2.95393300 & -2.48892800\end{array}$

$\begin{array}{llll}3.46037200 & -1.86913500 & -0.94952500\end{array}$

$\begin{array}{llll}1.33781500 & -0.94897400 & -1.84974800\end{array}$

$\begin{array}{llll}0.87159900 & -1.05190300 & -4.26848100\end{array}$

$\begin{array}{lll}0.12691100 & 0.90777100 & -4.42974400\end{array}$

$\begin{array}{lll}-0.11241200 & 1.37023200 & -2.73598800\end{array}$

$\begin{array}{llll}-2.62671700 & 1.22240300 & -3.12562100\end{array}$

$\begin{array}{lll}-1.35911800 & -0.71408500 & -2.08663600\end{array}$

$-2.84629400 \quad-1.15398500 \quad-2.94180800$

$\begin{array}{lll}-1.26197400 & -1.45478000 & -3.69032000\end{array}$

$-8.29873000 \quad-2.36374300 \quad-2.25022100$

$\begin{array}{lll}-9.64176200 & -2.92971800 & -3.21620400\end{array}$

$\begin{array}{llll}-8.09695500 & -4.62827900 & -1.17799900\end{array}$

$-9.47594700 \quad-5.19395600 \quad-2.12728700$

$\begin{array}{lll}-8.24051200 & -2.72143600 & 0.61148400\end{array}$

$\begin{array}{lll} & -1.53255700 & -0.32213200\end{array}$

$-8.01472900-2.46293600-0.31695300$

$\begin{array}{lll}-7.56163700 & 1.04057400 & -2.30204900\end{array}$

$\begin{array}{lll}-11.10149800 & 1.03761800 & 0.12032400\end{array}$

$\begin{array}{lll}-12.35275200 & -0.06362500 & -1.72337300\end{array}$

$\begin{array}{lll}-8.81487000 & 0.01254400 & -4.14582600\end{array}$

$\begin{array}{lll}-5.55741400 & -3.91390200 & -1.11735600\end{array}$

$\begin{array}{lll} & -2.76958700 & -2.30826200\end{array}$

$-2.64367500-3.35743100-1.88393400$

$\begin{array}{llll}-8.73127900 & -6.70056200 & 0.26822600\end{array}$

$\begin{array}{llll}-9.51526100 & -6.70055300 & 1.82816100\end{array}$

$\begin{array}{lll}-9.06030200 & -4.79379700 & 3.13814600\end{array}$

$\begin{array}{llll}-5.97283800 & -6.26909700 & 0.55507900\end{array}$

$\begin{array}{lll}-4.49180100 & -4.43597600 & 1.27876300\end{array}$

$2.93946100-4.00058300-0.23217900$

$\begin{array}{lll}1.28390000 & -3.79799000 & 0.78808600\end{array}$

$\begin{array}{llll}2.36844200 & -4.88969900 & 1.65577600\end{array}$

$2.19144600 \quad-4.67251600 \quad-2.09784400$

$\begin{array}{llll}0.98016700 & -5.94009000 & -2.32723300\end{array}$

$\begin{array}{lll}0.51933700 & -4.38969900 & -1.60959300\end{array}$

$\begin{array}{llll}0.56955600 & -6.53124900 & 1.44874900\end{array}$

$\begin{array}{lll}6.84562100 & -2.44120400 & 0.99056900\end{array}$

$\begin{array}{lll}8.67263800 & -0.24881500 & 3.08430100\end{array}$

$\begin{array}{lll}8.21164500 & 1.42214300 & 1.34288400\end{array}$

$\begin{array}{llll}6.95606800 & 0.40685700 & 0.63213500\end{array}$

$\begin{array}{llll}6.56507800 & 3.32657900 & 1.38122600\end{array}$

$\begin{array}{lll}5.32160200 & 2.26711000 & 0.69658500\end{array}$

$\begin{array}{llll}5.00396100 & 3.13440600 & 2.19781300\end{array}$

$\begin{array}{lll}7.78651500 & 2.55498900 & 3.63734700\end{array}$

$\begin{array}{lll}6.15493800 & 2.49193100 & 4.32078900 \\ 7.18548000 & 1.06017300 & 4.40420400\end{array}$

$\begin{array}{lll}5.53629000 & 0.73464800 & 2.64088800\end{array}$

$\begin{array}{llll}8.57050500 & -1.21072900 & 0.29166600\end{array}$

$\begin{array}{llll}9.29236900 & -3.88726200 & 0.21412300\end{array}$ 


\begin{tabular}{|c|c|c|c|}
\hline $\mathrm{H}$ & 12.25383700 & 1.19497600 & \\
\hline $\mathrm{H}$ & & -2.76369800 & -4.31007600 \\
\hline & .94173100 & -1.58236000 & -3.70252900 \\
\hline & & 2.68515400 & \\
\hline & 8.62200900 & & -1.70642100 \\
\hline & -4.68110000 & 4.57057800 & -2.1339 \\
\hline & -4.39511900 & 3.00309600 & -2.86682100 \\
\hline & -9.87566500 & 3.87659500 & -2.40673800 \\
\hline & -10.82368900 & 4.06447000 & -0.92962000 \\
\hline & -11.51797800 & 4.53802200 & \\
\hline & -10.77233200 & 6.55218600 & 780000 \\
\hline & -3.497 & & \\
\hline & -5.805 & 300 & 2.4 \\
\hline & -4.16005700 & 7.922 & \\
\hline & -5.22722 & & \\
\hline & -6.093 & & -0. \\
\hline & -6.8 & & \\
\hline & -3.43960500 & & \\
\hline & -4.18681 & & \\
\hline & -3.21 & & \\
\hline & -5.900 & & \\
\hline & 0.444 & & \\
\hline & 0.4 & & \\
\hline & $2.4 \mathrm{C}$ & & \\
\hline & 3.14 & & \\
\hline & 1.62 & & \\
\hline & 3.99 & & \\
\hline & & & \\
\hline & 2.21 & & \\
\hline & & & \\
\hline & 0.9 & & \\
\hline & 2.1 & -2.4 & \\
\hline & & & \\
\hline & & & \\
\hline & & -1.7 & \\
\hline & & & \\
\hline & 7.6 & -2.9 & \\
\hline & & & \\
\hline & & & -2 \\
\hline & -5.06 & & \\
\hline & -6.0 & 300 & \\
\hline & & & \\
\hline & 5.83 & & \\
\hline & -6.70 & & -3. \\
\hline & -8.864 & & \\
\hline & & & \\
\hline & & -0.7 & \\
\hline & 11.2 & & \\
\hline & 4.48 & -2.6 & 00 \\
\hline & & & \\
\hline & & & \\
\hline & 2.84 & -1.1 & -6.7 \\
\hline & -1.89 & -0.1 & \\
\hline & -6.85216100 & -4.21819700 & -3.26920600 \\
\hline & -8.57 & -8.97 & \\
\hline & -4.55823600 & -4.93 & -3.11492200 \\
\hline & -12.52437700 & -0.73736200 & -4.02097200 \\
\hline & 56400 & -1.76394900 & \\
\hline & & & \\
\hline & -5.14333700 & 4.65166100 & -4.42817200 \\
\hline & & & -4.40776600 \\
\hline & -2.90060800 & -5.39525400 & -3.23550000 \\
\hline & -3.90236800 & -6.15248800 & -2.05223300 \\
\hline
\end{tabular}

$\begin{array}{cccc}\mathrm{H} & -7.27708000 & -2.92067800 & -4.36054000 \\ \mathrm{H} & -8.08238300 & -4.48711100 & -4.48639400 \\ \mathrm{H} & -11.29043100 & -0.18821900 & -5.16330100 \\ \mathrm{H} & -2.46765300 & 1.49024100 & -5.65168200 \\ \mathrm{H} & -3.53018900 & 0.22010900 & -5.16610600 \\ \mathrm{H} & 1.37756700 & -2.06616500 & -6.49593700 \\ \mathrm{H} & 2.25383800 & 4.80056300 & -5.03903300 \\ \mathrm{H} & 7.33326600 & 6.26409900 & 0.25131000 \\ \mathrm{H} & 5.96515700 & 6.59253900 & -0.82105800 \\ \mathrm{H} & 9.05024200 & 4.84309100 & -3.82171300 \\ \mathrm{H} & 10.02209200 & 1.29319400 & 0.12036500 \\ \mathrm{H} & 5.60821900 & -3.89137800 & 3.11101200 \\ \mathrm{H} & 5.14968400 & -3.84363000 & 1.39765600 \\ \mathrm{H} & 4.97425800 & -2.28628900 & 6.90372100 \\ \mathrm{H} & 3.73542900 & -1.91489400 & 8.24917700 \\ \mathrm{H} & -0.78488200 & -8.23886200 & 0.05326800 \\ \mathrm{H} & 0.10292600 & -7.71059100 & -1.34957400 \\ \mathrm{H} & -7.90740600 & -8.41039200 & 2.68932000 \\ \mathrm{H} & -6.95731200 & -8.26747600 & 1.16568600 \\ \mathrm{H} & -9.24647900 & 7.58794900 & -2.94218800 \\ \mathrm{H} & -10.45515700 & 6.66126800 & -3.76761200 \\ \mathrm{H} & -4.01960100 & 7.60030700 & 4.29854000 \\ \mathrm{H} & -5.11576400 & 6.17030300 & 4.21935400 \\ \mathrm{H} & 1.95920400 & 5.38727200 & 4.87499400 \\ \mathrm{H} & -5.95941000 & 1.21499100 & 0.31183500 \\ & & & \end{array}$

\section{$\operatorname{Int} 7_{R}(+2.5 \mathrm{kcal} / \mathrm{mol})$}

$\begin{array}{cccc}\text { C } & 6.57332000 & 5.77876900 & -0.44337000 \\ \text { C } & 7.05408800 & 4.73777700 & -1.39976400 \\ \text { O } & 6.47262100 & 3.66071400 & -1.60212600 \\ \text { N } & 8.18293300 & 5.04964700 & -2.07072800 \\ \text { C } & 8.72088100 & 4.15386100 & -3.05953100 \\ \text { C } & 9.76507000 & 3.17271400 & -2.53788000 \\ \text { O } & 10.75759600 & 2.87182100 & -3.19152200 \\ \text { N } & 9.48969700 & 2.63966700 & -1.31202400 \\ \text { C } & 10.32327800 & 1.54565600 & -0.86204800 \\ \text { C } & 10.03079800 & 0.27580400 & -1.64977500 \\ \text { O } & 8.86666200 & -0.15397500 & -1.72229500 \\ \text { N } & 11.06253500 & -0.41828300 & -2.16383600 \\ \text { C } & 10.80632600 & -1.75406400 & -2.72986100 \\ \text { C } & 10.19270500 & -2.68153500 & -1.67549900 \\ \text { O } & 9.32754600 & -3.50258200 & -1.97781800 \\ \text { C } & 12.19392500 & -2.19111400 & -3.23589000 \\ \text { C } & 12.87943100 & -0.86068300 & -3.58325000 \\ \text { C } & 12.42134500 & 0.08444100 & -2.46575000 \\ \text { N } & 10.69573800 & -2.52191300 & -0.42902400 \\ \text { C } & 10.20665800 & -3.19486300 & 0.76003200 \\ \text { C } & 9.68992100 & -2.23292600 & 1.83846900 \\ \text { O } & 9.89168600 & -2.46260400 & 3.01939000 \\ \text { N } & 8.98657100 & -1.14279300 & 1.38749200 \\ \text { C } & 8.11530200 & -0.44342800 & 2.34044600 \\ \text { C } & 7.03201000 & -1.36361100 & 2.93884100 \\ \text { O } & 6.59761300 & -1.17610700 & 4.07407300 \\ \text { C } & 7.45595300 & 0.75906000 & 1.64073700 \\ \text { C } & 6.36812200 & 1.48675000 & 2.45245400 \\ \text { C } & 6.90104700 & 2.03135800 & 3.78177600 \\ \text { C } & 5.75547600 & 2.60280200 & 1.60226400 \\ \text { N } & 6.55798400 & -2.29666900 & 2.09176200 \\ \text { C } & 5.45236600 & -3.15302700 & 2.44990600 \\ \text { C } & 4.09167500 & -1.52903700 & 7.32342800\end{array}$




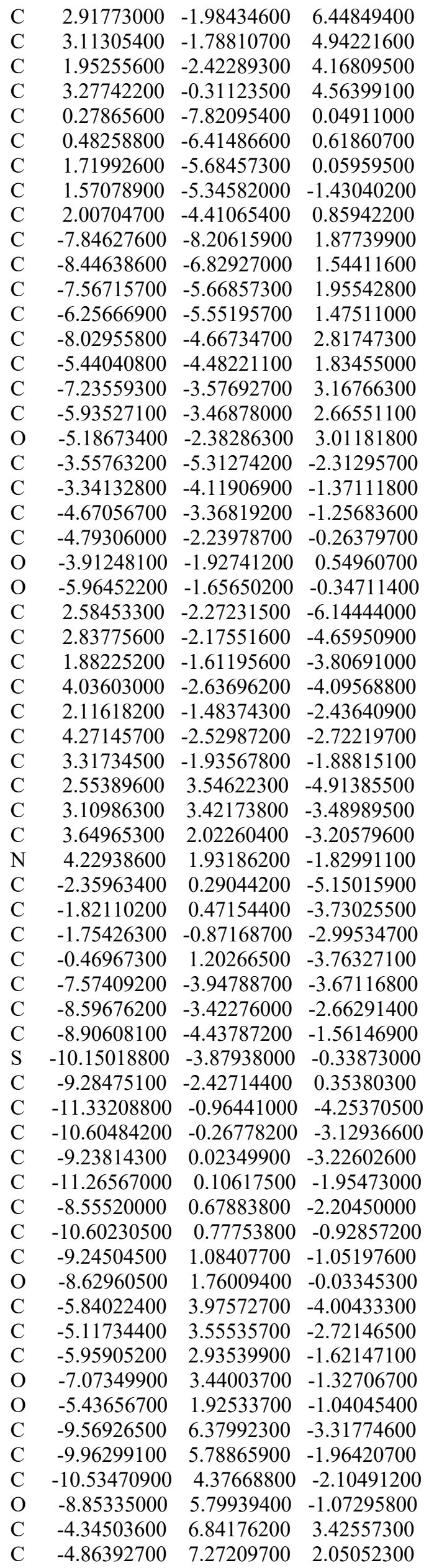

\begin{tabular}{|c|c|c|c|}
\hline & 5.16735500 & 6.11275000 & \\
\hline & -5.83338800 & 6.63326300 & \\
\hline & -3.90686900 & 5.30943800 & \\
\hline & 1.03413400 & 5.24874700 & 4.25128800 \\
\hline & 1.44362400 & 4.36686400 & 2.70110000 \\
\hline & 2.15169700 & 2.83786800 & 3.39881600 \\
\hline & 7.02314900 & -2.63071100 & -0.67156300 \\
\hline & 6.14430500 & -0.16051000 & -1.95001200 \\
\hline & -5.07539300 & -0.21805100 & \\
\hline & -3.15502400 & 421700 & 800 \\
\hline & -2.96396100 & 2.95286400 & 6.4 \\
\hline & -2.44302800 & 3.67883400 & \\
\hline & -3.09366100 & & \\
\hline & -2.37754200 & 20000 & -0.2 \\
\hline & -1.79139600 & 53700 & \\
\hline & -1.64163200 & 2000 & \\
\hline & -1.06809800 & & \\
\hline & -0.9 & & \\
\hline & 0.13 & & -0.1 \\
\hline & 1.36 & & \\
\hline & -1.92127300 & 500 & \\
\hline & 1.32187500 & 9200 & \\
\hline & -2.163 & & \\
\hline & -2.81789800 & & \\
\hline & 0.14900200 & -1.0 & \\
\hline & -1.1 & & \\
\hline & -3.5 & 2100 & \\
\hline & -6.3 & & \\
\hline & 2.54662100 & & \\
\hline & 2.52013100 & & \\
\hline $\mathrm{H}$ & -2.5 & & \\
\hline & -1.3 & & \\
\hline & 3.1 & & \\
\hline & -0.17 & & \\
\hline & -1.86 & 400 & 800 \\
\hline & -2.5 & 500 & \\
\hline & -0.45877900 & & \\
\hline & -2.26619700 & & \\
\hline & -2.12 & -0.8 & 900 \\
\hline & -2.06 & 08000 & \\
\hline & & & \\
\hline & -3.21 & & \\
\hline & $0.13 \xi$ & -1.97 & \\
\hline & -3.8 & 4900 & 7600 \\
\hline & -3.95 & & \\
\hline & -3.03 & & \\
\hline & -4.10 & & \\
\hline & -7.26303700 & & \\
\hline & & & \\
\hline & 3.4737 & 55500 & \\
\hline & & & \\
\hline & 4.44589700 & 1.76642100 & -3.91086500 \\
\hline & 2.87382400 & 1.25621600 & \\
\hline & & & \\
\hline & 2.33265600 & 727900 & -2.75654700 \\
\hline & & 20000 & \\
\hline & 3.27385100 & 3.16800500 & -5.64857400 \\
\hline & 4.77728700 & -3.11215900 & -4.73378300 \\
\hline & 5.17678500 & -2.94118600 & -2.28432800 \\
\hline $\mathrm{H}$ & 3.48604900 & -1.85991500 & -0.82019500 \\
\hline & & & \\
\hline $\mathrm{H}$ & 0.93366400 & -1.28825800 & -4.21834500 \\
\hline$H$ & 0.23874400 & 0.69795400 & -4.43059400 \\
\hline
\end{tabular}




\begin{tabular}{|c|c|c|c|}
\hline & -0.59456900 & & \\
\hline & -0.01645600 & 1.25667700 & -2.76901700 \\
\hline & & & -3.18019000 \\
\hline & -1.28756200 & -0.76918400 & -2.01080300 \\
\hline & -2.76766600 & -1.25499100 & -2.85466200 \\
\hline & -1.17626200 & -1.61133300 & -3.56256500 \\
\hline & -8.19792300 & -2.51410800 & -2.20355800 \\
\hline & -9.52622700 & -3.13118900 & -3.16040300 \\
\hline & -7.99124900 & -4.70642700 & 6600 \\
\hline & -9.31822600 & -5.36084400 & 5200 \\
\hline & -8.28550700 & -2.70409600 & 100 \\
\hline & -9.23349300 & & \\
\hline & -9.87 & 4600 & \\
\hline & -7.99 & 3900 & -0. \\
\hline & $-7.4 \mathrm{C}$ & & \\
\hline & -11.1 & 39500 & 300 \\
\hline & 2331200 & 35300 & 200 \\
\hline & -8.68 & -0.27 & \\
\hline & $-5.4^{\prime}$ & -4.0 & 900 \\
\hline & -4.94 & -2.9 & \\
\hline & -2.93 & & \\
\hline & -2.57 & -3.4 & \\
\hline & & & \\
\hline & -9.4 & -6.721 & \\
\hline & -9.0 & -4.727 & \\
\hline & -5.8 & -6.3 & \\
\hline & -4.41 & -4.42 & \\
\hline & -7.61 & -2.78 & \\
\hline & -4.5 & -2.2 & \\
\hline & 2.9 & -3.9 & \\
\hline & & -3.7 & \\
\hline & 2.14 & -4.6 & \\
\hline & 2.45 & -4.8 & \\
\hline & & -6.2 & \\
\hline & 0.71 & -4.6 & \\
\hline & & & \\
\hline & -0.40 & & \\
\hline & 0.56 & -6.4 & \\
\hline & 6.92 & -2.3 & \\
\hline & 8.726 & -0.1 & \\
\hline & 8.24 & & \\
\hline 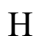 & & & \\
\hline & 6.51 & & \\
\hline $\mathrm{H}$ & & & \\
\hline & 4.96 & & \\
\hline & & 2.69 & \\
\hline & 6.12 & 2.6 & \\
\hline & & & \\
\hline & 5.57 & & \\
\hline & & -1.1 & \\
\hline & & -3.858 & \\
\hline & & & \\
\hline & 11.36419100 & -1.77781900 & 7000 \\
\hline & 13.0 & -0.000 & \\
\hline & 13.96707400 & -0.94106500 & -3.63 \\
\hline & 12.73760300 & -2.70186400 & 5700 \\
\hline & 12.51 & -0.48891200 & -4.54 \\
\hline & 12.36411100 & 1.12769500 & -2.777 \\
\hline & & -2.87862500 & \\
\hline & 10.07572200 & -1.68987400 & -3.54004300 \\
\hline & 8.53083700 & 2.66825700 & -0.98581200 \\
\hline & 8.68442500 & 5.89400200 & -1.83979400 \\
\hline & -4.64542900 & 4.44439800 & -2.28194400 \\
\hline
\end{tabular}

$\begin{array}{rrrr}\mathrm{H} & -3.40988700 & -0.01671300 & -5.17245800 \\ \mathrm{H} & 1.51761400 & -2.32681300 & -6.38272300\end{array}$

$\begin{array}{lll}-4.30827300 & 2.85793700 & -2.95699400\end{array}$

$\begin{array}{llll}-9.80322900 & 3.71264200 & -2.57933200\end{array}$

$\begin{array}{llll}-10.76472500 & 3.96108800 & -1.12056600\end{array}$

$\begin{array}{llll}-11.44542100 & 4.36674500 & -2.71341600\end{array}$

$\begin{array}{llll}-10.71632500 & 6.43545300 & -1.49499000\end{array}$

$\begin{array}{lll}-3.47105400 & 6.18851700 & 3.31842100\end{array}$

$\begin{array}{lll}-5.77394400 & 7.87222200 & 2.17623600\end{array}$

$\begin{array}{llll}-4.12680000 & 7.93553200 & 1.57444900\end{array}$

$\begin{array}{lll}-5.17291200 & 7.34139100 & -0.71164400\end{array}$

$\begin{array}{llll}-6.06150600 & 5.81307000 & -0.87726900\end{array}$

$\begin{array}{lll}-6.78147800 & 7.13518200 & 0.01483900\end{array}$

$\begin{array}{lll}-3.42479800 & 4.88692400 & 1.62247100\end{array}$

$\begin{array}{lll}-4.15146500 & 4.47717100 & 0.07061500\end{array}$

$\begin{array}{llll}-3.17208300 & 5.94546100 & 0.22543100\end{array}$

$\begin{array}{lll}-5.87456100 & 5.43174200 & 1.57919400\end{array}$

$\begin{array}{llll}0.55347600 & 6.18779000 & 3.96938100\end{array}$

$\begin{array}{llll}0.33915800 & 4.66100800 & 4.85506100\end{array}$

$\begin{array}{lll}2.45396800 & 2.21623600 & 2.55499900\end{array}$

$\begin{array}{llll}3.02808800 & 3.05286700 & 4.01628000\end{array}$

$\begin{array}{lll}1.40864000 & 2.30310000 & 3.99364600\end{array}$

$\begin{array}{lll}4.19587200 & 0.11127200 & 4.97812500\end{array}$

$\begin{array}{lll}3.31739900 & -0.19815100 & 3.47717600\end{array}$

$\begin{array}{llll}2.42658700 & 0.27831000 & 4.92862600\end{array}$

$\begin{array}{llll}1.82995200 & -3.48022800 & 4.42879100\end{array}$

$\begin{array}{llll}1.00768200 & -1.91551500 & 4.40429800\end{array}$

$\begin{array}{llll}2.11060600 & -2.34909200 & 3.09087100\end{array}$

$\begin{array}{llll}4.04295400 & -2.29765800 & 4.66579300\end{array}$

$\begin{array}{llll}2.72302300 & -3.04831600 & 6.64057200\end{array}$

$\begin{array}{llll}2.00642100 & -1.45183600 & 6.75893100\end{array}$

$\begin{array}{llll}6.73907100 & -1.79178600 & -1.07070000\end{array}$

$\begin{array}{llll}7.72336700 & -3.00042400 & -1.24139600\end{array}$

$\begin{array}{llll}7.09745400 & 0.03978300 & -2.04842900\end{array}$

$\begin{array}{llll}5.86843500 & -0.72444400 & -2.68956800\end{array}$

$\begin{array}{llll}-5.04809500 & 0.59001000 & -2.20330500\end{array}$

$\begin{array}{lll}-6.09526300 & -0.80382700 & 0.27341900\end{array}$

$\begin{array}{llll}7.88418300 & 3.55996900 & -3.44149300\end{array}$

$\begin{array}{llll}5.88886800 & 5.32818400 & 0.27516300\end{array}$

$\begin{array}{llll}-6.62043300 & 4.71255500 & -3.80264300\end{array}$

$\begin{array}{llll}-8.78158900 & 5.75832700 & -3.76331600\end{array}$

$\begin{array}{lll}-8.26532000 & 5.05690900 & -1.30074400\end{array}$

$\begin{array}{lll}4.28485200 & -0.46061700 & 7.17165800\end{array}$

$\begin{array}{llll}11.36789300 & 1.84556800 & -0.92664800\end{array}$

$\begin{array}{llll}4.55532200 & -2.57235100 & 2.67452100\end{array}$

$\begin{array}{llll}1.09585500 & -8.49268800 & 0.33138800\end{array}$

$\begin{array}{llll}3.07040800 & -3.15344500 & -6.57387400\end{array}$

$\begin{array}{llll}2.98538400 & -1.39587400 & -6.66949000\end{array}$

$\begin{array}{llll}-1.76192100 & -0.43462800 & -5.71560600\end{array}$

$-6.72773100 \quad-4.39879400 \quad-3.13920200$

$\begin{array}{lll}-8.45869800 & -9.00459400 & 1.44338200\end{array}$

$-4.42886400 \quad-5.11264300 \quad-2.94957100$

$\begin{array}{lll}-12.40962200 & -0.99227900 & -4.06975300\end{array}$

$\begin{array}{llll}-10.99413700 & -2.00079400 & -4.37370600\end{array}$

$\begin{array}{llll}-5.69497500 & -0.77412000 & -2.26216800\end{array}$

$\begin{array}{llll}-5.04787000 & 4.43110900 & -4.60735200\end{array}$

$\begin{array}{llll}-6.27622900 & 3.13175100 & -4.54904500\end{array}$

$-2.77017200 \quad-5.55553400 \quad-3.03513900$

$\begin{array}{llll}-3.77741300 & -6.27408600 & -1.83256000\end{array}$

$\begin{array}{llll}-7.14891000 & -3.14364500 & -4.28101000\end{array}$

$-7.94639000 \quad-4.71732800 \quad-4.35590700$

$\begin{array}{lll}-11.16807300 & -0.46069300 & -5.21151400\end{array}$

$\begin{array}{llll}-2.34932400 & 1.23993100 & -5.69596000\end{array}$ 


$\begin{array}{cccc}\mathrm{H} & 2.35293600 & 4.59214200 & -5.16918800 \\ \mathrm{H} & 7.38628500 & 6.26976800 & 0.10160100 \\ \mathrm{H} & 6.02474100 & 6.55302000 & -0.99152200 \\ \mathrm{H} & 9.13981400 & 4.71025400 & -3.90462500 \\ \mathrm{H} & 10.08773600 & 1.31716500 & 0.18066900 \\ \mathrm{H} & 5.68438900 & -3.78344700 & 3.31467200 \\ \mathrm{H} & 5.23796000 & -3.80010000 & 1.59724800 \\ \mathrm{H} & 5.01512200 & -2.04509300 & 7.04201300 \\ \mathrm{H} & 3.76467200 & -1.63099700 & 8.36405100 \\ \mathrm{H} & -0.66663100 & -8.26838900 & 0.36984800 \\ \mathrm{H} & 0.22930300 & -7.78712400 & -1.04469300 \\ \mathrm{H} & -7.80781200 & -8.37745800 & 2.95831600 \\ \mathrm{H} & -6.84700700 & -8.28537500 & 1.43748100 \\ \mathrm{H} & -9.17479600 & 7.40017800 & -3.25871400 \\ \mathrm{H} & -10.37321300 & 6.43862700 & -4.05882700 \\ \mathrm{H} & -4.00220900 & 7.69887100 & 4.01477700 \\ \mathrm{H} & -5.09148500 & 6.26186500 & 3.97944900 \\ \mathrm{H} & 1.93717600 & 5.46748900 & 4.82690700 \\ \mathrm{H} & -6.00097500 & 1.10405600 & 0.18385900\end{array}$

\section{$\operatorname{Int8}_{R}(+5.0 \mathrm{kcal} / \mathrm{mol})$}

$\begin{array}{lrrr}\mathrm{C} & -6.52878400 & -5.81569100 & -0.36666100 \\ \mathrm{C} & -7.01184700 & -4.79154100 & -1.34016500 \\ \mathrm{O} & -6.42903000 & -3.72002200 & -1.56538000 \\ \mathrm{~N} & -8.14348600 & -5.11330700 & -2.00178700 \\ \mathrm{C} & -8.68102500 & -4.23471700 & -3.00581300 \\ \mathrm{C} & -9.72688100 & -3.24629000 & -2.50188800 \\ \mathrm{O} & -10.71958900 & -2.95919700 & -3.16152800 \\ \mathrm{~N} & -9.45322200 & -2.69075900 & -1.28559900 \\ \mathrm{C} & -10.28951400 & -1.59071400 & -0.85636000 \\ \mathrm{C} & -9.99740500 & -0.33388400 & -1.66457600 \\ \mathrm{O} & -8.83374900 & 0.09487300 & -1.74397900 \\ \mathrm{~N} & -11.03051600 & 0.35083300 & -2.18918300 \\ \mathrm{C} & -10.77708100 & 1.67886000 & -2.77416400 \\ \mathrm{C} & -10.16794200 & 2.62417200 & -1.73311700 \\ \mathrm{O} & -9.30613200 & 3.44417300 & -2.04768800 \\ \mathrm{C} & -12.16502500 & 2.10473300 & -3.28878500 \\ \mathrm{C} & -12.84602200 & 0.76745900 & -3.61827500 \\ \mathrm{C} & -12.38726700 & -0.16016000 & -2.48643200 \\ \mathrm{~N} & -10.67174600 & 2.48172000 & -0.48487300 \\ \mathrm{C} & -10.18722400 & 3.17470000 & 0.69469500 \\ \mathrm{C} & -9.67081700 & 2.23024000 & 1.78853200 \\ \mathrm{O} & -9.88038700 & 2.47398400 & 2.96534700 \\ \mathrm{~N} & -8.96331000 & 1.13606500 & 1.35430600 \\ \mathrm{C} & -8.09565400 & 0.44876900 & 2.31893200 \\ \mathrm{C} & -7.00718600 & 1.37228300 & 2.90332700 \\ \mathrm{O} & -6.56875600 & 1.19333500 & 4.03867000 \\ \mathrm{C} & -7.44630900 & -0.77034000 & 1.63970000 \\ \mathrm{C} & -6.36417500 & -1.49557900 & 2.46080600 \\ \mathrm{C} & -6.89741300 & -2.00756800 & 3.80266400 \\ \mathrm{C} & -5.77245200 & -2.63708200 & 1.62980600 \\ \mathrm{~N} & -6.53666400 & 2.29905200 & 2.04814000 \\ \mathrm{C} & -5.43099300 & 3.16024200 & 2.39602000 \\ \mathrm{C} & -4.06528400 & 1.61096100 & 7.29239300 \\ \mathrm{C} & -2.89882500 & 2.06445600 & 6.40754000 \\ \mathrm{C} & -3.10142300 & 1.83325500 & 4.90751500 \\ \mathrm{C} & -1.97511200 & 2.49723800 & 4.10909300 \\ \mathrm{C} & -3.21106600 & 0.34388200 & 4.56104000 \\ \mathrm{C} & -0.27000200 & 7.80629600 & -0.07348600 \\ \mathrm{C} & -0.48969700 & 6.40791000 & 0.50484000\end{array}$

\begin{tabular}{|c|c|c|c|}
\hline & -1.77290800 & 5.72292400 & -0.00728300 \\
\hline & -1.72572500 & 5.44265100 & -1.51600000 \\
\hline & -2.03289900 & 4.42049700 & 0.75370400 \\
\hline & 7.85415100 & 8.23961000 & 1.74746300 \\
\hline & 8.45598800 & 6.85659200 & 1.44384400 \\
\hline & 7.56725100 & 5.71025700 & 1.87112000 \\
\hline & 6.27845100 & 5.57141100 & 1.34083600 \\
\hline & 7.99095400 & 4.74963700 & 2.79615200 \\
\hline & 5.44799700 & 4.51753300 & 1.70881500 \\
\hline & 7.18219000 & 3.67337500 & \\
\hline & 5.90600500 & 3.53704900 & 2.60021500 \\
\hline & 5.14561600 & 2.46361700 & 2.94288400 \\
\hline & 3.57262700 & 5.27407100 & -2.39943100 \\
\hline & 3.30726300 & 4.10737000 & -1.43248400 \\
\hline & 4.58454800 & 3.27426100 & -1.30040800 \\
\hline & 4.64911500 & 2.14860100 & -0.27438800 \\
\hline & 48400 & 2.03626500 & 19100 \\
\hline & 16100 & & \\
\hline & -2.56215600 & & \\
\hline & -2.81714700 & & -4.69879700 \\
\hline & -1.86 & 100 & 0900 \\
\hline & -4.02 & & \\
\hline & -2.09959200 & 3500 & 30600 \\
\hline & -4.26 & 4400 & 000 \\
\hline & -3.31 & & \\
\hline & -2.51596200 & & \\
\hline & -3.08319300 & -3.49100600 & -3.45264000 \\
\hline & -3.6 & & 000 \\
\hline & -4.21 & & \\
\hline & 1800 & 2100 & 59200 \\
\hline & & & -3.7 \\
\hline & 1.845 & 00 & 1900 \\
\hline & 0.51352100 & 94300 & -3.71224100 \\
\hline & 7.59238700 & 23300 & -3.73847100 \\
\hline & & & \\
\hline & 8.90 & & 200 \\
\hline & 10.03938100 & 3.75767900 & -0.31 \\
\hline & & & \\
\hline & 11.3 & & 200 \\
\hline & 10.663 & 1700 & 300 \\
\hline & 9.28615400 & -0.02575000 & -3.17538100 \\
\hline & 11.36 & & \\
\hline & & -0.6 & -2.1 \\
\hline & 10.73261900 & 4600 & -0.94537400 \\
\hline & & & \\
\hline & & & \\
\hline & & -4.0 & -3.9 \\
\hline & 5.151868 & 232200 & -2.68056400 \\
\hline & & & \\
\hline & & & \\
\hline & & 34000 & \\
\hline & 9.61483100 & -6.41597300 & -3.23506400 \\
\hline & & & \\
\hline & & -4.41 & \\
\hline & 8.91496400 & -5.82410800 & -0.98684900 \\
\hline & 4.39285000 & -6.79331000 & 3.51526800 \\
\hline & & & \\
\hline & & -6.06478900 & 1.18404200 \\
\hline & 5.83268200 & -6.60604300 & -0.12221400 \\
\hline & 4.02454700 & -5.17375000 & \\
\hline & & & \\
\hline & & -4.33231700 & 2.76605900 \\
\hline & -2.17127500 & -2.82835500 & 3.46558400 \\
\hline
\end{tabular}




\begin{tabular}{|c|c|c|c|}
\hline & -7.00537000 & 2.60325100 & \\
\hline & -6.11023600 & 0.13191800 & -1.97833600 \\
\hline & 33501900 & 0.16726500 & \\
\hline & & -0.49019300 & \\
\hline & 2.90560600 & -2.69725900 & 6.47434000 \\
\hline & & & \\
\hline & 3.02827500 & & \\
\hline & 2.31702800 & -1.45617500 & -0.11082400 \\
\hline & 1.70997900 & -2.80742600 & \\
\hline & 1.55193700 & -1.41607200 & \\
\hline & & & \\
\hline & 0.85540600 & -0.74488400 & \\
\hline & -0.19563700 & -1.50204100 & \\
\hline & & & \\
\hline & & & \\
\hline & -1.43934500 & & \\
\hline & & -0.6 & \\
\hline & & & \\
\hline & -0.28387500 & & \\
\hline & & & \\
\hline & & -3.3 & \\
\hline & & & \\
\hline & -2.6 & & \\
\hline & & & \\
\hline & & & \\
\hline & 1.29 & -3.3 & \\
\hline & -3.2 & & \\
\hline & & -0.0 & \\
\hline & & & \\
\hline & & & \\
\hline & & & \\
\hline & & & \\
\hline & & & \\
\hline & & & \\
\hline & -0.2 & -2.4 & \\
\hline & & -0.7 & \\
\hline & -0.2 & & \\
\hline & & & \\
\hline & & -2. & \\
\hline & & & \\
\hline & & & \\
\hline & & & \\
\hline & & & \\
\hline & -3.4 & & $-1 .($ \\
\hline & & & \\
\hline & & & \\
\hline & & & \\
\hline $\mathrm{H}$ & & & \\
\hline & & & \\
\hline & & & \\
\hline & 6300 & -3.2 & -5.615 \\
\hline $\mathrm{H}$ & & & \\
\hline & & & \\
\hline & & & \\
\hline $\mathrm{H}$ & -1.33 & 1.08877200 & -1.79959700 \\
\hline & & & \\
\hline & & & \\
\hline & & & \\
\hline $\mathrm{H}$ & 0.08797800 & -1.28368900 & -2.70522700 \\
\hline & & & \\
\hline & & & \\
\hline & & 1.20207700 & -2.9079940 \\
\hline & 1.25789100 & 1.55970000 & -3.56813900 \\
\hline
\end{tabular}$$
\mathrm{H}
$$
$\begin{array}{llll}\mathrm{H} & 10.82924000 & -3.99280900 & -1.05243700 \\ \mathrm{H} & 11.50779500 & -4.40874400 & -2.64250200\end{array}$ $\begin{array}{llll}\mathrm{H} & 11.50779500 & -4.40874400 & -2.64250200 \\ \mathrm{H} & 10.77115300 & -6.46870600 & -1.41870800\end{array}$ H $\quad 3.51562500 \quad-6.14352900 \quad 3.41112100$

$\begin{array}{llll}8.17935600 & 2.47541100 & -2.25888400\end{array}$ $\begin{array}{llll}9.53277500 & 3.06995500 & -3.19561000\end{array}$ $\begin{array}{llll}7.97980800 & 4.71071300 & -1.11780200\end{array}$ $\begin{array}{llll}9.39497600 & 5.27902300 & -2.01249000\end{array}$ $\begin{array}{lll}8.00813400 & 2.73835200 & 0.56361600\end{array}$ $\begin{array}{llll}8.99525000 & 1.54849700 & -0.34014500\end{array}$ $\begin{array}{llll}9.48595600 & 2.07629300 & 1.28506500\end{array}$ $\begin{array}{llll}8.16597500 & -2.45190000 & -0.35293700\end{array}$ $\begin{array}{llll}7.55863000 & -0.80316700 & -2.18022000\end{array}$ $\begin{array}{llll}11.28273000 & -1.16730100 & -0.07056500\end{array}$ $\begin{array}{llll}12.43353800 & 0.02350400 & -1.93051000\end{array}$ $\begin{array}{llll}8.70211300 & 0.30066300 & -4.03192300\end{array}$ $\begin{array}{llll}5.42543300 & 3.93099500 & -1.04534300\end{array}$ $\begin{array}{llll}4.83878300 & 2.84205100 & -2.27411300\end{array}$ $\begin{array}{llll}2.97995600 & 4.46605200 & -0.45158200\end{array}$ $\begin{array}{llll}2.49748400 & 3.47247100 & -1.80846500\end{array}$ $\begin{array}{llll}8.64595200 & 6.77921700 & 0.36555700\end{array}$ $\begin{array}{llll}9.43464300 & 6.75689200 & 1.92439700\end{array}$ $\begin{array}{llll}8.98825400 & 4.82996600 & 3.22009400\end{array}$ $\begin{array}{llll}5.90932800 & 6.30318000 & 0.62575200\end{array}$ $\begin{array}{llll}4.44693500 & 4.43409100 & 1.30319200\end{array}$ $\begin{array}{llll}7.53232600 & 2.91440800 & 3.84928400\end{array}$ $\begin{array}{lll}4.51932000 & 2.29237600 & 2.20388100\end{array}$ $\begin{array}{llll}-2.97567400 & 3.95697400 & 0.44918100\end{array}$ $\begin{array}{lll}-1.23852600 & 3.69646600 & 0.54550900\end{array}$ $\begin{array}{llll}-2.06802600 & 4.58051100 & 1.83573400\end{array}$ $\begin{array}{llll}-2.63860700 & 4.93994600 & -1.85061200\end{array}$ $\begin{array}{llll}-1.61388300 & 6.35692300 & -2.10559000\end{array}$ $-0.88344900 \quad 4.78225400 \quad-1.75632700$ $\begin{array}{llll}-2.61364200 & 6.40639600 & 0.18786400\end{array}$ $\begin{array}{llll}0.37133700 & 5.76807800 & 0.26446200\end{array}$ $\begin{array}{lll}-0.53222600 & 6.46463500 & 1.60013100\end{array}$ $\begin{array}{lll}-6.90446100 & 2.38640700 & 1.10516900\end{array}$ $\begin{array}{lll}-8.70958700 & 0.14262500 & 3.16621300\end{array}$ $\begin{array}{lll}-8.23997800 & -1.49117900 & 1.39409800\end{array}$ $\begin{array}{lll}-7.00905700 & -0.44415400 & 0.68609900\end{array}$ $\begin{array}{lll}-6.54661700 & -3.37449200 & 1.38426300\end{array}$ $\begin{array}{lll}-5.34716100 & -2.27174600 & 0.69159300\end{array}$ $\begin{array}{lll}-4.97574400 & -3.15277100 & 2.17394700\end{array}$ $\begin{array}{lll}-7.76383800 & -2.66616900 & 3.65638900\end{array}$ $\begin{array}{lll}-6.12668300 & -2.58421800 & 4.32427600\end{array}$ $\begin{array}{lll}-7.18473500 & -1.17538900 & 4.44724900\end{array}$ $\begin{array}{lll}-5.56211800 & -0.78029900 & 2.67544800\end{array}$ $\begin{array}{lll}-8.63106900 & 1.16932500 & 0.39550000\end{array}$ $\begin{array}{lll}-9.37558700 & 3.83500000 & 0.37722400\end{array}$ $\begin{array}{llll}-10.97221700 & 3.78563700 & 1.14622700\end{array}$ $\begin{array}{llll}-11.33676300 & 1.73655100 & -0.33953100\end{array}$ $\begin{array}{llll}-13.03742100 & -0.06470100 & -1.60778500\end{array}$ $\begin{array}{llll}-13.93379700 & 0.84380700 & -3.67475500\end{array}$ $\begin{array}{llll}-12.71147800 & 2.62522900 & -2.49496200\end{array}$ $\begin{array}{llll}-12.48151500 & 0.38304800 & -4.57610200\end{array}$ $\begin{array}{llll}-12.32674700 & -1.20773700 & -2.78330700\end{array}$ $\begin{array}{llll}-12.08875300 & 2.78043800 & -4.14166700\end{array}$ $\begin{array}{llll}-10.04511800 & 1.60493400 & -3.58230600\end{array}$ $\begin{array}{lll}-8.49465600 & -2.71103300 & -0.95808600\end{array}$ $-8.64743200 \quad-5.95087200 \quad-1.75223000$ $\begin{array}{llll}4.66861500 & -4.48213300 & -2.23471500\end{array}$ $\begin{array}{llll}4.34972700 & -2.89793200 & -2.91636500\end{array}$ $\begin{array}{llll}9.87073900 & -3.74547000 & -2.51480200\end{array}$ 


\begin{tabular}{|c|c|c|c|}
\hline & & -7.85663900 & \\
\hline & & -7.85723500 & \\
\hline & & & -0.62480300 \\
\hline & .08503500 & -5.79441800 & -0.80809600 \\
\hline & & -7.16516100 & \\
\hline & .61423600 & -4.72680900 & \\
\hline & .29200800 & -4.35046800 & 0.22810800 \\
\hline & .22446500 & -5.75252800 & \\
\hline & 6600 & -5.44161400 & 70800 \\
\hline & .47023300 & -6.12216800 & 4600 \\
\hline & -0.32790800 & -4.59000800 & 4800 \\
\hline & -2.48182000 & -2.20690 & \\
\hline & -3.04800800 & -3.0730 & 400 \\
\hline & -1.44917300 & -2.280 & 800 \\
\hline & -4.09 & -0.1105 & 3500 \\
\hline & -3.2777 & 0.2072 & \\
\hline & -2.32553900 & -0.19976 & 600 \\
\hline & -1.89 & & \\
\hline & -1.0074 & 2.0363 & 200 \\
\hline & -2.14 & & \\
\hline & -4.05 & & \\
\hline & -2.7198 & & \\
\hline & & & \\
\hline & -6.70 & & \\
\hline & -7.70 & 2.96237 & 900 \\
\hline & -7.06 & -0.066 & 500 \\
\hline & -5.82728200 & & 300 \\
\hline & 5.07856100 & -0.6117 & \\
\hline & & & \\
\hline & -7.8 & -3.6 & 600 \\
\hline & -5.8 & & \\
\hline & & -4.766 & \\
\hline & 8.827 & -5.796 & \\
\hline ( & & -5.1004 & \\
\hline & -4.25299800 & 0.5397 & \\
\hline 1 & & & \\
\hline & & & \\
\hline & -1.0 & 8.4 & \\
\hline & 500 & & \\
\hline & -2.957 & 1.26853 & \\
\hline & & & \\
\hline & & & \\
\hline & & 9.03206 & \\
\hline & & & \\
\hline & & & \\
\hline & 10.9 & & \\
\hline & 5.646 & 0.7591 & 900 \\
\hline & & -4.49818 & \\
\hline & & -3.19482 & \\
\hline & & & \\
\hline & 3.78968300 & & \\
\hline & & & \\
\hline & 7.96251900 & 4.660623 & \\
\hline & 11.1 & & \\
\hline & & -1.33029300 & -5.68649000 \\
\hline & 5300 & -0.063375 & \\
\hline & -1.49542500 & 2.21584900 & -6.42440200 \\
\hline & -2.31227600 & -4.68687800 & -5.11005500 \\
\hline & & -6.30083000 & \\
\hline & -5.97761400 & -6.59649100 & -0.90280700 \\
\hline & -9.09863100 & -4.80447400 & -3.84261900 \\
\hline & & -1.34411100 & 0.18320700 \\
\hline & -5.66457600 & 3.80256200 & 3.25154900 \\
\hline
\end{tabular}

$\begin{array}{cccc}\mathrm{H} & -5.21656900 & 3.79573600 & 1.53484700 \\ \mathrm{H} & -4.99014600 & 2.12044200 & 7.00366700 \\ \mathrm{H} & -3.73840800 & 1.72899000 & 8.33136000 \\ \mathrm{H} & 0.67412700 & 8.26090500 & 0.24050100 \\ \mathrm{H} & -0.22075100 & 7.75670000 & -1.16670000 \\ \mathrm{H} & 7.81534900 & 8.42652400 & 2.82576200 \\ \mathrm{H} & 6.85462900 & 8.30976800 & 1.30656100 \\ \mathrm{H} & 9.22313200 & -7.43633400 & -3.16104500 \\ \mathrm{H} & 10.41888900 & -6.48340600 & -3.97530000 \\ \mathrm{H} & 4.05248100 & -7.64273500 & 4.11694300 \\ \mathrm{H} & 5.13788800 & -6.20348900 & 4.06050600 \\ \mathrm{H} & -1.89179800 & -5.45755300 & 4.88189100 \\ \mathrm{H} & 5.91814900 & -1.48661700 & -0.10743900\end{array}$

\section{$\mathrm{TS5}_{R}(+18.0 \mathrm{kcal} / \mathrm{mol})$}

\begin{tabular}{|c|c|c|c|}
\hline & 6.70900100 & 5.57080200 & 1.69372600 \\
\hline & 7.14157800 & 5.01005400 & 0.38110100 \\
\hline & 6.41270300 & 4.30636800 & -0.32613000 \\
\hline & 8.39584400 & 5.34175900 & -0.01546500 \\
\hline & 8.89082700 & 4.90470300 & -1.28893300 \\
\hline & 9.89816600 & 3.76303700 & -1.24131900 \\
\hline & 10.69718300 & 3.58094000 & -2.15571900 \\
\hline & 9.82712200 & 2.93519000 & -0.16546900 \\
\hline & 10.60319200 & 1.71586000 & -0.21051300 \\
\hline & 10.21794700 & 0.85862400 & -1.41405200 \\
\hline & 9.03119300 & 0.57809800 & -1.62900500 \\
\hline & 11.21278100 & 0.30666200 & -2.14217900 \\
\hline & 10.86634100 & -0.71321000 & -3.14613200 \\
\hline & 10.21544500 & -1.93448800 & -2.48873200 \\
\hline & 9.33317200 & -2.56874100 & -3.06768100 \\
\hline & 12.21557900 & -1.00549800 & -3.82738300 \\
\hline & 12.95999600 & 0.33283300 & -3.71619500 \\
\hline & 12.57997900 & 0.84420900 & -2.32024800 \\
\hline & 10.71076600 & -2.24281000 & -1.26813600 \\
\hline & 10.17039400 & -3.24899500 & -0.37109200 \\
\hline & 9.62094200 & -2.65136600 & 0.93429800 \\
\hline $\mathrm{O}$ & 9.83489000 & -3.18822000 & 2.00906100 \\
\hline & 8.89154600 & -1.50016200 & 0.78704100 \\
\hline & 8.00341700 & -1.07902300 & 1.87183600 \\
\hline & 6.92757500 & -2.13024700 & 2.22315300 \\
\hline $\mathrm{O}$ & 6.42526000 & -2.16207100 & 3.34489300 \\
\hline & 7.28443700 & 0.22745200 & 1.48125200 \\
\hline & 6.68475300 & 0.98153400 & 2.68214400 \\
\hline & 7.76638500 & 1.78496900 & 3.41334000 \\
\hline $\mathrm{C}$ & 5.53452300 & 1.88934100 & 2.23866600 \\
\hline N & 6.53577000 & -2.90620700 & 1.19034700 \\
\hline $\mathrm{C}$ & 5.38979000 & -3.78362500 & 1.29538900 \\
\hline & 3.94197900 & -3.91745700 & 6.40641200 \\
\hline & 2.79469700 & -4.06219800 & 5.40171800 \\
\hline & 3.01256900 & -3.33434900 & 4.07249700 \\
\hline & 2.00163700 & -3.82515000 & 3.03198000 \\
\hline 0 & 2.95265600 & -1.80957600 & 4.22776400 \\
\hline $\mathrm{C}$ & 0.20340000 & -7.21670500 & -2.65470900 \\
\hline & 0.29418100 & -6.07103600 & -1.64030600 \\
\hline & 1.42240300 & -5.05026600 & -1.86696700 \\
\hline & 1.31087000 & -4.33672100 & -3.22136600 \\
\hline & 1.41524700 & -4.02178700 & -0.73181300 \\
\hline & -7.96660300 & -8.03444100 & -1.20571500 \\
\hline & -8.59974900 & -6.63844600 & -1.09448500 \\
\hline & -785230100 & -563506600 & -023999100 \\
\hline
\end{tabular}




\begin{tabular}{|c|c|c|c|}
\hline & -6.47937700 & -5.40426600 & -0.39223400 \\
\hline & -8.53428700 & -4.85753500 & 0.70529500 \\
\hline & -5.81614900 & -4.43072400 & 0.35512300 \\
\hline & -7.89151300 & -3.86920000 & 1.44569400 \\
\hline & -6.52606000 & -3.63221600 & 1.26110200 \\
\hline & -5.94018700 & -2.63833000 & 1.98430000 \\
\hline & -3.54099300 & -3.96481300 & -4.06498500 \\
\hline $\mathrm{C}$ & -2.99106700 & -3.32037700 & -2.78238800 \\
\hline & -4.02063600 & -2.49420100 & -2.02221100 \\
\hline & -3.48165100 & -1.81386600 & -0.77239200 \\
\hline $\mathrm{O}$ & -2.21198100 & -1.70279300 & -0.67 \\
\hline $\mathrm{O}$ & -4.30916500 & -1.38219100 & 0.072 \\
\hline $\mathrm{C}$ & 2.73126900 & 0.07909200 & -6.50616700 \\
\hline C & 2.94725300 & -0.33900800 & -5.07 \\
\hline & 2.00481500 & -0.02436300 & -4.08 \\
\hline 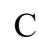 & 4.09637000 & -1.04347700 & \\
\hline & 2.20259500 & -0.37763600 & -2.75220800 \\
\hline & 4.29565900 & -1.41621300 & -3.35 \\
\hline & 3.35673800 & -1.06905500 & -2.38135400 \\
\hline & 2.75973800 & 5.11132900 & -3.33797900 \\
\hline 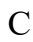 & 3.28421600 & 4.4672 & -2.05 \\
\hline $\mathrm{C}$ & 3.85276100 & 3.07615700 & 91900 \\
\hline $\mathbf{v}$ & 4.36766300 & 2.46122100 & 352600 \\
\hline & -2.19533400 & 2.2461 & 39400 \\
\hline & -1.73184900 & 1.93220400 & 782700 \\
\hline & -1.66548 & 0.4231 & -3.0 \\
\hline & -0.39720900 & 2.62290400 & -3.02 \\
\hline & -7.50487600 & -2.12655800 & 109600 \\
\hline & -8.59012900 & -1.9632 & 262200 \\
\hline & -8.97068400 & -3.29100500 & 308100 \\
\hline & -10.46811200 & -3.21577700 & 935300 \\
\hline C & -9.95587200 & -1.9650 & 950400 \\
\hline C & -11.20365000 & 0.95568900 & 509900 \\
\hline $\mathrm{C}$ & -10.61668000 & 1.27516900 & 514700 \\
\hline $\mathrm{C}$ & -9.24243900 & 1.49 & 366900 \\
\hline & -11.42298700 & 9700 & 720100 \\
\hline 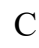 & -8.69496000 & 1.85 & 315900 \\
\hline $\mathrm{C}$ & -10.89354600 & 1.75113000 & 133800 \\
\hline & -9.53009900 & 2.01562100 & 33100 \\
\hline ) & -9.05320600 & 2.4 & 04500 \\
\hline c & -5.64602200 & 5.38363600 & 139900 \\
\hline C & -4.99634 & 4.50 & 18700 \\
\hline C & -5.939247 & 3.673 & 33500 \\
\hline & -7.04656500 & 4.09702900 & 603600 \\
\hline J & -5.45691800 & 2.49965100 & -0.23641200 \\
\hline C & -9.35338500 & 7.48254600 & -1.05514600 \\
\hline C & -9.85879900 & 6.536 & 08500 \\
\hline 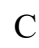 & -10.52508600 & 5.29643500 & -0.56335500 \\
\hline & -8.80655100 & 6.18158100 & 0.92350000 \\
\hline & -4.27747500 & 5.46866800 & 5.51426300 \\
\hline C & -4.76689500 & 6.36632400 & 44400 \\
\hline & -5.06148200 & 5.63269000 & 3.05149000 \\
\hline $\mathrm{C}$ & -5.72876100 & 6.58189000 & 2.04952400 \\
\hline $\mathrm{C}$ & -3.79503000 & 5.01836300 & 2.43997300 \\
\hline & 1.05742000 & 3.57079400 & 5.82381000 \\
\hline S & 2.03777200 & 3.49636700 & 4.28094000 \\
\hline $\mathrm{C}$ & 1.87729100 & 1.72009300 & 3.89372700 \\
\hline $\mathrm{O}$ & 7.04558500 & -2.20442700 & -1.54177200 \\
\hline & 6.31089600 & 0.54371600 & -1.96824800 \\
\hline & -6.85947500 & -0.91101800 & -0.60554600 \\
\hline & -3.26632700 & 0.04775900 & 2.38100600 \\
\hline & -3.08235300 & -3.76938700 & 6.43242500 \\
\hline & -3.93549200 & -2.93490000 & 5.70331500 \\
\hline
\end{tabular}

\begin{tabular}{|c|c|c|c|}
\hline & -3.02344600 & .47352600 & \\
\hline $\mathrm{C}$ & -2.19000100 & & \\
\hline & -3.40556700 & -1.95267500 & \\
\hline C & -2.01726200 & -1.78466600 & 4.74329500 \\
\hline & -0.92852000 & 0.99025600 & \\
\hline & -1.42164200 & -0.75925900 & 3.80626900 \\
\hline & 0.29117100 & 1.55034100 & \\
\hline & 1.48178500 & & \\
\hline & -2.07069500 & -0.77882100 & 800 \\
\hline & 138000 & -0.6 & \\
\hline & 37300 & -2.6 & 900 \\
\hline $\mathrm{C}$ & 49900 & 800 & 000 \\
\hline & 0.15 & -1.1 & \\
\hline $\mathrm{C}$ & 878800 & 7800 & \\
\hline C & 598700 & 6800 & \\
\hline $\mathrm{O}$ & 44000 & 5600 & 300 \\
\hline $\mathrm{C}$ & 57000 & -1.2 & \\
\hline $\mathrm{O}$ & & & \\
\hline $\mathrm{H}$ & 70000 & 7400 & \\
\hline $\mathrm{H}$ & 35900 & & \\
\hline $\mathrm{H}$ & 48800 & -0.5 & \\
\hline & 97800 & -0.9 & \\
\hline $\mathrm{H}$ & & & \\
\hline $\mathrm{H}$ & -2.5 & & \\
\hline $\mathrm{H}$ & -1.5 & & \\
\hline $\mathrm{H}$ & & & \\
\hline $\mathrm{H}$ & -2.3 & -1.8 & \\
\hline & 73900 & 400 & \\
\hline $\mathrm{H}$ & 0.3 & & \\
\hline $\mathrm{H}$ & -1.0 & -4.2 & \\
\hline $\mathrm{H}$ & & -2.2 & \\
\hline $\mathrm{H}$ & -3.8 & -0.2 & \\
\hline $\mathrm{H}$ & -2.9 & -5.2 & \\
\hline $\mathrm{H}$ & & & \\
\hline & -3.9 & & \\
\hline & -7.7 & 100 & \\
\hline $\mathrm{H}$ & & & \\
\hline & 3.5 & & \\
\hline & & & \\
\hline & 4.6 & & \\
\hline & & & \\
\hline & & & \\
\hline & 67600 & & \\
\hline & 2500 & 900 & \\
\hline $\mathrm{H}$ & & & \\
\hline & & -1.3 & \\
\hline & & -2.0 & \\
\hline & & -1.3 & \\
\hline & & & \\
\hline $\mathrm{H}$ & & & \\
\hline & & & \\
\hline $\mathrm{H}$ & -0.5 & 6000 & \\
\hline $\mathrm{H}$ & -0.03078500 & & \\
\hline $\mathrm{H}$ & & & \\
\hline $\mathrm{H}$ & 855900 & 2400 & \\
\hline & & -0.0 & \\
\hline $\mathrm{H}$ & -0.94954800 & -0.06068800 & -3.76427000 \\
\hline $\mathrm{H}$ & -8.24003900 & -1.27884200 & \\
\hline $\mathrm{H}$ & & -1.50747000 & \\
\hline $\mathrm{H}$ & -8.13760700 & -3.67558600 & -2.60275800 \\
\hline & & -4.04462900 & \\
\hline $\mathrm{H}$ & & -2.21419900 & -0.49687700 \\
\hline & -9.93147200 & -0.96586600 & -1.35463900 \\
\hline
\end{tabular}




\begin{tabular}{|c|c|c|c|}
\hline & & & \\
\hline & -8.33650000 & 3.10666100 & \\
\hline & & & \\
\hline & -11.52760000 & 1.86864900 & \\
\hline & -12.48998000 & 1.19985000 & \\
\hline & -8.58846400 & 1.39675300 & \\
\hline & -4.89453800 & -3.08527700 & -1.7322 \\
\hline & -4.40414700 & -1.69088200 & \\
\hline & -2.58043600 & -4.08795400 & \\
\hline & -2.14872700 & -2.66628500 & \\
\hline & -8.70859500 & -6.22620900 & -2.1 \\
\hline & & & \\
\hline & -9.6 & -5.0 & \\
\hline & -5.9 & -5.9 & \\
\hline & -4.7 & -4.29 & \\
\hline & -8.4 & -3.2 & \\
\hline & -5.1 & -2.3 & \\
\hline & & -3.3 & \\
\hline & 0.4 & -3.4 & \\
\hline & & -4.50 & \\
\hline & & -3.5 & \\
\hline & & -5.0 & \\
\hline & & & \\
\hline & 2.38 & -5.5 & \\
\hline & -0.6 & -5.5 & \\
\hline & & & \\
\hline & & -2.7 & \\
\hline & & & \\
\hline & & & \\
\hline & & -0.0 & \\
\hline & & & \\
\hline & & & \\
\hline & & & \\
\hline & & & \\
\hline & & & \\
\hline & & & \\
\hline & & & \\
\hline & & -1.25 & \\
\hline & & -3.7 & \\
\hline & & & \\
\hline & & -1.5 & \\
\hline & & & \\
\hline & & & \\
\hline & & & \\
\hline & & & \\
\hline & & & \\
\hline & $12 .($ & -1.3 & 700 \\
\hline & & & \\
\hline & & & \\
\hline & & & \\
\hline & -4.45 & & \\
\hline & & & \\
\hline & -9.83607800 & 4.77314100 & -1.2 \\
\hline & & & \\
\hline & $-11.41^{\prime}$ & 5.55993700 & 56900 \\
\hline & -10.5 & & \\
\hline & & & \\
\hline & -5.67480300 & & 200 \\
\hline & & & \\
\hline & & 7.45480400 & 1.86282800 \\
\hline & -5.90596900 & 6.08483600 & \\
\hline & & & \\
\hline & -3.28789300 & 4.33614200 & 3.12686600 \\
\hline
\end{tabular}

\begin{tabular}{|c|c|c|c|}
\hline & 03427900 & .44460900. & \\
\hline $\mathrm{H}$ & & & \\
\hline & -5.76478300 & & \\
\hline & 1.07255200 & 4.60630400 & 6.16939700 \\
\hline & 0.02201200 & 3.27227600 & \\
\hline & 2.44762100 & 1.54492100 & \\
\hline & 2.28662500 & & \\
\hline & 0.83537400 & & \\
\hline & 3.70992700 & & \\
\hline & 4800 & & \\
\hline & 12400 & 700 & 700 \\
\hline & 5600 & & \\
\hline & 2200 & -3.6 & \\
\hline & 300 & -3.3 & 100 \\
\hline & 4.02 & & \\
\hline & 1100 & -5.1 & 00 \\
\hline & & -3.7 & \\
\hline & 6.75 & -1.2 & \\
\hline & 7.72 & -2.3 & 100 \\
\hline & 7.26 & & \\
\hline & 6.06 & 0.3 & \\
\hline & -6.8 & -0.3 & \\
\hline & -6.8 & & \\
\hline & 8.02 & & \\
\hline & & & \\
\hline & -6.4 & & \\
\hline $\mathrm{H}$ & $-8.5^{\prime}$ & 6.9 & \\
\hline & $-8.1^{\prime}$ & & \\
\hline & 200 & -2.8 & \\
\hline & 400 & & 00 \\
\hline & & -3.2 & \\
\hline & 000 & -7.9 & \\
\hline & 3.21 & -0.6 & \\
\hline & & & \\
\hline & -1.5 & 1.7 & \\
\hline & -6.6 & -2.7 & \\
\hline & -8.55 & -8.6 & 300 \\
\hline & -4.40 & -3.4 & \\
\hline & 600 & & 200 \\
\hline & 1900 & & \\
\hline & & & \\
\hline & & & \\
\hline & -6.08 & & \\
\hline & -2.74 & -3.9 & 3900 \\
\hline & -3.78 & -5.0274 & \\
\hline & -7.0 & -1.1 & \\
\hline & $-7.8^{\prime}$ & -2.6 & 500 \\
\hline & 5500 & & \\
\hline & & & \\
\hline & -3.24 & & \\
\hline & & & \\
\hline & 2.579 & & -3.2 \\
\hline & 7.51681500 & & \\
\hline & & & \\
\hline & & 5.71000400 & -1.88 \\
\hline & 10.3 & & \\
\hline & 5.59217900 & -4.67880600 & 1.89298600 \\
\hline & 5500 & -4.10260100 & \\
\hline & & -4.32293700 & \\
\hline & 3.59088300 & -4.36413600 & \\
\hline & -0.75646700 & -7.72904000 & \\
\hline $\mathrm{H}$ & & -6.80725800 & -3.67157400 \\
\hline & -7.95535800 & -8.56973900 & -0.25056100 \\
\hline
\end{tabular}


$\mathrm{H} \quad-6.95896900 \quad-7.97826200 \quad-1.62965300$

$\mathrm{H} \quad-8.94485300 \quad 8.41016900 \quad-0.64011600$

$\mathrm{H} \quad-10.13905700 \quad 7.81124400 \quad-1.74304800$

$\mathrm{H} \quad-3.93500600 \quad 6.06104700 \quad 6.36925600$

$\mathrm{H} \quad-5.04457000 \quad 4.74913300 \quad 5.82084000$

$\begin{array}{llll}\mathrm{H} & 1.49241100 & 2.92974600 & 6.59479300\end{array}$

$\mathrm{H} \quad-6.06730400 \quad 1.92618800 \quad 0.41905100$

\section{$\mathrm{E}: \mathrm{P}_{R}(+0.8 \mathrm{kcal} / \mathrm{mol})$}

$\begin{array}{llll}\text { C } & 6.63733700 & 5.80054000 & -0.06729200\end{array}$

C $\quad 7.18454000 \quad 4.76735400 \quad-0.99863300$

O $\quad 6.78612300 \quad 3.59923700 \quad-1.05475200$

N $\quad 8.18859800 \quad 5.19656200 \quad-1.79622400$

C $\quad 8.78133300 \quad 4.31458600 \quad-2.76767100$

C $\quad 9.87613300 \quad 3.38910300 \quad-2.23640500$

O $\quad 10.88705100 \quad 3.14497000 \quad-2.88924500$

$\mathrm{N} \quad 9.62315700 \quad 2.84384600 \quad-1.01300800$

C $\quad 10.49913900 \quad 1.78224500 \quad-0.56467100$

C $\quad 10.23864700 \quad 0.51580800 \quad-1.36774900$

O $\quad 9.08177300 \quad 0.07371300 \quad-1.43558800$

$\mathrm{N} \quad 11.27692100 \quad-0.14859400 \quad-1.91200200$

$\begin{array}{llll}\text { C } & 11.02166800 & -1.45313600 & -2.54469100\end{array}$

$\begin{array}{llll}\text { C } & 10.37509400 & -2.43361800 & -1.56049000\end{array}$

O $\quad 9.54512600 \quad-3.25651500 \quad-1.94437300$

C $\quad 12.41581300 \quad-1.87242700 \quad-3.04394200$

C $\quad 13.09512100 \quad-0.52893700 \quad-3.35135500$

$\begin{array}{llll}\mathrm{C} & 12.62352300 & 0.38509900 & -2.21261300\end{array}$

$\begin{array}{llll}\mathrm{N} & 10.82090900 & -2.32558200 & -0.28736000\end{array}$

C $\quad 10.28512700 \quad-3.05516700 \quad 0.84574700$

$\begin{array}{llll}\text { C } & 9.68799700 & -2.15861200 & 1.93868500\end{array}$

O $\quad 9.83848800 \quad-2.45098700 \quad 3.11519600$

N $\quad 8.97661500 \quad-1.06673300 \quad 1.51612200$

$\begin{array}{llll}\text { C } & 8.05212100 & -0.44002400 & 2.47072900\end{array}$

C $\quad 6.98916400 \quad-1.43314200 \quad 2.98333200$

O $\quad 6.51892100 \quad-1.33928200 \quad 4.11632800$

$\begin{array}{llll}\text { C } & 7.37690300 & 0.78201800 & 1.82264400\end{array}$

$\begin{array}{llll}\text { C } & 6.27711900 & 1.44804000 & 2.67360800\end{array}$

$\begin{array}{llll}\text { C } & 6.78247000 & 1.86103300 & 4.06084900\end{array}$

$\begin{array}{llll}\text { C } & 5.69287300 & 2.64788600 & 1.92422300\end{array}$

$\begin{array}{llll}\mathrm{N} & 6.57978000 & -2.33538500 & 2.07078700\end{array}$

$\begin{array}{llll}\text { C } & 5.52018400 & -3.27020000 & 2.35728800\end{array}$

C $\quad 4.16608000 \quad-1.90298500 \quad 7.31070000$

$\begin{array}{llll}\text { C } & 2.97860900 & -2.35981800 & 6.45493300\end{array}$

$\begin{array}{llll}\text { C } & 3.07281000 & -2.05435200 & 4.95937600\end{array}$

$\begin{array}{llll}\text { C } & 1.90373600 & -2.72077800 & 4.22473300\end{array}$

$\begin{array}{llll}\text { C } & 3.12247400 & -0.54594500 & 4.68999500\end{array}$

C $\quad 0.34322900 \quad-7.80673300 \quad-0.27683200$

$\begin{array}{llll}\text { C } & 0.50679700 & -6.43456900 & 0.38412500\end{array}$

$\begin{array}{lllll}\text { C } & 1.65532600 & -5.57854600 & -0.17953100\end{array}$

$\begin{array}{llll}\text { C } & 1.37203700 & -5.10239600 & -1.61124600\end{array}$

$\begin{array}{llll}\text { C } & 1.92106000 & -4.37611000 & 0.73091800\end{array}$

$\begin{array}{llll}\text { C } & -7.77906200 & -8.28714400 & 1.53961900\end{array}$

$\begin{array}{llll}\text { C } & -8.47887300 & -6.95750300 & 1.24553200\end{array}$

C $\quad-7.72831000 \quad-5.67077900 \quad 1.54055200$

$\begin{array}{llll}\text { C } & -6.42065100 & -5.60612200 & 2.02627900\end{array}$

$\begin{array}{llll}\text { C } & -8.37063100 & -4.45686100 & 1.25256100\end{array}$

$\begin{array}{llll}\text { C } & -5.76167900 & -4.38136800 & 2.18069100\end{array}$

$\begin{array}{llll}\text { C } & -7.72154500 & -3.23291700 & 1.36798500\end{array}$

$\begin{array}{llll}\text { C } & -6.39727300 & -3.19096100 & 1.81342100\end{array}$

$\begin{array}{llll}\text { O } & -5.77202100 & -1.97382800 & 1.88307000\end{array}$
C $\quad-3.49661800 \quad-5.17876700 \quad-2.49955400$

C $\quad-2.77434500 \quad-4.22615900 \quad-1.53918900$

C $\quad-3.54755000 \quad-3.70286000 \quad-0.33684500$

$\begin{array}{llll}\text { C } & -2.72084000 & -2.83846100 & 0.58208500\end{array}$

$\begin{array}{llll}\text { O } & -3.10938700 & -2.41601700 & 1.66908300\end{array}$

$\begin{array}{llll}\text { O } & -1.49276100 & -2.57766100 & 0.12830500\end{array}$

C $\quad 2.64087800 \quad-1.94220800 \quad-6.17551000$

$\begin{array}{llll}\text { C } & 2.93126600 & -2.01300400 & -4.69709400\end{array}$

$\begin{array}{llll}2.06741000 & -1.42233200 & -3.76891100\end{array}$

$\begin{array}{llll}4.09248800 & -2.63621100 & -4.21905700\end{array}$

$\begin{array}{llll}2.35897100 & -1.42351000 & -2.40524500\end{array}$

$\begin{array}{llll}4.38846400 & -2.64903600 & -2.85356100\end{array}$

$\begin{array}{llll}3.52993700 & -2.02613400 & -1.94544100\end{array}$

$\begin{array}{llll}2.61188000 & 3.80415100 & -4.64277700\end{array}$

$3.42630500 \quad 3.53724300 \quad-3.37670100$

$\begin{array}{lll}3.91975100 & 2.08992500 & -3.34116100\end{array}$

$\begin{array}{llll}4.65928300 & 1.78673300 & -2.10743100\end{array}$

$\begin{array}{llll}-2.30161000 & 0.56501600 & -5.04237000\end{array}$

$\begin{array}{llll}-1.72722700 & 0.65714000 & -3.62353300\end{array}$

$-1.60742800 \quad-0.72151500 \quad-2.96641800$

$\begin{array}{lll}-0.37681000 & 1.38969000 & -3.65779900\end{array}$

$\begin{array}{lll}-7.51436200 & -3.74527100 & -3.77944300\end{array}$

$\begin{array}{llll}-8.65574600 & -3.28805100 & -2.86987900\end{array}$

$-9.28067800 \quad-4.44825700 \quad-2.09584700$

$-10.97415300 \quad-4.12792200 \quad-1.45383500$

$\begin{array}{lll}-10.78759800 & -2.57064700 & -0.51321400\end{array}$

$\begin{array}{lll}-11.27303900 & -0.73600700 & -4.20054700\end{array}$

$\begin{array}{lll}-10.61920700 & -0.05581800 & -3.02661200\end{array}$

$\begin{array}{llll}-9.22549100 & 0.06088400 & -2.95348500\end{array}$

$\begin{array}{llll}-11.37384500 & 0.45660500 & -1.96603800\end{array}$

$\begin{array}{llll}-8.60280400 & 0.65078900 & -1.85769000\end{array}$

$\begin{array}{llll}-10.76668300 & 1.06545500 & -0.86812000\end{array}$

$\begin{array}{llll}-9.37761900 & 1.17122000 & -0.81442700\end{array}$

$\begin{array}{lll}-8.79584800 & 1.78496900 & 0.27409900\end{array}$

$\begin{array}{llll}-5.78083400 & 4.18460900 & -3.70080900\end{array}$

$\begin{array}{llll}-5.07502100 & 3.66305900 & -2.44154600\end{array}$

$\begin{array}{llll}-5.93548900 & 2.93487000 & -1.43988700\end{array}$

$\begin{array}{llll}-7.04113000 & 3.34833400 & -1.07559500\end{array}$

$\begin{array}{llll}-5.35973800 & 1.84873100 & -0.97435000\end{array}$

$\begin{array}{llll}-9.50877200 & 6.54955700 & -2.88478700\end{array}$

$\begin{array}{llll}-9.93202200 & 5.88030100 & -1.57846800\end{array}$

$\begin{array}{llll}-10.62248000 & 4.53844700 & -1.82456900\end{array}$

$\begin{array}{llll}-8.81891600 & 5.71450000 & -0.70296900\end{array}$

$\begin{array}{lll}-4.27530400 & 6.65865200 & 3.86652100\end{array}$

$\begin{array}{lll}-4.82253400 & 7.26073600 & 2.56647300\end{array}$

$\begin{array}{llll}-5.56816000 & 6.30348500 & 1.62526400\end{array}$

$\begin{array}{llll}-5.96482200 & 7.04577400 & 0.34325500\end{array}$

$\begin{array}{lll}-4.75220100 & 5.05110100 & 1.29640700\end{array}$

$\begin{array}{lll}1.10452700 & 5.02577300 & 4.60071800\end{array}$

$\begin{array}{lll}1.01810200 & 4.12022200 & 3.01507500\end{array}$

$\begin{array}{lll}1.81361900 & 2.56416300 & 3.53156700\end{array}$

$\begin{array}{llll}7.09898300 & -2.66214200 & -0.67986000\end{array}$

$\begin{array}{llll}6.58660800 & -0.48083300 & -2.34889900\end{array}$

$\begin{array}{lll}-3.93414600 & -0.59612200 & -0.64133100\end{array}$

$\begin{array}{lll}-2.66156000 & 1.31344000 & 0.97102400\end{array}$

$\begin{array}{lll}-5.78333300 & 1.59061800 & 4.56236600\end{array}$

$\begin{array}{lll}-4.93550400 & 2.70032800 & 4.53346500\end{array}$

$\begin{array}{lll}-2.21044300 & 2.67360300 & 0.65222400\end{array}$

$\begin{array}{llll}-0.95434300 & 2.60231600 & -0.20854100\end{array}$

$\begin{array}{lll}-3.60214600 & 2.53855300 & 4.16442000\end{array}$

$\begin{array}{lll}-3.07972100 & 1.28109700 & 3.83706500\end{array}$

$\begin{array}{lll}0.05631800 & 1.60420600 & 0.31453600\end{array}$

$\begin{array}{lll}-1.67771400 & 1.13699300 & 3.29747000\end{array}$ 


\begin{tabular}{|c|c|c|c|}
\hline & 38909400 & 1.66237100 & 2100 \\
\hline & 2.32581400 & 0.72001900 & \\
\hline & -1.70950700 & & 1.83487000 \\
\hline & 1.90881900 & -0.33245800 & 1.12628900 \\
\hline & -3.94241000 & 0.17741300 & \\
\hline & -5.28054400 & 0.32416100 & 4.24959700 \\
\hline & 0.60706200 & -0.37700800 & 1.6068930 \\
\hline & -0.32815300 & 0.59968500 & 1.21353000 \\
\hline & & & \\
\hline & -6.36363500 & 0.67647700 & 1.00162800 \\
\hline & 2.84463100 & & \\
\hline & 3.63800400 & & -0.02196800 \\
\hline & & & \\
\hline & -2.95428200 & & \\
\hline & 3.68286100 & & \\
\hline & -1.08 & & \\
\hline & & & \\
\hline & -2.00 & & \\
\hline & -1.16077700 & & \\
\hline & -0.4 & & \\
\hline & & & \\
\hline & -3.57344900 & & \\
\hline & & & \\
\hline & -5.93 & & \\
\hline & & & \\
\hline & -3.54 & & \\
\hline & -7.5 & & \\
\hline & -1.2 & & \\
\hline & & & \\
\hline & -7.3 & & \\
\hline & & & \\
\hline & & & \\
\hline & & & \\
\hline & & & \\
\hline & 3.0 & & 000 \\
\hline & & & \\
\hline & & & \\
\hline & & & \\
\hline & & & \\
\hline & 4.76 & -3.1 & -4.9 \\
\hline & & & \\
\hline & & & 000 \\
\hline & & & \\
\hline & & & \\
\hline & & & \\
\hline & & & -3 \\
\hline & & 1.3 & -2.6 \\
\hline & & & \\
\hline & & & \\
\hline & & & \\
\hline $\mathrm{H}$ & & & 9900 \\
\hline & & & \\
\hline & & & \\
\hline H & & & \\
\hline $\mathrm{H}$ & -9.43239700 & -5.307 & -2.75785500 \\
\hline & & & \\
\hline & & & \\
\hline $\mathrm{H}$ & -11.78247900 & -2.12787900 & -0.45207300 \\
\hline & & & \\
\hline & & & \\
\hline & & & \\
\hline $\mathrm{H}$ & -12.45684500 & 0.37417700 & -1.99666600 \\
\hline & 064122500 & -0.33585600 & -3.7595020 \\
\hline
\end{tabular}

\section{$\mathrm{H}$}

$\mathrm{H}$

$\mathrm{H}$

$\mathrm{H}$

H

\section{$\mathrm{H}$
$\mathrm{H}$}

\section{$\mathrm{H}$
$\mathrm{H}$}

\section{$\mathrm{H}$
$\mathrm{H}$}

\section{$\mathrm{H}$
$\mathrm{H}$}

\section{$\mathrm{H}$}

$\begin{array}{rrr}-3.96766100 & -4.51305000 & 0.26738400 \\ -4.38991300 & -3.07051600 & -0.63666700\end{array}$ $\begin{array}{llll}-1.87348000 & -4.73652100 & -1.18249100\end{array}$ $-2.41317400 \quad-3.36844700 \quad-2.11362700$ $\begin{array}{llll}-8.74881500 & -6.93832100 & 0.18092300\end{array}$ $\begin{array}{lll}-9.43866100 & -6.93715200 & 1.77781000\end{array}$ $\begin{array}{lll}-9.39563200 & -4.48893100 & 0.89751400\end{array}$ $\begin{array}{lll}-5.89290900 & -6.51776200 & 2.28892100\end{array}$ $\begin{array}{lll}-4.74759600 & -4.34607500 & 2.56452000\end{array}$ $\begin{array}{lll}-8.21740100 & -2.30649400 & 1.09613300\end{array}$ $\begin{array}{lll}-4.79284400 & -2.10173700 & 1.85341400\end{array}$ $\begin{array}{llll}2.73879800 & -3.75685800 & 0.35697200\end{array}$ $\begin{array}{lll}1.03319200 & -3.73552700 & 0.78868400\end{array}$ $\begin{array}{llll}2.17218500 & -4.68743800 & 1.74987800\end{array}$ $\begin{array}{llll}2.22415800 & -4.55118200 & -2.01810200\end{array}$ $\begin{array}{llll}1.15702800 & -5.93350400 & -2.29032700\end{array}$ $\begin{array}{llll}0.51052600 & -4.42410000 & -1.62214900\end{array}$ $\begin{array}{llll}2.56209400 & -6.20279900 & -0.19716300\end{array}$ $\begin{array}{llll}-0.42712200 & -5.86320400 & 0.28442300\end{array}$ $\begin{array}{llll}0.65977000 & -6.57241900 & 1.46239300\end{array}$ $\begin{array}{llll}6.96466600 & -2.36164800 & 1.12841000\end{array}$ $\begin{array}{lll}8.62661300 & -0.14454600 & 3.34907400\end{array}$ $\begin{array}{llll}8.15509100 & 1.52709800 & 1.60048800\end{array}$ $\begin{array}{llll}6.94497900 & 0.48128800 & 0.85914200\end{array}$ $\begin{array}{llll}6.46328900 & 3.41155700 & 1.76465700\end{array}$ $\begin{array}{lll}5.29091000 & 2.36007700 & 0.95161200\end{array}$ $\begin{array}{lll}4.88157100 & 3.10392100 & 2.50160000\end{array}$ $\begin{array}{lll}7.65067000 & 2.52873300 & 3.97871600\end{array}$ $\begin{array}{llll}6.00037200 & 2.40323300 & 4.60309100\end{array}$ $\begin{array}{lll}7.05578200 & 0.98806700 & 4.65567100\end{array}$ $\begin{array}{llll}5.47280700 & 0.71673900 & 2.81509100\end{array}$ $\begin{array}{llll}8.72041800 & -1.02685900 & 0.53424600\end{array}$ $\begin{array}{llll}9.50567300 & -3.72237300 & 0.46869300\end{array}$ $\begin{array}{llll}11.05622600 & -3.66340900 & 1.32425500\end{array}$ $11.46017400 \quad-1.56990600 \quad-0.08934900$ $\begin{array}{llll}13.27718100 & 0.29650500 & -1.33614700\end{array}$ $14.18369900 \quad-0.60049800 \quad-3.40054500$ $12.95147200 \quad-2.39769300 \quad-2.24572200$ $\begin{array}{llll}12.73671800 & -0.13581500 & -4.30804300\end{array}$ $\begin{array}{llll}12.54419200 & 1.43318300 & -2.50449400\end{array}$ $12.35310300 \quad-2.54098300 \quad-3.90367200$ $\begin{array}{llll}10.31258100 & -1.34404800 & -3.37019200\end{array}$ $\begin{array}{llll}8.64843100 & 2.80218700 & -0.72442200\end{array}$ $\begin{array}{llll}8.56681900 & 6.12358600 & -1.67569300\end{array}$ $\begin{array}{llll}-4.64382700 & 4.51037400 & -1.89211800\end{array}$ $\begin{array}{llll}-4.24318700 & 3.00717500 & -2.71056200\end{array}$ $\begin{array}{llll}-9.95972300 & 3.85849300 & -2.37333800\end{array}$ $\begin{array}{llll}-10.86898000 & 4.06275300 & -0.87233500\end{array}$ $\begin{array}{llll}-11.54079600 & 4.65665600 & -2.40892200\end{array}$ $\begin{array}{llll}-10.61999900 & 6.54284700 & -1.03883700\end{array}$ $\begin{array}{lll}-3.41446400 & 6.00947200 & 3.67393500\end{array}$ $\begin{array}{llll}-5.50060900 & 8.08785700 & 2.81369100\end{array}$ $\begin{array}{lll}-3.98699900 & 7.71522600 & 2.01377000\end{array}$ $\begin{array}{llll}-5.06889000 & 7.32396500 & -0.22786800\end{array}$ $\begin{array}{llll}-6.61839000 & 6.44660400 & -0.29263400\end{array}$ $\begin{array}{lll}-6.50798400 & 7.96854600 & 0.57448900\end{array}$ $\begin{array}{lll}-4.50478600 & 4.46607300 & 2.18558900\end{array}$ $\begin{array}{llll}-5.31922000 & 4.39763500 & 0.62939900\end{array}$ $\begin{array}{lll}-3.81184300 & 5.32165000 & 0.79922500\end{array}$ $\begin{array}{lll}-6.48909500 & 5.97985700 & 2.13128000\end{array}$ $\begin{array}{llll}0.64373500 & 6.00369400 & 4.44664300\end{array}$

$\mathrm{H} \quad 0.55488900 \quad 4.49480700 \quad 5.38242800$

H $\quad 1.86258200 \quad 1.92157600 \quad 2.65453500$ 


\begin{tabular}{|c|c|c|c|}
\hline $\mathrm{H}$ & 2.82741200 & 2.74436200 & 3.89831800 \\
\hline $\mathrm{H}$ & 1.22648100 & 2.06251500 & 4.30371200 \\
\hline $\mathrm{H}$ & 4.01694200 & -0.09381400 & 5.12568500 \\
\hline $\mathrm{H}$ & 3.13933100 & -0.34606500 & 3.61796100 \\
\hline $\mathrm{H}$ & 2.24062600 & -0.04828700 & 5.11445600 \\
\hline $\mathrm{H}$ & 1.85768300 & -3.79310600 & 4.44761300 \\
\hline $\mathrm{H}$ & 0.94839500 & -2.28138800 & 4.54319100 \\
\hline $\mathrm{H}$ & 1.99378100 & -2.60172400 & 3.14567100 \\
\hline $\mathrm{H}$ & 4.01352600 & -2.47918300 & 4.59120000 \\
\hline $\mathrm{H}$ & 2.84918300 & -3.44299600 & 6.58704000 \\
\hline $\mathrm{H}$ & 2.05763900 & -1.90080300 & 6.84565800 \\
\hline $\mathrm{H}$ & 6.82024500 & -1.87587200 & -1.19035000 \\
\hline $\mathrm{H}$ & 7.85614200 & -3.03160500 & -1.16800600 \\
\hline $\mathrm{H}$ & 7.53154900 & -0.23940200 & -2.32708300 \\
\hline $\mathrm{H}$ & 6.40920600 & -0.97126000 & -3.16251000 \\
\hline $\mathrm{H}$ & -4.38041100 & -0.08526200 & -1.32684300 \\
\hline $\mathrm{H}$ & -6.14755300 & -0.26938900 & 1.13324300 \\
\hline $\mathrm{H}$ & 7.98874800 & 3.67981100 & -3.17853700 \\
\hline $\mathrm{H}$ & 5.93161500 & 5.33911900 & 0.62132200 \\
\hline $\mathrm{H}$ & -6.55919800 & 4.91042900 & -3.45799600 \\
\hline $\mathrm{H}$ & -8.72537700 & 5.94657700 & -3.36245500 \\
\hline $\mathrm{H}$ & -8.27375500 & 4.98020700 & -1.03115600 \\
\hline $\mathrm{H}$ & 4.33885600 & -0.82753700 & 7.19533700 \\
\hline $\mathrm{H}$ & 11.53388500 & 2.11635900 & -0.62484400 \\
\hline $\mathrm{H}$ & 4.58836900 & -2.75508900 & 2.60253200 \\
\hline $\mathrm{H}$ & 1.16737900 & -8.48207600 & -0.02786300 \\
\hline $\mathrm{H}$ & 3.13171000 & -2.75835000 & -6.71457300 \\
\hline $\mathrm{H}$ & 3.02086300 & -1.00431400 & -6.60133600 \\
\hline $\mathrm{H}$ & -1.71900100 & -0.13010900 & -5.65741800 \\
\hline $\mathrm{H}$ & -6.70524900 & -4.21442800 & -3.20935200 \\
\hline $\mathrm{H}$ & -8.36223800 & -9.09681400 & 1.08687500 \\
\hline $\mathrm{H}$ & -4.38200000 & -4.75790200 & -2.98037700 \\
\hline $\mathrm{H}$ & -12.35298500 & -0.82164300 & -4.05372700 \\
\hline $\mathrm{H}$ & -10.88583000 & -1.74804100 & -4.35011900 \\
\hline $\mathrm{H}$ & -3.36869200 & 0.08834800 & -0.20195600 \\
\hline $\mathrm{H}$ & -4.98944500 & 4.67152000 & -4.28029300 \\
\hline $\mathrm{H}$ & -6.21777800 & 3.37073800 & -4.28836800 \\
\hline $\mathrm{H}$ & -2.70945800 & -5.38363800 & -3.23474000 \\
\hline $\mathrm{H}$ & -3.71493000 & -6.16414300 & -2.07004300 \\
\hline $\mathrm{H}$ & -7.09009900 & -2.91028100 & -4.34711000 \\
\hline $\mathrm{H}$ & -7.88766800 & -4.47792100 & -4.50294300 \\
\hline $\mathrm{H}$ & -11.11044700 & -0.18255600 & -5.13074400 \\
\hline $\mathrm{H}$ & -2.29252800 & 1.54145700 & -5.53759500 \\
\hline $\mathrm{H}$ & -3.35234700 & 0.25920700 & -5.07901500 \\
\hline $\mathrm{H}$ & 1.57364200 & -1.98423500 & -6.41490900 \\
\hline $\mathrm{H}$ & 2.41069200 & 4.86195100 & -4.84293300 \\
\hline $\mathrm{H}$ & 7.45110300 & 6.26249300 & 0.50150400 \\
\hline $\mathrm{H}$ & 6.12639200 & 6.59178100 & -0.62559400 \\
\hline $\mathrm{H}$ & 9.19927100 & 4.91440100 & -3.58299200 \\
\hline $\mathrm{H}$ & 10.26337300 & 1.54044400 & 0.47553400 \\
\hline $\mathrm{H}$ & 5.75359900 & -3.94486900 & 3.18758200 \\
\hline $\mathrm{H}$ & 5.35359200 & -3.87128500 & 1.46086800 \\
\hline $\mathrm{H}$ & 5.08928800 & -2.40352300 & 7.00150500 \\
\hline $\mathrm{H}$ & 3.84061000 & -2.05914900 & 8.34505100 \\
\hline $\mathrm{H}$ & -0.60139900 & -8.27046900 & 0.02111800 \\
\hline $\mathrm{H}$ & 0.29256400 & -7.71586500 & -1.36762100 \\
\hline $\mathrm{H}$ & -7.73910100 & -8.51501400 & 2.61003200 \\
\hline $\mathrm{H}$ & -6.78045300 & -8.34374100 & 1.09468100 \\
\hline $\mathrm{H}$ & -9.11462800 & 7.56568400 & -2.77296900 \\
\hline $\mathrm{H}$ & -10.31414700 & 6.64726400 & -3.62046300 \\
\hline $\mathrm{H}$ & -3.93247400 & 7.48476900 & 4.49912500 \\
\hline $\mathrm{H}$ & -5.02155400 & 6.05152400 & 4.39046600 \\
\hline $\mathrm{H}$ & 2.14236000 & 5.16613600 & 4.91456500 \\
\hline
\end{tabular}


The intermediates and transition states in the "HPAA-first" mechanism.

\section{$\mathrm{E}: \mathrm{S}^{\prime}(+0.5 \mathrm{kcal} / \mathrm{mol})$}

$\begin{array}{lllll}\text { C } & 6.67847500 & 5.63761700 & 0.47545200\end{array}$

$\begin{array}{llll}\text { C } & 7.04857000 & 4.85980600 & -0.74308000\end{array}$

$\begin{array}{llll}\mathrm{O} & 6.30830100 & 4.01201300 & -1.25503900\end{array}$

$\begin{array}{llll}\mathrm{N} & 8.24961900 & 5.17099100 & -1.28351700\end{array}$

$\begin{array}{llll}\text { C } & 8.71445600 & 4.52147800 & -2.47524800\end{array}$

$\begin{array}{llll}\text { C } & 9.77018400 & 3.43685000 & -2.25223700\end{array}$

O $\quad 10.55546200 \quad 3.13003000 \quad-3.14220700$

$\mathrm{N} \quad 9.77265400 \quad 2.84010200 \quad-1.02471200$

$\begin{array}{llll}\text { C } & 10.61515700 & 1.67417200 & -0.83429000\end{array}$

$\begin{array}{llll}\text { C } & 10.04553900 & 0.46710200 & -1.57510100\end{array}$

$\begin{array}{llll}\mathrm{O} & 8.87702500 & 0.12796200 & -1.33292400\end{array}$

$\mathrm{N} \quad 10.83600200 \quad-0.23120800 \quad-2.41348300$

$\begin{array}{llll}\text { C } & 10.27895000 & -1.41716500 & -3.09192700\end{array}$

$\begin{array}{llll}\text { C } & 9.83733200 & -2.51168700 & -2.11851700\end{array}$

$\begin{array}{llll}\text { O } & 8.88834000 & -3.24473400 & -2.39080000\end{array}$

C $\quad 11.41907100 \quad-1.85504600 \quad-4.02995000$

$\begin{array}{llll}\text { C } & 12.12778800 & -0.53423600 & -4.36021200\end{array}$

$\begin{array}{llll}\text { C } & 12.09993700 & 0.23407400 & -3.03373600\end{array}$

$\begin{array}{llll}\mathrm{N} & 10.57979300 & -2.62070100 & -0.98983900\end{array}$

$\begin{array}{llll}\text { C } & 10.26952900 & -3.54662100 & 0.08345500\end{array}$

$\begin{array}{llll}\text { C } & 9.68733700 & -2.91432800 & 1.35471600\end{array}$

$\begin{array}{llll}\text { O } & 9.74373800 & -3.52307900 & 2.41194800\end{array}$

$\mathrm{N} \quad 9.09396200 \quad-1.69421800 \quad 1.21321300$

$\begin{array}{llll}\text { C } & 8.23012400 & -1.18414400 & 2.27862900\end{array}$

$\begin{array}{llll}\text { C } & 7.03183800 & -2.12005100 & 2.55808900\end{array}$

$\begin{array}{llll}\text { O } & 6.61395500 & -2.27531400 & 3.70306900\end{array}$

$\begin{array}{llll}\text { C } & 7.79876000 & 0.24482300 & 1.90617900\end{array}$

$\begin{array}{llll}\text { C } & 6.74034800 & 0.89757700 & 2.80794700\end{array}$

$\begin{array}{llll}\text { C } & 7.15692600 & 0.94131200 & 4.28131200\end{array}$

$\begin{array}{llll}\text { C } & 6.42498900 & 2.29764300 & 2.26262700\end{array}$

$\begin{array}{llll}\mathrm{N} & 6.49194500 & -2.71446800 & 1.47022000\end{array}$

$\begin{array}{llll}\text { C } & 5.39956500 & -3.66618700 & 1.57407800\end{array}$

$\begin{array}{llll}\text { C } & 4.20725100 & -3.01509800 & 6.71208300\end{array}$

$\begin{array}{llll}\text { C } & 3.01856400 & -3.31043400 & 5.78624100\end{array}$

$\begin{array}{llll}\text { C } & 3.30355900 & -3.03557500 & 4.30816300\end{array}$

$\begin{array}{llll}\text { C } & 2.17152100 & -3.56967900 & 3.42383200\end{array}$

$\begin{array}{llll}\text { C } & 3.56397300 & -1.54795300 & 4.05134100\end{array}$

$\begin{array}{llll}\text { C } & 0.04540500 & -7.65558200 & -1.54549700\end{array}$

$\begin{array}{llll}\text { C } & 0.24620500 & -6.38539300 & -0.70187100\end{array}$

$\begin{array}{llll}\text { C } & 1.18544300 & -5.33990100 & -1.33413100\end{array}$

$\begin{array}{llll}\text { C } & 0.52503700 & -4.64172700 & -2.53112800\end{array}$

$\begin{array}{llll}\text { C } & 1.62808600 & -4.30212700 & -0.29423600\end{array}$

$\begin{array}{llll}\text { C } & -8.03680100 & -8.23045400 & 0.41771400\end{array}$

$\begin{array}{llll}\text { C } & -8.62925700 & -6.81356000 & 0.36977400\end{array}$

$\begin{array}{llll}\text { C } & -7.72809700 & -5.77199400 & 0.98985900\end{array}$

$\begin{array}{llll}\text { C } & -6.43089100 & -5.57070800 & 0.50221100\end{array}$

$\begin{array}{llll}\text { C } & -8.14621800 & -4.98618700 & 2.06873500\end{array}$

$\begin{array}{llll}\text { C } & -5.57510000 & -4.63405100 & 1.07341200\end{array}$

$\begin{array}{llll}\text { C } & -7.31210400 & -4.02730900 & 2.63994000\end{array}$

$\begin{array}{llll}\text { C } & -6.02354300 & -3.84800400 & 2.13854900\end{array}$

$\begin{array}{llll}\text { O } & -5.21967400 & -2.90318900 & 2.72758500\end{array}$

$\begin{array}{llll}\text { C } & -3.78436100 & -4.65244200 & -3.24681200\end{array}$

$\begin{array}{llll}\text { C } & -3.53613700 & -3.64298700 & -2.11384600\end{array}$

$\begin{array}{llll}\text { C } & -4.85269000 & -2.91223000 & -1.81687800\end{array}$

$\begin{array}{llll}\text { C } & -4.91046700 & -1.82531500 & -0.75036900\end{array}$

$\begin{array}{llll}\text { O } & -6.05041100 & -1.40036900 & -0.46946800\end{array}$ $\begin{array}{lll}-3.80918600 & -1.39447700 & -0.23454000\end{array}$

$\begin{array}{lll}2.33491600 & -1.03643000 & -6.58358300\end{array}$

$\begin{array}{lll}2.56439600 & -0.99593100 & -5.09192000\end{array}$

$\begin{array}{lll}1.49152200 & -1.12730900 & -4.20333600\end{array}$

$\begin{array}{lll}3.85261800 & -0.84999400 & -4.55877300\end{array}$

$\begin{array}{lll}1.69017700 & -1.11210500 & -2.82116000\end{array}$

$\begin{array}{lll}4.06213200 & -0.84366000 & -3.18095100\end{array}$

$\begin{array}{lll}2.97801000 & -0.97367200 & -2.30646900\end{array}$

$\begin{array}{llll}2.48749900 & 4.42134000 & -4.22409800\end{array}$

$\begin{array}{llll}3.02732200 & 4.06917100 & -2.83813900\end{array}$

$\begin{array}{llll}3.41311900 & 2.60359000 & -2.67197200\end{array}$

$\begin{array}{llll}3.96209700 & 2.40030600 & -1.29642400\end{array}$

$\begin{array}{lll}-2.51255900 & 1.37971500 & -4.94983400\end{array}$

$\begin{array}{lll}-1.98527900 & 1.26272100 & -3.51325900\end{array}$

$\begin{array}{lll}-2.07933800 & -0.18104500 & -3.00846200\end{array}$

$\begin{array}{lll}-0.55781900 & 1.81629500 & -3.39614700\end{array}$

$\begin{array}{lll}-7.79694000 & -2.96085800 & -4.18333900\end{array}$

$\begin{array}{lll}-8.76077700 & -2.65857300 & -3.03667500\end{array}$

$\begin{array}{lll}-9.06653500 & -3.90408400 & -2.20305600\end{array}$

$\begin{array}{llll}-10.26659300 & -3.64064400 & -0.84546100\end{array}$

$\begin{array}{lll}-9.38987400 & -2.35192300 & 0.11113000\end{array}$

$\begin{array}{lll}-11.48956200 & 0.15496200 & -4.04861200\end{array}$

$\begin{array}{lll}-10.86273100 & 0.62257100 & -2.75896000\end{array}$

$\begin{array}{llll}-9.47331000 & 0.68358400 & -2.60009800\end{array}$

$\begin{array}{lll}-11.65082700 & 0.95563900 & -1.65148000\end{array}$

$\begin{array}{llll}-8.88566500 & 1.02617400 & -1.38464400\end{array}$

$\begin{array}{lll}-11.08432400 & 1.30919900 & -0.43064000\end{array}$

$\begin{array}{llll}-9.69253500 & 1.32735300 & -0.27490200\end{array}$

$\begin{array}{llll}-9.20276000 & 1.62648200 & 0.94984400\end{array}$

$\begin{array}{llll}-5.86561800 & 4.83279900 & -2.99275600\end{array}$

$\begin{array}{llll}-5.14849000 & 4.11947800 & -1.84286400\end{array}$

$\begin{array}{llll}-6.03512800 & 3.29153500 & -0.94247900\end{array}$

$\begin{array}{llll}-7.10161900 & 3.67766000 & -0.48457400\end{array}$

$\begin{array}{llll}-5.48986100 & 2.11217700 & -0.67731600\end{array}$

$\begin{array}{lll}-9.51147800 & 7.12874800 & -1.72997600\end{array}$

$\begin{array}{llll}-9.89198000 & 6.27790700 & -0.51697900\end{array}$

$\begin{array}{lll}-10.57096900 & 4.97019100 & -0.92486800\end{array}$

$\begin{array}{lll}-8.74513400 & 6.01978000 & 0.28915900\end{array}$

$\begin{array}{lll}-4.10427400 & 6.13320200 & 4.80909100\end{array}$

$\begin{array}{llll}-4.63351200 & 6.88847100 & 3.58338500\end{array}$

$\begin{array}{lll}-5.41474100 & 6.05766800 & 2.54982500\end{array}$

$\begin{array}{lll}-5.73561000 & 6.92343000 & 1.32532000\end{array}$

$\begin{array}{llll}-4.68773100 & 4.77669700 & 2.13060200\end{array}$

$\begin{array}{lll}1.25034300 & 4.29850600 & 5.13824200\end{array}$

$\begin{array}{llll}2.60525000 & 3.76997100 & 4.02715100\end{array}$

$\begin{array}{lll}3.16304800 & 2.28501500 & 4.92997200\end{array}$

$\begin{array}{lll}6.80486600 & -1.70049600 & -1.29232900\end{array}$

$\begin{array}{llll}5.15501600 & 0.29035600 & -0.22040300\end{array}$

$\begin{array}{rrr}-6.60768500 & 0.59710800 & 1.08183000\end{array}$

$\begin{array}{llll}4.74509400 & 3.05396400 & -1.13050700\end{array}$

$\begin{array}{llll}4.35628800 & 1.43599400 & -1.10236900\end{array}$

$\begin{array}{llll}4.18350900 & 2.29888700 & -3.38366500\end{array}$

$\begin{array}{llll}2.56286700 & 1.92933300 & -2.79188300\end{array}$

$\begin{array}{llll}3.91148300 & 4.68298700 & -2.62194000\end{array}$

$\begin{array}{llll}2.27501800 & 4.31815200 & -2.08043400\end{array}$

$\begin{array}{lll}1.54897400 & 3.90235000 & -4.43697500\end{array}$

$\begin{array}{lll}3.20505500 & 4.16893700 & -5.01152800\end{array}$

$\begin{array}{lll}4.70009300 & -0.75629000 & -5.23392700\end{array}$

$\begin{array}{lll}5.06857800 & -0.76750300 & -2.78418500\end{array}$

$\begin{array}{lll}3.14162500 & -0.96937600 & -1.23544100\end{array}$

$\begin{array}{lll}0.84070600 & -1.22221800 & -2.15492400\end{array}$

$\begin{array}{lll}0.48768700 & -1.24889400 & -4.59785400\end{array}$

$\mathrm{H} \quad 0.13070000 \quad 1.27382800 \quad-4.05332200$ 


\begin{tabular}{|c|c|c|c|}
\hline & & & \\
\hline & & 1.72411300 & \\
\hline & & & \\
\hline & -1.72018300 & -0.27825100 & \\
\hline & & -0.53273800 & \\
\hline & -1.49260600 & -0.85704700 & \\
\hline & -8.30253000 & & \\
\hline & -9.69627200 & -2.22699900 & \\
\hline & -8.14178600 & -4.30493200 & \\
\hline & -9.50 & -4.691 & \\
\hline & 7800 & -2.613 & \\
\hline & & -1.3 & \\
\hline & -9.8 & -2.3 & \\
\hline & -8.2 & 000 & \\
\hline & -7.80 & & \\
\hline & -11.7 & & \\
\hline & -12.7 & 0.91 & 7700 \\
\hline & -8.82 & & \\
\hline & -5.637 & -3.6 & \\
\hline & & & \\
\hline & & -4.1 & \\
\hline & -2.773 & -2.91 & \\
\hline & & & \\
\hline & -9.60 & -6.79 & \\
\hline & -9.15 & -5.1 & \\
\hline & & -6.1 & \\
\hline & -4.5 & -4.5 & \\
\hline & -7.6 & & \\
\hline & -4.4 & -2.7 & \\
\hline & 2.27 & -3.5 & -0.7 \\
\hline & & & \\
\hline & 2.17 & -4.7 & \\
\hline & 1.21 & -3.9 & \\
\hline & & -5.3 & \\
\hline & -0.34 & -4.0 & \\
\hline & & -5.8 & \\
\hline & -0.72 & -5.9 & \\
\hline & & -6.6 & \\
\hline & & -2.4 & \\
\hline & & -1.1 & \\
\hline & & & \\
\hline & & & \\
\hline & & & \\
\hline & & & \\
\hline & & & \\
\hline & & & \\
\hline & 300 & 1.48 & \\
\hline & & -0.068 & \\
\hline & & & \\
\hline & & -1.32 & 600 \\
\hline & & -4.252 & -0.3 \\
\hline & & & \\
\hline & & -1.936 & -0.8 \\
\hline & & -0.0 & \\
\hline & & -0.67 & -4.734 \\
\hline & 12.09726600 & -2.53 & 3500 \\
\hline $\mathrm{H}$ & & 0.021 & -5.1 \\
\hline & 12.06602200 & 2400 & 7500 \\
\hline & & & \\
\hline & & -1.13566200 & -3.65476000 \\
\hline & 8.93613300 & 2.90143100 & -0.46103400 \\
\hline & & & \\
\hline & -4.67326900 & 4.86488100 & -1.19179300 \\
\hline
\end{tabular}$$
\mathrm{H}
$$

$\mathrm{H}$
$\mathrm{H}$

$\mathrm{H}$

$\begin{array}{llrr}\mathrm{H} & -11.33721600 & 0.83578300 & -4.89185700\end{array}$

$\begin{array}{llll}\mathrm{H} & -11.33721600 & 0.83578300 & -4.89185700 \\ \mathrm{H} & -2.49283000 & 2.41702500 & -5.29868000\end{array}$

$\begin{array}{llll}\mathrm{H} & -3.57190500 & 1.10372400 & -5.00236800\end{array}$

$\begin{array}{rrrr}\mathrm{H} & -3.57190500 & 1.10372400 & -5.00236800 \\ \mathrm{H} & 1.26097800 & -1.01950300 & -6.79570600\end{array}$

$\begin{array}{llll}\mathrm{H} & 2.30823800 & 5.50105500 & -4.26142800\end{array}$ 


\begin{tabular}{|c|c|c|c|}
\hline & 7.51750000 & 5.99361000 & \\
\hline & 6.09256500 & 6.51275900 & 0.17169100 \\
\hline & 9.12612400 & 5.22630500 & -3.20556400 \\
\hline & 10.61788300 & 1.41623400 & 0.22816900 \\
\hline & 5.63680200 & -4.46086300 & 2.28920800 \\
\hline & 5.24332600 & -4.11702800 & 0.59172300 \\
\hline & 5.10957200 & -3.48517700 & 6.30590500 \\
\hline & 3.90529900 & -3.31665300 & 7.72121700 \\
\hline & -0.90301800 & -8.13975800 & -1.29086300 \\
\hline & -0.03157900 & -7.40538100 & -2.60893500 \\
\hline & -7.97561200 & -8.61507800 & 1.44069500 \\
\hline & -7.05222100 & -8.24167900 & -0.06062500 \\
\hline & -9.08960300 & 8.10891400 & 195300 \\
\hline & -10.33290400 & 7.35051800 & 1966200 \\
\hline & -3.72546400 & 6.84951200 & 65600 \\
\hline & -4.85207700 & 5.47083600 & 5.25941300 \\
\hline & 1.63425600 & 4.50851300 & 890400 \\
\hline & 805200 & 0.58 & 0700 \\
\hline & -0.20456800 & -0.66084000 & 11700 \\
\hline & 61000 & & 1300 \\
\hline & -2.77783700 & -2.96295 & 207200 \\
\hline & -0.28926600 & -0.97556400 & 4.19724600 \\
\hline & -2.88684400 & -3.540308 & 7200 \\
\hline( & 590400 & -1.94132500 & 88400 \\
\hline & 2579000 & -2.61112400 & 74800 \\
\hline$\zeta$ & 22500 & -2.31 & 7700 \\
\hline & 27400 & -1.3462 & 1100 \\
\hline & 29900 & 0.3920 & 700 \\
\hline $\mathrm{C}$ & 110100 & -0.12188 & 6200 \\
\hline & 154400 & 3.36006 & 7400 \\
\hline( & -1.6 & & 500 \\
\hline( & -2.53302000 & 1.92669 & 9800 \\
\hline C & -2.99255000 & & 5100 \\
\hline 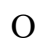 & 42600 & $0.2^{\prime}$ & 000 \\
\hline ( & -2.51231100 & 1.672567 & 5500 \\
\hline 0 & 7100 & 2.7 & 6400 \\
\hline $\mathrm{H}$ & 333000 & 0.51 & 0700 \\
\hline H & 2.87845100 & 1.63585 & \\
\hline $\mathrm{H}$ & 69500 & -2.4 & 8800 \\
\hline & 0.34271400 & -0.45378100 & 97300 \\
\hline & & & \\
\hline E & -1.27264000 & -2.17781100 & 93000 \\
\hline $\mathrm{F}$ & -1.01333500 & -1.10658400 & 402600 \\
\hline H & 1.24878300 & 0.82983300 & 02800 \\
\hline $\mathrm{F}$ & 0.22085700 & 1.20906300 & 74200 \\
\hline - & 2.07902200 & -1.16938500 & 4300 \\
\hline $\mathrm{H}$ & 1.51273000 & -0.06263800 & 3600 \\
\hline & -1.26457100 & 3.46105100 & 14900 \\
\hline 1 & -2.90046100 & 1.60000500 & 3.72027000 \\
\hline $\mathrm{H}$ & -3.92248000 & -0.30824400 & 0.91657800 \\
\hline $\mathrm{H}$ & -2.90291000 & 1.19138600 & -0.53986500 \\
\hline $\mathrm{H}$ & -1.23344100 & 3.01136100 & -0.72666300 \\
\hline & -0.08911500 & 4.45945000 & 1.30624300 \\
\hline $\mathrm{H}$ & 0.03628000 & 4.97328500 & 2.26717100 \\
\hline $\mathrm{H}$ & -0.40201000 & 5.25739500 & 0.60843300 \\
\hline $\mathrm{C}$ & 1.28993600 & 4.08529200 & 0.83391300 \\
\hline $\mathrm{H}$ & 2.05533100 & 4.87415000 & 0.98786600 \\
\hline $\mathrm{O}$ & 1.59812800 & 3.04778500 & 0.26433200 \\
\hline & 3.21468000 & 2.59738800 & -0.60989300 \\
\hline
\end{tabular}

\section{TS1' (+15.7 kcal/mol)}

C $\quad \begin{array}{llll}6.84565900 & 5.28010300 & 1.14340400\end{array}$

C $\quad 7.29606500 \quad 4.55565100 \quad-0.09250500$

O $\quad 6.70115700 \quad 3.60343700 \quad-0.59310500$

N $\quad 8.44599800 \quad 5.03430500 \quad-0.66066200$

C $\quad 8.82551300 \quad 4.56569000 \quad-1.96696600$

C $\quad 9.84234100 \quad 3.43372500 \quad-2.02746400$

O $\quad 10.16577900 \quad 2.96784900 \quad-3.11614800$

N $\quad 10.36680100 \quad 2.99576200 \quad-0.85160300$

C $\quad 11.13021700 \quad 1.75731000 \quad-0.87178700$

C $\quad 10.18906400 \quad 0.61930300 \quad-1.25993700$

O $\quad 9.20768600 \quad 0.39842900 \quad-0.52741500$

N $\quad 10.40997100 \quad-0.10484000 \quad-2.37328700$

C $\quad 9.35153800 \quad-1.04214500 \quad-2.80085500$

C $\quad 9.06966400 \quad-2.11491000 \quad-1.75365500$

$\begin{array}{llll}\text { O } & 7.91613500 & -2.50754600 & -1.53772700\end{array}$

C $\quad 9.87791700 \quad-1.58745300 \quad-4.13923800$

C $\quad 10.69103700 \quad-0.40898300 \quad-4.69261000$

C $\quad 11.38648200 \quad 0.16756400 \quad-3.45455500$

$\begin{array}{llll}\mathrm{N} & 10.15598800 & -2.60376700 & -1.12129900\end{array}$

C $\quad 10.09162500 \quad-3.64174700 \quad-0.10589500$

$\begin{array}{llll}\text { C } & 9.44277700 & -3.26491400 & 1.23334500\end{array}$

$\begin{array}{llll}\mathrm{O} & 9.28545100 & -4.14519600 & 2.06687600\end{array}$

N $\quad 8.99673600 \quad-1.99173300 \quad 1.38700000$

C $\quad 8.03527400 \quad-1.69183900 \quad 2.44853900$

$\begin{array}{llll}\text { C } & 6.85989200 & -2.68776500 & 2.40862400\end{array}$

O $\quad 6.35778300 \quad-3.11289800 \quad 3.43592200$

$\begin{array}{llll}\text { C } & 7.55901500 & -0.23688000 & 2.28532000\end{array}$

C $\quad 6.41440800 \quad 0.21348000 \quad 3.20579600$

$\begin{array}{llll}\text { C } & 6.74729200 & 0.04500200 & 4.69150900\end{array}$

$\begin{array}{llll}\text { C } & 6.02979100 & 1.66190000 & 2.87555900\end{array}$

N $\quad 6.38125900 \quad-2.97447100 \quad 1.15283900$

C $\quad 5.43363200 \quad-4.06471300 \quad 0.93540300$

C $\quad 4.32013900 \quad-4.13061200 \quad 6.13146900$

C $\quad 3.09645000 \quad-4.27417700 \quad 5.21081300$

C $\quad 3.26942000 \quad-3.67690000 \quad 3.81053500$

C $\quad 2.10145900 \quad-4.07168600 \quad 2.90016100$

C $\quad 3.45659700 \quad-2.15746300 \quad 3.86905800$

C $\quad-0.02451600 \quad-7.49759400 \quad-2.63382100$

C $\quad 0.21813700 \quad-6.35992700 \quad-1.63303600$

C $\quad 1.31644400 \quad-5.36335900 \quad-2.05362700$

C $\quad 0.88303300 \quad-4.50767900 \quad-3.25187700$

C $\quad 1.71253200 \quad-4.46261600 \quad-0.87698600$

$\begin{array}{llll}\text { C } & -8.08752400 & -8.23090100 & -0.64666000\end{array}$

C $\quad-8.70686100 \quad-6.82904500 \quad-0.50370400$

C $\quad-8.01125100 \quad-5.88392700 \quad 0.45259800$

C $\quad-6.77494700 \quad-5.29549100 \quad 0.15469600$

C $\quad-8.61972500 \quad-5.52657800 \quad 1.66199300$

C $\quad-6.17866100 \quad-4.36684500 \quad 1.00990300$

$\begin{array}{llll}\text { C } & -8.04622300 & -4.59704300 & 2.52430500\end{array}$

C $\quad-6.83133000 \quad-3.99307900 \quad 2.19159800$

O $\quad-6.33946600 \quad-3.04202200 \quad 3.04253700$

C $\quad-3.82821500 \quad-4.23078900 \quad-3.83408200$

C $\quad-3.57680300 \quad-3.39135900 \quad-2.57640900$

C $\quad-4.89205800 \quad-2.71084900 \quad-2.17806700$

C $\quad-4.90118600 \quad-1.84115300 \quad-0.93509200$

O $\quad-6.03778700 \quad-1.34335900 \quad-0.64164900$

O $\quad-3.83542400 \quad-1.69237800 \quad-0.28047900$

C $\quad 2.30330400 \quad-0.26425300 \quad-6.72041900$

C $\quad 2.61311500 \quad-0.50038800 \quad-5.26338700$

C $\quad 1.60321200 \quad-0.73626900 \quad-4.32387500$ 


\begin{tabular}{|c|c|c|c|}
\hline C & 57800 & 151000 & \\
\hline $\mathrm{C}$ & & -0.97501800 & \\
\hline $\mathrm{C}$ & 4.25524400 & -0.76827900 & \\
\hline $\mathrm{C}$ & 3.23752600 & & -2.55 \\
\hline & 2.57609300 & & \\
\hline 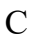 & & & \\
\hline $\mathrm{C}$ & & & \\
\hline & 48385800 & 2.13580200 & \\
\hline C & -2.48295000 & & \\
\hline & -1.92916500 & & \\
\hline & -2.09277100 & 900 & -2.9 \\
\hline C & -0.46999300 & & -3.16 \\
\hline & -7.82523700 & 800 & 100 \\
\hline & -8.78120000 & -2.1 & -3.2 \\
\hline & -9.15823700 & -3.5 & \\
\hline S & -10.33892 & 800 & -1.2 \\
\hline $\mathrm{C}$ & .36535700 & -2.3 & \\
\hline$C$ & -11.46526800 & 600 & -3.8 \\
\hline 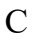 & -10.67594300 & & -2.5 \\
\hline & -9.28952 & & \\
\hline$C$ & -11.29803200 & & 400 \\
\hline & -8.54865700 & & \\
\hline$C$ & $-10.5^{\circ}$ & & \\
\hline & & & -0.2 \\
\hline & & & \\
\hline 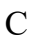 & -5.7534 & & \\
\hline & -5.0157 & & \\
\hline & & & \\
\hline & -6.97058 & & \\
\hline & & & \\
\hline & -9.34 & & \\
\hline & -9.70928 & & \\
\hline & -10.36767 & & 00 \\
\hline & -8.5596 & & \\
\hline & -3.8687 & & \\
\hline & -4.35 & & \\
\hline & 00 & & \\
\hline & & & \\
\hline & -3.78598 & & \\
\hline & 1.46003900 & & \\
\hline S & 2.56 & & \\
\hline & & & \\
\hline U & 6.50 & & \\
\hline $\mathrm{O}$ & 4.41 & & \\
\hline & & & \\
\hline & & & \\
\hline & & & -1.7 \\
\hline & & & \\
\hline $\mathrm{H}$ & 2.68 & & -2.6 \\
\hline & & & -2.47 \\
\hline & & & \\
\hline & & & -3.6 \\
\hline & & & \\
\hline & 4.74214500 & -0.33594700 & -5.53964700 \\
\hline & 5.29295700 & -0.78818200 & -3.17 \\
\hline & & & -1.51986900 \\
\hline & 1.10360500 & -1.16313500 & -2.27717200 \\
\hline & 0.56651200 & -0.74 & -4.6 \\
\hline & 0.17526800 & & -3.87269700 \\
\hline & -0.38610800 & 3.17851800 & -3.37755100 \\
\hline & & & -2.15527800 \\
\hline $\mathrm{H}$ & -2.51468200 & 2.23182400 & -2.56921500 \\
\hline & -1.73585200 & -0.04160200 & -1.91769300 \\
\hline
\end{tabular}

H $\quad-1.54236900 \quad-0.47175300 \quad-3.63025800$

$\mathrm{H} \quad-8.29303000 \quad-1.56749300 \quad-2.52825000$

$\mathrm{H} \quad-9.69049500 \quad-1.66476300 \quad-3.58455700$

$\mathrm{H} \quad-8.25884900 \quad-4.04336500 \quad-2.28341100$

$\mathrm{H} \quad-9.64397200 \quad-4.18805800 \quad-3.37324200$

$\mathrm{H} \quad-8.32176500 \quad-2.68397700 \quad-0.09671800$

$\mathrm{H} \quad-9.43196800 \quad-1.31113000 \quad-0.37485600$

$\mathrm{H} \quad-9.78242500 \quad-2.51212700 \quad 0.89370300$

$\mathrm{H} \quad-7.87899600 \quad 2.57589000 \quad 0.70688400$

$\mathrm{H} \quad-7.47237900 \quad 1.55262700 \quad-1.51492700$

$\mathrm{H} \quad-11.06105200 \quad 1.35461200 \quad 0.81079300$

$\mathrm{H} \quad-12.36997600 \quad 0.87332700 \quad-1.25314200$

$\mathrm{H} \quad-8.77738200 \quad 1.14280200 \quad-3.57753900$

$\mathrm{H} \quad-5.67843400 \quad-3.46115700 \quad-2.02903000$

$\mathrm{H} \quad-5.24974200 \quad-2.08664100 \quad-3.00482000$

$\mathrm{H} \quad-3.19565900 \quad-3.99700300 \quad-1.74813500$

$\mathrm{H} \quad-2.81779900 \quad-2.62638400 \quad-2.76665500$

$\mathrm{H} \quad-8.74979400 \quad-6.36418400 \quad-1.49608200$

$\mathrm{H} \quad-9.74947000 \quad-6.92959800 \quad-0.18494500$

H $\quad-9.58176100 \quad-5.96259000 \quad 1.91766100$

$\mathrm{H} \quad-6.26682300 \quad-5.55901300 \quad-0.77042000$

H $\quad-5.22221200 \quad-3.91894600 \quad 0.76294600$

$\mathrm{H} \quad-8.54036300 \quad-4.30095700 \quad 3.44363400$

$\mathrm{H} \quad-5.66816600 \quad-2.48421400 \quad 2.58501100$

H $\quad 2.49147400 \quad-3.74813400 \quad-1.16735800$

$\mathrm{H} \quad 0.84903000 \quad-3.88823200 \quad-0.51910600$

$\mathrm{H} \quad 2.08584100 \quad-5.05020900 \quad-0.03275300$

$\mathrm{H} \quad 1.67884200 \quad-3.82069200 \quad-3.55555800$

$\mathrm{H} \quad 0.61900800 \quad-5.11697600 \quad-4.12115200$

$\mathrm{H} \quad 0.00450000 \quad-3.90409500 \quad-2.99160200$

$\mathrm{H} \quad 2.20421600 \quad-5.94340400 \quad-2.35002000$

$\mathrm{H} \quad-0.71395600 \quad-5.79772000 \quad-1.48447100$

$\mathrm{H} \quad 0.47270500 \quad-6.78538400 \quad-0.65412600$

$\mathrm{H} \quad 7.00564600 \quad-2.78355300 \quad 0.37726100$

$\mathrm{H} \quad 8.50050900 \quad-1.84594600 \quad 3.42399700$

$\mathrm{H} \quad 8.42830400 \quad 0.41923600 \quad 2.42817200$

H $\quad 7.23815200 \quad-0.10165300 \quad 1.24847800$

$\mathrm{H} \quad \begin{array}{llll}6.87252800 & 2.34168400 & 3.06155400\end{array}$

$\mathrm{H} \quad 5.72298000 \quad 1.77337300 \quad 1.83032900$

$\mathrm{H} \quad 5.19380900 \quad 1.98624600 \quad 3.50250500$

H $\quad \begin{array}{llll}7.65957600 & 0.59467900 & 4.95678400\end{array}$

$\mathrm{H} \quad 5.93308700 \quad 0.43536800 \quad 5.31005800$

$\mathrm{H} \quad 6.88759200 \quad-1.00772400 \quad 4.94881200$

$\mathrm{H} \quad 5.54118800 \quad-0.40660900 \quad 2.98321700$

$\mathrm{H} \quad 9.14323700 \quad-1.29528000 \quad 0.66675400$

$\mathrm{H} \quad 9.50042500 \quad-4.48067000 \quad-0.48201000$

H $\quad 11.10095000 \quad-4.01012700 \quad 0.08411900$

$\mathrm{H} \quad 11.04319700 \quad-2.16550600 \quad-1.31953700$

$\mathrm{H} \quad 12.33065100 \quad-0.34822300 \quad-3.24433100$

H $\quad 11.40139900 \quad-0.70484200 \quad-5.46703100$

$\mathrm{H} \quad 10.52517300 \quad-2.45333000 \quad-3.96228800$

$\mathrm{H} \quad 10.02184000 \quad 0.34870500 \quad-5.11161300$

$\mathrm{H} \quad 11.55260700 \quad 1.23961000 \quad-3.53831500$

H $\quad 9.06210400 \quad-1.90484500 \quad-4.79027200$

H $\quad 8.41740600 \quad-0.49039100 \quad-2.93660500$

H $\quad 9.87838800 \quad 3.24343300 \quad-0.00263700$

$\mathrm{H} \quad 8.86295900 \quad 5.87654700 \quad-0.29238900$

$\mathrm{H} \quad-4.44537300 \quad 4.94911800 \quad-0.52081200$

$\mathrm{H} \quad-4.27638100 \quad 3.67859100 \quad-1.70446200$

H $\quad-9.69448600 \quad 4.54783900 \quad-0.79565400$

H $\quad-10.58228400 \quad 4.31158600 \quad 0.71085900$

$\mathrm{H} \quad-11.30100400 \quad 5.27394400 \quad-0.60140100$ 


\begin{tabular}{|c|c|c|c|}
\hline & -10.40099000 & 6.75261900 & \\
\hline & -3.02582800 & 4.69458300 & \\
\hline & -5.21705100 & & \\
\hline & -3.59953400 & 6.83971400 & 4.17180700 \\
\hline & -4.65595900 & & 1.85635200 \\
\hline & -5.80229700 & & 1.35931600 \\
\hline & -6.27993600 & 6.85971200 & 2.57466400 \\
\hline & -3.38632700 & & 3.44979300 \\
\hline & -4.17648700 & 3.83641400 & 51200 \\
\hline & -2.94735700 & 5.03068500 & 8600 \\
\hline & -5.70177200 & 4.69005700 & 2000 \\
\hline & 1.96208800 & & \\
\hline & 0.506 & & \\
\hline & 2.13 & -0.29 & 300 \\
\hline & 7900 & & \\
\hline & 0.61 & 0.4 & \\
\hline & 4.33 & -1.8 & \\
\hline & & & \\
\hline & 2.58 & -1.6 & 600 \\
\hline & 2.03 & -5.1 & \\
\hline & & & \\
\hline & 2.21 & -3.65 & \\
\hline & & & \\
\hline & 2.85 & -5.3 & \\
\hline & 2.21 & -3.8 & \\
\hline & 7.32 & & \\
\hline & 6.77 & -0.9 & 100 \\
\hline & 4.80 & & \\
\hline & & & \\
\hline & -6.1 & -0. & 900 \\
\hline & & & 000 \\
\hline & 6.14 & & \\
\hline & -6.4 & & \\
\hline & -8.6 & & -1.2 \\
\hline & -8.0 & & \\
\hline & & -3.0 & \\
\hline & 11.9 & & 700 \\
\hline & 4.41 & -3.7 & \\
\hline & 000 & -8.2 & 5700 \\
\hline & 2.70 & -1.0 & \\
\hline & 2.75 & & \\
\hline & -1.9 & & -5. \\
\hline & -6.98 & -2.99828700 & 0400 \\
\hline & -8.72 & -8.84 & \\
\hline & -4.71 & -3.82 & 5200 \\
\hline & -12.5 & & 9600 \\
\hline & 489900 & -0.19600900 & 04100 \\
\hline & -7.21020300 & 0.62856 & 9400 \\
\hline & -4.96 & & -2.6 \\
\hline & -6.24 & 4.55 & 200 \\
\hline & -3.07747500 & -4.23833900 & \\
\hline & & & \\
\hline & -7.38684200 & -1.41901700 & -4.78629400 \\
\hline & -8.25 & -2.85 & \\
\hline & -11.31362000 & 1.53974400 & -4.57062900 \\
\hline & -2.45096400 & 3.03927300 & -4.88604800 \\
\hline & -3.54703000 & 1.71229500 & 95900 \\
\hline & 1.22702300 & -0.20321300 & -6.91171100 \\
\hline & & & \\
\hline & 7.69905900 & 5.53876100 & 1.77895100 \\
\hline & 6.33251400 & 6.20579500 & 0.86182600 \\
\hline & 9.23738300 & 5.36209000 & -2.59697400 \\
\hline & 11.50010600 & 1.55905100 & 0.13595500 \\
\hline
\end{tabular}

\begin{tabular}{|c|c|c|c|}
\hline & 6786900 & -4.95599900 & \\
\hline $\mathrm{H}$ & .45507000 & -4.32352000 & \\
\hline & 5.20980200 & -4.55132500 & 5.64916800 \\
\hline & 4.02725000 & -4.56821600 & 7.09225800 \\
\hline & -0.97706200 & -7.99972300 & -2.43540700 \\
\hline & -0.11160000 & -7.09843100 & -3.64986400 \\
\hline & -8.01962600 & -8.75860300 & 5100 \\
\hline & -7.11026400 & -8.18932700 & 25700 \\
\hline & -8.90484600 & 8.22500100 & -0.202 \\
\hline & -10.17235900 & 424200 & 6000 \\
\hline & -3.46810100 & 05200 & 7400 \\
\hline & -4.62 & 4.6 & \\
\hline & & 3.4 & \\
\hline & -5.78797000 & 1800 & \\
\hline & -0.60225600 & -1.2 & \\
\hline & 1.68564000 & 458700 & \\
\hline & -4.177732 & -1.7 & \\
\hline & -0.54 & -1.8 & \\
\hline & -3.96 & 7200 & 4.7 \\
\hline & $-1.6^{\prime}$ & -2.4 & \\
\hline & & -2.4 & \\
\hline & -2.98 & -1.8 & \\
\hline & -1.8 & -1.2 & \\
\hline & 0.65 & -0.7 & \\
\hline & 0.47 & -0.1 & \\
\hline & & & \\
\hline & -0.936558 & & \\
\hline & & & \\
\hline & $-1.2^{\prime}$ & & \\
\hline & -2.08 & 3000 & \\
\hline & 0.01 & 5.0 & -0.8 \\
\hline & 0.83 & & \\
\hline & 1.54 & 9500 & \\
\hline & 2.50 & -0.0 & \\
\hline & -4.05 & 5000 & \\
\hline & & & \\
\hline & -4.742551 & -3.0 & \\
\hline & -1.63172700 & -2.8 & \\
\hline & -1.94680100 & -0.8 & 1.25 \\
\hline & 1.42470100 & 175000 & \\
\hline & 1.06 & 0.0 & \\
\hline $\mathrm{H}$ & 0.23899800 & -0.96 & \\
\hline & -0.35187700 & 92700 & \\
\hline & -1.34 & & \\
\hline & -2.778069 & 39200 & -0.2 \\
\hline & -1.645627 & & \\
\hline & 0.37985000 & 5.81404400 & -1.48118700 \\
\hline & 1.83411900 & 4.88288900 & \\
\hline & 1.29525500 & 2.83638200 & \\
\hline & 0.74840400 & 2.15496000 & 2.66839500 \\
\hline & & & \\
\hline $\mathrm{C}$ & 2.50611800 & 2.11656100 & 1.42719100 \\
\hline & 3.00087800 & 1.43699200 & 2.13385500 \\
\hline & & 2.70566000 & \\
\hline & 3.97850900 & 2.23364800 & -0.6194540 \\
\hline
\end{tabular}

\section{Int1' (+11.4 kcal/mol)}

$\begin{array}{llll}\text { C } & 6.90608100 & 5.05907700 & 1.23045500\end{array}$

$\begin{array}{llll}\text { C } & 7.36097600 & 4.33283800 & -0.00362400\end{array}$

O $\quad 6.77408900 \quad 3.37516700 \quad-0.49556800$ 


\begin{tabular}{|c|c|c|c|}
\hline & 8.50895000 & 4.82363600 & \\
\hline & 8.88083800 & 4.37531600 & -1.89004000 \\
\hline & 9.89759600 & 3.24356600 & -1.97770000 \\
\hline & 10.23755700 & 2.81707800 & -3.07748100 \\
\hline & 10.40789200 & 2.76549000 & -0.81065200 \\
\hline & 11.17061400 & 1.52938100 & -0.86918200 \\
\hline & 10.22839600 & 0.38607500 & -1.23988200 \\
\hline 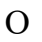 & 25665900 & 0.16957200 & -0.4 \\
\hline & 10.45729600 & -0.36049900 & -2.33760200 \\
\hline & 9.43647300 & -1.35815000 & -2.72246300 \\
\hline & 9.21404700 & -2.41828200 & -1.6 \\
\hline & 8.09461000 & -2.90603000 & 36500 \\
\hline 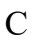 & 9.97210800 & -1.91659600 & 169800 \\
\hline & 10.71729700 & -0.71651100 & 5205800 \\
\hline 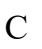 & 11.40175900 & -0.07014100 & 4214200 \\
\hline & 10.31191700 & -2.78802600 & 9000 \\
\hline & 10.27277800 & -3.76689000 & 2233900 \\
\hline 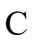 & 9.51908000 & -3.36372700 & 991900 \\
\hline D & 9.37845400 & -4.19 & \\
\hline & 8.98416600 & -2.11762300 & 260600 \\
\hline & 7.94585200 & -1.80 & \\
\hline 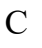 & 6.77291200 & -2.797 & \\
\hline U & 6.13032000 & -3.078 & 4000 \\
\hline & & -0.36 & 3000 \\
\hline ك & 6.33737400 & 0.15 & \\
\hline & 6.64423000 & 69400 & 200 \\
\hline C & & $1.61^{\circ}$ & \\
\hline & 74700 & 63800 & 700 \\
\hline & 6600 & 13200 & 400 \\
\hline C & 177300 & -4.40 & 36000 \\
\hline & 3.07989400 & -4.50 & \\
\hline C & 26000 & -3.76 & \\
\hline C & 59900 & -4.09932400 & 800 \\
\hline $\mathrm{C}$ & 3.21836700 & -2.24733400 & \\
\hline $\mathrm{C}$ & -0.02 & -7.6 & 000 \\
\hline & 0.22038400 & -6.52051900 & 200 \\
\hline & 70100 & 02900 & 100 \\
\hline C & 1.232062 & -4.79384600 & 300 \\
\hline $\mathrm{C}$ & 1.723114 & -4.69139400 & -0.8 \\
\hline $\mathrm{C}$ & -8.09 & 0000 & 500 \\
\hline & -8.70422500 & -6.95330100 & 109600 \\
\hline $\mathrm{C}$ & & & \\
\hline $\mathrm{C}$ & & -5.49400300 & 900 \\
\hline C & -8.60944400 & -5.73102900 & 1.66229200 \\
\hline C & -6.12470900 & -4.62898300 & 130600 \\
\hline $\mathrm{C}$ & -8.01034600 & -4.86486300 & 212500 \\
\hline $\mathrm{C}$ & -6.76904900 & -4.29329700 & 000 \\
\hline $\mathrm{O}$ & -6.24401400 & -3.41 & 586500 \\
\hline & -3.81669700 & -4.33500800 & -3.86212300 \\
\hline $\mathrm{C}$ & -3.55 & & \\
\hline $\mathrm{C}$ & -4.87554100 & -2.87103000 & -2.15751800 \\
\hline $\mathrm{C}$ & -4.86579700 & -2.03629000 & 352700 \\
\hline $\mathrm{O}$ & -5.97964900 & -1.49336200 & -0.59723300 \\
\hline $\mathrm{O}$ & -3.80207800 & -1.95706900 & -0.22182600 \\
\hline $\mathrm{C}$ & 2.33236300 & -0.36153400 & -6.70116800 \\
\hline $\mathrm{C}$ & 2.61221100 & -0.65802200 & -5.24766700 \\
\hline $\mathrm{C}$ & 1.57603000 & -0.91177100 & -4.34241900 \\
\hline $\mathrm{C}$ & 3.92944400 & -0.69473300 & -4.77116300 \\
\hline $\mathrm{C}$ & 1.84077100 & -1.17574800 & -2.99595300 \\
\hline $\mathrm{C}$ & 4.20360300 & -0.97452500 & -3.43445700 \\
\hline $\mathrm{C}$ & 3.15680600 & -1.20601600 & -2.53638800 \\
\hline $\mathrm{C}$ & & 4.66322100 & -3.53043400 \\
\hline $\mathrm{C}$ & 3.35131000 & 3.98956100 & -2.35621600 \\
\hline
\end{tabular}

\begin{tabular}{|c|c|c|c|}
\hline & 66922700 & 52169600 & \\
\hline & & & \\
\hline & -2.44186500 & & \\
\hline & -1.91751900 & 1.49848100 & \\
\hline & -2.14825000 & 0.01302400 & \\
\hline & -0.44312500 & 1.88742400 & -3.07 \\
\hline & -7.80502700 & -2.44496700 & \\
\hline & -8.75469900 & -2.28302200 & 597700 \\
\hline & -9.13117100 & -3.62867000 & \\
\hline & -10.28566900 & 3900 & -1.2 \\
\hline & -9.27249600 & 4000 & -0.0 \\
\hline & -11.42947600 & 1500 & -3 \\
\hline & -10.62314700 & 3700 & -2.5 \\
\hline & -9.23926000 & & -2.59 \\
\hline & -11.22697400 & & -1.2 \\
\hline & -8.48431400 & 91100 & -1.4 \\
\hline & -10.48911500 & & \\
\hline & -9.11 & & \\
\hline & -8.4 & & \\
\hline & -5.6 & & \\
\hline & -4.9 & & \\
\hline & -5.73 & & -0.2 \\
\hline & -6.8 & & \\
\hline & -5.1 & & \\
\hline & $-9.2^{7}$ & & \\
\hline & -9.6 & & \\
\hline & -10.3 & & \\
\hline & -8.4 & & \\
\hline & -3.80 & & \\
\hline & -4.32 & & \\
\hline & -4.7 & & \\
\hline & -5.42 & & \\
\hline & -3.6 & & \\
\hline & & & \\
\hline & 2.70 & & \\
\hline & 2.8 & & \\
\hline & 6.54 & & \\
\hline & 3.28 & & \\
\hline & -6.1 & & \\
\hline & & & \\
\hline & & & \\
\hline & & & \\
\hline & & & \\
\hline & 4.25 & 800 & \\
\hline & & & \\
\hline & & & \\
\hline & & & \\
\hline & 4.74 & -0.5 & \\
\hline & 5.22 & & \\
\hline & & & -1.4 \\
\hline & & & \\
\hline & & -0.90 & -4.6 \\
\hline & 0.18482100 & & \\
\hline & -033 & & \\
\hline & -0.06 & & -2.08 \\
\hline & & & \\
\hline & -1.85561900 & -0.25204800 & -1.91538200 \\
\hline & -3.20577200 & -0.24715400 & -3.03804900 \\
\hline & & & -3.62755900 \\
\hline & -8.26063000 & -1.67065700 & -2.51591600 \\
\hline & & & \\
\hline $\mathrm{H}$ & & -4.15630800 & -2.32218300 \\
\hline & -9.63412300 & -4.27017000 & -3.38869700 \\
\hline
\end{tabular}




\begin{tabular}{|c|c|c|c|}
\hline & -8.24075000 & -2.90000800 & \\
\hline & -9.30281900 & -1.48261700 & \\
\hline & -9.69239600 & & 0.92072600 \\
\hline & -7.79653100 & 2.39623500 & \\
\hline & -7.40989500 & & \\
\hline & -10.96334100 & 1.14017800 & 0.86317400 \\
\hline & -12.29621800 & 0.71518600 & -1.19637500 \\
\hline & -8.74017600 & 1.09690300 & \\
\hline & -5.64861300 & -3.63598800 & -2.00905800 \\
\hline & -5.25844500 & -2.23367300 & -2.962 \\
\hline & -3.15517600 & -4.14077100 & 38700 \\
\hline & -2.8129 & -2.7 & \\
\hline & -8.7320 & -6.4 & -1.5 \\
\hline & -9.75 & -7.0 & 2800 \\
\hline & -9.587 & 500 & \\
\hline & -6.239 & -5.73 & -0.7 \\
\hline & -5.148 & -4.2 & 0000 \\
\hline & -8.5 & & \\
\hline & -5.5 & -2.8 & 5500 \\
\hline & & -4.0 & \\
\hline & & -4.0 & \\
\hline & & -5.2 & \\
\hline & & & \\
\hline & & -5.4 & \\
\hline & 0.3 & -4.1 & -3 \\
\hline & & & \\
\hline & -0.67 & & 3300 \\
\hline & & & \\
\hline & & & \\
\hline & 8.3 & -1.9 & \\
\hline & & & \\
\hline & & -0.2 & \\
\hline & 6.920 & & \\
\hline & & & \\
\hline & & & \\
\hline & & & \\
\hline & & & \\
\hline & & -1.0 & \\
\hline & & -0.4 & 3100 \\
\hline & & & \\
\hline & & -4.6 & \\
\hline $\mathrm{H}$ & & & 5500 \\
\hline & 11.16 & -2.2 & 79200 \\
\hline $\mathrm{H}$ & & -0.5 & \\
\hline & 11.43 & -0.9 & 000 \\
\hline & 10.665 & -2.7 & \\
\hline & 10.0 & -0.0 & 78400 \\
\hline & & & 0800 \\
\hline & & -2.25 & \\
\hline $\mathrm{H}$ & & -0.85 & 1100 \\
\hline & & & \\
\hline & & & \\
\hline & -4.3828 & 4.824 & 9900 \\
\hline & & & \\
\hline & -9.639 & 4.43242800 & 43800 \\
\hline & -10.533 & 4.17740000 & 27200 \\
\hline & & & \\
\hline & -10.33536200 & 6.60745900 & 20800 \\
\hline & & & \\
\hline & -5.16897100 & 6.55853400 & 4.96534000 \\
\hline & -3.54731900 & 6.65146200 & 4.29937200 \\
\hline & -4.69467000 & 6.83181800 & 2.01123100 \\
\hline & -5.74833600 & 5.51416100 & 1.46192300 \\
\hline
\end{tabular}

\begin{tabular}{|c|c|c|c|}
\hline & 30351700 & 6.58864600 & \\
\hline $\mathrm{H}$ & & & \\
\hline & -4.03217300 & & 92539000 \\
\hline & -2.89222700 & 5.00922200 & 33616500 \\
\hline & -5.56595200 & & \\
\hline & 1.35694400 & 3.97953900 & 06237600 \\
\hline & 0.55729400 & 2.61681400 & \\
\hline & 3.49691100 & -0.05957700 & 1800 \\
\hline & 3.18844500 & & \\
\hline & & & \\
\hline & 4.146 & 27100 & 700 \\
\hline & 3.217 & -1.7 & \\
\hline & 2.365 & & \\
\hline & 1.761 & -5.1 & 000 \\
\hline & 0.93 & & \\
\hline & 1.872 & -3.6 & 700 \\
\hline & 3.9919 & & \\
\hline & 2.88 & & \\
\hline & 2.21 & -4.1 & 100 \\
\hline & 7.37 & & \\
\hline & 6.75 & -1.3 & \\
\hline & 3.7328 & & \\
\hline & 3.760 & & \\
\hline & -6.044 & & 300 \\
\hline & 7.98 & & \\
\hline & 6.20 & & \\
\hline $\mathrm{H}$ & -6.43 & & \\
\hline & -8.53 & & \\
\hline & -7.97 & & \\
\hline & 4.57 & & \\
\hline $\mathrm{H}$ & 11.9 & & 500 \\
\hline $\mathrm{H}$ & 4.46 & & \\
\hline & 0.77 & & \\
\hline $\mathrm{H}$ & 2.73 & & \\
\hline $\mathrm{H}$ & 2.79 & & \\
\hline & -1.89 & & \\
\hline & -6.962 & & \\
\hline & -8.736 & & \\
\hline $\mathrm{H}$ & -4.69 & & \\
\hline & 124001 & 2000 & \\
\hline & & & \\
\hline & & & \\
\hline & -4.903 & & \\
\hline & -6.19 & 700 & -2.89 \\
\hline & -3.066 & & \\
\hline & -4.0664 & & \\
\hline & -7.36 & & -4.77 \\
\hline & -8.238 & & \\
\hline & & & \\
\hline & -2.4047 & & -4.82 \\
\hline & -3.50 & & \\
\hline & 1.25621600 & -0.29271900 & -6.89 \\
\hline & 2.47208800 & & \\
\hline & 7.760 & & \\
\hline $\mathrm{H}$ & 6.40565 & & \\
\hline & 9.295 & & \\
\hline $\mathrm{H}$ & 11.57246800 & 1.31922800 & 0.12436800 \\
\hline & 5.67870500 & -5.17511900 & 1.48339800 \\
\hline & 5.41344400 & & -0.15693500 \\
\hline $\mathrm{H}$ & 5.22495900 & -4.82093100 & 5.61059800 \\
\hline $\mathrm{H}$ & 4.04312700 & & \\
\hline $\mathrm{H}$ & -0.98316000 & -8.13522400 & -2.51376800 \\
\hline & -0.11407700 & -7.22268800 & -3.7170300 \\
\hline
\end{tabular}




$\begin{array}{rrrr}\mathrm{H} & -8.02774900 & -8.89530800 & 0.22622900 \\ \mathrm{H} & -7.11644300 & -8.31189200 & -1.21432700 \\ \mathrm{H} & -8.83076400 & 8.09753700 & -0.06736100 \\ \mathrm{H} & -10.10176500 & 7.51604800 & -1.09060300 \\ \mathrm{H} & -3.40157200 & 5.66943700 & 6.59426000 \\ \mathrm{H} & -4.56066500 & 4.37265700 & 6.11756700 \\ \mathrm{H} & 1.91419700 & 3.47247900 & 6.67084700 \\ \mathrm{H} & -5.68874700 & 1.03869300 & 0.79595800 \\ \mathrm{C} & -0.69629700 & -0.87049900 & 2.87264800 \\ \mathrm{~N} & 0.71913100 & 2.01259600 & 0.78919000 \\ \mathrm{O} & -3.97824100 & -2.39405700 & 2.23178600 \\ \mathrm{C} & -0.56230500 & -1.17253100 & 4.22891700 \\ \mathrm{O} & -3.62334500 & -3.06214000 & 4.89409000 \\ \mathrm{C} & -1.54274200 & -1.91781400 & 4.89019600 \\ \mathrm{C} & -2.68804600 & -2.34449200 & 4.21986300 \\ \mathrm{C} & -2.84265300 & -2.00833500 & 2.85869700 \\ \mathrm{C} & -1.84252500 & -1.30222100 & 2.19664700 \\ \mathrm{C} & 0.35139200 & -0.06373900 & 2.14100300 \\ \mathrm{C} & -0.29859700 & 1.02144400 & 1.27514600 \\ \mathrm{C} & -0.25849200 & 4.77610800 & 0.49017400 \\ \mathrm{C} & -1.33984400 & 4.35653000 & -0.30105100 \\ \mathrm{C} & -1.52536700 & 4.85885100 & -1.58695600 \\ \mathrm{C} & -0.62952200 & 5.79596700 & -2.10435600 \\ \mathrm{O} & -0.83753500 & 6.22429400 & -3.38251100 \\ \mathrm{C} & 0.43420900 & 6.24874900 & -1.31650400 \\ \mathrm{C} & 0.61778400 & 5.73297200 & -0.03807600 \\ \mathrm{H} & 0.39179700 & 2.49535800 & -0.05568400 \\ \mathrm{H} & 1.62737600 & 1.52238300 & 0.56826200 \\ \mathrm{H} & -3.95617200 & -2.11193500 & 1.24698900 \\ \mathrm{H} & 0.31321700 & -0.83165700 & 4.77315000 \\ \mathrm{H} & -4.34983600 & -3.32237600 & 4.30270300 \\ \mathrm{H} & -1.44098500 & -2.17072100 & 5.94022800 \\ \mathrm{H} & -1.99398800 & -1.09876200 & 1.14238100 \\ \mathrm{H} & 0.97203500 & -0.70236200 & 1.49649300 \\ \mathrm{H} & 1.02861300 & 0.39397300 & 2.86969500 \\ \mathrm{H} & -0.77873500 & 0.59827400 & 0.39158500 \\ \mathrm{H} & -1.06335800 & 1.56321000 & 1.83417300 \\ \mathrm{H} & -2.06265900 & 3.64399800 & 0.08912900 \\ \mathrm{H} & -2.35783100 & 4.53496300 & -2.19793900 \\ \mathrm{H} & -0.16567800 & 6.87887200 & -3.61325500 \\ \mathrm{H} & 1.12091300 & 6.99548700 & -1.70687600 \\ \mathrm{H} & 1.46012000 & 6.06647500 & 0.55784200 \\ \mathrm{C} & 0.00158100 & 4.16729800 & 1.84652800 \\ \mathrm{H} & -0.91636500 & 3.74209700 & 2.25909500 \\ \mathrm{H} & 0.34656100 & 4.92267100 & 2.55898200 \\ \mathrm{C} & 1.08821100 & 3.08982500 & 1.81192500 \\ \mathrm{H} & 1.14635400 & 2.55561200 & 2.76588100 \\ \mathrm{O} & 2.30377600 & 3.64538900 & 1.44410900 \\ \mathrm{H} & 2.95215700 & 2.90752500 & 1.37220100\end{array}$

\section{Int2' (+7.2 kcal/mol)}

$\begin{array}{llll}\mathrm{C} & 6.92591600 & 4.99796000 & 1.29383400 \\ \mathrm{C} & 7.33117800 & 4.34465400 & 0.00913300 \\ \mathrm{O} & 6.63932200 & 3.51875200 & -0.58635700 \\ \mathrm{~N} & 8.53367700 & 4.74452500 & -0.50113900 \\ \mathrm{C} & 8.91052200 & 4.32156600 & -1.82217400 \\ \mathrm{C} & 9.92467200 & 3.18887300 & -1.92190700 \\ \mathrm{O} & 10.24282900 & 2.75655300 & -3.02531700 \\ \mathrm{~N} & 10.46208500 & 2.71858700 & -0.76258100 \\ \mathrm{C} & 11.21124000 & 1.47338800 & -0.83932800\end{array}$

C $\quad 10.24736100 \quad 0.36273400 \quad-1.24889800$

$\begin{array}{lllll}\text { O } & 9.24971200 & 0.17196600 & -0.53015600\end{array}$

N $\quad 10.47211600 \quad-0.37107900 \quad-2.35351200$

C $\quad 9.41855200 \quad-1.31537500 \quad-2.78298900$

C $\quad 9.11671100 \quad-2.37191100 \quad-1.72328300$

O $\quad 7.96261500 \quad-2.76915500 \quad-1.53584600$

C $\quad 9.97030000 \quad-1.88114500 \quad-4.10175900$

C $\quad 10.78073100 \quad-0.70604300 \quad-4.66616700$

C $\quad 11.45404400 \quad-0.09872500 \quad-3.42962600$

N $\quad 10.19219400 \quad-2.83709100 \quad-1.04982300$

C $\quad 10.11085300 \quad-3.84305200 \quad-0.00364000$

$\begin{array}{llll}\text { C } & 9.46519800 & -3.41831200 & 1.32451500\end{array}$

$\begin{array}{llll}\mathrm{O} & 9.35983200 & -4.25157200 & 2.21216800\end{array}$

$\begin{array}{llll}\mathrm{N} & 8.95599700 & -2.16157400 & 1.39615300\end{array}$

C $\quad 7.93397600 \quad-1.84837900 \quad 2.39323700$

C $\quad 6.77160500 \quad-2.85694500 \quad 2.33490900$

$\begin{array}{llll}\text { O } & 6.16293800 & -3.16011200 & 3.35557800\end{array}$

C $\quad 7.43597700 \quad-0.41525400 \quad 2.14246400$

$\begin{array}{llll}\text { C } & 6.28560200 & 0.08183400 & 3.03124300\end{array}$

$\begin{array}{llll}\text { C } & 6.58846600 & -0.04189700 & 4.52781500\end{array}$

$\begin{array}{llll}\text { C } & 5.95807300 & 1.53176200 & 2.64882500\end{array}$

N $\quad 6.43743700 \quad-3.30142800 \quad 1.09694600$

C $\quad 5.42528500 \quad-4.32718400 \quad 0.90999300$

$\begin{array}{llll}\text { C } & 4.29180800 & -4.47790100 & 6.09948900\end{array}$

C $\quad 3.04113100 \quad-4.55686600 \quad 5.19885500$

C $\quad 3.09558700 \quad-3.81022100 \quad 3.85719000$

C $\quad 1.85453900 \quad-4.12653500 \quad 3.01224900$

C $\quad 3.22752400 \quad-2.29515300 \quad 4.04039600$

$\begin{array}{llll}\text { C } & -0.05197500 & -7.64198800 & -2.74102300\end{array}$

C $\quad 0.19258100 \quad-6.53423600 \quad-1.70767000$

C $\quad 1.34739400 \quad-5.57115800 \quad-2.04632200$

C $\quad 1.01720400 \quad-4.68194800 \quad-3.25328300$

C $\quad 1.69933000 \quad-4.70135000 \quad-0.83205200$

C $\quad-8.12914400 \quad-8.33348600 \quad-0.79626500$

C $\quad-8.73121700 \quad-6.92881700 \quad-0.61673800$

$\begin{array}{llll}\text { C } & -8.02910200 & -6.04351500 & 0.38885900\end{array}$

$\begin{array}{llll}\text { C } & -6.76585900 & -5.49226800 & 0.13991100\end{array}$

C $\quad-8.64282700 \quad-5.72359600 \quad 1.60587800$

C $\quad-6.14332600 \quad-4.64005400 \quad 1.05302400$

$\begin{array}{llll}\text { C } & -8.04284200 & -4.87021100 & 2.52707900\end{array}$

$\begin{array}{llll}\text { C } & -6.79382700 & -4.30769200 & 2.24970300\end{array}$

O $\quad-6.26757000 \quad-3.44277000 \quad 3.16699200$

C $\quad-3.81982500 \quad-4.31709700 \quad-3.89484800$

C $\quad-3.56082900 \quad-3.51632700 \quad-2.61645900$

C $\quad-4.88122900 \quad-2.88005500 \quad-2.16826100$

C $\quad-4.87057400 \quad-2.05879700 \quad-0.89421600$

O $\quad-5.98364600 \quad-1.51235100 \quad-0.59919500$

$\begin{array}{llll}\mathrm{O} & -3.80955500 & -1.99570000 & -0.21855600\end{array}$

C $\quad 2.36010900-0.35792800 \quad-6.68648400$

C $\quad 2.64741000 \quad-0.61932700 \quad-5.22816400$

C $\quad \begin{array}{llll}\text { C } & 1.61742600 & -0.77971300 & -4.29494400\end{array}$

C $\quad 3.96986100 \quad-0.71630500 \quad-4.77484000$

C $\quad 1.89322800 \quad-1.00784100 \quad-2.94395100$

C $\quad 4.25377600 \quad-0.96480100 \quad-3.43384700$

C $\quad 3.21482400 \quad-1.09963900 \quad-2.50681800$

C $\quad 2.66961500 \quad 4.64831100 \quad-3.48753600$

C $\quad 3.36964900 \quad 3.98279200 \quad-2.29133200$

C $\quad 3.72433000 \quad 2.52314200 \quad-2.60097200$

$\mathrm{N} \quad 4.51220100 \quad 1.89054500 \quad-1.48995100$

C $\quad-2.41222200 \quad 1.87809400 \quad-4.63012500$

C $\quad-1.88867300 \quad 1.52522200 \quad-3.22941700$

C $\quad-2.08102000 \quad 0.03533100 \quad-2.92349900$

C $\quad-0.42754900 \quad 1.96184800 \quad-3.05955800$ 


\begin{tabular}{|c|c|c|c|}
\hline & -7.79653200 & -2.40479200 & -4.50142500 \\
\hline & -8.74850000 & -2.24534100 & -3.31681400 \\
\hline & -9.13173200 & -3.59279000 & -2.70519800 \\
\hline & -10.28910900 & -3.49193000 & -1.29063100 \\
\hline & -9.27685500 & -2.51804500 & -0.11873600 \\
\hline & -11.40887500 & 0.73463800 & -3.82697800 \\
\hline & -10.60790400 & 0.94654100 & -2.56672700 \\
\hline & -9.22247900 & 1.14733600 & -2.6196 \\
\hline & -11.21847600 & 0.93694300 & -1.30901100 \\
\hline & -8.47227200 & 1.35171100 & -1.46572700 \\
\hline & -10.48545100 & 1.15973100 & -0.14 \\
\hline & -9.11150900 & 1.38795200 & 31700 \\
\hline O & -8.41268800 & 1.63837600 & 0.93646100 \\
\hline C & -5.66155500 & 00800 & -2.10 \\
\hline 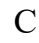 & -4.92137400 & 4.17558700 & 150000 \\
\hline & -5.72626100 & 3.226 & -0.21 \\
\hline $\mathrm{O}$ & -6.87752500 & 3.5747 & 381900 \\
\hline & -5.12 & 6000 & 0.07 \\
\hline 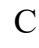 & -9.23869300 & 7.20 & -0.45 \\
\hline & -9.61703400 & 6.168 & 9900 \\
\hline & -10.28108100 & & \\
\hline & -8.47485000 & 5.79 & 1.37 \\
\hline & -3.80473100 & 7300 & 7300 \\
\hline C & -4.31778 & 5.91 & \\
\hline & -4.78072100 & 5.124 & 6900 \\
\hline & -5.390 & 6.06 & 2.34 \\
\hline & -3.64886 & 4.29 & \\
\hline & 1.50521600 & 3.06 & 6400 \\
\hline S & 2.77058900 & 1.963 & 5.01 \\
\hline C & 2.73288600 & 0.618564 & 6.24 \\
\hline O & 6.57752000 & -0.12694900 & -1.38 \\
\hline D & 3.12778400 & 8800 & 0.63 \\
\hline & 86600 & 0.16 & 1.15 \\
\hline & 5.18 & 2.55 & -1.0 \\
\hline $\mathrm{H}$ & 5.07 & 1.07 & -1.79 \\
\hline & 48900 & 2.45 & -3.50 \\
\hline $\mathrm{H}$ & 2.83661 & 1.90 & -2.76 \\
\hline & 4.29824600 & 4.51 & -2.05 \\
\hline 1 & 2.74227000 & 93600 & 5800 \\
\hline $\mathrm{H}$ & 1.687799 & 4.19 & -3.66 \\
\hline & 3.26290000 & $4.51 \mathrm{C}$ & -4.40 \\
\hline H & 4.78518900 & -0.60 & 22600 \\
\hline $\mathrm{H}$ & 5.28579400 & -1.04 & 56600 \\
\hline & 3.43377800 & -1.27283200 & 751700 \\
\hline $\mathrm{H}$ & 1.07331400 & -1.12361200 & -2.24237600 \\
\hline $\mathrm{I}$ & 0.58567400 & -0.72674500 & -4.62707500 \\
\hline $\mathrm{H}$ & 0.21352500 & 1.495 & 6100 \\
\hline $\mathrm{I}$ & -0.33007700 & 3.04760000 & 997800 \\
\hline & -0.03693000 & 1.67013900 & -2.07666400 \\
\hline $\mathrm{H}$ & -2.49008400 & 2.08412300 & -2.499 \\
\hline $\mathrm{H}$ & -1.77762700 & -0.21693000 & -1.90220700 \\
\hline & -3.13230700 & -0.25043800 & 047800 \\
\hline $\mathrm{H}$ & -1.50301100 & -0.59080600 & -3.61520900 \\
\hline & -8.25315800 & -1.63976500 & -2.55211900 \\
\hline $\mathrm{H}$ & -9.65519000 & -1.70479200 & -3.60372800 \\
\hline $\mathrm{H}$ & -8.23236600 & -4.12528400 & -2.37181900 \\
\hline & -9.63534200 & -4.22852300 & -3.44232400 \\
\hline $\mathrm{H}$ & -8.24307500 & -2.86752100 & -0.12845600 \\
\hline П & -9.31336400 & -1.45409700 & -0.35517000 \\
\hline $\mathrm{H}$ & -9.69235400 & -2.68548300 & 0.87607900 \\
\hline & -7.78715600 & 2.41343000 & 0.74428400 \\
\hline & & 1.47209000 & -1.51674200 \\
\hline & -10.96474600 & 1.17676700 & 0.82903800 \\
\hline
\end{tabular}

$\mathrm{H} \quad-12.28917000 \quad 0.76518300 \quad-1.23872800$

$\mathrm{H} \quad-8.71830500 \quad 1.13293200 \quad-3.58312400$

$\mathrm{H} \quad-5.64719500 \quad-3.65307300 \quad-2.02414800$

$\mathrm{H} \quad-5.27333000 \quad-2.23662100 \quad-2.96330200$

$\mathrm{H} \quad-3.14782100 \quad-4.13847800 \quad-1.81659900$

$\mathrm{H} \quad-2.82417500 \quad-2.72746500 \quad-2.79738300$

$\mathrm{H} \quad-8.74962600 \quad-6.42597600 \quad-1.59101000$

$\mathrm{H} \quad-9.78017300 \quad-7.02305600 \quad-0.31755200$

$\mathrm{H} \quad-9.62627300 \quad-6.13143400 \quad 1.82419800$

$\mathrm{H} \quad-6.25357300 \quad-5.72962200 \quad-0.79005100$

$\mathrm{H} \quad-5.16232100 \quad-4.22859100 \quad 0.84265000$

$\mathrm{H} \quad-8.53754700 \quad-4.60598400 \quad 3.45583100$

$\mathrm{H} \quad-5.55623400 \quad-2.91185000 \quad 2.74766800$

H $\quad 2.51635100 \quad-4.00820600 \quad-1.06433300$

H $\quad 0.83438900 \quad-4.10375900-0.51961600$

$\mathrm{H} \quad 2.00284400 \quad-5.31014700 \quad 0.02552100$

$\mathrm{H} \quad 1.84625100 \quad-4.00586900 \quad-3.48610400$

$\mathrm{H} \quad 0.80198000 \quad-5.26803100 \quad-4.15167100$

$\mathrm{H} \quad 0.13628600 \quad-4.06337000 \quad-3.04129500$

$\mathrm{H} \quad 2.23149000 \quad-6.17709500 \quad-2.29836600$

$\mathrm{H} \quad-0.72368800 \quad-5.93974800 \quad-1.58743900$

$\mathrm{H} \quad 0.38444300 \quad-6.99211200 \quad-0.72896900$

$\mathrm{H} \quad 7.06083700 \quad-3.11589800 \quad 0.32161700$

$\mathrm{H} \quad 8.35292200 \quad-1.95000800 \quad 3.39611700$

$\begin{array}{llll}\mathrm{H} & 8.29468200 & 0.26167100 & 2.24852300\end{array}$

H $\quad 7.11128200 \quad-0.34581100 \quad 1.09901200$

$\begin{array}{llll}\mathrm{H} & 6.80764200 & 2.18557300 & 2.88690000\end{array}$

$\mathrm{H} \quad 5.74958300 \quad 1.62816600 \quad 1.57810000$

$\mathrm{H} \quad 5.09018700 \quad 1.89945600 \quad 3.20343800$

$\mathrm{H} \quad \begin{array}{llll}7.52535700 & 0.47037000 & 4.78169800\end{array}$

$\mathrm{H} \quad 5.79148600 \quad 0.41994100 \quad 5.11938100$

$\mathrm{H} \quad 6.66978500 \quad-1.08999300 \quad 4.82425900$

H $\quad 5.40320300 \quad-0.53532000 \quad 2.82016300$

$\mathrm{H} \quad 9.08465600 \quad-1.50809500 \quad 0.63414100$

H $\quad 9.50280200 \quad-4.67973300 \quad-0.35839600$

$\mathrm{H} \quad 11.11218100 \quad-4.22655500 \quad 0.19750400$

$\mathrm{H} \quad 11.07780500 \quad-2.38667200 \quad-1.22606800$

H $\quad 12.40540300 \quad-0.59424400 \quad-3.20413600$

H $\quad 11.50520900 \quad-1.00937500 \quad-5.42454100$

$\mathrm{H} \quad 10.62304900 \quad-2.73694200 \quad-3.89764200$

H $\quad 10.10991300 \quad 0.03569400 \quad-5.11045300$

$\mathrm{H} \quad 11.60374900 \quad 0.97536800 \quad-3.52592100$

$\mathrm{H} \quad 9.16776800 \quad-2.21855300 \quad-4.75915500$

H $\quad 8.48547500 \quad-0.76820600 \quad-2.94077500$

$\mathrm{H} \quad 9.95842500 \quad 2.91337500 \quad 0.09174000$

$\mathrm{H} \quad 9.03298300 \quad 5.49690500 \quad-0.05031000$

$\mathrm{H} \quad-4.37106700 \quad 4.82976300 \quad-0.40202700$

$\mathrm{H} \quad-4.16857600 \quad 3.56423400 \quad-1.59837700$

$\mathrm{H} \quad-9.60661600 \quad 4.46003100 \quad-0.72716300$

$\mathrm{H} \quad-10.50798600 \quad 4.19933800 \quad 0.76732000$

$\mathrm{H} \quad-11.20827200 \quad 5.19559500 \quad-0.52928400$

$\mathrm{H} \quad-10.30951800 \quad 6.62677500 \quad 1.33060500$

$\mathrm{H} \quad-2.96597000 \quad 4.43039000 \quad 5.43647700$

$\mathrm{H} \quad-5.15387900 \quad 6.53709800 \quad 4.98096400$

$\mathrm{H} \quad-3.52798800 \quad 6.61885200 \quad 4.32313300$

$\mathrm{H} \quad-4.65037500 \quad 6.80843100 \quad 2.02361200$

$\mathrm{H} \quad-5.72470300 \quad 5.50795200 \quad 1.47305800$

$\mathrm{H} \quad-6.26516500 \quad 6.59367500 \quad 2.73558400$

$\mathrm{H} \quad-3.18179600 \quad 3.61524100 \quad 3.47832800$

$\mathrm{H} \quad-4.02444100 \quad 3.68972700 \quad 1.93576400$

$\mathrm{H} \quad-2.86963300 \quad 4.96624600 \quad 2.36993800$

$\mathrm{H} \quad-5.56491000 \quad 4.42477900 \quad 3.71664100$

H $\quad 1.42322200 \quad 3.93917100 \quad 5.10414300$ 


\begin{tabular}{|c|c|c|c|}
\hline $\mathrm{H}$ & 0.53547800 & 2.55797400 & \\
\hline $\mathrm{H}$ & .44297700 & & 92220700 \\
\hline & .02482300 & 0.98928300 & 23242300 \\
\hline & 1.73766300 & 0.17054000 & \\
\hline & 4.14606500 & -2.03305500 & 4.56942000 \\
\hline & 3.25549400 & -1.78888700 & 3.06645400 \\
\hline & 2.36608100 & -1.89764000 & 1700 \\
\hline & 1.75205100 & -5.20284900 & 2.83463000 \\
\hline & 0.93985000 & -3.77 & 3.50547100 \\
\hline & 9700 & -3.6 & \\
\hline & 7600 & -4.15 & 14700 \\
\hline & 2800 & -5.61 & 1700 \\
\hline & 1200 & -4.1 & 90700 \\
\hline & 7.43351100 & 0.19 & -1.052 \\
\hline & & -1.0 & 300 \\
\hline & 400 & 0.2 & 756800 \\
\hline & & & 940000 \\
\hline & 7300 & 200 & 8300 \\
\hline & & & 9000 \\
\hline & & & \\
\hline & 1200 & & 8500 \\
\hline & -8.497 & & \\
\hline & 600 & & \\
\hline & & -3.4 & 400 \\
\hline & & & 7400 \\
\hline & 600 & -3.9 & 1500 \\
\hline & 0.74 & -8.3 & 200 \\
\hline & & -1. & 700 \\
\hline & 900 & & -7.02 \\
\hline & & & \\
\hline & 900 & & 600 \\
\hline & $-8.7^{\prime}$ & -8.9 & 200 \\
\hline & 800 & -3.8 & 300 \\
\hline & 9800 & & 400 \\
\hline & 0200 & 200 & 200 \\
\hline & & & 700 \\
\hline & -4.8 & & 900 \\
\hline & & & \\
\hline & & & 100 \\
\hline & -4.075 & -5.3 & -3.7 \\
\hline & 400 & -1.4 & 7300 \\
\hline & -8.228 & -2.8 & 5200 \\
\hline & -11.24 & 200 & 100 \\
\hline & -2.3 & 2.9 & 200 \\
\hline & -3.478 & & 8500 \\
\hline & & & \\
\hline & & & 8800 \\
\hline & 7.779 & & \\
\hline & 200 & & 1700 \\
\hline & & & -2.43 \\
\hline & 7900 & & 100 \\
\hline & & -5.23 & 3400 \\
\hline & & & 5600 \\
\hline & 5.17904800 & -4.897 & 23000 \\
\hline & 3.99095400 & -4.92 & \\
\hline & -1.01018700 & -8.13 & -2.55506200 \\
\hline & -0.13165900 & -7.22320800 & -3.74970400 \\
\hline & -8.06990300 & -8.87910400 & 0.15099600 \\
\hline & & & -1.28252900 \\
\hline & -8.79071300 & 8.11872300 & -0.05429200 \\
\hline & -10.06010400 & 7.54883700 & -1.08602200 \\
\hline & -3.40151400 & 5.62888900 & 6.61701700 \\
\hline & -4.56474000 & 4.34025000 & 6.12844000 \\
\hline
\end{tabular}

$\begin{array}{lrrr}\mathrm{H} & 1.80058100 & 3.38010500 & 6.74948200 \\ \mathrm{H} & -5.70084800 & 1.03194200 & 0.76370500 \\ \mathrm{C} & -0.73327600 & -0.87483300 & 2.95235600 \\ \mathrm{~N} & 0.73344300 & 2.01046500 & 0.89115800 \\ \mathrm{O} & -4.00240600 & -2.40572200 & 2.24427300 \\ \mathrm{C} & -0.59590000 & -1.24337400 & 4.29223200 \\ \mathrm{O} & -3.64344900 & -3.18277200 & 4.87797100 \\ \mathrm{C} & -1.57092100 & -2.02253100 & 4.92237100 \\ \mathrm{C} & -2.71412400 & -2.42630600 & 4.23501500 \\ \mathrm{C} & -2.87107200 & -2.03132600 & 2.89059200 \\ \mathrm{C} & -1.88039500 & -1.28273000 & 2.26231400 \\ \mathrm{C} & 0.31387100 & -0.04289700 & 2.24942800 \\ \mathrm{C} & -0.30240600 & 1.09668200 & 1.42547300 \\ \mathrm{C} & -0.14186900 & 4.85687100 & 0.52473900 \\ \mathrm{C} & -1.23812100 & 4.45218800 & -0.25289500 \\ \mathrm{C} & -1.44726600 & 4.96767200 & -1.52988900 \\ \mathrm{C} & -0.55807400 & 5.90756100 & -2.05353100 \\ \mathrm{O} & -0.79090500 & 6.35838000 & -3.32363800 \\ \mathrm{C} & 0.52635000 & 6.34112700 & -1.28421000 \\ \mathrm{C} & 0.73073900 & 5.81027300 & -0.01307800 \\ \mathrm{H} & 0.39563400 & 2.45390500 & 0.03670900 \\ \mathrm{H} & 2.12213300 & 1.25231400 & 0.66166100 \\ \mathrm{H} & -3.96627100 & -2.12634800 & 1.26091500 \\ \mathrm{H} & 0.28047500 & -0.92619300 & 4.84994400 \\ \mathrm{H} & -4.37351300 & -3.41056400 & 4.27816100 \\ \mathrm{H} & -1.46558500 & -2.32212000 & 5.95986600 \\ \mathrm{H} & -2.03663100 & -1.02569000 & 1.22069100 \\ \mathrm{H} & 0.91411800 & -0.67122600 & 1.57333100 \\ \mathrm{H} & 1.00813600 & 0.37076000 & 2.99001400 \\ \mathrm{H} & -0.84996800 & 0.69176500 & 0.57150800 \\ \mathrm{H} & -1.03786000 & 1.63509900 & 2.03599500 \\ \mathrm{H} & -1.95294000 & 3.73651300 & 0.14477700 \\ \mathrm{H} & -2.29548200 & 4.65529900 & -2.12609600 \\ \mathrm{H} & -0.15410800 & 7.05353300 & -3.53351900 \\ \mathrm{H} & 1.20716800 & 7.09201600 & -1.67912200 \\ \mathrm{H} & 1.58520900 & 6.13364800 & 0.57220000 \\ & 0.12345300 & 4.24607800 & 1.87763000 \\ \mathrm{H} & -0.80992300 & 3.87957000 & 2.31266600 \\ \mathrm{H} & 1.12439700 & 3.08166800 & 1.83156800 \\ \mathrm{H} & 2.38963300 & 3.59916900 & 1.46357400 \\ \mathrm{H} & 2.597800 & 2.84126200 & 1.46341600\end{array}$

\section{TS2' (+18.8 $\mathrm{kcal} / \mathrm{mol})$}

$\begin{array}{cccc}\mathrm{C} & 7.06664600 & 5.13256100 & 1.41194300 \\ \mathrm{C} & 7.41172500 & 4.56028900 & 0.07426200 \\ \mathrm{O} & 6.63863300 & 3.84445600 & -0.56799500 \\ \mathrm{~N} & 8.63380500 & 4.92344500 & -0.40568300 \\ \mathrm{C} & 9.04629800 & 4.54687000 & -1.72556100 \\ \mathrm{C} & 10.09425000 & 3.43976400 & -1.83266400 \\ \mathrm{O} & 10.57753400 & 3.16827900 & -2.92673700 \\ \mathrm{~N} & 10.45551300 & 2.81386000 & -0.67844700 \\ \mathrm{C} & 11.26160800 & 1.60874000 & -0.77663900 \\ \mathrm{C} & 10.36781700 & 0.42567200 & -1.14664800 \\ \mathrm{O} & 9.41630200 & 0.15158400 & -0.40646800 \\ \mathrm{~N} & 10.64850800 & -0.30874100 & -2.24711200 \\ \mathrm{C} & 9.72465200 & -1.40330600 & -2.60488900 \\ \mathrm{C} & 9.61558500 & -2.47932900 & -1.52644400 \\ \mathrm{O} & 8.58139100 & -3.12571800 & -1.38303700\end{array}$


$\begin{array}{llll}\text { C } & -10.43136100 & 0.78776600 & -2.39438500\end{array}$

C $\quad-9.05066200 \quad 1.01781000 \quad-2.43238800$

C $\quad-11.06277300 \quad 0.80036500 \quad-1.14735500$

$\begin{array}{llll}\text { C } & -8.32250200 & 1.26570200 & -1.27412600\end{array}$

C $\quad-10.35193600 \quad 1.06462200 \quad 0.02254700$

$\begin{array}{llll}\text { C } & -8.98048300 & 1.31440900 & -0.03670700\end{array}$

O $\quad-8.30296800 \quad 1.58041400 \quad 1.12377800$

C $\quad-5.53985700 \quad 4.95790600 \quad-1.91347100$

C $\quad-4.75543100 \quad 4.07380300 \quad-0.92864000$

$\begin{array}{llll}\text { C } & -5.53619400 & 3.09791200 & -0.05706200\end{array}$

O $\quad-6.69239300 \quad 3.43881600 \quad 0.33614100$

$\begin{array}{llll}\mathrm{O} & -4.94141000 & 2.01694400 & 0.23475700\end{array}$

C $\quad-9.15321500 \quad 7.00209000 \quad-0.20349000$

$\begin{array}{llll}\text { C } & -9.48039700 & 5.95136900 & 0.85653100\end{array}$

$\begin{array}{llll}\text { C } & -10.13706100 & 4.71236600 & 0.24753400\end{array}$

$\begin{array}{llll}\text { O } & -8.30420600 & 5.59959600 & 1.57944300\end{array}$

C $\quad-3.63435600 \quad 4.87811100 \quad 5.96213600$

C $\quad-4.15573000 \quad 5.74779400 \quad 4.81810500$

C $\quad-4.63321900 \quad 4.95925200 \quad 3.58239300$

C $\quad-5.15061200 \quad 5.91709600 \quad 2.50393800$

C $\quad-3.54740300 \quad 4.03976200 \quad 3.00357500$

C $\quad 1.71795400 \quad 3.00918800 \quad 5.86774900$

S $\quad 2.30937100 \quad 2.14254600 \quad 4.36729000$

C $\quad \begin{array}{llll}1.09593200 & 0.78051900 & 4.25237500\end{array}$

$\begin{array}{llll}\text { O } & 5.03258400 & -0.24947000 & 0.24682800\end{array}$

O $\quad 2.50406300 \quad-0.29111600 \quad 1.20177800$

O $\quad-6.18030200 \quad-0.12664200 \quad 0.98331900$

$\mathrm{H} \quad 4.92579100 \quad 2.84025400 \quad-0.41940100$

$\mathrm{H} \quad 4.44096200 \quad 1.27768100 \quad-0.66584200$

$\mathrm{H} \quad 4.39912600 \quad 2.40119800 \quad-2.79532700$

H $\quad 2.73707100 \quad 2.12614000 \quad-2.27721400$

$\mathrm{H} \quad 4.35250600 \quad 4.70398500 \quad-1.84969100$

$\mathrm{H} \quad 2.73728900 \quad 4.43507400 \quad-1.21831200$

$\mathrm{H} \quad 1.83737900 \quad 4.27892900 \quad-3.58979600$

$\mathrm{H} \quad 3.46041900 \quad 4.63351100 \quad-4.20402700$

$\mathrm{H} \quad 4.90096000 \quad 0.15284000 \quad-5.20502200$

$\mathrm{H} \quad 5.30137500 \quad-0.29798100 \quad-2.79353000$

$\mathrm{H} \quad 3.47068400 \quad-1.10496300 \quad-1.35814500$

$\mathrm{H} \quad 1.21362700 \quad-1.53754700 \quad-2.35324400$

$\mathrm{H} \quad 0.83134700 \quad-1.12255700 \quad-4.75129700$

$\mathrm{H} \quad 0.48792600 \quad 1.58810200 \quad-3.86532100$

$\mathrm{H} \quad-0.16106800 \quad 3.07802600 \quad-3.15878500$

$\mathrm{H} \quad 0.28855600 \quad 1.71518200 \quad-2.11344300$

$\mathrm{H} \quad-2.17918300 \quad 1.79796400 \quad-2.35532100$

$\mathrm{H} \quad-1.02850000 \quad-0.49035300 \quad-2.30638500$

$\mathrm{H} \quad-2.60787000 \quad-0.48484700 \quad-3.06527800$

$\mathrm{H} \quad-1.13945800 \quad-0.50681600 \quad-4.07177700$

$\mathrm{H} \quad-8.07693200 \quad-1.75401000 \quad-2.47488800$

$\mathrm{H} \quad-9.40433100 \quad-1.76503800 \quad-3.61934100$

$\mathrm{H} \quad-8.16706600 \quad-4.23177900 \quad-2.30050200$

$\mathrm{H} \quad-9.45846300 \quad-4.29180600 \quad-3.50136800$

$\mathrm{H} \quad-8.52601500 \quad-2.93912600 \quad 0.09458400$

H $\quad-9.35889600 \quad-1.48313000 \quad-0.54264900$

H $\quad-10.13838100 \quad-2.49272700 \quad 0.68764500$

$\mathrm{H} \quad-7.62628200 \quad 2.30961500 \quad 0.91983000$

$\mathrm{H} \quad-7.25094900 \quad 1.41631300 \quad-1.31683500$

$\mathrm{H} \quad-10.84574700 \quad 1.09268100 \quad 0.98796600$

$\mathrm{H} \quad-12.13083100 \quad 0.60867600 \quad-1.09006900$

$\mathrm{H} \quad-8.53264500 \quad 0.99380900 \quad-3.38824400$

$\mathrm{H} \quad-3.91716500 \quad-3.95944700 \quad-0.53943800$

$\mathrm{H} \quad-5.06840900 \quad-4.15983100 \quad-1.83125600$

$\mathrm{H} \quad-2.23562500 \quad-4.30163800 \quad-2.10312000$

$\mathrm{H} \quad-2.62938800 \quad-2.70961800 \quad-2.71827500$ 


\begin{tabular}{|c|c|c|c|}
\hline $\mathrm{H}$ & -8.35134600 & -6.54430800 & \\
\hline & -9.42964800 & -7.30127700 & -0.32590400 \\
\hline & -8.52480700 & -7.58090900 & \\
\hline & -6.86490800 & -4.85308100 & -0.71301200 \\
\hline & -5.97400200 & -3.47423200 & \\
\hline & -7.61613100 & -6.18537600 & \\
\hline & -6.05138900 & -3.08471200 & 3.36080600 \\
\hline & 2.66632200 & & -0.94698700 \\
\hline & 0.92388600 & -3.96565100 & -0.6 \\
\hline & 403400 & -5.09220600 & \\
\hline & 343100 & -4.00045000 & -3. \\
\hline & 600 & -5.3666 & \\
\hline & & -4.14 & -3.2 \\
\hline & 700 & -6.0906 & -2. \\
\hline & 100 & -5.9137 & -1. \\
\hline & 0.6 & -6.95 & -0.7 \\
\hline & 7.4 & -3.04 & \\
\hline & & -1.9 & \\
\hline & 000 & 500 & \\
\hline & & -0.1 & \\
\hline & & & \\
\hline & 6.0 & & \\
\hline & & & \\
\hline & 100 & & \\
\hline & & & \\
\hline & & & \\
\hline & & -0.5 & \\
\hline & & & \\
\hline 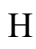 & 800 & -4.6 & \\
\hline & 200 & -3.7 & \\
\hline F & 500 & 500 & \\
\hline & 000 & 2700 & \\
\hline & 11.6 & -0.8 & \\
\hline $\mathrm{H}$ & & -2.6 & \\
\hline & 10. & -0.0 & \\
\hline & & & \\
\hline & & -2.3 & \\
\hline & 500 & -1.0 & -2 \\
\hline & & 900 & \\
\hline & & & \\
\hline & -4.20 & & \\
\hline & & 3.4 & -1 \\
\hline & -9.483 & 4.26 & -0. \\
\hline & & & \\
\hline & & 2500 & 900 \\
\hline & -10.1 & 6500 & \\
\hline $\mathrm{H}$ & -2.78 & 4.2795 & 00 \\
\hline & -4.983 & 6.37528500 & \\
\hline & -3.361 & & \\
\hline $\mathrm{H}$ & -4.347 & 6.5884 & \\
\hline & & & \\
\hline $\mathrm{H}$ & & & \\
\hline & -3.21058800 & 3.29067700 & 3.72701400 \\
\hline & & & \\
\hline & -2.67061600 & 4.62216200 & 2.68980100 \\
\hline & -5.473 & 4.32262500 & \\
\hline & & & \\
\hline & & 3.36396900 & \\
\hline & & & \\
\hline & 1.21199500 & 0.08860800 & 5.08760500 \\
\hline & 0.07376800 & 1.16813200 & 4.22956700 \\
\hline & & -1.88759500 & 4.82099500 \\
\hline & 3.38894300 & -1.49851300 & 3.44643500 \\
\hline
\end{tabular}

\section{$\mathrm{C}$}

$\mathrm{N}$
$\mathrm{O}$

$\begin{array}{llll}2.66603900 & -1.87075100 & 5.01620800\end{array}$

$\begin{array}{lll}2.10236000 & -4.94307700 & 2.80600800\end{array}$

$\begin{array}{llll}1.25624300 & -3.62209300 & 3.62496800\end{array}$

$\begin{array}{llll}2.20343900 & -3.29352200 & 2.17038300\end{array}$

$\begin{array}{llll}4.31014600 & -3.84565300 & 3.37169200\end{array}$

$\begin{array}{lll}3.27527400 & -5.57122600 & 4.85986300\end{array}$

$\begin{array}{lll}2.53870300 & -4.29024800 & 5.79988600\end{array}$

$\begin{array}{llll}4.18705900 & -0.41899000 & 0.72425900\end{array}$

$\begin{array}{llll}5.56669400 & -1.05419100 & 0.33315700\end{array}$

$\begin{array}{lll}1.88237400 & -0.88139100 & 0.75921500\end{array}$

$\begin{array}{llll}3.30806800 & 2.36742600 & -0.01024000\end{array}$

$\begin{array}{llll}-6.06231800 & -1.24462000 & -0.27625100\end{array}$

$\begin{array}{llll}8.15841200 & 4.17672700 & -2.24518800\end{array}$

$\begin{array}{llll}6.43478600 & 4.42135800 & 1.94742400\end{array}$

$-6.28129200 \quad 5.57575900-1.40453600$

$\begin{array}{llll}-8.40742700 & 6.58399000 & -0.89280000\end{array}$

$\begin{array}{lll}-7.78471300 & 4.97497200 & 1.04067900\end{array}$

$\begin{array}{llll}4.90246900 & -3.42700700 & 6.33744700\end{array}$

$\begin{array}{llll}12.06583400 & 1.78870900 & -1.48732800\end{array}$

$\begin{array}{llll}4.77359200 & -3.89715300 & 1.20945400\end{array}$

$\begin{array}{llll}1.16647400 & -8.31973500 & -2.77383100\end{array}$

$\begin{array}{llll}2.97592200 & -1.01541100 & -7.23636600\end{array}$

$3.02891100 \quad 0.72855200 \quad-6.96494500$

$\begin{array}{llll}-1.72558800 & 1.44212400 & -5.36243100\end{array}$

$-6.68724700 \quad-3.13821400 \quad-4.08121300$

$\begin{array}{lll}-8.33143100 & -9.09879100 & -1.45872600\end{array}$

$\begin{array}{lll}-4.39972800 & -3.84105000 & -4.23676400\end{array}$

$\begin{array}{lll}-12.27245700 & 0.44764700 & -3.46714100\end{array}$

$\begin{array}{llll}-10.88003000 & -0.38158800 & -4.16284100\end{array}$

$\begin{array}{llll}-7.06363800 & 0.24319500 & 1.20764600\end{array}$

$\begin{array}{llll}-4.75936400 & 5.59034900 & -2.34832900\end{array}$

$\begin{array}{llll}-6.02772100 & 4.37958700 & -2.70470900\end{array}$

$-2.75416100 \quad-4.31214300 \quad-4.65204200$

$\begin{array}{lll}-3.73378300 & -5.40582500 & -3.74432400\end{array}$

$\begin{array}{lll}-7.09870100 & -1.54328400 & -4.70234700\end{array}$

$-7.95117300 \quad-2.96733400 \quad-5.30528200$

$\begin{array}{llll}-11.06133200 & 1.35738200 & -4.37857300\end{array}$

$\begin{array}{llll}-2.21892500 & 2.97727700 & -4.66584900\end{array}$

$\begin{array}{llll}-3.29807900 & 1.63331200 & -4.58332500\end{array}$

$\begin{array}{llll}1.49315100 & -0.14977700 & -6.80729700\end{array}$

$\begin{array}{llll}2.61176500 & 5.82900900 & -3.19682500\end{array}$

$\begin{array}{llll}7.91850000 & 5.38120900 & 2.05329300\end{array}$

$\begin{array}{llll}6.47946200 & 6.04585600 & 1.26293500\end{array}$

$\begin{array}{llll}9.44596800 & 5.36890400 & -2.32990000\end{array}$

$\begin{array}{lll}11.69432900 & 1.39454300 & 0.20393200\end{array}$

$\begin{array}{llll}6.01699100 & -5.12429200 & 1.45798900\end{array}$

$\begin{array}{llll}5.74229700 & -4.45227400 & -0.16842200\end{array}$

$\begin{array}{llll}5.56584300 & -4.86211600 & 5.59239100\end{array}$

$\begin{array}{llll}4.38770800 & -4.94157300 & 7.03724100\end{array}$

$\begin{array}{llll}-0.60102800 & -8.11745900 & -2.58709800\end{array}$

$\begin{array}{llll}0.24959300 & -7.16569600 & -3.77298100\end{array}$

$\begin{array}{lll}-7.62554000 & -9.05517900 & 0.14915300\end{array}$

$\begin{array}{lll}-6.72753000 & -8.42685100 & -1.28070000\end{array}$

$\begin{array}{llll}-8.72317400 & 7.92353300 & 0.20357200\end{array}$

$\begin{array}{llll}-9.98607400 & 7.34107100 & -0.82908500\end{array}$

$\begin{array}{lll}-3.23881200 & 5.45482800 & 6.80479400\end{array}$

$\begin{array}{lll}-4.37629300 & 4.14809400 & 6.30378300\end{array}$

$\begin{array}{llll}1.77343400 & 2.34551000 & 6.73330100\end{array}$

$\begin{array}{lll}-5.66708300 & 0.71081600 & 0.72335900\end{array}$

$\begin{array}{lll}-1.68994200 & -0.90618100 & 1.42510200\end{array}$

$\begin{array}{lll}-0.72752100 & 2.72505600 & 0.67075100\end{array}$

$\begin{array}{lll}-5.04160800 & -1.81516700 & 2.67181800\end{array}$

C $\quad-0.95829600 \quad-2.00718500 \quad 1.87587300$ 


$\begin{array}{lrrr}\mathrm{O} & -3.48226000 & -4.05860600 & 3.51150500 \\ \mathrm{C} & -1.59107500 & -3.04680800 & 2.55327400 \\ \mathrm{C} & -2.96241800 & -3.01323300 & 2.82313000 \\ \mathrm{C} & -3.70209700 & -1.89206900 & 2.38468600 \\ \mathrm{C} & -3.06336600 & -0.87032200 & 1.68375600 \\ \mathrm{C} & -1.01472800 & 0.23235100 & 0.69823900 \\ \mathrm{C} & -1.54398500 & 1.60166700 & 1.13819400 \\ \mathrm{C} & -0.23567700 & 5.42261300 & 0.12262700 \\ \mathrm{C} & -1.24792100 & 6.25117700 & 0.62622000 \\ \mathrm{C} & -2.14866700 & 6.88811400 & -0.21842500 \\ \mathrm{C} & -2.05453300 & 6.70209400 & -1.60248700 \\ \mathrm{O} & -2.95477700 & 7.36111600 & -2.38426100 \\ \mathrm{C} & -1.06117400 & 5.86953300 & -2.12484200 \\ \mathrm{C} & -0.16259100 & 5.24343100 & -1.26215800 \\ \mathrm{H} & -0.98689200 & 3.15419100 & -0.20899600 \\ \mathrm{H} & 2.17662100 & 0.63944900 & 1.04387700 \\ \mathrm{H} & -5.47804000 & -1.11314400 & 2.10454700 \\ \mathrm{H} & 0.11518300 & -2.05918100 & 1.71525200 \\ \mathrm{H} & -4.44857600 & -3.99461300 & 3.63967500 \\ \mathrm{H} & -1.03345100 & -3.90964700 & 2.90097800 \\ \mathrm{H} & -3.65900800 & -0.03447900 & 1.34009700 \\ \mathrm{H} & -1.14300000 & 0.13723100 & -0.38546400 \\ \mathrm{H} & 0.05639700 & 0.20436200 & 0.90941200 \\ \mathrm{H} & -2.56021400 & 1.77251900 & 0.77122600 \\ \mathrm{H} & -1.57751500 & 1.65045900 & 2.23081500 \\ \mathrm{H} & -1.33944100 & 6.39398800 & 1.69850900 \\ \mathrm{H} & -2.93649100 & 7.52129000 & 0.17338000 \\ \mathrm{H} & -2.84447800 & 7.09094400 & -3.30538800 \\ \mathrm{H} & -0.98549800 & 5.71415700 & -3.19823200 \\ \mathrm{H} & 0.59942900 & 4.59555500 & -1.67771200 \\ \mathrm{C} & 0.70428400 & 4.68468100 & 1.04727800 \\ \mathrm{H} & 0.73787700 & 5.19033100 & 2.01806000 \\ \mathrm{H} & 1.72396000 & 4.69032100 & 0.66118600 \\ \mathrm{C} & 0.30126200 & 3.23471300 & 1.32915500 \\ \mathrm{H} & 0.36697800 & 2.91551300 & 2.36399100 \\ \mathrm{O} & 1.96915500 & 2.32928500 & 0.96627300 \\ \mathrm{H} & 2.36107000 & 2.48528700 & 1.84442800\end{array}$

\section{Int3' (+11.4 kcal/mol)}

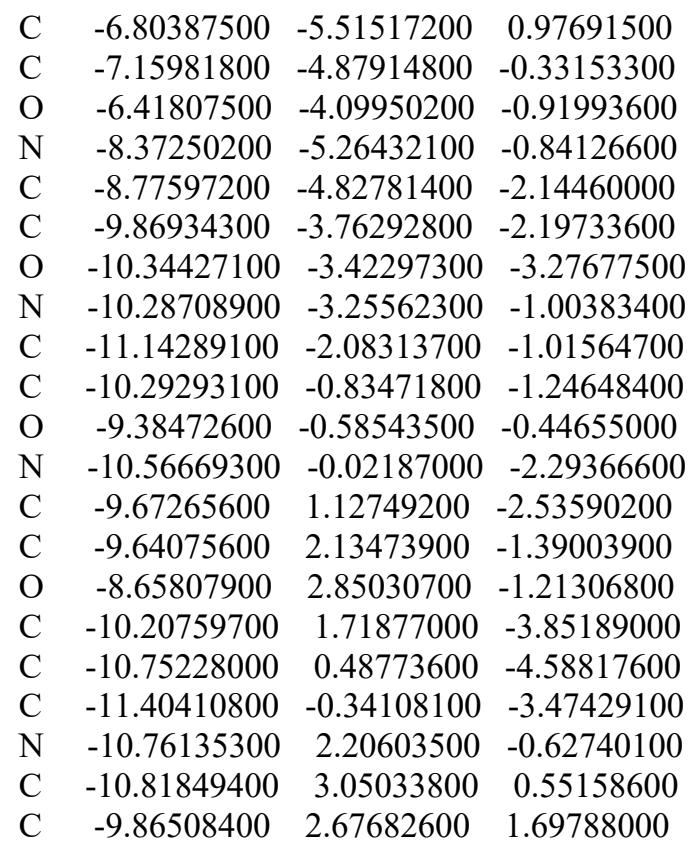

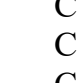

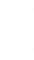

$$
\text { c }
$$ \\ ind}$$
\text { c }
$$

c

O

.

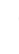

$-9.70502900$

$-9.22068700$

$-8.11331900$

$-7.02209900$

$-6.39104100$

$-7.53993700$

$-6.28834800$

$-6.50400200$

$-5.83445100$

$-6.74609500$

$-5.84752500$

$-4.80632000$

$-3.56921200$

$-3.51052700$

$-2.45177900$

$-3.24709400$

$-0.51860600$

$-0.71029800$

$-1.74169900$

$-1.28589400$

$-2.01588600$

7.47248100

8.02830200

7.05765400

5.85165600

7.32215600

4.96267800

6.45717100

5.27927800

4.47665400

3.45589300

3.16879100

4.45441100

4.40544600

5.41958400

3.49777300

$-2.43667100$

$-2.66388800$

$-1.60467400$

$-3.91042300$

$-1.77320500$

$-4.08633700$

$-3.01628400$

$-2.50149000$

$-2.87674900$

$-3.35711000$

$-3.68158000$

2.42609500

1.84933100

2.04339400

0.37335400

7.54709700

8.47754900

8.74384700

9.63612100

8.35746600

11.32640300

10.49192600

$\begin{array}{llll}9.45984300 & -1.24070400 & -2.49849900\end{array}$

$\begin{array}{llll}10.71267700 & 0.33140100 & -1.19700700\end{array}$

$\begin{array}{llll}8.67192700 & -1.54891900 & -1.39333900\end{array}$

$\begin{array}{llll}9.94400600 & 0.02666200 & -0.07709200\end{array}$

C

$3.47267400 \quad 2.61484100$

$1.48604100 \quad 1.61315900$

$2.28252500 \quad 2.42461900$

$2.61304700 \quad 3.42010800$

$-0.19022000 \quad 2.21187500$

$-0.59009800 \quad 3.01106700$

$\begin{array}{ll}-0.52350000 & 4.52628200\end{array}$

$\begin{array}{ll}-1.98492200 & 2.56315200\end{array}$

1.16890900

$3.89224700 \quad 6.20521300$

$4.22325600 \quad 5.34076400$

$3.59955900 \quad 3.94273800$

$4.30128300 \quad 3.08614700$

$2.09259800 \quad 4.00767900$

$\begin{array}{ll}.66373600 & -2.42176700\end{array}$

$4.69868700-3.15114300$

$4.44998400-0.75442500$

$8.74248100-0.30577400$

$7.31955600-0.10666200$

$6.39255000 \quad 0.59548200$

$6.02005900-0.01307800$

1.86706700

$4.96760000-2.48547800$

$4.57122700 \quad 1.84329500$

$3.65601000 \quad 2.45731700$

$4.61366700-3.63969600$

$3.75931800-2.40528500$

$3.02881100-2.00518000$

$-0.75603300$

$2.28923000-0.08594600$

$0.42006600-6.69879500$

$0.61577700 \quad-5.21396800$

$\begin{array}{ll}1.03237600 & -4.39745100 \\ 0.39110800 & -4.61262300\end{array}$

$1.20501800-3.02301500$

$0.57487900-3.24043700$

$0.97725300-2.43725000$

$-4.72291400-3.70926700$

$\begin{array}{ll}.15904700 & -2.33437000\end{array}$

$-2.27587000-0.98238100$

$-1.61774600-4.64841600$

$\begin{array}{ll}-1.38417900 & -3.24251700 \\ 0.06752200 & -2.78687500\end{array}$

$181000-3.17314000$

$2.78213100-3.05196700$

$4.09946900-2.32101000$

$3.92560700-0.73119800$

$8.91955300 \quad-0.92114100 \quad-0.16621000$ 


\begin{tabular}{|c|c|c|c|}
\hline & 19566900 & -1.20427000 & \\
\hline & 5.81658500 & -4.69859600 & -2.19669400 \\
\hline & 4.92759600 & -3.89282800 & -1.23361200 \\
\hline & 5.54045500 & -3.11774600 & -0.09084700 \\
\hline & 6.75477800 & -3.26686200 & 0.19171300 \\
\hline 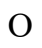 & 4.73418100 & -2.35311600 & 0.55012000 \\
\hline & 9.48779300 & -6.70235300 & -0.56341100 \\
\hline & 9.81774000 & -5.68527800 & 0.52858700 \\
\hline C & 10.56969500 & -4.47780200 & 234600 \\
\hline D & 8.63938200 & -5.26906300 & 1.21528600 \\
\hline 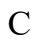 & 3.84021900 & -5.12693300 & 5.65017900 \\
\hline C & 4.49034400 & -6.08930800 & 4.636 \\
\hline 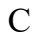 & 5.62828600 & -5.53065000 & 3.77542900 \\
\hline C & 6.16427600 & -6.62408700 & 2.84 \\
\hline & 5.21398800 & -4.29503700 & 2.97903500 \\
\hline 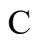 & -1.57634300 & -3.45083000 & 5.60122900 \\
\hline & -1.74675000 & -2.88051200 & 3.8 \\
\hline 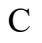 & -2.19474800 & -1.13225000 & 4.13776200 \\
\hline $\mathrm{O}$ & -4.81826400 & 0.35691300 & \\
\hline $\mathrm{O}$ & -2.17120200 & 0.35579900 & 93600 \\
\hline U & 5.69174200 & -0.02732800 & \\
\hline $\mathrm{H}$ & -4.44287400 & 5600 & -0.6 \\
\hline 11 & -4.04407900 & -1.32085400 & 367900 \\
\hline & -4.21988900 & -2.61468800 & -3.0 \\
\hline $\mathrm{H}$ & -2.57717900 & -2.04730500 & 502200 \\
\hline & -3.67558200 & -4.77409900 & -1.8 \\
\hline $\mathrm{H}$ & -2.01925200 & -4.233 & -1.6 \\
\hline 11 & -1.62260800 & -4.22087500 & 692800 \\
\hline [ & -3.32250100 & -4.60854000 & -4.4 \\
\hline $\mathrm{H}$ & -4.74699900 & 0.06 & -5.2 \\
\hline & -5.05293600 & 6600 & 94000 \\
\hline 11 & 953200 & 6900 & -1.3 \\
\hline $\mathrm{H}$ & -0.93368300 & 1.5199 & -2.4 \\
\hline & -0.63187500 & 4600 & -4.8 \\
\hline $\mathrm{H}$ & -0.24313800 & 1700 & 46000 \\
\hline 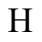 & 0.23229400 & -2.82423400 & 93100 \\
\hline 11 & -0.02068500 & -1.72576500 & -2.1 \\
\hline $\mathrm{H}$ & 2.40974000 & -2.025 & -2.5 \\
\hline & 1.63037000 & 0.22974400 & -1.7 \\
\hline $\mathrm{H}$ & 521700 & 30900 & 70000 \\
\hline (1) & 1.53967500 & 0.7629 & -3.4 \\
\hline 1 & 8.00563900 & 2.07778100 & -2.3 \\
\hline $\mathrm{H}$ & 9.42696700 & 2.32624600 & 84000 \\
\hline $\mathrm{H}$ & 7.79940900 & 4.61907700 & 28300 \\
\hline$\Pi$ & & & \\
\hline $\mathrm{H}$ & 7.40774600 & 3.56977600 & 406700 \\
\hline & 8.24279100 & 2.01103500 & -0.12543800 \\
\hline $\mathrm{H}$ & 8.68260000 & 3.02865100 & 1.26327300 \\
\hline $\mathrm{H}$ & 7.65276700 & -2.02237900 & 0.79249500 \\
\hline $\mathrm{H}$ & 7.89152000 & -2.29421000 & 40900 \\
\hline $\mathrm{H}$ & 10.13363800 & 0.50752300 & 637700 \\
\hline 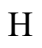 & 11.49515200 & 1.07909500 & 090900 \\
\hline $\mathrm{H}$ & 9.26750100 & -1.74925500 & -3.44034500 \\
\hline $\mathrm{H}$ & 5.26057000 & 3.75408200 & -1.83855800 \\
\hline $\mathrm{H}$ & 4.79972200 & 2.39021200 & -2.82376100 \\
\hline $\mathrm{H}$ & 2.80157300 & 4.38564800 & -1.58850000 \\
\hline $\mathrm{H}$ & 2.38163900 & 3.02660100 & -2.61167700 \\
\hline $\mathrm{H}$ & 8.28154800 & 6.89250400 & -1.08440600 \\
\hline $\mathrm{H}$ & 8.96749100 & 7.35140700 & 0.45427200 \\
\hline $\mathrm{H}$ & 8.24610500 & 6.14406300 & 2.36845200 \\
\hline $\mathrm{H}$ & 5.59552800 & 6.42748600 & -0.98878700 \\
\hline & 4.01639900 & 4.89312800 & 0.13805200 \\
\hline & 6.69281300 & 4.53625800 & 3.45249600 \\
\hline
\end{tabular}

3.99898800 $-2.73895600$ $-1.08922800$ $-2.40982600$ $-2.01993900$ $-1.13094700$ $-0.33840200$ $-2.68248800$ 0.24842900 $-1.00239700$ $-7.46470900$ $-8.47002000$ $-8.33811000$ $-7.29843800$ $-6.59752400$ $-5.65829200$ $-4.90655600$ $-7.35622900$ $-5.61999200$ $-6.68012500$ $-5.48735000$ $-9.35084000$ $-10.55869600$ $-11.84200400$ $-11.48570900$ $-12.43970000$ $-11.45805000$ $-11.01732800$ $-9.92915700$ $-11.37523300$ $-9.42828000$ $-8.64255800$

$-9.66496400$ $-8.91196400$ 4.21534400 4.30606100 9.99050300 10.73790500 11.53751100 10.43540000 3.00353700 4.88441700 3.70995800 5.38363100 7.02630700 6.46174800 4.77852700 6.06423600 4.47351700 6.43870300 $-1.30280200$ $-0.79556800$ $-2.29016000$ $-3.14843400$ $-1.41726000$ $-3.99926500$

$-3.27142800$ $-2.25594500$ $-2.73673100$ $-1.48301900$ $-2.30374100$ $-4.49001800$ $-3.51813700$
$3.14099500 \quad 1.78245200$ $3.68584400-1.05862000$ $3.94522100-0.45636400$ $4.95981800 \quad 0.12998100$ $3.94707200-3.45253900$ $5.37199600-3.99943300$ $4.17717200-2.96298800$ $5.96555400-2.12437200$ $5.98392200-1.28644800$ $6.89025800-0.46152400$ $\begin{array}{ll}2.64693400 & 0.45831700\end{array}$ $\begin{array}{ll}1.26258200 & 3.54986300\end{array}$ $\begin{array}{ll}-0.92435600 & 2.38725500\end{array}$ $\begin{array}{ll}-0.24043100 & 1.14569500\end{array}$ $\begin{array}{ll}-2.73724900 & 2.80498800\end{array}$ $\begin{array}{ll}-2.00191700 & 1.48390200\end{array}$ $-2.28027000 \quad 3.06471000$ $-1.14346100 \quad 4.83373100$ $\begin{array}{ll}-0.89382300 & 5.05789100\end{array}$ $0.50426900 \quad 4.85133500$ $0.10918800 \quad 2.75140300$ $0.87064700 \quad 0.81696300$ $\begin{array}{ll}4.07692200 & 0.28375500\end{array}$ $3.05532900 \quad 0.93267100$ $1.52373900-0.79039100$ $-0.02464600-3.29931700$ $\begin{array}{ll}0.73859000 & -5.38299200\end{array}$ $2.42497600-3.63668400$ $-0.08195500-5.03122000$ $-1.41237700-3.67506000$ $2.25355800-4.39625900$ $0.77433000-2.64374300$ $-3.36497400-0.21431500$ $-5.96329600-0.35410600$ $-4.57327400-0.75898300$ $-3.18759200-1.79714100$ $-3.99127900-0.82502900$ $\begin{array}{ll}-3.73827200 & 0.75495300\end{array}$ $-4.77023000-0.45323000$ $\begin{array}{ll}-6.16423200 & 1.29841600\end{array}$ $-4.56540200 \quad 5.21987700$ $-6.95536900 \quad 5.18230500$ $\begin{array}{ll}-6.50162800 & 3.97633000\end{array}$ $\begin{array}{ll}-6.93439900 & 2.13572000\end{array}$ $\begin{array}{ll}-6.27910200 & 2.26837000\end{array}$ $\begin{array}{ll}-7.51341000 & 3.41186100\end{array}$ $-3.51598600 \quad 3.61446600$ $\begin{array}{ll}-3.86253800 & 2.45138300\end{array}$ $-4.56572300 \quad 2.21742400$ $-5.23395000 \quad 4.45742500$ $-4.50733000 \quad 5.57527300$ $\begin{array}{ll}-2.88904200 & 6.11946700\end{array}$ $-0.66415700 \quad 3.15576900$ $-1.05871000 \quad 4.66373100$ $-0.61231100 \quad 4.70151500$ $1.58217300 \quad 4.61566600$ $1.64455200 \quad 3.01018500$ $\begin{array}{ll}1.89046100 & 4.43409400\end{array}$ $\begin{array}{ll}5.33764700 & 2.87106400\end{array}$ $4.32734000 \quad 3.59185800$ $3.78867900 \quad 2.12928900$ $3.73512400 \quad 3.48108000$ $5.31632000 \quad 5.23924400$ 


\begin{tabular}{|c|c|c|c|}
\hline & -2.65794100 & 3.93782000 & \\
\hline & -3.95612100 & & \\
\hline & & & \\
\hline & -1.44779100 & 0.97815400 & \\
\hline & -2.27214500 & -2.27507000 & -0.10571600 \\
\hline & 5.45520400 & 0.83032800 & \\
\hline & -7.89556000 & -4.39248000 & -2.62551200 \\
\hline & -6.19783600 & & \\
\hline & 6.56100300 & -5.30462100 & -1.67935600 \\
\hline & & -6.26805700 & 80000 \\
\hline & 8.10465000 & -4.69798400 & 8100 \\
\hline & -4.93537900 & & \\
\hline & -11.91832800 & 908400 & 8500 \\
\hline & -4.826 & 03700 & 400 \\
\hline & & & 5500 \\
\hline & -2.879 & 1.22 & 5700 \\
\hline & -2.856 & -0.52 & -7.0 \\
\hline & & & \\
\hline & 6.667 & & -3.9 \\
\hline & & & \\
\hline & & & \\
\hline & 12.38 & & \\
\hline & & & \\
\hline & & -0.1 & \\
\hline & 5.06 & -5.3 & -2.6 \\
\hline & & -4. & -2 \\
\hline & 2.71 & & -4.4 \\
\hline & & & \\
\hline & & & -4.6 \\
\hline & 7.96 & & -5.1 \\
\hline & 11.2 & & 3200 \\
\hline & & -2.6 & \\
\hline & 3.47 & -1.3 & -4 \\
\hline & & & 500 \\
\hline & -2.28 & -5.7 & 5700 \\
\hline & -7.65 & & \\
\hline 1 & & & \\
\hline & -9.13 & -5.6 & 7200 \\
\hline [ & -11.6 & & 4700 \\
\hline & & & \\
\hline & & & \\
\hline F & -5.70 & & \\
\hline & -4.54 & & \\
\hline & & & \\
\hline H & & & 900 \\
\hline & & & \\
\hline & 6.50 & & 1400 \\
\hline & & -7.6 & 4900 \\
\hline $\mathrm{H}$ & 10.3 & & 5700 \\
\hline $\mathrm{H}$ & & -5.7 & \\
\hline & & -4.39 & \\
\hline $\mathrm{H}$ & & & \\
\hline $\mathrm{H}$ & & -0.92822500 & 1.17062700 \\
\hline $\mathrm{C}$ & 0.93 & & \\
\hline $\mathrm{N}$ & 2.41861100 & -2.42897000 & 1.77281700 \\
\hline $\mathrm{O}$ & 9200 & 3.89264600 & \\
\hline $\mathrm{C}$ & & & \\
\hline $\mathrm{O}$ & 1.13303700 & & 2.96148200 \\
\hline $\mathrm{C}$ & & & \\
\hline $\mathrm{C}$ & 1.04691800 & & \\
\hline $\mathrm{C}$ & 1.13238600 & 3.11747000 & 1.66073700 \\
\hline $\mathrm{C}$ & & & 1.58755400 \\
\hline $\mathrm{C}$ & 1.00922900 & -0.53729400 & 2.64919700 \\
\hline
\end{tabular}

$\begin{array}{lrrr}\mathrm{C} & 2.34287300 & -0.97907000 & 2.04125900 \\ \mathrm{C} & 1.86680400 & -5.20977600 & 0.59372300 \\ \mathrm{C} & 2.69459800 & -6.31365500 & 0.35845900 \\ \mathrm{C} & 2.89957100 & -6.79662200 & -0.92992500 \\ \mathrm{C} & 2.27720300 & -6.16321800 & -2.00926800 \\ \mathrm{O} & 2.53948700 & -6.64594700 & -3.25763300 \\ \mathrm{C} & 1.43407200 & -5.06966200 & -1.78577600 \\ \mathrm{C} & 1.22684900 & -4.59888900 & -0.49215400 \\ \mathrm{H} & 3.25528800 & -2.67295200 & 1.15800300 \\ \mathrm{H} & -1.77251000 & -0.49557900 & 0.82436200 \\ \mathrm{H} & 2.09350300 & 3.41761500 & 0.07036500 \\ \mathrm{H} & 0.76438400 & 1.03995000 & 4.89680400 \\ \mathrm{H} & 1.34054600 & 5.42565800 & 2.06236000 \\ \mathrm{H} & 0.82413300 & 3.52654000 & 5.01095500 \\ \mathrm{H} & 1.14039600 & 1.26417500 & 0.61481700 \\ \mathrm{H} & 0.19798900 & -0.92454100 & 2.02116000 \\ \mathrm{H} & 0.87749200 & -0.97227000 & 3.64486400 \\ \mathrm{H} & 2.52424500 & -0.49013400 & 1.08092600 \\ \mathrm{H} & 3.19719700 & -0.71646600 & 2.67363800 \\ \mathrm{H} & 3.19528800 & -6.79761800 & 1.19155400 \\ \mathrm{H} & 3.55529000 & -7.63896100 & -1.11801000 \\ \mathrm{H} & 2.08595100 & -6.09819600 & -3.91201900 \\ \mathrm{H} & 0.93730600 & -4.58577900 & -2.62092400 \\ \mathrm{H} & 0.55219500 & -3.75984100 & -0.33521100 \\ \mathrm{C} & 1.59502700 & -4.75276200 & 2.00985100 \\ \mathrm{H} & 2.33833100 & -5.18164600 & 2.69554600 \\ \mathrm{H} & 0.62187000 & -5.12360000 & 2.35394600 \\ \mathrm{C} & 1.58527400 & -3.27744600 & 2.24657600 \\ \mathrm{H} & 0.79419700 & -2.89730200 & 2.88996300 \\ \mathrm{O} & -1.39192800 & -2.18659800 & 0.41640600 \\ \mathrm{H} & -1.53177700 & -2.73278900 & 1.20292600\end{array}$

\section{TS3' ( +22 kcal/mol)}

$\begin{array}{cccc}\text { C } & 7.00394000 & 5.72988100 & -0.68814400 \\ \text { C } & 7.42260600 & 4.74564900 & -1.73759600 \\ \text { O } & 6.70507000 & 3.82687200 & -2.11705600 \\ \text { N } & 8.66513800 & 4.96803600 & -2.27421400 \\ \text { C } & 9.10141000 & 4.19270900 & -3.39780900 \\ \text { C } & 10.17226400 & 3.13646700 & -3.13430500 \\ \text { O } & 10.66746100 & 2.52229000 & -4.07483900 \\ \text { N } & 10.55271400 & 2.95138400 & -1.83960000 \\ \text { C } & 11.39160900 & 1.80796300 & -1.52700500 \\ \text { C } & 10.52445000 & 0.55389200 & -1.42927800 \\ \text { O } & 9.61089800 & 0.53809600 & -0.59764800 \\ \text { N } & 10.78395000 & -0.51132400 & -2.22342500 \\ \text { C } & 9.85746900 & -1.66034000 & -2.16824900 \\ \text { C } & 9.79403300 & -2.34058400 & -0.80341200 \\ \text { O } & 8.79100900 & -2.96197300 & -0.46159300 \\ \text { C } & 10.37314300 & -2.58344700 & -3.28566500 \\ \text { C } & 10.95501400 & -1.59849800 & -4.30827500 \\ \text { C } & 11.63164800 & -0.53127300 & -3.43892800 \\ \text { N } & 10.90855000 & -2.24468400 & -0.03454300 \\ \text { C } & 10.93238600 & -2.75844900 & 1.32305700 \\ \text { C } & 9.97540300 & -2.07864100 & 2.31645800 \\ \text { O } & 9.77474200 & -2.60683100 & 3.40299600 \\ \text { N } & 9.37750900 & -0.93127800 & 1.91177100 \\ \text { C } & 8.26909000 & -0.37639400 & 2.67907200 \\ \text { C } & 7.13562400 & -1.39634300 & 2.85742100 \\ \text { O } & 6.47496300 & -1.40827800 & 3.88818000 \\ \text { C } & 7.76334300 & 0.89599500 & 1.97338400\end{array}$




\begin{tabular}{|c|c|c|c|}
\hline$\gamma$ & 6.50291800 & 1.54703800 & 56301800 \\
\hline & 6.65444800 & 1.91323200 & 4.04250100 \\
\hline & 6.14080400 & 2.76713900 & 1.70720000 \\
\hline & 6.86018800 & -2.19246700 & 1.77840800 \\
\hline & 5.90869000 & -3.29144700 & 1.91295500 \\
\hline 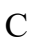 & 4.63940500 & -1.83340200 & 6.86230400 \\
\hline & 3.46366200 & -2.41927200 & 6.05451200 \\
\hline 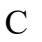 & 3.58230900 & -2.30518100 & 0300 \\
\hline $\mathrm{C}$ & 2.63665500 & -3.29400700 & 04300 \\
\hline 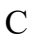 & 3.34689300 & -0.86788600 & 5200 \\
\hline $\mathrm{C}$ & 0.68011400 & -7.86141400 & -0.5556 \\
\hline 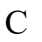 & 0.86742200 & -6.47050400 & 0000 \\
\hline$C$ & 1.78669100 & -5.53261700 & 520100 \\
\hline $\mathrm{C}$ & 1.11602500 & -5.07119800 & -2.03633200 \\
\hline $\mathrm{C}$ & 2.20081500 & -4.31700600 & 0.10265600 \\
\hline C & -7.41114600 & -8.29 & 8000 \\
\hline $\mathrm{C}$ & -8.00398900 & -6.89327300 & 5000 \\
\hline $\mathrm{C}$ & -7.18543700 & -5.73208600 & 9883700 \\
\hline $\mathrm{C}$ & -5.78611 & 000 & 300 \\
\hline $\mathrm{C}$ & -7.82816 & -4.60053200 & 2.2209 \\
\hline $\mathrm{C}$ & -5.05851 & -4.58 & 2.0536 \\
\hline $\mathrm{C}$ & -7.11898700 & -3.46955800 & 2.61840300 \\
\hline $\mathrm{C}$ & -5.72627500 & -3.44399000 & 2.51020700 \\
\hline $\mathrm{O}$ & -5.05911200 & -2.29 & 6100 \\
\hline $\mathrm{C}$ & -3.19134 & -5.2670 & -2.7611 \\
\hline $\mathrm{C}$ & -3.00626 & -4.12644300 & -1.7749 \\
\hline $\mathrm{C}$ & -4.40297 & -3.7 & -1.2 \\
\hline $\mathrm{C}$ & -4.49334 & 026000 & -0.3515 \\
\hline $\mathrm{O}$ & -5.6029 & -1.88 & -0.5 \\
\hline $\mathrm{O}$ & -3.60335 & -2.2721 & 5200 \\
\hline $\mathrm{C}$ & 2.89198 & -2.10858 & -6.5 \\
\hline $\mathrm{C}$ & 3.0574 & -1.8 & -5.10 \\
\hline $\mathrm{C}$ & 1.93895 & -1.89 & -4.2670 \\
\hline $\mathrm{C}$ & 4.30559 & -1.57 & -4.5 \\
\hline $\mathrm{C}$ & 2.0533 & -1.6 & -2.8 \\
\hline $\mathrm{C}$ & 4.42629400 & -1.35 & -3.1 \\
\hline $\mathrm{C}$ & 3.29897 & $-1.3 \xi$ & 400 \\
\hline $\mathrm{C}$ & 2.900872 & 3.66 & -5.1 \\
\hline $\mathrm{C}$ & 3.27685800 & 3.50 & -3.6 \\
\hline $\mathrm{C}$ & 3.7588 & 2.10 & -3.30 \\
\hline $\mathrm{N}$ & 4.07923100 & 2.05846500 & 1500 \\
\hline $\mathrm{C}$ & -2.02617 & & -5.4 \\
\hline $\mathrm{C}$ & -1.53247200 & 0.61 & -3.9 \\
\hline $\mathrm{C}$ & -1.63379500 & -0.68727900 & -3.17503500 \\
\hline $\mathrm{C}$ & -0.11140300 & 1.19416600 & 7900 \\
\hline $\mathrm{C}$ & -7.22647300 & -3.84509300 & -3.99952100 \\
\hline $\mathrm{C}$ & -8.24576000 & -3.35284600 & -2.9728 \\
\hline $\mathrm{C}$ & -8.55663200 & -4.40003500 & -1.90301500 \\
\hline S & -9.88981000 & -3.91653900 & -0.74332300 \\
\hline $\mathrm{C}$ & -9.18902 & & -0.088 \\
\hline $\mathrm{C}$ & -10.98499100 & -0.83504100 & -4.41151200 \\
\hline $\mathrm{C}$ & -10.26863500 & -0.14801100 & -3.27517100 \\
\hline $\mathrm{C}$ & -8.88777500 & 0.08023800 & -3.33592400 \\
\hline $\mathrm{C}$ & -10.94906200 & 0.28363000 & -2.13191900 \\
\hline $\mathrm{C}$ & -8.20599400 & 0.71775600 & -2.30334100 \\
\hline $\mathrm{C}$ & -10.28553900 & 0.93930500 & -1.09460900 \\
\hline $\mathrm{C}$ & -8.91161500 & 1.16772200 & -1.17867800 \\
\hline $\mathrm{O}$ & -8.27899300 & 1.81048700 & -0.14244700 \\
\hline $\mathrm{C}$ & -5.47514600 & 4.08097100 & -4.08962200 \\
\hline $\mathrm{C}$ & -4.73075000 & 3.62567600 & -2.82783400 \\
\hline $\mathrm{C}$ & -5.54165300 & 2.89782000 & -1.76927000 \\
\hline $\mathrm{O}$ & -6.60675100 & 3.42537300 & -1.33554100 \\
\hline $\mathrm{O}$ & -5.04985800 & 1.79664900 & -1.36446000 \\
\hline
\end{tabular}

\section{C}

$\mathrm{O}$

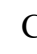

\section{.}

\section{$\mathrm{H}$
$\mathrm{H}$}$$
\mathrm{H}
$$$$
\mathrm{H}
$$

\section{H}

\section{$\mathrm{H}$}

\section{$\mathrm{H}$}

$\mathrm{H}$

H

$\begin{array}{rrr}-9.18462400 & 6.46855400 & -3.25390800 \\ -9.54343000 & 5.83058900 & -1.91257600\end{array}$ $\begin{array}{lll}-10.15066900 & 4.43854800 & -2.08549600\end{array}$ $\begin{array}{lll}-8.40025900 & 5.78140700 & -1.06194000\end{array}$ $\begin{array}{lll}-3.83528000 & 6.68403500 & 3.40740400\end{array}$ $\begin{array}{lll}-4.38502600 & 7.21896200 & 2.07947400\end{array}$ $\begin{array}{lll}-4.76364900 & 6.15291100 & 1.03823400\end{array}$ $\begin{array}{llll}-5.41514900 & 6.78821400 & -0.19436500\end{array}$ $\begin{array}{lll}-3.54861100 & 5.31646700 & 0.61678600\end{array}$ $\begin{array}{lll}1.54614800 & 5.05262700 & 4.08080600\end{array}$ $\begin{array}{lll}1.08890900 & 3.70604100 & 2.92361200\end{array}$ $\begin{array}{lll}2.35962600 & 2.46632200 & 3.35764700\end{array}$ $\begin{array}{lll}5.16298400 & -0.13752200 & 0.03817600\end{array}$ $\begin{array}{lll}2.53101500 & 0.11428800 & 0.89868700\end{array}$ $\begin{array}{lll}-6.01895000 & 0.39329900 & 0.52216900\end{array}$ $\begin{array}{llll}4.83243200 & 2.72099800 & -1.68026300\end{array}$ $\begin{array}{llll}4.44455900 & 1.14492400 & -1.59496900\end{array}$ $\begin{array}{llll}4.62293300 & 1.82860800 & -3.93055100\end{array}$ $\begin{array}{llll}2.97816400 & 1.36146000 & -3.50439500\end{array}$ $\begin{array}{llll}4.07371000 & 4.21628000 & -3.43030600\end{array}$ $\begin{array}{lll}2.41824000 & 3.75464200 & -3.04845700\end{array}$ $\begin{array}{lll}2.01447500 & 3.07740900 & -5.41809500\end{array}$ $\begin{array}{llll}3.71850100 & 3.34305400 & -5.81542400\end{array}$ $\begin{array}{lll}5.18791100 & -1.53782400 & -5.16712800\end{array}$ $\begin{array}{llll}5.39347700 & -1.13108100 & -2.71870600\end{array}$ $\begin{array}{llll}3.39925600 & -1.18283300 & -1.27760400\end{array}$ $\begin{array}{lll}1.16945300 & -1.68764600 & -2.26742600\end{array}$ $\begin{array}{lll}0.96414000 & -2.11413600 & -4.69535800\end{array}$ $\begin{array}{lll}0.60098300 & 0.51294800 & -4.41669300\end{array}$ $\begin{array}{lll}-0.06835200 & 2.15064400 & -4.47277900\end{array}$ $\begin{array}{llll}0.23488100 & 1.36757700 & -2.91524600\end{array}$ $\begin{array}{llll}-2.20436700 & 1.34352300 & -3.49742200\end{array}$ $\begin{array}{lll}-1.25206400 & -0.55232000 & -2.15664100\end{array}$ $\begin{array}{lll}-2.67244800 & -1.02796400 & -3.10559900\end{array}$ $\begin{array}{lll}-1.04670000 & -1.48625200 & -3.64190300\end{array}$ $\begin{array}{llll}-7.84951600 & -2.45803400 & -2.48556300\end{array}$ $\begin{array}{lll}-9.17544800 & -3.05180700 & -3.46425400\end{array}$ $\begin{array}{lll}-7.65617000 & -4.63115300 & -1.32098500\end{array}$ $\begin{array}{lll}-8.90435000 & -5.33397300 & -2.35817400\end{array}$ $\begin{array}{llll}-8.17082100 & -2.52520900 & 0.26917400\end{array}$ $\begin{array}{lll}-9.20916300 & -1.57275700 & -0.83829300\end{array}$ $\begin{array}{lll}-9.81807300 & -2.06168800 & 0.75192300\end{array}$ $\begin{array}{lll}-7.61257100 & 2.45640900 & -0.53968900\end{array}$ $\begin{array}{llll}-7.13191700 & 0.85207600 & -2.35395400\end{array}$ $\begin{array}{llll}-10.81999200 & 1.28642600 & -0.21678800\end{array}$ $\begin{array}{lll}-12.01900100 & 0.11148700 & -2.05292200\end{array}$ $\begin{array}{lll}-8.33044000 & -0.25826200 & -4.20612200\end{array}$ $\begin{array}{lll}-4.83267600 & -4.59423400 & -0.68997200\end{array}$ $\begin{array}{lll}-5.07284100 & -3.57678500 & -2.09519700\end{array}$ $\begin{array}{lll}-2.32839700 & -4.40985100 & -0.96608000\end{array}$ $\begin{array}{lll}-2.55908500 & -3.25999700 & -2.27309300\end{array}$ $\begin{array}{lll}-8.14627300 & -6.74855900 & 0.08247500\end{array}$ $\begin{array}{lll}-9.00936700 & -6.83849100 & 1.59275500\end{array}$ $\begin{array}{lll}-8.91200900 & -4.59251500 & 2.27736600\end{array}$ $\begin{array}{lll}-5.24038000 & -6.56888500 & 1.27589100\end{array}$ $\begin{array}{lll}-3.97338000 & -4.58995400 & 2.01053700\end{array}$ $\begin{array}{lll}-7.63140600 & -2.58768500 & 2.98781600\end{array}$ $\begin{array}{lll}-4.26809800 & -2.24194400 & 2.29126100\end{array}$ $\begin{array}{lll}2.84730200 & -3.64221400 & -0.46756700\end{array}$ $\begin{array}{lll}1.31702400 & -3.75002700 & 0.41725400\end{array}$ $\begin{array}{lll}2.73643500 & -4.62188500 & 1.00704600\end{array}$

$\begin{array}{llll}\mathrm{H} & 1.78917100 & -4.44627700 & -2.62935400\end{array}$ $\begin{array}{llll}\mathrm{H} & 0.80046400 & -5.91187300 & -2.66231700\end{array}$ 


\begin{tabular}{|c|c|c|c|}
\hline & 0.22404700 & -4.47342100 & \\
\hline & 2.69762600 & -6.09159000 & \\
\hline & -0.10326000 & -5.97135200 & \\
\hline & 1.26729600 & -6.58180200 & 1.08715500 \\
\hline & 7.59470900 & -2.32306800 & \\
\hline & 8.60548000 & -0.15580200 & 3.69446300 \\
\hline & 8.58628800 & & \\
\hline & 7.56232000 & 0.65307800 & 0.92608000 \\
\hline & & & \\
\hline & 5.99297400 & 2.47371200 & \\
\hline & 5.21798300 & 3.23876600 & \\
\hline & 7.51367300 & 2.57816000 & \\
\hline & 5.76130200 & 49300 & \\
\hline & 6.78515500 & & \\
\hline & 5.67951300 & & \\
\hline & 9.545 & -0.54 & \\
\hline & 10.64 & & \\
\hline & & & \\
\hline & 11.65096300 & -1.64316900 & \\
\hline & 12.6 & & \\
\hline & & & \\
\hline & 11.16 & & \\
\hline & 10.1 & & \\
\hline & 11.6 & & \\
\hline & & & \\
\hline & & & \\
\hline & 9.9 & & \\
\hline & 9.18 & & \\
\hline & -4.30 & & \\
\hline & -3.8 & & \\
\hline & -9.4 & & \\
\hline & -10.3 & & \\
\hline & 9700 & & \\
\hline & -10 & & \\
\hline & -2.9 & & 200 \\
\hline & -5.26 & & 2.277 \\
\hline & & & \\
\hline & -4.7 & & \\
\hline & -5.6 & & -0.9 \\
\hline & -6.3 & & \\
\hline & & & \\
\hline & -3.8 & & \\
\hline & & & \\
\hline 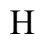 & & & \\
\hline & & & \\
\hline & & & \\
\hline$H$ & & & \\
\hline & & & \\
\hline & & & \\
\hline & & & \\
\hline $\mathrm{H}$ & & & \\
\hline & & & \\
\hline & & & \\
\hline & & & \\
\hline $\mathrm{H}$ & 2.56 & & \\
\hline & & & \\
\hline & & & \\
\hline $\mathrm{H}$ & 2.51963900 & -1.95331 & 6.37658300 \\
\hline & & & \\
\hline & & & \\
\hline & & & \\
\hline 11 & 2.64510600 & 2.24823100 & -1.03912800 \\
\hline & -5.71630800 & -1.03628400 & 0.0513540 \\
\hline
\end{tabular}

$\mathrm{H}$

H

\section{$\mathrm{H}$}

\section{$\mathrm{C}$}

\section{$\mathrm{N}$}

O

C

\section{$\mathrm{O}$}

C

\section{C}

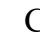

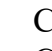

c

(

\section{(5)}

8.22793600 6.35735700 $-6.25200200$ $-8.40774400$ $-7.83188300$ 4.77186400 12.17633500 $.7440700-2.27823000$ $\begin{array}{llll}1.50683500 & -8.53606100 & -0.31519100\end{array}$ $\begin{array}{llll}3.34685000 & -3.05733700 & -6.89869400\end{array}$ $3.34235100 \quad-1.31334600 \quad-7.19561700$ $\begin{array}{llll}-1.42118700 & -0.28777700 & -5.98285000\end{array}$ $\begin{array}{llll}-6.37868600 & -4.32193300 & -3.49386500\end{array}$ $\begin{array}{lll}-8.04222600 & -9.05582100 & 0.94157200\end{array}$ $\begin{array}{lll}-4.07224100 & -5.05160700 & -3.38119300\end{array}$ $\begin{array}{lll}-12.06038700 & -0.89492700 & -4.22450800\end{array}$ $\begin{array}{lll}-10.61916500 & -1.85694400 & -4.56076300\end{array}$ $\begin{array}{llll}-6.96549000 & 0.65152300 & 0.54915500\end{array}$ $\begin{array}{llll}-4.69266500 & 4.55543700 & -4.69014900\end{array}$ $\begin{array}{llll}-5.92421500 & 3.25786800 & -4.65474200\end{array}$ $-2.41564300 \quad-5.48449200 \quad-3.50510400$ $\begin{array}{lll}-3.40391500 & -6.24210500 & -2.30979300\end{array}$ $\begin{array}{llll}-6.80963600 & -3.02118300 & -4.58843500\end{array}$ $\begin{array}{llll}-7.61277400 & -4.58966900 & -4.70274200\end{array}$ $\begin{array}{lll}-10.83718200 & -0.29855500 & -5.35386400\end{array}$ $\begin{array}{llll}-2.02311500 & 1.39713600 & -5.93674200\end{array}$ $\begin{array}{llll}-3.07828200 & 0.12568000 & -5.43833900\end{array}$ $\begin{array}{llll}1.82064800 & -2.15173600 & -6.81476700\end{array}$ $\begin{array}{llll}2.69710800 & 4.71861400 & -5.37864200\end{array}$ $\begin{array}{llll}7.82795400 & 6.20328000 & -0.14457100\end{array}$ $\begin{array}{llll}6.41275000 & 6.52102200 & -1.16287600\end{array}$ $\begin{array}{llll}9.50503000 & 4.77798000 & -4.23146700\end{array}$ $\begin{array}{llll}11.84838100 & 1.96241700 & -0.54598700\end{array}$ $\begin{array}{llll}6.15444300 & -3.95333000 & 2.75097500\end{array}$ $\begin{array}{llll}5.92133900 & -3.86526000 & 0.98501300\end{array}$ $\begin{array}{llll}5.55595100 & -2.34357500 & 6.54796900\end{array}$ $\begin{array}{llll}4.33085000 & -1.97231700 & 7.90517200\end{array}$ $-0.26082300 \quad-8.31906800-0.23331200$ $\begin{array}{llll}0.61134700 & -7.79133400 & -1.64647600\end{array}$ $\begin{array}{lll}-7.35596000 & -8.50198200 & 2.47624500\end{array}$ $\begin{array}{lll}-6.42150000 & -8.35912100 & 0.94232300\end{array}$ $\begin{array}{lll}-8.78691400 & 7.48542600 & -3.16634000\end{array}$ $\begin{array}{llll}-10.00173000 & 6.55492100 & -3.97812200\end{array}$ $\begin{array}{lll}-3.48696300 & 7.52007500 & 4.02037500\end{array}$ $\begin{array}{lll}-4.58004900 & 6.08879500 & 3.95407700\end{array}$ $\begin{array}{ccc}2.56128100 & 5.40442200 & 3.88172300\end{array}$ $\begin{array}{llll}-5.67908400 & 0.95714400 & -0.26278400\end{array}$ $\begin{array}{lll}-0.66947900 & -0.14593600 & 1.68094900\end{array}$ $\begin{array}{lll}-3.47998800 & 1.86538600 & 0.90923600\end{array}$ $\begin{array}{lll}-1.36611700 & -3.74476500 & 1.30389400\end{array}$ $\begin{array}{lll}-0.40826900 & -0.11574700 & 3.05618900\end{array}$ $\begin{array}{lll}-0.78411900 & -3.64065200 & 3.95403800\end{array}$ $\begin{array}{lll}-0.37871900 & -1.28833900 & 3.81327400\end{array}$ $\begin{array}{lll}-0.68653200 & -2.50723400 & 3.21329100\end{array}$ $\begin{array}{lll}-0.92931400 & -2.54721500 & 1.83209700\end{array}$ $\begin{array}{lll}-0.85980600 & -1.39205900 & 1.06864900\end{array}$ $\begin{array}{lll}-1.09111500 & 1.10779700 & 0.94469800\end{array}$ $\begin{array}{lll}-2.52955100 & 0.88542200 & 0.38524300\end{array}$ $\begin{array}{lll}-4.54760900 & 3.09726200 & 3.75716600\end{array}$ $\begin{array}{llll}-5.35580100 & 3.41055600 & 4.85795400\end{array}$ $\begin{array}{lll}-4.80376500 & 3.86377100 & 6.04948300\end{array}$ $\begin{array}{lll}-3.41450000 & 3.99239400 & 6.16986100\end{array}$ O $\quad-2.92767100 \quad 4.43198100 \quad 7.36373400$ 


$\begin{array}{rrrr}\mathrm{C} & -2.59269400 & 3.66674600 & 5.08848300 \\ \mathrm{C} & -3.16135400 & 3.22720400 & 3.89603600 \\ \mathrm{H} & -3.52486400 & 2.77629900 & 0.46188600 \\ \mathrm{H} & 2.14953900 & 0.84684200 & 0.34920200 \\ \mathrm{H} & -2.17874600 & -3.50553600 & 0.80915300 \\ \mathrm{H} & -0.26960400 & 0.84174300 & 3.54957500 \\ \mathrm{H} & -1.03808700 & -4.35026800 & 3.34115300 \\ \mathrm{H} & -0.19301300 & -1.26356300 & 4.88097700 \\ \mathrm{H} & -1.10667500 & -1.46082700 & 0.01456700 \\ \mathrm{H} & -0.41125200 & 1.35206500 & 0.12147200 \\ \mathrm{H} & -1.06855500 & 1.96246200 & 1.62533300 \\ \mathrm{H} & -2.57384200 & 0.97966600 & -0.70146400 \\ \mathrm{H} & -2.91439400 & -0.09905700 & 0.64470700 \\ \mathrm{H} & -6.43382900 & 3.30671300 & 4.77706400 \\ \mathrm{H} & -5.42889500 & 4.11410600 & 6.89917000 \\ \mathrm{H} & -1.96271000 & 4.45894800 & 7.32291200 \\ \mathrm{H} & -1.51139600 & 3.75411700 & 5.16324700 \\ \mathrm{H} & -2.49445200 & 2.98831100 & 3.07600500 \\ \mathrm{C} & -5.18857200 & 2.63662200 & 2.45231700 \\ \mathrm{H} & -5.33593400 & 3.46317100 & 1.74999900 \\ \mathrm{H} & -6.16861400 & 2.19821600 & 2.65014500 \\ \mathrm{C} & -4.32941400 & 1.59502500 & 1.84014300 \\ \mathrm{H} & -4.29356900 & 0.60368900 & 2.27817500 \\ \mathrm{O} & 1.73969600 & 2.21813500 & -0.56567000 \\ \mathrm{H} & 1.72914000 & 2.97255900 & 0.04042700\end{array}$

\section{Int4' (+3.0 kcal/mol)}

$\begin{array}{cccc}\mathrm{C} & 7.13280400 & 5.78228400 & 0.45905000 \\ \mathrm{C} & 7.56705500 & 4.98610400 & -0.73443600 \\ \mathrm{O} & 6.86796200 & 4.12560900 & -1.25777700 \\ \mathrm{~N} & 8.80223400 & 5.31317300 & -1.23182500 \\ \mathrm{C} & 9.23595300 & 4.74212600 & -2.47358800 \\ \mathrm{C} & 10.30465600 & 3.65343200 & -2.40009200 \\ \mathrm{O} & 10.78528100 & 3.20180100 & -3.43548700 \\ \mathrm{~N} & 10.70095300 & 3.25542600 & -1.15942300 \\ \mathrm{C} & 11.54531400 & 2.07810100 & -1.05496000 \\ \mathrm{C} & 10.68564800 & 0.82175900 & -1.18678900 \\ \mathrm{O} & 9.76495700 & 0.65686000 & -0.37981700 \\ \mathrm{~N} & 10.95738200 & -0.08613100 & -2.15371000 \\ \mathrm{C} & 10.03201600 & -1.22650200 & -2.31570600 \\ \mathrm{C} & 9.95074600 & -2.13884100 & -1.09527000 \\ \mathrm{O} & 8.94216200 & -2.80610300 & -0.87975600 \\ \mathrm{C} & 10.56558900 & -1.93670500 & -3.57154200 \\ \mathrm{C} & 11.15364300 & -0.78489000 & -4.39709600 \\ \mathrm{C} & 11.81744700 & 0.11136800 & -3.34447200 \\ \mathrm{~N} & 11.05788500 & -2.18769700 & -0.31088600 \\ \mathrm{C} & 11.06773300 & -2.93776700 & 0.93205600 \\ \mathrm{C} & 10.10662500 & -2.44131800 & 2.02468800 \\ \mathrm{O} & 9.89883700 & -3.15108800 & 3.00096300 \\ \mathrm{~N} & 9.51117900 & -1.24049300 & 1.82295300 \\ \mathrm{C} & 8.40091900 & -0.82120000 & 2.66867100 \\ \mathrm{C} & 7.26207000 & -1.85001300 & 2.66945400 \\ \mathrm{O} & 6.58882500 & -2.01974200 & 3.67811300 \\ \mathrm{C} & 7.90673500 & 0.55352900 & 2.18120500 \\ \mathrm{C} & 6.64433000 & 1.10807700 & 2.85768000 \\ \mathrm{C} & 6.78251200 & 1.22459800 & 4.37859900 \\ \mathrm{C} & 6.30382700 & 2.45641500 & 2.21058600 \\ \mathrm{~N} & 6.99163300 & -2.46414900 & 1.47595200 \\ \mathrm{C} & 6.02096000 & -3.55443700 & 1.43095400 \\ \mathrm{C} & 4.73920800 & -2.98789900 & 6.55650800\end{array}$

\begin{tabular}{|c|c|c|c|}
\hline & 3.56123400 & -3.38477800 & \\
\hline & 3.66863900 & -2.91672700 & 4.19091800 \\
\hline & 2.68287300 & -3.67947000 & 3.29966100 \\
\hline & 3.47577200 & -1.40272000 & 4.07776200 \\
\hline & 0.79446300 & -7.61135200 & -1.81583000 \\
\hline & 0.95071100 & -6.35037800 & -0.94912000 \\
\hline & 1.86474200 & -5.26254200 & -1.54291200 \\
\hline & 1.23529700 & -4.60786300 & -2.78008700 \\
\hline & 2.18593000 & -4.19630300 & -0.48793600 \\
\hline & -7.30290800 & -8.36526600 & 0.01768600 \\
\hline & -7.90979900 & -6.95314100 & 0.00575600 \\
\hline & -7.10337200 & -5.88139200 & 0.71575800 \\
\hline & -5.70973400 & -5.78993000 & 0.60697900 \\
\hline $\mathrm{C}$ & -7.75482100 & -4.89271500 & 1.46601400 \\
\hline & -4.99696100 & -4.74007900 & 87500 \\
\hline & -7.06172600 & -3.82733900 & 2.03455900 \\
\hline & -5.67727100 & -3.72784500 & 93900 \\
\hline & -5.03501900 & -2.64168200 & 2.41093200 \\
\hline & -3.06822700 & -4.66348100 & -3.54023200 \\
\hline 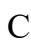 & -2.86949400 & -3.70521400 & 1800 \\
\hline & -4.26295400 & -3.27820800 & -1.89544100 \\
\hline & -4.31769300 & -2.27973600 & -0.78041000 \\
\hline & -5.37014300 & -1.50747000 & -0.82478000 \\
\hline & -3.46033800 & -2.21050500 & 0.11493700 \\
\hline & 3.02868300 & -0.88961900 & 0300 \\
\hline & 3.18783300 & -0.91300500 & -5.23610800 \\
\hline & 2.06102800 & 402800 & 4100 \\
\hline & 4.43761 & -0.77796900 & 8000 \\
\hline & 2.16882400 & -1.08749500 & 05900 \\
\hline & 4.55177000 & -0.80199900 & 88600 \\
\hline 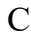 & 3.41588600 & -0.95224700 & -2.42382500 \\
\hline & 3.03986000 & 4.54266700 & 0700 \\
\hline & 3.48081100 & 29800 & 7900 \\
\hline & 3.97049000 & 2.66654100 & 8000 \\
\hline & & 2.34290000 & -1.44 \\
\hline & -1.88959400 & 1.41184100 & 2500 \\
\hline & -1.39907400 & 1.34435300 & -3.69951700 \\
\hline & -1.51459300 & -0.07488300 & 24900 \\
\hline & 0.02498200 & 1.90033900 & 0000 \\
\hline & -7.09865000 & -3.03961800 & -4.51959000 \\
\hline & -8.11539200 & -2.70232700 & 70500 \\
\hline & -8.43168100 & -3.87939000 & -2.50478900 \\
\hline & -9.71905100 & -3.51800600 & -1.250 \\
\hline & -8.94464600 & -2.10129900 & -0.39312900 \\
\hline & -10.85285400 & 0.00187900 & -4.40538200 \\
\hline & -10.18977500 & 0.51042200 & -3.15266000 \\
\hline & -8.81255300 & 0.76327500 & -3.12232200 \\
\hline & -10.92587200 & 0.76598600 & -1.99217500 \\
\hline & -8.19008000 & 1.27894700 & -1.98951900 \\
\hline & -10.32487500 & 1.30244600 & -0.85571600 \\
\hline & -8.95792700 & 1.58346800 & -0.85788700 \\
\hline & -8.40168400 & 2.15372800 & 0.25212900 \\
\hline & -5.33850100 & 4.77623200 & -3.21046300 \\
\hline & -4.64057400 & 4.10859400 & -2.02163900 \\
\hline & -5.50449600 & 3.30159200 & -1.06475600 \\
\hline & -6.62987500 & 3.75477000 & -0.70825400 \\
\hline & -4.99078600 & 2.21360400 & -0.66246500 \\
\hline & -9.04793100 & 6.98535600 & -1.97752200 \\
\hline & -9.45786000 & 6.16085100 & -0.75752900 \\
\hline & -10.11603700 & 4.84192000 & -1.16061200 \\
\hline & -8.34146700 & 5.93420700 & 0.09899400 \\
\hline & -3.72172200 & 6.01910900 & 4.63179700 \\
\hline & -4.24437600 & 6.74332600 & 3.38591300 \\
\hline
\end{tabular}




\begin{tabular}{|c|c|c|c|}
\hline $\mathrm{C}$ & -4.71609800 & & \\
\hline $\mathrm{C}$ & & & 1.06976600 \\
\hline $\mathrm{C}$ & -3.61275200 & 4.88070100 & 1.75112600 \\
\hline $\mathrm{C}$ & 1.66285700 & & 5.02094700 \\
\hline S & 1.40682800 & & \\
\hline $\mathrm{C}$ & 2.63553800 & .96346300 & 3.79458900 \\
\hline $\mathrm{O}$ & 5.37735700 & -0.16809800 & 8300 \\
\hline 0 & 2.76748100 & 6500 & 8800 \\
\hline $\mathrm{O}$ & -5.38793200 & & 5700 \\
\hline $\mathrm{H}$ & 5.11381700 & 2.9515 & 10900 \\
\hline$\pi$ & 4.70056900 & & 3500 \\
\hline $\mathrm{H}$ & & & 5000 \\
\hline $\mathrm{H}$ & 3.1765 & & 400 \\
\hline $\mathrm{H}$ & 4.2915 & & 200 \\
\hline $\mathrm{H}$ & 2.6526 & & -2.2 \\
\hline $\mathrm{H}$ & 2.1369 & & -4.6 \\
\hline $\mathrm{H}$ & 3.8225 & & -5.0 \\
\hline & 5.3267 & 00 & 300 \\
\hline & 5.519 & & \\
\hline $\mathrm{H}$ & & & \\
\hline & $1.2^{\prime}$ & & 900 \\
\hline $\mathrm{H}$ & & & 900 \\
\hline $\mathrm{H}$ & 0.734 & & 700 \\
\hline F & 0.068 & & -3.9 \\
\hline $\mathrm{H}$ & 0.37 & & 700 \\
\hline & -2.065 & & 400 \\
\hline $\mathrm{H}$ & -1.15 & & \\
\hline $\mathrm{H}$ & -2.5 & & 200 \\
\hline $\mathrm{H}$ & -0.91 & -0.7 & 300 \\
\hline $\mathrm{H}$ & -7.71 & & 900 \\
\hline & -9.0 & & 200 \\
\hline F & -7.5 & -4.2 & 300 \\
\hline $\mathrm{H}$ & -8.8 & -4.7 & 800 \\
\hline & -7.92 & -2.3 & 700 \\
\hline F & -8.94 & & \\
\hline & -9.5 & -1.5 & \\
\hline & -7.6 & & 800 \\
\hline $\mathrm{F}$ & -7.1 & 1.4 & 200 \\
\hline & -10.9 & & 1800 \\
\hline $\mathrm{F}$ & -11.9 & & \\
\hline & -8.2 & & 900 \\
\hline & -4.82505800 & -4.1 & 400 \\
\hline $\mathrm{H}$ & -4.8 & & \\
\hline F & -2.2918 & -4.1 & -1. \\
\hline & -2.3086 & -2.82 & 200 \\
\hline $\mathrm{H}$ & -8.057 & -6.6 & 900 \\
\hline & -8.9135 & -6.97 & 3100 \\
\hline $\mathrm{H}$ & -8.8339 & & \\
\hline $\mathrm{H}$ & -5.1573 & -6.5 & 9700 \\
\hline & -3.91528500 & -4.65 & 0300 \\
\hline & & & \\
\hline & -4.27466500 & -2.42401100 & 057900 \\
\hline & & & 8900 \\
\hline & 1.261 & -3.73 & 584000 \\
\hline & 2.70585500 & -4.63 & 40200 \\
\hline $\mathrm{H}$ & 1.906627 & & 16300 \\
\hline & 0.98973400 & -5.33882600 & 664200 \\
\hline & & -4.09 & 6300 \\
\hline & 2.80886400 & -5.73769600 & -1.85002200 \\
\hline & -0.03403800 & -5.89651500 & -0.77049300 \\
\hline & 1.32714700 & -6.63617600 & 0.04118900 \\
\hline & 7.73171000 & -2.49361500 & 0.77974400 \\
\hline & 8.73186100 & -0.77445900 & 3.70851000 \\
\hline
\end{tabular}

$\mathrm{H}$

$\mathrm{H}$

$\mathrm{H}$

$\mathrm{H}$

$\mathrm{H}$

$\mathrm{H}$

$\mathrm{H}$

$\mathrm{H}$

$\mathrm{H}$

$\mathrm{H}$

$\mathrm{H}$

$\mathrm{H}$

$\mathrm{H}$

$\mathrm{H}$

$\mathrm{H}$

$\mathrm{H}$

$\mathrm{H}$

$\mathrm{H}$

$\mathrm{H}$

$\mathrm{H}$

$\mathrm{H}$

$\mathrm{H}$ $\begin{array}{lll}8.73375800 & 1.26511100 & 2.30903400\end{array}$

$\begin{array}{lll}7.71477700 & 0.49028800 & 1.10672600\end{array}$

$\begin{array}{lll}7.10538400 & 3.18532200 & 2.39197700\end{array}$

$\begin{array}{lll}6.17368600 & 2.34391900 & 1.13092800\end{array}$

$\begin{array}{llll}5.37841900 & 2.87319600 & 2.62209000\end{array}$

$\begin{array}{lll}7.64530800 & 1.84649900 & 4.65083200\end{array}$

$\begin{array}{llll}5.89044800 & 1.68876700 & 4.81401200\end{array}$

$\begin{array}{llll}6.89996700 & 0.23990300 & 4.83615500\end{array}$

$\begin{array}{llll}5.81738900 & 0.42409800 & 2.64026000\end{array}$

$\begin{array}{llll}9.68206500 & -0.70241400 & 0.98002100\end{array}$

$\begin{array}{llll}10.77942800 & -3.97382200 & 0.74089300\end{array}$

$\begin{array}{llll}12.08383200 & -2.94376700 & 1.33247000\end{array}$

$\begin{array}{llll}11.80439500 & -1.53983100 & -0.51069000\end{array}$

$12.84028200-0.22136500 \quad-3.12955400$

$\begin{array}{llll}11.85999900 & -1.11868000 & -5.16032600\end{array}$

$\begin{array}{llll}11.35235300 & -2.64508200 & -3.28862600\end{array}$

$10.35201600 \quad-0.22929400 \quad-4.89424200$

$\begin{array}{llll}11.82367900 & 1.16289600 & -3.63248200\end{array}$

$\begin{array}{llll}9.77768000 & -2.49238000 & -4.08160200\end{array}$

$\begin{array}{llll}9.01507800 & -0.85175400 & -2.46704500\end{array}$

$\begin{array}{llll}10.07440900 & 3.44803600 & -0.38945800\end{array}$

$\begin{array}{llll}9.31061600 & 6.08340400 & -0.82514800\end{array}$

$\begin{array}{llll}-4.16466600 & 4.89236200 & -1.41669900\end{array}$

$\begin{array}{llll}-3.83796600 & 3.45106000 & -2.36745100\end{array}$

$\begin{array}{llll}-9.44332600 & 4.25397700 & -1.79432300\end{array}$

$\begin{array}{llll}-10.33864700 & 4.24539900 & -0.27322000\end{array}$

$\begin{array}{llll}-11.04388300 & 5.00907400 & -1.71794600\end{array}$

$\begin{array}{llll}-10.16604700 & 6.74339200 & -0.15397500\end{array}$

$\begin{array}{lll}-2.84873300 & 5.40477900 & 4.37853800\end{array}$

$\begin{array}{lll}-5.07718100 & 7.39911500 & 3.66813500\end{array}$

$\begin{array}{lll}-3.45427300 & 7.40497000 & 3.00299600\end{array}$

$\begin{array}{lll}-4.48033000 & 7.31460900 & 0.66910400\end{array}$

$\begin{array}{lll}-5.61138900 & 6.00559700 & 0.26527800\end{array}$

$\begin{array}{lll}-6.11212100 & 7.26928200 & 1.37115800\end{array}$

$\begin{array}{lll}-3.17533000 & 4.29434100 & 2.56573300\end{array}$

$\begin{array}{lll}-4.00605200 & 4.17448800 & 1.01502700\end{array}$

$\begin{array}{lll}-2.79779600 & 5.44693100 & 1.28369800\end{array}$

$\begin{array}{lll}-5.54813300 & 5.22084100 & 2.62193900\end{array}$

$\begin{array}{llll}0.96361500 & 5.12252900 & 4.98271700\end{array}$

$\begin{array}{lll}1.46292300 & 3.68987100 & 5.91509400\end{array}$

$\begin{array}{llll}2.64375100 & 1.31673500 & 2.91438300\end{array}$

$\begin{array}{lll}3.62984400 & 2.39538200 & 3.92537700\end{array}$

$\begin{array}{lll}2.37611200 & 1.36911200 & 4.67272000\end{array}$

$\begin{array}{llll}4.21859200 & -0.86392400 & 4.67213000\end{array}$

$\begin{array}{llll}3.57423200 & -1.06190100 & 3.04427000\end{array}$

$\begin{array}{llll}2.47671500 & -1.11219900 & 4.42364600\end{array}$

$\begin{array}{llll}2.97095100 & -4.73372400 & 3.20967800\end{array}$

$\begin{array}{llll}1.66794000 & -3.65660600 & 3.70405400\end{array}$

$\begin{array}{lll}2.64569100 & -3.25831100 & 2.28889700\end{array}$

$\begin{array}{lll}4.68437100 & -3.13141100 & 3.85462800\end{array}$

$\begin{array}{llll}3.47708700 & -4.48042400 & 5.66161900\end{array}$

$\begin{array}{llll}2.61811500 & -3.01019400 & 6.07208500\end{array}$

$\begin{array}{llll}4.50245600 & -0.20100800 & 0.49032200\end{array}$

$\begin{array}{lll}5.87010200 & -0.92612000 & 0.40577200\end{array}$

$\begin{array}{llll}2.00781700 & -0.57824800 & 1.15355500\end{array}$

$\begin{array}{llll}2.96971300 & 2.41231000 & -0.56598000\end{array}$

$\begin{array}{llll}-5.37296200 & -0.81549700 & -0.06588000\end{array}$

$\begin{array}{llll}8.36223700 & 4.28066300 & -2.94160600\end{array}$

$\begin{array}{llll}6.47628200 & 5.16598700 & 1.07397500\end{array}$

$\begin{array}{lll}-6.11780800 & 5.46362700 & -2.87284200\end{array}$

$\begin{array}{llll}-8.27303900 & 6.45024400 & -2.54004800\end{array}$

$\begin{array}{llll}-7.75374700 & 5.28839800 & -0.33310000\end{array}$

$\begin{array}{llll}4.88504600 & -1.90325100 & 6.57800500\end{array}$ 


\begin{tabular}{|c|c|c|c|}
\hline & 33170300 & 15067200 & \\
\hline & 5.02296500 & & \\
\hline & & & -1.68801400 \\
\hline & 3.48607100 & -1.76509300 & -7.21610800 \\
\hline & 3.47948700 & 0.00358000 & -7.18663700 \\
\hline & -1.28386000 & 0.80172400 & -5.83230900 \\
\hline & -6.25608300 & -3.59875700 & -4.09723400 \\
\hline & -7.92635200 & -9.04627900 & -0.57076800 \\
\hline & -3.94612200 & -4.34082100 & -4.11611500 \\
\hline & -11.92966100 & -0.12109400 & -4.262 \\
\hline & -10.44926300 & -0.96788700 & -4.71674000 \\
\hline & & 0.330 & \\
\hline & -4.55 & & -3.7 \\
\hline & -5.78 & 4.0 & -3.91 \\
\hline & -2.290 & -4.74 & 9100 \\
\hline & -3.283 & -5.70 & 8500 \\
\hline & -6.679 & -2.1 & -4.953 \\
\hline & -7.48 & -3.6 & \\
\hline & -10.70 & 600 & 5400 \\
\hline & -1.884 & & \\
\hline & -2.94 & & \\
\hline & 1.95 & -0.8 & 7000 \\
\hline & & & \\
\hline & 7.95 & & \\
\hline & 6.55 & & \\
\hline & 9.6 & & \\
\hline & 11.99 & & 0900 \\
\hline & & & \\
\hline & 6.0 & -3.9 & \\
\hline & 5.65 & -3.4 & \\
\hline & 4.42 & -3.3 & \\
\hline & -0.14 & -8.1 & \\
\hline & 0.728 & -7.3 & -2.8 \\
\hline & -7.25 & -8.7 & \\
\hline & -6.31 & -8.3 & 6800 \\
\hline & -8.64 & & \\
\hline & -9.86 & & -2 \\
\hline & -3.36 & & \\
\hline & -4.46 & & \\
\hline & & & \\
\hline & -5.45 & & \\
\hline & $-0.4 \mathrm{~S}$ & -0.1 & \\
\hline & -3.053 & & \\
\hline & -1.3 & & \\
\hline & -0.176 & -0.2 & \\
\hline & -0.786 & -3.8 & \\
\hline & -0.24 & -1.4 & 4900 \\
\hline & -0.654 & -2.6 & \\
\hline & -0.9 & -2.4 & \\
\hline & -0.838 & -1.2 & 300 \\
\hline & -0.69064100 & & \\
\hline & & & \\
\hline & -5.062 & 1.29294500 & \\
\hline & -5.73 & & \\
\hline & -7.057 & -0.03262200 & 3.61927800 \\
\hline & -7.713 & 1.07809700 & \\
\hline c & -8.9578 & 246700 & 2400 \\
\hline & -7.04723100 & 2.31790700 & 5700 \\
\hline & -5.7420 & & \\
\hline & -4.01321600 & 0.95812000 & 1.70671900 \\
\hline & 2.41743600 & 0.83230800 & 0.59605100 \\
\hline & -2.12977900 & -3.32342400 & 0.42715400 \\
\hline & 0.12586600 & 0.63743100 & 3.63025800 \\
\hline
\end{tabular}

$\begin{array}{lrrr}\mathrm{H} & -1.09916500 & -4.42887400 & 2.94087100 \\ \mathrm{H} & -0.00256500 & -1.58156900 & 4.75530300 \\ \mathrm{H} & -1.10897300 & -1.19690300 & -0.05868500 \\ \mathrm{H} & -0.14394800 & 1.32279200 & 0.10423900 \\ \mathrm{H} & -0.29366900 & 2.04152800 & 1.67753800 \\ \mathrm{H} & -2.36045700 & 2.46985000 & 0.31243300 \\ \mathrm{H} & -2.55235400 & 0.75199600 & 0.01652300 \\ \mathrm{H} & -5.22828300 & -0.81895200 & 4.40647400 \\ \mathrm{H} & -7.57699400 & -0.98322000 & 3.64485300 \\ \mathrm{H} & -9.01764800 & 1.52800800 & 1.79281500 \\ \mathrm{H} & -7.54829300 & 3.18349600 & 2.60238100 \\ \mathrm{H} & -5.22944500 & 3.36442000 & 3.41992800 \\ \mathrm{C} & -3.59671600 & 1.42142700 & 4.31113600 \\ \mathrm{H} & -3.21695900 & 0.53979500 & 4.84719200 \\ \mathrm{H} & -3.39825800 & 2.27379200 & 4.97472400 \\ \mathrm{C} & -2.70519800 & 1.59571900 & 3.12205600 \\ \mathrm{H} & -1.69928200 & 1.97176200 & 3.28811600 \\ \mathrm{O} & 2.06602800 & 2.33330100 & -0.08686600 \\ \mathrm{H} & 2.07124900 & 3.00862200 & 0.60572700\end{array}$

\section{Int5' (+4.8 kcal/mol)}

$\begin{array}{cccc}\mathrm{C} & 7.12919300 & 5.78364600 & 0.50558300 \\ \mathrm{C} & 7.56005800 & 5.00214700 & -0.69839700 \\ \mathrm{O} & 6.85374000 & 4.15677400 & -1.23655300 \\ \mathrm{~N} & 8.79934000 & 5.32440100 & -1.18766300 \\ \mathrm{C} & 9.23284000 & 4.76247200 & -2.43341600 \\ \mathrm{C} & 10.30095300 & 3.67275500 & -2.36756000 \\ \mathrm{O} & 10.78542000 & 3.23209500 & -3.40584100 \\ \mathrm{~N} & 10.69282500 & 3.26204200 & -1.12948100 \\ \mathrm{C} & 11.53574000 & 2.08287400 & -1.03459800 \\ \mathrm{C} & 10.67465600 & 0.82887100 & -1.17788300 \\ \mathrm{O} & 9.75108600 & 0.66003400 & -0.37510300 \\ \mathrm{~N} & 10.94914100 & -0.07307900 & -2.14945000 \\ \mathrm{C} & 10.02606100 & -1.21416500 & -2.31924300 \\ \mathrm{C} & 9.94421700 & -2.13259300 & -1.10327500 \\ \mathrm{O} & 8.93613300 & -2.80174500 & -0.89212100 \\ \mathrm{C} & 10.56375500 & -1.91694800 & -3.57748700 \\ \mathrm{C} & 11.15123000 & -0.75981600 & -4.39599800 \\ \mathrm{C} & 11.81102200 & 0.13255500 & -3.33745500 \\ \mathrm{~N} & 11.05051100 & -2.18398600 & -0.31766600 \\ \mathrm{C} & 11.05941300 & -2.93993400 & 0.92171000 \\ \mathrm{C} & 10.09983300 & -2.44764900 & 2.01761300 \\ \mathrm{O} & 9.89645600 & -3.16001000 & 2.99289000 \\ \mathrm{~N} & 9.50112500 & -1.24771100 & 1.82007200 \\ \mathrm{C} & 8.39062400 & -0.83411200 & 2.66846000 \\ \mathrm{C} & 7.25160100 & -1.86296100 & 2.66217400 \\ \mathrm{O} & 6.57840700 & -2.03900200 & 3.66972000 \\ \mathrm{C} & 7.89656400 & 0.54407100 & 2.19076900 \\ \mathrm{C} & 6.63377200 & 1.09330100 & 2.87104000 \\ \mathrm{C} & 6.77236800 & 1.19984900 & 4.39266600 \\ \mathrm{C} & 6.29144900 & 2.44533500 & 2.23268100 \\ \mathrm{~N} & 6.98177700 & -2.46948400 & 1.46468700 \\ \mathrm{C} & 6.01019400 & -3.55881500 & 1.41162400 \\ \mathrm{C} & 4.72676900 & -3.02722300 & 6.54046700 \\ \mathrm{C} & 3.54817700 & -3.41864700 & 5.62700500 \\ \mathrm{C} & 3.65928900 & -2.95193000 & 4.17290000 \\ \mathrm{C} & 2.67284800 & -3.71256100 & 3.28028600 \\ \mathrm{C} & 3.47126600 & -1.43740700 & 4.05783400 \\ \mathrm{C} & 0.78218800 & -7.58917200 & -1.86559800 \\ \mathrm{C} & 0.93527600 & -6.33312200 & -0.99123100\end{array}$




\begin{tabular}{|c|c|c|c|}
\hline $\mathrm{C}$ & 1.85001700 & -5.24142800 & 766060 \\
\hline & 1.22436600 & -4.58252900 & -2.81351200 \\
\hline & 2.16254500 & -4.17883600 & -0.51540600 \\
\hline & -7.31651900 & -8.35015900 & -0.04072700 \\
\hline & -7.92052900 & -6.93668200 & -0.04545300 \\
\hline$c$ & -7.08057000 & -5.88033800 & 0.64355400 \\
\hline 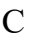 & -5.70879200 & -5.74710700 & 0.39310700 \\
\hline C & -7.66523700 & -4.95949700 & 1.52122300 \\
\hline $\mathrm{C}$ & -4.95676300 & -4.72289500 & 0.96521300 \\
\hline $\mathrm{C}$ & -6.93209100 & -3.91692000 & 3339200 \\
\hline C & -5.57514200 & -3.76899400 & 433100 \\
\hline D & -4.89224300 & -2.70393900 & 2.30190900 \\
\hline C & -3.07848800 & -4.62605300 & 7170500 \\
\hline $\mathrm{C}$ & -2.86605400 & -3.66758800 & -2.40392500 \\
\hline C & -4.23300400 & -3.09758100 & -2.01233400 \\
\hline $\mathrm{C}$ & -4.30362200 & -2.13548400 & 914400 \\
\hline $\mathrm{O}$ & -5.30188700 & -1.35510200 & -0.84175200 \\
\hline 0 & $-3.4221 \mathrm{c}$ & -2.16592500 & 420800 \\
\hline C & 3.02328500 & -0.8347 & -6.7 \\
\hline $\mathrm{C}$ & 3.18190300 & -0.86633700 & -5.23786000 \\
\hline 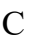 & 2.05 & -1.0418 & 57700 \\
\hline $\mathrm{C}$ & 4.43069900 & -0.72646700 & -4.6 \\
\hline $\mathrm{C}$ & 2.16403400 & -1.06492000 & 3219300 \\
\hline C & 4.54505 & -0.7602 & 504800 \\
\hline $\mathrm{C}$ & 3.41025800 & -0.92471500 & 2628100 \\
\hline $\mathrm{C}$ & 3.03732 & 4.5803 & -4.2 \\
\hline $\mathrm{C}$ & 3.482 & 4.14 & -2.8 \\
\hline $\mathrm{C}$ & 3.9703 & 2.6944 & 80200 \\
\hline $\mathrm{N}$ & 4000 & 2.3623 & 4800 \\
\hline $\mathrm{C}$ & -1.89407100 & 1.45898200 & +312300 \\
\hline $\mathrm{C}$ & -1.40191500 & 1.37743900 & -3.6 \\
\hline $\mathrm{C}$ & -1.5171 & -0.04612300 & 3004400 \\
\hline $\mathrm{C}$ & 0.02237900 & 1.93273000 & 5122000 \\
\hline $\mathrm{C}$ & -7.10613200 & -2.99303300 & -4.5 \\
\hline $\mathrm{C}$ & -8.099 & -2.66 & -3. \\
\hline $\mathrm{C}$ & -8.3965 & -3.8411 & 802900 \\
\hline$S$ & -9.6242 & -3.4713 & 700500 \\
\hline $\mathrm{C}$ & -8.77615100 & -2.09760000 & 2666600 \\
\hline $\mathrm{C}$ & -10.85868500 & 0.05038000 & -4.4 \\
\hline $\mathrm{C}$ & -10.23904700 & 0.56278 & 3434800 \\
\hline $\mathrm{C}$ & -8.86145300 & 0.79519200 & -3.05176900 \\
\hline $\mathrm{C}$ & -11.015 & & 40000 \\
\hline $\mathrm{C}$ & -8.27705900 & 1.305597 & 1200 \\
\hline $\mathrm{C}$ & -10.45275700 & 1.36085500 & -0.84520200 \\
\hline $\mathrm{C}$ & -9.08437200 & 1.62113900 & -0.79871300 \\
\hline $\mathrm{O}$ & -8.57321900 & 2.18597000 & 0.34246500 \\
\hline $\mathrm{C}$ & -5.34125100 & 4.81225900 & 1900 \\
\hline $\mathrm{C}$ & -4.60990500 & 4.13331100 & -2.01487300 \\
\hline $\mathrm{C}$ & -5.44424500 & 3.29109500 & -1.08569100 \\
\hline $\mathrm{O}$ & -6.51719100 & 3.66726200 & -0.59988800 \\
\hline $\mathrm{O}$ & -4.87492900 & 2.14082700 & -0.82627300 \\
\hline $\mathrm{C}$ & -9.04959400 & 7.01535500 & -1.92915100 \\
\hline $\mathrm{C}$ & -9.46779100 & 6.18389000 & -0.71776000 \\
\hline $\mathrm{C}$ & -10.15705900 & 4.88447900 & -1.12812900 \\
\hline $\mathrm{O}$ & -8.34805000 & 5.92286300 & 0.12918300 \\
\hline $\mathrm{C}$ & -3.72652900 & 5.99890000 & 4.67541500 \\
\hline $\mathrm{C}$ & -4.25220600 & 6.71893800 & 3.43030300 \\
\hline $\mathrm{C}$ & -4.73352200 & 5.79517400 & 2.29640200 \\
\hline $\mathrm{C}$ & -5.26711100 & 6.61761600 & 1.11854800 \\
\hline $\mathrm{C}$ & -3.63801200 & 4.83201500 & 1.81931800 \\
\hline $\mathrm{C}$ & 1.65633900 & 4.25845900 & 5.05461300 \\
\hline S & 1.42384900 & 3.27253900 & 3.53161200 \\
\hline $\mathrm{C}$ & 2.63555000 & 1.93841300 & 3.82772800 \\
\hline
\end{tabular}

$\mathrm{O}$

$\mathrm{H}$
$\mathrm{H}$

$\mathrm{H}$

\section{$\mathrm{H}$}

H

$\mathrm{H}$

$\mathrm{H}$

$\mathrm{H}$

$\mathrm{H}$

$\mathrm{H}$

$\mathrm{H}$

$\mathrm{H}$

$\mathrm{H}$

$\mathrm{H}$

$\mathrm{H}$

$\mathrm{H}$

$\mathrm{H}$

$\mathrm{H}$

$\mathrm{H}$

$\mathrm{H}$

$\mathrm{H}$

$\mathrm{H}$

$\mathrm{H}$

$\mathrm{H}$

$\mathrm{H}$

H

\section{$\mathrm{H}$}

H

$\mathrm{H}$

H

H

H

$\mathrm{H}$

$\mathrm{H}$

H

H

\section{$\mathrm{H}$}

$\mathrm{H}$

H

H

$\mathrm{H}$

H

\section{$\mathrm{H}$}

H

$\mathrm{H}$

$\mathrm{H}$

\section{$\mathrm{H}$}

H

H

$\mathrm{H}$

\section{$\mathrm{H}$}

H

$\mathrm{H}$

$\mathrm{H}$

$\mathrm{H}$

$\mathrm{H}$

$\mathrm{H}$

H $\begin{array}{llll}5.37615700 & -0.15680900 & 0.05283400\end{array}$ $\begin{array}{llll}2.77334000 & 0.02245800 & 1.02012300\end{array}$ $\begin{array}{lll}-5.37815000 & 0.41110800 & 0.84406100\end{array}$ $\begin{array}{llll}5.12016900 & 2.96695600 & -1.13973600\end{array}$ $\begin{array}{llll}4.69889300 & 1.40608400 & -1.34981000\end{array}$ $\begin{array}{llll}4.80347200 & 2.55108700 & -3.51772200\end{array}$ $\begin{array}{llll}3.17505100 & 2.00793600 & -3.12045900\end{array}$ $\begin{array}{llll}4.29456500 & 4.79372400 & -2.53188200\end{array}$ $\begin{array}{llll}2.65629500 & 4.25922500 & -2.16897300\end{array}$ $2.13336200 \quad 4.05068200 \quad-4.59706400$ $\begin{array}{llll}3.81769600 & 4.37725500 & -5.02316600\end{array}$ $\begin{array}{llll}5.31885000 & -0.58886600 & -5.22775200\end{array}$ $\begin{array}{llll}5.51197400 & -0.64838500 & -2.74385900\end{array}$ $\begin{array}{llll}3.50612600 & -0.92174500 & -1.34798400\end{array}$ $\begin{array}{lll}1.27521000 & -1.19574900 & -2.42366000\end{array}$ $\begin{array}{llll}1.08110800 & -1.16014000 & -4.88864400\end{array}$ $\begin{array}{llll}0.73110800 & 1.34811000 & -4.14622200\end{array}$ $\begin{array}{llll}0.06877700 & 2.97078600 & -3.89906200\end{array}$ $\begin{array}{llll}0.37428300 & 1.91412000 & -2.51381500\end{array}$ $\begin{array}{llll}-2.06714600 & 2.01426800 & -3.08514300\end{array}$ $\begin{array}{lll}-1.14564900 & -0.09633000 & -2.10077200\end{array}$ $\begin{array}{llll}-2.55703500 & -0.39016200 & -3.12850900\end{array}$ $\begin{array}{llll}-0.92995400 & -0.75431600 & -3.72522000\end{array}$ $\begin{array}{lll}-7.67253900 & -1.85473100 & -2.82088700\end{array}$ $-9.03965500 \quad-2.28142500 \quad-3.83448500$ $\begin{array}{llll}-7.47544700 & -4.19428600 & -2.01861000\end{array}$ $\begin{array}{llll}-8.82291100 & -4.68297700 & -3.05480000\end{array}$ $\begin{array}{llll}-7.73993900 & -2.36486900 & -0.11069400\end{array}$ $\begin{array}{llll}-8.81152200 & -1.18310200 & -0.91669400\end{array}$ $\begin{array}{lll}-9.30416100 & -1.92933800 & 0.61429900\end{array}$ $\begin{array}{lll}-7.74432900 & 2.67342400 & 0.12917300\end{array}$ $\begin{array}{llll}-7.20349200 & 1.43036000 & -1.83954800\end{array}$ $\begin{array}{llll}-11.06716300 & 1.59524900 & 0.01781900\end{array}$ $\begin{array}{llll}-12.08449100 & 0.63893000 & -2.03330100\end{array}$ $\begin{array}{llll}-8.22840200 & 0.56345400 & -3.90403200\end{array}$ $-4.91468000 \quad-3.91965100 \quad-1.75619600$ $\begin{array}{llll}-4.68172800 & -2.58932800 & -2.87193100\end{array}$ $-2.38282900 \quad-4.17135500 \quad-1.56198000$ $-2.20027600 \quad-2.84893700 \quad-2.69963900$ $\begin{array}{llll}-8.08885800 & -6.62658200 & -1.08604400\end{array}$ $\begin{array}{llll}-8.91330200 & -6.95506700 & 0.41616900\end{array}$ $\begin{array}{lll}-8.72638500 & -5.03685700 & 1.73720000\end{array}$ $\begin{array}{llll}-5.20657200 & -6.45363800 & -0.26194100\end{array}$ $\begin{array}{llll}-3.89179400 & -4.65251300 & 0.77919900\end{array}$ $\begin{array}{lll}-7.40947700 & -3.19369600 & 2.73460500\end{array}$ $\begin{array}{lll}-4.22050900 & -2.42786500 & 1.63713200\end{array}$ $\begin{array}{llll}2.79309600 & -3.38177700 & -0.92262400\end{array}$ $\begin{array}{llll}1.23465100 & -3.72413100 & -0.14764000\end{array}$ $\begin{array}{llll}2.67811400 & -4.61620900 & 0.34513300\end{array}$ $\begin{array}{llll}1.89614800 & -3.83591300 & -3.24545200\end{array}$ $\begin{array}{llll}0.98550300 & -5.31028300 & -3.59514700\end{array}$ $\begin{array}{llll}0.29219700 & -4.07238100 & -2.53945900\end{array}$ $\begin{array}{llll}2.79698600 & -5.71301000 & -1.88103900\end{array}$ $\begin{array}{llll}-0.04994700 & -5.88083900 & -0.81300800\end{array}$ $\begin{array}{llll}1.30968100 & -6.62432500 & -0.00164200\end{array}$ $\begin{array}{llll}7.72255800 & -2.49535600 & 0.76920400\end{array}$ $\begin{array}{lll}8.72187000 & -0.79489800 & 3.70847800\end{array}$ $\begin{array}{llll}8.72343200 & 1.25492200 & 2.32412200\end{array}$ $\begin{array}{llll}7.70453800 & 0.48850500 & 1.11589800\end{array}$ $\begin{array}{lll}7.09204300 & 3.17417800 & 2.41884200\end{array}$ $\begin{array}{llll}6.16151600 & 2.33889500 & 1.15235500\end{array}$ $\begin{array}{lll}5.36519000 & 2.85803900 & 2.64639500\end{array}$ $\begin{array}{lll}7.63512700 & 1.82008500 & 4.66893300\end{array}$ 


\begin{tabular}{|c|c|c|c|}
\hline $\mathrm{H}$ & 88024700 & 1.66069800 & \\
\hline $\mathrm{H}$ & & & \\
\hline & & & \\
\hline & 66872300 & -0.70733400 & 0.97801700 \\
\hline & 10.76903800 & & 0.72526500 \\
\hline & 12.07563700 & -2.95003300 & 1.32170100 \\
\hline & 11.79653500 & -1.53427000 & \\
\hline & 12.83426000 & -0.19895200 & 7700 \\
\hline & 4900 & -1.08829100 & \\
\hline & 400 & -2.62515600 & \\
\hline & 600 & -0.20352900 & 6300 \\
\hline & 400 & 1.18569300 & \\
\hline & & -2.47160800 & \\
\hline & 9.00 & -0.84065500 & 600 \\
\hline & & 3.44454800 & \\
\hline & 9.315 & 6.08201200 & -0.7 \\
\hline & -4.15 & 4.90970000 & \\
\hline & & & \\
\hline & -9.50 & 4.28805800 & -1.7 \\
\hline & 6000 & & \\
\hline & -11.0 & 5.07812300 & 5300 \\
\hline & -10.1 & 6.77334800 & 1400 \\
\hline & & & \\
\hline 1 & -5.0 & 7.3793 & \\
\hline & -3.4 & & \\
\hline & & & \\
\hline & -5.6 & $5.9^{-}$ & \\
\hline & & & 100 \\
\hline & -3.2 & 4.22841 & 800 \\
\hline $\mathrm{F}$ & -4.0 & & \\
\hline F & & & 100 \\
\hline & -5.5 & & 500 \\
\hline & & & \\
\hline & & & \\
\hline & 2.6 & & 00 \\
\hline & & & 600 \\
\hline & & & \\
\hline & & -0.90001400 & \\
\hline & & & 300 \\
\hline & & -1.1 & \\
\hline & & & \\
\hline & & & \\
\hline & & -3.29011000 & \\
\hline & 600 & -3.1 & 700 \\
\hline & & -4.51389400 & \\
\hline & & & \\
\hline & & -0.18 & 700 \\
\hline & & -0.92165600 & \\
\hline & & & \\
\hline & & & 07000 \\
\hline & & 803400 & \\
\hline & & 4.30556000 & -2.90434000 \\
\hline & & & 500900 \\
\hline & & & \\
\hline & -8.27650000 & 6.48198600 & -2.49640300 \\
\hline & & & \\
\hline & & -1.94276400 & 6.56871900 \\
\hline & 12.32273800 & 2.16122600 & -1.78212500 \\
\hline & & -3.18804100 & 1.64962700 \\
\hline & & -8.28953200 & -1.74119900 \\
\hline & & -1.70859100 & -7.22204200 \\
\hline & & 0.05995100 & -7.18371100 \\
\hline & -1.28993400 & 0.85342100 & -5.82505300 \\
\hline
\end{tabular}

H $\quad-7.93973200 \quad-9.02949800 \quad-0.63246500$

$\mathrm{H} \quad-3.95637200 \quad-4.29956100 \quad-4.14567700$

$\mathrm{H} \quad-11.93523100 \quad-0.09983300 \quad-4.29095700$

$\mathrm{H} \quad-10.42300600 \quad-0.90739500 \quad-4.71037700$

$\mathrm{H} \quad-6.12578300 \quad 0.27633800 \quad 1.44564100$

$\mathrm{H} \quad-4.55616800 \quad 5.38666200 \quad-3.67780400$

$\mathrm{H} \quad-5.79000200 \quad 4.10723200 \quad-3.88341200$

H $\quad-2.29981600 \quad-4.70619200 \quad-4.33958300$

$\mathrm{H} \quad-3.29353000 \quad-5.66695700 \quad-3.30590900$

$\mathrm{H} \quad-6.68657700 \quad-2.07616000 \quad-4.96792100$

$\mathrm{H} \quad-7.49178200 \quad-3.59565300 \quad-5.36925600$

$\mathrm{H} \quad-10.70665700 \quad 0.74955300 \quad-5.23466200$

$\mathrm{H} \quad-1.88822600 \quad 2.50318000 \quad-5.47212400$

$\mathrm{H} \quad-2.94677600 \quad 1.16432300 \quad-5.21748500$

$\mathrm{H} \quad 1.95259900 \quad-0.83454700 \quad-6.97281100$

$\mathrm{H} \quad 2.83612600 \quad 5.65598900 \quad-4.30347100$

$\mathrm{H} \quad 7.95237500 \quad 6.14800100 \quad 1.12867200$

H $\quad 6.54580200 \quad 6.64979700 \quad 0.17377400$

$\mathrm{H} \quad 9.64038700 \quad 5.48943100 \quad-3.14477700$

H $\quad 11.98918600 \quad 2.05277900 \quad-0.04048700$

H $\quad 6.25215800 \quad-4.36261700 \quad 2.11532300$

H $\quad 6.00347400 \quad-3.95810500 \quad 0.39626000$

$\mathrm{H} \quad 5.64422900 \quad-3.47174100 \quad 6.14063800$

$\mathrm{H} \quad 4.41513600 \quad-3.35231900 \quad 7.53954000$

H $\quad-0.16096000 \quad-8.09516400 \quad-1.63471900$

$\mathrm{H} \quad 0.71666200 \quad-7.32027200 \quad-2.92511000$

$\mathrm{H} \quad-7.26507500 \quad-8.75398800 \quad 0.97518600$

$\mathrm{H} \quad-6.32541000 \quad-8.33542400 \quad-0.50413800$

$\mathrm{H} \quad-8.65019500 \quad 7.99831700 \quad-1.65616300$

H $\quad-9.86412600 \quad 7.23451800 \quad-2.62754200$

$\mathrm{H} \quad-3.37201300 \quad 6.70819400 \quad 5.43069800$

$\mathrm{H} \quad-4.46823700 \quad 5.31451900 \quad 5.10156800$

$\mathrm{H} \quad 2.68011100 \quad 4.63516300 \quad 5.11722800$

H $\quad-5.31820500 \quad 1.53479200 \quad-0.11210800$

C $\quad-0.46672200 \quad-0.11835700 \quad 1.72569300$

$\begin{array}{llll}\mathrm{N} & -3.04822200 & 1.27349600 & 1.91105100\end{array}$

O $\quad-1.41494400 \quad-3.58285200 \quad 0.89649500$

C $\quad-0.15856200 \quad-0.28914600 \quad 3.08108800$

O $\quad-0.82261900 \quad-3.86348200 \quad 3.52135100$

C $\quad-0.24372500 \quad-1.54866700 \quad 3.68344500$

C $\quad-0.66739100 \quad-2.64731800 \quad 2.94111400$

C $\quad-0.94565600 \quad-2.49114600 \quad 1.57099700$

$\begin{array}{llll}\text { C } & -0.81878700 & -1.24498700 & 0.97406000\end{array}$

C $\quad-0.65822100 \quad 1.24477600 \quad 1.10218100$

C $\quad-2.14008200 \quad 1.46592300 \quad 0.76043000$

C $\quad-5.08454800 \quad 1.13062100 \quad 3.96661300$

$\begin{array}{llll}\text { C } & -5.73550900 & -0.10509000 & 3.97003900\end{array}$

$\begin{array}{llll}\text { C } & -7.05873900 & -0.20839600 & 3.54285800\end{array}$

C $\quad-7.74793600 \quad 0.92372000 \quad 3.08905100$

$\begin{array}{llll}\text { O } & -9.00779900 & 0.77143000 & 2.61954100\end{array}$

C $\quad-7.10707000 \quad 2.17649500 \quad 3.11374100$

C $\quad-5.79331300 \quad 2.26733500 \quad 3.55226300$

$\mathrm{H} \quad-4.00291300 \quad 0.88864900 \quad 1.66093600$

H $\quad 2.42372800 \quad 0.85501500 \quad 0.60777200$

$\mathrm{H} \quad-2.17992900 \quad-3.22797700 \quad 0.35872700$

$\mathrm{H} \quad 0.14720800 \quad 0.56625600 \quad 3.67503000$

$\mathrm{H} \quad-1.16871100 \quad-4.45044100 \quad 2.82719600$

$\mathrm{H} \quad-0.01306000 \quad-1.68556300 \quad 4.73409700$

$\mathrm{H} \quad-1.09327600 \quad-1.15020500 \quad-0.06997100$

H $\quad-0.09085500 \quad 1.36319500 \quad 0.17464900$

$\mathrm{H} \quad-0.29172200 \quad 2.03283200 \quad 1.76515000$

H $\quad-2.32203200 \quad 2.46857500 \quad 0.36323400$ 


$\begin{array}{rrrr}\mathrm{H} & -2.48116100 & 0.75060000 & 0.01051100 \\ \mathrm{H} & -5.19906200 & -1.01097400 & 4.23151700 \\ \mathrm{H} & -7.55923300 & -1.16796600 & 3.50762600 \\ \mathrm{H} & -9.12248600 & 1.42714000 & 1.90117700 \\ \mathrm{H} & -7.63874400 & 3.05992900 & 2.77504000 \\ \mathrm{H} & -5.30304500 & 3.23572100 & 3.55420700 \\ \mathrm{C} & -3.62005400 & 1.25564900 & 4.30173800 \\ \mathrm{H} & -3.23727000 & 0.35060400 & 4.79499700 \\ \mathrm{H} & -3.43041200 & 2.07462500 & 5.00832600 \\ \mathrm{C} & -2.72006400 & 1.48736500 & 3.13043600 \\ \mathrm{H} & -1.72054500 & 1.86566400 & 3.32550800 \\ \mathrm{O} & 2.07411300 & 2.37181500 & -0.06127400 \\ \mathrm{H} & 2.09646600 & 3.03097400 & 0.64651100\end{array}$

\section{TS4' (+28.4 kcal/mol)}

$\begin{array}{lccc}\mathrm{C} & 7.17060900 & 5.71014200 & 0.52072100 \\ \mathrm{C} & 7.59793000 & 4.93655200 & -0.68924700 \\ \mathrm{O} & 6.90454800 & 4.07530800 & -1.21805500 \\ \mathrm{~N} & 8.82248200 & 5.27970500 & -1.19783000 \\ \mathrm{C} & 9.24876700 & 4.71145600 & -2.44413100 \\ \mathrm{C} & 10.31314400 & 3.61868300 & -2.36927200 \\ \mathrm{O} & 10.78697100 & 3.15384000 & -3.40169700 \\ \mathrm{~N} & 10.71323900 & 3.23484700 & -1.12523100 \\ \mathrm{C} & 11.55042500 & 2.05465600 & -1.00478300 \\ \mathrm{C} & 10.68482200 & 0.80085800 & -1.12544900 \\ \mathrm{O} & 9.76106800 & 0.64671900 & -0.31997900 \\ \mathrm{~N} & 10.96031100 & -0.11570500 & -2.08379300 \\ \mathrm{C} & 10.03702400 & -1.25723800 & -2.24364200 \\ \mathrm{C} & 9.95170300 & -2.16709500 & -1.02282600 \\ \mathrm{O} & 8.95423900 & -2.85878900 & -0.83174200 \\ \mathrm{C} & 10.57395300 & -1.97412700 & -3.49439700 \\ \mathrm{C} & 11.16738500 & -0.82737200 & -4.32257600 \\ \mathrm{C} & 11.82775900 & 0.07235900 & -3.27103700 \\ \mathrm{~N} & 11.03812800 & -2.18605800 & -0.21015700 \\ \mathrm{C} & 11.03044700 & -2.92442700 & 1.03978200 \\ \mathrm{C} & 10.01959900 & -2.44764100 & 2.09579300 \\ \mathrm{O} & 9.79088400 & -3.16354800 & 3.06287600 \\ \mathrm{~N} & 9.40611800 & -1.25881600 & 1.87526100 \\ \mathrm{C} & 8.25678800 & -0.87238700 & 2.68473600 \\ \mathrm{C} & 7.14119000 & -1.92543400 & 2.62863400 \\ \mathrm{O} & 6.42528000 & -2.12167500 & 3.60216300 \\ \mathrm{C} & -7.74284500 & 0.49607800 & 2.19884000 \\ \mathrm{C} & -7.17730700 & -5.89419700 & 0.76340400 \\ \mathrm{C} & 6.45293700 & 1.00358700 & 2.86328000 \\ \mathrm{C} & 6.58667700 & 1.12477300 & 4.38463600 \\ \mathrm{C} & 6.05342400 & 2.33584700 & 2.21669800 \\ \mathrm{~N} & 6.93707800 & -2.52868800 & 1.41611100 \\ \mathrm{C} & 5.99182200 & -3.63519200 & 1.31604100 \\ \mathrm{C} & 4.74391700 & -3.15960900 & 6.45915200 \\ \mathrm{C} & 3.52425900 & -3.54373900 & 5.58815400 \\ \mathrm{C} & 3.48585700 & -3.01043900 & 4.15264400 \\ \mathrm{C} & 2.38207300 & -3.70689700 & 3.34705600 \\ \mathrm{C} & 3.29234400 & -1.49371900 & 4.12099000 \\ \mathrm{C} & 0.71556900 & -7.58745800 & -1.97883500 \\ \mathrm{C} & 0.87649500 & -6.34579200 & -1.08904100 \\ \mathrm{C} & 1.90146900 & -5.30825700 & -1.58237100 \\ \mathrm{C} & -7.37640400 & -4.64401200 & -2.89194000 \\ \mathrm{C} & -4.24373600 & -0.50310400 \\ \mathrm{C} & -8.31469500 & -0.11254000 \\ \mathrm{C} & & & \\ \mathrm{C} & & & \\ \mathrm{C} & & & \end{array}$

\begin{tabular}{|c|c|c|c|}
\hline & -5.78644600 & -5.95475000 & 0.90571300 \\
\hline & -7.84445100 & -4.82130000 & 1.37376300 \\
\hline & -5.07980400 & -4.96249700 & 1.58932400 \\
\hline & -7.15356700 & -3.81758100 & 2.04586300 \\
\hline & -5.76086300 & -3.87057700 & 2.13127700 \\
\hline & -5.10282300 & -2.84171700 & 2.75576400 \\
\hline & -3.13331900 & -4.57703700 & -3.62215000 \\
\hline & -2.89205200 & -3.71114900 & -2.39527500 \\
\hline & -4.11322900 & -3.90908600 & -1.46377100 \\
\hline & -4.45354500 & -2.71644500 & -0.61629700 \\
\hline & -5.48147200 & -2.07025700 & -0.77535000 \\
\hline & -3.57497700 & -2.34804600 & 0.31851800 \\
\hline & 2.97347000 & -0.78795200 & -6.78514200 \\
\hline & 3.13420500 & -0.85821500 & -5.28091100 \\
\hline & 2.00943500 & -1.06008900 & -4.47188900 \\
\hline & 4.38180600 & -0.72517000 & -4.65448600 \\
\hline & 2.11592400 & -1.11108300 & -3.08139300 \\
\hline & 4.49518300 & -0.78759900 & -3.26545100 \\
\hline & 3.36024200 & -0.97412400 & -2.47157000 \\
\hline & 3.04106100 & 4.59583200 & 4900 \\
\hline & 3.54287300 & 4.13822800 & -2.88037300 \\
\hline & 3.96862500 & 2.66907700 & -2.83850400 \\
\hline & 4.44747900 & 2.32 & 2100 \\
\hline & -1.91737400 & 1.51977400 & -5.12537000 \\
\hline & -1.39707500 & 6700 & 56900 \\
\hline & -1.53534200 & -0.05870400 & -3.19349000 \\
\hline & 0.04134400 & 1.90707500 & -3.57887200 \\
\hline & -7.15706900 & -2.9033 & 20200 \\
\hline & -8.17913300 & -2.57704400 & -3.45572100 \\
\hline & -8.52122200 & -3.77076400 & -2.56601800 \\
\hline & -9.84675900 & -3.42955200 & -1.34532500 \\
\hline & -9.07689300 & -2.05442300 & -0.41934800 \\
\hline & -10.88680400 & 0.16417800 & -4.35027200 \\
\hline & -10.24345700 & 2500 & -3.08058100 \\
\hline & -8.86685600 & 0.8984 & -3.02933100 \\
\hline & -10.99066900 & 6200 & -1.923 \\
\hline & -8.25672900 & 1.39523000 & 8175500 \\
\hline & -10.40108700 & 1.41193600 & 86000 \\
\hline & -9.03315600 & 1.68609500 & -0.75338600 \\
\hline & -8.49248900 & 2.24751500 & 0.36897800 \\
\hline & -5.32884400 & 4.87171000 & -3.09449900 \\
\hline & -4.57459200 & 4.14756200 & -1.96636000 \\
\hline & -5.40184900 & 3.26424800 & -1.06878800 \\
\hline & -6.40961800 & 3.66985300 & -0.47950600 \\
\hline & -4.92283300 & 2.04617600 & -0.97857300 \\
\hline & -9.01381900 & 7.08455100 & -1.79681200 \\
\hline & -9.42616500 & 6.23617700 & -0.59524700 \\
\hline & -10.11266400 & 4.94059900 & -1.02159900 \\
\hline & -8.30521600 & 5.96961500 & 0.24749500 \\
\hline & -3.65749600 & 5.94786600 & 4.76097000 \\
\hline & -4.19273200 & 6.70632000 & 3.54145700 \\
\hline & -4.56039400 & 5.82051000 & 2.33818300 \\
\hline & -5.25940800 & 6.63944600 & 1.24794600 \\
\hline & -3.33813600 & 5.10026900 & 1.75193600 \\
\hline & 1.71548000 & 4.16542400 & 5.08441400 \\
\hline & 1.62720100 & 3.27799900 & 3.48944800 \\
\hline & 2.53865600 & 1.76434500 & 3.94207900 \\
\hline & 5.38001900 & -0.26158600 & -0.06193300 \\
\hline & 2.84484400 & 0.03094200 & 1.02719200 \\
\hline & -5.54549900 & 0.21010000 & 0.59777000 \\
\hline & 5.27173400 & 2.88056000 & -1.26911600 \\
\hline & 4.73502800 & 1.34662100 & -1.42537500 \\
\hline & 4.73222700 & 2.48382200 & -3.61009800 \\
\hline
\end{tabular}




\begin{tabular}{|c|c|c|c|}
\hline & & & \\
\hline $\mathrm{H}$ & & & \\
\hline & & & \\
\hline & 11784200 & 4.07847700 & -4.53441600 \\
\hline & & & \\
\hline & 5.26944900 & -0.56824200 & -5.26295500 \\
\hline & 5.45940500 & -0.68016900 & -2.77 \\
\hline & 45979600 & -0.98910200 & 0700 \\
\hline & & -1.2617 & \\
\hline & & 200 & -4. \\
\hline & 0.7189 & & 900 \\
\hline & & 2.9 & \\
\hline & & & \\
\hline & 00 & 2.0 & 500 \\
\hline & & -0.1 & \\
\hline $\mathrm{H}$ & -2.58326200 & -0.37 & 1000 \\
\hline & -0.964 & -0.7 & \\
\hline $\mathrm{H}$ & -7.77 & -1.7 & \\
\hline 1 & -9.099 & -2.1 & 200 \\
\hline & & & \\
\hline $\mathrm{H}$ & -8.8932 & -4.6 & 000 \\
\hline & -8.0691 & -2.3 & -0 . \\
\hline $\mathrm{H}$ & -9.0 & -1.1 & \\
\hline $\mathrm{H}$ & -9.6 & -1.8 & \\
\hline & -7.6 & & \\
\hline $\mathrm{H}$ & & & \\
\hline 1 & -10.9 & & 300 \\
\hline $\mathrm{H}$ & -12. & & \\
\hline $\mathrm{H}$ & -8.255 & & \\
\hline & -3.947 & -4.7 & \\
\hline $\mathrm{H}$ & & & \\
\hline $\mathrm{H}$ & -1.967 & & \\
\hline $\mathrm{H}$ & & & \\
\hline $\mathrm{H}$ & & & \\
\hline $\mathrm{H}$ & -8.9 & -6.9 & \\
\hline $\mathrm{H}$ & -8.9 & & 400 \\
\hline $\mathrm{H}$ & & & \\
\hline $\mathrm{H}$ & & & \\
\hline $\mathrm{H}$ & & & 00 \\
\hline & -4.30 & & \\
\hline & & & \\
\hline & & & \\
\hline & 2.543 & -4.6 & \\
\hline & 2.19 & -3.9 & 300 \\
\hline $\mathrm{H}$ & & -5.3 & \\
\hline $\mathrm{H}$ & 0.510 & & \\
\hline $\mathrm{H}$ & & & \\
\hline & -0.093 & & \\
\hline & & & \\
\hline & & -2.5 & \\
\hline & & & \\
\hline $\mathrm{H}$ & 8547 & & 400 \\
\hline & & & \\
\hline & & & \\
\hline & & & \\
\hline & & & \\
\hline & & 1.76561200 & 90500 \\
\hline & 5.68629600 & 1.56 & \\
\hline & & & \\
\hline & & 0.28561400 & 2.64847400 \\
\hline & & -0.71068400 & \\
\hline & & & 0.85100200 \\
\hline & 12.03305000 & -2.89550000 & 1.47203500 \\
\hline
\end{tabular}

H $\quad 11.77529000 \quad-1.52356100 \quad-0.39568800$

$\begin{array}{llll}\mathrm{H} & 12.84826400 & -0.26234600 & -3.04831800\end{array}$

$\mathrm{H} \quad 11.87674700 \quad-1.16572700 \quad-5.08100900$

$\mathrm{H} \quad 11.35839400 \quad-2.68256300 \quad-3.20525700$

$\mathrm{H} \quad 10.36909200 \quad-0.27238200 \quad-4.82577600$

H $\quad 11.83835000 \quad 1.12186700 \quad-3.56569000$

$\mathrm{H} \quad 9.78684200 \quad-2.53093800 \quad-4.00432100$

$\mathrm{H} \quad 9.02015600 \quad-0.88404100 \quad-2.39992500$

$\mathrm{H} \quad 10.09099700 \quad 3.44280500 \quad-0.35601400$

$\mathrm{H} \quad \begin{array}{llll}9.33170700 & 6.04966800 & -0.79172500\end{array}$

$\mathrm{H} \quad-4.11753000 \quad 4.90020000 \quad-1.31261700$

$\mathrm{H} \quad-3.76368900 \quad 3.54484400 \quad-2.38129100$

$\mathrm{H} \quad-9.46111400 \quad 4.35524700 \quad-1.68072500$

$\mathrm{H} \quad-10.33646300 \quad 4.32638600 \quad-0.14729400$

$\mathrm{H} \quad-11.04266300 \quad 5.13960500 \quad-1.56420000$

$\mathrm{H} \quad-10.11451800 \quad 6.81504700 \quad 0.03286300$

$\mathrm{H} \quad-2.79303300 \quad 5.33234000 \quad 4.48534700$

$\mathrm{H} \quad-5.07891600 \quad 7.28354800 \quad 3.83363900$

$\mathrm{H} \quad-3.44446700 \quad 7.44334400 \quad 3.21583600$

$\mathrm{H} \quad-4.61684400 \quad 7.46430700 \quad 0.91403000$

$\mathrm{H} \quad-5.49322600 \quad 6.01824200 \quad 0.37974900$

H $\quad-6.20672900 \quad 7.05887200 \quad 1.59515800$

$\mathrm{H} \quad-2.77748800 \quad 4.53682100 \quad 2.50376200$

$\mathrm{H} \quad-3.64289400 \quad 4.38721400 \quad 0.98039900$

$\mathrm{H} \quad-2.64685700 \quad 5.82035400 \quad 1.29680700$

$\mathrm{H} \quad-5.26879200 \quad 5.06033300 \quad 2.69339700$

$\mathrm{H} \quad 1.18201900 \quad 5.11053200 \quad 4.96645700$

$\mathrm{H} \quad 1.23790900 \quad 3.58227800 \quad 5.87558200$

$\mathrm{H} \quad 2.60361000 \quad 1.13716500 \quad 3.05168900$

$\mathrm{H} \quad 3.54882200 \quad 2.00947800 \quad 4.27575100$

$\mathrm{H} \quad 2.01674900 \quad 1.21696000 \quad 4.72957700$

$\mathrm{H} \quad 4.07838100 \quad-0.97735400 \quad 4.67833800$

H $\quad 3.31024800 \quad-1.11420300 \quad 3.09643600$

$\mathrm{H} \quad 2.32176900 \quad-1.22433100 \quad 4.55882400$

$\mathrm{H} \quad 2.57505300 \quad-4.78207000 \quad 3.25133200$

$\mathrm{H} \quad 1.40304100 \quad-3.58932100 \quad 3.82465200$

$\mathrm{H} \quad 2.30620800 \quad-3.29101400 \quad 2.33590800$

$\mathrm{H} \quad 4.45304600 \quad-3.22474500 \quad 3.69257500$

$\mathrm{H} \quad 3.46881200 \quad-4.64112400 \quad 5.55859000$

$\mathrm{H} \quad 2.60572300 \quad-3.21840500 \quad 6.10043400$

$\mathrm{H} \quad 4.51815000 \quad-0.25171900 \quad 0.40472700$

H $\quad 5.86782100 \quad-1.01064700 \quad 0.31698500$

H $\quad 2.04286200 \quad-0.49493700 \quad 0.91196600$

H $\quad 3.24491400 \quad 2.53527600 \quad-0.39452300$

$\mathrm{H} \quad-5.62314300 \quad-0.66824000 \quad 0.14849400$

$\mathrm{H} \quad 8.37303100 \quad 4.25848700 \quad-2.91549100$

$\mathrm{H} \quad \begin{array}{llll}6.51362500 & 5.08396400 & 1.12514900\end{array}$

$\mathrm{H} \quad-6.09158400 \quad 5.54812400 \quad-2.70431100$

$\mathrm{H} \quad-8.24720100 \quad 6.55588800 \quad-2.37754500$

$\mathrm{H} \quad-7.73232300 \quad 5.32334300 \quad-0.19554400$

$\mathrm{H} \quad 4.89052300 \quad-2.07584300 \quad 6.49529200$

H $\quad 12.34011200 \quad 2.11387400 \quad-1.75121700$

H $\quad 4.98069400 \quad-3.28964800 \quad 1.54008900$

H $\quad 1.54208700 \quad-8.29442200 \quad-1.86493600$

H $\quad 3.42362400 \quad-1.65140800 \quad-7.28719900$

H $\quad 3.42847400 \quad 0.11583400 \quad-7.20274500$

$\mathrm{H} \quad-1.33250000 \quad 0.92389900 \quad-5.83316300$

$\mathrm{H} \quad-6.31450200 \quad-3.46574200 \quad-4.12480800$

$\mathrm{H} \quad-8.01466400 \quad-8.96857600 \quad-0.71470800$

$\mathrm{H} \quad-4.02680500 \quad-4.20689500 \quad-4.14039600$

$\mathrm{H} \quad-11.96383300 \quad 0.02735900 \quad-4.22253800$

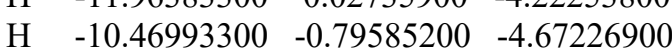

H $\quad-6.10771700 \quad 0.21161600 \quad 1.38671500$ 


\begin{tabular}{|c|c|c|c|}
\hline $\mathrm{H}$ & -4.54279700 & 5.44696700 & \\
\hline $\mathrm{H}$ & & & -3.80797400 \\
\hline & -2.36201100 & -4.65156900 & -4.39765900 \\
\hline & & -5.61891700 & 45500 \\
\hline & -6.73326700 & -1.98402500 & -4.96449800 \\
\hline & -7.55176900 & -3.49265500 & 70500 \\
\hline & -10.73524400 & 0.87258700 & 57600 \\
\hline & -1.90600600 & 2.56773200 & 12200 \\
\hline & & & 5600 \\
\hline & & -0.77671700 & -7.0 \\
\hline & 707900 & & -4.2 \\
\hline & & & \\
\hline & 6.59420300 & & \\
\hline & 9.657259 & & -3.1 \\
\hline & 12.001482 & 400 & 300 \\
\hline & 300 & -4.44 & \\
\hline & & -4.01 & \\
\hline & 5.65572 & -3.6 & \\
\hline & 4.43616 & -3.4 & \\
\hline & -0.22 & -8.0 & \\
\hline & & -7.30472 & -3. \\
\hline & -7.3 & -8.73248 & \\
\hline & & -8.3 & \\
\hline & -8.6 & & \\
\hline & & & \\
\hline & -3.2 & & \\
\hline & -4.4 & & \\
\hline & & & \\
\hline & -5.35 & & 400 \\
\hline C & & & \\
\hline & & & \\
\hline C & -1.3 & -3.4 & 200 \\
\hline C & -0.6 & & \\
\hline C & -1.1 & & \\
\hline C & -0.6 & -0.9 & \\
\hline $\mathrm{C}$ & & -2.1 & \\
\hline C & -0.9 & -2.3 & \\
\hline C & & & \\
\hline $\mathrm{C}$ & & & \\
\hline $\mathrm{C}$ & -1.9 & & \\
\hline $\mathrm{C}$ & & & \\
\hline $\mathrm{C}$ & & -0.0 & \\
\hline $\mathrm{C}$ & -6.9 & & \\
\hline $\mathrm{C}$ & -7.4 & & \\
\hline $\mathrm{O}$ & -8.74 & & \\
\hline $\mathrm{C}$ & & & \\
\hline $\mathrm{C}$ & -5.12 & & \\
\hline $\mathrm{H}$ & -3.4 & & \\
\hline $\mathrm{H}$ & 2.6 & & \\
\hline $\mathrm{H}$ & -2.75 & -2.92947300 & 3000 \\
\hline $\mathrm{H}$ & & & \\
\hline $\mathrm{H}$ & -1.25656600 & -3.9619 & \\
\hline $\mathrm{H}$ & -0.65 & -0.81 & \\
\hline $\mathrm{H}$ & -0.68707300 & -1.32344800 & 77000 \\
\hline $\mathrm{H}$ & -0.11583500 & 1.08164700 & 0400 \\
\hline $\mathrm{H}$ & & 2.06814 & \\
\hline $\mathrm{H}$ & -1.95374600 & 2.89041300 & 0.34047000 \\
\hline $\mathrm{H}$ & -2.4411 & 1.48100700 & 08100 \\
\hline $\mathrm{H}$ & & & \\
\hline $\mathrm{H}$ & -7.61835300 & -0.66121000 & 3.92819700 \\
\hline $\mathrm{H}$ & & & 2.12334600 \\
\hline $\mathrm{H}$ & -6.88139000 & 3.31641300 & 2.49755600 \\
\hline & -4.46603000 & 2.99836200 & 2.85979400 \\
\hline
\end{tabular}

$\begin{array}{lrrr}\mathrm{C} & -3.15722300 & 0.70432300 & 3.77372300 \\ \mathrm{H} & -3.04431800 & -0.38147000 & 3.80020100 \\ \mathrm{H} & -2.77672100 & 1.06704700 & 4.73684800 \\ \mathrm{C} & -2.24787900 & 1.26802700 & 2.68017400 \\ \mathrm{H} & -1.78996400 & 2.22609600 & 2.93278400 \\ \mathrm{O} & 2.45164700 & 2.54482600 & 0.25230700 \\ \mathrm{H} & 2.68630400 & 3.14184900 & 0.97647600\end{array}$

\section{Int6' (+21.7 kcal/mol)}

\begin{tabular}{|c|c|c|c|}
\hline $\mathrm{C}$ & 7.14928600 & 5.78799500 & \\
\hline $\mathrm{C}$ & & 5.05739400 & -0.56204400 \\
\hline $\mathrm{O}$ & 87165700 & 4.21771000 & -1.11840200 \\
\hline N & 8.79486800 & 5.41699500 & -1.05878800 \\
\hline $\mathrm{C}$ & 9.23149700 & & -2.31596600 \\
\hline $\mathrm{C}$ & 10.30953400 & 3.80249600 & 7900 \\
\hline $\mathrm{O}$ & 10.79447300 & 3.36312900 & -3.2 \\
\hline $\mathrm{N}$ & 10.70822200 & 3.4016 & 2000 \\
\hline $\mathrm{C}$ & 11.55912000 & 2.2301 & -0. \\
\hline $\mathrm{C}$ & .70689500 & & -1. \\
\hline $\mathrm{O}$ & 9.79099700 & 0.791 & 200 \\
\hline $\mathrm{N}$ & 476000 & & 3900 \\
\hline $\mathrm{C}$ & 10.07 & -1.07 & 4900 \\
\hline $\mathrm{C}$ & 10.00323300 & -2.01 & 2800 \\
\hline $\mathrm{O}$ & & -2.72 & -0.8 \\
\hline $\mathrm{C}$ & 10.60764600 & -1.7 & 6600 \\
\hline $\mathrm{C}$ & & & \\
\hline $\mathrm{C}$ & & & -3 \\
\hline $\mathrm{N}$ & 11.0 & -2.0 & 9700 \\
\hline C & & -2.7 & \\
\hline $\mathrm{C}$ & 10.0 & -2.3 & 1600 \\
\hline $\mathrm{O}$ & 9.81 & -3.10 & \\
\hline $\mathrm{N}$ & & -1.1 & \\
\hline $\mathrm{C}$ & 8.24 & -0.82 & \\
\hline $\mathrm{C}$ & & & \\
\hline $\mathrm{O}$ & & -2.1 & 400 \\
\hline $\mathrm{C}$ & 7.70 & 0.5 & 2.2 \\
\hline $\mathrm{C}$ & 6.38 & & 900 \\
\hline $\mathrm{C}$ & 6.46 & & 500 \\
\hline $\mathrm{C}$ & 5.94 & & 500 \\
\hline $\mathrm{N}$ & & -2.4 & 100 \\
\hline $\mathrm{C}$ & 6.04 & -3.5 & \\
\hline $\mathrm{C}$ & & & \\
\hline $\mathrm{C}$ & & -3.6 & \\
\hline $\mathrm{C}$ & 3.50 & -3.0 & 400 \\
\hline $\mathrm{C}$ & 2.36 & -3.6 & 3800 \\
\hline $\mathrm{C}$ & 3.35 & -1.4 & 100 \\
\hline $\mathrm{C}$ & 0.79 & -7.4 & -2.1 \\
\hline $\mathrm{C}$ & 1.01 & -6.2 & -1.2 \\
\hline $\mathrm{C}$ & 29200 & -5.3 & 5300 \\
\hline $\mathrm{C}$ & & & \\
\hline $\mathrm{C}$ & 2.48073100 & -4.32 & 9700 \\
\hline $\mathrm{C}$ & -7.28 & -8.33 & 5600 \\
\hline $\mathrm{C}$ & -7.71256100 & -6.86609400 & -0.14081200 \\
\hline $\mathrm{C}$ & -6.70716300 & -5.99556600 & 6900 \\
\hline $\mathrm{C}$ & -5.44090900 & -6.4361 & 50700 \\
\hline $\mathrm{C}$ & -7.04793700 & -4.66728400 & 46400 \\
\hline $\mathrm{C}$ & -4.54608300 & -5.59320700 & 1.67897900 \\
\hline $\mathrm{C}$ & -6.17197100 & & 1.57864100 \\
\hline $\mathrm{C}$ & -4.90779300 & -4.27600100 & 1.96867500 \\
\hline 0 & -4.08306200 & -3.39947800 & 2.61788100 \\
\hline
\end{tabular}




\begin{tabular}{|c|c|c|c|}
\hline 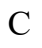 & -3.07655300 & -4.47334800 & -3.71792700 \\
\hline & -2.78246500 & -3.65678700 & -2.47268100 \\
\hline & -3.99986900 & -3.77332700 & -1.51434000 \\
\hline & -4.30493000 & -2.47875500 & -0.82005900 \\
\hline D & -5.35898200 & -1.87900100 & -0.93562700 \\
\hline & -3.31268000 & -1.95824500 & -0.07854400 \\
\hline & 2.99556100 & -0.55425700 & -6.78992200 \\
\hline & 3.14805700 & -0.68775100 & -5.28827400 \\
\hline & 2.02374100 & -0.96493700 & -4.50024500 \\
\hline & 4.38429100 & -0.54054600 & -4.643 \\
\hline & 2.12008200 & -1.07833600 & -3.1 \\
\hline & 4.48763500 & -0.66143800 & 3600 \\
\hline & 3.35330100 & -0.92486400 & -2.484 \\
\hline $\mathrm{C}$ & 3.02231500 & 4.76278100 & -4.12151900 \\
\hline - & 3.49937600 & 4.26566800 & -2.75296000 \\
\hline 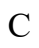 & 3.96935600 & 2.80978400 & -2.75812800 \\
\hline N & 4.40775000 & 2.42035000 & -1.40693600 \\
\hline & -1.91 & 1.66968800 & -5.0 \\
\hline $\mathrm{C}$ & -1.41417000 & 1.50486300 & -3.62081200 \\
\hline $\mathrm{C}$ & -1.57365900 & 0.05595600 & -3.14461800 \\
\hline$c$ & 0.02746600 & 2.00547700 & 49500 \\
\hline $\mathrm{C}$ & -7.11453900 & -2.80976600 & -4.59376000 \\
\hline $\mathrm{C}$ & -8.14636600 & -2.47753200 & -3.50783000 \\
\hline $\mathrm{C}$ & -8.40965700 & 562400 & 393900 \\
\hline 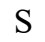 & -9.68611700 & -3.22295900 & -1.26467200 \\
\hline $\mathrm{C}$ & -8.88136100 & -1.81340100 & -0.41831900 \\
\hline $\mathrm{C}$ & -10.86898600 & 0.22095600 & 1483800 \\
\hline $\mathrm{C}$ & -10.28082600 & 0.73095000 & 2948500 \\
\hline $\mathrm{C}$ & -8.93261800 & 1.09618100 & $-2.9^{\prime}$ \\
\hline $\mathrm{C}$ & -11.04475800 & 0.88815300 & -1.86915200 \\
\hline $\mathrm{C}$ & -8.36781500 & 1.63050200 & -1.82636400 \\
\hline $\mathrm{C}$ & -10.49808900 & 1.42914600 & -0.70700300 \\
\hline $\mathrm{C}$ & -9.15954500 & 1.82323200 & -0.68830500 \\
\hline $\mathrm{O}$ & -8.67622400 & 2.41856500 & 0.43944800 \\
\hline $\mathrm{C}$ & -5.34789000 & 4.94023400 & -2.9 \\
\hline $\mathrm{C}$ & -4.59620100 & 4.22068700 & 260700 \\
\hline $\mathrm{C}$ & -5.43214300 & 512800 & -0.98075600 \\
\hline $\mathrm{O}$ & -6.41179100 & 3.67240200 & -0.33007500 \\
\hline $\mathrm{O}$ & -5.01249400 & 2.04670500 & 1600 \\
\hline $\mathrm{C}$ & -9.04910300 & 7.0 & 669800 \\
\hline $\mathrm{C}$ & -9.49667300 & 6.23083200 & -0.40378100 \\
\hline $\mathrm{C}$ & -10.27710600 & 5.00140600 & -0.86345800 \\
\hline $\mathrm{O}$ & -8.38990300 & 5.872851 & 077000 \\
\hline $\mathrm{C}$ & -3.67469000 & 5.82832000 & 4.93342500 \\
\hline $\mathrm{C}$ & -4.23112800 & 6.59697000 & 3.73308100 \\
\hline $\mathrm{C}$ & -4.77352100 & 5.71172000 & 2.59822000 \\
\hline $\mathrm{C}$ & -5.35527100 & 6.57301600 & 1.47251000 \\
\hline $\mathrm{C}$ & -3.71254100 & 4.75742300 & 2.03621300 \\
\hline $\mathrm{C}$ & 1.71320600 & 4.08216000 & 5.20485500 \\
\hline S & 1.44156800 & 3.24591000 & 3.59994100 \\
\hline $\mathrm{C}$ & 2.26827800 & 1.65885700 & 3.94544400 \\
\hline $\mathrm{O}$ & 5.44168800 & -0.20298300 & -0.11329800 \\
\hline $\mathrm{O}$ & 2.92778700 & -0.01852700 & 1.04557800 \\
\hline $\mathrm{O}$ & -6.19379500 & 0.33294100 & 0.34847600 \\
\hline $\mathrm{H}$ & 5.19144100 & 3.00911000 & -1.12533600 \\
\hline $\mathrm{H}$ & 4.75052800 & 1.45967200 & -1.38182600 \\
\hline 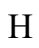 & 4.76842200 & 2.68325100 & -3.50491900 \\
\hline $\mathrm{H}$ & 3.15252000 & 2.14421900 & -3.05703400 \\
\hline $\mathrm{H}$ & 4.33083900 & 4.89172000 & -2.40211200 \\
\hline $\mathrm{H}$ & 2.69667700 & 4.36593000 & -2.01228300 \\
\hline $\mathrm{H}$ & 2.11062000 & 4.24583900 & -4.43741800 \\
\hline $\mathrm{H}$ & 3.78419200 & 4.58777400 & -4.89013800 \\
\hline $\mathrm{H}$ & 5.27134300 & -0.32436000 & -5.23425700 \\
\hline
\end{tabular}

$\mathrm{H}$
$\mathrm{H}$

$\mathrm{H}$

$\mathrm{H}$

$\mathrm{H}$
$\mathrm{H}$

$\mathrm{H}$

$\mathrm{H}$

$\mathrm{H}$

H

$\mathrm{H}$

$\mathrm{H}$

$\mathrm{H}$
$\mathrm{H}$

$\mathrm{H}$

H

$\mathrm{H}$

$$
\begin{aligned}
& \mathrm{H} \\
& \mathrm{H}
\end{aligned}
$$

\section{$\mathrm{H}$}

$\mathrm{H}$

$\mathrm{H}$

$\mathrm{H}$

$\mathrm{H}$
$\mathrm{H}$

$\mathrm{H}$

$\mathrm{H}$

$\mathrm{H}$

$\mathrm{H}$

H

$\mathrm{H}$

H

H

$\mathrm{H}$

\section{$\mathrm{H}$}

H

H

$\mathrm{H}$

H

H

H

$\mathrm{H}$

$\mathrm{H}$

H

H

$\mathrm{H}$

$\mathrm{H}$

H

H

H

H

\section{$\mathrm{H}$}

H

$\mathrm{H}$

$\mathrm{H}$

\section{H}

H

H $\quad 11.81113900 \quad-1.34468600 \quad-0.34827000$

$\begin{array}{lllll}\mathrm{H} & 12.86917900 & -0.04362900 & -2.99743100\end{array}$

$\begin{array}{lllll}\mathrm{H} & 11.89416800 & -0.91314600 & -5.04365700\end{array}$

H $\quad 11.39965000 \quad-2.47061800 \quad-3.19515100$

H $\quad 10.38068200 \quad-0.03764000 \quad-4.76222300$

H $\quad 11.84788400 \quad 1.34323900 \quad-3.48483900$ 


\begin{tabular}{|c|c|c|c|}
\hline $\mathrm{H}$ & .82198700 & -2.31674200 & \\
\hline & 9.05070400 & -0.70970600 & \\
\hline & 10.07871600 & 3.58822800 & -0.24989800 \\
\hline & 9.30554600 & 6.17006000 & -0.62390400 \\
\hline & -4.19222600 & 4.97722800 & \\
\hline & -3.75487100 & 3.65531200 & -2.21954000 \\
\hline & -9.68082800 & 4.39804200 & -1.55682000 \\
\hline & -10.52983100 & 4.37077000 & -0.00938900 \\
\hline & -11.19894100 & 5.28905100 & -1.38002500 \\
\hline & -10.14142400 & 6.83509400 & 0.2467 \\
\hline & -2.80674200 & 5.22704100 & \\
\hline & -5.037 & & \\
\hline & -3.44 & 7.250 & \\
\hline & -4.581 & 7.234 & \\
\hline & -5.7 & 5.953 & \\
\hline & -6.183 & 7.19409 & \\
\hline & -3.261 & 4.12718 & \\
\hline & -4.16 & & \\
\hline & -2.90080600 & 5.31 & \\
\hline & -5.587 & & \\
\hline & 1.24 & & \\
\hline & 1.24 & & \\
\hline & & & \\
\hline & 3.30 & & \\
\hline & 1.74 & 1.10 & \\
\hline & 4.16 & & \\
\hline & 3.3 & & \\
\hline & 2.4 & & \\
\hline & 2.4 & & \\
\hline & 1.3 & -3.4 & \\
\hline & 2.29 & -3.2 & \\
\hline & & -3.23 & \\
\hline & 3.52 & -4.70 & \\
\hline & 2.6 & -3.3 & \\
\hline & & -0.2 & \\
\hline & & & \\
\hline & & -0.6 & \\
\hline & 3.10 & 2.47 & -0. \\
\hline & -5.8 & -0.5 & 100 \\
\hline & & & \\
\hline & 6.50 & & \\
\hline & -6.1 & & -2 \\
\hline & -8.28 & & \\
\hline & -7.84 & & \\
\hline & & -2.16 & \\
\hline & 12.3 & & \\
\hline & 5.029 & -3.260 & 900 \\
\hline & & -8.2 & -2.0760 \\
\hline & 3.4 & -1.3 & -7. \\
\hline & 3.44 & & -7.166 \\
\hline & -1.316 & & -5.7 \\
\hline & & & \\
\hline & -7.97744900 & -8.90521600 & -0.92072200 \\
\hline & -3.97 & -4.07 & -4.208 \\
\hline & -11.94191100 & 0.03 & -4.21975900 \\
\hline & -10.398 & -0.71 & -4.63850100 \\
\hline & -5.82715900 & 0.369 & \\
\hline & -4.56729200 & 5.53446700 & -3.43057700 \\
\hline & & & \\
\hline & -2.30486900 & -4.52205800 & -4.49547100 \\
\hline & -3.28955700 & -5.52337000 & -3.49151600 \\
\hline & -6.69884100 & -1.87660200 & -4.98881300 \\
\hline & -7.50556700 & -3.38074600 & -5.44165000 \\
\hline
\end{tabular}

\begin{tabular}{|c|c|c|c|}
\hline & 10.72428900 & & \\
\hline & -1.90992900 & 2.72553300 & \\
\hline & -2.96496600 & & \\
\hline & 1.92314100 & -0.54679500 & \\
\hline & & & \\
\hline & 7.97682200 & 6.12942400 & \\
\hline & 6.55873300 & 6.66488800 & \\
\hline & 9.63283300 & 5.63718300 & \\
\hline & 12.01109400 & 2.21296500 & \\
\hline & 6.29619600 & -4.41364800 & \\
\hline & 6.08916900 & -3.92978900 & \\
\hline & & -3.678 & \\
\hline & 4.49964 & -3.61 & \\
\hline & -0.14116000 & -8.00 & \\
\hline & & -7.18 & \\
\hline & -7.22442500 & -8.7776 & 0.6 \\
\hline & -6.296533 & -8.3014 & -0.7 \\
\hline & -8.6 & & \\
\hline & -9.8 & 7.33 & -2.2 \\
\hline & & & \\
\hline & -4.4 & 5.12 & \\
\hline & 2.78166 & 4.200 & \\
\hline & -5.5 & & \\
\hline & -0.56870 & 0.18 & \\
\hline & -3.1 & & \\
\hline & -0.7 & -3.20 & \\
\hline & -1.1 & 0.00 & \\
\hline & & -3.7 & \\
\hline & -1.18319 & -1.39 & \\
\hline & -1.01841 & -2.42 & \\
\hline & -0.6 & -2.2 & \\
\hline & -0.30 & -0.88 & \\
\hline & -0.73 & 1.54 & \\
\hline & -2.2 & & \\
\hline & -5.1 & 0.3 & \\
\hline & -6.1 & -0.6 & \\
\hline & & -0.36 & \\
\hline & -7.8 & 0.92 & \\
\hline & -9.1 & & \\
\hline & -6.88 & & \\
\hline & & & \\
\hline & & & \\
\hline & & & \\
\hline & -2.53 & -2.55 & 300 \\
\hline & -0.60938 & & \\
\hline & & -4.22 & \\
\hline & -1.4 & -1.57 & \\
\hline & -0.00972800 & -0.74 & \\
\hline & -0.0 & & \\
\hline & -0.49114800 & 2.30 & \\
\hline & -2.56498100 & 2.69 & \\
\hline & -2.41689400 & 1.3510 & $-0.4 c$ \\
\hline & -5.80026200 & -1.66 & \\
\hline & & & \\
\hline & -9.17744000 & 1.80864900 & \\
\hline & & 2.95 & \\
\hline & -4.80431000 & 2.43677200 & 3.10607400 \\
\hline & -3.66605300 & -0.07151700 & \\
\hline & & -1.13314200 & 3.39527800 \\
\hline $\mathrm{H}$ & -3.33338800 & 0.01618300 & 4.69121400 \\
\hline & & 0.69606700 & \\
\hline $\mathrm{H}$ & -2.52802200 & 1.70996400 & 3.15687100 \\
\hline & 2.24274200 & 2.37523400 & 0.13103300 \\
\hline
\end{tabular}




\section{TS5' (+41.5 kcal/mol)}

\begin{tabular}{|c|c|c|c|}
\hline & 7.01400500 & 5.84041000 & 0.46628900 \\
\hline & 7.42458000 & 5.04554600 & -0.73773000 \\
\hline y & 6.71738700 & 4.17986900 & -1.24303100 \\
\hline & 8.64689400 & 5.38344000 & -1.26015900 \\
\hline & 9.06078500 & 4.83111700 & -2.51741400 \\
\hline C & 10.12743200 & 3.73855200 & -2.48384800 \\
\hline O & 10.58340900 & 3.30761200 & -3.53895500 \\
\hline $\mathbf{N}$ & 10.54428000 & 3.31317600 & -1.26013800 \\
\hline & 11.38526000 & 2.12995100 & -1.18407200 \\
\hline$\tau$ & 10.50427500 & 0.88352200 & -1.25523800 \\
\hline 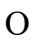 & 9.66739000 & 0.7108 & -0.361 \\
\hline 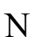 & 10.65104500 & 0.00331100 & -2.27317000 \\
\hline 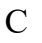 & 9.66765400 & -1.09367400 & -2.38823200 \\
\hline C & 9.67773600 & -2.07002200 & -1.21710500 \\
\hline 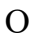 & 8.67900900 & -2.7366 & -0.95476700 \\
\hline $\mathrm{C}$ & 10.02486000 & -1.756 & 58000 \\
\hline 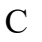 & 10.59981400 & 0900 & -4.55095100 \\
\hline 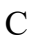 & 11.41809100 & 1800 & -3.52637200 \\
\hline N & 10.8409 & -2.17 & -0.52676000 \\
\hline 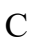 & 10.92608100 & 7700 & 0.67827100 \\
\hline 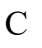 & 10.01657500 & -2.53 & 1.83621700 \\
\hline $\mathrm{O}$ & 9.80592700 & -3.310 & 2.76185000 \\
\hline $\mathbf{v}$ & 9.469 & -1.29 & 1.75049400 \\
\hline $\mathrm{C}$ & 8.410 & -0.90 & 2.670 \\
\hline 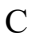 & 7.248 & -1.90 & 2.66253400 \\
\hline O & 6.641 & -2.16 & 3.69395500 \\
\hline $\mathrm{C}$ & 7.9329 & 0.50 & 2.28158100 \\
\hline $\mathrm{C}$ & 6.70717700 & 1.03 & 3.04055900 \\
\hline $\mathrm{C}$ & 6.927 & 1.10 & 4.554 \\
\hline $\mathrm{C}$ & 6.29669200 & 2.39 & 2.46436500 \\
\hline $\mathrm{N}$ & 6.8786 & -2.40 & 1.43546900 \\
\hline $\mathrm{C}$ & 5.924 & -3.50 & 4100 \\
\hline 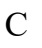 & 4.741 & -2.99 & 35300 \\
\hline C & 3.511 & -3.20 & 5.61470300 \\
\hline $\mathrm{C}$ & 3.60350900 & -2.60 & 4.20475600 \\
\hline $\mathrm{C}$ & 2.36717200 & -2.96 & 3.37048000 \\
\hline $\mathrm{C}$ & 3.80 & -1.08 & 4.24557400 \\
\hline 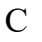 & 0.63782000 & -7.53 & -1.82340500 \\
\hline $\mathrm{C}$ & & & -0.91993800 \\
\hline $\mathrm{C}$ & 2.053 & -5.42 & -1.45732100 \\
\hline $\mathrm{C}$ & 1.56321500 & -4.60170000 & -2.65572100 \\
\hline $\mathrm{C}$ & 2.587 & -4.49149500 & -0.36056200 \\
\hline $\mathrm{C}$ & -7.42225900 & -8.30 & 0.15813800 \\
\hline $\mathrm{C}$ & -7.86964600 & -6.8 & 0.27248500 \\
\hline $\mathrm{C}$ & -6.83416000 & -5.95 & 0.93513300 \\
\hline $\mathrm{C}$ & -5.58614500 & 20900 & 1.37377100 \\
\hline $\mathrm{C}$ & -7.119 & 3500 & 1.09643400 \\
\hline $\mathrm{C}$ & -4.63907000 & -5.53985100 & 1.92048600 \\
\hline $\mathrm{C}$ & -6.187 & -3.709 & 1.63021600 \\
\hline $\mathrm{C}$ & -4.93625900 & -4.18555700 & 2.03329300 \\
\hline $\mathrm{O}$ & -4.02069700 & -3.25866700 & 2.47830700 \\
\hline $\mathrm{C}$ & -3.25799000 & -4.56935300 & -3.44265400 \\
\hline $\mathrm{C}$ & -2.68007000 & -3.78849400 & -2.25619300 \\
\hline $\mathrm{C}$ & -3.58574100 & -3.69394200 & -0.99883100 \\
\hline $\mathrm{C}$ & -4.25229300 & -2.34456700 & -0.84201400 \\
\hline $\mathrm{O}$ & -4.92497200 & -1.81956000 & -1.72229400 \\
\hline م & -4.08660700 & -1.67712300 & 0.30985100 \\
\hline
\end{tabular}

\begin{tabular}{|c|c|c|c|}
\hline & 2.77450300 & -0.75960600 & 700 \\
\hline & 3.06661300 & -0.79378700 & -5.23987900 \\
\hline & 2.08402300 & -1.16104500 & -4.31412300 \\
\hline & 4.34776300 & -0.48639500 & -4.75767000 \\
\hline & 2.36613400 & -1.21822900 & -2.94782400 \\
\hline & 4.63815800 & -0.55990600 & -3.39594800 \\
\hline & 3.64680300 & -0.92697800 & -2.48218000 \\
\hline & 2.83031700 & 4.64757000 & -4.24238900 \\
\hline & 3.20581600 & 4.18969000 & -2.81917900 \\
\hline & 3.62070600 & 2.71822100 & -2.73738200 \\
\hline & 4.10915500 & 2.34820100 & -1.38379100 \\
\hline & -2.11305500 & 1.52358900 & -5.01756400 \\
\hline & -1.46240200 & 1.25845300 & -3.66996900 \\
\hline $\mathrm{C}$ & -1.65443100 & -0.21989800 & -3.31130900 \\
\hline & 0.01256400 & 1.67535600 & -3.73440900 \\
\hline & -7.30736700 & -2.93762200 & -4.32791300 \\
\hline & -8.33141200 & -2.58021600 & -3.24790000 \\
\hline 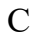 & -8.61871900 & -3.72473900 & -2.27965100 \\
\hline & -9.85615200 & -3.31404300 & -0.98885400 \\
\hline $\mathrm{C}$ & -9.01393000 & -1.88998400 & 753500 \\
\hline & -11.05913800 & 0.10011500 & -4.11062900 \\
\hline & -10.43139500 & 0.63542100 & -2.85709200 \\
\hline & -9.08832700 & 1.02177400 & -2.86947300 \\
\hline c & -11.14489600 & 0.77603000 & -1.66370000 \\
\hline & -8.47243200 & 1.53355100 & 82000 \\
\hline 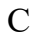 & -10.54501800 & 1.29803500 & -0.51842400 \\
\hline & -9.20457500 & 1.67883800 & \\
\hline 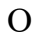 & -8.64206800 & 2.18800700 & 34200 \\
\hline & -5.52488900 & 4.86547700 & -2.97294000 \\
\hline & -4.74094400 & 4.21045800 & 94900 \\
\hline $\mathrm{C}$ & -5.53678300 & 3.27922400 & 537000 \\
\hline & -6.55916900 & 3.63595900 & 15800 \\
\hline 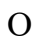 & -5.03122000 & 2.06884700 & 86400 \\
\hline$C$ & -9.21059400 & 7.05965300 & -1.64625900 \\
\hline C & & & \\
\hline & -10.27964300 & 4.91386400 & 32700 \\
\hline U & -8.45930200 & 5.97248300 & 0.39469800 \\
\hline & -3.75754700 & 6.02693100 & 05800 \\
\hline $\mathrm{C}$ & -4.33352700 & 6.78225200 & 3.64303400 \\
\hline & -4.63913500 & 5.90920000 & 2.41313500 \\
\hline c & -5.42577200 & 6.69981900 & 36900 \\
\hline & -3.36111500 & 5.32950500 & 1.79356600 \\
\hline - & & 4.29377700 & \\
\hline & 1.70857100 & 2.81618400 & 4.04714600 \\
\hline & 0.94761100 & 1.61192700 & 5.18704900 \\
\hline & 5.44249400 & -0.02211000 & 0.09444600 \\
\hline & 1.50628400 & 0.07257400 & 2.36118400 \\
\hline & -6.26655300 & 0.03866800 & -0.12238100 \\
\hline & 4.85583800 & 2.99971200 & -1.12973200 \\
\hline & 4.57771800 & 1.44305900 & -1.44930100 \\
\hline & 4.40001800 & 2.52842200 & -3.48896000 \\
\hline & 2.78350600 & 2.06142700 & -2.98971100 \\
\hline & 4.04338400 & 4.80049700 & -2.45529900 \\
\hline & 2.36819400 & 4.36946500 & -2.13369200 \\
\hline & 1.94260400 & 4.12348500 & -4.60963100 \\
\hline & 3.64805900 & 4.45041700 & -4.94438000 \\
\hline & 5.12401400 & -0.19558400 & -5.46169500 \\
\hline & 5.63708500 & -0.33164700 & -3.03399800 \\
\hline & 3.89015700 & -0.97175800 & -1.42645100 \\
\hline & 1.59014900 & -1.51109800 & -2.24906300 \\
\hline & 1.08723900 & -1.41026100 & -4.66423700 \\
\hline & 0.52351300 & 1.16637300 & -4.55799700 \\
\hline & 0.10222400 & 2.75464700 & -3.90022000 \\
\hline
\end{tabular}




\begin{tabular}{|c|c|c|c|}
\hline & & & \\
\hline & & 1.86339000 & \\
\hline & & & \\
\hline & -2.71853300 & -0.47108300 & \\
\hline & -1.19562800 & -0.86400800 & \\
\hline & -7.94797400 & -1.72975700 & -2.67772300 \\
\hline & -9.27148100 & -2.25031300 & 983500 \\
\hline & -7.69256100 & -4.04099800 & 375600 \\
\hline & -9.02765000 & -4.59498800 & \\
\hline & -7.9563 & & -0.0 \\
\hline & -9.1075 & -0.9928 & -0.8 \\
\hline & -9.47 & -1.7136 & \\
\hline & -7.79 & & \\
\hline & -7.42 & & -1.7 \\
\hline & -11.10 & & \\
\hline & -12.18 & & 5500 \\
\hline & -8.50 & & \\
\hline & -2.98 & & \\
\hline & -4.391 & -4.4 & \\
\hline & -1.74 & & \\
\hline & & & \\
\hline & -8.08 & -6.4 & \\
\hline & & & \\
\hline & -8.09 & -4.2 & \\
\hline & -5.32 & -7.4 & \\
\hline & -3.66 & -5.5 & \\
\hline & -6.41 & -2.6 & \\
\hline & & & \\
\hline & & & -0.7 \\
\hline & 1.76 & -3.8 & \\
\hline & 3.02 & & \\
\hline & & & \\
\hline & 1.16 & -5.2 & \\
\hline & & & \\
\hline & 2.88 & & \\
\hline & & & \\
\hline & & & \\
\hline & & -2.3 & \\
\hline & 8.78 & -0.9 & \\
\hline & & & \\
\hline & & & \\
\hline & & & \\
\hline & & & \\
\hline & & & \\
\hline & & & \\
\hline & & & \\
\hline & & & \\
\hline & & & \\
\hline & & -0.7 & \\
\hline & & & 700 \\
\hline & & & \\
\hline & & & \\
\hline & 12.42 & -0.2168 & 63600 \\
\hline & & -0.9 & \\
\hline $\mathrm{H}$ & 10.78 & -2.52707800 & 36700 \\
\hline & & $0.0355^{7}$ & -4.9 \\
\hline $\mathrm{H}$ & 11.47 & & 6800 \\
\hline & 9.154 & -2.23308500 & -4.18 \\
\hline & & -0.67257000 & \\
\hline 11 & 9.94487700 & 3.50936900 & -0.46983700 \\
\hline & 9.15418400 & 6.15812100 & -0.86027300 \\
\hline & & & -1.17602500 \\
\hline & -3.88579900 & 3.65761700 & -2.21440500 \\
\hline
\end{tabular}

\section{$\mathrm{H}$}

$\mathrm{H}$

\section{$\mathrm{H}$}

\section{H}

$\mathrm{H}$
$\mathrm{H}$

$\mathrm{H}$

$\mathrm{H}$
$\mathrm{H}$

\section{$\mathrm{H}$}

$\mathrm{H}$

\begin{tabular}{|c|c|c|}
\hline-9.63727600 & 4.33035600 & -1.50171200 \\
\hline-10.47811000 & 4.30329100 & 0.05087000 \\
\hline-11.22313300 & 5.10039400 & -1.35582700 \\
\hline-10.27976500 & 6.79899700 & 0.20283700 \\
\hline-2.89459300 & 5.41899400 & 4.55262200 \\
\hline-5.25224800 & 7.29838200 & 3.94931700 \\
\hline-3.63015300 & 7.57106200 & 3.33909500 \\
\hline-4.85401100 & 7.57666100 & 1.03175500 \\
\hline-5.63767000 & 6.08250400 & 0.48563800 \\
\hline-6.39195200 & 7.04095100 & 1.74200100 \\
\hline-2.75285800 & 4.77187300 & 2.50999800 \\
\hline-3.58990200 & 4.62573600 & 0.99002400 \\
\hline-2.74168500 & 6.13658300 & 1.38128700 \\
\hline-5.27500300 & 5.07643600 & 2.74518000 \\
\hline 2.06462100 & 5.12711400 & 4.55799800 \\
\hline 0.59814900 & 4.53521800 & 5.36796800 \\
\hline 0.89905900 & 0.66023600 & 4.66063500 \\
\hline 1.55262900 & 1.49619400 & 6.08948300 \\
\hline-0.06352400 & 1.92919500 & 5.45319600 \\
\hline 4.73466300 & -0.82728000 & 4.75840100 \\
\hline 3.88742300 & -0.66831700 & 3.23526400 \\
\hline 2.97753800 & -0.58029800 & 4.75017200 \\
\hline 2.19585100 & -4.04513900 & 3.32844200 \\
\hline 1.45168000 & -2.51871200 & 3.78079400 \\
\hline 2.47972400 & -2.64544700 & 2.32217000 \\
\hline 4.48775000 & -3.03031200 & 3.72695500 \\
\hline 3.31899800 & -4.28289100 & 5.52192700 \\
\hline 2.62680300 & -2.78722900 & 6.11851500 \\
\hline 4.70848300 & 0.29128000 & 0.64779100 \\
\hline 5.81718300 & -0.80135700 & 0.54143500 \\
\hline 1.79208100 & -0.85581400 & 2.38748600 \\
\hline 3.28769700 & 1.88802200 & 0.03092900 \\
\hline-5.87977300 & -0.56770500 & -0.79763200 \\
\hline 8.17911500 & 4.37609200 & -2.97741400 \\
\hline 6.36808200 & 5.22330200 & 1.09245800 \\
\hline-6.29442400 & 5.54281400 & -2.59657800 \\
\hline-8.44717800 & 6.53232200 & -2.23219500 \\
\hline-7.90155500 & 5.30342300 & -0.03444400 \\
\hline 4.95213000 & -1.92048000 & 6.61386300 \\
\hline 12.13103900 & 2.18852400 & -1.97430900 \\
\hline 4.91462700 & -3.16330400 & 1.59675900 \\
\hline 1.44172800 & -8.27493200 & -1.75905300 \\
\hline 3.20465800 & -1.63284700 & -7.22742400 \\
\hline 3.20764500 & 0.12924900 & -7.19288600 \\
\hline-1.59062400 & 0.95403500 & -5.79732500 \\
\hline-6.45968800 & -3.47681200 & -3.89117900 \\
\hline-8.11706500 & -8.91290500 & -0.42881700 \\
\hline-4.16066700 & -4.10794100 & -3.85076700 \\
\hline-12.13177200 & -0.07000600 & -3.98355300 \\
\hline-10.61003600 & -0.85041500 & -4.41666500 \\
\hline-5.89875600 & -0.28370200 & 0.71572900 \\
\hline-4.75054600 & 5.44243700 & -3.48847600 \\
\hline-5.98665400 & 4.16209400 & -3.67373100 \\
\hline-2.49611700 & -4.64498900 & -4.22750500 \\
\hline-3.46813100 & -5.61069800 & -3.17716500 \\
\hline-6.89688800 & -2.01894600 & -4.76024600 \\
\hline-7.70825900 & -3.53814700 & -5.15037200 \\
\hline-10.92547700 & 0.80298700 & -4.93860300 \\
\hline-2.11495900 & 2.56783600 & -5.34453100 \\
\hline-3.16667400 & 1.22660700 & -5.07312900 \\
\hline 1.69959700 & -0.76014800 & -6.93089700 \\
\hline 2.62789200 & 5.72308500 & -4.25856500 \\
\hline 7.84929600 & 6.20419400 & 1.07358200 \\
\hline
\end{tabular}




\begin{tabular}{|c|c|c|c|}
\hline & 6.43002100 & & \\
\hline & 9.45388100 & 5.56150700 & -3.23391300 \\
\hline & 11.88935900 & & \\
\hline & & & \\
\hline & 5.92964600 & -3.89232800 & \\
\hline & 5.65143300 & & \\
\hline & & -3.32166700 & \\
\hline & & & \\
\hline & & & -2.8804870 \\
\hline & & & \\
\hline & & & \\
\hline & & & \\
\hline & -10.0 & & \\
\hline & & & \\
\hline & & & \\
\hline & & & \\
\hline & & & \\
\hline & & & \\
\hline & & & \\
\hline & & & \\
\hline & & & \\
\hline & & & \\
\hline & & & \\
\hline & & & \\
\hline & & & \\
\hline & & & \\
\hline & & & \\
\hline & & & \\
\hline & & & \\
\hline & & & \\
\hline & & & \\
\hline & & & \\
\hline & & & \\
\hline & & & \\
\hline & & & \\
\hline & & & \\
\hline & & & \\
\hline & & & \\
\hline & & & \\
\hline & & & \\
\hline & & & \\
\hline & & & \\
\hline & & & \\
\hline & & & \\
\hline & & & \\
\hline & & & \\
\hline & & & \\
\hline & & & \\
\hline & & & \\
\hline & & & \\
\hline & & & \\
\hline & & & \\
\hline & & & \\
\hline & & & \\
\hline & & & \\
\hline & & 2.08116400 & \\
\hline & & & \\
\hline & & 2.16680900 & \\
\hline
\end{tabular}

\section{Int7' (+15.6 kcal/mol)}

C $\quad-7.04584300 \quad-5.79677200 \quad 0.49408900$

C $\quad-7.45131200 \quad-5.03110200 \quad-0.72500900$

O $\quad-6.73588300 \quad-4.17900000 \quad-1.25841300$

$\mathrm{N} \quad-8.66333900 \quad-5.36259700 \quad-1.24466700$

C $\quad-9.10236900 \quad-4.77752900 \quad-2.47884300$

C $\quad-10.17094100 \quad-3.68962500 \quad-2.38088400$

O $\quad-10.65351700 \quad-3.21968800 \quad-3.40547300$

$\mathrm{N} \quad-10.55406300 \quad-3.30967200 \quad-1.13033600$

C $\quad-11.38529000 \quad-2.12501400 \quad-1.00122600$

C $\quad-10.52373700 \quad-0.87358100 \quad-1.16873800$

O $\quad-9.55370800 \quad-0.72001500 \quad-0.41744900$

$\mathrm{N} \quad-10.85785300 \quad 0.04483100 \quad-2.10378800$

C $\quad-9.97301600 \quad 1.21281100 \quad-2.28107400$

C $\quad-9.85262600 \quad 2.07855100 \quad-1.02995600$

O $\quad-8.83635400 \quad 2.73409200 \quad-0.81609200$

C $\quad-10.59720600 \quad 1.94903100 \quad-3.47888200$

C $\quad-11.20808800 \quad 0.80996700 \quad-4.30617200$

C $\quad-11.78360500 \quad-0.13853700 \quad-3.24696100$

$\begin{array}{llll}\mathrm{N} & -10.93902800 & 2.09726700 & -0.21697800\end{array}$

$\begin{array}{llll}\text { C } & -10.93898300 & 2.83258500 & 1.03426500\end{array}$

$\begin{array}{llll}\text { C } & -9.96899400 & 2.32868900 & 2.11288600\end{array}$

$\begin{array}{llll}\text { O } & -9.77014900 & 3.02295400 & 3.10094200\end{array}$

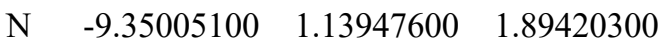

C $\quad-8.22396700 \quad 0.75969800 \quad 2.73850300$

$\begin{array}{llll}\text { C } & -7.09661200 & 1.80541600 & 2.69213100\end{array}$

O $\quad-6.40187100 \quad 1.99961400 \quad 3.67990800$

C $\quad-7.72169900 \quad-0.63373600 \quad 2.31592400$

C $\quad-6.43781500 \quad-1.12327700 \quad 3.00688800$

C $\quad-6.57387500 \quad-1.19415000 \quad 4.53100500$

C $\quad-6.02859000 \quad-2.47894600 \quad 2.41687100$

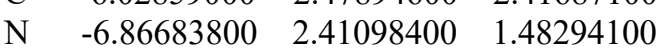

C $\quad-5.94524800 \quad 3.54668600 \quad 1.41419900$

$\begin{array}{llll}\text { C } & -4.74436600 & 3.01805500 & 6.56313200\end{array}$

C $\quad-3.50620500 \quad 3.35513500 \quad 5.69478400$

C $\quad-3.45270800 \quad 2.80307700 \quad 4.26166600$

C $\quad-2.28720000 \quad 3.42366600 \quad 3.47869600$

C $\quad-3.34732100 \quad 1.27420500 \quad 4.24215900$

$\begin{array}{llll}\text { C } & -0.66743400 & 7.57773900 & -1.78064100\end{array}$

C $\quad-0.98889800 \quad 6.38094700 \quad-0.88482100$

C $\quad-2.13744900 \quad 5.51978800 \quad-1.44728100$

C $\quad-1.64696500 \quad 4.69154100 \quad-2.64106000$

C $\quad-2.72954900 \quad 4.59745900 \quad-0.37342200$

$\begin{array}{llll}\text { C } & 7.40051600 & 8.34325300 & 0.17372500\end{array}$

$\begin{array}{llll}\text { C } & 7.82937300 & 6.87678400 & 0.28739900\end{array}$

$\begin{array}{llll}\text { C } & 6.77263900 & 5.99525200 & 0.93631500\end{array}$

$\begin{array}{llll}\text { C } & 5.53345400 & 6.47216800 & 1.37907600\end{array}$

C $\quad 7.02550700 \quad 4.62345200 \quad 1.07885900$

C $\quad 4.56624600 \quad 5.61755600 \quad 1.91625300$

$\begin{array}{llll}\text { C } & 6.07330300 & 3.75918700 & 1.60203600\end{array}$

C $\quad 4.83132000 \quad 4.25449500 \quad 2.01483000$

O $\quad 3.90781100 \quad 3.34569600 \quad 2.46326400$

C $\quad 3.22037700 \quad 4.61658500 \quad-3.42230100$

C $\quad 2.58626400 \quad 3.84061800 \quad-2.25566600$

C $\quad 3.40806700 \quad 3.70726200 \quad-0.95044500$

C $\quad 4.14647400 \quad 2.39053700 \quad-0.81930800$

O $\quad 4.84893700 \quad 1.91188000 \quad-1.70406500$

$\begin{array}{llll}\mathrm{O} & 4.02040400 & 1.70347200 & 0.32285300\end{array}$

$\begin{array}{llll}\mathrm{C} & -2.82700700 & 0.82059200 & -6.69112700\end{array}$

C $\quad-3.09659400 \quad 0.85234900 \quad-5.20553600$

C $\quad-2.11249400 \quad 1.27264400 \quad-4.30389400$ 


\begin{tabular}{|c|c|c|c|}
\hline & -4.36127300 & 0.51233100 & \\
\hline & -2.38659400 & 1.38652200 & -2.93939200 \\
\hline & -4.64197800 & 0.62774900 & -3.33907800 \\
\hline & -3.65775800 & 1.08041700 & -2.45512800 \\
\hline & -2.87798900 & -4.59358000 & -4.22618600 \\
\hline 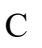 & -3.42580700 & -4.20957400 & -2.83558100 \\
\hline & -3.80087000 & -2.73177900 & -2.68840200 \\
\hline $\mathbf{N}$ & -4.44315400 & -2.48140500 & -1.35058700 \\
\hline C & 2.06502500 & -1.47113100 & -5.01025000 \\
\hline & 1.46905500 & -1.27644000 & -3.61 \\
\hline c & 1.6844 & 0.16392700 & 95900 \\
\hline & -0.01501000 & -1.67131500 & 62800 \\
\hline 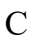 & 7.26507500 & 2.98419700 & 76500 \\
\hline C & 8.29258600 & 2.62226500 & -3.249 \\
\hline 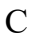 & 8.56494000 & 3.75506400 & 29000 \\
\hline & 9.817 & 3.34742400 & 36000 \\
\hline C & 9.00754800 & 1.89982800 & 86800 \\
\hline 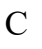 & 11.015 & -0.05708200 & -4.1 \\
\hline $\mathrm{C}$ & 10.41 & 37200 & -2.87 \\
\hline 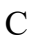 & 9.071 & -0.99518800 & -2.86920100 \\
\hline $\mathrm{C}$ & 11.15 & 34600 & -1.70 \\
\hline $\mathrm{C}$ & 8.480 & -1.5370 & $-1.73^{\prime}$ \\
\hline $\mathrm{C}$ & 10.57915100 & -1.32482400 & -0.55767900 \\
\hline C & 9.23 & -1.7113 & -0.57 \\
\hline 0 & 8.710 & -2.25985000 & 13900 \\
\hline 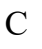 & 5.481 & -4.82137600 & -2.98 \\
\hline $\mathrm{C}$ & 4.72 & -4.1 & -1.8 \\
\hline 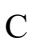 & 5.537 & -3.233 & 75000 \\
\hline O & 6.536 & -3.6 & -0.33 \\
\hline $\mathrm{O}$ & 5.077 & -2.006726 & -0.99 \\
\hline 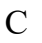 & 9.170 & -7.02209600 & -1.6 \\
\hline $\mathrm{C}$ & 9.576 & -6.1969 & $-0.4 c$ \\
\hline $\mathrm{C}$ & 10.29268500 & -4.908 & -0.8 \\
\hline $\mathrm{O}$ & 8.44959600 & $-5.930 S$ & \\
\hline $\mathrm{C}$ & 3.741 & -6.0 & \\
\hline 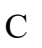 & 4.318 & -6.7 & 000 \\
\hline C & 4.65 & -5.8 & 5400 \\
\hline $\mathrm{C}$ & 5.418 & -6.68 & 1700 \\
\hline $\mathrm{C}$ & 3.406 & -5.2438 & 1.8 \\
\hline $\mathrm{C}$ & -1.647 & -4.2 & 80000 \\
\hline 5 & -1.212 & -3.45663600 & 39900 \\
\hline $\mathrm{C}$ & -1.2 & -1.7 & \\
\hline $\mathrm{O}$ & -5.20 & -0.04 & 600 \\
\hline $\mathrm{O}$ & -2.450 & 0.09949300 & 91300 \\
\hline $\mathrm{O}$ & 6.25590000 & 0.0226 & -0.129 \\
\hline $\mathrm{H}$ & -5.26797200 & -3.091 & 27600 \\
\hline $\mathrm{H}$ & -4.75729300 & -1.50549800 & -1.21 \\
\hline $\mathrm{H}$ & -4.51233700 & -2.41664400 & -3.45491800 \\
\hline $\mathrm{H}$ & -2.92947800 & -2.07750200 & -2.76035900 \\
\hline $\mathrm{H}$ & -4.31828000 & -4.81 & -2.62 \\
\hline $\mathrm{H}$ & -2.68492600 & -4.45829300 & -2.06623600 \\
\hline $\mathrm{H}$ & -1.94944000 & -4.06258900 & -4.45253500 \\
\hline $\mathrm{H}$ & -3.60230900 & -4.36727900 & -5.01531900 \\
\hline $\mathrm{H}$ & -5.13596600 & 0.18078200 & -5.38703800 \\
\hline $\mathrm{H}$ & -5.63464100 & 0.39612100 & -2.96129400 \\
\hline $\mathrm{H}$ & -3.90625300 & 1.21479800 & -1.41037500 \\
\hline $\mathrm{H}$ & -1.62272100 & 1.76264500 & -2.26786400 \\
\hline $\mathrm{H}$ & -1.12715600 & 1.54009200 & -4.67364500 \\
\hline $\mathrm{H}$ & -0.57049600 & -1.06808600 & -4.36099500 \\
\hline $\mathrm{H}$ & -0.13243200 & -2.72580300 & -3.91039500 \\
\hline $\mathrm{H}$ & -0.48887600 & -1.52462500 & -2.65929600 \\
\hline $\mathrm{H}$ & & -1.94222200 & -2.91882500 \\
\hline & 1.30129800 & 0.32333100 & -2.13374800 \\
\hline
\end{tabular}
$\begin{array}{lrrr}\mathrm{H} & 10.50580400 & -4.30524500 & 0.02566900 \\ \mathrm{H} & 11.23136700 & -5.12042900 & -1.38144200\end{array}$

$\begin{array}{llll}2.74877300 & 0.41802100 & -3.12797600\end{array}$ $\begin{array}{llll}1.18002700 & 0.87318100 & -3.81157400\end{array}$ $\begin{array}{llll}7.91881100 & 1.75916600 & -2.69209400\end{array}$ $\begin{array}{llll}9.23724500 & 2.31090800 & -3.70580700\end{array}$ $\begin{array}{llll}7.63576600 & 4.04614900 & -1.75834500\end{array}$ $\begin{array}{llll}8.95587600 & 4.64115000 & -2.77535100\end{array}$ $\begin{array}{llll}7.95022900 & 2.10342400 & -0.03816700\end{array}$ $\begin{array}{llll}9.10035000 & 1.01373300 & -0.83476600\end{array}$ $\begin{array}{llll}9.48889000 & 1.71111300 & 0.74758300\end{array}$ $\begin{array}{lll}7.83188100 & -2.65396600 & 0.34764800\end{array}$ $\begin{array}{llll}7.43504600 & -1.79376200 & -1.75012300\end{array}$ $\begin{array}{llll}11.15563000 & -1.46173300 & 0.35109700\end{array}$ $12.19686100 \quad-0.47181100-1.68145000$ $\begin{array}{llll}8.47352800 & -0.86246200 & -3.76735800\end{array}$ $\begin{array}{llll}2.73039900 & 3.83061600 & -0.10423700\end{array}$ $\begin{array}{llll}4.16412000 & 4.49863000 & -0.88328700\end{array}$ $\begin{array}{llll}1.64720900 & 4.32729100 & -1.97789500\end{array}$ $\begin{array}{llll}2.30663200 & 2.84147000 & -2.60318000\end{array}$ $\begin{array}{llll}8.04917100 & 6.47183000 & -0.70871800\end{array}$ $\begin{array}{llll}8.76798700 & 6.78954500 & 0.84862000\end{array}$ $\begin{array}{llll}7.98784200 & 4.23558500 & 0.76027600\end{array}$ $\begin{array}{llll}5.29368000 & 7.52658600 & 1.30802300\end{array}$ $\begin{array}{lll}3.60448200 & 6.00807400 & 2.23664000\end{array}$ $\begin{array}{llll}6.26756700 & 2.69774000 & 1.67648300\end{array}$ $\begin{array}{lll}3.01603400 & 3.73981800 & 2.62879500\end{array}$ $\begin{array}{llll}-3.52426000 & 3.96708400 & -0.79276300\end{array}$ $\begin{array}{lll}-1.93824400 & 3.95864900 & 0.03117900\end{array}$ $\begin{array}{llll}-3.15921200 & 5.17554500 & 0.45305900\end{array}$ $\begin{array}{llll}-2.46365100 & 4.12302900 & -3.09584700\end{array}$ $\begin{array}{llll}-1.20294400 & 5.31566400 & -3.42333600\end{array}$ $\begin{array}{llll}-0.88262500 & 3.98668400 & -2.29581000\end{array}$ $\begin{array}{llll}-2.93104500 & 6.19873300 & -1.79832500\end{array}$ $\begin{array}{llll}-0.11614800 & 5.73668600 & -0.73831800\end{array}$ $\begin{array}{lll}-1.26358900 & 6.74734200 & 0.11298400\end{array}$ $\begin{array}{llll}-7.63656300 & 2.43499600 & 0.81897600\end{array}$ $\begin{array}{lll}-8.54656900 & 0.75521400 & 3.78148500\end{array}$ $\begin{array}{lll}-8.53398800 & -1.34978100 & 2.50174200\end{array}$ $\begin{array}{lll}-7.55650600 & -0.63006500 & 1.23399800\end{array}$ $\begin{array}{lll}-6.77408000 & -3.24851100 & 2.65822900\end{array}$ $\begin{array}{lll}-5.93476200 & -2.41387000 & 1.32903100\end{array}$ $\begin{array}{lll}-5.06462200 & -2.80833500 & 2.81911400\end{array}$ $\begin{array}{llll}-7.41356300 & -1.83790900 & 4.82278700\end{array}$ $\begin{array}{lll}-5.66505300 & -1.60973300 & 4.97946000\end{array}$ $\begin{array}{lll}-6.72743400 & -0.19991500 & 4.95503100\end{array}$ $\begin{array}{lll}-5.63824100 & -0.41198900 & 2.77565900\end{array}$ $\begin{array}{lll}-9.49670600 & 0.61841700 & 1.03642600\end{array}$ $\begin{array}{llll}-10.65545200 & 3.87187500 & 0.85270200\end{array}$ $\begin{array}{lll}-11.95100500 & 2.83151000 & 1.44449300\end{array}$ $\begin{array}{llll}-11.70027000 & 1.47123900 & -0.42958100\end{array}$ $\begin{array}{llll}-12.80043700 & 0.15635100 & -2.96091600\end{array}$ $\begin{array}{llll}-11.96844600 & 1.15048200 & -5.01212900\end{array}$ $\begin{array}{lll}-11.38088300 & 2.62918200 & -3.12727300\end{array}$ $\begin{array}{llll}-10.42637600 & 0.29261200 & -4.87160500\end{array}$ $-11.77928800 \quad-1.18024200 \quad-3.56847200$ $\begin{array}{llll}-9.85491000 & 2.53960600 & -4.01728300\end{array}$ $\begin{array}{llll}-8.95650100 & 0.87399200 & -2.50290800\end{array}$ $-9.92304100 \quad-3.51268500-0.36750500$ $-9.19868500 \quad-6.10288100-0.81654300$ $\begin{array}{llll}4.34628300 & -4.96335600 & -1.16489900\end{array}$ $\begin{array}{llll}3.85824600 & -3.61468600 & -2.19509700\end{array}$ $\begin{array}{llll}9.66723500 & -4.30779600 & -1.52789400\end{array}$ 


\begin{tabular}{|c|c|c|c|}
\hline & 10.24841900 & -6.79718800 & \\
\hline & 2.87955100 & -5.39495900 & \\
\hline & & & \\
\hline & 3.60201100 & -7.52881300 & \\
\hline & 4.81517200 & -7.53529800 & 1.01285700 \\
\hline & 5.65795900 & -6.06386800 & 0.49061500 \\
\hline & .36967600 & -7.06880100 & 7900 \\
\hline & 2.82640700 & -4.65864500 & \\
\hline & 3.66998200 & -4.54771700 & 385200 \\
\hline & 59100 & -6.022 & 200 \\
\hline & 5.323 & -5.07 & 700 \\
\hline & & & \\
\hline & -0.87 & -4.0 & \\
\hline & -0.919 & 4700 & 700 \\
\hline & -2.204 & & \\
\hline & -0.47 & -1.5 & \\
\hline & -4.20 & & \\
\hline & -3.31 & & \\
\hline & -2.42 & 0.9 & \\
\hline & -2.37 & & \\
\hline & -1.3 & & \\
\hline & -2.25 & & \\
\hline & -4.39 & & \\
\hline & -3.41 & & \\
\hline & -2.60 & & \\
\hline & -4.3 & & \\
\hline & -5.790 & & \\
\hline & -2.344 & & \\
\hline & -3.776 & -2.6 & \\
\hline & 5.823 & & \\
\hline & -8.23 & & \\
\hline & -6.41 & & \\
\hline & 6.25 & -5.5 & \\
\hline 1 & & -6. & -2.2 \\
\hline & 7.897 & & \\
\hline & -4.909 & & \\
\hline & -12.19 & & 4000 \\
\hline & -4.927 & & \\
\hline & -1.46 & & 200 \\
\hline & -3.258 & & \\
\hline & -3.268 & & \\
\hline & & -0.8 & \\
\hline & & & \\
\hline & & & \\
\hline & & & \\
\hline & 12.0 & & \\
\hline & 10.5 & 600 & 3300 \\
\hline & & & \\
\hline & & -5.3 & \\
\hline & & -4.11 & -3.6 \\
\hline & & & \\
\hline & & & \\
\hline & & 2.06 & \\
\hline & & & \\
\hline & 10.87805800 & 41800 & -4.96106700 \\
\hline & & -2.51 & \\
\hline & 3.11859200 & -1.17 & -5.06 \\
\hline & -1.75285200 & 0.82 & -6.90 \\
\hline & & -5.66 & \\
\hline & -7.87877500 & -6.16095700 & 1.10413000 \\
\hline & -6.44712800 & -6.66153400 & 0.18671000 \\
\hline & -9.49847700 & -5.50506700 & -3.19607800 \\
\hline & -11.80454700 & -2.09935000 & 0.00783200 \\
\hline
\end{tabular}

\begin{tabular}{|c|c|c|c|}
\hline & 6.19941500 & 4.35077800 & \\
\hline & -5.96620000 & & 0.39634600 \\
\hline & -5.65550900 & 3.46168500 & .14831500 \\
\hline & -4.44895300 & 3.34335300 & .56685400 \\
\hline & 0.27162300 & 8.08466400 & -1.53478800 \\
\hline & -0.58470600 & 7.30875000 & \\
\hline & 7.33282000 & 8.74758000 & \\
\hline & 6.41714600 & 8.32795400 & -0.30566000 \\
\hline & 8.76717100 & -8.00540000 & 38500 \\
\hline & 9.99618800 & -7.24140600 & \\
\hline & 3.37507200 & -6.71480400 & \\
\hline & 4.47674200 & -5.32176200 & \\
\hline & -2.62137300 & -3.92962700 & 0900 \\
\hline & $5.631^{\prime}$ & -1.28352800 & \\
\hline & 0.747 & -0.04623 & \\
\hline & 2.733 & -1.955 & \\
\hline & 0.235 & & \\
\hline & 1.39709800 & -0.011280 & \\
\hline & 1.27603300 & 3.656154 & \\
\hline & 1.54 & & \\
\hline & 1.10 & 2.383 & \\
\hline & 0.50 & 2.404 & \\
\hline & 0.28 & & \\
\hline & 0.57 & -1.399 & \\
\hline & & & \\
\hline & 4.366 & -0.99483 & \\
\hline & 4.929 & 0.28426 & \\
\hline & 6.27 & & \\
\hline & 7.08 & -0.573 & \\
\hline & & & \\
\hline & 6.540 & -1.865 & \\
\hline & 5.20320800 & -2.0571 & \\
\hline & 3.45 & -1.295 & \\
\hline & -2.49 & -1.6736 & \\
\hline & 3.651 & 2.2304 & \\
\hline $\mathrm{H}$ & -1.63 & 0.55 & \\
\hline & 0.85323500 & 4.17980100 & \\
\hline & & & \\
\hline $\mathrm{H}$ & -0.11 & 1.097 & \\
\hline & 0.22 & -1.306 & \\
\hline & -0.19 & -1.96965900 & 100 \\
\hline & 1.634 & -3.29199500 & \\
\hline & 2.49 & -2.013 & \\
\hline & 4.32293600 & 1.14189 & \\
\hline & 6.713 & 1.48326700 & \\
\hline $\mathrm{H}$ & 8.68091800 & & \\
\hline $\mathrm{H}$ & 7.16285300 & -2.71276700 & 14100 \\
\hline & 4.78564000 & -3.05495000 & \\
\hline & 2.91076900 & -1.28718100 & 3.76683600 \\
\hline & 2.50357300 & -0.57335400 & 4.48910800 \\
\hline & 2.85364100 & -2.27297700 & 4.23960700 \\
\hline & 1.99853200 & -1.33526000 & 2.51552700 \\
\hline & & -2.03796500 & \\
\hline $\mathrm{O}$ & -2.68173500 & -2.62669800 & 0.67492600 \\
\hline $\mathrm{H}$ & -2.56527700 & -3.07816700 & 1.52893900 \\
\hline
\end{tabular}

\section{Int8' (+9.1 kcal/mol)}

C $\quad-6.97339200 \quad-5.84248900 \quad 0.34600800$

C $\quad-7.38547500 \quad-5.02818200 \quad-0.84029400$

O $\quad-6.66957400 \quad-4.16018500 \quad-1.34705200$ 
$\mathrm{N} \quad-8.60458300 \quad-5.33981600 \quad-1.36188500$

C $\quad-9.04322500 \quad-4.73354800 \quad-2.58536500$

C $\quad-10.11599600 \quad-3.64976300 \quad-2.48125200$

O $\quad-10.59534100 \quad-3.17557800 \quad-3.50579300$

$\mathrm{N} \quad-10.50908200 \quad-3.27637600 \quad-1.23193700$

C $\quad-11.35386100 \quad-2.10006700 \quad-1.10966600$

C $\quad-10.50300600 \quad-0.83945300 \quad-1.25987900$

$\begin{array}{lllll}\mathrm{O} & -9.54005600 & -0.68706100 & -0.49938700\end{array}$

$\begin{array}{llll}\mathrm{N} & -10.83328200 & 0.08978400 & -2.18489800\end{array}$

$\begin{array}{lllll}\text { C } & -9.94420500 & 1.25902900 & -2.34188400\end{array}$

C $\quad-9.82729700 \quad 2.10701900 \quad-1.07702200$

$\begin{array}{lllll}\text { O } & -8.80078800 & 2.73457600 & -0.83129300\end{array}$

$\begin{array}{llll}\text { C } & -10.56078300 & 2.00933200 & -3.53456000\end{array}$

C $\quad-11.16072500 \quad 0.87918000 \quad-4.38239200$

C $\quad-11.74351800 \quad-0.08533700 \quad-3.34142800$

$\begin{array}{llll}\mathrm{N} & -10.93107700 & 2.14289600 & -0.28680800\end{array}$

$\begin{array}{llll}\text { C } & -10.93985700 & 2.86454500 & 0.97283800\end{array}$

$\begin{array}{llll}\text { C } & -10.00912700 & 2.31939500 & 2.06649700\end{array}$

$\begin{array}{llll}\mathrm{O} & -9.81619600 & 2.98753700 & 3.07359200\end{array}$

$\begin{array}{llll}\mathrm{N} & -9.41642200 & 1.12084400 & 1.83333900\end{array}$

$\begin{array}{llll}\text { C } & -8.32366900 & 0.67385400 & 2.68663400\end{array}$

$\begin{array}{llll}\text { C } & -7.18002400 & 1.69968000 & 2.73845600\end{array}$

$\begin{array}{llll}\text { O } & -6.52301400 & 1.83439900 & 3.76331000\end{array}$

$\begin{array}{llll}\text { C } & -7.83513600 & -0.69417000 & 2.17681100\end{array}$

$\begin{array}{llll}\text { C } & -6.57722200 & -1.26957200 & 2.84212700\end{array}$

C $\quad-6.70028500 \quad-1.39038700 \quad 4.36391700$

$\begin{array}{llll}\text { C } & -6.25165000 & -2.62383100 & 2.19933300\end{array}$

$\begin{array}{llll}\mathrm{N} & -6.89043200 & 2.35888900 & 1.57523600\end{array}$

$\begin{array}{llll}\text { C } & -5.94044500 & 3.47018000 & 1.59601000\end{array}$

$\begin{array}{llll}\text { C } & -4.72583300 & 2.76714300 & 6.72094700\end{array}$

$\begin{array}{llll}\text { C } & -3.50207800 & 3.05181600 & 5.82685900\end{array}$

$\begin{array}{llll}\text { C } & -3.58265400 & 2.48901700 & 4.40206600\end{array}$

C $\quad-2.42140700 \quad 2.97995600 \quad 3.52749100$

$\begin{array}{llll}\text { C } & -3.63808200 & 0.95768300 & 4.40931000\end{array}$

C $\quad-0.69898700 \quad 7.65166200 \quad-1.46248600$

$\begin{array}{llll}\text { C } & -1.03519100 & 6.42670400 & -0.61746300\end{array}$

C $\quad-2.41981400 \quad 5.84016300 \quad-0.95833600$

C $\quad-2.43919800 \quad 5.22716700 \quad-2.36426000$

$\begin{array}{llll}\text { C } & -2.87161800 & 4.81147600 & 0.08406800\end{array}$

$\begin{array}{llll}\text { C } & 7.36695100 & 8.40740200 & 0.50489900\end{array}$

$\begin{array}{llll}\text { C } & 7.77653800 & 6.93150600 & 0.57938600\end{array}$

$\begin{array}{llll}\text { C } & 6.70162500 & 6.03767700 & 1.18583300\end{array}$

$\begin{array}{llll}\text { C } & 5.41030300 & 6.47277500 & 1.51097900\end{array}$

$\begin{array}{llll}\text { C } & 6.99189900 & 4.68202200 & 1.39699900\end{array}$

$\begin{array}{llll}\text { C } & 4.42807100 & 5.59333900 & 1.98074400\end{array}$

C $\quad 6.02759200 \quad 3.79665500 \quad 1.85817100$

$\begin{array}{llll}\text { C } & 4.72532200 & 4.23933200 & 2.13382600\end{array}$

$\begin{array}{llll}\text { O } & 3.79862400 & 3.29890100 & 2.48419800\end{array}$

C $\quad 3.20800200 \quad 4.77981300 \quad-3.21515800$

C $\quad 2.62447700 \quad 3.91776300 \quad-2.09090100$

C $\quad 3.48672900 \quad 3.74172700 \quad-0.82065100$

C $\quad 4.24896100 \quad 2.43166200 \quad-0.75428900$

O $\quad 4.88908300 \quad 1.96571900 \quad-1.69372600$

O $\quad 4.21674300 \quad 1.74941500 \quad 0.39140300$

C $\quad-2.81776900 \quad 1.05743300 \quad-6.60611300$

$\begin{array}{llll}\text { C } & -2.98374900 & 1.12850700 & -5.10259900\end{array}$

C $\quad-1.93963900 \quad 1.62947900 \quad-4.31279400$

C $\quad-4.15914600 \quad 0.71831900 \quad-4.45936400$

C $\quad-2.05633500 \quad 1.72472700 \quad-2.92622100$

C $\quad-4.28463800 \quad 0.81260100 \quad-3.07127700$

$\begin{array}{lllll}\text { C } & -3.23286200 & 1.31322000 & -2.29991500\end{array}$

C $\quad-2.82418600 \quad-4.44087000 \quad-4.33553700$

C $\quad-3.33275700 \quad-4.12076800 \quad-2.91478500$$$
\text { C }
$$

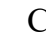

C

C$$
\text { O }
$$

$\begin{array}{lll}-3.68012300 & -2.64907100 & -2.67584100\end{array}$ $\begin{array}{lll}-4.30366300 & -2.46789500 & -1.31986300\end{array}$ $\begin{array}{lll}2.09424700 & -1.25613000 & -5.01630900\end{array}$ $\begin{array}{lll}1.52298400 & -1.10735500 & -3.60752000\end{array}$ $\begin{array}{lll}1.77872900 & 0.30552500 & -3.07186300\end{array}$ $\begin{array}{lll}0.02925800 & -1.46027200 & -3.60450900\end{array}$ $\begin{array}{llll}7.26259500 & 3.21116600 & -4.18351100\end{array}$ $\begin{array}{lll}8.28840500 & 2.81745300 & -3.11492600\end{array}$ $\begin{array}{llll}8.53445600 & 3.90428200 & -2.06964900\end{array}$ $\begin{array}{llll}9.77081300 & 3.44405100 & -0.79328600\end{array}$ $\begin{array}{llll}8.92923700 & 1.99267100 & -0.06674400\end{array}$ $\begin{array}{llll}11.03584300 & 0.19304800 & -4.10304000\end{array}$ $\begin{array}{lll}10.44112800 & -0.41155800 & -2.86864600\end{array}$ $\begin{array}{llll}9.11774300 & -0.85806900 & -2.89496400\end{array}$ $\begin{array}{lll}11.16948500 & -0.56972200 & -1.68747700\end{array}$ $\begin{array}{lll}8.53808900 & -1.46078800 & -1.78971100\end{array}$ $\begin{array}{lll}10.60486800 & -1.17858100 & -0.56861600\end{array}$ $\begin{array}{lll}9.28822100 & -1.63203200 & -0.62240700\end{array}$ $\begin{array}{lll}8.77324400 & -2.24972400 & 0.48356600\end{array}$ $\begin{array}{lll}5.53962800 & -4.65015300 & -3.11960000\end{array}$ $\begin{array}{lll}4.76334300 & -4.06230800 & -1.92684900\end{array}$ $\begin{array}{lll}5.56431000 & -3.16289400 & -1.02325200\end{array}$ $\begin{array}{lll}6.59234800 & -3.54624800 & -0.45258500\end{array}$ $\begin{array}{llll}5.06216500 & -1.95582500 & -0.95001400\end{array}$ $\begin{array}{llll}9.24714400 & -6.86832000 & -1.89777300\end{array}$ $\begin{array}{llll}9.65705200 & -6.08621200 & -0.65093400\end{array}$ $\begin{array}{llll}10.38358400 & -4.79173200 & -1.00784200\end{array}$ $\begin{array}{lll}8.53161900 & -5.84164500 & 0.18928000\end{array}$ $3.82370300 \quad-6.12257000 \quad 4.66029800$ $\begin{array}{lll}4.42837800 & -6.85401300 & 3.45297900\end{array}$ $\begin{array}{lll}4.72413100 & -5.96879900 & 2.23150200\end{array}$ $\begin{array}{lll}5.54851400 & -6.72978200 & 1.18786900\end{array}$ $\begin{array}{lll}3.43461300 & -5.43265600 & 1.60144300\end{array}$ $\begin{array}{lll}-1.57754500 & -4.43815800 & 5.02398600\end{array}$ $\begin{array}{lll}-0.97287100 & -3.61923200 & 3.50740400\end{array}$ $\begin{array}{lll}-1.16478500 & -1.87745800 & 4.02135300\end{array}$ $\begin{array}{lll}-5.03320600 & -0.07581600 & 0.06824100\end{array}$ $\begin{array}{lll}-2.55994800 & 0.03829600 & 1.36412700\end{array}$ $\begin{array}{llll}6.33081100 & 0.03782100 & -0.17796500\end{array}$ $-5.13190100 \quad-3.07741800-1.21776100$ $\begin{array}{lll}-4.61272200 & -1.49544900 & -1.12824400\end{array}$ $\begin{array}{lll}-4.39711800 & -2.28021500 & -3.41278900\end{array}$ $\begin{array}{lll}-2.80184500 & -2.00199700 & -2.72224900\end{array}$ $\begin{array}{lll}-4.22866600 & -4.72124200 & -2.71093300\end{array}$ $\begin{array}{llll}-2.57661500 & -4.42644700 & -2.18095700\end{array}$ $\begin{array}{lll}-1.90639100 & -3.89381600 & -4.56794900\end{array}$ $\begin{array}{lll}-3.57357100 & -4.19301600 & -5.09371700\end{array}$ $\begin{array}{llll}-4.98449400 & 0.33094400 & -5.05291200\end{array}$ $\begin{array}{llll}-5.20591900 & 0.51069100 & -2.58136800\end{array}$ $\begin{array}{lll}-3.33817800 & 1.38855200 & -1.22434300\end{array}$ $\begin{array}{lll}-1.23965000 & 2.12895100 & -2.33774400\end{array}$ $\begin{array}{lll}-1.01933100 & 1.95184800 & -4.79238400\end{array}$ $-0.52365500 \quad-0.82008500-4.30050200$ $\begin{array}{lll}-0.12780500 & -2.50362700 & -3.90365400\end{array}$ $\begin{array}{lll}-0.41374800 & -1.31846600 & -2.61464200\end{array}$ $\begin{array}{lll}2.04435300 & -1.81362200 & -2.94619300\end{array}$ $\begin{array}{llll}1.41272500 & 0.42139700 & -2.04974400\end{array}$ $\begin{array}{lll}2.84787500 & 0.53848100 & -3.06600700\end{array}$ $\begin{array}{lll}1.27333800 & 1.05272100 & -3.69687700\end{array}$ $\begin{array}{llll}7.92425200 & 1.92187500 & -2.60369300\end{array}$ $\begin{array}{lll}9.24237600 & 2.54387400 & -3.57577400\end{array}$ $\begin{array}{llll}7.59640700 & 4.16311900 & -1.56354500\end{array}$ $\begin{array}{llll}8.92459700 & 4.81770500 & -2.53182300\end{array}$ 


\begin{tabular}{|c|c|c|c|}
\hline & & .23453500 & \\
\hline & & 1.13282600 & \\
\hline & & & \\
\hline & 9496700 & -2.63596600 & 0.25781900 \\
\hline & & -1.77468200 & -1.82529900 \\
\hline & 11.17345800 & -1.31240800 & 0.34564000 \\
\hline & 12.19612900 & -0.21801500 & -1.64221000 \\
\hline & & -0.73054500 & -3.79995100 \\
\hline & .85054800 & 3.82409400 & \\
\hline & .23393200 & 0500 & 7900 \\
\hline & .67062600 & 4.352 & -1.77 \\
\hline & & & \\
\hline & & & -0.4 \\
\hline & 00 & 600 & \\
\hline & & & \\
\hline & 5.1378 & & \\
\hline & 3.4253 & & \\
\hline & & & \\
\hline & 300 & & \\
\hline & & & \\
\hline & -2.16 & & \\
\hline & -2.945 & & 700 \\
\hline & & & \\
\hline & -2.2364 & & -3 . \\
\hline & -1.6822 & 000 & -2 \\
\hline 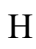 & -3.13 & & -0 . \\
\hline & -0.27311 & & -0 . \\
\hline & -1.01213 & & \\
\hline & -7.6 & & \\
\hline & -8.6 & & \\
\hline & -8.6 & & 200 \\
\hline & -7.647 & & \\
\hline & -7.000 & -3.3 & 500 \\
\hline H & -6.246 & 600 & 000 \\
\hline & -5.264 & 600 & 600 \\
\hline & -7.565 & & \\
\hline & & & \\
\hline & -6.80 & -0.4 & 700 \\
\hline & -5.7 & 600 & 200 \\
\hline & -9.558 & & \\
\hline & & 2900 & 400 \\
\hline & & 200 & 500 \\
\hline & -11.700 & 0100 & 0300 \\
\hline & & & \\
\hline & & 9800 & 500 \\
\hline & -11.3 & 59400 & 2000 \\
\hline & -10.371 & 0100 & 300 \\
\hline & -11.72905400 & 45200 & 1400 \\
\hline & & & \\
\hline & -8.92756800 & & 3400 \\
\hline & -9.88194000 & 32200 & 5000 \\
\hline & & & \\
\hline & 4.39522100 & -4.89531600 & 1500 \\
\hline & & -3.50 & \\
\hline & 9.76658100 & -4.16634800 & -1.66240300 \\
\hline & 10.59731100 & -4.21600500 & 50600 \\
\hline & & & 1500 \\
\hline & 10.32439700 & -6.71100900 & 76400 \\
\hline & & & \\
\hline & 5.35504300 & -7.35148700 & 3.76697500 \\
\hline & 3.74727600 & -7.65750200 & 3.13603100 \\
\hline & & -7.62071300 & 0.84347600 \\
\hline & 5.75678400 & -6.10094500 & 0.31839100 \\
\hline
\end{tabular}

$\mathrm{H}$

$\mathrm{H}$
$\mathrm{H}$

$\mathrm{H}$

$\mathrm{H}$

H

\section{$\mathrm{H}$}

H

$\mathrm{H}$

$\mathrm{H}$

$\mathrm{H}$

\section{$\mathrm{H}$}

\section{H}

$$
\mathrm{H}
$$

$\mathrm{H}$

$\mathrm{H}$

\section{$\mathrm{H}$}

$\begin{array}{llll}6.51810400 & -7.04604200 & 1.58042600\end{array}$ $\begin{array}{llll}2.82382500 & -4.85797500 & 2.30111200\end{array}$ $\begin{array}{llll}3.65519400 & -4.75268500 & 0.77685100\end{array}$ $\begin{array}{llll}2.83008100 & -6.26423000 & 1.21511500\end{array}$ $\begin{array}{llll}5.32613000 & -5.11434600 & 2.57094700\end{array}$ $\begin{array}{llll}-1.52315300 & -5.51536000 & 4.85657300\end{array}$ $\begin{array}{lll}-0.93687000 & -4.17783700 & 5.86944800\end{array}$ $\begin{array}{lll}-0.84585800 & -1.24616300 & 3.19009400\end{array}$ $\begin{array}{llll}-2.20689200 & -1.66337200 & 4.26658800\end{array}$ $\begin{array}{llll}-0.52638600 & -1.66355400 & 4.88138100\end{array}$ $\begin{array}{lll}-4.39054500 & 0.57397000 & 5.09955700\end{array}$ $\begin{array}{lll}-3.92870200 & 0.57177300 & 3.42643400\end{array}$ $\begin{array}{lll}-2.66597600 & 0.53461300 & 4.69362800\end{array}$ $\begin{array}{lll}-2.38182900 & 4.07284500 & 3.48430300\end{array}$ $\begin{array}{lll}-1.45079400 & 2.63348400 & 3.90258400\end{array}$ $\begin{array}{lll}-2.51693000 & 2.62689300 & 2.49263400\end{array}$ $\begin{array}{lll}-4.52388000 & 2.83688600 & 3.97242700\end{array}$ $\begin{array}{lll}-3.35256900 & 4.13785400 & 5.76657200\end{array}$ $\begin{array}{llll}-2.60092800 & 2.65465600 & 6.31747100\end{array}$ $\begin{array}{lll}-4.20526300 & 0.10947700 & 0.55693600\end{array}$ $\begin{array}{lll}-5.65162800 & 0.62974100 & 0.31455000\end{array}$ $\begin{array}{lll}-1.70066100 & 0.31064100 & 0.96013900\end{array}$ $\begin{array}{llll}-3.65332000 & -2.69581100 & -0.52336400\end{array}$ $\begin{array}{llll}5.91184300 & 0.67477200 & -0.80375700\end{array}$ $\begin{array}{llll}-8.17199600 & -4.25329900 & -3.03764000\end{array}$ $\begin{array}{llll}-6.33503600 & -5.23615900 & 0.98995400\end{array}$ $\begin{array}{llll}6.31777200 & -5.33887400 & -2.78286200\end{array}$ $\begin{array}{llll}8.47874100 & -6.31486600 & -2.45147300\end{array}$ $\begin{array}{llll}7.96993400 & -5.16572900 & -0.22374600\end{array}$ $\begin{array}{llll}-4.92167200 & 1.68988900 & 6.76977000\end{array}$ $\begin{array}{lll}-12.15659500 & -2.17402100 & -1.84057000\end{array}$ $\begin{array}{lll}-4.93812800 & 3.11051200 & 1.83260900\end{array}$ $\begin{array}{llll}-1.49303100 & 8.40308300 & -1.36629700\end{array}$ $\begin{array}{llll}-3.28078600 & 1.91499800 & -7.10693300\end{array}$ $\begin{array}{llll}-3.25132800 & 0.14596500 & -7.02779100\end{array}$ $\begin{array}{llll}1.53588400 & -0.64209400 & -5.73288900\end{array}$ $\begin{array}{llll}6.41110900 & 3.72589100 & -3.72526700\end{array}$ $\begin{array}{llll}8.06979400 & 9.01655600 & -0.06598200\end{array}$ $\begin{array}{llll}4.11284600 & 4.34583200 & -3.64773400\end{array}$ $\begin{array}{llll}12.10667500 & 0.38173400 & -3.98670900\end{array}$ $\begin{array}{llll}10.56090400 & 1.14525700 & -4.36232700\end{array}$ $\begin{array}{llll}6.04178600 & 0.34576100 & 0.69664700\end{array}$ $\begin{array}{llll}4.76623900 & -5.21226900 & -3.65274000\end{array}$ $\begin{array}{llll}5.99283100 & -3.91735700 & -3.79532600\end{array}$ $\begin{array}{llll}2.44056300 & 4.88055000 & -3.99181200\end{array}$ $\begin{array}{llll}3.41209300 & 5.81169500 & -2.91050400\end{array}$ $\begin{array}{llll}6.85587300 & 2.30693100 & -4.64863300\end{array}$ $\begin{array}{llll}7.65497000 & 3.84547500 & -4.98445600\end{array}$ $10.90193500 \quad-0.47837400 \quad-4.95655800$ $\begin{array}{llll}2.10139400 & -2.28672800 & -5.38296700\end{array}$ $3.14554100-0.94955200 \quad-5.06594500$ $\begin{array}{llll}-1.74402100 & 1.07361100 & -6.82018400\end{array}$ $\begin{array}{llll}-2.61390700 & -5.51362700 & -4.39356500\end{array}$ $\begin{array}{lll}-7.80241200 & -6.23438500 & 0.94399800\end{array}$ $-6.37724100 \quad-6.69331400 \quad-0.00291700$ $-9.43529900 \quad-5.43811700 \quad-3.32737600$ $\begin{array}{llll}-11.78743100 & -2.08210900 & -0.10642700\end{array}$ $\begin{array}{lll}-6.19898700 & 4.24713800 & 2.32379500\end{array}$ $\begin{array}{llll}-5.92238400 & 3.91330600 & 0.60007800\end{array}$ $\begin{array}{lll}-5.64057600 & 3.21947600 & 6.32367200\end{array}$ $\begin{array}{lll}-4.43055000 & 3.05972300 & 7.73494000\end{array}$ $\begin{array}{llll}0.23688800 & 8.15675100 & -1.19995700\end{array}$ $\begin{array}{llll}-0.61591100 & 7.42112500 & -2.52931400\end{array}$ 


$\begin{array}{rrrr}\mathrm{H} & 7.29814800 & 8.77514200 & 1.53311200 \\ \mathrm{H} & 6.38303200 & 8.40223900 & 0.02626900 \\ \mathrm{H} & 8.85182700 & -7.86353900 & -1.66548000 \\ \mathrm{H} & 10.07329700 & -7.05702100 & -2.59180200 \\ \mathrm{H} & 3.46401100 & -6.86317200 & 5.38560400 \\ \mathrm{H} & 4.55462000 & -5.45175000 & 5.12306000 \\ \mathrm{H} & -2.61108900 & -4.15641600 & 5.24034900 \\ \mathrm{H} & 5.64843700 & -1.23058400 & -0.49130900 \\ \mathrm{C} & 0.55288700 & -0.41521000 & 0.72163400 \\ \mathrm{~N} & 2.64426500 & -2.20752100 & 1.31329100 \\ \mathrm{O} & -0.10009500 & 3.09697700 & -0.18560300 \\ \mathrm{C} & 1.34889100 & -0.21052500 & 1.86012900 \\ \mathrm{O} & 1.38871000 & 3.47979300 & 1.90951600 \\ \mathrm{C} & 1.60427500 & 1.08975300 & 2.31645800 \\ \mathrm{C} & 1.12039600 & 2.21952000 & 1.63131900 \\ \mathrm{C} & 0.31835600 & 1.97977500 & 0.47578800 \\ \mathrm{C} & 0.01626700 & 0.69645300 & 0.03854000 \\ \mathrm{C} & 0.37080900 & -1.83328300 & 0.26033700 \\ \mathrm{C} & 1.73078800 & -2.58150200 & 0.22652300 \\ \mathrm{C} & 4.38261200 & -1.04996200 & 3.31511800 \\ \mathrm{C} & 4.97748700 & 0.20772000 & 3.46283500 \\ \mathrm{C} & 6.33775400 & 0.39601200 & 3.20685600 \\ \mathrm{C} & 7.13545400 & -0.67134400 & 2.77721800 \\ \mathrm{O} & 8.44558600 & -0.43882300 & 2.51080600 \\ \mathrm{C} & 6.55300300 & -1.94119000 & 2.64118700 \\ \mathrm{C} & 5.20076700 & -2.11301200 & 2.90637600 \\ \mathrm{H} & 3.36676000 & -1.59967100 & 0.93841000 \\ \mathrm{H} & -2.78265600 & -1.74056000 & 1.27576200 \\ \mathrm{H} & 3.88727700 & 2.27316400 & 1.17592100 \\ \mathrm{H} & -2.54755700 & 0.41513400 & 2.25577900 \\ \mathrm{H} & 0.27971500 & 3.81318100 & 0.37779300 \\ \mathrm{H} & 2.22792800 & 1.25830500 & 3.18529100 \\ \mathrm{H} & -0.54720100 & 0.55986600 & -0.88060300 \\ \mathrm{H} & -0.10665000 & -1.87663400 & -0.72239200 \\ \mathrm{H} & -0.30032300 & -2.37791000 & 0.93778300 \\ \mathrm{H} & 1.53549400 & -3.66195900 & 0.26307000 \\ \mathrm{H} & 2.23657800 & -2.37604600 & -0.72117400 \\ \mathrm{H} & 4.38736800 & 1.06481100 & 3.77128400 \\ \mathrm{H} & 6.80301200 & 1.36410100 & 3.34988900 \\ \mathrm{H} & 8.74679000 & -1.14069500 & 1.89663400 \\ \mathrm{H} & 7.16021300 & -2.79142000 & 2.35184300 \\ \mathrm{H} & 4.75866900 & -3.09491400 & 2.79230400 \\ \mathrm{C} & 2.92850200 & -1.32764000 & 3.62453300 \\ \mathrm{H} & 2.52795300 & -0.56215300 & 4.29614600 \\ \mathrm{H} & 2.88266800 & -2.27424700 & 4.17368100 \\ \mathrm{C} & 1.98093500 & -1.48024000 & 2.41293000 \\ \mathrm{H} & 1.18011100 & -2.14543700 & 2.75324800 \\ \mathrm{O} & -3.00128300 & -2.66766400 & 1.03908400 \\ \mathrm{H} & -2.46257300 & -3.22830700 & 1.62464700\end{array}$

\section{$\mathrm{E}: \mathrm{P}^{\prime}(+4.2 \mathrm{kcal} / \mathrm{mol})$}

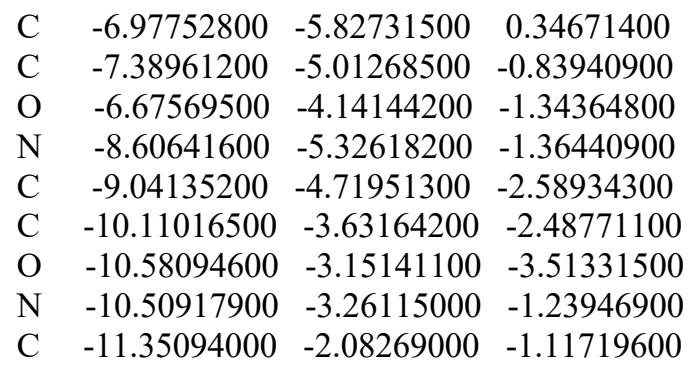

C $\quad-10.49619000 \quad-0.82386700 \quad-1.26107500$

O $\quad-9.53663300 \quad-0.67468600 \quad-0.49536900$

$\begin{array}{llll}\mathrm{N} & -10.81902500 & 0.10668900 & -2.18731500\end{array}$

C $\quad-9.92514200 \quad 1.27263800 \quad-2.34127300$

C $\quad-9.80845000 \quad 2.12006100 \quad-1.07630000$

$\begin{array}{llll}\text { O } & -8.78001700 & 2.74415600 & -0.82846700\end{array}$

C $\quad-10.53504500 \quad 2.02574200 \quad-3.53563400$

C $\quad-11.13628700 \quad 0.89810400 \quad-4.38581900$

C $\quad-11.72604200 \quad-0.06461000 \quad-3.34723600$

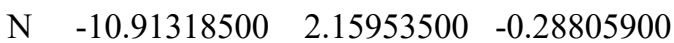

$\begin{array}{llll}\text { C } & -10.92246200 & 2.88232600 & 0.97097000\end{array}$

$\begin{array}{llll}\text { C } & -9.98676800 & 2.34083400 & 2.06202400\end{array}$

O $\quad-9.78330200 \quad 3.01459400 \quad 3.06330400$

$\begin{array}{llll}\mathrm{N} & -9.40116600 & 1.13771900 & 1.83353900\end{array}$

$\begin{array}{llll}\text { C } & -8.30685000 & 0.69176000 & 2.68497500\end{array}$

$\begin{array}{llll}\text { C } & -7.16062900 & 1.71495500 & 2.72871400\end{array}$

$\begin{array}{llll}\text { O } & -6.49674200 & 1.84882800 & 3.74943500\end{array}$

C $\quad-7.82386100 \quad-0.68045600 \quad 2.18057800$

C $\quad-6.56445100 \quad-1.25511200 \quad 2.84386500$

C $\quad-6.68136700 \quad-1.36791800 \quad 4.36680500$

$\begin{array}{llll}\text { C } & -6.24377700 & -2.61312100 & 2.20671600\end{array}$

N $\quad-6.87581400 \quad 2.37335400 \quad 1.56380100$

C $\quad-5.92670400 \quad 3.48557400 \quad 1.58081400$

$\begin{array}{llll}\text { C } & -4.71988900 & 2.79009300 & 6.70845000\end{array}$

$\begin{array}{llll}\text { C } & -3.49370800 & 3.07747900 & 5.81840200\end{array}$

C $\quad-3.57022700 \quad 2.51642000 \quad 4.39298800$

C $\quad-2.40925000 \quad 3.01143100 \quad 3.52044700$

$\begin{array}{llll}\text { C } & -3.62086800 & 0.98507200 & 4.39818600\end{array}$

C $\quad-0.67270400 \quad 7.64991800 \quad-1.47916400$

C $\quad-1.01180000 \quad 6.42550800 \quad-0.63439700$

C $\quad-2.40859300 \quad 5.85899600 \quad-0.95920700$

C $\quad-2.45629600 \quad 5.24707600 \quad-2.36488100$

$\begin{array}{llll}\text { C } & -2.87065100 & 4.84126200 & 0.08898000\end{array}$

$\begin{array}{llll}\text { C } & 7.39204500 & 8.39306900 & 0.49720600\end{array}$

$\begin{array}{llll}\text { C } & 7.85015900 & 6.93385300 & 0.57991300\end{array}$

$\begin{array}{llll}\text { C } & 6.81360500 & 6.01189000 & 1.20438400\end{array}$

$\begin{array}{llll}\text { C } & 5.59086900 & 6.45310000 & 1.72362000\end{array}$

C $\quad 7.06219000 \quad 4.63295400 \quad 1.21947900$

C $\quad 4.62005800 \quad 5.55272500 \quad 2.17396600$

$\begin{array}{llll}\text { C } & 6.10743200 & 3.72708700 & 1.66013500\end{array}$

C $\quad 4.85962800 \quad 4.18265600 \quad 2.10764600$

O $\quad 3.86937400 \quad 3.27683800 \quad 2.40868400$

C $\quad 3.22994200 \quad 4.76748400 \quad-3.22085200$

$\begin{array}{llll}\text { C } & 2.66368400 & 3.90361900 & -2.08982300\end{array}$

C $\quad 3.55493800 \quad 3.74879200 \quad-0.83697300$

C $\quad 4.22850000 \quad 2.38068800 \quad-0.68860300$

O $\quad 4.89407900 \quad 1.92727700 \quad-1.65361500$

$\begin{array}{llll}\text { O } & 4.09838400 & 1.76703100 & 0.43453000\end{array}$

C $\quad-2.79882800 \quad 1.05124200 \quad-6.61282900$

C $\quad-2.97060100 \quad 1.12512700 \quad-5.11054200$

C $\quad-1.92827000 \quad 1.62275800 \quad-4.31671400$

C $\quad-4.15105100 \quad 0.72108800 \quad-4.47235900$

C $\quad-2.05202300 \quad 1.72122200 \quad-2.93097000$

C $\quad-4.28351700 \quad 0.81830200 \quad-3.08525200$

C $\quad-3.23349000 \quad 1.31629300 \quad-2.30959900$

C $\quad-2.81903800 \quad-4.44314100 \quad-4.33206200$

C $\quad-3.33898300 \quad-4.11842800 \quad-2.91740700$

C $\quad-3.68864000 \quad-2.64585500 \quad-2.68965600$

$\mathrm{N} \quad-4.31950300 \quad-2.45902300 \quad-1.33787800$

C $\quad 2.10633700 \quad-1.26925300 \quad-5.01270800$

C $\quad 1.53373600 \quad-1.11090900 \quad-3.60550000$

C $\quad \begin{array}{llll}\text { C } & 1.80328200 & 0.30148200 & -3.07481500\end{array}$

C $\quad 0.03649600 \quad-1.45054500 \quad-3.60966500$ 


\begin{tabular}{|c|c|c|c|}
\hline $\mathrm{C}$ & 28293600 & & \\
\hline C & & & \\
\hline & & & -2.10928700 \\
\hline & 9.83927800 & 3.39524100 & -0.83285300 \\
\hline & 8.97603300 & & 3100 \\
\hline & 11.04971700 & 0.16258000 & -4.09063900 \\
\hline & 10.43739400 & -0.43124300 & 864400 \\
\hline & 9.08947500 & -0.79946100 & 785500 \\
\hline & 11.17509100 & -0.65184600 & 317500 \\
\hline 0 & .49153800 & -1.37969300 & 6900 \\
\hline & 10.59437200 & -1.24282600 & 42500 \\
\hline & 9.25066000 & & 77200 \\
\hline & 40300 & & 8600 \\
\hline & 300 & -4.6 & 1600 \\
\hline C & 400 & -4.0 & 0700 \\
\hline & 5.550 & & 4300 \\
\hline & 6.585 & -3.5 & 3500 \\
\hline & & & 6000 \\
\hline & 700 & -6.8 & 6700 \\
\hline 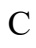 & 9.63 & & -0 . \\
\hline & 500 & & 4500 \\
\hline & 300 & -5 & 9500 \\
\hline & & & 3000 \\
\hline & 600 & & 400 \\
\hline & 4.71 & & 000 \\
\hline & & & 5100 \\
\hline & 200 & & \\
\hline 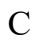 & -1.58 & & 500 \\
\hline$S$ & & & \\
\hline & -1.1 & & 400 \\
\hline & 800 & & 8000 \\
\hline$U$ & 500 & & 9200 \\
\hline & 400 & & 200 \\
\hline r & 200 & & 5700 \\
\hline & -4.62 & 9200 & 4700 \\
\hline & & & \\
\hline H & -2.81 & & 9900 \\
\hline & -4.23 & 5200 & 7500 \\
\hline & 200 & 100 & 8200 \\
\hline & -1.89750000 & & 6600 \\
\hline & -3.56083200 & & 600 \\
\hline & -4.97 & & \\
\hline & & & 2400 \\
\hline & & & \\
\hline 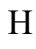 & & & 6700 \\
\hline & -1.0034 & & \\
\hline & -0.50 & 5400 & 3500 \\
\hline & -0.12797100 & & 52300 \\
\hline & -0.41589600 & & 5800 \\
\hline & & 30000 & 7300 \\
\hline & & & \\
\hline & & & \\
\hline & 1.32186700 & & 31800 \\
\hline & & & 6600 \\
\hline & 9.24535200 & 2.46294600 & 09300 \\
\hline & 8800 & 4.19270400 & 6000 \\
\hline & & & 73400 \\
\hline & 7.90877800 & 2.14454500 & 45900 \\
\hline & & & 27200 \\
\hline $\mathrm{H}$ & 9.38682900 & 1.74359300 & 0.86269600 \\
\hline & 7.83367700 & -2.58251300 & 0.26885700 \\
\hline & & -1.62302200 & -1.77705600 \\
\hline & 11.16812000 & -1.42288200 & 0.32954500 \\
\hline
\end{tabular}

$\begin{array}{llll}12.22120000 & -0.36095500 & -1.66090000\end{array}$ $\begin{array}{llll}8.49300000 & -0.62028400 & -3.75880100\end{array}$ $\begin{array}{llll}2.96879700 & 3.94293300 & 0.06083200\end{array}$ $\begin{array}{llll}4.36035200 & 4.49265300 & -0.84982100\end{array}$ $\begin{array}{llll}1.70199700 & 4.32871800 & -1.77977300\end{array}$ $\begin{array}{llll}2.43150300 & 2.91394200 & -2.49322800\end{array}$ $\begin{array}{llll}8.08043700 & 6.55379300 & -0.42327500\end{array}$ $\begin{array}{llll}8.79171400 & 6.86158400 & 1.13948000\end{array}$ $\begin{array}{llll}8.01941100 & 4.27270200 & 0.85628100\end{array}$ $\begin{array}{lll}5.36039200 & 7.51215100 & 1.75736200\end{array}$ $\begin{array}{lll}3.65820300 & 5.90933200 & 2.53026800\end{array}$ $\begin{array}{llll}6.29863300 & 2.66331100 & 1.61628000\end{array}$ $\begin{array}{lll}2.22083500 & 3.53097000 & 2.26969400\end{array}$ $\begin{array}{llll}-3.87086200 & 4.47122400 & -0.15505000\end{array}$ $\begin{array}{lll}-2.19668600 & 3.98151900 & 0.12581600\end{array}$ $\begin{array}{llll}-2.90963300 & 5.28606300 & 1.08946300\end{array}$ $\begin{array}{llll}-3.43858700 & 4.81102600 & -2.57495400\end{array}$ $\begin{array}{llll}-2.24776100 & 5.98805000 & -3.14198500\end{array}$ $\begin{array}{llll}-1.71677700 & 4.44608200 & -2.45652500\end{array}$ $\begin{array}{llll}-3.11239000 & 6.70505300 & -0.93208000\end{array}$ $\begin{array}{llll}-0.25746000 & 5.64356500 & -0.79315100\end{array}$ $\begin{array}{llll}-0.97024000 & 6.69266900 & 0.42914100\end{array}$ $\begin{array}{lll}-7.60721300 & 2.42498800 & 0.85927300\end{array}$ $\begin{array}{lll}-8.65342500 & 0.62659800 & 3.71863700\end{array}$ $\begin{array}{lll}-8.65640400 & -1.38713800 & 2.29929300\end{array}$ $\begin{array}{lll}-7.64064000 & -0.60553400 & 1.10448800\end{array}$ $\begin{array}{llll}-6.99585300 & -3.35861000 & 2.49774500\end{array}$ $\begin{array}{lll}-6.23730100 & -2.54073300 & 1.11502600\end{array}$ $\begin{array}{lll}-5.25921900 & -2.97542300 & 2.51497500\end{array}$ $\begin{array}{lll}-7.54685500 & -1.98103400 & 4.64860100\end{array}$ $\begin{array}{lll}-5.78864400 & -1.84450400 & 4.78644200\end{array}$ $\begin{array}{lll}-6.78359600 & -0.38294600 & 4.82625700\end{array}$ $\begin{array}{lll}-5.73224600 & -0.58155300 & 2.61271300\end{array}$ $\begin{array}{llll}-9.54996600 & 0.63812700 & 0.96374500\end{array}$ $\begin{array}{lll}-10.61102500 & 3.91655100 & 0.80688800\end{array}$ $\begin{array}{lll}-11.94258600 & 2.90063900 & 1.36019500\end{array}$ $\begin{array}{llll}-11.68530800 & 1.55547400 & -0.52441400\end{array}$ $\begin{array}{llll}-12.74798100 & 0.22390300 & -3.07347200\end{array}$ $\begin{array}{llll}-11.88717800 & 1.24895400 & -5.09678800\end{array}$ $\begin{array}{llll}-11.32237400 & 2.70173400 & -3.18409700\end{array}$ $\begin{array}{llll}-10.34751200 & 0.38839000 & -4.94825600\end{array}$ $\begin{array}{llll}-11.71398600 & -1.10256800 & -3.68095800\end{array}$ $\begin{array}{llll}-9.78621400 & 2.62266000 & -4.05784100\end{array}$ $\begin{array}{llll}-8.90918300 & 0.92991600 & -2.55903600\end{array}$ $\begin{array}{llll}-9.88906500 & -3.46821800 & -0.46861200\end{array}$ $\begin{array}{llll}-9.13491200 & -6.08495400 & -0.96076800\end{array}$ $\begin{array}{llll}4.41422200 & -4.90538200 & -1.29498000\end{array}$ $\begin{array}{llll}3.88844400 & -3.53464400 & -2.27568900\end{array}$ $9.72001900-4.17617400 \quad-1.64829800$ $10.54801300 \quad-4.20978700 \quad-0.09041000$ $\begin{array}{llll}11.28887500 & -4.98314200 & -1.51339400\end{array}$ $10.30444000 \quad-6.70417500 \quad-0.01251200$ $\begin{array}{llll}2.95105500 & -5.51479200 & 4.37990300\end{array}$ $\begin{array}{lll}5.34972400 & -7.34834200 & 3.78785100\end{array}$ $\begin{array}{llll}3.74319100 & -7.66679600 & 3.16020400\end{array}$ $\begin{array}{llll}5.01132700 & -7.63354600 & 0.86449300\end{array}$ $\begin{array}{llll}5.75420300 & -6.11030900 & 0.33809200\end{array}$ $\begin{array}{llll}6.51452600 & -7.04526700 & 1.60704500\end{array}$ $\begin{array}{llll}2.79972100 & -4.88493800 & 2.30028000\end{array}$ $\begin{array}{llll}3.63633300 & -4.78758100 & 0.77622700\end{array}$ $\begin{array}{llll}2.82169000 & -6.30134800 & 1.22641000\end{array}$ $\begin{array}{lll}5.30176200 & -5.11443200 & 2.57838700\end{array}$ $\begin{array}{llll}-1.54398100 & -5.50176600 & 4.85111200\end{array}$ 


\begin{tabular}{|c|c|c|c|}
\hline $\mathrm{H}$ & -0.91952100 & -4.17875400 & \\
\hline $\mathrm{H}$ & & & \\
\hline & -2.19796900 & & \\
\hline & -0.49115300 & & 7924000 \\
\hline & -4.35638100 & & \\
\hline & -3.93552700 & 0.60095200 & 42215700 \\
\hline & -2.64178800 & & \\
\hline & -2.38000900 & & \\
\hline & -1.43796400 & & \\
\hline & -2.4 & & \\
\hline & -4.5 & 2.86178900 & 87800 \\
\hline & & & \\
\hline & -2.5 & & \\
\hline & -4.2 & & \\
\hline & & & \\
\hline & -1.7 & 0.288 & \\
\hline & -3.6 & -2.691 & \\
\hline & & & \\
\hline & -8.1 & -4.2 & 300 \\
\hline & & & \\
\hline & & -5.3 & \\
\hline & 8.4 & -6.3 & -2.4 \\
\hline & & -5.1 & -0.2 \\
\hline & -4.9 & $1.712^{\prime}$ & \\
\hline & -12.1 & & 500 \\
\hline & -4.9 & & \\
\hline & -1.4 & & \\
\hline & & & \\
\hline & -3.2 & & \\
\hline & & -0.6 & \\
\hline & & & \\
\hline & & & \\
\hline & 4.1 & & \\
\hline $\mathrm{H}$ & & & \\
\hline & 10.5 & & 000 \\
\hline 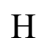 & & & \\
\hline & & & \\
\hline & & & \\
\hline & & & \\
\hline & & & \\
\hline & & & \\
\hline & & & \\
\hline & 10.9 & & 7400 \\
\hline & & & \\
\hline & & -0.9 & \\
\hline $\mathrm{H}$ & -1.72 & & \\
\hline $\mathrm{H}$ & -2.6 & -5.5 & -4 \\
\hline & -7.80 & & \\
\hline & & & \\
\hline $\mathrm{H}$ & -9.4 & -5.424 & 49000 \\
\hline & -11.7 & & 557700 \\
\hline $\mathrm{H}$ & -6.18 & 4.26426700 & 72100 \\
\hline $\mathrm{H}$ & -5.91217300 & & \\
\hline $\mathrm{H}$ & -5.6 & & \\
\hline $\mathrm{H}$ & -4.42 & & 48200 \\
\hline $\mathrm{H}$ & & & 86300 \\
\hline $\mathrm{H}$ & -0.588 & & -2.54509600 \\
\hline $\mathrm{H}$ & & 8.76292500 & 1.52479600 \\
\hline $\mathrm{H}$ & & & \\
\hline $\mathrm{H}$ & & -7.88491700 & -1.64097200 \\
\hline $\mathrm{H}$ & 10.07025700 & -7.08262700 & -2.56726600 \\
\hline $\mathrm{H}$ & & -6.86020900 & 5.40143400 \\
\hline & 4.54522400 & -5.45155900 & 5.13761800 \\
\hline
\end{tabular}

$\begin{array}{lrrr}\mathrm{H} & -2.60968000 & -4.13709000 & 5.27464600 \\ \mathrm{H} & 5.58198900 & -1.12419800 & -0.51429000 \\ \mathrm{C} & 0.54948500 & -0.46173300 & 0.70784000 \\ \mathrm{~N} & 2.64357600 & -2.24187400 & 1.32923500 \\ \mathrm{O} & -0.15847400 & 3.01474800 & -0.22372900 \\ \mathrm{C} & 1.32706700 & -0.26602900 & 1.86381700 \\ \mathrm{O} & 1.26631800 & 3.43404300 & 1.98814900 \\ \mathrm{C} & 1.55037300 & 1.03322800 & 2.32802300 \\ \mathrm{C} & 1.04541700 & 2.13261700 & 1.63814200 \\ \mathrm{C} & 0.28384200 & 1.93234000 & 0.47609400 \\ \mathrm{C} & 0.00950500 & 0.63975000 & 0.02817500 \\ \mathrm{C} & 0.39181500 & -1.87678800 & 0.23005800 \\ \mathrm{C} & 1.76334300 & -2.60530200 & 0.21441300 \\ \mathrm{C} & 4.32704700 & -1.05872100 & 3.36477100 \\ \mathrm{C} & 4.88453500 & 0.21584000 & 3.51680000 \\ \mathrm{C} & 6.23052300 & 0.45356600 & 3.24034800 \\ \mathrm{C} & 7.05142700 & -0.58335100 & 2.78302800 \\ \mathrm{O} & 8.34574600 & -0.31035300 & 2.48254300 \\ \mathrm{C} & 6.51395500 & -1.87385100 & 2.66275600 \\ \mathrm{C} & 5.17339400 & -2.09449200 & 2.94653000 \\ \mathrm{H} & 3.38941500 & -1.64469500 & 0.98425800 \\ \mathrm{H} & -2.80457700 & -1.75416000 & 1.27194200 \\ \mathrm{H} & 3.92423300 & 2.56604600 & 1.64500000 \\ \mathrm{H} & -2.57734000 & 0.41207600 & 2.25970800 \\ \mathrm{H} & 0.20402300 & 3.79257100 & 0.23828600 \\ \mathrm{H} & 2.15974200 & 1.21588900 & 3.20379500 \\ \mathrm{H} & -0.53502900 & 0.50690300 & -0.90144100 \\ \mathrm{H} & -0.06809400 & -1.91522100 & -0.76022700 \\ \mathrm{H} & -0.28507700 & -2.42576700 & 0.89626700 \\ \mathrm{H} & 1.57694500 & -3.68756200 & 0.22357200 \\ \mathrm{H} & 2.28866200 & -2.37350700 & -0.71560600 \\ \mathrm{H} & 4.27312200 & 1.05209100 & 3.84075300 \\ \mathrm{H} & 6.65587200 & 1.44268600 & 3.36086600 \\ \mathrm{H} & 8.65663300 & -1.01263100 & 1.87415800 \\ \mathrm{H} & 7.14605900 & -2.70090300 & 2.36122500 \\ \mathrm{H} & 4.76397400 & -3.09100800 & 2.83432200 \\ \mathrm{H} & 2.87819500 & -1.37760600 & 3.65338000 \\ \mathrm{H} & 2.44562000 & -0.63375600 & 4.33105600 \\ \mathrm{H} & 1.95761600 & -1.53285900 & 2.42159400 \\ \mathrm{H} & -2.99775500 & -2.67941600 & 1.01231000 \\ \mathrm{H} & -3.24150800 & 1.62817000\end{array}$

